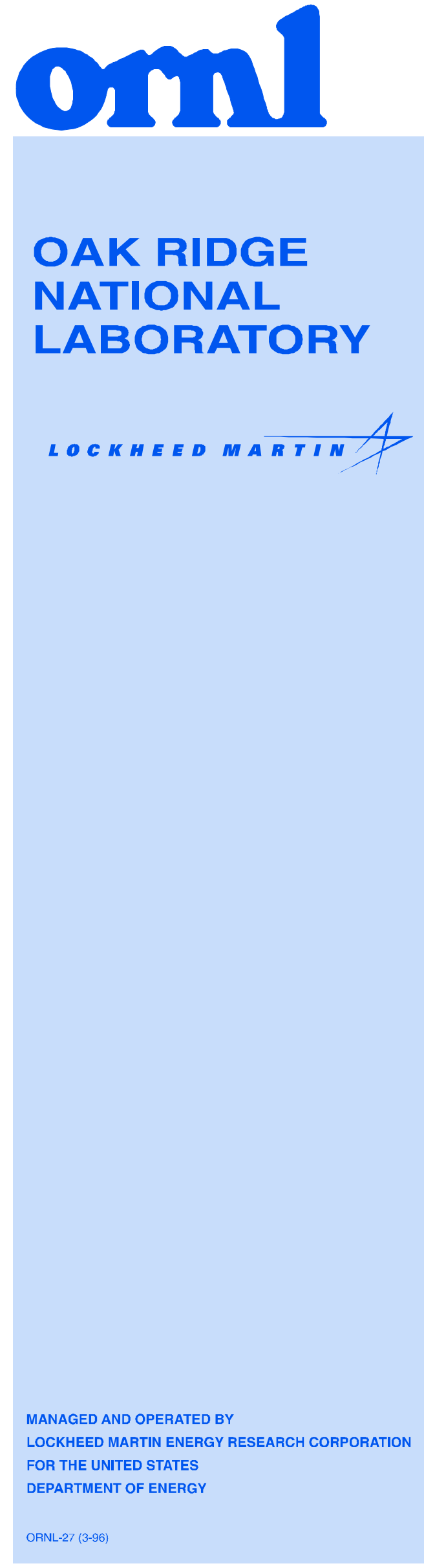

ORNL/TM-1999/299

\title{
Neutronics Benchmarks of Mixed-Oxide Fuels using the SCALE/CENTRM Sequence
}

\author{
D. F. Hollenbach
}

P. B. Fox 
This report has been reproduced directly from the best available copy.

Available to DOE and DOE contractors form the Office of Scientific and Technical Information, P.O. Box 62, Oak Ridge, TN 37831; prices available from (615) 576-8401.

Available to the public from the National Technical Information Service, U.S. Department of Commerce, 5285 Port Royal Rd., Springfield, VA 22161.

This report was prepared as an account of work sponsored by an agency of the United States Government. Neither the United States nor any agency thereof, nor any of their employees, makes any warranty, express or implied, or assumes any legal liability or responsibility for the accuracy, completeness, or usefulness of any information, apparatus, product, or process disclosed, or represents that its use would not infringe privately owned rights. Reference herein to any specific commercial product, process, or service by trade name, trademark, manufacturer, or otherwise, does not necessarily constitute or imply its endorsement, recommendation, or favoring by the United States Government or any agency thereof. The views and opinions of authors expressed herein do not necessarily state or reflect those of the United States Government or any agency thereof. 
ORNL/TM-1999/168

Computational Physics and Engineering Division

\section{Neutronics Benchmarks of Mixed-Oxide Fuels using the SCALE/CENTRM Sequence}

D. F. Hollenbach

P. B. Fox

Date Published: February 2000

Prepared by the

OAK RIDGE NATIONAL LABORATORY

Oak Ridge, Tennessee 37831-6370

managed by

LOCKHEED MARTIN ENERGY RESEARCH CORP.

for the

U.S. DEPARTMENT OF ENERGY

under contract DE-AC05-96OR22464 



\section{CONTENTS}

\section{Page}

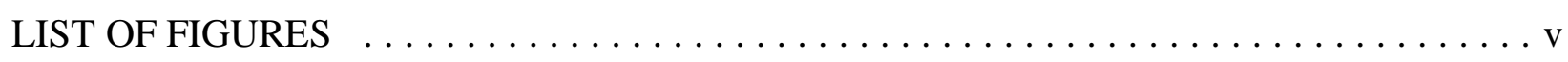

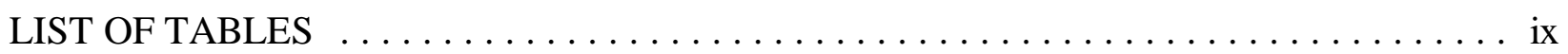

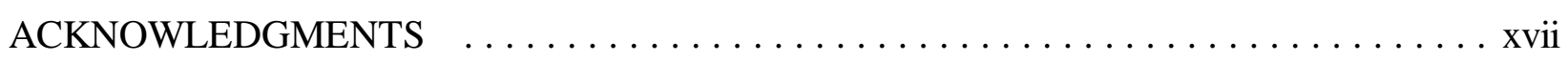

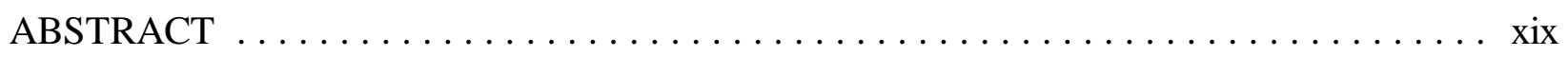

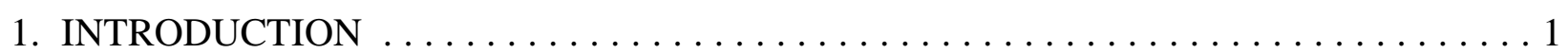

1.1 THE SCALE 5.0 SEQUENCES $\ldots \ldots \ldots \ldots \ldots \ldots \ldots \ldots \ldots \ldots \ldots \ldots \ldots$

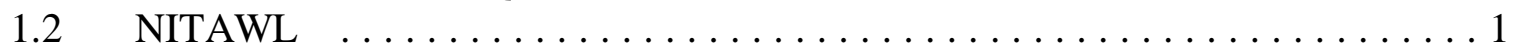

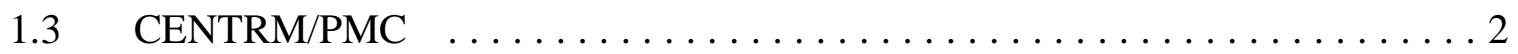

2. MIX-COMP-THERM-2 (PNL-30 to PNL-35) $\ldots \ldots \ldots \ldots \ldots \ldots \ldots \ldots \ldots \ldots \ldots$

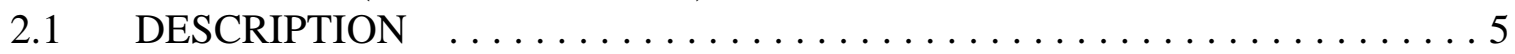

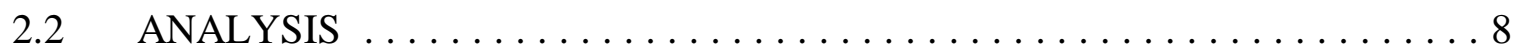

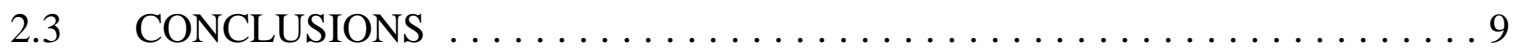

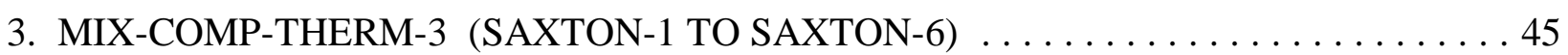

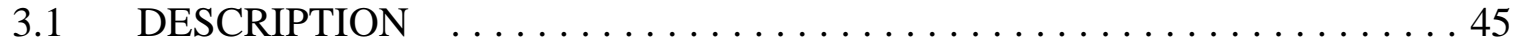

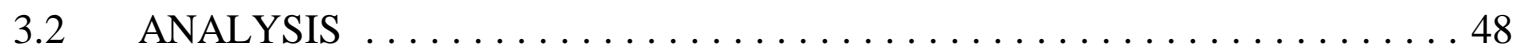

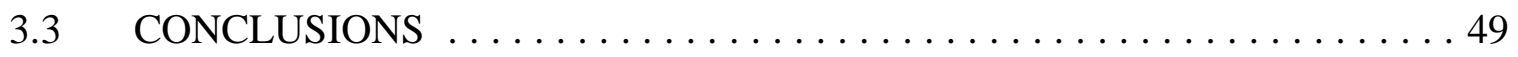

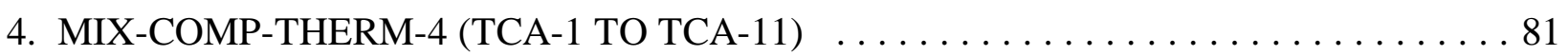

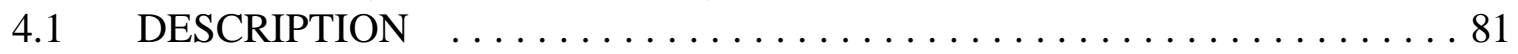

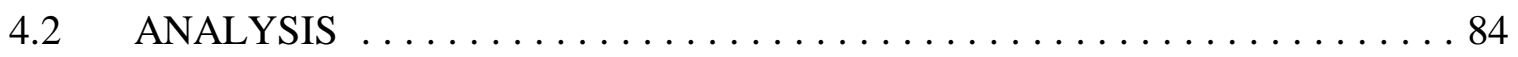

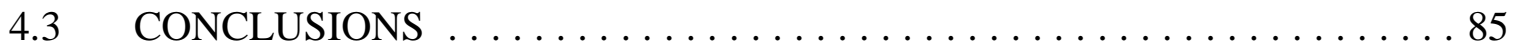

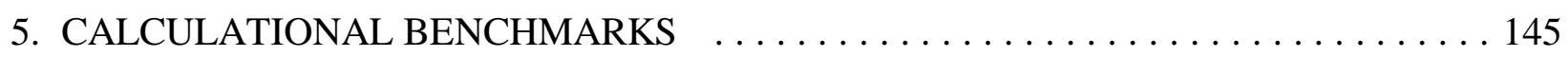

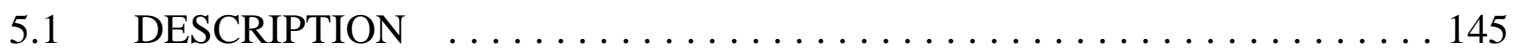

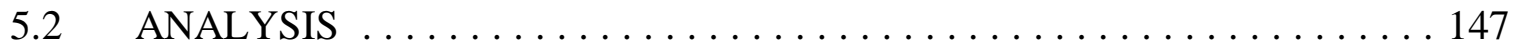

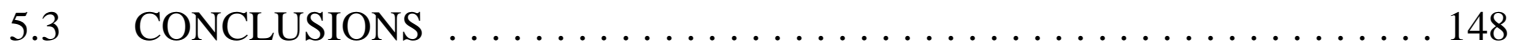

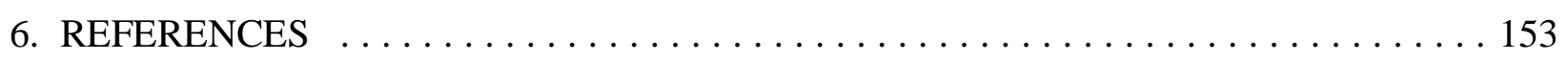





\section{LIST OF FIGURES}

\begin{tabular}{|c|c|}
\hline Figure & $\underline{\text { Page }}$ \\
\hline 2.1 & Schematic of the PNL fuel rods with reflector \\
\hline 2.2 & $\begin{array}{lll}\text { Pin layout of benchmark case PNL-30 } \ldots . . . \\
\end{array}$ \\
\hline 2.3 & Pin layout of benchmark cases PNL-31 and PNL-33 \\
\hline 2.4 & Pin layout of benchmark case PNL-32 $\ldots \ldots \ldots$ \\
\hline 2.5 & Pin layout of benchmark case PNL-34 \\
\hline 2.6 & Pin layout of benchmark case PNL-35 . . . . . . . . . . . \\
\hline $2.7 \mathrm{a}$ & $\begin{array}{l}\text { Pin-power distribution for CENTRM benchmark PNL-30. Value in } \\
\text { parentheses is the percent standard deviation. } \ldots \ldots \ldots \ldots \ldots \ldots\end{array}$ \\
\hline $2.7 \mathrm{~b}$ & $\begin{array}{l}\text { Pin-power distribution for NITAWL benchmark PNL-30. Value in } \\
\text { parentheses is the percent standard deviation. . . . . . . . . . . }\end{array}$ \\
\hline $2.8 \mathrm{a}$ & $\begin{array}{l}\text { Pin-power distribution for CENTRM benchmark PNL-31. Value in } \\
\text { parentheses is the percent standard deviation. . . . . . . . . . . }\end{array}$ \\
\hline $2.8 \mathrm{~b}$ & $\begin{array}{l}\text { Pin-power distribution for NITAWL benchmark PNL-31. Value in } \\
\text { parentheses is the percent standard deviation. . . . . . . . . . . . . }\end{array}$ \\
\hline $2.9 \mathrm{a}$ & $\begin{array}{l}\text { Pin-power distribution for CENTRM benchmark PNL-32 } \\
\text { with standard deviation between } 0.59 \text { and } 0.87 \% \text { of the value }\end{array}$ \\
\hline $2.9 \mathrm{~b}$ & $\begin{array}{l}\text { Pin-power distribution for NITAWL benchmark PNL-32 } \\
\text { with standard deviation between } 0.57 \text { and } 0.90 \% \text { of the value } \ldots\end{array}$ \\
\hline $2.10 \mathrm{a}$ & $\begin{array}{l}\text { Pin-power distribution for CENTRM benchmark PNL-33. Value in } \\
\text { parentheses is the percent standard deviation. . . . . . . . . . }\end{array}$ \\
\hline $2.10 \mathrm{~b}$ & $\begin{array}{l}\text { Pin-power distribution for NITAWL benchmark PNL-33. Value in } \\
\text { parentheses is the percent standard deviation. . . . . . . . . . . }\end{array}$ \\
\hline $2.11 \mathrm{a}$ & $\begin{array}{l}\text { Pin-power distribution for CENTRM benchmark PNL-34. Value in } \\
\text { parentheses is the percent standard deviation. . . . . . . . . . . . }\end{array}$ \\
\hline $2.11 \mathrm{~b}$ & $\begin{array}{l}\text { Pin-power distribution for NITAWL benchmark PNL-34. Value in } \\
\text { parentheses is the percent standard deviation. . . . . . . . . . . . }\end{array}$ \\
\hline $2.12 \mathrm{a}$ & $\begin{array}{l}\text { Pin-power distribution for CENTRM benchmark PNL-35. Value in } \\
\text { parentheses is the percent standard deviation. . . . . . . . . . }\end{array}$ \\
\hline $2.12 \mathrm{~b}$ & $\begin{array}{l}\text { Pin-power distribution for NITAWL benchmark PNL-35. Value in } \\
\text { parentheses is the percent standard deviation. . . . . . . . . . . }\end{array}$ \\
\hline 3.1 & Schematic of the SAXTON fuel rods with reflector $\ldots \ldots \ldots \ldots$ \\
\hline 3.2 & Pin layout of benchmark case SAXTON-1 . . . . . . \\
\hline 3.3 & Pin layout of benchmark case SAXTON-2 \\
\hline 3.4 & Pin layout of benchmark case SAXTON-3 \\
\hline 3.5 & Pin layout of benchmark case SAXTON-4 . . . . \\
\hline 3.6 & Pin layout of benchmark cases SAXTON-5 and $6 \ldots \ldots \ldots$ \\
\hline $3.7 \mathrm{a}$ & $\begin{array}{l}\text { Pin-power distribution for CENTRM benchmark SAXTON-1. Value in } \\
\text { parentheses is the percent standard deviation. . . . . . . . . . . . . }\end{array}$ \\
\hline
\end{tabular}




\section{LIST OF FIGURES (continued)}

\begin{tabular}{|c|c|}
\hline $3.7 \mathrm{~b}$ & $\begin{array}{l}\text { Pin-power distribution for NITAWL benchmark SAXTON-1. Value in } \\
\text { parentheses is the percent standard deviation. . . . . . . . . . . . }\end{array}$ \\
\hline 3.8a & $\begin{array}{l}\text { Pin-power distribution for CENTRM benchmark SAXTON-2. Value in } \\
\text { parentheses is the percent standard deviation. . . . . . . . . . . . . . }\end{array}$ \\
\hline $3.8 \mathrm{~b}$ & 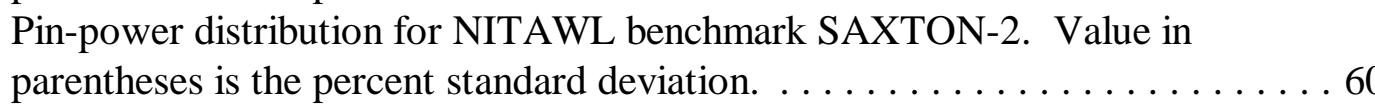 \\
\hline $3.9 \mathrm{a}$ & 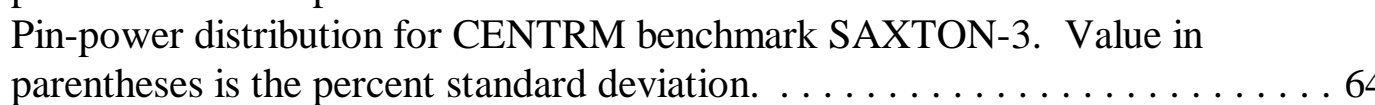 \\
\hline $3.9 \mathrm{~b}$ & $\begin{array}{l}\text { Pin-power distribution for NITAWL benchmark SAXTON-3. Value in } \\
\text { parentheses is the percent standard deviation. . . . . . . . . . . . . . }\end{array}$ \\
\hline $3.10 \mathrm{a}$ & 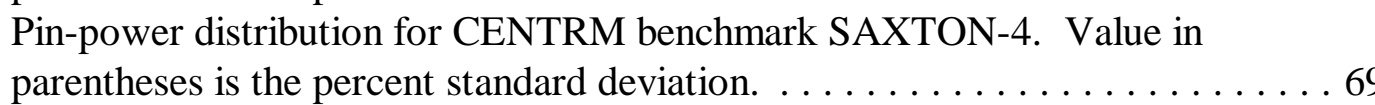 \\
\hline $3.10 \mathrm{~b}$ & 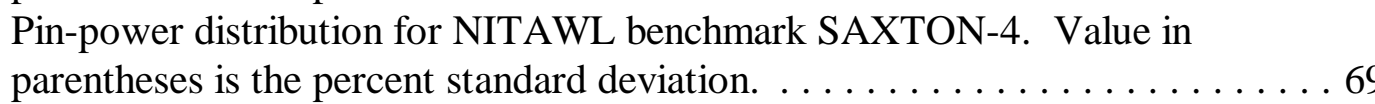 \\
\hline $3.11 \mathrm{a}$ & 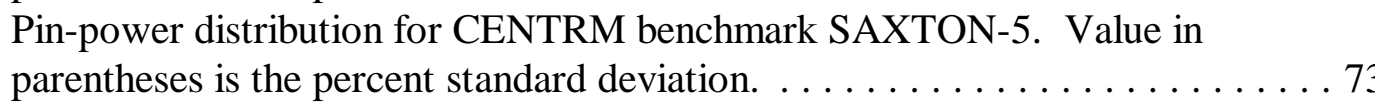 \\
\hline $3.11 \mathrm{~b}$ & $\begin{array}{l}\text { Pin-power distribution for NITAWL Benchmark SAXTON-5. Value in } \\
\text { parentheses is the percent standard deviation. . . . . . . . . . . . . . . }\end{array}$ \\
\hline $3.12 \mathrm{a}$ & $\begin{array}{l}\text { Pin-power distribution for CENTRM benchmark SAXTON-6. Value in } \\
\text { parentheses is the percent standard deviation. . . . . . . . . . . }\end{array}$ \\
\hline $3.12 \mathrm{~b}$ & $\begin{array}{l}\text { Pin-power distribution for NITAWL benchmark SAXTON-6. Value in } \\
\text { parentheses is the percent standard deviation. } \ldots \ldots \ldots \ldots \ldots \ldots \ldots\end{array}$ \\
\hline 4.1 & Schematic of fuel rods and bottom reflector $\ldots \ldots \ldots \ldots$ \\
\hline 4.2 & Pin layout of benchmark cases TCA-1, TCA-2, and TCA-3 \\
\hline 4.3 & Pin lavout of benchmark cases TCA-4, TCA- 5 ,TCA- 6 , TCA-10, and TCA-11. 88 \\
\hline 4.4 & Pin lavout of benchmark cases TCA-7, TCA-8, and TCA-9 $\ldots \ldots \ldots \ldots$ \\
\hline $4.5 \mathrm{a}$ & $\begin{array}{l}\text { Pin-power distribution for CENTRM benchmark TCA-1. Value in } \\
\text { parentheses is the percent standard deviation. } \ldots \ldots \ldots \ldots \ldots \text {. }\end{array}$ \\
\hline $4.5 \mathrm{~b}$ & $\begin{array}{l}\text { Pin-power distribution for NITAWL benchmark TCA-1. Value in } \\
\text { parentheses is the percent standard deviation. . . . . . . . . . }\end{array}$ \\
\hline $4.6 \mathrm{a}$ & $\begin{array}{l}\text { Pin-power distribution for CENTRM benchmark TCA-2. Value in } \\
\text { parentheses is the percent standard deviation. . . . . . . . . . }\end{array}$ \\
\hline $4.6 \mathrm{~b}$ & $\begin{array}{l}\text { Pin-power distribution for NITAWL benchmark TCA-2. Value in } \\
\text { parentheses is the percent standard deviation. . . . . . . . . }\end{array}$ \\
\hline $4.7 \mathrm{a}$ & $\begin{array}{l}\text { Pin-power distribution for CENTRM benchmark TCA-3. Value in } \\
\text { parentheses is the percent standard deviation. . . . . . . . . . . }\end{array}$ \\
\hline $4.7 \mathrm{~b}$ & $\begin{array}{l}\text { Pin-power distribution for NITAWL benchmark TCA-3. Value in } \\
\text { parentheses is the percent standard deviation. . . . . . . . . . }\end{array}$ \\
\hline
\end{tabular}




\section{LIST OF FIGURES (continued)}

\begin{tabular}{|c|c|}
\hline $4.8 \mathrm{a}$ & $\begin{array}{l}\text { Pin-power distribution for CENTRM benchmark TCA-4. Value in } \\
\text { parentheses is the percent standard deviation. . . . . . . . . . }\end{array}$ \\
\hline $4.8 \mathrm{~b}$ & $\begin{array}{l}\text { Pin-power distribution for NITAWL benchmark TCA-4. Value in } \\
\text { parentheses is the percent standard deviation. . . . . . . . . . }\end{array}$ \\
\hline $4.9 \mathrm{a}$ & $\begin{array}{l}\text { Pin-power distribution for CENTRM benchmark TCA-5. Value in } \\
\text { parentheses is the percent standard deviation. . . . . . . . . . . }\end{array}$ \\
\hline $4.9 b$ & $\begin{array}{l}\text { Pin-power distribution for NITAWL benchmark TCA-5. Value in } \\
\text { parentheses is the percent standard deviation. . . . . . . . . }\end{array}$ \\
\hline $4.10 \mathrm{a}$ & $\begin{array}{l}\text { Pin-power distribution for CENTRM benchmark TCA-6. Value in } \\
\text { parentheses is the percent standard deviation. . . . . . . . . . . }\end{array}$ \\
\hline $4.10 \mathrm{~b}$ & $\begin{array}{l}\text { Pin-power distribution for NITAWL benchmark TCA-6. Value in } \\
\text { parentheses is the percent standard deviation. . . . . . . . . . . }\end{array}$ \\
\hline $4.11 \mathrm{a}$ & $\begin{array}{l}\text { Pin-power distribution for CENTRM benchmark TCA-7. Value in } \\
\text { parentheses is the percent standard deviation. . . . . . . . . }\end{array}$ \\
\hline $4.11 b$ & $\begin{array}{l}\text { Pin-power distribution for NITAWL benchmark TCA-7. Value in } \\
\text { parentheses is the percent standard deviation. . . . . . . . . . }\end{array}$ \\
\hline $4.12 \mathrm{a}$ & $\begin{array}{l}\text { Pin-power distribution for CENTRM benchmark TCA-8. Value in } \\
\text { parentheses is the percent standard deviation. . . . . . . . . . . }\end{array}$ \\
\hline $4.12 b$ & $\begin{array}{l}\text { Pin-power distribution for NITAWL benchmark TCA-8. Value in } \\
\text { parentheses is the percent standard deviation. . . . . . . . . . . }\end{array}$ \\
\hline $4.13 \mathrm{a}$ & $\begin{array}{l}\text { Pin-power distribution for CENTRM benchmark TCA-9. Value in } \\
\text { parentheses is the percent standard deviation. . . . . . . . . . }\end{array}$ \\
\hline $4.13 b$ & $\begin{array}{l}\text { Pin-power distribution for NITAWL benchmark TCA-9. Value in } \\
\text { parentheses is the percent standard deviation. . . . . . . . . . }\end{array}$ \\
\hline $4.14 \mathrm{a}$ & $\begin{array}{l}\text { Pin-power distribution for CENTRM benchmark TCA-10. Value in } \\
\text { parentheses is the percent standard deviation. . . . . . . . . . }\end{array}$ \\
\hline $4.14 b$ & $\begin{array}{l}\text { Pin-power distribution for NITAWL benchmark TCA-10. Value in } \\
\text { parentheses is the percent standard deviation. . . . . . . . . . }\end{array}$ \\
\hline $4.15 \mathrm{a}$ & $\begin{array}{l}\text { Pin-power distribution for CENTRM benchmark TCA-11. Value in } \\
\text { parentheses is the percent standard deviation. . . . . . . . . . . }\end{array}$ \\
\hline $4.15 b$ & $\begin{array}{l}\text { Pin-power distribution for NITAWL benchmark TCA-11. Value in } \\
\text { parentheses is the percent standard deviation. . . . . . . . . . . }\end{array}$ \\
\hline
\end{tabular}





\section{LIST OF TABLES}

$\underline{\text { Table }}$

Page

2.1

2.2

2.3

2.4

2.5

2.6

2.7

2.8

2.9

2.10

2.11

2.12

2.13

2.14

2.15

2.16

2.17

2.18

2.19

2.20

2.21

2.22

2.23

2.24

2.25

2.26

2.27

2.28

2.29

2.30

2.31

2.32

2.33

2.34

2.35

2.36

2.37

Lattice description for benchmarks $\ldots \ldots \ldots \ldots \ldots \ldots \ldots \ldots \ldots \ldots$

Constant benchmark atom densities $\ldots \ldots \ldots \ldots \ldots \ldots \ldots \ldots \ldots \ldots$

Moderator atom densities . . . . . . . . . . . . . . . . . . . 8

Comparison of $k_{\text {off }}$ and EALCF from CENTRM and NITAWL $\ldots \ldots \ldots \ldots 10$

PNL-30 CENTRM reaction rates and fluxes $\ldots \ldots \ldots \ldots \ldots \ldots \ldots \ldots$

PNL-30 NITAWL reaction rates and fluxes $\ldots \ldots \ldots \ldots \ldots \ldots \ldots \ldots$

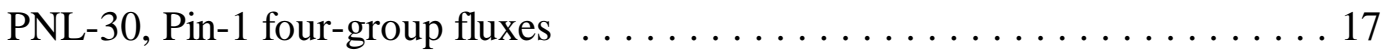

PNL-30, Pin-1 four-group U-235 cross sections $\ldots \ldots \ldots \ldots \ldots \ldots \ldots \ldots$

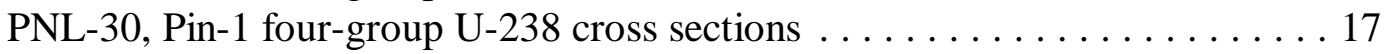

PNL-30, Pin-1 four-group Pu-239 cross sections $\ldots \ldots \ldots \ldots \ldots \ldots \ldots 17$

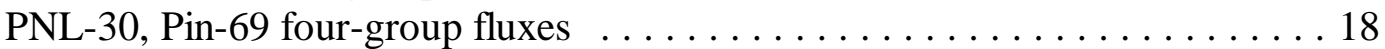

PNL-30, Pin-69 four-group U-235 cross sections $\ldots \ldots \ldots \ldots \ldots \ldots \ldots$

PNL-30, Pin-69 four-group U-238 cross sections . . . . . . . . . . . . 18

PNL-30, Pin-691 four-group Pu-239 cross sections $\ldots \ldots \ldots \ldots \ldots \ldots . . \ldots$

PNL-31 CENTRM reaction rates and fluxes $\ldots \ldots \ldots \ldots \ldots \ldots \ldots \ldots 21$

PNL-31 NITAWL reaction rates and fluxes $\ldots \ldots \ldots \ldots \ldots \ldots \ldots \ldots 21$

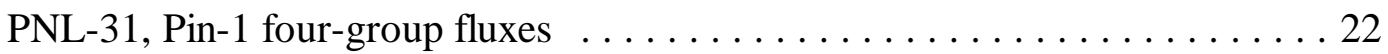

PNL-31, Pin-1 four-group U-235 cross sections $\ldots \ldots \ldots \ldots \ldots \ldots \ldots \ldots 22$

PNL-31, Pin-1 four-group U-238 cross sections . . . . . . . . . . . . 22

PNL-31, Pin-1 four-group Pu-239 cross sections $\ldots \ldots \ldots \ldots \ldots \ldots \ldots 22$

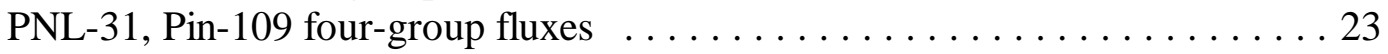

PNL-31, Pin-109 four-group U-235 cross sections . . . . . . . . . . . . 23

PNL-31, Pin-109 four-group U-238 cross sections . . . . . . . . . . . . 23

PNL-31, Pin-109 four-group Pu-239 cross sections $\ldots \ldots \ldots \ldots \ldots \ldots 23$

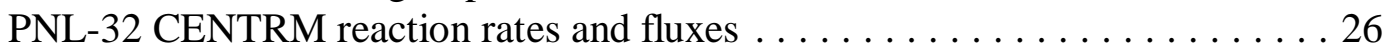

PNL-32 NITAWL reaction rates and fluxes $\ldots \ldots \ldots \ldots \ldots \ldots \ldots \ldots . \ldots \ldots$

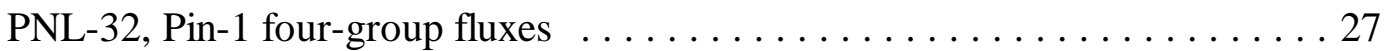

PNL-32, Pin-1 four-group U-235 cross sections $\ldots \ldots \ldots \ldots \ldots \ldots \ldots \ldots 27$

PNL-32, Pin-1 four-group U-238 cross sections . . . . . . . . . . . . . 27

PNL-32, Pin-1 four-group Pu-239 cross sections . . . . . . . . . . . . 27

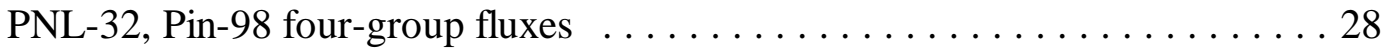

PNL-32, Pin-98 four-group U-235 cross sections . . . . . . . . . . . . 28

PNL-32, Pin-98 four-group U-238 cross sections . ................. 28

PNL-32, Pin-98 four-group Pu-239 cross sections . . . . . . . . . . . . . 28

PNL-33 CENTRM reaction rates and fluxes $\ldots \ldots \ldots \ldots \ldots \ldots \ldots \ldots \ldots \ldots$

PNL-33 NITAWL reaction rates and fluxes $\ldots \ldots \ldots \ldots \ldots \ldots \ldots \ldots \ldots \ldots$

PNL-33, Pin-1 four-group fluxes $\ldots \ldots \ldots \ldots \ldots \ldots \ldots \ldots \ldots \ldots \ldots \ldots \ldots \ldots$

ix 


\section{LIST OF TABLES (continued)}

\begin{tabular}{|c|c|}
\hline 2.38 & PNL-33, Pin-1 four-group U-235 cross sections \\
\hline 2.39 & PNL-33, Pin-1 four-group U-238 cross sections \\
\hline 2.40 & PNL-33, Pin-1 four-group Pu-239 cross sections \\
\hline 2.41 & PNL-33, Pin-109 four-group fluxes $\ldots \ldots \ldots$ \\
\hline 2.42 & PNL-33, Pin-109 four-group U-235 cross sections \\
\hline 2.43 & PNL-33, Pin-109 four-group U-238 cross sections \\
\hline 2.44 & PNL-33, Pin-109 four-group Pu-239 cross sections \\
\hline 2.45 & PNL-34 CENTRM reaction rates and fluxes ...... \\
\hline 2.46 & PNL-34 CENTRM reaction rates and fluxes \\
\hline 2.47 & PNL-34, Pin-1 four-group fluxes $\ldots \ldots \ldots$ \\
\hline 2.48 & PNL-34, Pin-1 four-group U-235 cross sections \\
\hline 2.49 & PNL-34, Pin-1 four-group U-238 cross sections. \\
\hline 2.50 & PNL-34, Pin-1 four-group Pu-239 cross sections \\
\hline 2.51 & PNL-34, Pin-27 four-group fluxes $\ldots \ldots \ldots$ \\
\hline 2.52 & PNL-34, Pin-27 four-group U-235 cross sections \\
\hline 2.53 & PNL-34, Pin-27 four-group U-238 cross sections \\
\hline 2.54 & PNL-34, Pin-27 four-group Pu-239 cross sections \\
\hline 2.55 & PNL-35 CENTRM reaction rates and fluxes ..... \\
\hline 2.56 & PNL-35 NITAWL reaction rates and fluxes \\
\hline 2.57 & PNL-35, Pin-1 four-group fluxes $\ldots \ldots \ldots$ \\
\hline 2.58 & PNL-35, Pin-1 four-group U-235 cross sections \\
\hline 2.59 & PNL-35, Pin-1 four-group U-238 cross sections \\
\hline 2.60 & PNL-35, Pin-1 four-group Pu-239 cross sections \\
\hline 2.61 & PNL-35, Pin-99 four-group fluxes $\ldots \ldots \ldots$ \\
\hline 2.62 & PNL-35, Pin-99 four-group U-235 cross sections \\
\hline 2.63 & PNL-35, Pin-99 four-group U-238 cross sections, \\
\hline 2.64 & PNL-35, Pin-99 four-group Pu-239 cross sections \\
\hline 3.1 & Lattice description for SAXTON benchmark cases \\
\hline 3.2 & Constant benchmark atom densities $\ldots \ldots \ldots \ldots$ \\
\hline 3.3 & Moderator atom densities $\ldots \ldots \ldots \ldots \ldots \ldots \ldots \ldots$ \\
\hline 3.4 & Comparison of $k_{\text {off }}$ and EALCF from CENTRM and NITAWL \\
\hline 3.5 & SAXTON-1 CENTRM reaction rates and fluxes $\ldots \ldots \ldots$ \\
\hline 3.6 & SAXTON-1 NITAWL reaction rates and fluxes \\
\hline 3.7 & SAXTON-1, Pin-1 four-group fluxes $\ldots \ldots \ldots$ \\
\hline 3.8 & SAXTON-1, Pin-1 four-group U-235 cross sections \\
\hline 3.9 & SAXTON-1, Pin-1 four-group U-238 cross sections. \\
\hline 3.10 & SAXTON-1, Pin-1 four-group Pu-239 cross sections \\
\hline 3.11 & SAXTON-1, Pin-132 four-group fluxes $\ldots \ldots \ldots$ \\
\hline 3.12 & SAXTON-1, Pin-132 four-group U-235 cross sections \\
\hline
\end{tabular}




\section{LIST OF TABLES (continued)}

\begin{tabular}{|c|c|}
\hline 3.13 & SAXTON-1, Pin-132 four-group U-238 cross sections \\
\hline 3.14 & SAXTON-1, Pin-132 four-group Pu-239 cross sections . \\
\hline 3.15 & SAXTON-2 CENTRM reaction rates and fluxes . . . . . . \\
\hline 3.16 & SAXTON-2 NITAWL reaction rates and fluxes \\
\hline 3.17 & SAXTON-2, Pin-1 four-group fluxes $\ldots \ldots \ldots$ \\
\hline 3.18 & SAXTON-2, Pin-1 four-group U-235 cross sections \\
\hline 3.19 & SAXTON-2, Pin-1 four-group U-238 cross sections. \\
\hline 3.20 & SAXTON-2, Pin-1 four-group Pu-239 cross sections \\
\hline 3.21 & SAXTON-2, Pin-55 four-group fluxes $\ldots \ldots \ldots$ \\
\hline 3.22 & SAXTON-2, Pin-55 four-group U-235 cross sections \\
\hline 3.23 & SAXTON-2, Pin-55 four-group U-238 cross sections. \\
\hline 3.24 & SAXTON-2, Pin-55 four-group $\mathrm{Pu}-239$ cross sections \\
\hline 3.25 & SAXTON-3 CENTRM reaction rates and fluxes . . . . \\
\hline 3.26 & SAXTON-3 NITAWL reaction rates and fluxes \\
\hline 3.27 & SAXTON-3, Pin-1 four-group fluxes $\ldots \ldots \ldots$ \\
\hline 3.28 & SAXTON-3, Pin-1 four-group U-235 cross sections \\
\hline 3.29 & SAXTON-3, Pin-1 four-group U-239 cross sections. \\
\hline 3.30 & SAXTON-3, Pin-1 four-group Pu-239 cross sections \\
\hline 3.31 & SAXTON-3, Pin-66 four-group fluxes $\ldots \ldots \ldots$ \\
\hline 3.32 & SAXTON-3, Pin-66 four-group U-235 cross sections. \\
\hline 3.33 & SAXTON-3, Pin-66 four-group U-238 cross sections \\
\hline 3.34 & SAXTON-3, Pin-66 four-group $\mathrm{Pu}-239$ cross sections . \\
\hline 3.35 & SAXTON-4 CENTRM reaction rates and fluxes . . . . . . \\
\hline 3.36 & SAXTON-4 NITAWL reaction rates and fluxes \\
\hline 3.37 & SAXTON-4, Pin-1 four-group fluxes ....... \\
\hline 3.38 & SAXTON-4, Pin-1 four-group U-235 cross sections. \\
\hline 3.39 & SAXTON-4, Pin-1 four-group U-238 cross sections. \\
\hline 3.40 & SAXTON-4, Pin-1 four-group Pu-239 cross sections \\
\hline 3.41 & SAXTON-4, Pin-28 four-group fluxes $\ldots \ldots \ldots \ldots$ \\
\hline 3.42 & SAXTON-4, Pin-28 four-group U-235 cross sections. \\
\hline 3.43 & SAXTON-4, Pin-28 four-group U-239 cross sections \\
\hline 3.44 & SAXTON-4, Pin-28 four-group $\mathrm{Pu}-239$ cross sections \\
\hline 3.45 & SAXTON-5 CENTRM reaction rates and fluxes . . . . \\
\hline 3.46 & SAXTON-5 NITAWL reaction rates and fluxes \\
\hline 3.47 & SAXTON-5, Pin-1 four-group fluxes $\ldots \ldots \ldots$ \\
\hline 3.48 & SAXTON-5, Pin-1 four-group U-235 cross sections \\
\hline 3.49 & SAXTON-5, Pin-1 four-group U-239 cross sections \\
\hline 3.50 & SAXTON-5, Pin-1 four-group Pu-239 cross sections \\
\hline 3.51 & SAXTON-5, Pin-21 four-group fluxes $\ldots \ldots \ldots \ldots$ \\
\hline
\end{tabular}




\section{LIST OF TABLES (continued)}

\begin{tabular}{|c|c|}
\hline 3.52 & SAXTON-5, Pin-21 four-group U-235 cross sections \\
\hline 3.53 & SAXTON-5, Pin-21 four-group U-238 cross sections \\
\hline 3.54 & SAXTON-5, Pin-21 four-group Pu-239 cross sections \\
\hline 3.55 & SAXTON-6 CENTRM reaction rates and fluxes. ..... \\
\hline 3.56 & SAXTON-6 NITAWL reaction rates and fluxes \\
\hline 3.57 & SAXTON-6, Pin-1 four-group fluxes $\ldots \ldots$ \\
\hline 3.58 & SAXTON-6, Pin-1 four-group U-235 cross sections \\
\hline 3.59 & SAXTON-6, Pin-1 four-group U-238 cross sections, \\
\hline 3.60 & SAXTON-6, Pin-1 four-group Pu-239 cross sections \\
\hline 3.61 & SAXTON-6, Pin-21 four-group fluxes $\ldots \ldots \ldots \ldots$ \\
\hline 3.62 & SAXTON-6, Pin-21 four-group U-235 cross sections \\
\hline 3.63 & SAXTON-6, Pin-21 four-group U-239 cross sections \\
\hline 3.64 & SAXTON-6, Pin-21 four-group Pu-239 cross sections \\
\hline 4.1 & Lattice description for benchmarks . . . . . . . . \\
\hline 4.2 & Benchmark atom densities ...... \\
\hline 4.3 & Pu-241 and Am-241 atom densities $\ldots \ldots \ldots \ldots \ldots$ \\
\hline 4.4 & Comparison of $k_{\text {off }}$ and EALCF from CENTRM and NITAWL. \\
\hline 4.5 & Selected reaction rates for CENTRM case TCA-1 $\ldots \ldots \ldots$ \\
\hline 4.6 & Selected reaction rates for NITAWL case TCA-1 \\
\hline 4.7 & TCA-1, Pin-1 four-group fluxes $\ldots \ldots \ldots \ldots$ \\
\hline 4.8 & TCA-1, Pin-1 four-group U-235 cross sections \\
\hline 4.9 & TCA-1, Pin-1 four-group U-238 cross sections. \\
\hline 4.10 & TCA-1, Pin-1 four-group Pu-239 cross sections \\
\hline 4.11 & TCA-1, Pin-78 four-group fluxes $\ldots \ldots \ldots \ldots$ \\
\hline 4.12 & TCA-1, Pin-78 four-group U-235 cross sections \\
\hline 4.13 & TCA-1, Pin-78 four-group U-238 cross sections \\
\hline 4.14 & TCA-1, Pin- 78 four-group $\mathrm{Pu}-239$ cross sections \\
\hline 4.15 & Selected reaction rates for CENTRM case TCA- 2 \\
\hline 4.16 & Selected reaction rates for NITAWL case TCA-2 \\
\hline 4.17 & TCA-2, Pin-1 four-group fluxes $\ldots \ldots \ldots \ldots$ \\
\hline 4.18 & TCA-2, Pin-1 four-group U-235 cross sections \\
\hline 4.19 & TCA-2, Pin-1 four-group U-238 cross sections, \\
\hline 4.20 & TCA-2, Pin-1 four-group Pu-239 cross sections \\
\hline 4.21 & TCA-2, Pin-78 four-group fluxes $\ldots \ldots \ldots$ \\
\hline 4.22 & TCA-2, Pin-78 four-group U-235 cross sections \\
\hline 4.23 & TCA-2, Pin- 78 four-group U-238 cross sections. \\
\hline 4.24 & TCA-2, Pin- 78 four-group Pu-239 cross sections \\
\hline 4.25 & Selected reaction rates for CENTRM case TCA-3 \\
\hline
\end{tabular}




\section{LIST OF TABLES (continued)}

\begin{tabular}{|c|c|c|}
\hline 4.26 & Selected reaction rates for NITAWL case TCA-3 & \\
\hline 4.27 & TCA-3, Pin-1 four-group fluxes $\ldots \ldots \ldots \ldots$ & 103 \\
\hline 4.28 & TCA-3, Pin-1 four-group U-235 cross sections & 103 \\
\hline 4.29 & TCA-3, Pin-1 four-group U-238 cross sections & 10 \\
\hline 4.30 & TCA-3, Pin-1 four-group $\mathrm{Pu}-239$ cross sections & \\
\hline 4.31 & TCA-3, Pin-78 four-group fluxes $\ldots \ldots \ldots$ & \\
\hline 4.32 & TCA-3, Pin-78 four-group U-235 cross sections & \\
\hline 4.33 & TCA-3, Pin-78 four-group U-238 cross sections, & \\
\hline 4.34 & TCA-3, Pin-78 four-group Pu-239 cross sections & \\
\hline 4.35 & Selected reaction rates for CENTRM case TCA- 4 & 107 \\
\hline 4.36 & Selected reaction rates for NITAWL case TCA-4 & \\
\hline 4.37 & TCA-4, Pin-1 four-group fluxes $\ldots \ldots \ldots \ldots$ & \\
\hline 4.38 & TCA-4, Pin- 1 four-group U- 235 cross sections & \\
\hline 4.39 & TCA-4, Pin-1 four-group U-238 cross sections. & \\
\hline 4.40 & TCA-4, Pin-1 four-group $\mathrm{Pu}-239$ cross sections & \\
\hline 4.41 & TCA-4, Pin-66 four-group fluxes $\ldots \ldots \ldots$ & \\
\hline 4.42 & TCA-4, Pin-66 four-group U-235 cross sections & 1 \\
\hline 4.43 & TCA-4, Pin-66 four-group U-238 cross sections. & \\
\hline 4.44 & TCA-4, Pin- 66 four-group Pu-239 cross sections & \\
\hline 4.45 & Selected reaction rates for CENTRM case TCA-5 & 11 \\
\hline 4.46 & Selected reaction rates for NITAWL case TCA-5 & 11. \\
\hline 4.47 & TCA-5, Pin-1 four-group fluxes . . . . . . . . . . . & 11 \\
\hline 4.48 & TCA-5, Pin-1 four-group U-235 cross sections & 11 \\
\hline 4.49 & TCA-5, Pin-1 four-group U-238 cross sections. & 1 \\
\hline 4.50 & TCA-5, Pin-1 four-group Pu-239 cross sections & \\
\hline 4.51 & TCA-5, Pin-66 four-group fluxes $\ldots \ldots \ldots \ldots$ & \\
\hline 4.52 & TCA-5, Pin- 66 four-group U-235 cross sections & 11 \\
\hline 4.53 & TCA-5, Pin-66 four-group U-238 cross sections. & 11 \\
\hline 4.54 & TCA-5, Pin- 66 four-group Pu-239 cross sections & 11 \\
\hline 4.55 & Selected reaction rates for CENTRM case TCA-6 & 11 \\
\hline 4.56 & Selected reaction rates for NITAWL case TCA- 6 & 11 \\
\hline 4.57 & TCA-6, Pin-1 four-group fluxes . . . . . . . . . . . & \\
\hline 4.58 & TCA-6, Pin- 1 four-group U-235 cross sections & 11 \\
\hline 4.59 & TCA-6, Pin-1 four-group U-238 cross sections. & 11 \\
\hline 4.60 & TCA-6, Pin- 1 four-group $\mathrm{Pu}-239$ cross sections & 11 \\
\hline 4.61 & TCA- 6 , Pin-66 four-group fluxes $\ldots \ldots \ldots$ & 11 \\
\hline 4.62 & TCA-6, Pin-66 four-group U-235 cross sections & \\
\hline 4.63 & TCA-6, Pin-66 four-group U-238 cross sections. & \\
\hline 4.64 & TCA-6, Pin- 66 four-group Pu- 239 cross sections & \\
\hline
\end{tabular}




\section{LIST OF TABLES (continued)}

\begin{tabular}{|c|c|c|}
\hline 4.65 & Selected reaction rates for CENTRM case TCA-7 & \\
\hline 4.66 & Selected reaction rates for NITAWL case TCA-7 & 122 \\
\hline 4.67 & TCA-7, Pin-1 four-group fluxes $\ldots \ldots \ldots \ldots$ & 123 \\
\hline 4.68 & TCA-7, Pin-1 four-group U-235 cross sections & 123 \\
\hline 4.69 & TCA-7, Pin-1 four-group U-238 cross sections. & 123 \\
\hline 4.70 & TCA-7, Pin-1 four-group Pu-239 cross sections & 123 \\
\hline 4.71 & TCA-7, Pin-55 four-group fluxes $\ldots \ldots \ldots$ & 124 \\
\hline 4.72 & TCA-7, Pin-55 four-group U-235 cross sections & 124 \\
\hline 4.73 & TCA-7, Pin-55 four-group U-238 cross sections & 124 \\
\hline 4.74 & TCA-7, Pin-55 four-group Pu-239 cross sections & 124 \\
\hline 4.75 & Selected reaction rates for CENTRM case TCA-8 & 127 \\
\hline 4.76 & Selected reaction rates for NITAWL case TCA-8 & 127 \\
\hline 4.77 & TCA-8, Pin-1 four-group fluxes $\ldots \ldots \ldots \ldots$ & 128 \\
\hline 4.78 & TCA- 8 , Pin- 1 four-group U- 235 cross sections & 128 \\
\hline 4.79 & TCA-8, Pin-1 four-group U-238 cross sections. & 128 \\
\hline 4.80 & TCA-8, Pin-1 four-group Pu-239 cross sections & 128 \\
\hline 4.81 & TCA-8, Pin-55 four-group fluxes $\ldots \ldots \ldots$ & 129 \\
\hline 4.82 & TCA-8, Pin-55 four-group U-235 cross sections & 129 \\
\hline 4.83 & TCA-8, Pin-55 four-group U-238 cross sections & 129 \\
\hline 4.84 & TCA-8, Pin-55 four-group Pu-239 cross sections & 129 \\
\hline 4.85 & Selected reaction rates for CENTRM case TCA-9 & 132 \\
\hline 4.86 & Selected reaction rates for NITAWL case TCA-9 & 132 \\
\hline 4.87 & TCA-9, Pin-1 four-group fluxes $\ldots \ldots \ldots \ldots$ & 133 \\
\hline 4.88 & TCA-9, Pin-1 four-group U-235 cross sections & 133 \\
\hline 4.89 & TCA-9, Pin-1 four-group U-238 cross sections. & 133 \\
\hline 4.90 & TCA-9, Pin-1 four-group Pu-239 cross sections & 133 \\
\hline 4.91 & TCA-9, Pin-55 four-group fluxes $\ldots \ldots \ldots$ & 134 \\
\hline 4.92 & TCA-9, Pin-55 four-group U-235 cross sections & 134 \\
\hline 4.93 & TCA-9, Pin-55 four-group U-238 cross sections. & 134 \\
\hline 4.94 & TCA-9, Pin-55 four-group $\mathrm{Pu}-239$ cross sections . & 134 \\
\hline 4.95 & Selected reaction rates for CENTRM case TCA-10 & 137 \\
\hline 4.96 & Selected reaction rates for NITAWL case TCA-10 & 137 \\
\hline 4.97 & TCA-10, Pin-1 four-group fluxes . . . . . . . . . . . & 138 \\
\hline 4.98 & TCA-10, Pin-1 four-group U-235 cross sections & 138 \\
\hline 4.99 & TCA-10, Pin-1 four-group U-238 cross sections. & 138 \\
\hline 4.100 & TCA-10, Pin-1 four-group Pu-239 cross sections & 138 \\
\hline 4.101 & TCA-10, Pin-66 four-group fluxes $\ldots \ldots \ldots$. . . & 139 \\
\hline 4.102 & TCA-10, Pin-66 four-group U-235 cross sections. & 139 \\
\hline
\end{tabular}




\section{LIST OF TABLES (continued)}

\begin{tabular}{|c|c|}
\hline 4.103 & TCA-10, Pin-66 four-group U-238 cross sections. \\
\hline 4.104 & TCA-10, Pin-66 four-group $\mathrm{Pu}-239$ cross sections \\
\hline 4.105 & Selected reaction rates for CENTRM case TCA-11 \\
\hline 4.106 & Selected reaction rates for NITAWL case TCA-11 \\
\hline 4.107 & TCA-11, Pin-1 four-group fluxes $\ldots \ldots \ldots \ldots$ \\
\hline 4.108 & TCA-11, Pin-1 four-group U-235 cross sections \\
\hline 4.109 & TCA-11, Pin-1 four-group U-238 cross sections \\
\hline 4.110 & TCA-11, Pin-1 four-group Pu-239 cross sections \\
\hline 4.111 & TCA-11, Pin-66 four-group fluxes $\ldots . . . \ldots$. \\
\hline 4.112 & TCA-11, Pin-66 four-group U-235 cross sections \\
\hline 4.113 & TCA-11, Pin-66 four-group U-238 cross sections \\
\hline 4.114 & TCA-11, Pin-66 four-group Pu-239 cross sections \\
\hline 5.1 & State parameters for the calculational benchmark variants \\
\hline 5.2 & Basic fuel isotopic composition for each computational benchmark variant . \\
\hline 5.3 & Nonfuel material isotopic composition $\ldots \ldots \ldots \ldots \ldots \ldots \ldots$ \\
\hline $5.4 \mathrm{a}$ & $k_{\Lambda}$ and EALCF values and differences for the variant cases \\
\hline $5.4 \mathrm{~b}$ & $k_{\Lambda}$ and EALCF values and differences for the variant cases (cont.) \\
\hline $5.5 \mathrm{a}$ & Buckled $k_{\lrcorner}$and EALCF values and differences for the variant cases \\
\hline $5.5 \mathrm{~b}$ & Buckled $k_{4}$ and EALCF values and differences for the variant cases (cont.) \\
\hline
\end{tabular}





\section{ACKNOWLEDGMENTS}

This work is supported jointly by the DOE Nuclear Criticality Safety Program and the DOE Fissile Material Disposition Program.

The author would like to thank the following individuals for their contributions: M. L. Williams, Louisiana State University, who wrote CENTRM and was available whenever problems were encountered with the code; L. M. Petrie for his assistance in implementing CENTRM into the SCALE sequence; W. C. Jordan and R. Q. Wright for testing and often breaking the code; M. E. Dunn and N. M. Greene for help creating the point cross-section library used by CENTRM; and J. B. Anderson for preparing this report. 



\section{ABSTRACT}

The purpose of this study is to determine and document the reactor physics parameters (multiplication factors, spatially dependent flux ratios, and spacially dependent reaction rates ) for several distinct sets of problems using two distinct resonance cross-section processing techniques. In SCALE, by default, resonances are processed using NITAWL, which utilizes the Nordheim Integral Treatment. The results produced using this sequence are considered to be the base results. A second set of results are produced by replacing NITAWL with CENTRM/PMC. CENTRM produces point-wise fluxes for a given geometry configuration and set of isotopes. Using these fluxes, PMC produces problem-dependent self-shielding cross sections. Both sequences use ENDF/B-V cross-section data. 



\section{INTRODUCTION}

This report examines four sets of problems that are used to evaluate the differences between the NITAWL resonance processor and the CENTRM/PMC resonance processor. All the problems are predominantly thermal, with the energy of the average lethargy causing fission (EALCF) being below $1 \mathrm{eV}$. They are all water-moderated fuel rod lattices of various dimensions and fuel materials. The objective of this report is to compare the differences between the NITAWL and CENTRM/PMC resolved-resonance processors for the class of problems presented in this report. The existence of severe resonance overlap when mixing uranium and plutonium could cause a significant problem with the NITAWL results. CENTRM/PMC does not contain this potential problem, which is a limitation of the Nordheim Integral Treatment.

All problem were processed using SCALE5.0, which allows one to choose which resolved resonance processor will be used to self-shield the nuclides, NITAWL or CENTRM/PMC. SCALE5.0 is a developmental version of the SCALE code system. The current version of SCALE, SCALE4.4, does not contain CENTRM but is essentially the same in all other aspects to SCALE5.0. SCALE5.0 has the ability to use either NITAWL or CENTRM/PMC as the resolved-resonance processor. All problems were run first using CENTRM/PMC and then using NITAWL. The results were then analyzed and compared.1,2

\subsection{THE SCALE 5.0 SEQUENCES}

SCALE 5.0 contains two distinct sequences used for processing cross-section data. The original sequence consisting of the codes CSAS, BONAMI, and NITAWL process all resolved resonance data in NITAWL. The new computational sequence added to SCALE5.0 uses the following sequence to process the cross sections: CSAS, BONAMI, NITAWL, CENTRM, PMC, WORKER. CENTRM/PMC process the resolved resonances cross sections in the unit cell. All other resolved resonance cross sections are processed using NITAWL. To calculate the desired physics parameters, the three experimental benchmark sets then use KENO-V.a and the set of computational benchmarks uses XSDRNPM. Many of the physics parameters are compared with direct experimental data and others are compared only with the same parameters calculated using difference codes and cross sections.

\subsection{NITAWL}

NITAWL performs problem-dependent resonance self-shielding using the Nordheim Integral Treatment, which involves solving for the energy dependence of the neutron flux in a material region containing a resonance absorber and a maximum of two moderating materials. The resonance selfshielding is made with reference to infinite dilution cross-section values. The material region is either infinite or a one-dimensional (1-D) slab, cylinder, or sphere surrounded by moderator. In the moderator, the neutron flux is assumed to be spatially flat and slowly varying with energy. The presence of more than one fuel region in the moderator, such as a lattice, is accounted for using a Dancoff factor. 
There are many problems where the Nordheim Integral Treatment may not be appropriate because of the following assumptions:

1. Each resonance nuclide can be treated without consideration of other resonance nuclides present in the system.

2. The neutron flux is spatially uniform in the absorber and moderator regions.

3. Neutron transport into and out of the absorber region can be treated with first-flight escape probabilities.

4. The presence of other absorber lumps in the system can be accounted for with a Dancoff factor which corresponds to the first-flight transmission probability across the moderator. A Dancoff factor of zero corresponds to a single lump in the moderator. A Dancoff factor of 1 corresponds to an infinite medium of the absorber material with no moderator outside the absorber lump.

\subsection{CENTRM/PMC}

The new resolved-resonance processor is composed of two codes that are executed sequentially. The first code, CENTRM, is a discrete-ordinance code that produces a point-wise continuous-energy flux spectrum for a given infinite or 1-D problem. Subsequently, PMC uses this point flux spectrum to collapse point cross sections to a problem-dependent multigroup cross-section set. To generate the flux profile and produce new group cross sections, the two codes use a pointwise continuous cross-section library that contain all the nuclides present in the selected multigroup cross-section library. All the cases in this report were analyzed using the 238-group ENDF/B-V multigroup cross-section library.

CENTRM has several options that determine the range over which the flux spectrum will be generated and the parameters used for generating the flux spectrum. Although pointwise continuous in energy, the flux spectrum is generated on a specified 1-D spacial mesh. For all cases in this report, the spatial mesh is over an infinite cylinder that is automatically generated by the code. The flux spectrum is generated from $20 \mathrm{MeV}$ to $1.0 \mathrm{E}-5 \mathrm{eV}$ over the spatial mesh using a default quadrature of 8. CENTRM uses the specified quadrature to generate Legendre coefficients for the scattering component of the pointwise flux.

CENTRM is capable of using a fixed or fission input source to generate the flux profile. For simplicity and because the benchmarks are fission problems, a spacially flat fission source is assumed as an initial guess. Two other parameters that can affect the flux are the point convergence criterion and the tolerance used for thinning the pointwise cross sections. These parameters are both set to default values of 0.0001 for the convergence criterion and 0.0025 for the thinning tolerance.

In order for CENTRM to generate the flux profile it first combines the pointwise continuous-energy cross sections of all nuclides in each zone. Each zone contains only one material but is divided into many intervals, with each interval being a mesh point where the entire energy spectrum of the flux is calculated. There is no code limit on the number of materials and 1-D zones. However, for large problems involving many materials and zones, computer memory and CPU speed may be a limiting factor.

By default, the flux profile is generated using an $S_{N}$ calculation over the entire spectrum. The option exists to perform several other types of calculations independently over the fast, thermal, and 
pointwise ranges. Over all energy ranges, scattering for the pointwise continuous cross sections is assumed to be elastic and isotropic in the center of mass system. Consequently, no scattering data are contained in the point library, only 1-D cross sections.

PMC replaces the cross sections for specified nuclides and energy groups in the problem-dependent multigroup library. PMC reads the pointwise flux file, the pointwise continuous cross sections, and the problem-dependent multigroup library; generates new multigroup data from the point data; and finally rewrites the multigroup library with the new multigroup data. PMC can produce multigroup cross sections over three ranges for all nuclides: the entire point library range, the resolved resonance range, or the pointwise flux range. For the problems, in this report PMC produces multigroup cross sections over the resolved resonance range of each nuclide in the unit cell data.

PMC has three methods for computing the scattering component of the new multigroup cross sections. The $\mathrm{P}_{\mathrm{N}}$ components of the original multigroup scattering cross sections can be renormalized to the new 1-D scattering cross sections. The $\mathrm{P}_{\mathrm{N}}$ components of the original multigroup scattering cross sections can be renormalized using the scalar flux. Finally, new $\mathrm{P}_{\mathrm{N}}$ components for the scattering cross section can be computed using the pointwise flux moments. For the problems in this report, the first option of renormalizing the $\mathrm{P}_{\mathrm{N}}$ components using the new 1-D cross sections is used.

A set of pointwise continuous cross sections are available for use by CENTRM and PMC. The point cross sections used in the report were generated using the AMPX code system from the ENDF/B-V nuclear data library. The library contains all the nuclides present in both the 238-group and 44-group ENDF/B-V multigroup libraries. The library contains the total, elastic scattering, and radiative capture cross sections for each nuclide. If a nuclide is fissionable, the fission cross section is also present. Also, if a nuclide contains inelastic data at higher energies, inelastic data are also included in the library. The pointwise continuous library is divided up into files, with each file containing all the cross-section data for one nuclide. The ENDF/B-V pointwise library was generated to be used with the ENDF/B-V multigroup libraries. Using it with any other type of library, such as one generated using ENDF/B-VI data, could produce incorrect results. 



\section{MIX-COMP-THERM-2 (PNL-30 to PNL-35)}

\subsection{DESCRIPTION}

This section describes a set of six critical experiments, each consisting of a square-pitched array of mixed plutonium-uranium fuel rods submerged in water surrounded by a water reflector. The water contains boron concentrations from 0.9 to $767.2 \mathrm{ppm}$. This set of experiments is contained in the International Handbook of Evaluated Criticality Safety Benchmark Experiments. 3

This set of experiments was performed between December 1975 and March 1976 at the Plutonium Recycle Critical Facility at Pacific Northwest Laboratory. The benchmark experiments are light-water-moderated critical assemblies consisting of a core array supported by upper and lower lattice plates. The lattice plates are $2.54 \mathrm{~cm}$ thick and located at the top and bottom of the fuel region - The reactor is brought to critical by raising the water level in the tank, thus avoiding the use of control rods. The fuel rods sit on a support plate above the bottom of the tank. The tank is wide enough to assume an infinite moderator on the sides and bottom $(\sim 30 \mathrm{~cm}$ of water).

All fuel rods have the same physical dimensions. A schematic diagram of the fuel rods and bottom reflector is given in Fig. 2.1. Each fuel rod has an active fuel length of $86.6775 \mathrm{~cm}, 0.5 \mathrm{~cm}$ of $\mathrm{UO}_{2}$ at the bottom of the fuel, a $0.6985-\mathrm{cm}$ cladding plug on the bottom and a 0.8255 -cm cladding plug on the top. The fuel has a radius of $0.64135 \mathrm{~cm}$. The cladding has an outside radius of 0.71755 $\mathrm{cm}$. A $0.3175-\mathrm{cm}$ water gap is located between the top of the aluminum support plate and the bottom of the lower lattice plate. The aluminum support plate is $2.8575 \mathrm{~cm}$ thick. There is a $0.889-\mathrm{cm}$ water gap between the bottom of the lead plate and the top of the upper lattice plate. The lead plate is $0.9525 \mathrm{~cm}$ thick. The top reflector varies depending on the water level for the particular problem.

The primary differences between the six benchmarks are lattice pitch, number of rods in the lattice, water level, and boron concentrations. All other benchmark characteristics are constant. There are three different lattice pitches, with each pitch used in two problems: $1.778 \mathrm{~cm}, 2.20914 \mathrm{~cm}$, and $2.51447 \mathrm{~cm}$. The fuel is arranged in a square-pitched lattice. The characteristics of each of the four lattices are given in Table 2.1. Table 2.2 contains the atom densities for all the materials in the problem except B-10 and B-11. The atom densities in Table 2.2 are constant for all benchmarks. Table 2.3 contain the atom densities of B-10 and B-11 for each benchmark. All material temperatures are assumed to be $23^{\circ} \mathrm{C}(295 \mathrm{~K})$.

As shown in Table 2.1, the six benchmark problems change by moderator boron concentration, number of fuel rods, lattice pitch and upper-reflector thickness. To simplify the accumulation of power densities, advantage was taken in the symmetry of the problem whenever possible. The entire problem was explicitly modeled, but instead of having a separate unit for each pin, 1/8th symmetry was used for every problem except PNL-32. Due to an irregular placement of outer rods, this problem needed to be modeled using a unit for each pin. The lattice is then filled from the 1/8th section by inserting additional pins in a mirror image. Figures 2.2 through 2.6 show the lattice map for each problem. Figure 2.4 shows a full-lattice mapping; the other figures contain only 1/8th lattices. The remaining lattice can be extrapolated from these $1 / 8$ th sections. 


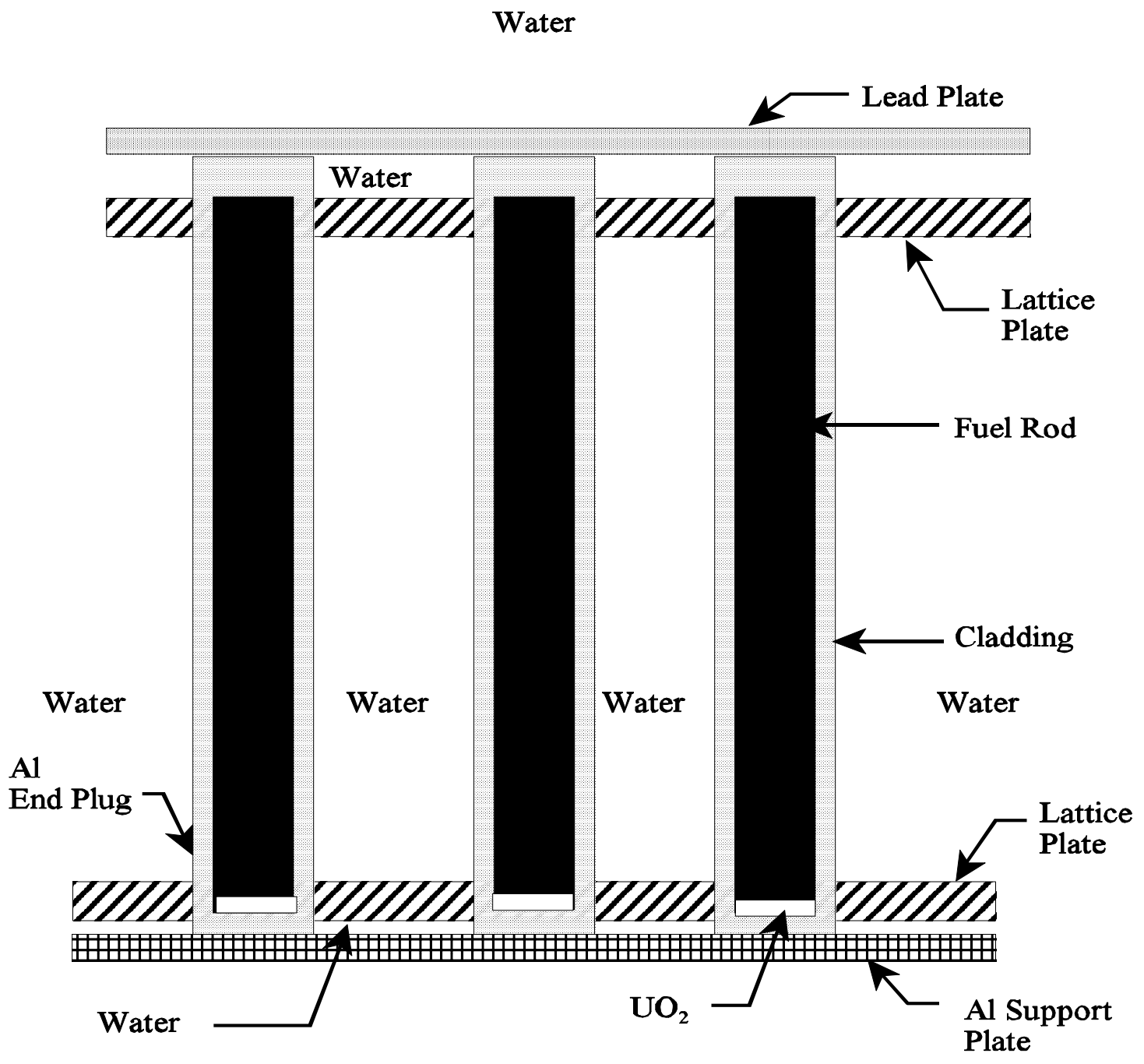

Water

Fig. 2.1. Schematic of the PNL fuel rods with reflector. 
Table 2.1. Lattice description for benchmarks

\begin{tabular}{ccccc}
\hline $\begin{array}{c}\text { Benchmark } \\
\text { No. }\end{array}$ & $\begin{array}{c}\text { Boron con. } \\
(\mathrm{ppm})\end{array}$ & $\begin{array}{c}\text { Critical } \\
\text { No. rods }\end{array}$ & $\begin{array}{c}\text { Lattice } \\
\text { pitch } \\
(\mathrm{cm})\end{array}$ & $\begin{array}{c}\text { Water level } \\
\text { above lead } \\
(\mathrm{cm})\end{array}$ \\
\hline 30 & 1.7 & 469 & 1.778 & 13.462 \\
31 & 687.9 & 761 & 1.778 & 13.462 \\
32 & 0.9 & 195 & 2.20914 & 3.937 \\
33 & 1090.4 & 761 & 2.20914 & 13.462 \\
34 & 1.6 & 160 & 2.51447 & 0.508 \\
35 & 767.2 & 689 & 2.51447 & 13.462 \\
\hline
\end{tabular}

Table 2.2. Constant benchmark atom densities

\begin{tabular}{cccccc}
\hline Material & Isotope & $\begin{array}{c}\text { Atom density } \\
\text { (atoms/barn-cm) }\end{array}$ & Material & Isotope & $\begin{array}{c}\text { Atom density } \\
\text { (atoms/barn-cm) }\end{array}$ \\
\hline Fuel & $\mathrm{U}-234$ & $1.2458 \times 10^{-6}$ & Lattice plate & $\mathrm{Si}$ & $1.3742 \times 10^{-2}$ \\
$\mathrm{UO}_{2}-\mathrm{PuO}_{2}$ & $\mathrm{U}-235$ & $1.4886 \times 10^{-4}$ & Support plate & $\mathrm{Fe}$ & $4.5919 \times 10^{-2}$ \\
$(9.54 \mathrm{~g} / \mathrm{cc})$ & $\mathrm{U}-236$ & $2.0936 \times 10^{-9}$ & & $\mathrm{Cu}$ & $1.1532 \times 10^{-4}$ \\
& $\mathrm{U}-238$ & $2.0936 \times 10^{-2}$ & & $\mathrm{Mn}$ & $9.6395 \times 10^{-4}$ \\
& $\mathrm{Pu}-238$ & $3.8836 \times 10^{-8}$ & & $\mathrm{Mg}$ & $1.2388 \times 10^{-4}$ \\
& $\mathrm{Pu}-239$ & $3.9462 \times 10^{-4}$ & & $\mathrm{Cr}$ & $1.7409 \times 10^{-3}$ \\
& $\mathrm{Pu}-240$ & $3.3206 \times 10^{-5}$ & & $\mathrm{Zn}$ & $1.6617 \times 10^{-2}$ \\
& $\mathrm{Pu}-241$ & $1.6081 \times 10^{-6}$ & & $\mathrm{Ti}$ & $4.6052 \times 10^{-4}$ \\
& $\mathrm{Pu}-242$ & $1.1882 \times 10^{-7}$ & & $\mathrm{Al}$ & $1.5025 \times 10^{-3}$ \\
& $\mathrm{Am}-241$ & $1.4954 \times 10^{-6}$ & & & \\
& $\mathrm{O}-16$ & $4.3779 \times 10^{-2}$ & & & \\
& $\mathrm{U}-234$ & $1.2406 \times 10^{-6}$ & Cladding & $\mathrm{Sn}$ & $4.8328 \times 10^{-4}$ \\
Natural UO $\mathrm{UO}_{2}$ & $\mathrm{U}-235$ & $1.4824 \times 10^{-4}$ & & $\mathrm{Fe}$ & $9.5642 \times 10^{-5}$ \\
& $\mathrm{U}-236$ & $2.0848 \times 10^{-9}$ & & $\mathrm{Cr}$ & $7.6093 \times 10^{-5}$ \\
& $\mathrm{U}-238$ & $2.0525 \times 10^{-2}$ & & $\mathrm{Ni}$ & $3.0336 \times 10^{-5}$ \\
& $\mathrm{O}-16$ & $4.1943 \times 10^{-2}$ & & $\mathrm{Zr}$ & $4.2621 \times 10^{-2}$ \\
& & & & $\mathrm{~Pb}$ & $3.2174 \times 10^{-2}$ \\
& & & & \\
\hline
\end{tabular}


Table 2.3. Moderator atom densities

\begin{tabular}{ccccc}
\hline $\begin{array}{c}\text { Benchmark } \\
\text { No. }\end{array}$ & \multicolumn{4}{c}{ Atom densities ( atoms/barn-cm ) } \\
\cline { 2 - 5 } & $\mathrm{H}$ & $\mathrm{O}$ & $\mathrm{B}-10$ & $\mathrm{~B}-11$ \\
\hline PNL-30 & $6.6706 \times 10^{-2}$ & $3.3353 \times 10^{-2}$ & $1.8706 \times 10^{-8}$ & $7.5770 \times 10^{-8}$ \\
PNL-31 & $6.6605 \times 10^{-2}$ & $3.3400 \times 10^{-2}$ & $7.5838 \times 10^{-6}$ & $3.0718 \times 10^{-5}$ \\
PNL-32 & $6.6706 \times 10^{-2}$ & $3.3353 \times 10^{-2}$ & $9.9034 \times 10^{-9}$ & $4.0114 \times 10^{-8}$ \\
PNL-33 & $6.6672 \times 10^{-2}$ & $3.3427 \times 10^{-2}$ & $1.2034 \times 10^{-5}$ & $4.8746 \times 10^{-5}$ \\
PNL-34 & $6.6706 \times 10^{-2}$ & $3.3353 \times 10^{-2}$ & $1.7606 \times 10^{-8}$ & $7.1313 \times 10^{-8}$ \\
PNL-35 & $6.6682 \times 10^{-2}$ & $3.3405 \times 10^{-2}$ & $8.4597 \times 10^{-5}$ & $3.4266 \times 10^{-5}$ \\
\hline
\end{tabular}

\subsection{ANALYSIS}

All six computational benchmarks in this section were processed twice using SCALE 5.0. The set labeled NITAWL uses the NITAWL resonance processor to self-shield the resolved resonance region for all nuclides in the fuel and clad. An identical set, labeled CENTRM, replaces NITAWL with the CENTRM/PMC code sequence for the nuclides in the unit cell. The NITAWL and CENTRM results are then compared.

Table 2.4 contains the $k_{e f f}$ and energy of the average lethargy causing fission (EALCF). The $k_{e f f}$ values for all the benchmark cases are close to 1.0: the worst NITAWL benchmark is $0.90 \%$ high, and the worst CENTRM case is $0.68 \%$ high. A small negative bias does appear between NITAWL and CENTRM, with the CENTRM cases about $0.25 \%$ lower on average than the NITAWL cases.

The EALCF is also shown in this table. The EALCF values listed are from the CENTRM cases. EALCF is the energy of the average lethargy causing fission and is calculated by summing the lethargies of the neutrons that undego fission, dividing the value by the number of fissions, and converting this value to energy. For all cases, the difference between this value for CENTRM and NITAWL was less than $0.1 \%$. Both CENTRM and NITAWL produce excellent and consistent results for the $k_{\text {eff }}$ and fission energy for this set of problems.

Additional parameters calculated for each problem include: pin-power distributions; absortption, $\mathrm{G}_{\mathrm{f}}$, and fission reaction rates and fluxes in the pin fuel, clad, and moderator; and four group cross sections and fluxes for a corner outside pin and the innermost pin. Each of these sets of data are calculated using both CENTRM and NITAWL. All the data for each case are contained in Figs. 2.7a through $2.12 \mathrm{~b}$ and Tables 2.5 through 2.64 .

The pin-power distributions for this problem assumed 1/8th core symmetry with a surrounding reflector for all benchmark cases except PNL-32. Because of a lack of symmetry in two outer pins the entire assembly needed to be modeled. The pin-power data consist of a value and a standard deviation for each pin. The values for the pin powers are actually in units of fissions per $\mathrm{cm}^{3}$-s-source particle $\times 10^{-5}$. The value in parentheses is the percent standard deviation of the pin-power value.

In most cases the CENTRM and NITAWL results for each benchmark case fall within 2 standard deviations. The peak-to-low power changes with change in pitch and boron concentration. 
The peak-to-low power ratio changed from a low of 1.86 for the smallest pitch with no boron added to a high of 4.19 at the largest pitch with added boron. The addition of boron significantly shifts the flux profile to the center of the assembly by depressing the thermal flux in the moderator.

The reaction rates, total fluxes, and flux ratios for the CENTRM and NITAWL cases of each benchmark case are also in good agreement, seldom varying by more than $1 \%$. These values are included for a corner pin and the center pin for each case. All the reaction rates and fluxes increase as the pins approach the center of the assembly if the outer two layers of the arrays are excluded. The lowest power pin occurs in either the outer layer or the next inner layer of the array. The flux ratios over the fuel, clad, and moderator are relatively constant for a given pitch and boron concentration. However, as the pitch increases, the proportion of the total pin flux in the moderator increases.

Finally, four-group fluxes and cross sections were calculated for selected nuclides in the fuel region of the same corner and center pins. The macroscopic cross sections listed include the radiative capture, fission, and nu*fission cross sections for U-235, U-238, and Pu-239. The four groups are collapsed from the 238-group multigroup cross-section set using the flux profile calculated in KENOV.a as follows: group 1 is from $20 \mathrm{Mev}$ to $9.5 \mathrm{keV}$, group 2 is from $9.5 \mathrm{keV}$ to $3.0 \mathrm{eV}$, group 3 is from $3 \mathrm{eV}$ to $0.4 \mathrm{eV}$, and group 4 is from $0.4 \mathrm{eV}$ to $10^{-5} \mathrm{eV}$. The $0.4 \mathrm{eV}$ was chosen as a boundary because it is the cadmium cutoff energy. Groups 2 and 3 contain the resolved resonance regions for most of the nuclides used in these cases. Most of the cross-section data for the CENTRM and NITAWL cases of a given benchmark case agree within $1 \%$.

\subsection{CONCLUSIONS}

For this set of benchmark cases, using either NITAWL and CENTRM as the resonance region processor produces acceptable results of the $k_{\text {eff }}$ values. All other values produced using CENTRM and NITAWL are also consistent with each. For all cases, the $k_{\text {eff }}$ 's produced using CENTRM are slightly lower, $\sim 0.25 \%$, than those produced using NITAWL to do the resonance self-shielding. As a result, the average $k_{\text {eff }}$ of all the CENTRM cases is closer to 1.0 than the average $k_{\text {eff }}$ of all the NITAWL cases for this set of benchmarks. The pin-power distributions, fluxes, reactions rates, and macroscopic cross sections all agree to approximately $1 \%$ between CENTRM and NITAWL.

The presence of boron in the moderator depresses the fraction of the flux in the moderator, thus creating a larger difference between the highest- and lowest-power pins. No significant differences were identified between the results produced by NITAWL and CENTRM for these benchmark cases. 
Table 2.4. Comparison of $k_{\text {eff }}$ and EALCF from CENTRM and NITAWL

\begin{tabular}{ccccc}
\hline $\begin{array}{c}\text { CASE } \\
\text { MIX-COMP-THERM- }\end{array}$ & $\begin{array}{c}\text { NITAWL } \\
k_{\text {eff }}( \pm \mathrm{F})\end{array}$ & $\begin{array}{c}\text { CENTRM } \\
k_{\text {eff }}( \pm \mathrm{F})\end{array}$ & $\%$ DIFF & $\begin{array}{c}\text { EALCF } \\
(\mathrm{eV})\end{array}$ \\
\hline PNL-30 & $1.000(0.0005)^{a}$ & $0.9966(0.0004)$ & -0.34 & 0.575 \\
PNL-31 & $1.0026(0.0004)$ & $0.9987(0.0003)$ & -0.39 & 0.768 \\
PNL-32 & $1.0021(0.0004)$ & $1.0000(0.0004)$ & -0.21 & 0.193 \\
PNL-33 & $1.0090(0.0003)$ & $1.0064(0.0004)$ & -0.26 & 0.282 \\
PNL-34 & $1.0046(0.0004)$ & $1.0020(0.0005)$ & -0.26 & 0.138 \\
PNL-35 & $1.0079(0.0004)$ & $1.0068(0.0004)$ & -0.11 & 0.182 \\
\hline
\end{tabular}

${ }^{a}$ Values in parentheses is the percent standard deviation.

\begin{tabular}{|c|c|c|c|c|c|c|c|c|c|c|c|}
\hline & & & & & & & & & & & 69 \\
\hline & & & & & & & & & & 67 & 68 \\
\hline & & & & & & & & & 64 & 65 & 66 \\
\hline & & & & & & & & 60 & 61 & 62 & 63 \\
\hline & & & & & & & 55 & 56 & 57 & 58 & 59 \\
\hline & & & & & & 49 & 50 & 51 & 52 & 53 & 54 \\
\hline & & & & & 42 & 43 & 44 & 45 & 46 & 47 & 48 \\
\hline & & & & 34 & 35 & 36 & 37 & 38 & 39 & 40 & 41 \\
\hline & & & 25 & 26 & 27 & 28 & 29 & 30 & 31 & 32 & 33 \\
\hline & & $\mathrm{M}$ & 16 & 17 & 18 & 19 & 20 & 21 & 22 & 23 & 24 \\
\hline & $\mathrm{M}$ & $\mathrm{M}$ & $\mathrm{M}$ & 8 & 9 & 10 & 11 & 12 & 13 & 14 & 15 \\
\hline$M$ & $\mathrm{M}$ & $\mathrm{M}$ & $\mathrm{M}$ & $\mathrm{M}$ & 1 & 2 & 3 & 4 & 5 & 6 & 7 \\
\hline
\end{tabular}

Fig. 2.2. Pin layout of benchmark case PNL-30. 


\begin{tabular}{|c|c|c|c|c|c|c|c|c|c|c|c|}
\hline & & & & & & & & & & & 109 \\
\hline & & & & & & & & & & 107 & 108 \\
\hline & & & & & & & & & 104 & 105 & 106 \\
\hline & & & & & & & & 100 & 101 & 102 & 103 \\
\hline & & & & & & & 95 & 96 & 97 & 98 & 99 \\
\hline & & & & & & 89 & 90 & 91 & 92 & 93 & 94 \\
\hline & & & & & 82 & 83 & 84 & 85 & 86 & 87 & 88 \\
\hline & & & & 74 & 75 & 76 & 77 & 78 & 79 & 80 & 81 \\
\hline & & & 65 & 66 & 67 & 68 & 69 & 70 & 71 & 72 & 73 \\
\hline & & 55 & 56 & 57 & 58 & 59 & 60 & 61 & 62 & 63 & 64 \\
\hline & 44 & 45 & 46 & 47 & 48 & 49 & 50 & 51 & 52 & 53 & 54 \\
\hline 32 & 33 & 34 & 35 & 36 & 37 & 38 & 39 & 40 & 41 & 42 & 43 \\
\hline M & 21 & 22 & 23 & 24 & 25 & 26 & 27 & 28 & 29 & 30 & 31 \\
\hline M & $\mathrm{M}$ & $\mathrm{M}$ & 12 & 13 & 14 & 15 & 16 & 17 & 18 & 19 & 20 \\
\hline M & $\mathrm{M}$ & $\mathrm{M}$ & $\mathrm{M}$ & $\mathrm{M}$ & 5 & 6 & 7 & 8 & 9 & 10 & 11 \\
\hline $\mathrm{M}$ & $\mathrm{M}$ & $\mathrm{M}$ & $\mathrm{M}$ & $\mathrm{M}$ & $\mathrm{M}$ & $\mathrm{M}$ & $\mathrm{M}$ & 1 & 2 & 3 & 4 \\
\hline
\end{tabular}

Fig. 2.3. Pin layout of benchmark cases PNL-31 and PNL-33. 


\begin{tabular}{|l|l|l|l|l|l|l|l|l|l|l|l|l|l|l|l|l|}
\hline $\mathrm{M}$ & $\mathrm{M}$ & $\mathrm{M}$ & $\mathrm{M}$ & $\mathrm{M}$ & $\mathrm{M}$ & $\mathrm{M}$ & 193 & 194 & 195 & $\mathrm{M}$ & $\mathrm{M}$ & $\mathrm{M}$ & $\mathrm{M}$ & $\mathrm{M}$ & $\mathrm{M}$ & $\mathrm{M}$ \\
\hline $\mathrm{M}$ & $\mathrm{M}$ & $\mathrm{M}$ & $\mathrm{M}$ & $\mathrm{M}$ & $\mathrm{M}$ & 187 & 188 & 189 & 190 & 191 & 192 & $\mathrm{M}$ & $\mathrm{M}$ & $\mathrm{M}$ & $\mathrm{M}$ & $\mathrm{M}$ \\
\hline $\mathrm{M}$ & $\mathrm{M}$ & $\mathrm{M}$ & $\mathrm{M}$ & 178 & 179 & 180 & 181 & 182 & 183 & 184 & 185 & 186 & $\mathrm{M}$ & $\mathrm{M}$ & $\mathrm{M}$ & $\mathrm{M}$ \\
\hline $\mathrm{M}$ & $\mathrm{M}$ & $\mathrm{M}$ & 167 & 168 & 169 & 170 & 171 & 172 & 173 & 174 & 175 & 176 & 177 & $\mathrm{M}$ & $\mathrm{M}$ & $\mathrm{M}$ \\
\hline $\mathrm{M}$ & $\mathrm{M}$ & 154 & 155 & 156 & 157 & 158 & 159 & 160 & 161 & 162 & 163 & 164 & 165 & 166 & $\mathrm{M}$ & $\mathrm{M}$ \\
\hline $\mathrm{M}$ & 139 & 140 & 141 & 142 & 143 & 144 & 145 & 146 & 147 & 148 & 149 & 150 & 151 & 152 & 153 & $\mathrm{M}$ \\
\hline $\mathrm{M}$ & 124 & 125 & 126 & 127 & 128 & 129 & 130 & 131 & 132 & 133 & 134 & 135 & 136 & 137 & 138 & $\mathrm{M}$ \\
\hline 107 & 108 & 109 & 110 & 111 & 112 & 113 & 114 & 115 & 116 & 117 & 118 & 119 & 120 & 121 & 122 & 123 \\
\hline 90 & 91 & 92 & 93 & 94 & 95 & 96 & 97 & 98 & 99 & 100 & 101 & 102 & 103 & 104 & 105 & 106 \\
\hline 73 & 74 & 75 & 76 & 77 & 78 & 79 & 80 & 81 & 82 & 83 & 84 & 85 & 86 & 87 & 88 & 89 \\
\hline $\mathrm{M}$ & 58 & 59 & 60 & 61 & 62 & 63 & 64 & 65 & 66 & 67 & 68 & 69 & 70 & 71 & 72 & $\mathrm{M}$ \\
\hline $\mathrm{M}$ & 43 & 44 & 45 & 46 & 47 & 48 & 49 & 50 & 51 & 52 & 53 & 54 & 55 & 56 & 57 & $\mathrm{M}$ \\
\hline $\mathrm{M}$ & $\mathrm{M}$ & 30 & 31 & 32 & 33 & 34 & 35 & 36 & 37 & 38 & 39 & 40 & 41 & 42 & $\mathrm{M}$ & $\mathrm{M}$ \\
\hline $\mathrm{M}$ & $\mathrm{M}$ & $\mathrm{M}$ & 19 & 20 & 21 & 22 & 23 & 24 & 25 & 26 & 27 & 28 & 29 & $\mathrm{M}$ & $\mathrm{M}$ & $\mathrm{M}$ \\
\hline $\mathrm{M}$ & $\mathrm{M}$ & $\mathrm{M}$ & $\mathrm{M}$ & 10 & 11 & 12 & 13 & 14 & 15 & 16 & 17 & 18 & $\mathrm{M}$ & $\mathrm{M}$ & $\mathrm{M}$ & $\mathrm{M}$ \\
\hline $\mathrm{M}$ & $\mathrm{M}$ & $\mathrm{M}$ & $\mathrm{M}$ & $\mathrm{M}$ & 4 & 5 & 6 & 7 & 8 & 9 & $\mathrm{M}$ & $\mathrm{M}$ & $\mathrm{M}$ & $\mathrm{M}$ & $\mathrm{M}$ & $\mathrm{M}$ \\
\hline $\mathrm{M}$ & $\mathrm{M}$ & $\mathrm{M}$ & $\mathrm{M}$ & $\mathrm{M}$ & $\mathrm{M}$ & $\mathrm{M}$ & 1 & 2 & 3 & $\mathrm{M}$ & $\mathrm{M}$ & $\mathrm{M}$ & $\mathrm{M}$ & $\mathrm{M}$ & $\mathrm{M}$ & $\mathrm{M}$ \\
\hline
\end{tabular}

Fig. 2.4. Pin layout of benchmark case PNL-32.

\begin{tabular}{|c|c|c|c|c|c|c|c|c|}
\hline & & & & & & & & 27 \\
\hline & & & & & & & 25 & 26 \\
\hline & & & & & & 22 & 23 & 24 \\
\hline & & & & & 18 & 19 & 20 & 21 \\
\hline & & & & 13 & 14 & 15 & 16 & 17 \\
\hline & & & $\mathrm{M}$ & 8 & 9 & 10 & 11 & 12 \\
\hline & & $\mathrm{M}$ & $\mathrm{M}$ & $\mathrm{M}$ & 4 & 5 & 6 & 7 \\
\hline & $\mathrm{M}$ & $\mathrm{M}$ & $\mathrm{M}$ & $\mathrm{M}$ & $\mathrm{M}$ & $\mathrm{M}$ & 2 & 3 \\
\hline $\mathrm{M}$ & $\mathrm{M}$ & $\mathrm{M}$ & $\mathrm{M}$ & $\mathrm{M}$ & $\mathrm{M}$ & $\mathrm{M}$ & $\mathrm{M}$ & 1 \\
\hline
\end{tabular}

Fig. 2.5. Pin layout of benchmark case PNL-34. 


\begin{tabular}{|c|c|c|c|c|c|c|c|c|c|c|}
\hline & & & & & & & & & & 99 \\
\hline & & & & & & & & & 97 & 98 \\
\hline & & & & & & & & 94 & 95 & 96 \\
\hline & & & & & & & 90 & 91 & 92 & 93 \\
\hline & & & & & & 85 & 86 & 87 & 88 & 89 \\
\hline & & & & & 79 & 80 & 81 & 82 & 83 & 84 \\
\hline & & & & 72 & 73 & 74 & 75 & 76 & 77 & 78 \\
\hline & & & 64 & 65 & 66 & 67 & 68 & 69 & 70 & 71 \\
\hline & & 55 & 56 & 57 & 58 & 59 & 60 & 61 & 62 & 63 \\
\hline & 45 & 46 & 47 & 48 & 49 & 50 & 51 & 52 & 53 & 54 \\
\hline 34 & 35 & 36 & 37 & 38 & 39 & 40 & 41 & 42 & 43 & 44 \\
\hline $\mathrm{M}$ & 24 & 25 & 26 & 27 & 28 & 29 & 30 & 31 & 32 & 33 \\
\hline $\mathrm{M}$ & $\mathrm{M}$ & 15 & 16 & 17 & 18 & 19 & 20 & 21 & 22 & 23 \\
\hline $\mathrm{M}$ & $\mathrm{M}$ & $\mathrm{M}$ & 7 & 8 & 9 & 10 & 11 & 12 & 13 & 14 \\
\hline $\mathrm{M}$ & $\mathrm{M}$ & $\mathrm{M}$ & $\mathrm{M}$ & $\mathrm{M}$ & 1 & 2 & 3 & 4 & 5 & 6 \\
\hline
\end{tabular}

Fig. 2.6. Pin layout of benchmark case PNL-35. 


\begin{tabular}{|c|c|c|c|c|c|c|c|c|c|c|c|}
\hline & & & & & & & & & & & $\begin{array}{c}2.475 \\
(0.91)\end{array}$ \\
\hline & & & & & & & & & & $\begin{array}{l}2.458 \\
(0.46)\end{array}$ & $\begin{array}{r}2.460 \\
(0.46)\end{array}$ \\
\hline & & & & & & & & & $\begin{array}{l}2.413 \\
(0.48)\end{array}$ & $\begin{array}{r}2.427 \\
(0.31)\end{array}$ & $\begin{array}{c}2.423 \\
(0.44)\end{array}$ \\
\hline & & & & & & & & $\begin{array}{r}2.235 \\
(0.48)\end{array}$ & $\begin{array}{l}2.337 \\
(0.35)\end{array}$ & $\begin{array}{c}2.354 \\
(0.35)\end{array}$ & $\begin{array}{r}2.386 \\
(0.43)\end{array}$ \\
\hline & & & & & & & $\begin{array}{r}2.095 \\
(0.46)\end{array}$ & $\begin{array}{c}2.183 \\
(0.35)\end{array}$ & $\begin{array}{l}2.220 \\
(0.32)\end{array}$ & $\begin{array}{c}2.275 \\
(0.33)\end{array}$ & $\begin{array}{c}2.293 \\
(0.46)\end{array}$ \\
\hline & & & & & & $\begin{array}{r}1.883 \\
(0.57)\end{array}$ & $\begin{array}{c}1.982 \\
(0.35)\end{array}$ & $\begin{array}{l}2.074 \\
(0.32)\end{array}$ & $\begin{array}{l}2.127 \\
(0.36)\end{array}$ & $\begin{array}{r}2.150 \\
(0.35)\end{array}$ & $\begin{array}{r}2.168 \\
(0.49)\end{array}$ \\
\hline & & & & & $\begin{array}{l}1.636 \\
(0.58)\end{array}$ & $\begin{array}{l}1.761 \\
(0.41)\end{array}$ & $\begin{array}{c}1.860 \\
(0.35)\end{array}$ & $\begin{array}{l}1.947 \\
(0.35)\end{array}$ & $\begin{array}{l}1.999 \\
(0.36)\end{array}$ & $\begin{array}{l}2.033 \\
(0.35)\end{array}$ & $\begin{array}{c}2.042 \\
(0.49)\end{array}$ \\
\hline & & & & $\begin{array}{l}1.423 \\
(0.62)\end{array}$ & $\begin{array}{c}1.534 \\
(0.40)\end{array}$ & $\begin{array}{r}1.616 \\
(0.35)\end{array}$ & $\begin{array}{r}1.707 \\
(0.41)\end{array}$ & $\begin{array}{r}1.792 \\
(0.38)\end{array}$ & $\begin{array}{l}1.833 \\
(0.35)\end{array}$ & $\begin{array}{c}1.874 \\
(0.35)\end{array}$ & $\begin{array}{c}1.877 \\
(0.50)\end{array}$ \\
\hline & & & $\begin{array}{l}1.530 \\
(0.56)\end{array}$ & $\begin{array}{l}1.381 \\
(0.46)\end{array}$ & $\begin{array}{r}1.416 \\
(0.43)\end{array}$ & $\begin{array}{l}1.483 \\
(0.40)\end{array}$ & $\begin{array}{l}1.557 \\
(0.42)\end{array}$ & $\begin{array}{l}1.624 \\
(0.38)\end{array}$ & $\begin{array}{r}1.670 \\
(0.33)\end{array}$ & $\begin{array}{r}1.710 \\
(0.38)\end{array}$ & $\begin{array}{c}1.714 \\
(0.55)\end{array}$ \\
\hline & & 0 & $\begin{array}{l}2.213 \\
(0.35)\end{array}$ & $\begin{array}{l}1.510 \\
(0.44)\end{array}$ & $\begin{array}{c}1.358 \\
(0.41)\end{array}$ & $\begin{array}{l}1.367 \\
(0.43)\end{array}$ & $\begin{array}{c}1.423 \\
(0.42)\end{array}$ & $\begin{array}{l}1.493 \\
(0.39)\end{array}$ & $\begin{array}{l}1.530 \\
0.43)\end{array}$ & $\begin{array}{c}1.566 \\
(0.39)\end{array}$ & $\begin{array}{c}1.566 \\
(0.55)\end{array}$ \\
\hline & 0 & 0 & 0 & $\begin{array}{l}2.160 \\
(0.37)\end{array}$ & $\begin{array}{l}1.519 \\
(0.43)\end{array}$ & $\begin{array}{l}1.391 \\
(0.43)\end{array}$ & $\begin{array}{c}1.437 \\
(0.44)\end{array}$ & $\begin{array}{l}1.480 \\
(0.39)\end{array}$ & $\begin{array}{l}1.539 \\
(0.40)\end{array}$ & $\begin{array}{l}1.566 \\
(0.39)\end{array}$ & $\begin{array}{r}1.573 \\
(0.54)\end{array}$ \\
\hline 0 & 0 & 0 & 0 & 0 & $\begin{array}{l}2.311 \\
(0.40)\end{array}$ & $\begin{array}{l}2.001 \\
(0.43)\end{array}$ & $\begin{array}{l}2.035 \\
(0.39)\end{array}$ & $\begin{array}{l}2.124 \\
(0.40)\end{array}$ & $\begin{array}{r}2.197 \\
(0.36)\end{array}$ & $\begin{array}{l}2.225 \\
(0.39)\end{array}$ & $\begin{array}{c}2.254 \\
(0.57)\end{array}$ \\
\hline
\end{tabular}

Fig. 2.7a. Pin-power distribution for CENTRM benchmark PNL-30. Value in parentheses is the percent standard deviation. 


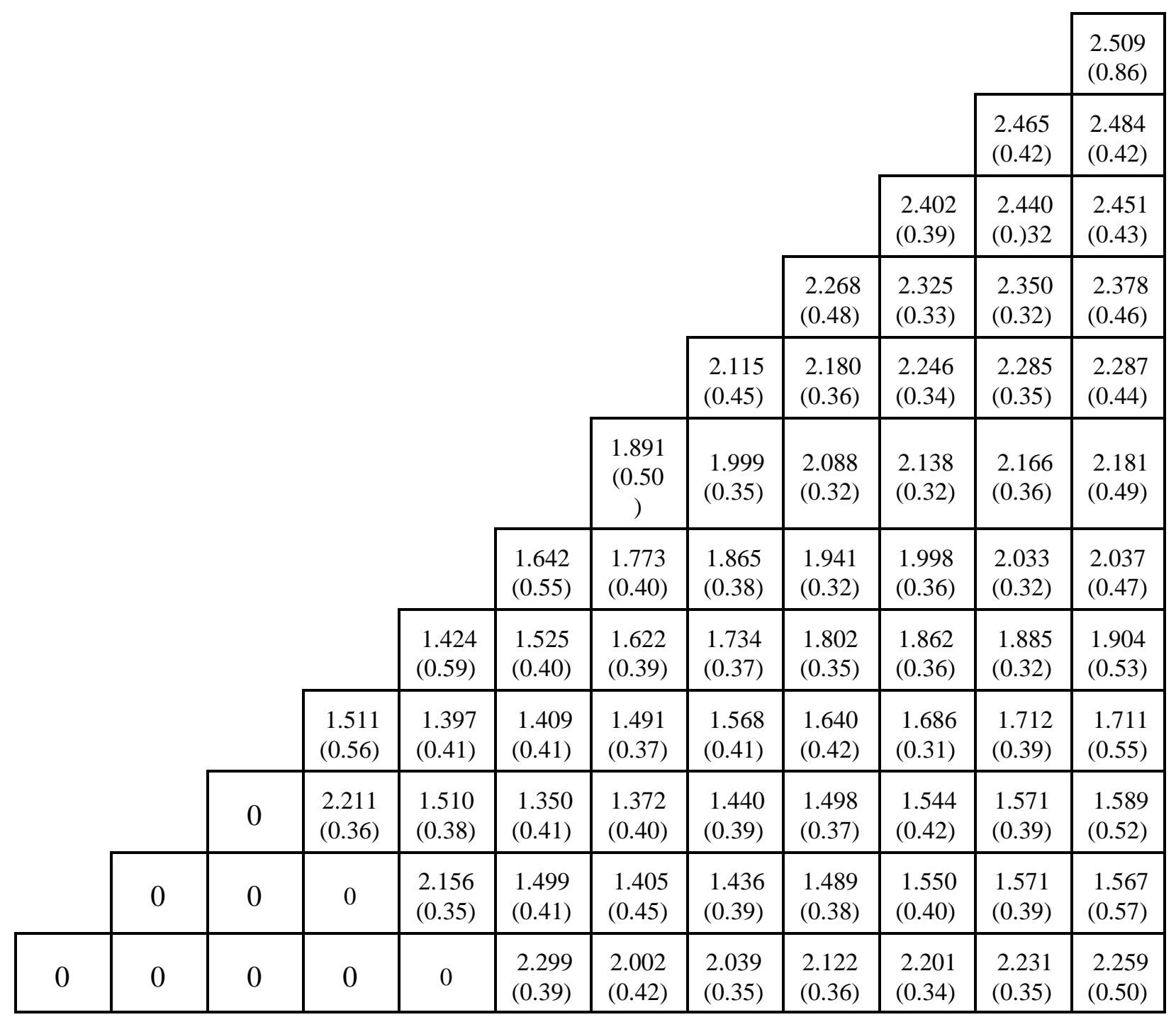

Fig. 2.7b. Pin-power distribution for NITAWL benchmark PNL-30. Value in parentheses is the percent standard deviation. 
Table 2.5. PNL-30 CENTRM reaction rates and fluxes

\begin{tabular}{|c|c|c|c|c|c|c|}
\hline Pin & Region & $\begin{array}{c}\mathrm{G}_{\mathrm{a}} \mathrm{M} \\
\left(\mathrm{cm}^{-3}-\mathrm{s}^{-1}\right)\end{array}$ & $\begin{array}{c}\mathcal{G}_{\mathrm{f}} \mathrm{M} \\
\left(\mathrm{cm}^{-3}-\mathrm{s}^{-1}\right)\end{array}$ & $\begin{array}{c}\mathrm{G}_{\mathrm{f}} \mathrm{M} \\
\left(\mathrm{cm}^{-3}-\mathrm{s}^{-1}\right)\end{array}$ & $\begin{array}{c}\mathrm{M} \\
\left(\mathrm{cm}-\mathrm{s}^{-1}\right)\end{array}$ & $M / M_{t}$ \\
\hline \multirow{3}{*}{1} & Fuel & $1.417 \mathrm{E}-05$ & $2.311 \mathrm{E}-05$ & $8.244 \mathrm{E}-06$ & $1.385 \mathrm{E}-2$ & 0.412 \\
\hline & Clad & $2.864 \mathrm{E}-07$ & 0.0 & 0.0 & $2.672 \mathrm{E}-3$ & 0.079 \\
\hline & Mod. & $5.922 \mathrm{E}-07$ & 0.0 & 0.0 & $1.710 \mathrm{E}-2$ & 0.509 \\
\hline \multirow{3}{*}{69} & Fuel & $1.809 \mathrm{E}-05$ & $2.453 \mathrm{E}-05$ & $8.727 \mathrm{E}-06$ & $3.412 \mathrm{E}-2$ & 0.410 \\
\hline & Clad & 4.701E-07 & 0.0 & 0.0 & $6.638 \mathrm{E}-3$ & 0.080 \\
\hline & Mod. & $5.476 \mathrm{E}-07$ & 0.0 & 0.0 & 4.237E-2 & 0.510 \\
\hline
\end{tabular}

Table 2.6. PNL-30 NITAWL reaction rates and fluxes

\begin{tabular}{ccccccc}
\hline Pin & Region & $\begin{array}{c}\mathrm{G}_{\mathrm{a}} \mathrm{M} \\
\left(\mathrm{cm}^{-3}-\mathrm{s}^{-1}\right)\end{array}$ & $\begin{array}{c}\mathrm{G}_{\mathrm{f}} \mathrm{M} \\
\left(\mathrm{cm}^{-3}-\mathrm{s}^{-1}\right)\end{array}$ & $\begin{array}{c}\mathrm{G}_{\mathrm{f}} \mathrm{M} \\
\left(\mathrm{cm}^{-3}-\mathrm{s}^{-1}\right)\end{array}$ & $\begin{array}{c}\mathrm{M} \\
\left(\mathrm{cm} \mathrm{-} \mathrm{s}^{-1}\right)\end{array}$ & $\mathrm{MM}_{\mathrm{t}}$ \\
\hline \multirow{2}{*}{1} & Fuel & $1.407 \mathrm{E}-05$ & $2.297 \mathrm{E}-05$ & $8.192 \mathrm{E}-06$ & $1.384 \mathrm{E}-2$ & 0.412 \\
& Clad & $2.802 \mathrm{E}-07$ & 0.0 & 0.0 & $2.660 \mathrm{E}-3$ & 0.079 \\
& Mod. & $5.895 \mathrm{E}-07$ & 0.0 & 0.0 & $1.707 \mathrm{E}-2$ & 0.509 \\
\hline \multirow{2}{*}{69} & Fuel & $1.835 \mathrm{E}-05$ & $2.515 \mathrm{E}-05$ & $8.948 \mathrm{E}-06$ & $3.395 \mathrm{E}-2$ & 0.410 \\
& Clad & $4.678 \mathrm{E}-07$ & 0.0 & 0.0 & $6.629 \mathrm{E}-3$ & 0.080 \\
& Mod. & $5.644 \mathrm{E}-07$ & 0.0 & 0.0 & $4.232 \mathrm{E}-2$ & 0.510 \\
\hline
\end{tabular}


Table 2.7. PNL-30, Pin-1 four-group fluxes

\begin{tabular}{ccc}
\hline Group & $\begin{array}{c}\text { CENTRM M } \\
\left(\mathrm{cm}^{-2}-\mathrm{s}^{-1}\right)\end{array}$ & $\begin{array}{c}\text { NITAWL M } \\
\left(\mathrm{cm}^{-2}-\mathrm{s}^{-1}\right)\end{array}$ \\
\hline 1 & $7.308 \mathrm{E}-05$ & $7.296 \mathrm{E}-05$ \\
2 & $2.292 \mathrm{E}-05$ & $2.291 \mathrm{E}-05$ \\
3 & $5.145 \mathrm{E}-06$ & $5.269 \mathrm{E}-06$ \\
4 & $2.251 \mathrm{E}-05$ & $2.239 \mathrm{E}-05$ \\
\hline
\end{tabular}

Table 2.8. PNL-30, Pin-1 four-group U-235 cross sections

\begin{tabular}{cccccccc}
\hline Group & \multicolumn{3}{c}{ CENTRM cross sections $\left(\mathrm{cm}^{-1}\right)$} & \multicolumn{2}{c}{ NITAWL cross sections $\left(\mathrm{cm}^{-1}\right)$} \\
\hline & $\frac{\mathrm{G}_{\mathrm{a}}}{2.31102 \mathrm{E}-04}$ & $\frac{\mathrm{G}_{\mathrm{f}}}{5.17496 \mathrm{E}-04}$ & $\frac{\mathrm{G}_{\mathrm{f}}}{2.00508 \mathrm{E}-04}$ & $\frac{\mathrm{G}_{\mathrm{a}}}{2.30990 \mathrm{E}-04}$ & $\frac{\mathrm{G}_{\mathrm{f}}}{5.17470 \mathrm{E}-04}$ & $\frac{\mathrm{G}_{\mathrm{f}}}{2.00433 \mathrm{E}-04}$ \\
1 & $5.24167 \mathrm{E}-03$ & $8.10227 \mathrm{E}-03$ & $3.32510 \mathrm{E}-03$ & $5.22152 \mathrm{E}-03$ & $8.04138 \mathrm{E}-03$ & $3.30010 \mathrm{E}-03$ \\
2 & $8.11652 \mathrm{E}-03$ & $1.64870 \mathrm{E}-02$ & $6.76610 \mathrm{E}-03$ & $8.06149 \mathrm{E}-03$ & $1.64181 \mathrm{E}-02$ & $6.73784 \mathrm{E}-03$ \\
3 & $7.34638 \mathrm{E}-02$ & $1.52885 \mathrm{E}-01$ & $6.27423 \mathrm{E}-02$ & $7.34430 \mathrm{E}-02$ & $1.52842 \mathrm{E}-01$ & $6.27253 \mathrm{E}-02$ \\
\hline
\end{tabular}

Table 2.9. PNL-30, Pin-1 four-group U-238 cross sections

\begin{tabular}{cccccccc}
\hline Group & \multicolumn{3}{c}{ CENTRM cross sections $\left(\mathrm{cm}^{-1}\right)$} & \multicolumn{3}{c}{ NITAWL cross sections $\left(\mathrm{cm}^{-1}\right)$} \\
\hline & $\frac{\mathrm{G}_{\mathrm{a}}}{}$ & $\frac{\mathrm{G}_{\mathrm{f}}}{1.78395 \mathrm{E}-03}$ & $\frac{1.18456 \mathrm{E}-02}{4.21512 \mathrm{E}-03}$ & $\frac{\mathrm{G}_{\mathrm{f}}}{4.78829 \mathrm{E}-03}$ & $\frac{\mathrm{G}_{\mathrm{f}}}{1.18801 \mathrm{E}-02}$ & $\overline{\mathrm{G}}_{\mathrm{f}}$ \\
\hline & $4.22404 \mathrm{E}-03$ \\
3 & $1.10308 \mathrm{E}-02$ & $3.66348 \mathrm{E}-06$ & $2.34636 \mathrm{E}-06$ & $4.43898 \mathrm{E}-02$ & $5.54963 \mathrm{E}-06$ & $2.29918 \mathrm{E}-06$ \\
4 & $4.21801 \mathrm{E}-02$ & $1.98117 \mathrm{E}-07$ & $8.20835 \mathrm{E}-08$ & $4.21730 \mathrm{E}-02$ & $1.98085 \mathrm{E}-07$ & $8.20706 \mathrm{E}-08$ \\
\hline
\end{tabular}

Table 2.10. PNL-30, Pin-1 four-group Pu-239 cross sections

\begin{tabular}{cccccccc}
\hline Group & \multicolumn{3}{c}{ CENTRM cross sections $\left(\mathrm{cm}^{-1}\right)$} & \multicolumn{3}{c}{ NITAWL cross sections $\left(\mathrm{cm}^{-1}\right)$} \\
\hline & $\frac{\mathrm{G}_{\mathrm{a}}}{7.31247 \mathrm{E}-04}$ & $\frac{\mathrm{G}_{\mathrm{f}}}{2.10623 \mathrm{E}-03}$ & $\frac{\mathrm{G}_{\mathrm{f}}}{6.81955 \mathrm{E}-04}$ & $\frac{\mathrm{G}_{\mathrm{a}}}{7.31013 \mathrm{E}-04}$ & $\frac{\mathrm{G}_{\mathrm{f}}}{2.10628 \mathrm{E}-03}$ & $\frac{\mathrm{G}_{\mathrm{f}}}{6.81768 \mathrm{E}-04}$ \\
1 & $1.46556 \mathrm{E}-02$ & $2.42259 \mathrm{E}-02$ & $8.41230 \mathrm{E}-03$ & $1.42800 \mathrm{E}-02$ & $2.35796 \mathrm{E}-02$ & $8.18791 \mathrm{E}-03$ \\
2 & $4.46091 \mathrm{E}-02$ & $8.80453 \mathrm{E}-02$ & $3.05734 \mathrm{E}-02$ & $4.45318 \mathrm{E}-02$ & $8.79178 \mathrm{E}-02$ & $3.05291 \mathrm{E}-02$ \\
3 & $3.81701 \mathrm{E}-01$ & $7.65540 \mathrm{E}-01$ & $2.64839 \mathrm{E}-01$ & $3.80931 \mathrm{E}-01$ & $7.64203 \mathrm{E}-01$ & $2.64376 \mathrm{E}-01$ \\
\hline
\end{tabular}


Table 2.11. PNL-30, Pin-69 four-group fluxes

\begin{tabular}{ccc}
\hline Group & $\begin{array}{c}\text { CENTRM M } \\
\left(\mathrm{cm}^{-2}-\mathrm{s}^{-1}\right)\end{array}$ & $\begin{array}{c}\text { NITAWL M } \\
\left(\mathrm{cm}^{-2}-\mathrm{s}^{-1}\right)\end{array}$ \\
\hline 1 & $1.978 \mathrm{E}-04$ & $1.958 \mathrm{E}-04$ \\
2 & $7.342 \mathrm{E}-05$ & $7.309 \mathrm{E}-05$ \\
3 & $1.445 \mathrm{E}-05$ & $1.458 \mathrm{E}-05$ \\
4 & $1.901 \mathrm{E}-05$ & $1.966 \mathrm{E}-05$ \\
\hline
\end{tabular}

Table 2.12. PNL-30, Pin-69 four-group U-235 cross sections

\begin{tabular}{cccccccc}
\hline Group & \multicolumn{3}{c}{ CENTRM cross sections $\left(\mathrm{cm}^{-1}\right)$} & \multicolumn{3}{c}{ NITAWL cross sections $\left(\mathrm{cm}^{-1}\right)$} \\
\hline & $\frac{\mathrm{G}_{\mathrm{a}}}{2.40583 \mathrm{E}-04}$ & $\frac{\mathrm{G}_{\mathrm{f}}}{5.25394 \mathrm{E}-04}$ & $\frac{\mathrm{G}_{\mathrm{f}}}{2.05274 \mathrm{E}-04}$ & $\frac{\mathrm{G}_{\mathrm{a}}}{2.40289 \mathrm{E}-04}$ & $\frac{\mathrm{G}_{\mathrm{f}}}{5.25082 \mathrm{E}-04}$ & $\frac{\mathrm{G}_{\mathrm{f}}}{2.05125 \mathrm{E}-04}$ \\
1 & $5.09194 \mathrm{E}-03$ & $7.87946 \mathrm{E}-03$ & $3.23366 \mathrm{E}-03$ & $4.90525 \mathrm{E}-03$ & $7.60379 \mathrm{E}-03$ & $3.12052 \mathrm{E}-03$ \\
2 & $8.03087 \mathrm{E}-03$ & $1.62800 \mathrm{E}-02$ & $6.68118 \mathrm{E}-03$ & $8.07572 \mathrm{E}-03$ & $1.64173 \mathrm{E}-02$ & $6.73752 \mathrm{E}-03$ \\
3 & $6.44140 \mathrm{E}-02$ & $1.33756 \mathrm{E}-01$ & $5.48922 \mathrm{E}-02$ & $6.49323 \mathrm{E}-02$ & $1.34838 \mathrm{E}-01$ & $5.53362 \mathrm{E}-02$ \\
\hline
\end{tabular}

Table 2.13. PNL-30, Pin-69 four-group U-238 cross sections

\begin{tabular}{|c|c|c|c|c|c|c|}
\hline \multirow[t]{2}{*}{ Group } & \multicolumn{3}{|c|}{ CENTRM cross sections $\left(\mathrm{cm}^{-1}\right)$} & \multicolumn{3}{|c|}{ NITAWL cross sections $\left(\mathrm{cm}^{-1}\right)$} \\
\hline & $\mathrm{G}_{\mathrm{a}}$ & $<\mathrm{G}_{\mathrm{f}}$ & $\mathrm{G}_{\mathrm{f}}$ & $\mathrm{G}_{\mathrm{a}}$ & $\mathbb{G}_{\mathrm{f}}$ & $\mathrm{G}_{\mathrm{f}}$ \\
\hline 1 & $6.48013 \mathrm{E}-03$ & $1.00639 \mathrm{E}-02$ & $3.58425 \mathrm{E}-03$ & $6 . \overline{50153 \mathrm{E}-03}$ & $\overline{1.01355 \mathrm{E}-02}$ & $\overline{3.61266 \mathrm{E}-03}$ \\
\hline 2 & 4.33496E-02 & $5.86890 \mathrm{E}-06$ & $2.43146 \mathrm{E}-06$ & 4.25035E-02 & $5.67595 \mathrm{E}-06$ & 2.35151E-06 \\
\hline 3 & $1.10028 \mathrm{E}-02$ & $3.78364 \mathrm{E}-08$ & $1.56763 \mathrm{E}-08$ & $1.10397 \mathrm{E}-02$ & $3.81740 \mathrm{E}-08$ & $1.58162 \mathrm{E}-08$ \\
\hline 4 & $3.74989 \mathrm{E}-02$ & $1.75656 \mathrm{E}-07$ & $7.27780 \mathrm{E}-08$ & $3.77530 \mathrm{E}-02$ & $1.76873 \mathrm{E}-07$ & $7.32818 \mathrm{E}-08$ \\
\hline
\end{tabular}

Table 2.14. PNL-30, Pin-69 four-group Pu-239 cross sections

\begin{tabular}{cccccccc}
\hline Group & \multicolumn{3}{c}{ CENTRM cross sections $\left(\mathrm{cm}^{-1}\right)$} & \multicolumn{3}{c}{ NITAWL cross sections $\left(\mathrm{cm}^{-1}\right)$} \\
\hline & $\frac{\mathrm{G}_{\mathrm{a}}}{7.34312 \mathrm{E}-04}$ & $\frac{\mathrm{G}_{\mathrm{f}}}{2.06900 \mathrm{E}-03}$ & $\frac{\mathrm{G}_{\mathrm{f}}}{6.75303 \mathrm{E}-04}$ & $\frac{\mathrm{G}_{\mathrm{a}}}{7.34377 \mathrm{E}-04}$ & $\frac{\mathrm{G}_{\mathrm{f}}}{2.07025 \mathrm{E}-03}$ & $\frac{\mathrm{G}_{\mathrm{f}}}{6.75577 \mathrm{E}-04}$ \\
1 & $1.41486 \mathrm{E}-02$ & $2.33093 \mathrm{E}-02$ & $8.09402 \mathrm{E}-03$ & $1.38472 \mathrm{E}-02$ & $2.29011 \mathrm{E}-02$ & $7.95226 \mathrm{E}-03$ \\
2 & $4.37500 \mathrm{E}-02$ & $8.64152 \mathrm{E}-02$ & $3.00072 \mathrm{E}-02$ & $4.41986 \mathrm{E}-02$ & $8.73169 \mathrm{E}-02$ & $3.03204 \mathrm{E}-02$ \\
3 & $4.24647 \mathrm{E}-01$ & $8.20289 \mathrm{E}-01$ & $2.83994 \mathrm{E}-01$ & $4.24708 \mathrm{E}-01$ & $8.21267 \mathrm{E}-01$ & $2.84326 \mathrm{E}-01$ \\
\hline
\end{tabular}




\begin{tabular}{|c|c|c|c|c|c|c|c|c|c|c|c|}
\hline & & & & & & & & & & & $\begin{array}{c}1.824 \\
(0.90)\end{array}$ \\
\hline & & & & & & & & & & $\begin{array}{l}1.821 \\
(0.58)\end{array}$ & $\begin{array}{l}1.818 \\
(0.53)\end{array}$ \\
\hline & & & & & & & & & $\begin{array}{l}1.780 \\
(0.50)\end{array}$ & $\begin{array}{c}1.785 \\
(0.32)\end{array}$ & $\begin{array}{l}1.817 \\
(0.48)\end{array}$ \\
\hline & & & & & & & & $\begin{array}{c}1.693 \\
(0.56)\end{array}$ & $\begin{array}{r}1.725 \\
(038 .)\end{array}$ & $\begin{array}{c}1.759 \\
(0.36)\end{array}$ & $\begin{array}{r}1.764 \\
(0.47)\end{array}$ \\
\hline & & & & & & & $\begin{array}{l}1.611 \\
(055 .)\end{array}$ & $\begin{array}{r}1.650 \\
(0.34)\end{array}$ & $\begin{array}{l}1.683 \\
(0.35)\end{array}$ & $\begin{array}{l}1.706 \\
(0.36)\end{array}$ & $\begin{array}{c}1.687 \\
(0.50)\end{array}$ \\
\hline & & & & & & $\begin{array}{c}1.492 \\
(0.52)\end{array}$ & $\begin{array}{c}1.535 \\
(0.38)\end{array}$ & $\begin{array}{r}1.601 \\
(0.35)\end{array}$ & $\begin{array}{c}1.624 \\
(0.40)\end{array}$ & $\begin{array}{c}1.633 \\
(0.38)\end{array}$ & $\begin{array}{c}1.634 \\
(052 .)\end{array}$ \\
\hline & & & & & $\begin{array}{r}1.338 \\
(0.58)\end{array}$ & $\begin{array}{r}1.419 \\
(0.41)\end{array}$ & $\begin{array}{r}1.476 \\
(0.41)\end{array}$ & $\begin{array}{r}1.509 \\
(0.35)\end{array}$ & $\begin{array}{r}1.550 \\
(0.37)\end{array}$ & $\begin{array}{r}1.562 \\
(0.41)\end{array}$ & $\begin{array}{l}1.570 \\
(0.57)\end{array}$ \\
\hline & & & & $\begin{array}{c}1.202 \\
(064 .)\end{array}$ & $\begin{array}{l}1.271 \\
(0.42)\end{array}$ & $\begin{array}{l}1.332 \\
(0.39)\end{array}$ & $\begin{array}{r}1.385 \\
(0.40)\end{array}$ & $\begin{array}{c}1.437 \\
(0.36)\end{array}$ & $\begin{array}{r}1.460 \\
(0.44)\end{array}$ & $\begin{array}{c}1.488 \\
(0.40)\end{array}$ & $\begin{array}{l}1.500 \\
(0.51)\end{array}$ \\
\hline & & & $\begin{array}{l}1.018 \\
(0.65)\end{array}$ & $\begin{array}{l}1.098 \\
(0.46)\end{array}$ & $\begin{array}{c}1.175 \\
(0.42)\end{array}$ & $\begin{array}{l}1.245 \\
(0.48)\end{array}$ & $\begin{array}{l}1.304 \\
(0.38)\end{array}$ & $\begin{array}{l}1.346 \\
(0.40)\end{array}$ & $\begin{array}{l}1.359 \\
(0.40)\end{array}$ & $\begin{array}{c}1.393 \\
(0.40)\end{array}$ & $\begin{array}{l}1.393 \\
(0.59)\end{array}$ \\
\hline & & $\begin{array}{c}0.861 \\
(0.74)\end{array}$ & $\begin{array}{l}0.930 \\
(0.45)\end{array}$ & $\begin{array}{l}1.016 \\
(0.48)\end{array}$ & $\begin{array}{l}1.078 \\
(0.45)\end{array}$ & $\begin{array}{l}1.139 \\
(0.45)\end{array}$ & $\begin{array}{r}1.195 \\
(0.46)\end{array}$ & $\begin{array}{r}1.228 \\
(0.40)\end{array}$ & $\begin{array}{l}1.261 \\
(0.41)\end{array}$ & $\begin{array}{l}1.282 \\
(0.45)\end{array}$ & $\begin{array}{r}1.288 \\
(0.58)\end{array}$ \\
\hline & $\begin{array}{c}0.719 \\
(0.74)\end{array}$ & $\begin{array}{c}0.777 \\
(0.57)\end{array}$ & $\begin{array}{c}0.849 \\
(0.55)\end{array}$ & $\begin{array}{r}0.919 \\
(0.46)\end{array}$ & $\begin{array}{l}0.978 \\
(0.53)\end{array}$ & $\begin{array}{r}1.026 \\
(0.43)\end{array}$ & $\begin{array}{l}1.088 \\
(0.43)\end{array}$ & $\begin{array}{l}1.121 \\
(0.49)\end{array}$ & $\begin{array}{l}1.157 \\
(0.44)\end{array}$ & $\begin{array}{l}1.164 \\
(0.45)\end{array}$ & $\begin{array}{c}1.181 \\
(0.66)\end{array}$ \\
\hline $\begin{array}{l}0.924 \\
(0.72)\end{array}$ & $\begin{array}{r}0.737 \\
(0.59)\end{array}$ & $\begin{array}{c}0.737 \\
(0.54)\end{array}$ & $\begin{array}{c}0.771 \\
(0.57)\end{array}$ & $\begin{array}{r}0.822 \\
(0.52)\end{array}$ & $\begin{array}{r}0.872 \\
(0.47)\end{array}$ & $\begin{array}{l}0.918 \\
(0.48)\end{array}$ & $\begin{array}{r}0.965 \\
(0.46)\end{array}$ & $\begin{array}{l}1.011 \\
(0.51)\end{array}$ & $\begin{array}{c}1.043 \\
(0.47)\end{array}$ & $\begin{array}{r}1.054 \\
(0.45)\end{array}$ & $\begin{array}{r}1.056 \\
(0.64)\end{array}$ \\
\hline 0 & $\begin{array}{r}0.985 \\
(0.52)\end{array}$ & $\begin{array}{c}0.860 \\
(0.51)\end{array}$ & $\begin{array}{l}0.772 \\
(0.55)\end{array}$ & $\begin{array}{c}0.756 \\
(0.56)\end{array}$ & $\begin{array}{r}0.787 \\
(0.57)\end{array}$ & $\begin{array}{l}0.818 \\
(0.53)\end{array}$ & $\begin{array}{l}0.862 \\
(0.51)\end{array}$ & $\begin{array}{l}0.888 \\
(0.48)\end{array}$ & $\begin{array}{c}0.916 \\
(0.53)\end{array}$ & $\begin{array}{l}0.932 \\
(0.42)\end{array}$ & $\begin{array}{r}0.944 \\
(0.67)\end{array}$ \\
\hline 0 & 0 & 0 & $\begin{array}{c}1.046 \\
(0.49)\end{array}$ & $\begin{array}{l}0.900 \\
(0.48)\end{array}$ & $\begin{array}{l}0.789 \\
(0.52)\end{array}$ & $\begin{array}{r}0.782 \\
(0.57)\end{array}$ & $\begin{array}{c}0.792 \\
(0.56)\end{array}$ & $\begin{array}{c}0.788 \\
(0.56)\end{array}$ & $\begin{array}{r}0.803 \\
(0.49)\end{array}$ & $\begin{array}{r}0.820 \\
(0.51)\end{array}$ & $\begin{array}{c}0.821 \\
(0.70)\end{array}$ \\
\hline 0 & 0 & 0 & 0 & 0 & $\begin{array}{r}1.065 \\
(0.45)\end{array}$ & $\begin{array}{c}0.946 \\
(0.50)\end{array}$ & $\begin{array}{l}0.890 \\
(0.55)\end{array}$ & $\begin{array}{l}0.781 \\
(0.59)\end{array}$ & $\begin{array}{l}0.745 \\
(0.55)\end{array}$ & $\begin{array}{c}0.751 \\
(0.60)\end{array}$ & $\begin{array}{l}0.764 \\
(0.79)\end{array}$ \\
\hline 0 & 0 & 0 & 0 & 0 & 0 & 0 & 0 & $\begin{array}{l}1.051 \\
(0.45)\end{array}$ & $\begin{array}{l}0.917 \\
(0.54)\end{array}$ & $\begin{array}{l}0.911 \\
(0.50)\end{array}$ & $\begin{array}{r}0.903 \\
(0.77)\end{array}$ \\
\hline
\end{tabular}

Fig. 2.8a. Pin-power distribution for CENTRM benchmark PNL-31. Value in parentheses is percent standard deviation. 


\begin{tabular}{|c|c|c|c|c|c|c|c|c|c|c|c|}
\hline & & & & & & & & & & & $\begin{array}{l}1.829 \\
(1.01)\end{array}$ \\
\hline & & & & & & & & & & $\begin{array}{l}1.827 \\
(0.51)\end{array}$ & $\begin{array}{r}1.832 \\
(0.53)\end{array}$ \\
\hline & & & & & & & & & $\begin{array}{l}1.775 \\
(0.50)\end{array}$ & $\begin{array}{c}1.798 \\
(0.35)\end{array}$ & $\begin{array}{l}1.794 \\
(0.52)\end{array}$ \\
\hline & & & & & & & & $\begin{array}{c}1.722 \\
(0.57)\end{array}$ & $\begin{array}{c}1.742 \\
(0.35)\end{array}$ & $\begin{array}{c}1.776 \\
(0.37)\end{array}$ & $\begin{array}{r}1.785 \\
(0.51)\end{array}$ \\
\hline & & & & & & & $\begin{array}{l}1.612 \\
(0.53)\end{array}$ & $\begin{array}{r}1.666 \\
(0.36)\end{array}$ & $\begin{array}{l}1.698 \\
(0.38)\end{array}$ & $\begin{array}{l}1.716 \\
(0.35)\end{array}$ & $\begin{array}{l}1.724 \\
(0.50)\end{array}$ \\
\hline & & & & & & $\begin{array}{r}1.502 \\
(0.53)\end{array}$ & $\begin{array}{r}1.549 \\
(0.38)\end{array}$ & $\begin{array}{c}1.593 \\
(0.40)\end{array}$ & $\begin{array}{r}1.640 \\
(0.35)\end{array}$ & $\begin{array}{c}1.644 \\
(0.37)\end{array}$ & $\begin{array}{l}1.646 \\
(0.56)\end{array}$ \\
\hline & & & & & $\begin{array}{c}1.353 \\
(0.57)\end{array}$ & $\begin{array}{c}1.418 \\
(0.43)\end{array}$ & $\begin{array}{r}1.474 \\
(0.37)\end{array}$ & $\begin{array}{l}1.525 \\
(0.38)\end{array}$ & $\begin{array}{l}1.566 \\
(0.38)\end{array}$ & $\begin{array}{c}1.580 \\
(0.38)\end{array}$ & $\begin{array}{c}1.572 \\
(0.51)\end{array}$ \\
\hline & & & & $\begin{array}{c}1.192 \\
(0.61)\end{array}$ & $\begin{array}{c}1.274 \\
(0.44)\end{array}$ & $\begin{array}{c}1.343 \\
(0.39)\end{array}$ & $\begin{array}{c}1.402 \\
(0.41)\end{array}$ & $\begin{array}{r}1.435 \\
(0.38)\end{array}$ & $\begin{array}{c}1.478 \\
(0.41)\end{array}$ & $\begin{array}{c}1.493 \\
(0.37)\end{array}$ & $\begin{array}{r}1.486 \\
(0.58)\end{array}$ \\
\hline & & & $\begin{array}{l}1.017 \\
(0.66)\end{array}$ & $\begin{array}{l}1.107 \\
(0.47)\end{array}$ & $\begin{array}{l}1.177 \\
(0.43)\end{array}$ & $\begin{array}{l}1.252 \\
(0.40)\end{array}$ & $\begin{array}{l}1.299 \\
(0.39)\end{array}$ & $\begin{array}{l}1.359 \\
(0.42)\end{array}$ & $\begin{array}{l}1.381 \\
(0.44)\end{array}$ & $\begin{array}{l}1.384 \\
(0.40)\end{array}$ & $\begin{array}{l}1.399 \\
(0.55)\end{array}$ \\
\hline & & $\begin{array}{r}0.860 \\
(0.75)\end{array}$ & $\begin{array}{l}0.929 \\
(0.51)\end{array}$ & $\begin{array}{l}1.010 \\
(0.46)\end{array}$ & $\begin{array}{l}1.079 \\
(0.46)\end{array}$ & $\begin{array}{l}1.145 \\
(0.45)\end{array}$ & $\begin{array}{l}1.202 \\
(0.41)\end{array}$ & $\begin{array}{r}1.245 \\
(0.43)\end{array}$ & $\begin{array}{l}1.272 \\
(0.39)\end{array}$ & $\begin{array}{l}1.295 \\
(0.43)\end{array}$ & $\begin{array}{l}1.297 \\
(0.59)\end{array}$ \\
\hline & $\begin{array}{l}0.722 \\
(0.79)\end{array}$ & $\begin{array}{l}0.771 \\
(0.55)\end{array}$ & $\begin{array}{l}0.841 \\
(0.55)\end{array}$ & $\begin{array}{l}0.915 \\
(0.51)\end{array}$ & $\begin{array}{l}0.977 \\
(0.43)\end{array}$ & $\begin{array}{l}1.041 \\
(0.47)\end{array}$ & $\begin{array}{l}1.106 \\
(0.47)\end{array}$ & $\begin{array}{l}1.131 \\
(0.43)\end{array}$ & $\begin{array}{c}1.162 \\
(0.43)\end{array}$ & $\begin{array}{r}1.179 \\
(0.48)\end{array}$ & $\begin{array}{c}1.181 \\
(0.60)\end{array}$ \\
\hline $\begin{array}{l}0.937 \\
(0.68)\end{array}$ & $\begin{array}{c}0.736 \\
(0.61)\end{array}$ & $\begin{array}{c}0.728 \\
(0.54)\end{array}$ & $\begin{array}{c}0.766 \\
(0.58)\end{array}$ & $\begin{array}{c}0.825 \\
(0.55)\end{array}$ & $\begin{array}{l}0.880 \\
(0.47)\end{array}$ & $\begin{array}{l}0.917 \\
(0.49)\end{array}$ & $\begin{array}{c}0.982 \\
(0.47)\end{array}$ & $\begin{array}{c}1.013 \\
(0.45)\end{array}$ & $\begin{array}{l}1.031 \\
(0.46)\end{array}$ & $\begin{array}{c}1.049 \\
(0.45)\end{array}$ & $\begin{array}{r}1.064 \\
(0.66)\end{array}$ \\
\hline 0 & $\begin{array}{r}0.991 \\
(0.51)\end{array}$ & $\begin{array}{l}0.853 \\
(0.52)\end{array}$ & $\begin{array}{l}0.774 \\
(0.57)\end{array}$ & $\begin{array}{l}0.771 \\
(0.53)\end{array}$ & $\begin{array}{l}0.795 \\
(0.53)\end{array}$ & $\begin{array}{l}0.827 \\
(0.53)\end{array}$ & $\begin{array}{l}0.866 \\
(0.48)\end{array}$ & $\begin{array}{l}0.891 \\
(0.52)\end{array}$ & $\begin{array}{l}0.918 \\
(0.52)\end{array}$ & $\begin{array}{l}0.925 \\
(0.44)\end{array}$ & $\begin{array}{c}0.936 \\
(0.67)\end{array}$ \\
\hline 0 & 0 & 0 & $\begin{array}{c}1.039 \\
(0.54)\end{array}$ & $\begin{array}{c}0.888 \\
(0.51)\end{array}$ & $\begin{array}{l}0.787 \\
(0.50)\end{array}$ & $\begin{array}{c}0.778 \\
(0.47)\end{array}$ & $\begin{array}{l}0.787 \\
(0.60)\end{array}$ & $\begin{array}{l}0.798 \\
(0.55)\end{array}$ & $\begin{array}{c}0.803 \\
(0.52)\end{array}$ & $\begin{array}{c}0.811 \\
(0.51)\end{array}$ & $\begin{array}{r}0.817 \\
(0.72)\end{array}$ \\
\hline 0 & 0 & 0 & 0 & 0 & $\begin{array}{l}1.064 \\
(0.49)\end{array}$ & $\begin{array}{c}0.952 \\
(0.45)\end{array}$ & $\begin{array}{r}0.896 \\
(0.51)\end{array}$ & $\begin{array}{l}0.782 \\
(0.53)\end{array}$ & $\begin{array}{l}0.751 \\
(0.55)\end{array}$ & $\begin{array}{r}0.757 \\
(0.55)\end{array}$ & $\begin{array}{l}0.744 \\
(0.80)\end{array}$ \\
\hline 0 & 0 & 0 & 0 & 0 & 0 & 0 & 0 & $\begin{array}{r}1.052 \\
(0.48)\end{array}$ & $\begin{array}{l}0.917 \\
(0.52)\end{array}$ & $\begin{array}{l}0.905 \\
(0.49)\end{array}$ & $\begin{array}{r}0.914 \\
(0.71)\end{array}$ \\
\hline
\end{tabular}

Fig. 2.8b. Pin power distribution for NITAWL benchmark PNL-31. Value in parentheses is percent standard deviation. 
Table 2.15. PNL-31 CENTRM reaction rates and fluxes

\begin{tabular}{|c|c|c|c|c|c|c|}
\hline Pin & Region & $\begin{array}{c}\mathrm{G}_{\mathrm{a}} \mathrm{M} \\
\left(\mathrm{cm}^{-3}-\mathrm{s}^{-1}\right)\end{array}$ & $\begin{array}{c}\mathcal{G}_{\mathrm{f}} \mathrm{M} \\
\left(\mathrm{cm}^{-3}-\mathrm{s}^{-1}\right)\end{array}$ & $\begin{array}{c}\mathrm{G}_{\mathrm{f}} \mathrm{M} \\
\left(\mathrm{cm}^{-3}-\mathrm{s}^{-1}\right)\end{array}$ & $\begin{array}{c}\mathrm{M} \\
\left(\mathrm{cm}-\mathrm{s}^{-1}\right)\end{array}$ & $\mathrm{M}_{\mathrm{t}}$ \\
\hline \multirow{3}{*}{1} & Fuel & $6.732 \mathrm{E}-06$ & $1.0493 \mathrm{E}-05$ & $3.738 \mathrm{E}-06$ & $7.748 \mathrm{E}-3$ & 0.412 \\
\hline & Clad & $1.407 \mathrm{E}-07$ & 0.0 & 0.0 & $1.494 \mathrm{E}-3$ & 0.079 \\
\hline & Mod. & $5.801 \mathrm{E}-07$ & 0.0 & 0.0 & $9.563 \mathrm{E}-3$ & 0.509 \\
\hline \multirow{3}{*}{109} & Fuel & $1.352 \mathrm{E}-05$ & $1.821 \mathrm{E}-05$ & $6.477 \mathrm{E}-06$ & $2.577 \mathrm{E}-2$ & 0.410 \\
\hline & Clad & $3.513 \mathrm{E}-07$ & 0.0 & 0.0 & $5.039 \mathrm{E}-3$ & 0.081 \\
\hline & Mod. & $8.806 \mathrm{E}-07$ & 0.0 & 0.0 & $3.197 \mathrm{E}-2$ & 0.509 \\
\hline
\end{tabular}

Table 2.16. PNL-31 NITAWL reaction rates and fluxes

\begin{tabular}{|c|c|c|c|c|c|c|}
\hline Pin & Region & $\begin{array}{c}\mathrm{G}_{\mathrm{a}} \mathrm{M} \\
\left(\mathrm{cm}^{-3}-\mathrm{s}^{-1}\right)\end{array}$ & $\begin{array}{c}\mathrm{G}_{\mathrm{f}} \mathrm{M} \\
\left(\mathrm{cm}^{-3}-\mathrm{s}^{-1}\right)\end{array}$ & $\begin{array}{c}\mathrm{G}_{\mathrm{f}} \mathrm{M} \\
\left(\mathrm{cm}^{-3}-\mathrm{s}^{-1}\right)\end{array}$ & $\begin{array}{c}\mathrm{M} \\
\left(\mathrm{cm}-\mathrm{s}^{-1}\right)\end{array}$ & MVM \\
\hline \multirow{3}{*}{1} & Fuel & $6.745 \mathrm{E}-06$ & $1.049 \mathrm{E}-05$ & $3.740 \mathrm{E}-06$ & $7.754 \mathrm{E}-3$ & 0.412 \\
\hline & Clad & $1.404 \mathrm{E}-07$ & 0.0 & 0.0 & $1.500 \mathrm{E}-3$ & 0.079 \\
\hline & Mod. & $5.820 \mathrm{E}-07$ & 0.0 & 0.0 & $9.588 \mathrm{E}-3$ & 0.509 \\
\hline \multirow{3}{*}{109} & Fuel & $1.362 \mathrm{E}-05$ & $1.838 \mathrm{E}-05$ & $6.538 \mathrm{E}-06$ & $2.583 \mathrm{E}-2$ & 0.411 \\
\hline & Clad & $3.478 \mathrm{E}-07$ & 0.0 & 0.0 & $5.033 \mathrm{E}-3$ & 0.080 \\
\hline & Mod. & $8.960 \mathrm{E}-07$ & 0.0 & 0.0 & $3.200 \mathrm{E}-2$ & 0.509 \\
\hline
\end{tabular}


Table 2.17. PNL-31, Pin-1 four-group fluxes

\begin{tabular}{ccc}
\hline Group & $\begin{array}{c}\text { CENTRM M } \\
\left(\mathrm{cm}^{-2}-\mathrm{s}^{-1}\right)\end{array}$ & $\begin{array}{c}\text { NITAWL M } \\
\left(\mathrm{cm}^{-2}-\mathrm{s}^{-1}\right)\end{array}$ \\
\hline 1 & $4.151 \mathrm{E}-05$ & $4.145 \mathrm{E}-05$ \\
2 & $1.455 \mathrm{E}-05$ & $1.468 \mathrm{E}-05$ \\
3 & $3.350 \mathrm{E}-06$ & $3.351 \mathrm{E}-06$ \\
4 & $9.754 \mathrm{E}-06$ & $9.744 \mathrm{E}-06$ \\
\hline
\end{tabular}

Table 2.18. PNL-31, Pin-1 four-group U-235 cross sections

\begin{tabular}{cccccccc}
\hline Group & \multicolumn{3}{c}{ CENTRM cross sections $\left(\mathrm{cm}^{-1}\right)$} & \multicolumn{3}{c}{ NITAWL cross sections $\left(\mathrm{cm}^{-1}\right)$} \\
\hline & $\frac{\mathrm{G}_{\mathrm{a}}}{2.34277 \mathrm{E}-04}$ & $\frac{\mathrm{G}_{\mathrm{f}}}{5.20559 \mathrm{E}-04}$ & $\frac{\mathrm{G}_{\mathrm{f}}}{2.02137 \mathrm{E}-04}$ & $\frac{\mathrm{G}_{\mathrm{a}}}{2.34433 \mathrm{E}-04}$ & $\frac{\mathrm{G}_{\mathrm{f}}}{5.20890 \mathrm{E}-04}$ & $\frac{\mathrm{G}_{\mathrm{f}}}{2.02275 \mathrm{E}-04}$ \\
1 & $5.31166 \mathrm{E}-03$ & $8.16900 \mathrm{E}-03$ & $3.35248 \mathrm{E}-03$ & $5.27939 \mathrm{E}-03$ & $8.08910 \mathrm{E}-03$ & $3.31969 \mathrm{E}-03$ \\
2 & $8.11675 \mathrm{E}-03$ & $1.65287 \mathrm{E}-02$ & $6.78319 \mathrm{E}-03$ & $8.06329 \mathrm{E}-03$ & $1.64082 \mathrm{E}-02$ & $6.73381 \mathrm{E}-03$ \\
3 & $7.08071 \mathrm{E}-02$ & $1.47273 \mathrm{E}-01$ & $6.04396 \mathrm{E}-02$ & $7.10855 \mathrm{E}-02$ & $1.47857 \mathrm{E}-01$ & $6.06792 \mathrm{E}-02$ \\
\hline
\end{tabular}

Table 2.19. PNL-31, Pin-1 four-group U-238 cross sections

\begin{tabular}{|c|c|c|c|c|c|c|}
\hline Group & \multicolumn{3}{|c|}{ CENTRM cross sections $\left(\mathrm{cm}^{-1}\right)$} & \multicolumn{3}{|c|}{ NITAWL cross sections $\left(\mathrm{cm}^{-1}\right)$} \\
\hline & $\mathrm{G}_{\mathrm{a}}$ & $<\mathrm{G}_{\mathrm{f}}$ & $\mathrm{G}_{\mathrm{f}}$ & $\mathrm{G}_{\mathrm{a}}$ & $\mathbb{G}_{\mathrm{f}}$ & $\mathrm{G}_{\mathrm{f}}$ \\
\hline 1 & $6.72455 \mathrm{E}-03$ & $\overline{1.13885 \mathrm{E}-02}$ & $\overline{4.04980 \mathrm{E}-03}$ & $6 . \overline{74729 \mathrm{E}-03}$ & $\overline{1.14467 \mathrm{E}-02}$ & $\overline{4.07385 \mathrm{E}-03}$ \\
\hline 2 & $4.56936 \mathrm{E}-02$ & $5.60056 \mathrm{E}-06$ & 2.32029E-06 & 4.55851E-02 & 5.56831E-06 & 2.30692E-06 \\
\hline 3 & $1.10516 \mathrm{E}-02$ & $3.83036 \mathrm{E}-08$ & $1.58698 \mathrm{E}-08$ & 1.10407E-02 & 3.82095E-08 & $1.58309 \mathrm{E}-08$ \\
\hline 4 & 4.08111E-02 & $1.91551 \mathrm{E}-07$ & 7.93633E-08 & 4.09499E-02 & $1.92215 \mathrm{E}-07$ & $7.96381 \mathrm{E}-08$ \\
\hline
\end{tabular}

Table 2.20. PNL-31, Pin-1 four-group Pu-239 cross sections

\begin{tabular}{cccccccc}
\hline Group & \multicolumn{3}{c}{ CENTRM cross sections $\left(\mathrm{cm}^{-1}\right)$} & \multicolumn{3}{c}{ NITAWL cross sections $\left(\mathrm{cm}^{-1}\right)$} \\
\hline & $\frac{\mathrm{G}_{\mathrm{a}}}{7.32515 \mathrm{E}-04}$ & $\frac{\mathrm{G}_{\mathrm{f}}}{2.09589 \mathrm{E}-03}$ & $\frac{\mathrm{G}_{\mathrm{f}}}{6.79949 \mathrm{E}-04}$ & $\frac{7.32859 \mathrm{E}-04}{2.09690 \mathrm{E}-03}$ & $\frac{\mathrm{G}_{\mathrm{a}}}{6.80273 \mathrm{E}-04}$ \\
1 & $1.48948 \mathrm{E}-02$ & $2.45228 \mathrm{E}-02$ & $8.51540 \mathrm{E}-03$ & $1.45238 \mathrm{E}-02$ & $2.40236 \mathrm{E}-02$ & $8.34210 \mathrm{E}-03$ \\
2 & $4.46260 \mathrm{E}-02$ & $8.81024 \mathrm{E}-02$ & $3.05931 \mathrm{E}-02$ & $4.49138 \mathrm{E}-02$ & $8.86079 \mathrm{E}-02$ & $3.07687 \mathrm{E}-02$ \\
3 & $3.92873 \mathrm{E}-01$ & $7.79195 \mathrm{E}-01$ & $2.69624 \mathrm{E}-01$ & $3.93098 \mathrm{E}-01$ & $7.80112 \mathrm{E}-01$ & $2.69936 \mathrm{E}-01$ \\
\hline
\end{tabular}


Table 2.21. PNL-31, Pin-109 four-group fluxes

\begin{tabular}{ccc}
\hline Group & $\begin{array}{c}\text { CENTRM M } \\
\left(\mathrm{cm}^{-2}-\mathrm{s}^{-1}\right)\end{array}$ & $\begin{array}{c}\text { NITAWL M } \\
\left(\mathrm{cm}^{-2}-\mathrm{s}^{-1}\right)\end{array}$ \\
\hline 1 & $1.485 \mathrm{E}-04$ & $1.486 \mathrm{E}-04$ \\
2 & $5.674 \mathrm{E}-05$ & $5.667 \mathrm{E}-05$ \\
3 & $1.105 \mathrm{E}-05$ & $1.126 \mathrm{E}-05$ \\
4 & $1.379 \mathrm{E}-05$ & $1.412 \mathrm{E}-05$ \\
\hline
\end{tabular}

Table 2.22. PNL-31, Pin-109 four-group U-235 cross sections

\begin{tabular}{ccccccc}
\hline Group & \multicolumn{2}{c}{ CENTRM cross sections $\left(\mathrm{cm}^{-1}\right)$} & \multicolumn{2}{c}{ NITAWL cross sections $\left(\mathrm{cm}^{-1}\right)$} \\
\hline & $\frac{\mathrm{G}_{\mathrm{a}}}{2.40011 \mathrm{E}-04}$ & $\frac{\mathrm{G}_{\mathrm{f}}}{5.25238 \mathrm{E}-04}$ & $\frac{\mathrm{G}_{\mathrm{f}}}{2.05020 \mathrm{E}-04}$ & $\frac{\mathrm{G}_{\mathrm{a}}}{2.41044 \mathrm{E}-04}$ & $\frac{\mathrm{G}_{\mathrm{f}}}{5.25948 \mathrm{E}-04}$ & $\frac{\mathrm{G}_{\mathrm{f}}}{2.05500 \mathrm{E}-04}$ \\
1 & $5.08433 \mathrm{E}-03$ & $7.90435 \mathrm{E}-03$ & $3.24387 \mathrm{E}-03$ & $5.05763 \mathrm{E}-03$ & $7.83928 \mathrm{E}-03$ & $3.21717 \mathrm{E}-03$ \\
2 & $7.87603 \mathrm{E}-03$ & $1.60055 \mathrm{E}-02$ & $6.56854 \mathrm{E}-03$ & $7.93289 \mathrm{E}-03$ & $1.60894 \mathrm{E}-02$ & $6.60295 \mathrm{E}-03$ \\
3 & $6.39052 \mathrm{E}-02$ & $1.32666 \mathrm{E}-01$ & $5.44450 \mathrm{E}-02$ & $6.44026 \mathrm{E}-02$ & $1.33734 \mathrm{E}-01$ & $5.48830 \mathrm{E}-02$ \\
\hline
\end{tabular}

Table 2.23. PNL-31, Pin-109 four-group U-238 cross sections

\begin{tabular}{|c|c|c|c|c|c|c|}
\hline \multirow[t]{2}{*}{ Group } & \multicolumn{3}{|c|}{ CENTRM cross sections $\left(\mathrm{cm}^{-1}\right)$} & \multicolumn{3}{|c|}{ NITAWL cross sections $\left(\mathrm{cm}^{-1}\right)$} \\
\hline & $\mathrm{G}_{\mathrm{a}}$ & $\mathcal{G}_{\mathrm{f}}$ & $\mathrm{G}_{\mathrm{f}}$ & $\mathrm{G}_{\mathrm{a}-}$ & $\mathrm{G}_{\mathrm{f}}$ & $\mathrm{G}_{\mathrm{f}}$ \\
\hline 1 & $6 . \overline{52904 \mathrm{E}-03}$ & $\overline{1.02652 \mathrm{E}-02}$ & $\overline{3.65253 \mathrm{E}-03}$ & $6 . \overline{48723 \mathrm{E}-03}$ & $\overline{1.00441 \mathrm{E}-02}$ & $\overline{3.57473 \mathrm{E}-03}$ \\
\hline 2 & 4.26228E-02 & $5.78745 \mathrm{E}-06$ & 2.39771E-06 & 4.25107E-02 & 5.49843E-06 & 2.27798E-06 \\
\hline 3 & $1.10023 \mathrm{E}-02$ & $3.78561 \mathrm{E}-08$ & $1.56844 \mathrm{E}-08$ & $1.09805 \mathrm{E}-02$ & $3.77877 \mathrm{E}-08$ & $1.56562 \mathrm{E}-08$ \\
\hline 4 & 3.72074E-02 & $1.74257 \mathrm{E}-07$ & 7.21979E-08 & 3.74882E-02 & $1.75595 \mathrm{E}-07$ & 7.27521E-08 \\
\hline
\end{tabular}

Table 2.24. PNL-31, Pin-109 four-group Pu-239 cross sections

\begin{tabular}{cccccccc}
\hline Group & \multicolumn{3}{c}{ CENTRM cross sections $\left(\mathrm{cm}^{-1}\right)$} & \multicolumn{3}{c}{ NITAWL cross sections $\left(\mathrm{cm}^{-1}\right)$} \\
\hline & $\frac{\mathrm{G}_{\mathrm{a}}}{7.34579 \mathrm{E}-04}$ & $\frac{\mathrm{G}_{\mathrm{f}}}{2.07331 \mathrm{E}-03}$ & $\frac{\mathrm{G}_{\mathrm{f}}}{6.76047 \mathrm{E}-04}$ & $\frac{\mathrm{G}_{\mathrm{a}}}{7.34616 \mathrm{E}-04}$ & $\frac{\mathrm{G}_{\mathrm{f}}}{2.06809 \mathrm{E}-03}$ & $\frac{\mathrm{G}_{\mathrm{f}}}{6.74966 \mathrm{E}-04}$ \\
1 & $1.42795 \mathrm{E}-02$ & $2.36502 \mathrm{E}-02$ & $8.21241 \mathrm{E}-03$ & $1.41529 \mathrm{E}-02$ & $2.32927 \mathrm{E}-02$ & $8.08827 \mathrm{E}-03$ \\
3 & $4.25902 \mathrm{E}-02$ & $8.43169 \mathrm{E}-02$ & $2.92786 \mathrm{E}-02$ & $4.25863 \mathrm{E}-02$ & $8.42498 \mathrm{E}-02$ & $2.92554 \mathrm{E}-02$ \\
4 & $4.32807 \mathrm{E}-01$ & $8.32922 \mathrm{E}-01$ & $2.88390 \mathrm{E}-01$ & $4.26617 \mathrm{E}-01$ & $8.23669 \mathrm{E}-01$ & $2.85166 \mathrm{E}-01$ \\
\hline
\end{tabular}




\begin{tabular}{|c|c|c|c|c|c|c|c|c|c|c|c|c|c|c|c|c|}
\hline 0 & 0 & 0 & 0 & 0 & 0 & 0 & 4.104 & 3.592 & 3.967 & 0 & 0 & 0 & 0 & 0 & 0 & 0 \\
\hline 0 & 0 & 0 & 0 & 0 & 0 & 4.526 & 3.498 & 3.291 & 3.407 & 3.866 & 4.466 & 0 & 0 & 0 & 0 & 0 \\
\hline 0 & 0 & 0 & 0 & 4.931 & 4.345 & 3.900 & 3.723 & 3.716 & 3.703 & 3.685 & 3.685 & 4.587 & 0 & 0 & 0 & 0 \\
\hline 0 & 0 & 0 & 4.714 & 3.978 & 4.001 & 4.116 & 4.282 & 4.343 & 4.308 & 4.142 & 3.941 & 3.917 & 4.729 & 0 & 0 & 0 \\
\hline 0 & 0 & 4.627 & 3.919 & 4.040 & 4.348 & 4.602 & 4.824 & 4.909 & 4.867 & 4.690 & 4.406 & 4.053 & 3.954 & 4.586 & 0 & 0 \\
\hline 0 & 4.571 & 3.828 & 3.932 & 4.394 & 4.732 & 5.084 & 5.314 & 5.470 & 5.314 & 5.046 & 4.789 & 4.388 & 3.947 & 3.705 & 4.529 & 0 \\
\hline 0 & 3.922 & 3.663 & 4.307 & 4.687 & 5.147 & 5.525 & 5.761 & 5.808 & 5.730 & 5.474 & 5.132 & 4.724 & 4.133 & 3.716 & 3.894 & 0 \\
\hline 4.157 & 3.430 & 3.833 & 4.318 & 4.889 & 5.371 & 5.758 & 5.973 & 6.000 & 5.910 & 5.721 & 5.336 & 4.926 & 4.334 & 3.776 & 3.452 & 4.097 \\
\hline 3.718 & 3.346 & 3.822 & 4.366 & 4.891 & 5.430 & 5.794 & 6.009 & 6.086 & 6.014 & 5.818 & 5.526 & 4.983 & 4.389 & 3.801 & 3.329 & 3.647 \\
\hline 4.092 & 3.451 & 3.831 & 4.371 & 4.841 & 5.391 & 5.711 & 5.923 & 5.990 & 5.928 & 5.707 & 5.408 & 4.879 & 4.329 & 3.765 & 3.512 & 4.149 \\
\hline 0 & 3.884 & 3.703 & 4.188 & 4.675 & 5.207 & 5.526 & 5.712 & 5.786 & 5.739 & 5.492 & 5.143 & 4.681 & 4.107 & 3.701 & 3.953 & 0 \\
\hline 0 & 4.583 & 3.803 & 4.002 & 4.472 & 4.861 & 5.180 & 5.299 & 5.429 & 5.395 & 5.173 & 4.764 & 4.385 & 3.921 & 3.733 & 4.522 & 0 \\
\hline 0 & 0 & 4.695 & 3.920 & 4.100 & 4.404 & 4.715 & 4.886 & 4.992 & 4.915 & 4.709 & 4.402 & 4.043 & 3.925 & 4.545 & 0 & 0 \\
\hline 0 & 0 & 0 & 4.721 & 3.921 & 3.954 & 4.219 & 4.362 & 4.377 & 4.325 & 4.198 & 3.946 & 3.951 & 4.748 & 0 & 0 & 0 \\
\hline 0 & 0 & 0 & 0 & 4.639 & 3.762 & 3.701 & 3.821 & 3.812 & 3.789 & 3.926 & 4.415 & 4.865 & 0 & 0 & 0 & 0 \\
\hline 0 & 0 & 0 & 0 & 0 & 4.516 & 3.925 & 3.438 & 3.393 & 3.471 & 4.512 & 0 & 0 & 0 & 0 & 0 & 0 \\
\hline 0 & 0 & 0 & 0 & 0 & 0 & 0 & 4.100 & 3.642 & 4.175 & 0 & 0 & 0 & 0 & 0 & 0 & 0 \\
\hline
\end{tabular}

Fig. 2.9a. Pin-power distribution for CENTRM benchmark PNL-32 with standard deviation between 0.59 and $0.87 \%$ of the value. 


\begin{tabular}{|c|c|c|c|c|c|c|c|c|c|c|c|c|c|c|c|c|}
\hline 0 & 0 & 0 & 0 & 0 & 0 & 0 & 4.193 & 3.696 & 4.139 & 0 & 0 & 0 & 0 & 0 & 0 & 0 \\
\hline 0 & 0 & 0 & 0 & 0 & 0 & 4.568 & 3.565 & 3.354 & 3.466 & 3.987 & 4.530 & 0 & 0 & 0 & 0 & 0 \\
\hline 0 & 0 & 0 & 0 & 4.902 & 4.409 & 3.981 & 3.841 & 3.804 & 3.753 & 3.706 & 3.767 & 4.624 & 0 & 0 & 0 & 0 \\
\hline 0 & 0 & 0 & 4.739 & 3.971 & 4.071 & 4.190 & 4.304 & 4.352 & 4.301 & 4.177 & 3.952 & 3.930 & 4.705 & 0 & 0 & 0 \\
\hline 0 & 0 & 4.625 & 3.956 & 4.080 & 4.417 & 4.773 & 4.884 & 4.914 & 4.907 & 4.720 & 4.427 & 4.010 & 3.991 & 4.684 & 0 & 0 \\
\hline 0 & 4.612 & 3.769 & 4.039 & 4.470 & 4.913 & 5.191 & 5.404 & 5.433 & 5.357 & 5.142 & 4.790 & 4.319 & 3.940 & 3.779 & 4.489 & 0 \\
\hline 0 & 3.884 & 3.700 & 4.150 & 4.792 & 5.200 & 5.595 & 5.759 & 5.763 & 5.729 & 5.484 & 5.192 & 4.683 & 4.164 & 3.727 & 3.874 & 0 \\
\hline 4.153 & 3.500 & 3.842 & 4.455 & 4.989 & 5.431 & 5.783 & 5.974 & 5.974 & 5.953 & 5.726 & 5.346 & 4.840 & 4.324 & 3.784 & 3.470 & 4.059 \\
\hline 3.735 & 3.352 & 3.825 & 4.417 & 4.978 & 5.524 & 5.756 & 6.026 & 6.099 & 5.988 & 5.801 & 5.425 & 4.969 & 4.412 & 3.818 & 3.329 & 3.711 \\
\hline 4.211 & 3.533 & 3.823 & 4.416 & 4.969 & 5.395 & 5.781 & 5.929 & 5.958 & 5.856 & 5.731 & 5.360 & 4.817 & 4.315 & 3.757 & 3.404 & 4.099 \\
\hline 0 & 3.894 & 3.705 & 4.182 & 4.755 & 5.208 & 5.492 & 5.682 & 5.749 & 5.595 & 5.475 & 5.095 & 4.677 & 4.078 & 3.680 & 3.876 & 0 \\
\hline 0 & 4.558 & 3.742 & 4.015 & 4.413 & 4.846 & 5.195 & 5.336 & 5.383 & 5.265 & 5.059 & 4.737 & 4.377 & 3.944 & 3.712 & 4.465 & 0 \\
\hline 0 & 0 & 4.614 & 3.980 & 4.088 & 4.380 & 4.731 & 4.941 & 4.880 & 4.853 & 4.659 & 4.358 & 4.024 & 3.882 & 4.574 & 0 & 0 \\
\hline 0 & 0 & 0 & 4.789 & 3.910 & 3.973 & 4.142 & 4.350 & 4.423 & 4.348 & 4.130 & 3.973 & 3.904 & 4.683 & 0 & 0 & 0 \\
\hline 0 & 0 & 0 & 0 & 4.625 & 3.768 & 3.664 & 3.837 & 3.803 & 3.735 & 3.920 & 4.301 & 4.912 & 0 & 0 & 0 & 0 \\
\hline 0 & 0 & 0 & 0 & 0 & 4.516 & 3.907 & 3.442 & 3.317 & 3.414 & 4.463 & 0 & 0 & 0 & 0 & 0 & 0 \\
\hline 0 & 0 & 0 & 0 & 0 & 0 & 0 & 4.045 & 3.626 & 4.106 & 0 & 0 & 0 & 0 & 0 & 0 & 0 \\
\hline
\end{tabular}

Fig. 2.9b. Pin-power distribution for NITAWL benchmark PNL-32 with standard deviation between 0.57 and $0.90 \%$ of the values. 
Table 2.25. PNL-32 CENTRM reaction rates and fluxes

\begin{tabular}{|c|c|c|c|c|c|c|}
\hline Pin & Region & $\begin{array}{c}\mathrm{G}_{\mathrm{a}} \mathrm{M} \\
\left(\mathrm{cm}^{-3}-\mathrm{s}^{-1}\right)\end{array}$ & $\begin{array}{c}\mathcal{G}_{\mathrm{f}} \mathrm{M} \\
\left(\mathrm{cm}^{-3}-\mathrm{s}^{-1}\right)\end{array}$ & $\begin{array}{c}\mathrm{G}_{\mathrm{f}} \mathrm{M} \\
\left(\mathrm{cm}^{-3}-\mathrm{s}^{-1}\right)\end{array}$ & $\begin{array}{c}\mathrm{M} \\
\left(\mathrm{cm}-\mathrm{s}^{-1}\right)\end{array}$ & $\mathrm{M} / \mathrm{M}$ \\
\hline \multirow{3}{*}{1} & Fuel & $2.451 \mathrm{E}-05$ & $4.086 \mathrm{E}-05$ & $1.457 \mathrm{E}-05$ & $2.060 \mathrm{E}-2$ & 0.267 \\
\hline & Clad & 4.627E-07 & 0.0 & 0.0 & $3.943 \mathrm{E}-3$ & 0.051 \\
\hline & Mod. & $1.135 \mathrm{E}-06$ & 0.0 & 0.0 & $5.259 \mathrm{E}-2$ & 0.682 \\
\hline \multirow{3}{*}{98} & Fuel & $3.946 \mathrm{E}-05$ & $6.076 \mathrm{E}-05$ & $2.164 \mathrm{E}-05$ & $4.729 \mathrm{E}-2$ & 0.266 \\
\hline & Clad & $8.113 \mathrm{E}-07$ & 0.0 & 0.0 & $9.173 \mathrm{E}-3$ & 0.052 \\
\hline & Mod. & $1.536 \mathrm{E}-06$ & 0.0 & 0.0 & $1.121 \mathrm{E}-2$ & 0.682 \\
\hline
\end{tabular}

Table 2.26. PNL-32 NITAWL reaction rates and fluxes

\begin{tabular}{|c|c|c|c|c|c|c|}
\hline Pin & Region & $\begin{array}{c}\mathrm{G}_{\mathrm{a}} \mathrm{M} \\
\left(\mathrm{cm}^{-3}-\mathrm{s}^{-1}\right)\end{array}$ & $\begin{array}{c}\mathrm{G}_{\mathrm{f}} \mathrm{M} \\
\left(\mathrm{cm}^{-3}-\mathrm{s}^{-1}\right)\end{array}$ & $\begin{array}{c}\mathrm{G}_{\mathrm{f}} \mathrm{M} \\
\left(\mathrm{cm}^{-3}-\mathrm{s}^{-1}\right)\end{array}$ & $\begin{array}{c}\mathrm{M} \\
\left(\mathrm{cm}-\mathrm{s}^{-1}\right)\end{array}$ & $\mathrm{M}_{\mathrm{t}}$ \\
\hline \multirow{3}{*}{1} & Fuel & $2.429 \mathrm{E}-05$ & $4.063 \mathrm{E}-05$ & $1.449 \mathrm{E}-05$ & $2.033 \mathrm{E}-2$ & 0.266 \\
\hline & Clad & $4.624 \mathrm{E}-07$ & 0.0 & 0.0 & $3.930 \mathrm{E}-3$ & 0.051 \\
\hline & Mod. & $1.136 \mathrm{E}-06$ & 0.0 & 0.0 & $5.223 \mathrm{E}-2$ & 0.683 \\
\hline \multirow{3}{*}{98} & Fuel & $3.963 \mathrm{E}-05$ & $6.092 \mathrm{E}-05$ & $2.169 \mathrm{E}-05$ & $4.753 \mathrm{E}-2$ & 0.267 \\
\hline & Clad & $8.007 \mathrm{E}-07$ & 0.0 & 0.0 & $9.195 \mathrm{E}-3$ & 0.052 \\
\hline & Mod. & $1.550 \mathrm{E}-06$ & 0.0 & 0.0 & $1.214 \mathrm{E}-1$ & 0.681 \\
\hline
\end{tabular}


Table 2.27. PNL-32, Pin-1 four-group fluxes

\begin{tabular}{ccc}
\hline Group & $\begin{array}{c}\text { CENTRM M } \\
\left(\mathrm{cm}^{-2}-\mathrm{s}^{-1}\right)\end{array}$ & $\begin{array}{c}\text { NITAWL M } \\
\left(\mathrm{cm}^{-2}-\mathrm{s}^{-1}\right)\end{array}$ \\
\hline 1 & $1.048 \mathrm{E}-04$ & $1.029 \mathrm{E}-04$ \\
2 & $3.087 \mathrm{E}-05$ & $3.073 \mathrm{E}-05$ \\
3 & $7.654 \mathrm{E}-06$ & $7.310 \mathrm{E}-06$ \\
4 & $4.050 \mathrm{E}-05$ & $4.057 \mathrm{E}-05$ \\
\hline
\end{tabular}

Table 2.28. PNL-32, Pin-1 four-group U-235 cross sections

\begin{tabular}{cccccccc}
\hline Group & \multicolumn{3}{c}{ CENTRM cross sections $\left(\mathrm{cm}^{-1}\right)$} & \multicolumn{3}{c}{ NITAWL cross sections $\left(\mathrm{cm}^{-1}\right)$} \\
\hline & $\frac{\mathrm{G}_{\mathrm{a}}}{4.28226 \mathrm{E}-04}$ & $\frac{\leftarrow_{\mathrm{f}}}{5.15645 \mathrm{E}-04}$ & $\frac{\mathrm{G}_{\mathrm{f}}}{1.99145 \mathrm{E}-04}$ & $\frac{\mathrm{G}_{\mathrm{a}}}{2.28198 \mathrm{E}-04}$ & $\frac{\mathrm{G}_{\mathrm{f}}}{5.16018 \mathrm{E}-04}$ & $\frac{\mathrm{G}_{\mathrm{f}}}{1.99123 \mathrm{E}-04}$ \\
1 & $5.29819 \mathrm{E}-03$ & $8.08102 \mathrm{E}-03$ & $3.31639 \mathrm{E}-03$ & $5.44312 \mathrm{E}-03$ & $8.33186 \mathrm{E}-03$ & $3.41933 \mathrm{E}-03$ \\
2 & $8.13381 \mathrm{E}-03$ & $1.64731 \mathrm{E}-02$ & $6.76041 \mathrm{E}-03$ & $8.07192 \mathrm{E}-03$ & $1.63713 \mathrm{E}-02$ & $6.71862 \mathrm{E}-03$ \\
3 & $7.46578 \mathrm{E}-02$ & $1.55396 \mathrm{E}-01$ & $6.37731 \mathrm{E}-02$ & $7.43303 \mathrm{E}-02$ & $1.54714 \mathrm{E}-01$ & $6.34932 \mathrm{E}-02$ \\
\hline
\end{tabular}

Table 2.29. PNL-32, Pin-1 four-group U-238 cross sections

\begin{tabular}{|c|c|c|c|c|c|c|}
\hline \multirow[t]{2}{*}{ Group } & \multicolumn{3}{|c|}{ CENTRM cross sections $\left(\mathrm{cm}^{-1}\right)$} & \multicolumn{3}{|c|}{ NITAWL cross sections $\left(\mathrm{cm}^{-1}\right)$} \\
\hline & $\mathrm{G}_{\mathrm{a}}$ & $\mathcal{G}_{\mathrm{f}}$ & $\mathrm{G}_{\mathrm{f}}$ & $\mathrm{G}_{\mathrm{a}}$ & $\mathcal{G}_{\mathrm{f}}$ & $\mathrm{G}_{\mathrm{f}}$ \\
\hline 1 & $6.95603 \mathrm{E}-03$ & $1.26008 \mathrm{E}-02$ & $\overline{4.48409 \mathrm{E}-03}$ & $6.96190 \mathrm{E}-03$ & $\overline{1.26902 \mathrm{E}-02}$ & $\overline{4.50424 \mathrm{E}-03}$ \\
\hline 2 & 4.76213E-02 & $5.91756 \mathrm{E}-06$ & $2.45162 \mathrm{E}-06$ & 4.49401E-02 & $5.50696 \mathrm{E}-06$ & $2.28152 \mathrm{E}-06$ \\
\hline 3 & $1.10199 \mathrm{E}-02$ & $3.80020 \mathrm{E}-08$ & $1.57448 \mathrm{E}-08$ & $1.10245 \mathrm{E}-02$ & 3.79821E-08 & $1.57367 \mathrm{E}-08$ \\
\hline 4 & 4.27798E-02 & 2.00991E-07 & 8.32743E-08 & 4.26310E-02 & 2.00282E-07 & 8.29805E-08 \\
\hline
\end{tabular}

Table 2.30. PNL-32, Pin-1 four-group Pu-239 cross sections

\begin{tabular}{cccccccc}
\hline Group & \multicolumn{3}{c}{ CENTRM cross sections $\left(\mathrm{cm}^{-1}\right)$} & \multicolumn{3}{c}{ NITAWL cross sections $\left(\mathrm{cm}^{-1}\right)$} \\
\hline & $\frac{\mathrm{G}_{\mathrm{a}}}{\mathrm{7.30986 \textrm {E } - 0 4}}$ & $\frac{\mathrm{G}_{\mathrm{f}}}{2.12139 \mathrm{E}-03}$ & $\frac{\mathrm{G}_{\mathrm{f}}}{6.84695 \mathrm{E}-04}$ & $\overline{7.30230 \mathrm{E}-04}$ & $\frac{\mathrm{G}_{\mathrm{f}}}{2.12081 \mathrm{E}-03}$ & $\frac{\mathrm{G}_{\mathrm{f}}}{6.84023 \mathrm{E}-04}$ \\
1 & $1.53809 \mathrm{E}-02$ & $2.53859 \mathrm{E}-02$ & $8.81516 \mathrm{E}-03$ & $1.48494 \mathrm{E}-02$ & $2.44809 \mathrm{E}-02$ & $8.50088 \mathrm{E}-03$ \\
2 & $4.46591 \mathrm{E}-02$ & $8.80834 \mathrm{E}-02$ & $3.05866 \mathrm{E}-02$ & $4.50509 \mathrm{E}-02$ & $8.87866 \mathrm{E}-02$ & $3.08308 \mathrm{E}-02$ \\
3 & $3.79625 \mathrm{E}-01$ & $7.64299 \mathrm{E}-01$ & $2.64388 \mathrm{E}-01$ & $3.77128 \mathrm{E}-01$ & $7.59495 \mathrm{E}-01$ & $2.62726 \mathrm{E}-01$ \\
\hline
\end{tabular}


Table 2.31. PNL-32, Pin-98 four-group fluxes

\begin{tabular}{ccc}
\hline Group & $\begin{array}{c}\text { CENTRM M } \\
\left(\mathrm{cm}^{-2}-\mathrm{s}^{-1}\right)\end{array}$ & $\begin{array}{c}\text { NITAWL M } \\
\left(\mathrm{cm}^{-2}-\mathrm{s}^{-1}\right)\end{array}$ \\
\hline 1 & $2.586 \mathrm{E}-04$ & $2.586 \mathrm{E}-04$ \\
2 & $8.866 \mathrm{E}-05$ & $8.976 \mathrm{E}-05$ \\
3 & $1.956 \mathrm{E}-05$ & $2.042 \mathrm{E}-05$ \\
4 & $5.545 \mathrm{E}-05$ & $5.552 \mathrm{E}-05$ \\
\hline
\end{tabular}

Table 2.32. PNL-32, Pin-98 four-group U-235 cross sections

\begin{tabular}{cccccccc}
\hline Group & \multicolumn{2}{c}{ CENTRM cross sections $\left(\mathrm{cm}^{-1}\right)$} & \multicolumn{3}{c}{ NITAWL cross sections $\left(\mathrm{cm}^{-1}\right)$} \\
\hline & $\frac{\mathrm{G}_{\mathrm{a}}}{4.34086 \mathrm{E}-04}$ & $\frac{\mathrm{G}_{\mathrm{f}}}{5.20599 \mathrm{E}-04}$ & $\frac{\mathrm{G}_{\mathrm{f}}}{2.02092 \mathrm{E}-04}$ & $2.34329 \mathrm{E}-04$ & $\frac{\mathrm{G}_{\mathrm{f}}}{5.21021 \mathrm{E}-04}$ & $\frac{\mathrm{G}_{\mathrm{f}}}{2.02251 \mathrm{E}-04}$ \\
1 & $5.15153 \mathrm{E}-03$ & $7.96071 \mathrm{E}-03$ & $3.26701 \mathrm{E}-03$ & $5.21152 \mathrm{E}-03$ & $7.99933 \mathrm{E}-03$ & $3.28285 \mathrm{E}-03$ \\
2 & $8.14944 \mathrm{E}-03$ & $1.65985 \mathrm{E}-02$ & $6.81186 \mathrm{E}-03$ & $8.16239 \mathrm{E}-03$ & $1.66200 \mathrm{E}-02$ & $6.82071 \mathrm{E}-03$ \\
3 & $7.06334 \mathrm{E}-02$ & $1.46886 \mathrm{E}-01$ & $6.02804 \mathrm{E}-02$ & $7.03727 \mathrm{E}-02$ & $1.46337 \mathrm{E}-01$ & $6.00555 \mathrm{E}-02$ \\
\hline
\end{tabular}

Table 2.33. PNL-32, Pin-98 four-group U-238 cross sections

\begin{tabular}{cccccccc}
\hline Group & \multicolumn{3}{c}{ CENTRM cross sections $\left(\mathrm{cm}^{-1}\right)$} & \multicolumn{3}{c}{ NITAWL cross sections $\left(\mathrm{cm}^{-1}\right)$} \\
\hline & $\frac{\mathrm{G}_{\mathrm{a}}}{}$ & $\frac{\mathrm{G}_{\mathrm{f}}}{1.75406 \mathrm{E}-03}$ & $\frac{1.14805 \mathrm{E}-02}{4}$ & $\frac{\mathrm{G}_{\mathrm{f}}}{4.08322 \mathrm{E}-03}$ & $\frac{6.78241 \mathrm{E}-03}{1.15393 \mathrm{E}-02}$ & $\frac{\mathrm{G}_{\mathrm{f}}}{4.10572 \mathrm{E}-03}$ \\
2 & $4.75024 \mathrm{E}-02$ & $5.74765 \mathrm{E}-06$ & $2.38123 \mathrm{E}-06$ & $4.63517 \mathrm{E}-02$ & $5.42737 \mathrm{E}-06$ & $2.24854 \mathrm{E}-06$ \\
3 & $1.10577 \mathrm{E}-02$ & $3.83380 \mathrm{E}-08$ & $1.58842 \mathrm{E}-08$ & $1.10567 \mathrm{E}-02$ & $3.83610 \mathrm{E}-08$ & $1.58937 \mathrm{E}-08$ \\
4 & $4.07001 \mathrm{E}-02$ & $1.91006 \mathrm{E}-07$ & $7.91378 \mathrm{E}-08$ & $4.05670 \mathrm{E}-02$ & $1.90373 \mathrm{E}-07$ & $7.88750 \mathrm{E}-08$ \\
\hline
\end{tabular}

Table 2.34. PNL-32, Pin-98 four-group Pu-239 cross sections

\begin{tabular}{cccccccc}
\hline Group & \multicolumn{3}{c}{ CENTRM cross sections $\left(\mathrm{cm}^{-1}\right)$} & \multicolumn{3}{c}{ NITAWL cross sections $\left(\mathrm{cm}^{-1}\right)$} \\
\hline & $\frac{\mathrm{G}_{\mathrm{a}}}{7.32645 \mathrm{E}-04}$ & $\frac{\mathrm{G}_{\mathrm{f}}}{2.09793 \mathrm{E}-03}$ & $\frac{\mathrm{G}_{\mathrm{f}}}{6.80410 \mathrm{E}-04}$ & $\frac{\mathrm{G}_{\mathrm{a}}}{7.32954 \mathrm{E}-04}$ & $\frac{\mathrm{G}_{\mathrm{f}}}{2.09852 \mathrm{E}-03}$ & $\frac{\mathrm{G}_{\mathrm{f}}}{6.80530 \mathrm{E}-04}$ \\
1 & $1.46368 \mathrm{E}-02$ & $2.41261 \mathrm{E}-02$ & $8.37768 \mathrm{E}-03$ & $1.46065 \mathrm{E}-02$ & $2.41771 \mathrm{E}-02$ & $8.39539 \mathrm{E}-03$ \\
2 & $4.63098 \mathrm{E}-02$ & $9.11738 \mathrm{E}-02$ & $3.16598 \mathrm{E}-02$ & $4.55958 \mathrm{E}-02$ & $8.98849 \mathrm{E}-02$ & $3.12122 \mathrm{E}-02$ \\
3 & $3.98803 \mathrm{E}-01$ & $7.88748 \mathrm{E}-01$ & $2.72941 \mathrm{E}-01$ & $3.99072 \mathrm{E}-01$ & $7.88716 \mathrm{E}-01$ & $2.72936 \mathrm{E}-01$ \\
\hline
\end{tabular}




\begin{tabular}{|c|c|c|c|c|c|c|c|c|c|c|c|}
\hline & & & & & & & & & & & $\begin{array}{l}2.024 \\
(1.15)\end{array}$ \\
\hline & & & & & & & & & & $\begin{array}{l}2.037 \\
(0.53)\end{array}$ & $\begin{array}{l}2.025 \\
(0.53)\end{array}$ \\
\hline & & & & & & & & & $\begin{array}{c}1.958 \\
(0.50)\end{array}$ & $\begin{array}{c}2.001 \\
(0.40)\end{array}$ & $\begin{array}{l}2.009 \\
(0.55)\end{array}$ \\
\hline & & & & & & & & $\begin{array}{l}1.872 \\
(0.50)\end{array}$ & $\begin{array}{l}1.935 \\
(0.32)\end{array}$ & $\begin{array}{l}1.955 \\
(0.40)\end{array}$ & $\begin{array}{l}1.973 \\
(0.54)\end{array}$ \\
\hline & & & & & & & $\begin{array}{l}1.783 \\
(0.57)\end{array}$ & $\begin{array}{l}1.848 \\
(0.41)\end{array}$ & $\begin{array}{l}1.869 \\
(0.37)\end{array}$ & $\begin{array}{c}1.905 \\
(0.40)\end{array}$ & $\begin{array}{l}1.904 \\
(0.51)\end{array}$ \\
\hline & & & & & & $\begin{array}{c}1.626 \\
(0.54)\end{array}$ & $\begin{array}{c}1.702 \\
(0.39)\end{array}$ & $\begin{array}{l}1.760 \\
(0.37)\end{array}$ & $\begin{array}{l}1.801 \\
(0.37)\end{array}$ & $\begin{array}{c}1.832 \\
(0.41)\end{array}$ & $\begin{array}{l}1.848 \\
(0.47)\end{array}$ \\
\hline & & & & & $\begin{array}{l}1.461 \\
(0.56)\end{array}$ & $\begin{array}{l}1.539 \\
(0.42)\end{array}$ & $\begin{array}{c}1.624 \\
(0.37)\end{array}$ & $\begin{array}{l}1.661 \\
(0.37)\end{array}$ & $\begin{array}{l}1.707 \\
(0.41)\end{array}$ & $\begin{array}{l}1.721 \\
(0.37)\end{array}$ & $\begin{array}{l}1.746 \\
(0.55)\end{array}$ \\
\hline & & & & $\begin{array}{l}1.275 \\
(0.64)\end{array}$ & $\begin{array}{l}1.368 \\
(0.47)\end{array}$ & $\begin{array}{c}1.450 \\
(0.43)\end{array}$ & $\begin{array}{l}1.509 \\
(0.45)\end{array}$ & $\begin{array}{l}1.564 \\
(0.43)\end{array}$ & $\begin{array}{c}1.599 \\
(0.37)\end{array}$ & $\begin{array}{l}1.636 \\
(0.40)\end{array}$ & $\begin{array}{l}1.638 \\
(0.55)\end{array}$ \\
\hline & & & $\begin{array}{c}1.065 \\
(0.68)\end{array}$ & $\begin{array}{l}1.155 \\
(0.47)\end{array}$ & $\begin{array}{l}1.251 \\
(0.50)\end{array}$ & $\begin{array}{l}1.341 \\
(0.45)\end{array}$ & $\begin{array}{c}1.403 \\
(0.43)\end{array}$ & $\begin{array}{l}1.448 \\
(0.39)\end{array}$ & $\begin{array}{l}1.485 \\
(0.43)\end{array}$ & $\begin{array}{l}1.512 \\
(0.41)\end{array}$ & $\begin{array}{l}1.518 \\
(0.58)\end{array}$ \\
\hline & & $\begin{array}{c}0.850 \\
(0.79)\end{array}$ & $\begin{array}{c}0.954 \\
(0.52)\end{array}$ & $\begin{array}{l}1.040 \\
(0.51)\end{array}$ & $\begin{array}{c}1.130 \\
(051 .)\end{array}$ & $\begin{array}{c}1.204 \\
(0.45)\end{array}$ & $\begin{array}{c}1.258 \\
(0.45)\end{array}$ & $\begin{array}{l}1.323 \\
(0.45)\end{array}$ & $\begin{array}{l}1.358 \\
(0.43)\end{array}$ & $\begin{array}{l}1.373 \\
(0.42)\end{array}$ & $\begin{array}{l}1.386 \\
(0.60)\end{array}$ \\
\hline & $\begin{array}{c}0.639 \\
(0.83)\end{array}$ & $\begin{array}{c}0.738 \\
(0.57)\end{array}$ & $\begin{array}{l}0.846 \\
(0.54)\end{array}$ & $\begin{array}{c}0.910 \\
(0.58)\end{array}$ & $\begin{array}{c}1.008 \\
(0.49)\end{array}$ & $\begin{array}{c}1.077 \\
(0.48)\end{array}$ & $\begin{array}{l}1.137 \\
(0.49)\end{array}$ & $\begin{array}{l}1.182 \\
(0.53)\end{array}$ & $\begin{array}{l}1.213 \\
(0.50)\end{array}$ & $\begin{array}{c}1.231 \\
(0.48)\end{array}$ & $\begin{array}{l}1.240 \\
(0.72)\end{array}$ \\
\hline $\begin{array}{c}0.564 \\
(1.07)\end{array}$ & $\begin{array}{c}0.554 \\
(0.78)\end{array}$ & $\begin{array}{c}0.633 \\
(0.62)\end{array}$ & $\begin{array}{c}0.712 \\
(0.59)\end{array}$ & $\begin{array}{c}0.793 \\
(0.58)\end{array}$ & $\begin{array}{r}0.866 \\
(0.60)\end{array}$ & $\begin{array}{c}0.931 \\
(0.48)\end{array}$ & $\begin{array}{l}0.991 \\
(0.45)\end{array}$ & $\begin{array}{l}1.039 \\
(0.53)\end{array}$ & $\begin{array}{c}1.078 \\
(0.48)\end{array}$ & $\begin{array}{c}1.092 \\
(0.54)\end{array}$ & $\begin{array}{l}1.099 \\
(0.62)\end{array}$ \\
\hline 0 & $\begin{array}{c}0.565 \\
(0.69)\end{array}$ & $\begin{array}{c}0.594 \\
(0.73)\end{array}$ & $\begin{array}{c}0.618 \\
(0.63)\end{array}$ & $\begin{array}{c}0.663 \\
(0.64)\end{array}$ & $\begin{array}{c}0.731 \\
(0.57)\end{array}$ & $\begin{array}{c}0.786 \\
(0.54)\end{array}$ & $\begin{array}{c}0.838 \\
(0.57)\end{array}$ & $\begin{array}{l}0.883 \\
(0.53)\end{array}$ & $\begin{array}{r}0.932 \\
(0.52)\end{array}$ & $\begin{array}{r}0.942 \\
(0.51)\end{array}$ & $\begin{array}{l}0.937 \\
(0.74)\end{array}$ \\
\hline 0 & 0 & 0 & $\begin{array}{l}0.621 \\
(0.69)\end{array}$ & $\begin{array}{c}0.628 \\
(0.59)\end{array}$ & $\begin{array}{c}0.628 \\
(0.52)\end{array}$ & $\begin{array}{c}0.658 \\
(0.61)\end{array}$ & $\begin{array}{l}0.702 \\
(0.63)\end{array}$ & $\begin{array}{l}0.735 \\
(0.62)\end{array}$ & $\begin{array}{c}0.761 \\
(0.54)\end{array}$ & $\begin{array}{l}0.783 \\
(0.62)\end{array}$ & $\begin{array}{l}0.783 \\
(0.86)\end{array}$ \\
\hline 0 & 0 & 0 & 0 & 0 & $\begin{array}{c}0.622 \\
(0.64)\end{array}$ & $\begin{array}{c}0.618 \\
(0.65)\end{array}$ & $\begin{array}{l}0.639 \\
(0.63)\end{array}$ & $\begin{array}{l}0.619 \\
(0.63)\end{array}$ & $\begin{array}{c}0.623 \\
(0.66)\end{array}$ & $\begin{array}{l}0.641 \\
(0.63)\end{array}$ & $\begin{array}{l}0.659 \\
(0.97)\end{array}$ \\
\hline 0 & 0 & 0 & 0 & 0 & 0 & 0 & 0 & $\begin{array}{l}0.610 \\
(0.66)\end{array}$ & $\begin{array}{c}0.570 \\
(0.66)\end{array}$ & $\begin{array}{l}0.585 \\
(0.75)\end{array}$ & $\begin{array}{l}0.604 \\
(0.90)\end{array}$ \\
\hline
\end{tabular}

Fig. 2.10a. Pin-power distribution for CENTRM benchmark PNL-33. Value in parentheses is percent standard deviation. 


\begin{tabular}{|c|c|c|c|c|c|c|c|c|c|c|c|}
\hline & & & & & & & & & & & $\begin{array}{l}2.037 \\
(0.95)\end{array}$ \\
\hline & & & & & & & & & & $\begin{array}{l}2.052 \\
(0.57)\end{array}$ & $\begin{array}{c}2.057 \\
(0.51)\end{array}$ \\
\hline & & & & & & & & & $\begin{array}{l}2.004 \\
(0.50)\end{array}$ & $\begin{array}{l}2.007 \\
(0.40)\end{array}$ & $\begin{array}{r}2.018 \\
(0.53)\end{array}$ \\
\hline & & & & & & & & $\begin{array}{l}1.884 \\
(0.57)\end{array}$ & $\begin{array}{l}1.950 \\
(0.36)\end{array}$ & $\begin{array}{c}1.981 \\
(0.38)\end{array}$ & $\begin{array}{l}1.981 \\
(0.51)\end{array}$ \\
\hline & & & & & & & $\begin{array}{c}1.783 \\
(0.53)\end{array}$ & $\begin{array}{c}1.833 \\
(0.41)\end{array}$ & $\begin{array}{l}1.883 \\
(0.35)\end{array}$ & $\begin{array}{l}1.917 \\
(0.38)\end{array}$ & $\begin{array}{l}1.917 \\
(0.54)\end{array}$ \\
\hline & & & & & & $\begin{array}{l}1.646 \\
(0.55)\end{array}$ & $\begin{array}{c}1.711 \\
(0.38)\end{array}$ & $\begin{array}{l}1.772 \\
(0.37)\end{array}$ & $\begin{array}{c}1.804 \\
(0.40)\end{array}$ & $\begin{array}{c}1.823 \\
(0.40)\end{array}$ & $\begin{array}{c}1.827 \\
(0.49)\end{array}$ \\
\hline & & & & & $\begin{array}{l}1.466 \\
(0.59)\end{array}$ & $\begin{array}{c}1.553 \\
(0.39)\end{array}$ & $\begin{array}{l}1.610 \\
(0.40)\end{array}$ & $\begin{array}{l}1.670 \\
(0.43)\end{array}$ & $\begin{array}{l}1.718 \\
(0.41)\end{array}$ & $\begin{array}{l}1.736 \\
(0.37)\end{array}$ & $\begin{array}{c}1.751 \\
(0.50)\end{array}$ \\
\hline & & & & $\begin{array}{l}1.245 \\
(0.60)\end{array}$ & $\begin{array}{c}1.362 \\
(0.43)\end{array}$ & $\begin{array}{l}1.456 \\
(0.39)\end{array}$ & $\begin{array}{l}1.527 \\
(0.40)\end{array}$ & $\begin{array}{c}1.572 \\
(0.41)\end{array}$ & $\begin{array}{c}1.604 \\
(0.41)\end{array}$ & $\begin{array}{c}1.643 \\
(0.40)\end{array}$ & $\begin{array}{l}1.638 \\
(0.54)\end{array}$ \\
\hline & & & $\begin{array}{l}1.067 \\
(0.72)\end{array}$ & $\begin{array}{l}1.155 \\
(0.45)\end{array}$ & $\begin{array}{c}1.257 \\
(0.46)\end{array}$ & $\begin{array}{l}1.330 \\
(0.48)\end{array}$ & $\begin{array}{c}1.403 \\
(0.43)\end{array}$ & $\begin{array}{l}1.447 \\
(0.43)\end{array}$ & $\begin{array}{l}1.491 \\
(0.43)\end{array}$ & $\begin{array}{l}1.509 \\
(0.42)\end{array}$ & $\begin{array}{l}1.528 \\
(0.55)\end{array}$ \\
\hline & & $\begin{array}{l}0.839 \\
(0.75)\end{array}$ & $\begin{array}{c}0.943 \\
(0.60)\end{array}$ & $\begin{array}{l}1.050 \\
(0.52)\end{array}$ & $\begin{array}{c}1.135 \\
(0.48)\end{array}$ & $\begin{array}{l}1.220 \\
(0.47)\end{array}$ & $\begin{array}{c}1.283 \\
(0.45)\end{array}$ & $\begin{array}{c}1.321 \\
(0.48)\end{array}$ & $\begin{array}{l}1.371 \\
(0.47)\end{array}$ & $\begin{array}{c}1.393 \\
(0.45)\end{array}$ & $\begin{array}{c}1.391 \\
(0.60)\end{array}$ \\
\hline & $\begin{array}{l}0.632 \\
(0.82)\end{array}$ & $\begin{array}{c}0.731 \\
(0.65)\end{array}$ & $\begin{array}{l}0.824 \\
(0.57)\end{array}$ & $\begin{array}{c}0.919 \\
(0.50)\end{array}$ & $\begin{array}{l}1.004 \\
(0.50)\end{array}$ & $\begin{array}{l}1.069 \\
(0.49)\end{array}$ & $\begin{array}{c}1.141 \\
(0.47)\end{array}$ & $\begin{array}{c}1.181 \\
(0.52)\end{array}$ & $\begin{array}{r}1.220 \\
(0.44)\end{array}$ & $\begin{array}{l}1.247 \\
(0.44)\end{array}$ & $\begin{array}{c}1.249 \\
(063 .)\end{array}$ \\
\hline $\begin{array}{l}0.565 \\
(0.98)\end{array}$ & $\begin{array}{l}0.561 \\
(0.63)\end{array}$ & $\begin{array}{c}0.631 \\
(0.62)\end{array}$ & $\begin{array}{c}0.703 \\
(0.54)\end{array}$ & $\begin{array}{l}0.788 \\
(0.53)\end{array}$ & $\begin{array}{l}0.880 \\
(0.54)\end{array}$ & $\begin{array}{l}0.938 \\
(0.51)\end{array}$ & $\begin{array}{l}0.990 \\
(0.53)\end{array}$ & $\begin{array}{l}1.042 \\
(0.53)\end{array}$ & $\begin{array}{l}1.071 \\
(0.53)\end{array}$ & $\begin{array}{c}1.099 \\
(0.50)\end{array}$ & $\begin{array}{c}1.088 \\
(0.73)\end{array}$ \\
\hline 0 & $\begin{array}{l}0.566 \\
(0.64)\end{array}$ & $\begin{array}{c}0.586 \\
(0.69)\end{array}$ & $\begin{array}{l}0.617 \\
(0.62)\end{array}$ & $\begin{array}{r}0.672 \\
(0.68)\end{array}$ & $\begin{array}{l}0.729 \\
(0.58)\end{array}$ & $\begin{array}{l}0.795 \\
(0.53)\end{array}$ & $\begin{array}{c}0.845 \\
(0.55)\end{array}$ & $\begin{array}{l}0.886 \\
(0.53)\end{array}$ & $\begin{array}{l}0.922 \\
(0.55)\end{array}$ & $\begin{array}{c}0.941 \\
(0.55)\end{array}$ & $\begin{array}{r}0.947 \\
(0.78)\end{array}$ \\
\hline 0 & 0 & 0 & $\begin{array}{c}0.619 \\
(0.69)\end{array}$ & $\begin{array}{l}0.618 \\
(0.67)\end{array}$ & $\begin{array}{r}0.625 \\
(0.68)\end{array}$ & $\begin{array}{l}0.665 \\
(0.63)\end{array}$ & $\begin{array}{l}0.708 \\
(0.54)\end{array}$ & $\begin{array}{l}0.737 \\
(0.61)\end{array}$ & $\begin{array}{l}0.763 \\
(0.54)\end{array}$ & $\begin{array}{c}0.776 \\
(0.62)\end{array}$ & $\begin{array}{l}0.797 \\
(0.82)\end{array}$ \\
\hline 0 & 0 & 0 & 0 & 0 & $\begin{array}{l}0.621 \\
(0.67)\end{array}$ & $\begin{array}{c}0.619 \\
(0.68)\end{array}$ & $\begin{array}{l}0.637 \\
(0.67)\end{array}$ & $\begin{array}{l}0.618 \\
(0.65)\end{array}$ & $\begin{array}{r}0.629 \\
(0.68)\end{array}$ & $\begin{array}{c}0.634 \\
(0.69)\end{array}$ & $\begin{array}{l}0.642 \\
(0.97)\end{array}$ \\
\hline 0 & 0 & 0 & 0 & 0 & 0 & 0 & 0 & $\begin{array}{l}0.608 \\
(0.65)\end{array}$ & $\begin{array}{l}0.579 \\
(0.62)\end{array}$ & $\begin{array}{l}0.588 \\
(0.72)\end{array}$ & $\begin{array}{c}0.590 \\
(0.96)\end{array}$ \\
\hline
\end{tabular}

Fig. 2.10b. Pin-power distribution for NITAWL benchmark PNL-33. Value in parentheses is percent standard deviation. 
Table 2.35. PNL-33 CENTRM reaction rates and fluxes

\begin{tabular}{ccccccc}
\hline Pin & Region & $\begin{array}{c}\mathrm{G}_{\mathrm{a}} \mathrm{M} \\
\left(\mathrm{cm}^{-3}-\mathrm{s}^{-1}\right)\end{array}$ & $\begin{array}{c}\mathrm{G}_{\mathrm{f}} \mathrm{M} \\
\left(\mathrm{cm}^{-3}-\mathrm{s}^{-1}\right)\end{array}$ & $\begin{array}{c}\mathrm{G}_{\mathrm{f}} \mathrm{M} \\
\left(\mathrm{cm}^{-3}-\mathrm{s}^{-1}\right)\end{array}$ & $\begin{array}{c}\mathrm{M} \\
\left(\mathrm{cm}^{-1}\right)\end{array}$ & $\mathrm{M}_{\mathrm{t}}$ \\
\hline \multirow{2}{*}{1} & Fuel & $3.818 \mathrm{E}-06$ & $6.043 \mathrm{E}-06$ & $2.152 \mathrm{E}-06$ & $3.853 \mathrm{E}-3$ & 0.267 \\
& Clad & $7.376 \mathrm{E}-08$ & 0.0 & 0.0 & $7.423 \mathrm{E}-4$ & 0.051 \\
& Mod. & $4.770 \mathrm{E}-07$ & 0.0 & 0.0 & $9.836 \mathrm{E}-3$ & 0.682 \\
\hline \multirow{2}{*}{109} & Fuel & $1.360 \mathrm{E}-05$ & $2.034 \mathrm{E}-05$ & $7.243 \mathrm{E}-06$ & $1.705 \mathrm{E}-2$ & 0.265 \\
& Clad & $2.788 \mathrm{E}-07$ & 0.0 & 0.0 & $3.401 \mathrm{E}-3$ & 0.051 \\
& Mod. & $1.498 \mathrm{E}-06$ & 0.0 & 0.0 & $4.516 \mathrm{E}-2$ & 0.684 \\
\hline
\end{tabular}

Table 2.36. PNL-33 NITAWL reaction rates and fluxes

\begin{tabular}{ccccccc}
\hline Pin & Region & $\begin{array}{c}\mathrm{G}_{\mathrm{a}} \mathrm{M} \\
\left(\mathrm{cm}^{-3}-\mathrm{s}^{-1}\right)\end{array}$ & $\begin{array}{c}\mathrm{G}_{\mathrm{f}} \mathrm{M} \\
\left(\mathrm{cm}^{-3}-\mathrm{s}^{-1}\right)\end{array}$ & $\begin{array}{c}\mathrm{G}_{\mathrm{f}} \mathrm{M} \\
\left(\mathrm{cm}^{-3}-\mathrm{s}^{-1}\right)\end{array}$ & $\begin{array}{c}\mathrm{M} \\
\left(\mathrm{cm}^{-1}\right)\end{array}$ & $\mathrm{M}_{\mathrm{t}}$ \\
\hline \multirow{2}{*}{1} & Fuel & $3.812 \mathrm{E}-06$ & $6.048 \mathrm{E}-06$ & $2.155 \mathrm{E}-06$ & $3.867 \mathrm{E}-3$ & 0.267 \\
& Clad & $7.253 \mathrm{E}-08$ & 0.0 & 0.0 & $7.426 \mathrm{E}-4$ & 0.052 \\
& Mod. & $4.787 \mathrm{E}-07$ & 0.0 & 0.0 & $9.858 \mathrm{E}-3$ & 0.681 \\
\hline \multirow{2}{*}{109} & Fuel & $1.375 \mathrm{E}-05$ & $2.067 \mathrm{E}-05$ & $7.357 \mathrm{E}-06$ & $1.779 \mathrm{E}-2$ & 0.268 \\
& Clad & $2.783 \mathrm{E}-07$ & 0.0 & 0.0 & $3.442 \mathrm{E}-3$ & 0.052 \\
& Mod. & $1.505 \mathrm{E}-06$ & 0.0 & 0.0 & $4.521 \mathrm{E}-2$ & 0.680 \\
\hline
\end{tabular}


Table 2.37. PNL-33, Pin-1 four-group fluxes

\begin{tabular}{ccc}
\hline Group & $\begin{array}{c}\text { CENTRM M } \\
\left(\mathrm{cm}^{-2}-\mathrm{s}^{-1}\right)\end{array}$ & $\begin{array}{c}\text { NITAWL M } \\
\left(\mathrm{cm}^{-2}-\mathrm{s}^{-1}\right)\end{array}$ \\
\hline 1 & $2.014 \mathrm{E}-05$ & $2.016 \mathrm{E}-05$ \\
2 & $6.869 \mathrm{E}-06$ & $6.908 \mathrm{E}-06$ \\
3 & $1.672 \mathrm{E}-06$ & $1.719 \mathrm{E}-06$ \\
4 & $5.718 \mathrm{E}-06$ & $5.745 \mathrm{E}-06$ \\
\hline
\end{tabular}

Table 2.38. PNL-33, Pin-1 four-group U-235 cross sections

\begin{tabular}{ccccccc}
\hline Group & \multicolumn{2}{c}{ CENTRM cross sections $\left(\mathrm{cm}^{-1}\right)$} & \multicolumn{3}{c}{ NITAWL cross sections $\left(\mathrm{cm}^{-1}\right)$} \\
\hline & $\frac{\mathrm{G}_{\mathrm{a}}}{4.31933 \mathrm{E}-04}$ & $\frac{\leftarrow_{\mathrm{f}}}{5.20055 \mathrm{E}-04}$ & $\frac{\mathrm{G}_{\mathrm{f}}}{2.01129 \mathrm{E}-04}$ & $\frac{\mathrm{G}_{\mathrm{a}}}{2.31159 \mathrm{E}-04}$ & $\frac{\mathrm{G}_{\mathrm{f}}}{5.18686 \mathrm{E}-04}$ & $\frac{\mathrm{G}_{\mathrm{f}}}{2.00672 \mathrm{E}-04}$ \\
1 & $5.35943 \mathrm{E}-03$ & $8.22495 \mathrm{E}-03$ & $3.37546 \mathrm{E}-03$ & $5.34155 \mathrm{E}-03$ & $8.22719 \mathrm{E}-03$ & $3.37636 \mathrm{E}-03$ \\
2 & $8.26154 \mathrm{E}-03$ & $1.68721 \mathrm{E}-02$ & $6.92416 \mathrm{E}-03$ & $8.25026 \mathrm{E}-03$ & $1.68098 \mathrm{E}-02$ & $6.89860 \mathrm{E}-03$ \\
3 & $7.10700 \mathrm{E}-02$ & $1.47828 \mathrm{E}-01$ & $6.06675 \mathrm{E}-02$ & $7.10641 \mathrm{E}-02$ & $1.47819 \mathrm{E}-01$ & $6.06639 \mathrm{E}-02$ \\
\hline
\end{tabular}

Table 2.39. PNL-33, Pin-1 four-group U-238 cross sections

\begin{tabular}{|c|c|c|c|c|c|c|}
\hline \multirow[t]{2}{*}{ Group } & \multicolumn{3}{|c|}{ CENTRM cross sections $\left(\mathrm{cm}^{-1}\right)$} & \multicolumn{3}{|c|}{ NITAWL cross sections $\left(\mathrm{cm}^{-1}\right)$} \\
\hline & $\mathrm{G}_{\mathrm{a}}$ & $\mathcal{G}_{\mathrm{f}}$ & $\mathrm{G}_{\mathrm{f}}$ & $\mathrm{G}_{\mathrm{a}}$ & $<\mathrm{G}_{\mathrm{f}}$ & $\mathrm{G}_{\mathrm{f}}$ \\
\hline 1 & $6.93024 \mathrm{E}-03$ & $1.22507 \mathrm{E}-02$ & $\overline{4.34474 \mathrm{E}-03}$ & $6.91791 \mathrm{E}-03$ & $\overline{1.22576 \mathrm{E}-02}$ & $\overline{4.35689 \mathrm{E}-03}$ \\
\hline 2 & 4.93469E-02 & $5.63616 \mathrm{E}-06$ & 2.33504E-06 & 4.72956E-02 & $5.71788 \mathrm{E}-06$ & 2.36889E-06 \\
\hline 3 & $1.10965 \mathrm{E}-02$ & 3.87293E-08 & 1.60462E-08 & $1.10697 \mathrm{E}-02$ & $3.84758 \mathrm{E}-08$ & $1.59412 \mathrm{E}-08$ \\
\hline 4 & 4.09384E-02 & $1.92170 \mathrm{E}-07$ & 7.96200E-08 & 4.09467E-02 & $1.92207 \mathrm{E}-07$ & 7.96350E-08 \\
\hline
\end{tabular}

Table 2.40. PNL-33, Pin-1 four-group Pu-239 cross sections

\begin{tabular}{cccccccc}
\hline Group & \multicolumn{3}{c}{ CENTRM cross sections $\left(\mathrm{cm}^{-1}\right)$} & \multicolumn{3}{c}{ NITAWL cross sections $\left(\mathrm{cm}^{-1}\right)$} \\
\hline & $\frac{\mathrm{G}_{\mathrm{a}}}{7.32468 \mathrm{E}-04}$ & $\frac{\mathrm{G}_{\mathrm{f}}}{2.11213 \mathrm{E}-03}$ & $\frac{\mathrm{G}_{\mathrm{f}}}{6.82512 \mathrm{E}-04}$ & $\frac{\mathrm{G}_{\mathrm{a}}}{7.31890 \mathrm{E}-04}$ & $\frac{\mathrm{G}_{\mathrm{f}}}{2.11186 \mathrm{E}-03}$ & $\frac{\mathrm{G}_{\mathrm{f}}}{6.82669 \mathrm{E}-04}$ \\
1 & $1.55242 \mathrm{E}-02$ & $2.53875 \mathrm{E}-02$ & $8.81569 \mathrm{E}-03$ & $1.47320 \mathrm{E}-02$ & $2.43541 \mathrm{E}-02$ & $8.45686 \mathrm{E}-03$ \\
2 & $4.75761 \mathrm{E}-02$ & $9.35353 \mathrm{E}-02$ & $3.24798 \mathrm{E}-02$ & $4.58342 \mathrm{E}-02$ & $9.03415 \mathrm{E}-02$ & $3.13708 \mathrm{E}-02$ \\
3 & $3.92576 \mathrm{E}-01$ & $7.79160 \mathrm{E}-01$ & $2.69612 \mathrm{E}-01$ & $3.91025 \mathrm{E}-01$ & $7.76590 \mathrm{E}-01$ & $2.68717 \mathrm{E}-01$ \\
\hline
\end{tabular}


Table 2.41. PNL-33, Pin-109 four-group fluxes

\begin{tabular}{ccc}
\hline Group & $\begin{array}{c}\text { CENTRM M } \\
\left(\mathrm{cm}^{-2}-\mathrm{s}^{-1}\right)\end{array}$ & $\begin{array}{c}\text { NITAWL M } \\
\left(\mathrm{cm}^{-2}-\mathrm{s}^{-1}\right)\end{array}$ \\
\hline 1 & $9.567 \mathrm{E}-05$ & $9.796 \mathrm{E}-05$ \\
2 & $3.454 \mathrm{E}-05$ & $3.486 \mathrm{E}-05$ \\
3 & $8.083 \mathrm{E}-06$ & $7.903 \mathrm{E}-06$ \\
4 & $1.794 \mathrm{E}-05$ & $1.809 \mathrm{E}-05$ \\
\hline
\end{tabular}

Table 2.42. PNL-33, Pin-109 four-group U-235 cross sections

\begin{tabular}{ccccccc}
\hline Group & \multicolumn{2}{c}{ CENTRM cross sections $\left(\mathrm{cm}^{-1}\right)$} & \multicolumn{3}{c}{ NITAWL cross sections $\left(\mathrm{cm}^{-1}\right)$} \\
\hline & $\frac{\mathrm{G}_{\mathrm{a}}}{4.35819 \mathrm{E}-04}$ & $\frac{\leftarrow_{\mathrm{f}}}{5.23015 \mathrm{E}-04}$ & $\frac{\mathrm{G}_{\mathrm{f}}}{2.03090 \mathrm{E}-04}$ & $\frac{\mathrm{G}_{\mathrm{a}}}{2.36391 \mathrm{E}-04}$ & $\frac{\mathrm{G}_{\mathrm{f}}}{5.23032 \mathrm{E}-04}$ & $\frac{\mathrm{G}_{\mathrm{f}}}{2.03334 \mathrm{E}-04}$ \\
1 & $5.23295 \mathrm{E}-03$ & $8.08842 \mathrm{E}-03$ & $3.31943 \mathrm{E}-03$ & $5.36724 \mathrm{E}-03$ & $8.21076 \mathrm{E}-03$ & $3.36962 \mathrm{E}-03$ \\
2 & $8.20877 \mathrm{E}-03$ & $1.67738 \mathrm{E}-02$ & $6.88382 \mathrm{E}-03$ & $8.26627 \mathrm{E}-03$ & $1.68900 \mathrm{E}-02$ & $6.93152 \mathrm{E}-03$ \\
3 & $6.90920 \mathrm{E}-02$ & $1.43635 \mathrm{E}-01$ & $5.89465 \mathrm{E}-02$ & $6.84549 \mathrm{E}-02$ & $1.42274 \mathrm{E}-01$ & $5.83881 \mathrm{E}-02$ \\
\hline
\end{tabular}

Table 2.43. PNL-33, Pin-109 four-group U-238 cross sections

\begin{tabular}{|c|c|c|c|c|c|c|}
\hline \multirow[t]{2}{*}{ Group } & \multicolumn{3}{|c|}{ CENTRM cross sections $\left(\mathrm{cm}^{-1}\right)$} & \multicolumn{3}{|c|}{ NITAWL cross sections $\left(\mathrm{cm}^{-1}\right)$} \\
\hline & $\mathrm{G}_{\mathrm{a}}$ & $\leftarrow_{\mathrm{f}}$ & $\mathrm{G}_{\mathrm{f}}$ & $\mathrm{G}_{\mathrm{a}}$ & $<\mathrm{G}_{\mathrm{f}}$ & $\mathrm{G}_{\mathrm{f}}$ \\
\hline 1 & $6.78959 \mathrm{E}-03$ & $\overline{1.14474 \mathrm{E}-02}$ & $\overline{4.06739 \mathrm{E}-03}$ & $6.75365 \mathrm{E}-03$ & $1.12640 \mathrm{E}-02$ & $\overline{4.00886 \mathrm{E}-03}$ \\
\hline 2 & 4.77122E-02 & $5.87328 \mathrm{E}-06$ & $2.43327 \mathrm{E}-06$ & 4.72083E-02 & 5.94071E-06 & $2.46121 \mathrm{E}-06$ \\
\hline 3 & $1.10759 \mathrm{E}-02$ & $3.86960 \mathrm{E}-08$ & $1.60324 \mathrm{E}-08$ & $1.11162 \mathrm{E}-02$ & $3.88436 \mathrm{E}-08$ & $1.60936 \mathrm{E}-08$ \\
\hline 4 & $3.99038 \mathrm{E}-02$ & $1.87192 \mathrm{E}-07$ & $7.75574 \mathrm{E}-08$ & $3.95563 \mathrm{E}-02$ & $1.85520 \mathrm{E}-07$ & $7.68646 \mathrm{E}-08$ \\
\hline
\end{tabular}

Table 2.44. PNL-33, Pin-109 four-group Pu-239 cross sections

\begin{tabular}{cccccccc}
\hline Group & \multicolumn{3}{c}{ CENTRM cross sections $\left(\mathrm{cm}^{-1}\right)$} & \multicolumn{3}{c}{ NITAWL cross sections $\left(\mathrm{cm}^{-1}\right)$} \\
\hline & $\frac{\mathrm{G}_{\mathrm{a}}}{7.33828 \mathrm{E}-04}$ & $\frac{\mathrm{G}_{\mathrm{f}}}{2.09652 \mathrm{E}-03}$ & $\frac{\mathrm{G}_{\mathrm{f}}}{6.79997 \mathrm{E}-04}$ & $\frac{\mathrm{G}_{\mathrm{a}}}{7.33983 \mathrm{E}-04}$ & $\frac{\mathrm{G}_{\mathrm{f}}}{2.09254 \mathrm{E}-03}$ & $\frac{\mathrm{G}_{\mathrm{f}}}{6.79479 \mathrm{E}-04}$ \\
1 & $1.54018 \mathrm{E}-02$ & $2.52039 \mathrm{E}-02$ & $8.75191 \mathrm{E}-03$ & $1.50735 \mathrm{E}-02$ & $2.48369 \mathrm{E}-02$ & $8.62447 \mathrm{E}-03$ \\
2 & $4.51535 \mathrm{E}-02$ & $8.91184 \mathrm{E}-02$ & $3.09459 \mathrm{E}-02$ & $4.89102 \mathrm{E}-02$ & $9.59904 \mathrm{E}-02$ & $3.33323 \mathrm{E}-02$ \\
3 & $4.06251 \mathrm{E}-01$ & $7.98367 \mathrm{E}-01$ & $2.76308 \mathrm{E}-01$ & $4.12182 \mathrm{E}-01$ & $8.07020 \mathrm{E}-01$ & $2.79323 \mathrm{E}-01$ \\
\hline
\end{tabular}




\begin{tabular}{|c|c|c|c|c|c|c|c|c|}
\hline & & & & & & & & $\begin{array}{c}7.799 \\
(0.66)\end{array}$ \\
\hline & & & & & & & $\begin{array}{c}7.518 \\
(0.37)\end{array}$ & $\begin{array}{c}7.649 \\
(0.35)\end{array}$ \\
\hline & & & & & & $\begin{array}{l}6.835 \\
(0.40)\end{array}$ & $\begin{array}{c}7.190 \\
(0.27)\end{array}$ & $\begin{array}{r}7.306 \\
(0.37)\end{array}$ \\
\hline & & & & & $\begin{array}{l}5.772 \\
(0.44)\end{array}$ & $\begin{array}{l}6.293 \\
(0.30)\end{array}$ & $\begin{array}{l}6.617 \\
(0.26)\end{array}$ & $\begin{array}{c}6.772 \\
(0.35)\end{array}$ \\
\hline & & & & $\begin{array}{l}4.766 \\
(0.47)\end{array}$ & $\begin{array}{l}5.125 \\
(0.31)\end{array}$ & $\begin{array}{l}5.558 \\
(0.30)\end{array}$ & $\begin{array}{l}5.878 \\
(0.29)\end{array}$ & $\begin{array}{l}5.991 \\
(0.39)\end{array}$ \\
\hline & & & 0 & $\begin{array}{l}5.095 \\
(0.29)\end{array}$ & $\begin{array}{l}4.633 \\
(0.33)\end{array}$ & $\begin{array}{l}4.852 \\
(0.31)\end{array}$ & $\begin{array}{l}5.064 \\
(0.30)\end{array}$ & $\begin{array}{l}5.107 \\
(0.46)\end{array}$ \\
\hline & & 0 & 0 & 0 & $\begin{array}{l}5.015 \\
(0.27)\end{array}$ & $\begin{array}{l}4.688 \\
(0.34)\end{array}$ & $\begin{array}{l}4.382 \\
(0.33)\end{array}$ & $\begin{array}{l}4.282 \\
(0.47)\end{array}$ \\
\hline & 0 & 0 & 0 & 0 & 0 & 0 & $\begin{array}{l}4.431 \\
(0.34)\end{array}$ & $\begin{array}{l}3.764 \\
(0.52)\end{array}$ \\
\hline 0 & 0 & 0 & 0 & 0 & 0 & 0 & 0 & $\begin{array}{l}4.082 \\
(0.48)\end{array}$ \\
\hline
\end{tabular}

Fig. 2.11a. Pin-power distribution for CENTRM benchmark PNL-34. Value in parentheses is percent standard deviation. 


\begin{tabular}{|c|c|c|c|c|c|c|c|c|}
\hline & & & & & & & & $\begin{array}{l}7.789 \\
(0.58)\end{array}$ \\
\hline & & & & & & & $\begin{array}{c}7.559 \\
(0.29)\end{array}$ & $\begin{array}{l}7.692 \\
(0.25)\end{array}$ \\
\hline & & & & & & $\begin{array}{l}6.856 \\
(0.30)\end{array}$ & $\begin{array}{l}7.211 \\
(0.20)\end{array}$ & $\begin{array}{c}7.342 \\
(0.28)\end{array}$ \\
\hline & & & & & $\begin{array}{l}5.778 \\
(0.31)\end{array}$ & $\begin{array}{l}6.319 \\
(0.21)\end{array}$ & $\begin{array}{l}6.661 \\
(0.21)\end{array}$ & $\begin{array}{c}6.794 \\
(0.32)\end{array}$ \\
\hline & & & & $\begin{array}{l}4.790 \\
(0.33)\end{array}$ & $\begin{array}{l}5.142 \\
(0.25)\end{array}$ & $\begin{array}{l}5.607 \\
(0.23)\end{array}$ & $\begin{array}{c}5.928 \\
(0.22)\end{array}$ & $\begin{array}{l}5.991 \\
(0.32)\end{array}$ \\
\hline & & & 0 & $\begin{array}{l}5.108 \\
(0.24)\end{array}$ & $\begin{array}{l}4.634 \\
(0.25)\end{array}$ & $\begin{array}{l}4.865 \\
(0.21)\end{array}$ & $\begin{array}{l}5.047 \\
(0.23)\end{array}$ & $\begin{array}{l}5.134 \\
(0.33)\end{array}$ \\
\hline & & 0 & 0 & 0 & $\begin{array}{l}5.009 \\
(0.25)\end{array}$ & $\begin{array}{l}4.689 \\
(0.24)\end{array}$ & $\begin{array}{l}4.367 \\
(0.26)\end{array}$ & $\begin{array}{l}4.295 \\
(0.36)\end{array}$ \\
\hline & 0 & 0 & 0 & 0 & 0 & 0 & $\begin{array}{l}4.412 \\
(0.26)\end{array}$ & $\begin{array}{l}3.775 \\
(0.40)\end{array}$ \\
\hline 0 & 0 & 0 & 0 & 0 & 0 & 0 & 0 & $\begin{array}{l}4.055 \\
(0.41)\end{array}$ \\
\hline
\end{tabular}

Fig. 2.11b. Pin-power distribution for NITAWL benchmark PNL-34. Value in parentheses is percent standard deviation. 
Table 2.45. PNL-34 CENTRM reaction rates and fluxes

\begin{tabular}{|c|c|c|c|c|c|c|}
\hline Pin & Region & $\begin{array}{c}\mathrm{G}_{\mathrm{a}} \mathrm{M} \\
\left(\mathrm{cm}^{-3}-\mathrm{s}^{-1}\right)\end{array}$ & $\begin{array}{c}\mathcal{G}_{\mathrm{f}} \mathrm{M} \\
\left(\mathrm{cm}^{-3}-\mathrm{s}^{-1}\right)\end{array}$ & $\begin{array}{c}\mathrm{G}_{\mathrm{f}} \mathrm{M} \\
\left(\mathrm{cm}^{-3}-\mathrm{s}^{-1}\right)\end{array}$ & $\begin{array}{c}\mathrm{M} \\
\left(\mathrm{cm}-\mathrm{s}^{-1}\right)\end{array}$ & $\mathrm{M}_{\mathrm{t}}$ \\
\hline \multirow{3}{*}{1} & Fuel & $2.378 \mathrm{E}-05$ & $4.072 \mathrm{E}-05$ & $1.453 \mathrm{E}-05$ & $1.638 \mathrm{E}-2$ & 0.205 \\
\hline & Clad & $4.283 \mathrm{E}-07$ & 0.0 & 0.0 & $3.115 \mathrm{E}-3$ & 0.039 \\
\hline & Mod. & $1.208 \mathrm{E}-06$ & 0.0 & 0.0 & $6.042 \mathrm{E}-2$ & 0.756 \\
\hline \multirow{3}{*}{27} & Fuel & $4.850 \mathrm{E}-05$ & $7.778 \mathrm{E}-05$ & $2.772 \mathrm{E}-05$ & $4.812 \mathrm{E}-2$ & 0.206 \\
\hline & Clad & $9.350 \mathrm{E}-07$ & 0.0 & 0.0 & $9.315 \mathrm{E}-3$ & 0.039 \\
\hline & Mod. & $2.117 \mathrm{E}-06$ & 0.0 & 0.0 & $1.767 \mathrm{E}-1$ & 0.755 \\
\hline
\end{tabular}

Table 2.46. PNL-34 NITAWL reaction rates and fluxes

\begin{tabular}{|c|c|c|c|c|c|c|}
\hline Pin & Region & $\begin{array}{c}\mathrm{G}_{\mathrm{a}} \mathrm{M} \\
\left(\mathrm{cm}^{-3}-\mathrm{s}^{-1}\right)\end{array}$ & $\begin{array}{c}\mathcal{G}_{\mathrm{f}} \mathrm{M} \\
\left(\mathrm{cm}^{-3}-\mathrm{s}^{-1}\right)\end{array}$ & $\begin{array}{c}\mathrm{G}_{\mathrm{f}} \mathrm{M} \\
\left(\mathrm{cm}^{-3}-\mathrm{s}^{-1}\right)\end{array}$ & $\begin{array}{c}\mathrm{M} \\
\left(\mathrm{cm}-\mathrm{s}^{-1}\right)\end{array}$ & $\mathrm{M} / \mathrm{M}_{\mathrm{t}}$ \\
\hline \multirow{3}{*}{1} & Fuel & $2.359 \mathrm{E}-05$ & $4.048 \mathrm{E}-05$ & $1.444 \mathrm{E}-05$ & $1.632 \mathrm{E}-2$ & 0.205 \\
\hline & Clad & $4.263 \mathrm{E}-07$ & 0.0 & 0.0 & $3.103 \mathrm{E}-3$ & 0.039 \\
\hline & Mod. & $1.204 \mathrm{E}-06$ & 0.0 & 0.0 & $6.010 \mathrm{E}-2$ & 0.756 \\
\hline \multirow{3}{*}{27} & Fuel & $4.865 \mathrm{E}-05$ & $7.795 \mathrm{E}-5$ & $2.778 \mathrm{E}-05$ & $4.833 \mathrm{E}-2$ & 0.206 \\
\hline & Clad & $9.241 \mathrm{E}-07$ & 0.0 & 0.0 & $9.314 \mathrm{E}-3$ & 0.040 \\
\hline & Mod. & $2.122 \mathrm{E}-06$ & 0.0 & 0.0 & $1.768 \mathrm{E}-1$ & 0.754 \\
\hline
\end{tabular}


Table 2.47. PNL-34, Pin-1 four-group fluxes

\begin{tabular}{ccc}
\hline Group & $\begin{array}{c}\text { CENTRM M } \\
\left(\mathrm{cm}^{-2}-\mathrm{s}^{-1}\right)\end{array}$ & $\begin{array}{c}\text { NITAWL M } \\
\left(\mathrm{cm}^{-2}-\mathrm{s}^{-1}\right)\end{array}$ \\
\hline 1 & $7.853 \mathrm{E}-05$ & $7.833 \mathrm{E}-05$ \\
2 & $2.067 \mathrm{E}-05$ & $2.085 \mathrm{E}-05$ \\
3 & $5.292 \mathrm{E}-06$ & $5.285 \mathrm{E}-06$ \\
4 & $4.175 \mathrm{E}-05$ & $4.127 \mathrm{E}-05$ \\
\hline
\end{tabular}

Table 2.48. PNL-34, Pin-1 four-group U-235 cross sections

\begin{tabular}{|c|c|c|c|c|c|c|}
\hline \multirow[t]{2}{*}{ Group } & \multicolumn{3}{|c|}{ CENTRM cross sections $\left(\mathrm{cm}^{-1}\right)$} & \multicolumn{3}{|c|}{ NITAWL cross sections $\left(\mathrm{cm}^{-1}\right)$} \\
\hline & $\mathrm{G}_{2}$ & $\varangle G_{i}$ & $\mathrm{G}_{\mathrm{f}}$ & G & $\ll \mathrm{G}_{\mathrm{f}}$ & $\mathrm{G}_{\mathrm{f}}$ \\
\hline 1 & $2 . \overline{24131 \mathrm{E}-04}$ & $\overline{5.12577 \mathrm{E}-04}$ & $1.97109 \mathrm{E}-04$ & $2 . \overline{23704 \overline{\mathrm{E}-04}}$ & $\overline{5.11854 \mathrm{E}-04}$ & $1.96816 \mathrm{E}-04$ \\
\hline 2 & $5.42283 \mathrm{E}-03$ & $8.35085 \mathrm{E}-03$ & $3.42710 \mathrm{E}-03$ & $5.42556 \mathrm{E}-03$ & $8.28643 \mathrm{E}-03$ & $3.40068 \mathrm{E}-03$ \\
\hline 3 & $8.31740 \mathrm{E}-03$ & $1.69753 \mathrm{E}-02$ & $6.96653 \mathrm{E}-03$ & $8.31298 \mathrm{E}-03$ & $1.69590 \mathrm{E}-02$ & $6.95981 \mathrm{E}-03$ \\
\hline 4 & 7.54771E-02 & $1.57143 \mathrm{E}-01$ & $6.44898 \mathrm{E}-02$ & $7.58120 \mathrm{E}-02$ & $1.57838 \mathrm{E}-01$ & $6.47754 \mathrm{E}-02$ \\
\hline
\end{tabular}

Table 2.49. PNL-34, Pin-1 four-group U-238 cross sections

\begin{tabular}{cccccccc}
\hline Group & \multicolumn{3}{c}{ CENTRM cross sections $\left(\mathrm{cm}^{-1}\right)$} & \multicolumn{3}{c}{ NITAWL cross sections $\left(\mathrm{cm}^{-1}\right)$} \\
\hline & $\frac{\mathrm{G}_{\mathrm{a}}}{}$ & $7.08275 \mathrm{E}-03$ & $\frac{\mathrm{G}_{\mathrm{f}}}{1.34026 \mathrm{E}-02}$ & $\frac{\mathrm{G}_{\mathrm{f}}}{4.75965 \mathrm{E}-03}$ & $\frac{\mathrm{G}_{\mathrm{a}}}{7.06095 \mathrm{E}-03}$ & $\frac{\varangle_{\mathrm{f}}}{1.33747 \mathrm{E}-02}$ & $\frac{\mathrm{G}_{\mathrm{f}}}{4.74910 \mathrm{E}-03}$ \\
2 & $5.08244 \mathrm{E}-02$ & $6.00968 \mathrm{E}-06$ & $2.48979 \mathrm{E}-06$ & $4.81132 \mathrm{E}-02$ & $5.51775 \mathrm{E}-06$ & $2.28598 \mathrm{E}-06$ \\
3 & $1.11012 \mathrm{E}-02$ & $3.87466 \mathrm{E}-08$ & $1.60534 \mathrm{E}-08$ & $1.11191 \mathrm{E}-02$ & $3.89584 \mathrm{E}-08$ & $1.61412 \mathrm{E}-08$ \\
4 & $4.32220 \mathrm{E}-02$ & $2.03118 \mathrm{E}-07$ & $8.41557 \mathrm{E}-08$ & $4.33792 \mathrm{E}-02$ & $2.03865 \mathrm{E}-07$ & $8.44652 \mathrm{E}-08$ \\
\hline
\end{tabular}

Table 2.50. PNL-34, Pin-1 four-group Pu-239 cross sections

\begin{tabular}{cccccccc}
\hline Group & \multicolumn{3}{c}{ CENTRM cross sections $\left(\mathrm{cm}^{-1}\right)$} & \multicolumn{3}{c}{ NITAWL cross sections $\left(\mathrm{cm}^{-1}\right)$} \\
\hline & $\frac{\mathrm{G}_{\mathrm{a}}}{}$ & $7.29345 \mathrm{E}-04$ & $\frac{\mathrm{G}_{\mathrm{f}}}{2.13767 \mathrm{E}-03}$ & $\frac{\mathrm{G}_{\mathrm{f}}}{6.87363 \mathrm{E}-04}$ & $\frac{\mathrm{G}_{\mathrm{a}}}{7.28644 \mathrm{E}-04}$ & $\frac{\varangle_{\mathrm{f}}}{2.13663 \mathrm{E}-03}$ & $\frac{\mathrm{G}_{\mathrm{f}}}{6.86999 \mathrm{E}-04}$ \\
2 & $1.53106 \mathrm{E}-02$ & $2.52664 \mathrm{E}-02$ & $8.77363 \mathrm{E}-03$ & $1.54151 \mathrm{E}-02$ & $2.54295 \mathrm{E}-02$ & $8.83026 \mathrm{E}-03$ \\
3 & $4.67356 \mathrm{E}-02$ & $9.20261 \mathrm{E}-02$ & $3.19556 \mathrm{E}-02$ & $4.76683 \mathrm{E}-02$ & $9.37614 \mathrm{E}-02$ & $3.25584 \mathrm{E}-02$ \\
4 & $3.72031 \mathrm{E}-01$ & $7.53188 \mathrm{E}-01$ & $2.60517 \mathrm{E}-01$ & $3.73795 \mathrm{E}-01$ & $7.56765 \mathrm{E}-01$ & $2.61754 \mathrm{E}-01$ \\
\hline
\end{tabular}


Table 2.51. PNL-34, Pin-27 four-group fluxes

\begin{tabular}{ccc}
\hline Group & $\begin{array}{c}\text { CENTRM M } \\
\left(\mathrm{cm}^{-2}-\mathrm{s}^{-1}\right)\end{array}$ & $\begin{array}{c}\text { NITAWL M } \\
\left(\mathrm{cm}^{-2}-\mathrm{s}^{-1}\right)\end{array}$ \\
\hline 1 & $2.549 \mathrm{E}-04$ & $2.550 \mathrm{E}-04$ \\
2 & $8.111 \mathrm{E}-05$ & $8.287 \mathrm{E}-05$ \\
3 & $1.947 \mathrm{E}-05$ & $1.947 \mathrm{E}-05$ \\
4 & $7.406 \mathrm{E}-05$ & $7.415 \mathrm{E}-05$ \\
\hline
\end{tabular}

Table 2.52. PNL-34, Pin-27 four-group U-235 cross sections

\begin{tabular}{cccccccc}
\hline Group & \multicolumn{3}{c}{ CENTRM cross sections $\left(\mathrm{cm}^{-1}\right)$} & \multicolumn{3}{c}{ NITAWL cross sections $\left(\mathrm{cm}^{-1}\right)$} \\
\hline & $\frac{\mathrm{G}_{\mathrm{a}}}{2.32268 \mathrm{E}-04}$ & $\frac{\mathrm{G}_{\mathrm{f}}}{5.19584 \mathrm{E}-04}$ & $\frac{\mathrm{G}_{\mathrm{f}}}{2.01251 \mathrm{E}-04}$ & $\frac{\mathrm{G}_{\mathrm{a}}}{2.31678 \mathrm{E}-04}$ & $\frac{\mathrm{G}_{\mathrm{f}}}{5.19278 \mathrm{E}-04}$ & $\frac{\mathrm{G}_{\mathrm{f}}}{2.00931 \mathrm{E}-04}$ \\
1 & $5.33129 \mathrm{E}-03$ & $8.13383 \mathrm{E}-03$ & $3.33805 \mathrm{E}-03$ & $5.40039 \mathrm{E}-03$ & $8.25888 \mathrm{E}-03$ & $3.38937 \mathrm{E}-03$ \\
2 & $8.21787 \mathrm{E}-03$ & $1.67382 \mathrm{E}-02$ & $6.86918 \mathrm{E}-03$ & $8.24787 \mathrm{E}-03$ & $1.68440 \mathrm{E}-02$ & $6.91263 \mathrm{E}-03$ \\
3 & $7.26200 \mathrm{E}-02$ & $1.51090 \mathrm{E}-01$ & $6.20057 \mathrm{E}-02$ & $7.26900 \mathrm{E}-02$ & $1.51227 \mathrm{E}-01$ & $6.20624 \mathrm{E}-02$ \\
\hline
\end{tabular}

Table 2.53. PNL-34, Pin-27 four-group U-238 cross sections

\begin{tabular}{cccccccc}
\hline Group & \multicolumn{3}{c}{ CENTRM cross sections $\left(\mathrm{cm}^{-1}\right)$} & \multicolumn{3}{c}{ NITAWL cross sections $\left(\mathrm{cm}^{-1}\right)$} \\
\hline & $\frac{\mathrm{G}_{\mathrm{a}}}{6.87156 \mathrm{E}-03}$ & $\frac{\mathrm{G}_{\mathrm{f}}}{1.19877 \mathrm{E}-02}$ & $\frac{\mathrm{G}_{\mathrm{f}}}{4.26261 \mathrm{E}-03}$ & $\frac{6.88984 \mathrm{E}-03}{1.21250 \mathrm{E}-02}$ & $\frac{\mathrm{G}_{\mathrm{a}}}{4.30392 \mathrm{E}-03}$ \\
1 & $4.88942 \mathrm{E}-02$ & $5.93992 \mathrm{E}-06$ & $2.46088 \mathrm{E}-06$ & $4.94199 \mathrm{E}-02$ & $5.55323 \mathrm{E}-06$ & $2.30068 \mathrm{E}-06$ \\
2 & $1.10643 \mathrm{E}-02$ & $3.83905 \mathrm{E}-08$ & $1.59059 \mathrm{E}-08$ & $1.11102 \mathrm{E}-02$ & $3.88583 \mathrm{E}-08$ & $1.60997 \mathrm{E}-08$ \\
3 & $4.17166 \mathrm{E}-02$ & $1.95887 \mathrm{E}-07$ & $8.11600 \mathrm{E}-08$ & $4.17541 \mathrm{E}-02$ & $1.96065 \mathrm{E}-07$ & $8.12334 \mathrm{E}-08$ \\
\hline
\end{tabular}

Table 2.54. PNL-34, Pin-27 four-group Pu-239 cross sections

\begin{tabular}{cccccccc}
\hline Group & \multicolumn{3}{c}{ CENTRM cross sections $\left(\mathrm{cm}^{-1}\right)$} & \multicolumn{3}{c}{ NITAWL cross sections $\left(\mathrm{cm}^{-1}\right)$} \\
\hline & $\frac{\mathrm{G}_{\mathrm{a}}}{7.32585 \mathrm{E}-04}$ & $\frac{\mathrm{G}_{\mathrm{f}}}{2.10845 \mathrm{E}-03}$ & $\frac{\mathrm{G}_{\mathrm{f}}}{6.82303 \mathrm{E}-04}$ & $\frac{\mathrm{G}_{\mathrm{a}}}{7.32086 \mathrm{E}-04}$ & $\frac{\mathrm{G}_{\mathrm{f}}}{2.11047 \mathrm{E}-03}$ & $\frac{\mathrm{G}_{\mathrm{f}}}{6.82341 \mathrm{E}-04}$ \\
1 & $1.52932 \mathrm{E}-02$ & $2.51849 \mathrm{E}-02$ & $8.74535 \mathrm{E}-03$ & $1.50659 \mathrm{E}-02$ & $2.45838 \mathrm{E}-02$ & $8.53662 \mathrm{E}-03$ \\
2 & $4.71772 \mathrm{E}-02$ & $9.27560 \mathrm{E}-02$ & $3.22091 \mathrm{E}-02$ & $4.72429 \mathrm{E}-02$ & $9.29723 \mathrm{E}-02$ & $3.22843 \mathrm{E}-02$ \\
3 & $3.90512 \mathrm{E}-01$ & $7.78732 \mathrm{E}-01$ & $2.69434 \mathrm{E}-01$ & $3.90550 \mathrm{E}-01$ & $7.78815 \mathrm{E}-01$ & $2.69459 \mathrm{E}-01$ \\
\hline
\end{tabular}




\begin{tabular}{|c|c|c|c|c|c|c|c|c|c|c|}
\hline & & & & & & & & & & $\begin{array}{l}2.316 \\
(1.24)\end{array}$ \\
\hline & & & & & & & & & $\begin{array}{l}2.296 \\
(0.53)\end{array}$ & $\begin{array}{l}2.287 \\
(0.52)\end{array}$ \\
\hline & & & & & & & & $\begin{array}{l}2.231 \\
(0.55)\end{array}$ & $\begin{array}{l}2.256 \\
(0.39)\end{array}$ & $\begin{array}{l}2.273 \\
(0.58)\end{array}$ \\
\hline & & & & & & & $\begin{array}{l}2.099 \\
(0.52)\end{array}$ & $\begin{array}{l}2.175 \\
(0.40)\end{array}$ & $\begin{array}{l}2.223 \\
(0.43)\end{array}$ & $\begin{array}{c}2.208 \\
(0.54)\end{array}$ \\
\hline & & & & & & $\begin{array}{l}1.961 \\
(0.55)\end{array}$ & $\begin{array}{c}2.034 \\
(0.41)\end{array}$ & $\begin{array}{l}2.098 \\
(0.41)\end{array}$ & $\begin{array}{l}2.134 \\
(0.38)\end{array}$ & $\begin{array}{l}2.135 \\
(0.61)\end{array}$ \\
\hline & & & & & $\begin{array}{c}1.756 \\
(0.64)\end{array}$ & $\begin{array}{l}1.872 \\
(0.43)\end{array}$ & $\begin{array}{l}1.941 \\
(0.42)\end{array}$ & $\begin{array}{l}1.996 \\
(0.39)\end{array}$ & $\begin{array}{l}2.057 \\
(0.38)\end{array}$ & $\begin{array}{r}2.047 \\
(0.55)\end{array}$ \\
\hline & & & & $\begin{array}{c}1.554 \\
(0.67)\end{array}$ & $\begin{array}{c}1.667 \\
(0.45)\end{array}$ & $\begin{array}{l}1.768 \\
(0.42)\end{array}$ & $\begin{array}{c}1.831 \\
(0.46)\end{array}$ & $\begin{array}{c}1.888 \\
(0.45)\end{array}$ & $\begin{array}{c}1.915 \\
(0.41)\end{array}$ & $\begin{array}{c}1.936 \\
(0.60)\end{array}$ \\
\hline & & & $\begin{array}{l}1.322 \\
(0.73)\end{array}$ & $\begin{array}{c}1.441 \\
(0.50)\end{array}$ & $\begin{array}{c}1.550 \\
(0.43)\end{array}$ & $\begin{array}{l}1.640 \\
(0.45)\end{array}$ & $\begin{array}{c}1.704 \\
(0.46)\end{array}$ & $\begin{array}{c}1.742 \\
(0.40)\end{array}$ & $\begin{array}{c}1.797 \\
(0.43)\end{array}$ & $\begin{array}{c}1.788 \\
(0.57)\end{array}$ \\
\hline & & $\begin{array}{l}1.050 \\
(0.80)\end{array}$ & $\begin{array}{l}1.196 \\
(0.58)\end{array}$ & $\begin{array}{c}1.301 \\
(0.46)\end{array}$ & $\begin{array}{c}1.414 \\
(0.47)\end{array}$ & $\begin{array}{c}1.498 \\
(0.52)\end{array}$ & $\begin{array}{l}1.567 \\
(0.46)\end{array}$ & $\begin{array}{l}1.606 \\
(0.46)\end{array}$ & $\begin{array}{l}1.640 \\
(0.43)\end{array}$ & $\begin{array}{l}1.640 \\
(0.61)\end{array}$ \\
\hline & $\begin{array}{c}0.798 \\
(0.83)\end{array}$ & $\begin{array}{c}0.924 \\
(0.60)\end{array}$ & $\begin{array}{c}1.047 \\
(0.60)\end{array}$ & $\begin{array}{c}1.159 \\
(0.53)\end{array}$ & $\begin{array}{c}1.254 \\
(0.49)\end{array}$ & $\begin{array}{l}1.331 \\
(0.51)\end{array}$ & $\begin{array}{r}1.400 \\
(0.49)\end{array}$ & $\begin{array}{l}1.441 \\
(0.49)\end{array}$ & $\begin{array}{c}1.475 \\
(0.45)\end{array}$ & $\begin{array}{c}1.488 \\
(0.64)\end{array}$ \\
\hline $\begin{array}{c}0.657 \\
(0.98)\end{array}$ & $\begin{array}{r}0.680 \\
(0.66)\end{array}$ & $\begin{array}{c}0.780 \\
(0.67)\end{array}$ & $\begin{array}{c}0.888 \\
(0.60)\end{array}$ & $\begin{array}{c}1.002 \\
(0.60)\end{array}$ & $\begin{array}{c}1.099 \\
(0.58)\end{array}$ & $\begin{array}{l}1.163 \\
(0.52)\end{array}$ & $\begin{array}{l}1.236 \\
(0.55)\end{array}$ & $\begin{array}{l}1.270 \\
(0.55)\end{array}$ & $\begin{array}{c}1.299 \\
(0.48)\end{array}$ & $\begin{array}{l}1.291 \\
(0.76)\end{array}$ \\
\hline 0 & $\begin{array}{l}0.645 \\
(0.64)\end{array}$ & $\begin{array}{r}0.667 \\
(0.70)\end{array}$ & $\begin{array}{l}0.755 \\
(0.71)\end{array}$ & $\begin{array}{c}0.838 \\
(0.66)\end{array}$ & $\begin{array}{c}0.926 \\
(0.58)\end{array}$ & $\begin{array}{l}1.004 \\
(0.57)\end{array}$ & $\begin{array}{c}1.052 \\
(0.58)\end{array}$ & $\begin{array}{c}1.091 \\
(0.50)\end{array}$ & $\begin{array}{c}1.122 \\
(0.56)\end{array}$ & $\begin{array}{c}1.114 \\
(0.73)\end{array}$ \\
\hline 0 & 0 & $\begin{array}{r}0.605 \\
(0.73)\end{array}$ & $\begin{array}{c}0.620 \\
(0.71)\end{array}$ & $\begin{array}{l}0.685 \\
(0.66)\end{array}$ & $\begin{array}{c}0.759 \\
(0.64)\end{array}$ & $\begin{array}{l}0.821 \\
(0.68)\end{array}$ & $\begin{array}{c}0.868 \\
(0.53)\end{array}$ & $\begin{array}{r}0.902 \\
(0.62)\end{array}$ & $\begin{array}{l}0.924 \\
(0.56)\end{array}$ & $\begin{array}{c}0.929 \\
(0.81)\end{array}$ \\
\hline 0 & 0 & 0 & $\begin{array}{c}0.562 \\
(0.81)\end{array}$ & $\begin{array}{c}0.591 \\
(0.78)\end{array}$ & $\begin{array}{r}0.617 \\
(0.75)\end{array}$ & $\begin{array}{l}0.649 \\
(0.79)\end{array}$ & $\begin{array}{l}0.696 \\
(0.66)\end{array}$ & $\begin{array}{l}0.736 \\
(0.69)\end{array}$ & $\begin{array}{c}0.753 \\
(0.64)\end{array}$ & $\begin{array}{l}0.760 \\
(0.98)\end{array}$ \\
\hline 0 & 0 & 0 & 0 & 0 & $\begin{array}{c}0.553 \\
(0.78)\end{array}$ & $\begin{array}{l}0.558 \\
(0.83)\end{array}$ & $\begin{array}{c}0.591 \\
(0.70)\end{array}$ & $\begin{array}{c}0.619 \\
(0.71)\end{array}$ & $\begin{array}{l}0.642 \\
(0.75)\end{array}$ & $\begin{array}{l}0.645 \\
(1.12)\end{array}$ \\
\hline
\end{tabular}

Fig. 2.12a. Pin-power distribution for CENTRM benchmark PNL-35. Value in parentheses is percent standard deviation. 


\begin{tabular}{|c|c|c|c|c|c|c|c|c|c|c|}
\hline & & & & & & & & & & $\begin{array}{l}2.333 \\
(0.98)\end{array}$ \\
\hline & & & & & & & & & $\begin{array}{l}2.308 \\
(0.50)\end{array}$ & $\begin{array}{l}2.324 \\
(0.46)\end{array}$ \\
\hline & & & & & & & & $\begin{array}{l}2.251 \\
(0.49)\end{array}$ & $\begin{array}{l}2.275 \\
(0.36)\end{array}$ & $\begin{array}{l}2.283 \\
(0.54)\end{array}$ \\
\hline & & & & & & & $\begin{array}{l}2.133 \\
(0.50)\end{array}$ & $\begin{array}{l}2.193 \\
(0 .) 36\end{array}$ & $\begin{array}{l}2.202 \\
(0.36)\end{array}$ & $\begin{array}{l}2.210 \\
(0.48)\end{array}$ \\
\hline & & & & & & $\begin{array}{c}1.973 \\
(0.50)\end{array}$ & $\begin{array}{l}2.044 \\
(0.36)\end{array}$ & $\begin{array}{r}2.110 \\
(0.35)\end{array}$ & $\begin{array}{l}2.131 \\
(0.37)\end{array}$ & $\begin{array}{l}2.142 \\
(0.54)\end{array}$ \\
\hline & & & & & $\begin{array}{c}1.790 \\
(0.55)\end{array}$ & $\begin{array}{c}1.887 \\
(0.38)\end{array}$ & $\begin{array}{c}1.950 \\
(0.40)\end{array}$ & $\begin{array}{l}2.004 \\
(0.36)\end{array}$ & $\begin{array}{l}2.038 \\
(0.35)\end{array}$ & $\begin{array}{l}2.050 \\
(0.48)\end{array}$ \\
\hline & & & & $\begin{array}{c}1.583 \\
(0.60)\end{array}$ & $\begin{array}{c}1.688 \\
(0.41)\end{array}$ & $\begin{array}{c}1.765 \\
(0.40)\end{array}$ & $\begin{array}{c}1.827 \\
(0.40)\end{array}$ & $\begin{array}{l}1.890 \\
(0.38)\end{array}$ & $\begin{array}{c}1.916 \\
(0.37)\end{array}$ & $\begin{array}{l}1.941 \\
(0.52)\end{array}$ \\
\hline & & & $\begin{array}{c}1.329 \\
(0.66)\end{array}$ & $\begin{array}{r}1.460 \\
(0.45)\end{array}$ & $\begin{array}{c}1.552 \\
(0.42)\end{array}$ & $\begin{array}{c}1.636 \\
(0.38)\end{array}$ & $\begin{array}{c}1.692 \\
(0.38)\end{array}$ & $\begin{array}{c}1.766 \\
(0.39)\end{array}$ & $\begin{array}{c}1.772 \\
(0.40)\end{array}$ & $\begin{array}{l}1.802 \\
(0.60)\end{array}$ \\
\hline & & $\begin{array}{l}1.065 \\
(0.73)\end{array}$ & $\begin{array}{c}1.212 \\
(0.48)\end{array}$ & $\begin{array}{c}1.315 \\
(0.50)\end{array}$ & $\begin{array}{c}1.409 \\
(0.45)\end{array}$ & $\begin{array}{l}1.482 \\
(0.42)\end{array}$ & $\begin{array}{l}1.566 \\
(0.44)\end{array}$ & $\begin{array}{l}1.603 \\
(0.42)\end{array}$ & $\begin{array}{l}1.622 \\
(0.42)\end{array}$ & $\begin{array}{l}1.645 \\
(0.59)\end{array}$ \\
\hline & $\begin{array}{l}8.130 \\
(0.80)\end{array}$ & $\begin{array}{r}9.340 \\
(0.58)\end{array}$ & $\begin{array}{c}1.060 \\
(0.54)\end{array}$ & $\begin{array}{c}1.164 \\
(0.47)\end{array}$ & $\begin{array}{c}1.252 \\
(0.50)\end{array}$ & $\begin{array}{c}1.329 \\
(0.47)\end{array}$ & $\begin{array}{c}1.391 \\
(0.45)\end{array}$ & $\begin{array}{l}1.433 \\
(0.44)\end{array}$ & $\begin{array}{c}1.479 \\
(0.41)\end{array}$ & $\begin{array}{l}1.488 \\
(0.61)\end{array}$ \\
\hline $\begin{array}{l}6.750 \\
(0.89)\end{array}$ & $\begin{array}{l}6.790 \\
(0.62)\end{array}$ & $\begin{array}{c}7.980 \\
(0.63)\end{array}$ & $\begin{array}{c}9.02 \\
(0.55)\end{array}$ & $\begin{array}{c}1.011 \\
(0.53)\end{array}$ & $\begin{array}{c}1.095 \\
(0.48)\end{array}$ & $\begin{array}{c}1.159 \\
(0.50)\end{array}$ & $\begin{array}{c}1.220 \\
(0.46)\end{array}$ & $\begin{array}{c}1.258 \\
(0.46)\end{array}$ & $\begin{array}{l}1.301 \\
(0.47)\end{array}$ & $\begin{array}{l}1.315 \\
(0.61)\end{array}$ \\
\hline 0 & $\begin{array}{r}0.651 \\
(0.64)\end{array}$ & $\begin{array}{c}0.663 \\
(0.67)\end{array}$ & $\begin{array}{c}0.742 \\
(0.60)\end{array}$ & $\begin{array}{l}0.837 \\
(0.60)\end{array}$ & $\begin{array}{c}0.927 \\
(0.54)\end{array}$ & $\begin{array}{c}0.994 \\
(0.54)\end{array}$ & $\begin{array}{l}1.033 \\
(0.52)\end{array}$ & $\begin{array}{l}1.087 \\
(0.53)\end{array}$ & $\begin{array}{c}1.101 \\
(0.49)\end{array}$ & $\begin{array}{l}1.123 \\
(0.72)\end{array}$ \\
\hline 0 & 0 & $\begin{array}{l}0.607 \\
(0.74)\end{array}$ & $\begin{array}{c}0.616 \\
(0.66)\end{array}$ & $\begin{array}{r}0.677 \\
(0.65)\end{array}$ & $\begin{array}{c}0.765 \\
(0.57)\end{array}$ & $\begin{array}{r}0.819 \\
(0.56)\end{array}$ & $\begin{array}{c}0.870 \\
(0.59)\end{array}$ & $\begin{array}{l}0.900 \\
(0.53)\end{array}$ & $\begin{array}{c}0.920 \\
(0.59)\end{array}$ & $\begin{array}{l}0.921 \\
(0.77)\end{array}$ \\
\hline 0 & 0 & 0 & $\begin{array}{c}0.565 \\
(0.68)\end{array}$ & $\begin{array}{l}0.591 \\
(0.65)\end{array}$ & $\begin{array}{c}0.606 \\
(0.66)\end{array}$ & $\begin{array}{r}0.653 \\
(0.63)\end{array}$ & $\begin{array}{l}0.686 \\
(0.66)\end{array}$ & $\begin{array}{l}0.717 \\
(0.59)\end{array}$ & $\begin{array}{l}0.744 \\
(0.59)\end{array}$ & $\begin{array}{l}0.755 \\
(0.87)\end{array}$ \\
\hline 0 & 0 & 0 & 0 & 0 & $\begin{array}{c}0.557 \\
(0.69)\end{array}$ & $\begin{array}{c}0.554 \\
(0.69)\end{array}$ & $\begin{array}{c}0.600 \\
(0.73)\end{array}$ & $\begin{array}{r}0.615 \\
(0.71)\end{array}$ & $\begin{array}{r}0.640 \\
(0.70)\end{array}$ & $\begin{array}{c}0.650 \\
(0.96)\end{array}$ \\
\hline
\end{tabular}

Fig. 2.12b. Pin-power distribution for NITAWL benchmark PNL-35. Value in parentheses is percent standard deviation. 
Table 2.55. PNL-35 CENTRM reaction rates and fluxes

\begin{tabular}{ccccccc}
\hline Pin & Region & $\begin{array}{c}\mathrm{G}_{\mathrm{a}} \mathrm{M} \\
\left(\mathrm{cm}^{-3}-\mathrm{s}^{-1}\right)\end{array}$ & $\begin{array}{c}\mathrm{G}_{\mathrm{f}} \mathrm{M} \\
\left(\mathrm{cm}^{-3}-\mathrm{s}^{-1}\right)\end{array}$ & $\begin{array}{c}\mathrm{G}_{\mathrm{f}} \mathrm{M} \\
\left(\mathrm{cm}^{-3}-\mathrm{s}^{-1}\right)\end{array}$ & $\begin{array}{c}\mathrm{M} \\
\left(\mathrm{cm}^{-1}\right)\end{array}$ & $\mathrm{M}_{\mathrm{t}}$ \\
\hline \multirow{2}{*}{1} & Fuel & $3.409 \mathrm{E}-06$ & $5.555 \mathrm{E}-06$ & $1.980 \mathrm{E}-06$ & $3.018 \mathrm{E}-3$ & 0.205 \\
& Clad & $6.266 \mathrm{E}-08$ & 0.0 & 0.0 & $5.795 \mathrm{E}-4$ & 0.039 \\
& Mod. & $3.753 \mathrm{E}-07$ & 0.0 & 0.0 & $1.113 \mathrm{E}-2$ & 0.756 \\
\hline \multirow{2}{*}{99} & Fuel & $1.478 \mathrm{E}-05$ & $2.314 \mathrm{E}-05$ & $8.243 \mathrm{E}-06$ & $1.578 \mathrm{E}-2$ & 0.206 \\
& Clad & $2.862 \mathrm{E}-07$ & 0.0 & 0.0 & $3.049 \mathrm{E}-3$ & 0.039 \\
& Mod. & $1.467 \mathrm{E}-06$ & 0.0 & 0.0 & $5.794 \mathrm{E}-2$ & 0.755 \\
\hline
\end{tabular}

Table 2.56. PNL-35 NITAWL reaction rates and fluxes

\begin{tabular}{|c|c|c|c|c|c|c|}
\hline Pin & Region & $\begin{array}{c}\mathrm{G}_{\mathrm{a}} \mathrm{M} \\
\left(\mathrm{cm}^{-3}-\mathrm{s}^{-1}\right)\end{array}$ & $\begin{array}{c}\mathcal{G}_{\mathrm{f}} \mathrm{M} \\
\left(\mathrm{cm}^{-3}-\mathrm{s}^{-1}\right)\end{array}$ & $\begin{array}{c}\mathrm{G}_{\mathrm{f}} \mathrm{M} \\
\left(\mathrm{cm}^{-3}-\mathrm{s}^{-1}\right)\end{array}$ & $\begin{array}{c}\mathrm{M} \\
\left(\mathrm{cm}-\mathrm{s}^{-1}\right)\end{array}$ & $\mathrm{M}_{\mathrm{t}}$ \\
\hline \multirow{3}{*}{1} & Fuel & $3.418 \mathrm{E}-06$ & $5.589 \mathrm{E}-06$ & $1.992 \mathrm{E}-06$ & $3.030 \mathrm{E}-3$ & 0.206 \\
\hline & Clad & $6.288 \mathrm{E}-08$ & 0.0 & 0.0 & $5.820 \mathrm{E}-4$ & 0.040 \\
\hline & Mod. & $3.743 \mathrm{E}-07$ & 0.0 & 0.0 & $1.107 \mathrm{E}-2$ & 0.754 \\
\hline \multirow{3}{*}{99} & Fuel & $1.486 \mathrm{E}-05$ & $2.341 \mathrm{E}-05$ & 8.338E-06 & $1.603 \mathrm{E}-2$ & 0.207 \\
\hline & Clad & $2.817 \mathrm{E}-07$ & 0.0 & 0.0 & $3.073 \mathrm{E}-3$ & 0.040 \\
\hline & Mod. & $1.487 \mathrm{E}-06$ & 0.0 & 0.0 & $5.834 \mathrm{E}-2$ & 0.753 \\
\hline
\end{tabular}


Table 2.57. PNL-35, Pin-1 four-group fluxes

\begin{tabular}{ccc}
\hline Group & $\begin{array}{c}\text { CENTRM M } \\
\left(\mathrm{cm}^{-2}-\mathrm{s}^{-1}\right)\end{array}$ & $\begin{array}{c}\text { NITAWL M } \\
\left(\mathrm{cm}^{-2}-\mathrm{s}^{-1}\right)\end{array}$ \\
\hline 1 & $1.534 \mathrm{E}-05$ & $1.545 \mathrm{E}-05$ \\
2 & $4.935 \mathrm{E}-06$ & $4.897 \mathrm{E}-06$ \\
3 & $1.254 \mathrm{E}-06$ & $1.264 \mathrm{E}-06$ \\
4 & $5.413 \mathrm{E}-06$ & $5.443 \mathrm{E}-06$ \\
\hline
\end{tabular}

Table 2.58. PNL-35, Pin-1 four-group U-235 cross sections

\begin{tabular}{cccccccc}
\hline Group & \multicolumn{3}{c}{ CENTRM cross sections $\left(\mathrm{cm}^{-1}\right)$} & \multicolumn{3}{c}{ NITAWL cross sections $\left(\mathrm{cm}^{-1}\right)$} \\
\hline & $\frac{\mathrm{G}_{\mathrm{a}}}{4.29059 \mathrm{E}-04}$ & $\frac{\varangle_{\mathrm{f}}}{5.17093 \mathrm{E}-04}$ & $\frac{\mathrm{G}_{\mathrm{f}}}{1.99589 \mathrm{E}-04}$ & $\frac{2.29390 \mathrm{E}-04}{\mathrm{G}}$ & $\frac{\mathrm{G}_{\mathrm{f}}}{5.17691 \mathrm{E}-04}$ & $\frac{\mathrm{G}_{\mathrm{f}}}{1.99788 \mathrm{E}-04}$ \\
1 & $5.45929 \mathrm{E}-03$ & $8.37664 \mathrm{E}-03$ & $3.43769 \mathrm{E}-03$ & $5.46541 \mathrm{E}-03$ & $8.36718 \mathrm{E}-03$ & $3.43382 \mathrm{E}-03$ \\
2 & $8.30534 \mathrm{E}-03$ & $1.69238 \mathrm{E}-02$ & $6.94538 \mathrm{E}-03$ & $8.28758 \mathrm{E}-03$ & $1.68623 \mathrm{E}-02$ & $6.92012 \mathrm{E}-03$ \\
3 & $7.30078 \mathrm{E}-02$ & $1.51919 \mathrm{E}-01$ & $6.23460 \mathrm{E}-02$ & $7.27680 \mathrm{E}-02$ & $1.51408 \mathrm{E}-01$ & $6.21367 \mathrm{E}-02$ \\
\hline
\end{tabular}

Table 2.59. PNL-35, Pin-1 four-group U-238 cross sections

\begin{tabular}{|c|c|c|c|c|c|c|}
\hline \multirow[t]{2}{*}{ Group } & \multicolumn{3}{|c|}{ CENTRM cross sections $\left(\mathrm{cm}^{-1}\right)$} & \multicolumn{3}{|c|}{ NITAWL cross sections $\left(\mathrm{cm}^{-1}\right)$} \\
\hline & G & $\varangle \mathrm{G}_{\mathrm{f}}$ & $\mathrm{G}_{\mathrm{f}}$ & G & $\ll \mathrm{G}_{\mathrm{f}}$ & $\mathrm{G}_{\mathrm{f}}$ \\
\hline 1 & $6.96200 \mathrm{E}-03$ & $\overline{1.25983 \mathrm{E}-02}$ & $\overline{4.46724 \mathrm{E}-03}$ & $6 . \overline{97774 \mathrm{E}-03}$ & $\overline{1.26269 \mathrm{E}-02}$ & $\overline{4.474} \overline{53 \mathrm{E}-03}$ \\
\hline 2 & $5.05345 \mathrm{E}-02$ & $5.59431 \mathrm{E}-06$ & $2.31769 \mathrm{E}-06$ & $4.85328 \mathrm{E}-02$ & $5.78528 \mathrm{E}-06$ & 2.39681E-06 \\
\hline 3 & $1.11140 \mathrm{E}-02$ & $3.88695 \mathrm{E}-08$ & $1.61043 \mathrm{E}-08$ & $1.10876 \mathrm{E}-02$ & 3.87287E-08 & $1.60460 \mathrm{E}-08$ \\
\hline 4 & 4.19366E-02 & $1.96955 \mathrm{E}-07$ & $8.16021 \mathrm{E}-08$ & 4.18109E-02 & $1.96348 \mathrm{E}-07$ & 8.13505E-08 \\
\hline
\end{tabular}

Table 2.60. PNL-35, Pin-1 four-group Pu-239 cross sections

\begin{tabular}{cccccccc}
\hline Group & \multicolumn{3}{c}{ CENTRM cross sections $\left(\mathrm{cm}^{-1}\right)$} & \multicolumn{3}{c}{ NITAWL cross sections $\left(\mathrm{cm}^{-1}\right)$} \\
\hline & $\frac{\mathrm{G}_{\mathrm{a}}}{}$ & $7.31004 \mathrm{E}-04$ & $\frac{\mathrm{G}_{\mathrm{f}}}{2.12002 \mathrm{E}-03}$ & $\frac{\mathrm{G}_{\mathrm{f}}}{6.83898 \mathrm{E}-04}$ & $\frac{\mathrm{G}_{\mathrm{a}}}{7.31003 \mathrm{E}-04}$ & $\frac{\mathrm{G}_{\mathrm{f}}}{2.11949 \mathrm{E}-03}$ & $\frac{\mathrm{G}_{\mathrm{f}}}{6.83599 \mathrm{E}-04}$ \\
2 & $1.57027 \mathrm{E}-02$ & $2.57978 \mathrm{E}-02$ & $8.95814 \mathrm{E}-03$ & $1.48272 \mathrm{E}-02$ & $2.46432 \mathrm{E}-02$ & $8.55722 \mathrm{E}-03$ \\
3 & $4.68010 \mathrm{E}-02$ & $9.21946 \mathrm{E}-02$ & $3.20143 \mathrm{E}-02$ & $4.68134 \mathrm{E}-02$ & $9.21519 \mathrm{E}-02$ & $3.19994 \mathrm{E}-02$ \\
4 & $3.84903 \mathrm{E}-01$ & $7.69989 \mathrm{E}-01$ & $2.66394 \mathrm{E}-01$ & $3.86636 \mathrm{E}-01$ & $7.72458 \mathrm{E}-01$ & $2.67253 \mathrm{E}-01$ \\
\hline
\end{tabular}


Table 2.61. PNL-35, Pin-99 four-group fluxes

\begin{tabular}{ccc}
\hline Group & $\begin{array}{c}\text { CENTRM M } \\
\left(\mathrm{cm}^{-2}-\mathrm{s}^{-1}\right)\end{array}$ & $\begin{array}{c}\text { NITAWL M } \\
\left(\mathrm{cm}^{-2}-\mathrm{s}^{-1}\right)\end{array}$ \\
\hline 1 & $8.383 \mathrm{E}-05$ & $8.501 \mathrm{E}-05$ \\
2 & $2.858 \mathrm{E}-05$ & $2.911 \mathrm{E}-05$ \\
3 & $6.805 \mathrm{E}-06$ & $7.098 \mathrm{E}-06$ \\
4 & $2.163 \mathrm{E}-05$ & $2.187 \mathrm{E}-05$ \\
\hline
\end{tabular}

Table 2.62. PNL-35, Pin-99 four-group U-235 cross sections

\begin{tabular}{cccccccc}
\hline Group & \multicolumn{3}{c}{ CENTRM cross sections $\left(\mathrm{cm}^{-1}\right)$} & \multicolumn{3}{c}{ NITAWL cross sections $\left(\mathrm{cm}^{-1}\right)$} \\
\hline & $\frac{\mathrm{G}_{\mathrm{a}}}{2.33860 \mathrm{E}-04}$ & $\frac{<\mathrm{G}_{\mathrm{f}}}{5.21028 \mathrm{E}-04}$ & $\frac{\mathrm{G}_{\mathrm{f}}}{2.02116 \mathrm{E}-04}$ & $\frac{\mathrm{G}_{\mathrm{a}}}{2.31754 \mathrm{E}-04}$ & $\frac{\mathrm{G}_{\mathrm{f}}}{5.19152 \mathrm{E}-04}$ & $\frac{\mathrm{G}_{\mathrm{f}}}{2.00942 \mathrm{E}-04}$ \\
1 & $5.36679 \mathrm{E}-03$ & $8.26977 \mathrm{E}-03$ & $3.39384 \mathrm{E}-03$ & $5.36675 \mathrm{E}-03$ & $8.27252 \mathrm{E}-03$ & $3.39496 \mathrm{E}-03$ \\
2 & $8.17348 \mathrm{E}-03$ & $1.67070 \mathrm{E}-02$ & $6.85640 \mathrm{E}-03$ & $8.15523 \mathrm{E}-03$ & $1.66066 \mathrm{E}-02$ & $6.81524 \mathrm{E}-03$ \\
3 & $7.08756 \mathrm{E}-02$ & $1.47418 \mathrm{E}-01$ & $6.04988 \mathrm{E}-02$ & $7.06159 \mathrm{E}-02$ & $1.46859 \mathrm{E}-01$ & $6.02693 \mathrm{E}-02$ \\
\hline
\end{tabular}

Table 2.63. PNL-35, Pin-99 four-group U-238 cross sections

\begin{tabular}{cccccccc}
\hline Group & \multicolumn{3}{c}{ CENTRM cross sections $\left(\mathrm{cm}^{-1}\right)$} & \multicolumn{3}{c}{ NITAWL cross sections $\left(\mathrm{cm}^{-1}\right)$} \\
\hline & $\frac{\mathrm{G}_{\mathrm{a}}}{4.85097 \mathrm{E}-03}$ & $\frac{\mathrm{G}_{\mathrm{f}}}{1.17780 \mathrm{E}-02}$ & $\frac{\mathrm{G}_{\mathrm{f}}}{4.19479 \mathrm{E}-03}$ & $\frac{6.87710 \mathrm{E}-03}{1.20714 \mathrm{E}-02}$ & $\frac{\mathrm{G}_{\mathrm{a}}}{4.28595 \mathrm{E}-03}$ \\
1 & $5.03681 \mathrm{E}-02$ & $5.57278 \mathrm{E}-06$ & $2.30878 \mathrm{E}-06$ & $4.60701 \mathrm{E}-02$ & $5.75479 \mathrm{E}-06$ & $2.38419 \mathrm{E}-06$ \\
2 & $1.10871 \mathrm{E}-02$ & $3.86935 \mathrm{E}-08$ & $1.60313 \mathrm{E}-08$ & $1.10723 \mathrm{E}-02$ & $3.85789 \mathrm{E}-08$ & $1.59839 \mathrm{E}-08$ \\
3 & $4.08300 \mathrm{E}-02$ & $1.91640 \mathrm{E}-07$ & $7.94004 \mathrm{E}-08$ & $4.06994 \mathrm{E}-02$ & $1.91015 \mathrm{E}-07$ & $7.91408 \mathrm{E}-08$ \\
\hline
\end{tabular}

Table 2.64. PNL-35, Pin-99 four-group Pu-239 cross sections

\begin{tabular}{cccccccc}
\hline Group & \multicolumn{3}{c}{ CENTRM cross sections $\left(\mathrm{cm}^{-1}\right)$} & \multicolumn{3}{c}{ NITAWL cross sections $\left(\mathrm{cm}^{-1}\right)$} \\
\hline & $\frac{\mathrm{G}_{\mathrm{a}}}{7.33474 \mathrm{E}-04}$ & $\frac{\mathrm{G}_{\mathrm{f}}}{2.10363 \mathrm{E}-03}$ & $\frac{\mathrm{G}_{\mathrm{f}}}{6.81678 \mathrm{E}-04}$ & $\overline{7.31953 \mathrm{E}-04}$ & $\frac{\mathrm{G}_{\mathrm{f}}}{2.10893 \mathrm{E}-03}$ & $\frac{\mathrm{G}_{\mathrm{f}}}{6.82076 \mathrm{E}-04}$ \\
1 & $1.48751 \mathrm{E}-02$ & $2.48097 \mathrm{E}-02$ & $8.61505 \mathrm{E}-03$ & $1.48705 \mathrm{E}-02$ & $2.44772 \mathrm{E}-02$ & $8.49959 \mathrm{E}-03$ \\
2 & $4.59681 \mathrm{E}-02$ & $9.06342 \mathrm{E}-02$ & $3.14723 \mathrm{E}-02$ & $4.52842 \mathrm{E}-02$ & $8.93873 \mathrm{E}-02$ & $3.10394 \mathrm{E}-02$ \\
3 & $3.95694 \mathrm{E}-01$ & $7.84138 \mathrm{E}-01$ & $2.71343 \mathrm{E}-01$ & $3.95679 \mathrm{E}-01$ & $7.83429 \mathrm{E}-01$ & $2.71097 \mathrm{E}-01$ \\
\hline
\end{tabular}





\section{MIX-COMP-THERM-3 (SAXTON-1 TO SAXTON-6)}

\subsection{DESCRIPTION}

This section describes a set of six critical experiments, each consisting of a square-pitched array of mixed plutonium-uranium fuel rods submerged in water surrounded by a water reflector. The fuel rods are identical in all cases. Criticality is controlled by varying the number of rods, rod pitch, boron in water, and water level. This set of experiments is contained in the International Handbook of Evaluated Criticality Safety Benchmark Experiments. ${ }^{3}$

This set of experiments was performed in 1965 at the in the Critical Reactor Experiment Facility at the Westinghouse Reactor Evaluation Center. The benchmark experiments are light-watermoderated critical assemblies consisting of a core array supported by upper, middle, and lower lattice plates. The lower and middle lattice plates are $0.635 \mathrm{~cm}$ thick. The upper lattice plate is $1.27 \mathrm{~cm}$ thick. The middle lattice plate was not included in the models to facilitate the accumulation of pinpower distributions. The reactor is brought to critical by raising the water level in the tank, thus avoiding the use of control rods. The fuel rods sit on a support plate above the bottom of the tank. The tank is wide enough to assume an infinite moderator on the sides and bottom $(\sim 30 \mathrm{~cm}$ of water).

All fuel rods have the same physical dimensions. A schematic diagram of the fuel rods and bottom reflector is given in Fig. 3.1. Each fuel rod has an active fuel length of $92.964 \mathrm{~cm}$, a 1.905$\mathrm{cm}$-long cladding plug on the bottom and a 4.320-cm-long cladding plug on the top. The fuel has a diameter of $0.856996 \mathrm{~cm}$. The cladding outside diameter is $0.99314 \mathrm{~cm}$ with a $0.059055-\mathrm{cm}$-thick wall. This leaves a $0.009017-\mathrm{cm}$ gap between the fuel and cladding.

The bottom of the fuel rod and lower lattice plate rest on a $2.54-\mathrm{cm}$ aluminum support plate. Between the bottom of the aluminum support plate and the top of a 5.08-cm aluminum slab is 6.35 $\mathrm{cm}$ of water. The space between the top of the lower lattice plate and bottom of the middle lattice plate is $46.99 \mathrm{~cm}$. The space between the top of the middle lattice plate and bottom of the top lattice plate is $47.625 \mathrm{~cm}$. To simplify the problem the middle lattice plate was removed and replaced with water. The water level varies with each problem but is always between the middle and upper lattice plates. The tank is large enough to assume an infinite water reflector on the sides and bottom. An infinite water reflector can be effectively modeled using $30 \mathrm{~cm}$ of water, which is used to model the reflector for this set of benchmarks.

The primary differences between the six benchmarks are lattice pitch, number of rods in the lattice, water level, and for problem 3, boron density in the water. The physical characteristics of each benchmark case, including moderator temperature, are given in Table 3.1. The atom densities for all the materials in the problem except B-10, B-11 H, and $\mathrm{O}$ in the moderator are contained in Table 3.2. The atom densities in Table 3.2 are constant for all benchmarks. Table 3.3 contain the atom densities of B-10, B-11, $\mathrm{H}$, and $\mathrm{O}$ in the moderator for each benchmark.

The lattice is filled from the $1 / 8$ th or $1 / 4$ th section by inserting additional pins in a mirror image. Figures 3.2 through 3.6 show the lattice map for each problem. Figure 3.2 shows a 1/4th lattice mapping while the other figures contain only $1 / 8$ th lattices. This difference exists because SAXTON1 contains a different number of pins in the $\mathrm{X}$ and $\mathrm{Y}$ directions. 
Table 3.1. Lattice description for SAXTON benchmark cases

\begin{tabular}{cccccc}
\hline $\begin{array}{c}\text { Benchmark } \\
\text { No. }\end{array}$ & $\begin{array}{c}\text { Boron con. } \\
(\mathrm{ppm})\end{array}$ & Lattice & $\begin{array}{c}\text { Lattice } \\
\text { pitch } \\
(\mathrm{cm})\end{array}$ & $\begin{array}{c}\text { Water level } \\
\text { from bottom } \\
\text { of fuel } \\
(\mathrm{cm})\end{array}$ & $\begin{array}{c}\text { Water } \\
\text { temp. } \\
\left({ }^{\circ} \mathrm{C}\right)\end{array}$ \\
\hline 1 & 0.0 & $22 \times 23$ & 1.3208 & 82.90 & 25.8 \\
2 & 0.0 & $19 \times 19$ & 1.4224 & 80.80 & 17.0 \\
3 & 337 & $21 \times 21$ & 1.4224 & 88.06 & 18.0 \\
4 & 0.0 & $13 \times 13$ & 1.86789 & 68.41 & 24.1 \\
5 & 0.0 & $12 \times 12$ & 2.01158 & 76.76 & 16.1 \\
6 & 0.0 & $11 \times 11$ & 2.6416 & 79.50 & 19.9 \\
\hline
\end{tabular}

Table 3.2. Constant benchmark atom densities

\begin{tabular}{|c|c|c|c|c|c|}
\hline Material & Isotope & $\begin{array}{c}\text { Atom density } \\
\text { (atoms/barn-cm) }\end{array}$ & Material & Isotope & $\begin{array}{c}\text { Atom density } \\
\text { (atoms/barn-cm) }\end{array}$ \\
\hline $\begin{array}{c}\text { Fuel } \\
\mathrm{UO}_{2}-\mathrm{PuO}_{2}\end{array}$ & $\begin{array}{c}\mathrm{U}-234 \\
\mathrm{U}-235 \\
\mathrm{U}-238 \\
\mathrm{Pu}-239 \\
\mathrm{Pu}-240 \\
\mathrm{Pu}-241 \\
\mathrm{Pu}-242 \\
\mathrm{Am}-241 \\
\mathrm{O}-16\end{array}$ & $\begin{array}{l}4.6590 \times 10^{-6} \\
1.5301 \times 10^{-4} \\
2.1097 \times 10^{-2} \\
1.3526 \times 10^{-3} \\
1.2759 \times 10^{-4} \\
1.1407 \times 10^{-5} \\
6.0318 \times 10^{-7} \\
1.7783 \times 10^{-6} \\
4.3779 \times 10^{-2}\end{array}$ & $\begin{array}{c}\text { Al lattice plate, } \\
\text { Al support } \\
\text { plate, Al slab } \\
(2.69 \mathrm{gm} / \mathrm{cc})\end{array}$ & $\begin{array}{c}\mathrm{Sn} \\
\mathrm{Fe} \\
\mathrm{Cr} \\
\mathrm{O} \\
\mathrm{Zr}\end{array}$ & $\begin{array}{l}4.6590 \times 10^{-4} \\
1.4148 \times 10^{-4} \\
7.5977 \times 10^{-5} \\
2.9630 \times 10^{-4} \\
4.2517 \times 10^{-2} \\
6.0039 \times 10^{-2}\end{array}$ \\
\hline
\end{tabular}




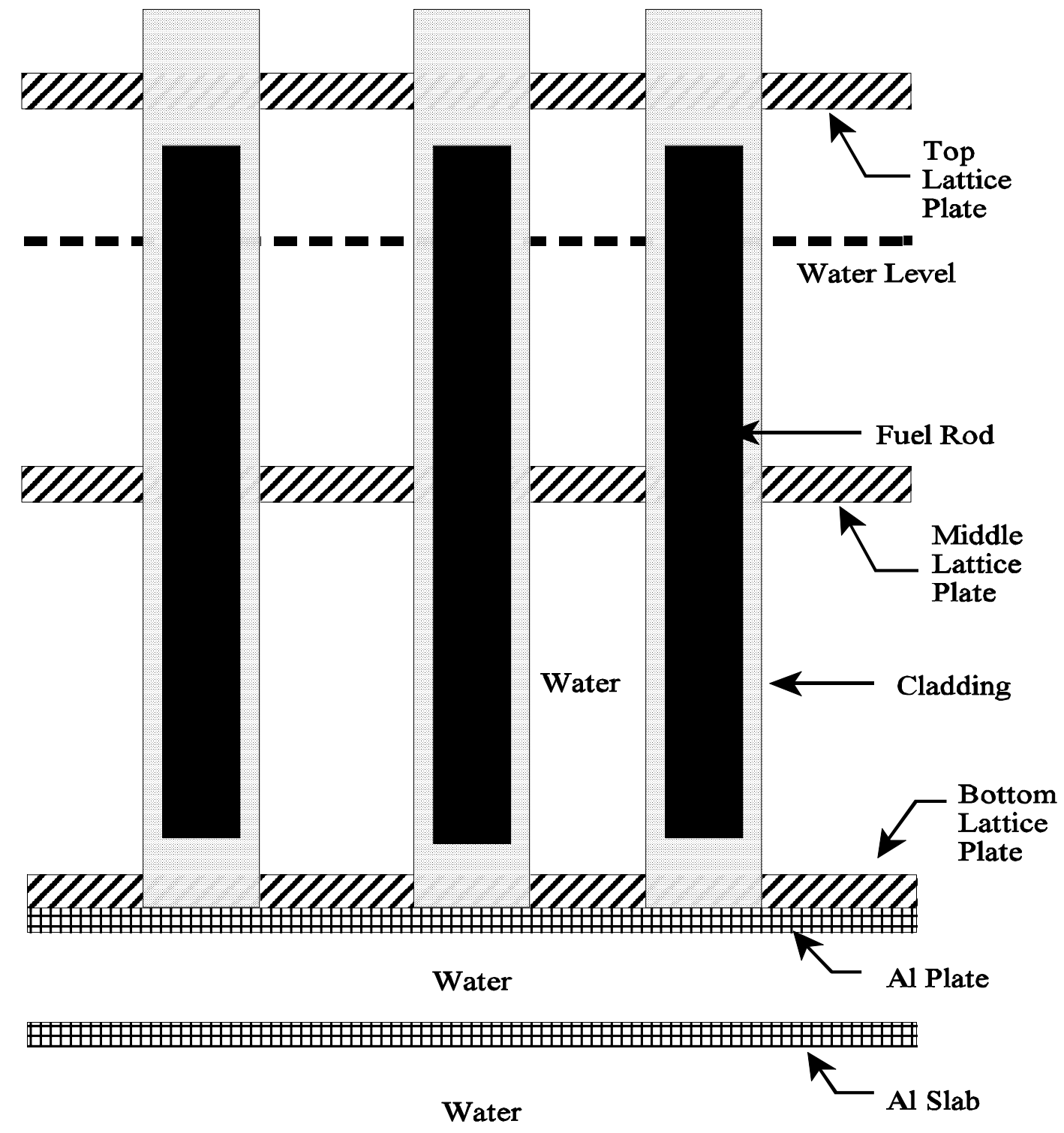

Fig. 3.1. Schematic of the SAXTON fuel rods with reflector. 
Table 3.3. Moderator atom densities

\begin{tabular}{ccccc}
\hline \multirow{2}{*}{$\begin{array}{c}\text { Benchmark } \\
\text { No. }\end{array}$} & $\mathrm{4}$ & $\mathrm{O}$ & $\mathrm{B}-10$ & $\mathrm{~B}-11$ \\
\cline { 2 - 5 } & $\mathrm{H}$ & 0.0 & 0.0 \\
\hline SAXTON-1 & $6.6643 \times 10^{-2}$ & $3.3322 \times 10^{-2}$ & 0.0 & 0.0 \\
SAXTON-2 & $6.6781 \times 10^{-2}$ & $3.3390 \times 10^{-2}$ & 0.070 \\
SAXTON-3 & $6.6751 \times 10^{-2}$ & $3.3404 \times 10^{-2}$ & $3.7338 \times 10^{-6}$ & $1.5029 \times 10^{-5}$ \\
SAXTON-4 & $6.6673 \times 10^{-2}$ & $3.3336 \times 10^{-2}$ & 0.0 & 0.0 \\
SAXTON-5 & $6.6783 \times 10^{-2}$ & $3.3392 \times 10^{-2}$ & 0.0 & 0.0 \\
SAXTON-6 & $6.6737 \times 10^{-2}$ & $3.3368 \times 10^{-2}$ & 0.0 & 0.0 \\
\hline
\end{tabular}

\subsection{ANALYSIS}

All six computational benchmarks in this section were processed twice using SCALE 5.0. The set labeled NITAWL uses the NITAWL resonance processor to self-shield the resolved resonance region for all nuclides in the unit cell. An identical set, labeled CENTRM, replaces NITAWL with the CENTRM/PMC code sequence. The NITAWL and CENTRM results are then compared.

Table 3.4 contains the $k_{\text {eff }}$ and Energy of the Average Lethargy Causing Fission (EALCF). The $k_{\text {eff }}$ values for all the benchmark cases are very close to 1.0, the worst NITAWL benchmark being $0.68 \%$ high and the worst CENTRM case being $0.59 \%$ high. There appears to be a small negative bias between NITAWL and CENTRM, the CENTRM cases being about $0.3 \%$ lower on average than the NITAWL cases. The EALCF values listed are from the CENTRM cases. For all cases, the difference between this value for CENTRM and NITAWL was less than 0.1\%. Both CENTRM and NITAWL produce excellent results for the $k_{\text {eff }}$ and EALCF for this set of problems.

Also, calculated for each problem are the pin-power distributions; absorption, $\mathcal{G}_{\mathrm{f}}$, and fission reaction rates and fluxes in the pin fuel, clad, and moderator; and four group cross sections and fluxes for a corner outside pin and the center pin. Each set of data is calculated using both CENTRM and NITAWL. All the data for each case are contained in the following Figs. 3.7a through 3.12b and Tables 2.5 through 3.64.

The pin-power distributions for the first problem assume 1/4th core symmetry, the pin-power distributions for the remaining problems assume 1/8th core symmetry, with a surrounding reflector in all problems. The pin-power data consist of a value and a standard deviation for each pin. The values for the pin power are actually in units of fissions per $\mathrm{cm}^{3}$-source particle $\times 10^{-5}$. The value in parentheses is the percent standard deviation of the pin-power value. The pin-power distributions in the 1/8th or full-core representations are the portion of the pin covered by water. The value off to the side in the pin-power distributions represents all the fuel not covered by water. Separating the fuel rod was necessary to model this in KENO-V.a.

In most cases the CENTRM and NITAWL results for each benchmark case agree within 2 standard deviations. The peak-to-low power changes with respect to pitch. The peak-to-low power ratio initially decreased with increasing pitch, starting with a high of 2.6 for SAXTON-1 and decreasing to a low of 1.8 for SAXTON-5. A further increase in pitch, as shown in SAXTON-6, 
increases the ratio to 2.5. The small pitch in SAXTON-1 and -2 cause the array to be under moderated. This under moderation results in the highest power pin being on the edge of the array and the lowest power pin being several pins in from the edge where the thermal flux is low due to lack of moderator and the fast flux is also low because it is not deep within the array.

The total fluxes, and flux ratios for the CENTRM and NITAWL cases of each benchmark case, are also in good agreement, seldom varying by more than $1 \%$. These values are included for a corner pin and the center pin for each case. The reaction rates in the fuel and cladding are also in good agreement. However, the reaction rates for the moderator appear to be significantly different, with the CENTRM value being almost a factor of 2 higher for all cases.

Finally, four-group fluxes and cross sections were calculated for selected nuclides in the fuel region of the same corner and center pins. The macroscopic cross sections listed include the radiative capture, fission, and nu*fission cross sections for U-235, U-238, and Pu-239. The four groups are collapsed from the 238-group multigroup cross-section set using the flux profile calculated in KENOV.a as follows: group 1 is from $20 \mathrm{Mev}$ to $9.5 \mathrm{keV}$, group 2 is from $9.5 \mathrm{keV}$ to $3.0 \mathrm{eV}$, group 3 is from $3 \mathrm{eV}$ to $0.4 \mathrm{eV}$, and group 4 is from $0.4 \mathrm{eV}$ to $10^{-5} \mathrm{eV}$. The $0.4 \mathrm{eV}$ was chosen as a boundary because it is the cadmium cutoff energy. Groups 2 and 3 contain the resolved resonance regions for most of the nuclides used in these cases. Most of the cross-section data for the CENTRM and NITAWL cases of a given benchmark case agree within $1 \%$.

\subsection{CONCLUSIONS}

For this set of benchmark cases using either NITAWL and CENTRM as the resonance region processor produces acceptable results of the $k_{\text {eff }}$ values. All other values produced using CENTRM and NITAWL are also consistent with each. For all cases, the $k_{\text {eff }} s$ produced using CENTRM are slightly lower $(\sim 0.3 \%)$ than those produced using NITAWL to do the resonance self-shielding. As a result the average $k_{\text {eff }}$ of all the CENTRM cases is closer to 1.0 than the average $k_{\text {eff }}$ of all the NITAWL cases for this set of benchmarks. The pin-power distributions, fluxes, most reactions rates, and macroscopic cross sections generally agree to approximately $1 \%$ between CENTRM and NITAWL. The only significant discrepancy is the absorption reaction rate for the moderator. CENTRM calculates a value that is about a factor of 2 higher than the same value using NITAWL. 
Table 3.4 Comparison of $k_{\text {eff }}$ and EALCF from CENTRM and NITAWL ${ }^{a}$

\begin{tabular}{ccccc}
\hline $\begin{array}{c}\text { CASE } \\
\text { MIX-COMP-THERM-3 }\end{array}$ & $\begin{array}{c}\text { NITAWL } \\
k_{\text {eff }}( \pm \mathrm{F})\end{array}$ & $\begin{array}{c}\text { CENTRM } \\
k_{\text {eff }}( \pm \mathrm{F})\end{array}$ & \% DIFF & $\begin{array}{c}\text { EALCF } \\
(\mathrm{eV})\end{array}$ \\
\hline SAXTON-1 & $1.0046(0.0005)$ & $1.0007(0.0004)$ & -0.39 & 0.881 \\
SAXTON-2 & $1.0058(0.0004)$ & $1.0012(0.0004)$ & -0.46 & 0.537 \\
SAXTON-3 & $1.0054(0.0004)$ & $1.0017(0.0004)$ & -0.37 & 0.633 \\
SAXTON-4 & $1.0056(0.0004)$ & $1.0029(0.0004)$ & -0.27 & 0.186 \\
SAXTON-5 & $1.0062(0.0004)$ & $1.0035(0.0004)$ & -0.27 & 0.154 \\
SAXTON-6 & $1.0068(0.0005)$ & $1.0059(0.0004)$ & -0.09 & 0.100 \\
\hline
\end{tabular}

${ }^{a}$ Value in parentheses is percent standard deviation. 


\begin{tabular}{|l|c|c|c|c|c|c|c|c|c|c|c|}
\hline 121 & 122 & 123 & 124 & 125 & 126 & 127 & 128 & 129 & 130 & 131 & 132 \\
\hline 109 & 110 & 111 & 112 & 113 & 114 & 115 & 116 & 117 & 118 & 119 & 120 \\
\hline 97 & 98 & 99 & 100 & 101 & 102 & 103 & 104 & 105 & 106 & 107 & 108 \\
\hline 85 & 86 & 87 & 88 & 89 & 90 & 91 & 92 & 93 & 94 & 95 & 96 \\
\hline 73 & 74 & 75 & 76 & 77 & 78 & 79 & 80 & 81 & 82 & 83 & 84 \\
\hline 61 & 62 & 63 & 64 & 65 & 66 & 67 & 68 & 69 & 70 & 71 & 72 \\
\hline 49 & 50 & 51 & 52 & 53 & 54 & 55 & 56 & 57 & 58 & 59 & 60 \\
\hline 37 & 38 & 39 & 40 & 41 & 42 & 43 & 44 & 45 & 46 & 47 & 48 \\
\hline 25 & 26 & 27 & 28 & 29 & 30 & 31 & 32 & 33 & 34 & 35 & 36 \\
\hline 13 & 14 & 15 & 16 & 17 & 18 & 19 & 20 & 21 & 22 & 23 & 24 \\
\hline 1 & 2 & 3 & 4 & 5 & 6 & 7 & 8 & 9 & 10 & 11 & 12 \\
\hline
\end{tabular}

Fig.3.2. Pin layout of benchmark case SAXTON-1. 


\begin{tabular}{|c|c|c|c|c|c|c|c|c|c|}
\hline & 55 \\
\hline & & & & & & & \multirow[b]{2}{*}{50} & 53 & 54 \\
\hline & & & & & & & & 51 & 52 \\
\hline & & & & & & 46 & 47 & 48 & 49 \\
\hline & & & & & 41 & 42 & 43 & 44 & 45 \\
\hline & & & & 35 & 36 & 37 & 38 & 39 & 40 \\
\hline & & & 28 & 29 & 30 & 31 & 32 & 33 & 34 \\
\hline & & 20 & 21 & 22 & 23 & 24 & 25 & 26 & 27 \\
\hline & 11 & 12 & 13 & 14 & 15 & 16 & 17 & 18 & 19 \\
\hline 1 & 2 & 3 & 4 & 5 & 6 & 7 & 8 & 9 & 10 \\
\hline
\end{tabular}

Fig. 3.3. Pin layout of benchmark case SAXTON-2.

\begin{tabular}{|c|c|c|c|c|c|c|c|c|c|c|}
\hline & & & & & & & & & & 66 \\
\hline & & & & & & & & & 64 & 65 \\
\hline & & & & & & & & 61 & 62 & 63 \\
\hline & & & & & & & 57 & 58 & 59 & 60 \\
\hline & & & & & & 52 & 53 & 54 & 55 & 56 \\
\hline & & & & & 46 & 47 & 48 & 49 & 50 & 51 \\
\hline & & & & 39 & 40 & 41 & 42 & 43 & 44 & 45 \\
\hline & & & 31 & 32 & 33 & 34 & 35 & 36 & 37 & 38 \\
\hline & & 22 & 23 & 24 & 25 & 26 & 27 & 28 & 29 & 30 \\
\hline & 12 & 13 & 14 & 15 & 16 & 17 & 18 & 19 & 20 & 21 \\
\hline 1 & 2 & 3 & 4 & 5 & 6 & 7 & 8 & 9 & 10 & 11 \\
\hline
\end{tabular}

Fig. 3.4. Pin layout of benchmark case SAXTON-3. 


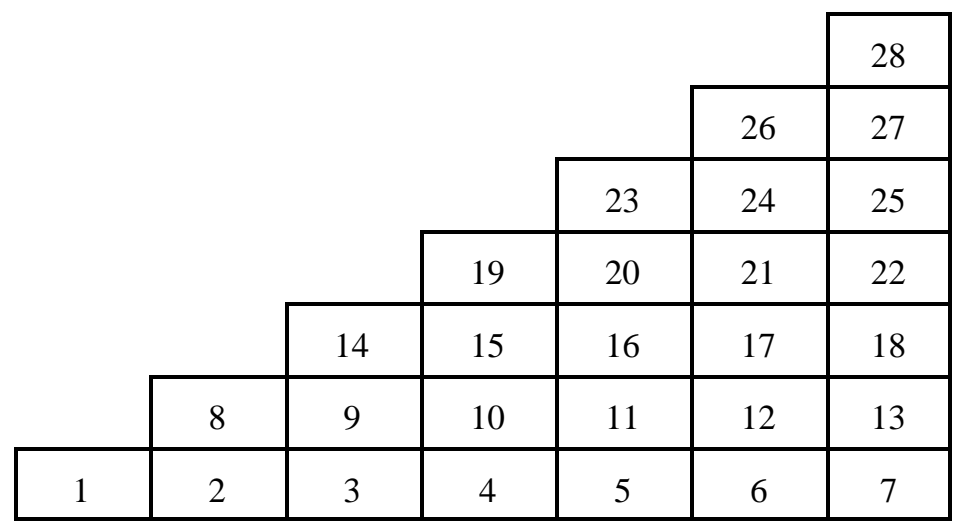

Fig. 3.5. Pin layout of benchmark case SAXTON-4.

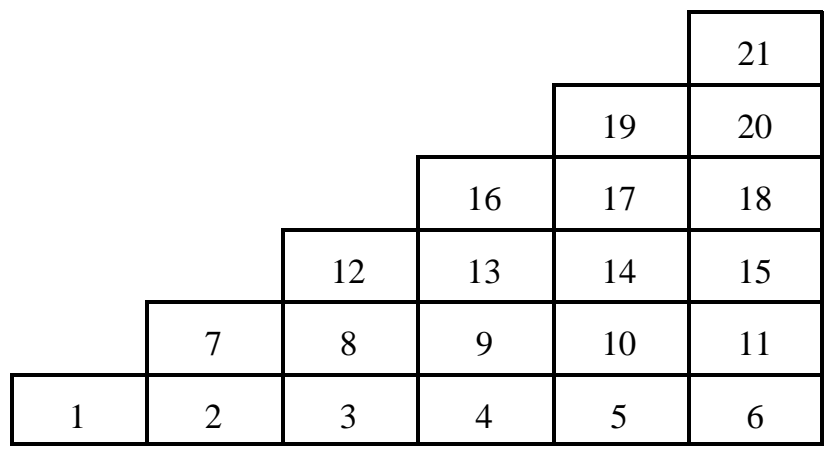

Fig. 3.6. Pin layout of benchmark cases SAXTON-5 and 6. 


\begin{tabular}{|c|c|c|c|c|c|c|c|c|c|c|c|}
\hline & & & & & & $\begin{array}{r}0.150 \\
(0.73)\end{array}$ & & & & & \\
\hline $\begin{array}{l}6.485 \\
(0.53)\end{array}$ & $\begin{array}{l}3.658 \\
(0.59)\end{array}$ & $\begin{array}{l}3.513 \\
(0.64)\end{array}$ & $\begin{array}{l}3.704 \\
(0.60)\end{array}$ & $\begin{array}{l}3.968 \\
(0.55)\end{array}$ & $\begin{array}{l}4.199 \\
(0.57)\end{array}$ & $\begin{array}{l}4.486 \\
(0.55)\end{array}$ & $\begin{array}{l}4.633 \\
(0.49)\end{array}$ & $\begin{array}{l}4.770 \\
(0.54)\end{array}$ & $\begin{array}{l}4.894 \\
(0.55)\end{array}$ & $\begin{array}{l}4.914 \\
(0.50)\end{array}$ & $\begin{array}{l}4.971 \\
(0.73)\end{array}$ \\
\hline $\begin{array}{l}6.446 \\
(0.50)\end{array}$ & $\begin{array}{l}3.716 \\
(0.58)\end{array}$ & $\begin{array}{l}3.461 \\
(0.61)\end{array}$ & $\begin{array}{c}3.693 \\
(0.61)\end{array}$ & $\begin{array}{l}3.957 \\
(0.59)\end{array}$ & $\begin{array}{l}4.222 \\
(0.55)\end{array}$ & $\begin{array}{l}4.421 \\
(0.57)\end{array}$ & $\begin{array}{l}4.570 \\
(0.57)\end{array}$ & $\begin{array}{l}4.687 \\
(0.51)\end{array}$ & $\begin{array}{l}4.871 \\
(0.51)\end{array}$ & $\begin{array}{l}4.858 \\
(0.53)\end{array}$ & $\begin{array}{l}4.962 \\
(0.66)\end{array}$ \\
\hline $\begin{array}{l}6.319 \\
(0.48)\end{array}$ & $\begin{array}{l}3.630 \\
(0.63)\end{array}$ & $\begin{array}{l}3.434 \\
(0.62)\end{array}$ & $\begin{array}{l}3.601 \\
(0.61)\end{array}$ & $\begin{array}{l}3.837 \\
(0.54)\end{array}$ & $\begin{array}{l}4.133 \\
(0.57)\end{array}$ & $\begin{array}{l}4.381 \\
(0.56)\end{array}$ & $\begin{array}{l}4.493 \\
(0.49)\end{array}$ & $\begin{array}{l}4.664 \\
(0.56)\end{array}$ & $\begin{array}{l}4.782 \\
(0.56)\end{array}$ & $\begin{array}{l}4.846 \\
(0.52)\end{array}$ & $\begin{array}{l}4.847 \\
(0.71)\end{array}$ \\
\hline $\begin{array}{l}6.148 \\
(0.50)\end{array}$ & $\begin{array}{l}3.531 \\
(0.62)\end{array}$ & $\begin{array}{l}3.368 \\
(0.59)\end{array}$ & $\begin{array}{l}3.524 \\
(0.66)\end{array}$ & $\begin{array}{l}3.729 \\
(0.56)\end{array}$ & $\begin{array}{l}3.986 \\
(0.58)\end{array}$ & $\begin{array}{l}4.175 \\
(0.52)\end{array}$ & $\begin{array}{l}4.397 \\
(0.55)\end{array}$ & $\begin{array}{l}4.503 \\
(0.50)\end{array}$ & $\begin{array}{l}4.649 \\
(0.51)\end{array}$ & $\begin{array}{l}4.680 \\
(0.52)\end{array}$ & $\begin{array}{l}4.705 \\
(0.75)\end{array}$ \\
\hline $\begin{array}{l}5.884 \\
(0.49)\end{array}$ & $\begin{array}{l}3.410 \\
(0.63)\end{array}$ & $\begin{array}{l}3.176 \\
(0.67)\end{array}$ & $\begin{array}{c}3.433 \\
(0.59)\end{array}$ & $\begin{array}{l}3.686 \\
(0.59)\end{array}$ & $\begin{array}{c}3.876 \\
(0.58)\end{array}$ & $\begin{array}{l}4.087 \\
(0.61)\end{array}$ & $\begin{array}{l}4.247 \\
(0.55)\end{array}$ & $\begin{array}{l}4.338 \\
(0.54)\end{array}$ & $\begin{array}{l}4.491 \\
(0.54)\end{array}$ & $\begin{array}{l}4.514 \\
(0.53)\end{array}$ & $\begin{array}{l}4.556 \\
(0.75)\end{array}$ \\
\hline $\begin{array}{l}5.635 \\
(0.50)\end{array}$ & $\begin{array}{l}3.234 \\
(0.67)\end{array}$ & $\begin{array}{l}3.058 \\
(0.64)\end{array}$ & $\begin{array}{l}3.231 \\
(0.66)\end{array}$ & $\begin{array}{l}3.496 \\
(0.60)\end{array}$ & $\begin{array}{l}3.668 \\
(0.54)\end{array}$ & $\begin{array}{l}3.879 \\
(0.60)\end{array}$ & $\begin{array}{l}4.029 \\
(0.57)\end{array}$ & $\begin{array}{l}4.171 \\
(0.52)\end{array}$ & $\begin{array}{l}4.254 \\
(0.57)\end{array}$ & $\begin{array}{l}4.283 \\
(0.51)\end{array}$ & $\begin{array}{l}4.344 \\
(0.75)\end{array}$ \\
\hline $\begin{array}{l}5.340 \\
(0.53)\end{array}$ & $\begin{array}{l}3.066 \\
(0.66)\end{array}$ & $\begin{array}{l}2.898 \\
(0.63)\end{array}$ & $\begin{array}{l}3.037 \\
(0.71)\end{array}$ & $\begin{array}{l}3.280 \\
(0.62)\end{array}$ & $\begin{array}{l}3.477 \\
(0.56)\end{array}$ & $\begin{array}{l}3.662 \\
(0.61)\end{array}$ & $\begin{array}{l}3.794 \\
(0.59)\end{array}$ & $\begin{array}{l}3.908 \\
(0.61)\end{array}$ & $\begin{array}{l}4.018 \\
(0.57)\end{array}$ & $\begin{array}{l}4.067 \\
(0.56)\end{array}$ & $\begin{array}{l}4.085 \\
(0.75)\end{array}$ \\
\hline $\begin{array}{l}4.988 \\
(0.55)\end{array}$ & $\begin{array}{l}2.822 \\
(0.70)\end{array}$ & $\begin{array}{l}2.693 \\
(0.72)\end{array}$ & $\begin{array}{l}2.864 \\
(0.66)\end{array}$ & $\begin{array}{l}3.054 \\
(0.70)\end{array}$ & $\begin{array}{l}3.264 \\
(0.61)\end{array}$ & $\begin{array}{l}3.412 \\
(0.61)\end{array}$ & $\begin{array}{l}3.552 \\
(0.61)\end{array}$ & $\begin{array}{l}3.652 \\
(0.59)\end{array}$ & $\begin{array}{l}3.747 \\
(0.59)\end{array}$ & $\begin{array}{l}3.772 \\
(0.58)\end{array}$ & $\begin{array}{l}3.830 \\
(0.84)\end{array}$ \\
\hline $\begin{array}{l}4.609 \\
(0.58)\end{array}$ & $\begin{array}{l}4.609 \\
(0.67)\end{array}$ & $\begin{array}{l}2.520 \\
(0.72)\end{array}$ & $\begin{array}{l}2.716 \\
(0.71)\end{array}$ & $\begin{array}{l}2.878 \\
(0.67)\end{array}$ & $\begin{array}{l}3.057 \\
(0.67)\end{array}$ & $\begin{array}{l}3.187 \\
(0.62)\end{array}$ & $\begin{array}{l}3.360 \\
(0.58)\end{array}$ & $\begin{array}{l}3.473 \\
(0.62)\end{array}$ & $\begin{array}{l}3.531 \\
(0.59)\end{array}$ & $\begin{array}{l}3.574 \\
(0.70)\end{array}$ & $\begin{array}{l}3.571 \\
(0.88)\end{array}$ \\
\hline $\begin{array}{l}4.611 \\
(0.62)\end{array}$ & $\begin{array}{l}2.773 \\
(0.75)\end{array}$ & $\begin{array}{l}2.701 \\
(0.67)\end{array}$ & $\begin{array}{l}2.827 \\
(0.68)\end{array}$ & $\begin{array}{l}3.079 \\
(0.65)\end{array}$ & $\begin{array}{l}3.255 \\
(0.69)\end{array}$ & $\begin{array}{l}3.427 \\
(0.66)\end{array}$ & $\begin{array}{l}3.557 \\
(0.63)\end{array}$ & $\begin{array}{l}3.717 \\
(0.60)\end{array}$ & $\begin{array}{l}3.757 \\
(0.54)\end{array}$ & $\begin{array}{c}3.81 \\
(0.62)\end{array}$ & $\begin{array}{l}3.815 \\
(0.88)\end{array}$ \\
\hline $\begin{array}{l}6.090 \\
(0.50)\end{array}$ & $\begin{array}{l}4.599 \\
(0.56)\end{array}$ & $\begin{array}{l}4.683 \\
(0.52)\end{array}$ & $\begin{array}{l}5.010 \\
(0.56)\end{array}$ & $\begin{array}{l}5.313 \\
(0.51)\end{array}$ & $\begin{array}{l}5.712 \\
(0.53)\end{array}$ & $\begin{array}{l}5.943 \\
(0.52)\end{array}$ & $\begin{array}{c}6.227 \\
(0.49)\end{array}$ & $\begin{array}{l}6.425 \\
(0.51)\end{array}$ & $\begin{array}{l}6.539 \\
(0.50)\end{array}$ & $\begin{array}{l}6.636 \\
(0.48)\end{array}$ & $\begin{array}{l}6.635 \\
(0.67)\end{array}$ \\
\hline
\end{tabular}

Fig. 3.7a. Pin-power distribution for CENTRM benchmark SAXTON-1. Value in parentheses is the percent standard deviation. 


\begin{tabular}{|c|c|c|c|c|c|c|c|c|c|c|c|}
\hline & & & & & & $\begin{array}{l}4.967 \\
(0.78)\end{array}$ & & & & & \\
\hline $\begin{array}{l}6.502 \\
(0.50)\end{array}$ & $\begin{array}{l}3.746 \\
(0.64)\end{array}$ & $\begin{array}{l}3.555 \\
(0.60)\end{array}$ & $\begin{array}{l}3.711 \\
(0.62)\end{array}$ & $\begin{array}{l}4.013 \\
(0.54)\end{array}$ & $\begin{array}{l}4.274 \\
(0.56)\end{array}$ & $\begin{array}{l}4.457 \\
(0.58)\end{array}$ & $\begin{array}{l}4.689 \\
(0.54)\end{array}$ & $\begin{array}{l}4.801 \\
(0.55)\end{array}$ & $\begin{array}{l}4.913 \\
(0.55)\end{array}$ & $\begin{array}{l}4.997 \\
(0.49)\end{array}$ & $\begin{array}{l}4.967 \\
(0.78)\end{array}$ \\
\hline $\begin{array}{l}6.441 \\
(0.49)\end{array}$ & $\begin{array}{c}3.706 \\
(0.59)\end{array}$ & $\begin{array}{c}3.493 \\
(0.67)\end{array}$ & $\begin{array}{l}3.699 \\
(0.65)\end{array}$ & $\begin{array}{c}3.939 \\
(0.63)\end{array}$ & $\begin{array}{l}4.244 \\
(0.56)\end{array}$ & $\begin{array}{l}4.422 \\
(0.53)\end{array}$ & $\begin{array}{l}4.641 \\
(0.55)\end{array}$ & $\begin{array}{l}4.780 \\
(0.55)\end{array}$ & $\begin{array}{l}4.840 \\
(0.51)\end{array}$ & $\begin{array}{l}4.917 \\
(0.49)\end{array}$ & $\begin{array}{l}4.912 \\
(0.74)\end{array}$ \\
\hline $\begin{array}{l}6.347 \\
(0.45)\end{array}$ & $\begin{array}{l}3.634 \\
(0.54)\end{array}$ & $\begin{array}{l}3.368 \\
(0.65)\end{array}$ & $\begin{array}{l}3.599 \\
(0.59)\end{array}$ & $\begin{array}{c}3.884 \\
(0.61)\end{array}$ & $\begin{array}{l}4.115 \\
(0.57)\end{array}$ & $\begin{array}{l}4.390 \\
(0.51)\end{array}$ & $\begin{array}{l}4.502 \\
(0.52)\end{array}$ & $\begin{array}{l}4.684 \\
(0.54)\end{array}$ & $\begin{array}{l}4.757 \\
(0.56)\end{array}$ & $\begin{array}{l}4.854 \\
(0.51)\end{array}$ & $\begin{array}{l}4.879 \\
(0.78)\end{array}$ \\
\hline $\begin{array}{l}6.197 \\
(0.52)\end{array}$ & $\begin{array}{l}3.522 \\
(0.63)\end{array}$ & $\begin{array}{l}3.356 \\
(0.64)\end{array}$ & $\begin{array}{l}3.356 \\
(0.59)\end{array}$ & $\begin{array}{l}3.836 \\
(0.60)\end{array}$ & $\begin{array}{l}4.052 \\
(0.56)\end{array}$ & $\begin{array}{l}4.279 \\
(0.57)\end{array}$ & $\begin{array}{l}4.446 \\
(0.54)\end{array}$ & $\begin{array}{l}4.547 \\
(0.54)\end{array}$ & $\begin{array}{l}4.614 \\
(0.55)\end{array}$ & $\begin{array}{l}4.711 \\
(0.49)\end{array}$ & $\begin{array}{l}4.737 \\
(0.76)\end{array}$ \\
\hline $\begin{array}{l}5.882 \\
(0.52)\end{array}$ & $\begin{array}{l}3.391 \\
(0.62)\end{array}$ & $\begin{array}{l}3.212 \\
(0.63)\end{array}$ & $\begin{array}{l}3.408 \\
(0.64)\end{array}$ & $\begin{array}{l}3.685 \\
(0.61)\end{array}$ & $\begin{array}{l}3.868 \\
(0.56)\end{array}$ & $\begin{array}{l}4.112 \\
(0.57)\end{array}$ & $\begin{array}{l}4.229 \\
(0.55)\end{array}$ & $\begin{array}{l}4.343 \\
(049 .)\end{array}$ & $\begin{array}{l}4.500 \\
(0.54)\end{array}$ & $\begin{array}{l}4.497 \\
(0.57)\end{array}$ & $\begin{array}{l}4.520 \\
(0.76)\end{array}$ \\
\hline $\begin{array}{l}5.619 \\
(0.54)\end{array}$ & $\begin{array}{c}3.243 \\
(0.70)\end{array}$ & $\begin{array}{l}3.060 \\
(0.66)\end{array}$ & $\begin{array}{l}3.260 \\
(0.61)\end{array}$ & $\begin{array}{l}3.477 \\
(0.64)\end{array}$ & $\begin{array}{l}3.708 \\
(0.59)\end{array}$ & $\begin{array}{c}3.880 \\
(0.57)\end{array}$ & $\begin{array}{l}4.050 \\
(0.60)\end{array}$ & $\begin{array}{l}4.189 \\
(0.60)\end{array}$ & $\begin{array}{l}4.265 \\
(0.55)\end{array}$ & $\begin{array}{l}4.321 \\
(0.58)\end{array}$ & $\begin{array}{l}4.305 \\
(0.71)\end{array}$ \\
\hline $\begin{array}{l}5.353 \\
(0.51)\end{array}$ & $\begin{array}{l}3.065 \\
(0.67)\end{array}$ & $\begin{array}{l}2.880 \\
(0.64)\end{array}$ & $\begin{array}{l}3.041 \\
(0.64)\end{array}$ & $\begin{array}{l}3.296 \\
(0.66)\end{array}$ & $\begin{array}{l}3.479 \\
(0.62)\end{array}$ & $\begin{array}{l}3.650 \\
(0.59)\end{array}$ & $\begin{array}{l}3.879 \\
(0.60)\end{array}$ & $\begin{array}{l}3.922 \\
(0.67)\end{array}$ & $\begin{array}{l}3.990 \\
(0.52)\end{array}$ & $\begin{array}{l}4.087 \\
(0.54)\end{array}$ & $\begin{array}{l}4.126 \\
(0.78)\end{array}$ \\
\hline $\begin{array}{l}4.982 \\
(0.54)\end{array}$ & $\begin{array}{l}2.844 \\
(0.73)\end{array}$ & $\begin{array}{l}2.673 \\
(0.69)\end{array}$ & $\begin{array}{l}2.857 \\
(0.73)\end{array}$ & $\begin{array}{l}3.063 \\
(0.66)\end{array}$ & $\begin{array}{l}3.264 \\
(0.62)\end{array}$ & $\begin{array}{l}3.422 \\
(0.68)\end{array}$ & $\begin{array}{l}3.577 \\
(0.62)\end{array}$ & $\begin{array}{l}3.698 \\
(0.59)\end{array}$ & $\begin{array}{l}3.787 \\
(0.62)\end{array}$ & $\begin{array}{l}3.847 \\
(0.59)\end{array}$ & $\begin{array}{l}3.823 \\
(0.83)\end{array}$ \\
\hline $\begin{array}{l}4.689 \\
(0.60)\end{array}$ & $\begin{array}{l}2.684 \\
(0.76)\end{array}$ & $\begin{array}{l}2.526 \\
(0.71)\end{array}$ & $\begin{array}{l}2.697 \\
(0.68)\end{array}$ & $\begin{array}{c}2.904 \\
(0.69)\end{array}$ & $\begin{array}{l}3.064 \\
(0.68)\end{array}$ & $\begin{array}{c}3.243 \\
(0.65)\end{array}$ & $\begin{array}{l}3.361 \\
(0.64)\end{array}$ & $\begin{array}{l}3.478 \\
(0.62)\end{array}$ & $\begin{array}{l}3.538 \\
(0.61)\end{array}$ & $\begin{array}{c}3.578 \\
(0.63)\end{array}$ & $\begin{array}{l}3.626 \\
(0.89)\end{array}$ \\
\hline $\begin{array}{l}4.621 \\
(0.58)\end{array}$ & $\begin{array}{l}2.801 \\
(0.72)\end{array}$ & $\begin{array}{l}2.677 \\
(0.75)\end{array}$ & $\begin{array}{l}2.839 \\
(0.78)\end{array}$ & $\begin{array}{l}3.079 \\
(0.61)\end{array}$ & $\begin{array}{l}3.258 \\
(0.64)\end{array}$ & $\begin{array}{l}3.417 \\
(0.65)\end{array}$ & $\begin{array}{l}3.558 \\
(0.61)\end{array}$ & $\begin{array}{l}3.735 \\
(0.53)\end{array}$ & $\begin{array}{l}3.801 \\
(0.60)\end{array}$ & $\begin{array}{l}3.882 \\
(0.63)\end{array}$ & $\begin{array}{l}3.872 \\
(0.86)\end{array}$ \\
\hline $\begin{array}{l}6.047 \\
(0.48)\end{array}$ & $\begin{array}{l}4.602 \\
(0.56)\end{array}$ & $\begin{array}{l}4.618 \\
(0.58)\end{array}$ & $\begin{array}{l}4.932 \\
(0.58)\end{array}$ & $\begin{array}{l}5.360 \\
(0.51)\end{array}$ & $\begin{array}{l}5.681 \\
(0.53)\end{array}$ & $\begin{array}{l}6.037 \\
(0.46)\end{array}$ & $\begin{array}{l}6.282 \\
(0.44)\end{array}$ & $\begin{array}{l}6.440 \\
(0.48)\end{array}$ & $\begin{array}{l}6.562 \\
(0.47)\end{array}$ & $\begin{array}{l}6.694 \\
(0.48)\end{array}$ & $\begin{array}{c}6.750 \\
(0.68)\end{array}$ \\
\hline
\end{tabular}

Fig. 3.7b. Pin-power distribution for NITAWL benchmark SAXTON-1. Value in parentheses is the percent standard deviation. 
Table 3.5. SAXTON-1 CENTRM reaction rates and fluxes

\begin{tabular}{|c|c|c|c|c|c|c|}
\hline Pin & Region & $\begin{array}{c}\mathrm{G}_{\mathrm{a}} \mathrm{M} \\
\left(\mathrm{cm}^{-3}-\mathrm{s}^{-1}\right)\end{array}$ & $\begin{array}{c}\mathcal{G}_{\mathrm{f}} \mathrm{M} \\
\left(\mathrm{cm}^{-3}-\mathrm{s}^{-1}\right)\end{array}$ & $\begin{array}{c}\mathrm{G}_{\mathrm{f}} \mathbf{M} \\
\left(\mathrm{cm}^{-3}-\mathrm{s}^{-1}\right)\end{array}$ & $\begin{array}{c}\mathrm{M} \\
\left(\mathrm{cm}-\mathrm{s}^{-1}\right)\end{array}$ & $\mathrm{M}_{\mathrm{t}}$ \\
\hline \multirow{3}{*}{1} & Fuel & $3.400 \mathrm{E}-05$ & $6.075 \mathrm{E}-05$ & $2.123 \mathrm{E}-05$ & $7.209 \mathrm{E}-03$ & 0.344 \\
\hline & Clad & $3.137 \mathrm{E}-07$ & 0.0 & 0.0 & $2.114 \mathrm{E}-03$ & 0.101 \\
\hline & Mod. & $1.303 \mathrm{E}-06$ & 0.0 & 0.0 & $1.162 \mathrm{E}-02$ & 0.555 \\
\hline \multirow{3}{*}{132} & Fuel & $3.446 \mathrm{E}-05$ & $5.001 \mathrm{E}-05$ & $1.747 \mathrm{E}-05$ & $1.905 \mathrm{E}-02$ & 0.339 \\
\hline & Clad & $5.304 \mathrm{E}-07$ & 0.0 & 0.0 & $5.690 \mathrm{E}-03$ & 0.101 \\
\hline & Mod. & $9.171 \mathrm{E}-07$ & 0.0 & 0.0 & $3.141 \mathrm{E}-02$ & 0.559 \\
\hline
\end{tabular}

Table 3.6. SAXTON-1 NITAWL reaction rates and fluxes

\begin{tabular}{ccccccc}
\hline \multirow{2}{*}{ Pin } & Region & $\begin{array}{c}\mathrm{G}_{\mathrm{a}} \mathrm{M} \\
\left(\mathrm{cm}^{-3}-\mathrm{s}^{-1}\right)\end{array}$ & $\begin{array}{c}\mathrm{G}_{\mathrm{f}} \mathrm{M} \\
\left(\mathrm{cm}^{-3}-\mathrm{s}^{-1}\right)\end{array}$ & $\begin{array}{c}\mathrm{G}_{\mathrm{f}} \mathrm{M} \\
\left(\mathrm{cm}^{-3}-\mathrm{s}^{-1}\right)\end{array}$ & $\begin{array}{c}\mathrm{M} \\
\left(\mathrm{cm}-\mathrm{s}^{-1}\right)\end{array}$ & $\mathrm{M}_{\mathrm{t}}$ \\
\hline \multirow{2}{*}{1} & Fuel & $3.407 \mathrm{E}-05$ & $6.097 \mathrm{E}-05$ & $2.131 \mathrm{E}-05$ & $7.160 \mathrm{E}-03$ & 0.344 \\
& Clad & $3.038 \mathrm{E}-07$ & 0.0 & 0.0 & $2.102 \mathrm{E}-03$ & 0.101 \\
& Mod. & $6.505 \mathrm{E}-07$ & 0.0 & 0.0 & $1.157 \mathrm{E}-02$ & 0.556 \\
\hline \multirow{2}{*}{132} & Fuel & $3.445 \mathrm{E}-05$ & $4.998 \mathrm{E}-05$ & $1.746 \mathrm{E}-05$ & $1.895 \mathrm{E}-02$ & 0.338 \\
& Clad & $5.131 \mathrm{E}-07$ & 0.0 & 0.0 & $5.682 \mathrm{E}-03$ & 0.101 \\
& Mod. & $4.648 \mathrm{E}-07$ & 0.0 & 0.0 & $3.143 \mathrm{E}-02$ & 0.561 \\
\hline
\end{tabular}


Table 3.7. SAXTON-1, Pin-1 four-group fluxes

\begin{tabular}{ccc}
\hline Group & $\begin{array}{c}\text { CENTRM M } \\
\left(\mathrm{cm}^{-2}-\mathrm{s}^{-1}\right)\end{array}$ & $\begin{array}{c}\text { NITAWL M } \\
\left(\mathrm{cm}^{-2}-\mathrm{s}^{-1}\right)\end{array}$ \\
\hline 1 & $9.920 \mathrm{E}-05$ & $9.850 \mathrm{E}-05$ \\
2 & $2.598 \mathrm{E}-05$ & $2.555 \mathrm{E}-05$ \\
3 & $5.448 \mathrm{E}-06$ & $5.416 \mathrm{E}-06$ \\
4 & $2.012 \mathrm{E}-05$ & $2.026 \mathrm{E}-05$ \\
\hline
\end{tabular}

Table 3.8. SAXTON-1, Pin-1 four-group U-235 cross sections

\begin{tabular}{ccccccc}
\hline Group & \multicolumn{3}{c}{ CENTRM cross sections $\left(\mathrm{cm}^{-1}\right)$} & \multicolumn{2}{c}{ NITAWL cross sections $\left(\mathrm{cm}^{-1}\right)$} \\
\hline & $\frac{\mathrm{G}_{\mathrm{a}}}{2.32297 \mathrm{E}-04}$ & $\frac{\mathrm{G}_{\mathrm{f}}}{5.28702 \mathrm{E}-04}$ & $\frac{\mathrm{G}_{\mathrm{f}}}{2.03588 \mathrm{E}-04}$ & $\frac{\mathrm{G}_{\mathrm{a}}}{2.31560 \mathrm{E}-04}$ & $\frac{\mathrm{G}_{\mathrm{f}}}{5.28129 \mathrm{E}-04}$ & $\frac{\mathrm{G}_{\mathrm{f}}}{2.03214 \mathrm{E}-04}$ \\
1 & $5.36940 \mathrm{E}-03$ & $8.29315 \mathrm{E}-03$ & $3.40344 \mathrm{E}-03$ & $5.43045 \mathrm{E}-03$ & $8.32423 \mathrm{E}-03$ & $3.41618 \mathrm{E}-03$ \\
2 & $7.84363 \mathrm{E}-03$ & $1.58948 \mathrm{E}-02$ & $6.52311 \mathrm{E}-03$ & $7.88612 \mathrm{E}-03$ & $1.60166 \mathrm{E}-02$ & $6.57304 \mathrm{E}-03$ \\
3 & $7.43305 \mathrm{E}-02$ & $1.54727 \mathrm{E}-01$ & $6.34985 \mathrm{E}-02$ & $7.41841 \mathrm{E}-02$ & $1.54426 \mathrm{E}-01$ & $6.33751 \mathrm{E}-02$ \\
\hline
\end{tabular}

Table 3.9. SAXTON-1, Pin-1 four-group U-238 cross sections

\begin{tabular}{cccccccc}
\hline Group & \multicolumn{3}{c}{ CENTRM cross sections $\left(\mathrm{cm}^{-1}\right)$} & \multicolumn{3}{c}{ NITAWL cross sections $\left(\mathrm{cm}^{-1}\right)$} \\
\hline & $\frac{\mathrm{G}_{\mathrm{a}}}{7.21435 \mathrm{E}-03}$ & $\frac{\mathrm{G}_{\mathrm{f}}}{1.34429 \mathrm{E}-02}$ & $\frac{\mathrm{G}_{\mathrm{f}}}{4.77316 \mathrm{E}-03}$ & $\frac{\mathrm{G}_{\mathrm{a}}}{7.22446 \mathrm{E}-03}$ & $\frac{\mathrm{G}_{\mathrm{f}}}{1.35539 \mathrm{E}-02}$ & $\frac{\mathrm{G}_{\mathrm{f}}}{4.80883 \mathrm{E}-03}$ \\
1 & $5.31239 \mathrm{E}-02$ & $5.82914 \mathrm{E}-06$ & $2.41498 \mathrm{E}-06$ & $5.09276 \mathrm{E}-02$ & $5.71143 \mathrm{E}-06$ & $2.36622 \mathrm{E}-06$ \\
2 & $1.12361 \mathrm{E}-02$ & $3.85156 \mathrm{E}-08$ & $1.59577 \mathrm{E}-08$ & $1.12440 \mathrm{E}-02$ & $3.86270 \mathrm{E}-08$ & $1.60039 \mathrm{E}-08$ \\
3 & $4.26642 \mathrm{E}-02$ & $2.00382 \mathrm{E}-07$ & $8.30224 \mathrm{E}-08$ & $4.25934 \mathrm{E}-02$ & $2.00045 \mathrm{E}-07$ & $8.28825 \mathrm{E}-08$ \\
\hline
\end{tabular}

Table 3.10. SAXTON-1, Pin-1 four-group Pu-239 cross sections

\begin{tabular}{ccccccc}
\hline Group & \multicolumn{2}{c}{ CENTRM cross sections $\left(\mathrm{cm}^{-1}\right)$} & \multicolumn{2}{c}{ NITAWL cross sections $\left(\mathrm{cm}^{-1}\right)$} \\
\hline & $\frac{\mathrm{G}_{\mathrm{a}}}{2.51521 \mathrm{E}-03}$ & $\frac{\mathrm{G}_{\mathrm{f}}}{7.34395 \mathrm{E}-03}$ & $\frac{\mathrm{G}_{\mathrm{f}}}{2.36412 \mathrm{E}-03}$ & $\frac{\mathrm{G}_{\mathrm{a}}}{2.51413 \mathrm{E}-03}$ & $\frac{\mathrm{G}_{\mathrm{f}}}{7.35233 \mathrm{E}-03}$ & $\frac{\mathrm{G}_{\mathrm{f}}}{2.36542 \mathrm{E}-03}$ \\
1 & $4.31528 \mathrm{E}-02$ & $7.26582 \mathrm{E}-02$ & $2.52301 \mathrm{E}-02$ & $4.23284 \mathrm{E}-02$ & $7.17029 \mathrm{E}-02$ & $2.48984 \mathrm{E}-02$ \\
2 & $1.42157 \mathrm{E}-01$ & $2.82164 \mathrm{E}-01$ & $9.79800 \mathrm{E}-02$ & $1.43048 \mathrm{E}-01$ & $2.83783 \mathrm{E}-01$ & $9.85430 \mathrm{E}-02$ \\
3 & $1.26177 \mathrm{E}+00$ & $2.54082 \mathrm{E}+00$ & $8.78966 \mathrm{E}-01$ & $1.25963 \mathrm{E}+00$ & $2.53651 \mathrm{E}+00$ & $8.77483 \mathrm{E}-01$ \\
\hline
\end{tabular}


Table 3.11. SAXTON-1, Pin-132 four-group fluxes

\begin{tabular}{ccc}
\hline Group & $\begin{array}{c}\text { CENTRM M } \\
\left(\mathrm{cm}^{-2}-\mathrm{s}^{-1}\right)\end{array}$ & $\begin{array}{c}\text { NITAWL M } \\
\left(\mathrm{cm}^{-2}-\mathrm{s}^{-1}\right)\end{array}$ \\
\hline 1 & $2.750 \mathrm{E}-04$ & $2.726 \mathrm{E}-04$ \\
2 & $9.570 \mathrm{E}-05$ & $9.640 \mathrm{E}-05$ \\
3 & $1.651 \mathrm{E}-05$ & $1.607 \mathrm{E}-05$ \\
4 & $1.110 \mathrm{E}-05$ & $1.112 \mathrm{E}-05$ \\
\hline
\end{tabular}

Table 3.12. SAXTON-1, Pin-132 four-group U-235 cross sections

\begin{tabular}{ccccccc}
\hline Group & \multicolumn{2}{c}{ CENTRM cross sections $\left(\mathrm{cm}^{-1}\right)$} & \multicolumn{3}{c}{ NITAWL cross sections $\left(\mathrm{cm}^{-1}\right)$} \\
\hline & $\frac{\mathrm{G}_{\mathrm{a}}}{2.45122 \mathrm{E}-04}$ & $\frac{\varangle_{\mathrm{f}}}{5.39109 \mathrm{E}-04}$ & $\frac{\mathrm{G}_{\mathrm{f}}}{2.10038 \mathrm{E}-04}$ & $\frac{\mathrm{G}_{\mathrm{a}}}{2.44993 \mathrm{E}-04}$ & $\frac{\mathrm{G}_{\mathrm{f}}}{5.38844 \mathrm{E}-04}$ & $\frac{\mathrm{G}_{\mathrm{f}}}{2.09938 \mathrm{E}-04}$ \\
1 & $5.15441 \mathrm{E}-03$ & $7.97880 \mathrm{E}-03$ & $3.27443 \mathrm{E}-03$ & $5.09111 \mathrm{E}-03$ & $7.85028 \mathrm{E}-03$ & $3.22168 \mathrm{E}-03$ \\
2 & $7.58571 \mathrm{E}-03$ & $1.52693 \mathrm{E}-02$ & $6.26638 \mathrm{E}-03$ & $7.63367 \mathrm{E}-03$ & $1.54170 \mathrm{E}-02$ & $6.32701 \mathrm{E}-03$ \\
3 & $6.21247 \mathrm{E}-02$ & $1.28918 \mathrm{E}-01$ & $5.29069 \mathrm{E}-02$ & $6.23526 \mathrm{E}-02$ & $1.29385 \mathrm{E}-01$ & $5.30985 \mathrm{E}-02$ \\
\hline
\end{tabular}

Table 3.13. SAXTON-1, Pin-132 four-group U-238 cross sections

\begin{tabular}{|c|c|c|c|c|c|c|}
\hline \multirow[t]{2}{*}{ Group } & \multicolumn{3}{|c|}{ CENTRM cross sections $\left(\mathrm{cm}^{-1}\right)$} & \multicolumn{3}{|c|}{ NITAWL cross sections $\left(\mathrm{cm}^{-1}\right)$} \\
\hline & $\mathrm{G}_{\mathrm{a}}$ & $<\mathrm{G}_{\mathrm{f}}$ & $\mathrm{G}_{\mathrm{f}}$ & $\mathrm{G}_{\mathrm{a}}$ & $\mathrm{G}_{\mathrm{f}}$ & $\mathrm{G}_{\mathrm{f}}$ \\
\hline 1 & $6.80382 \mathrm{E}-03$ & $1.10027 \mathrm{E}-02$ & $3.91622 \mathrm{E}-03$ & $6 . \overline{78188 \mathrm{E}-03}$ & $1.09576 \mathrm{E}-02$ & $3.89839 \mathrm{E}-03$ \\
\hline 2 & 4.89435E-02 & $6.26429 \mathrm{E}-06$ & $2.59527 \mathrm{E}-06$ & 4.75272E-02 & $6.04762 \mathrm{E}-06$ & $2.50550 \mathrm{E}-06$ \\
\hline 3 & $1.11343 \mathrm{E}-02$ & 3.75412E-08 & $1.55540 \mathrm{E}-08$ & 1.11699E-02 & $3.77752 \mathrm{E}-08$ & 1.56510E-08 \\
\hline 4 & $3.63750 \mathrm{E}-02$ & $1.70185 \mathrm{E}-07$ & $7.05107 \mathrm{E}-08$ & $3.64831 \mathrm{E}-02$ & $1.70704 \mathrm{E}-07$ & $7.07262 \mathrm{E}-08$ \\
\hline
\end{tabular}

Table 3.14. SAXTON-1, Pin-132 four-group Pu-239 cross sections

\begin{tabular}{cccccccc}
\hline Group & \multicolumn{3}{c}{ CENTRM cross sections $\left(\mathrm{cm}^{-1}\right)$} & \multicolumn{3}{c}{ NITAWL cross sections $\left(\mathrm{cm}^{-1}\right)$} \\
\hline & $\frac{\mathrm{G}_{\mathrm{a}}}{2.52991 \mathrm{E}-03}$ & $\frac{\mathrm{G}_{\mathrm{f}}}{7.17411 \mathrm{E}-03}$ & $\frac{\mathrm{G}_{\mathrm{f}}}{2.33467 \mathrm{E}-03}$ & $\frac{\mathrm{G}_{\mathrm{a}}}{2.52913 \mathrm{E}-03}$ & $\frac{\mathrm{G}_{\mathrm{f}}}{7.17146 \mathrm{E}-03}$ & $\frac{\mathrm{G}_{\mathrm{f}}}{2.33393 \mathrm{E}-03}$ \\
1 & $4.10734 \mathrm{E}-02$ & $6.89915 \mathrm{E}-02$ & $2.39569 \mathrm{E}-02$ & $3.95781 \mathrm{E}-02$ & $6.68722 \mathrm{E}-02$ & $2.32210 \mathrm{E}-02$ \\
2 & $1.30973 \mathrm{E}-01$ & $2.61059 \mathrm{E}-01$ & $9.06517 \mathrm{E}-02$ & $1.35790 \mathrm{E}-01$ & $2.70087 \mathrm{E}-01$ & $9.37866 \mathrm{E}-02$ \\
3 & $1.45288 \mathrm{E}+00$ & $2.78304 \mathrm{E}+00$ & $9.63703 \mathrm{E}-01$ & $1.45769 \mathrm{E}+00$ & $2.79201 \mathrm{E}+00$ & $9.66808 \mathrm{E}-01$ \\
\hline
\end{tabular}




\begin{tabular}{|c|c|c|c|c|c|c|c|c|c|}
\hline & & & & & & & & & $\begin{array}{l}7.032 \\
(0.91)\end{array}$ \\
\hline & $\begin{array}{l}0.171 \\
(0.75)\end{array}$ & & & & & & & $\begin{array}{l}7.088 \\
(0.42)\end{array}$ & $\begin{array}{l}7.085 \\
(0.46)\end{array}$ \\
\hline & & & & & & & $\begin{array}{l}6.809 \\
(0.46)\end{array}$ & $\begin{array}{l}6.904 \\
(0.32)\end{array}$ & $\begin{array}{l}6.960 \\
(0.49)\end{array}$ \\
\hline & & & & & & $\begin{array}{l}6.408 \\
(0.49)\end{array}$ & $\begin{array}{l}6.587 \\
(0.33)\end{array}$ & $\begin{array}{l}6.701 \\
(0.33)\end{array}$ & $\begin{array}{l}6.721 \\
(0.48)\end{array}$ \\
\hline & & & & & $\begin{array}{l}5.874 \\
(0.49)\end{array}$ & $\begin{array}{l}6.137 \\
(0.35)\end{array}$ & $\begin{array}{l}6.268 \\
(0.37)\end{array}$ & $\begin{array}{l}6.459 \\
(0.35)\end{array}$ & $\begin{array}{l}6.431 \\
(0.48)\end{array}$ \\
\hline & & & & $\begin{array}{l}5.267 \\
(0.53)\end{array}$ & $\begin{array}{l}5.498 \\
(0.34)\end{array}$ & $\begin{array}{l}5.807 \\
(0.36)\end{array}$ & $\begin{array}{l}5.922 \\
(0.34)\end{array}$ & $\begin{array}{c}6.082 \\
(0.36)\end{array}$ & $\begin{array}{r}6.127 \\
(0.49)\end{array}$ \\
\hline & & & $\begin{array}{l}4.501 \\
(0.61)\end{array}$ & $\begin{array}{l}4.877 \\
(0.40)\end{array}$ & $\begin{array}{l}5.169 \\
(0.39)\end{array}$ & $\begin{array}{l}5.362 \\
(0.37)\end{array}$ & $\begin{array}{l}5.580 \\
(0.37)\end{array}$ & $\begin{array}{l}5.629 \\
(0.38)\end{array}$ & $\begin{array}{l}5.695 \\
(0.52)\end{array}$ \\
\hline & & $\begin{array}{l}3.937 \\
(0.61)\end{array}$ & $\begin{array}{l}4.239 \\
(0.41)\end{array}$ & $\begin{array}{l}4.533 \\
(0.40)\end{array}$ & $\begin{array}{l}4.826 \\
(0.42)\end{array}$ & $\begin{array}{l}5.017 \\
(0.39)\end{array}$ & $\begin{array}{l}5.191 \\
(0.39)\end{array}$ & $\begin{array}{l}5.256 \\
(0.37)\end{array}$ & $\begin{array}{l}5.308 \\
(0.56)\end{array}$ \\
\hline & $\begin{array}{l}4.056 \\
(0.65)\end{array}$ & $\begin{array}{l}4.036 \\
(0.42)\end{array}$ & $\begin{array}{l}4.308 \\
(0.40)\end{array}$ & $\begin{array}{l}4.628 \\
(0.40)\end{array}$ & $\begin{array}{l}4.898 \\
(0.37)\end{array}$ & $\begin{array}{l}5.101 \\
(0.39)\end{array}$ & $\begin{array}{l}5.261 \\
(0.38)\end{array}$ & $\begin{array}{l}5.374 \\
(0.35)\end{array}$ & $\begin{array}{l}5.422 \\
(0.51)\end{array}$ \\
\hline $\begin{array}{l}7.779 \\
(0.47)\end{array}$ & $\begin{array}{c}6.169 \\
(0.37)\end{array}$ & $\begin{array}{l}6.394 \\
(0.34)\end{array}$ & $\begin{array}{l}6.876 \\
(0.36)\end{array}$ & $\begin{array}{c}7.378 \\
(0.34)\end{array}$ & $\begin{array}{c}7.815 \\
(0.30)\end{array}$ & $\begin{array}{l}8.167 \\
(0.33)\end{array}$ & $\begin{array}{l}8.417 \\
(0.30)\end{array}$ & $\begin{array}{l}8.574 \\
(0.31)\end{array}$ & $\begin{array}{l}8.669 \\
(0.41)\end{array}$ \\
\hline
\end{tabular}

Fig. 3.8a. Pin-power distribution for CENTRM benchmark SAXTON-2. Value in parentheses is percent standard deviation. 


\begin{tabular}{|c|c|c|c|c|c|c|c|c|c|}
\hline \multirow[t]{9}{*}{$\begin{array}{c}0.172 \\
(0.72)\end{array}$} & & & & & & & & & $\begin{array}{c}7.111 \\
(0.85)\end{array}$ \\
\hline & & & & & & & & $\begin{array}{l}7.073 \\
(0.45)\end{array}$ & $\begin{array}{l}7.107 \\
(0.46)\end{array}$ \\
\hline & & & & & & & $\begin{array}{l}6.803 \\
(0.46)\end{array}$ & $\begin{array}{l}6.929 \\
(0.32)\end{array}$ & $\begin{array}{c}7.015 \\
(0.47)\end{array}$ \\
\hline & & & & & & $\begin{array}{l}6.476 \\
(0.49)\end{array}$ & $\begin{array}{l}6.655 \\
(0.35)\end{array}$ & $\begin{array}{l}6.786 \\
(0.34)\end{array}$ & $\begin{array}{l}6.773 \\
(0.44)\end{array}$ \\
\hline & & & & & $\begin{array}{l}5.890 \\
(0.52)\end{array}$ & $\begin{array}{l}6.194 \\
(0.36)\end{array}$ & $\begin{array}{l}6.362 \\
(0.34)\end{array}$ & $\begin{array}{l}6.449 \\
(0.34)\end{array}$ & $\begin{array}{l}6.504 \\
(0.46)\end{array}$ \\
\hline & & & & $\begin{array}{l}5.255 \\
(0.53)\end{array}$ & $\begin{array}{l}5.527 \\
(0.39)\end{array}$ & $\begin{array}{l}5.826 \\
(0.36)\end{array}$ & $\begin{array}{l}5.985 \\
(0.35)\end{array}$ & $\begin{array}{l}6.159 \\
(0.37)\end{array}$ & $\begin{array}{c}6.139 \\
(0.50)\end{array}$ \\
\hline & & & $\begin{array}{l}4.556 \\
(0.57)\end{array}$ & $\begin{array}{l}4.871 \\
(0.39)\end{array}$ & $\begin{array}{l}5.198 \\
(0.36)\end{array}$ & $\begin{array}{l}5.449 \\
(0.38)\end{array}$ & $\begin{array}{l}5.562 \\
(0.37)\end{array}$ & $\begin{array}{l}5.690 \\
(0.38)\end{array}$ & $\begin{array}{l}5.706 \\
(0.48)\end{array}$ \\
\hline & & $\begin{array}{c}3.988 \\
(0.57)\end{array}$ & $\begin{array}{l}4.229 \\
(0.40)\end{array}$ & $\begin{array}{l}4.534 \\
(0.40)\end{array}$ & $\begin{array}{l}4.839 \\
(040 .)\end{array}$ & $\begin{array}{l}5.022 \\
(0.42)\end{array}$ & $\begin{array}{l}5.209 \\
(0.39)\end{array}$ & $\begin{array}{l}5.298 \\
(0.39)\end{array}$ & $\begin{array}{l}5.338 \\
(054 .)\end{array}$ \\
\hline & $\begin{array}{l}4.073 \\
(0.62)\end{array}$ & $\begin{array}{l}3.989 \\
(0.46)\end{array}$ & $\begin{array}{l}4.314 \\
(0.38)\end{array}$ & $\begin{array}{l}4.651 \\
(0.40)\end{array}$ & $\begin{array}{l}4.909 \\
(0.36)\end{array}$ & $\begin{array}{l}5.172 \\
(0.39)\end{array}$ & $\begin{array}{l}5.260 \\
(040 .)\end{array}$ & $\begin{array}{l}5.401 \\
(0.36)\end{array}$ & $\begin{array}{l}5.401 \\
(048 .)\end{array}$ \\
\hline $\begin{array}{l}7.878 \\
(0.40)\end{array}$ & $\begin{array}{c}6.193 \\
(0.34)\end{array}$ & $\begin{array}{l}6.355 \\
(0.33)\end{array}$ & $\begin{array}{l}6.870 \\
(0.34)\end{array}$ & $\begin{array}{c}7.353 \\
(0.32)\end{array}$ & $\begin{array}{l}7.839 \\
(0.32)\end{array}$ & $\begin{array}{l}8.201 \\
(0.30)\end{array}$ & $\begin{array}{l}8.457 \\
(0.29)\end{array}$ & $\begin{array}{l}8.657 \\
(0.29)\end{array}$ & $\begin{array}{l}8.684 \\
(0.44)\end{array}$ \\
\hline
\end{tabular}

Fig. 3.8b. Pin-power distribution for NITAWL benchmark SAXTON-2. Value in parentheses is percent standard deviation. 
Table 3.15. SAXTON-2 CENTRM reaction rates and fluxes

\begin{tabular}{|c|c|c|c|c|c|c|}
\hline Pin & Region & $\begin{array}{c}\mathrm{G}_{\mathrm{a}} \mathrm{M} \\
\left(\mathrm{cm}^{-3}-\mathrm{s}^{-1}\right)\end{array}$ & $\begin{array}{c}\mathcal{G}_{\mathrm{f}} \mathrm{M} \\
\left(\mathrm{cm}^{-3}-\mathrm{s}^{-1}\right)\end{array}$ & $\begin{array}{c}\mathrm{G}_{\mathrm{f}} \mathrm{M} \\
\left(\mathrm{cm}^{-3}-\mathrm{s}^{-1}\right)\end{array}$ & $\begin{array}{c}\mathrm{M} \\
\left(\mathrm{cm}-\mathrm{s}^{-1}\right)\end{array}$ & $\mathrm{MM}_{\mathrm{t}}$ \\
\hline \multirow{3}{*}{1} & Fuel & $4.361 \mathrm{E}-05$ & $7.811 \mathrm{E}-05$ & $2.729 \mathrm{E}-05$ & $8.590 \mathrm{E}-03$ & 0.296 \\
\hline & Clad & $3.882 \mathrm{E}-07$ & 0.0 & 0.0 & $2.531 \mathrm{E}-03$ & 0.087 \\
\hline & Mod. & $1.731 \mathrm{E}-06$ & 0.0 & 0.0 & $1.793 \mathrm{E}-02$ & 0.617 \\
\hline \multirow{3}{*}{55} & Fuel & $4.675 \mathrm{E}-05$ & $7.033 \mathrm{E}-05$ & $2.457 \mathrm{E}-05$ & $2.148 \mathrm{E}-02$ & 0.293 \\
\hline & Clad & $6.310 \mathrm{E}-07$ & 0.0 & 0.0 & $6.403 \mathrm{E}-03$ & 0.087 \\
\hline & Mod. & $1.351 \mathrm{E}-06$ & 0.0 & 0.0 & 4.552E-02 & 0.620 \\
\hline
\end{tabular}

Table 3.16. SAXTON-2 NITAWL reaction rates and fluxes

\begin{tabular}{ccccccc}
\hline \multirow{2}{*}{ Pin } & Region & $\begin{array}{c}\mathrm{G}_{\mathrm{a}} \mathrm{M} \\
\left(\mathrm{cm}^{-3}-\mathrm{s}^{-1}\right)\end{array}$ & $\begin{array}{c}\mathrm{G}_{\mathrm{f}} \mathrm{M} \\
\left(\mathrm{cm}^{-3}-\mathrm{s}^{-1}\right)\end{array}$ & $\begin{array}{c}\mathrm{G}_{\mathrm{f}} \mathrm{M} \\
\left(\mathrm{cm}^{-3}-\mathrm{s}^{-1}\right)\end{array}$ & $\begin{array}{c}\mathrm{M} \\
\left(\mathrm{cm}^{-1}\right)\end{array}$ & $\mathrm{M}_{\mathrm{t}}$ \\
\hline \multirow{2}{*}{1} & Fuel & $4.393 \mathrm{E}-05$ & $7.895 \mathrm{E}-05$ & $2.759 \mathrm{E}-05$ & $8.596 \mathrm{E}-03$ & 0.296 \\
& Clad & $3.874 \mathrm{E}-07$ & 0.0 & 0.0 & $2.523 \mathrm{E}-03$ & 0.087 \\
& Mod. & $8.732 \mathrm{E}-07$ & 0.0 & 0.0 & $1.790 \mathrm{E}-02$ & 0.617 \\
\hline \multirow{2}{*}{55} & Fuel & $4.723 \mathrm{E}-05$ & $7.130 \mathrm{E}-05$ & $2.491 \mathrm{E}-05$ & $2.145 \mathrm{E}-02$ & 0.292 \\
& Clad & $6.079 \mathrm{E}-07$ & 0.0 & 0.0 & $6.411 \mathrm{E}-03$ & 0.087 \\
& Mod. & $6.803 \mathrm{E}-07$ & 0.0 & 0.0 & $4.565 \mathrm{E}-02$ & 0.621 \\
\hline
\end{tabular}


Table 3.17. SAXTON-2, Pin-1 four-group fluxes

\begin{tabular}{ccc}
\hline Group & $\begin{array}{c}\text { CENTRM M } \\
\left(\mathrm{cm}^{-2}-\mathrm{s}^{-1}\right)\end{array}$ & $\begin{array}{c}\text { NITAWL M } \\
\left(\mathrm{cm}^{-2}-\mathrm{s}^{-1}\right)\end{array}$ \\
\hline 1 & $1.210 \mathrm{E}-04$ & $1.209 \mathrm{E}-04$ \\
2 & $3.068 \mathrm{E}-05$ & $3.075 \mathrm{E}-05$ \\
3 & $6.650 \mathrm{E}-06$ & $6.426 \mathrm{E}-06$ \\
4 & $2.598 \mathrm{E}-05$ & $2.635 \mathrm{E}-05$ \\
\hline
\end{tabular}

Table 3.18. SAXTON-2, Pin-1 four-group U-235 cross sections

\begin{tabular}{cccccccc}
\hline Group & \multicolumn{3}{c}{ CENTRM cross sections $\left(\mathrm{cm}^{-1}\right)$} & \multicolumn{3}{c}{ NITAWL cross sections $\left(\mathrm{cm}^{-1}\right)$} \\
\hline & $\frac{\mathrm{G}_{\mathrm{a}}}{2.31295 \mathrm{E}-04}$ & $\frac{\mathrm{G}_{\mathrm{f}}}{5.28168 \mathrm{E}-04}$ & $\frac{\mathrm{G}_{\mathrm{f}}}{2.03137 \mathrm{E}-04}$ & $\frac{\mathrm{G}_{\mathrm{a}}}{2.31046 \mathrm{E}-04}$ & $\frac{\mathrm{G}_{\mathrm{f}}}{5.27611 \mathrm{E}-04}$ & $\frac{\mathrm{G}_{\mathrm{f}}}{2.02942 \mathrm{E}-04}$ \\
1 & $5.50818 \mathrm{E}-03$ & $8.46995 \mathrm{E}-03$ & $3.47598 \mathrm{E}-03$ & $5.34062 \mathrm{E}-03$ & $8.20683 \mathrm{E}-03$ & $3.36800 \mathrm{E}-03$ \\
2 & $7.88307 \mathrm{E}-03$ & $1.60481 \mathrm{E}-02$ & $6.58600 \mathrm{E}-03$ & $7.88335 \mathrm{E}-03$ & $1.59830 \mathrm{E}-02$ & $6.55932 \mathrm{E}-03$ \\
3 & $7.48945 \mathrm{E}-02$ & $1.55911 \mathrm{E}-01$ & $6.39846 \mathrm{E}-02$ & $7.47985 \mathrm{E}-02$ & $1.55708 \mathrm{E}-01$ & $6.39009 \mathrm{E}-02$ \\
\hline
\end{tabular}

Table 3.19. SAXTON-2, Pin-1 four-group U-238 cross sections

\begin{tabular}{cccccccc}
\hline Group & \multicolumn{3}{c}{ CENTRM cross sections $\left(\mathrm{cm}^{-1}\right)$} & \multicolumn{3}{c}{ NITAWL cross sections $\left(\mathrm{cm}^{-1}\right)$} \\
\hline & $\frac{\mathrm{G}_{\mathrm{a}}}{7.27299 \mathrm{E}-03}$ & $\frac{\mathrm{G}_{\mathrm{f}}}{1.37200 \mathrm{E}-02}$ & $\frac{\mathrm{G}_{\mathrm{f}}}{4.87017 \mathrm{E}-03}$ & $\frac{\mathrm{G}_{\mathrm{a}}}{7.25772 \mathrm{E}-03}$ & $\frac{\mathrm{G}_{\mathrm{f}}}{1.36913 \mathrm{E}-02}$ & $\frac{\mathrm{G}_{\mathrm{f}}}{4.86098 \mathrm{E}-03}$ \\
1 & $5.45685 \mathrm{E}-02$ & $6.04055 \mathrm{E}-06$ & $2.50257 \mathrm{E}-06$ & $5.15366 \mathrm{E}-02$ & $5.85011 \mathrm{E}-06$ & $2.42368 \mathrm{E}-06$ \\
2 & $1.12498 \mathrm{E}-02$ & $3.86744 \mathrm{E}-08$ & $1.60235 \mathrm{E}-08$ & $1.12126 \mathrm{E}-02$ & $3.84197 \mathrm{E}-08$ & $1.59180 \mathrm{E}-08$ \\
3 & $4.29376 \mathrm{E}-02$ & $2.01691 \mathrm{E}-07$ & $8.35643 \mathrm{E}-08$ & $4.28896 \mathrm{E}-02$ & $2.01461 \mathrm{E}-07$ & $8.34691 \mathrm{E}-08$ \\
\hline
\end{tabular}

Table 3.20. SAXTON-2, Pin-1 four-group Pu-239 cross sections

\begin{tabular}{ccccccc}
\hline Group & \multicolumn{3}{c}{ CENTRM cross sections $\left(\mathrm{cm}^{-1}\right)$} & \multicolumn{3}{c}{ NITAWL cross sections $\left(\mathrm{cm}^{-1}\right)$} \\
\hline & $\frac{\mathrm{G}_{\mathrm{a}}}{2.51538 \mathrm{E}-03}$ & $\frac{\mathrm{G}_{\mathrm{f}}}{7.36394 \mathrm{E}-03}$ & $\frac{\mathrm{G}_{\mathrm{f}}}{2.36798 \mathrm{E}-03}$ & $\frac{\mathrm{G}_{\mathrm{a}}}{2.51302 \mathrm{E}-03}$ & $\frac{\mathrm{G}_{\mathrm{f}}}{7.35725 \mathrm{E}-03}$ & $\frac{\mathrm{G}_{\mathrm{f}}}{2.36609 \mathrm{E}-03}$ \\
1 & $4.36441 \mathrm{E}-02$ & $7.27138 \mathrm{E}-02$ & $2.52495 \mathrm{E}-02$ & $4.25694 \mathrm{E}-02$ & $7.20315 \mathrm{E}-02$ & $2.50126 \mathrm{E}-02$ \\
2 & $1.44153 \mathrm{E}-01$ & $2.85821 \mathrm{E}-01$ & $9.92499 \mathrm{E}-02$ & $1.40219 \mathrm{E}-01$ & $2.78403 \mathrm{E}-01$ & $9.66744 \mathrm{E}-02$ \\
3 & $1.26117 \mathrm{E}+00$ & $2.54337 \mathrm{E}+00$ & $8.79827 \mathrm{E}-01$ & $1.26115 \mathrm{E}+00$ & $2.54272 \mathrm{E}+00$ & $8.79601 \mathrm{E}-01$ \\
\hline
\end{tabular}


Table 3.21. SAXTON-2, Pin-55 four-group fluxes

\begin{tabular}{ccc}
\hline Group & $\begin{array}{c}\text { CENTRM M } \\
\left(\mathrm{cm}^{-2}-\mathrm{s}^{-1}\right)\end{array}$ & $\begin{array}{c}\text { NITAWL M } \\
\left(\mathrm{cm}^{-2}-\mathrm{s}^{-1}\right)\end{array}$ \\
\hline 1 & $3.168 \mathrm{E}-04$ & $3.145 \mathrm{E}-04$ \\
2 & $1.074 \mathrm{E}-04$ & $1.086 \mathrm{E}-04$ \\
3 & $1.945 \mathrm{E}-05$ & $1.953 \mathrm{E}-05$ \\
4 & $1.722 \mathrm{E}-05$ & $1.751 \mathrm{E}-05$ \\
\hline
\end{tabular}

Table 3.22 SAXTON-2, Pin-55 four-group U-235 cross sections

\begin{tabular}{cccccccc}
\hline Group & \multicolumn{2}{c}{ CENTRM cross sections $\left(\mathrm{cm}^{-1}\right)$} & \multicolumn{2}{c}{ NITAWL cross sections $\left(\mathrm{cm}^{-1}\right)$} \\
\hline & $\frac{\mathrm{G}_{\mathrm{a}}}{2.42818 \mathrm{E}-04}$ & $\frac{\mathrm{G}_{\mathrm{f}}}{5.37235 \mathrm{E}-04}$ & $\frac{\mathrm{G}_{\mathrm{f}}}{2.08871 \mathrm{E}-04}$ & $\frac{\mathrm{G}_{\mathrm{a}}}{2.42331 \mathrm{E}-04}$ & $\frac{\mathrm{G}_{\mathrm{f}}}{5.36480 \mathrm{E}-04}$ & $\frac{\mathrm{G}_{\mathrm{f}}}{2.08578 \mathrm{E}-04}$ \\
1 & $5.28301 \mathrm{E}-03$ & $8.16913 \mathrm{E}-03$ & $3.35254 \mathrm{E}-03$ & $5.21227 \mathrm{E}-03$ & $8.02944 \mathrm{E}-03$ & $3.29521 \mathrm{E}-03$ \\
3 & $7.65558 \mathrm{E}-03$ & $1.53980 \mathrm{E}-02$ & $6.31918 \mathrm{E}-03$ & $7.58530 \mathrm{E}-03$ & $1.53401 \mathrm{E}-02$ & $6.29544 \mathrm{E}-03$ \\
4 & $6.42364 \mathrm{E}-02$ & $1.33386 \mathrm{E}-01$ & $5.47403 \mathrm{E}-02$ & $6.47836 \mathrm{E}-02$ & $1.34511 \mathrm{E}-01$ & $5.52022 \mathrm{E}-02$ \\
\hline
\end{tabular}

Table 3.23. SAXTON-2, Pin-55 four-group U-238 cross sections

\begin{tabular}{cccccccc}
\hline Group & \multicolumn{3}{c}{ CENTRM cross sections $\left(\mathrm{cm}^{-1}\right)$} & \multicolumn{3}{c}{ NITAWL cross sections $\left(\mathrm{cm}^{-1}\right)$} \\
\hline & $\frac{\mathrm{G}_{\mathrm{a}}}{4}$ & $\frac{\mathrm{G}_{\mathrm{f}}}{1.14866 \mathrm{E}-02}$ & $\frac{\mathrm{G}_{\mathrm{f}}}{4.08747 \mathrm{E}-03}$ & $\frac{\mathrm{G}_{\mathrm{a}}}{6.87320 \mathrm{E}-03}$ & $\frac{\mathrm{G}_{\mathrm{f}}}{1.14586 \mathrm{E}-02}$ & $\frac{\mathrm{G}_{\mathrm{f}}}{4.07844 \mathrm{E}-03}$ \\
2 & $5.89564 \mathrm{E}-03$ & $1.14845 \mathrm{E}-02$ & $5.98081 \mathrm{E}-06$ & $2.47782 \mathrm{E}-06$ & $5.07872 \mathrm{E}-02$ & $5.96084 \mathrm{E}-06$ & $2.46955 \mathrm{E}-06$ \\
3 & $1.11261 \mathrm{E}-02$ & $3.76231 \mathrm{E}-08$ & $1.55879 \mathrm{E}-08$ & $1.11419 \mathrm{E}-02$ & $3.76606 \mathrm{E}-08$ & $1.56035 \mathrm{E}-08$ \\
4 & $3.74682 \mathrm{E}-02$ & $1.75440 \mathrm{E}-07$ & $7.26882 \mathrm{E}-08$ & $3.77212 \mathrm{E}-02$ & $1.76632 \mathrm{E}-07$ & $7.31816 \mathrm{E}-08$ \\
\hline
\end{tabular}

Table 3.24. SAXTON-2, Pin-55 four-group Pu-239 cross sections

\begin{tabular}{cccccccc}
\hline Group & \multicolumn{3}{c}{ CENTRM cross sections $\left(\mathrm{cm}^{-1}\right)$} & \multicolumn{2}{c}{ NITAWL cross sections $\left(\mathrm{cm}^{-1}\right)$} \\
\hline & $\frac{\mathrm{G}_{\mathrm{a}}}{2.52700 \mathrm{E}-03}$ & $\frac{\mathrm{G}_{\mathrm{f}}}{7.20417 \mathrm{E}-03}$ & $\frac{\mathrm{G}_{\mathrm{f}}}{2.33954 \mathrm{E}-03}$ & $\frac{\mathrm{G}_{\mathrm{a}}}{2.52549 \mathrm{E}-03}$ & $\frac{\mathrm{G}_{\mathrm{f}}}{7.20372 \mathrm{E}-03}$ & $\frac{\mathrm{G}_{\mathrm{f}}}{2.33961 \mathrm{E}-03}$ \\
1 & $4.23034 \mathrm{E}-02$ & $7.11892 \mathrm{E}-02$ & $2.47201 \mathrm{E}-02$ & $4.01978 \mathrm{E}-02$ & $6.76656 \mathrm{E}-02$ & $2.34965 \mathrm{E}-02$ \\
2 & $1.33769 \mathrm{E}-01$ & $2.66117 \mathrm{E}-01$ & $9.24077 \mathrm{E}-02$ & $1.31144 \mathrm{E}-01$ & $2.61407 \mathrm{E}-01$ & $9.07727 \mathrm{E}-02$ \\
3 & $1.41690 \mathrm{E}+00$ & $2.73612 \mathrm{E}+00$ & $9.47311 \mathrm{E}-01$ & $1.43265 \mathrm{E}+00$ & $2.76589 \mathrm{E}+00$ & $9.57596 \mathrm{E}-01$ \\
\hline
\end{tabular}




\begin{tabular}{|c|c|c|c|c|c|c|c|c|c|c|}
\hline \multirow[t]{10}{*}{$\begin{array}{l}0.186 \\
(0.99) \\
\end{array}$} & & & & & & & & & & $\begin{array}{l}5.900 \\
(0.99)\end{array}$ \\
\hline & & & & & & & & & $\begin{array}{l}5.929 \\
(0.48)\end{array}$ & $\begin{array}{l}5.958 \\
(0.50)\end{array}$ \\
\hline & & & & & & & & $\begin{array}{l}5.672 \\
(0.51)\end{array}$ & $\begin{array}{l}5.819 \\
(0.31)\end{array}$ & $\begin{array}{l}5.884 \\
(0.49)\end{array}$ \\
\hline & & & & & & & $\begin{array}{l}5.436 \\
(0.48)\end{array}$ & $\begin{array}{l}5.585 \\
(0.33)\end{array}$ & $\begin{array}{l}5.666 \\
(0.33)\end{array}$ & $\begin{array}{l}5.630 \\
(0.49)\end{array}$ \\
\hline & & & & & & $\begin{array}{l}5.000 \\
(0.54)\end{array}$ & $\begin{array}{l}5.206 \\
(0.35)\end{array}$ & $\begin{array}{l}5.373 \\
(0.33)\end{array}$ & $\begin{array}{l}5.425 \\
(0.33)\end{array}$ & $\begin{array}{l}5.486 \\
(0.55)\end{array}$ \\
\hline & & & & & $\begin{array}{l}4.473 \\
(0.49)\end{array}$ & $\begin{array}{l}4.718 \\
(0.35)\end{array}$ & $\begin{array}{l}4.935 \\
(0.37)\end{array}$ & $\begin{array}{l}5.060 \\
(0.34)\end{array}$ & $\begin{array}{l}5.148 \\
(0.34)\end{array}$ & $\begin{array}{l}5.191 \\
(0 .) 55\end{array}$ \\
\hline & & & & $\begin{array}{l}3.921 \\
(0.58)\end{array}$ & $\begin{array}{l}4.189 \\
(0.42)\end{array}$ & $\begin{array}{l}4.440 \\
(0.38)\end{array}$ & $\begin{array}{l}4.590 \\
(0.38)\end{array}$ & $\begin{array}{l}4.750 \\
(0.36)\end{array}$ & $\begin{array}{l}4.833 \\
(0.34)\end{array}$ & $\begin{array}{l}4.836 \\
(0.52)\end{array}$ \\
\hline & & & $\begin{array}{l}3.331 \\
(0.66)\end{array}$ & $\begin{array}{l}3.599 \\
(0.45)\end{array}$ & $\begin{array}{l}3.842 \\
(0.38)\end{array}$ & $\begin{array}{l}4.096 \\
(0.43)\end{array}$ & $\begin{array}{l}4.271 \\
(0.41)\end{array}$ & $\begin{array}{l}4.357 \\
(0.41)\end{array}$ & $\begin{array}{l}4.399 \\
(0.42)\end{array}$ & $\begin{array}{l}4.447 \\
(0.52)\end{array}$ \\
\hline & & $\begin{array}{l}2.821 \\
(0.75)\end{array}$ & $\begin{array}{l}3.029 \\
(0.47)\end{array}$ & $\begin{array}{l}3.314 \\
(0.50)\end{array}$ & $\begin{array}{l}3.555 \\
(0.43)\end{array}$ & $\begin{array}{c}3.722 \\
(0.39)\end{array}$ & $\begin{array}{l}3.898 \\
(0.40)\end{array}$ & $\begin{array}{l}3.976 \\
(0.39)\end{array}$ & $\begin{array}{l}4.078 \\
(0.39)\end{array}$ & $\begin{array}{l}4.096 \\
(0.57)\end{array}$ \\
\hline & $\begin{array}{l}2.693 \\
(0.80)\end{array}$ & $\begin{array}{l}2.750 \\
(0.51)\end{array}$ & $\begin{array}{l}2.987 \\
(0.44)\end{array}$ & $\begin{array}{r}3.235 \\
(0.47)\end{array}$ & $\begin{array}{c}3.464 \\
(0.40)\end{array}$ & $\begin{array}{c}3.672 \\
(0.41)\end{array}$ & $\begin{array}{l}3.860 \\
(0.41)\end{array}$ & $\begin{array}{l}3.906 \\
(0.40)\end{array}$ & $\begin{array}{l}3.977 \\
(0.41)\end{array}$ & $\begin{array}{c}3.972 \\
(0.56)\end{array}$ \\
\hline $\begin{array}{l}4.495 \\
(0.57)\end{array}$ & $\begin{array}{l}3.726 \\
(0.48)\end{array}$ & $\begin{array}{c}3.956 \\
(0.41)\end{array}$ & $\begin{array}{l}4.320 \\
(0.37)\end{array}$ & $\begin{array}{l}4.667 \\
(0.37)\end{array}$ & $\begin{array}{l}5.028 \\
(0.37)\end{array}$ & $\begin{array}{l}5.272 \\
(0.37)\end{array}$ & $\begin{array}{l}5.506 \\
(0.38)\end{array}$ & $\begin{array}{l}5.679 \\
(0.38)\end{array}$ & $\begin{array}{l}5.763 \\
(0.36)\end{array}$ & $\begin{array}{l}5.781 \\
(0.51)\end{array}$ \\
\hline
\end{tabular}

Fig. 3.9a. Pin-power distribution for CENTRM benchmark SAXTON-3. Value in parentheses is percent standard deviation. 


\begin{tabular}{|c|c|c|c|c|c|c|c|c|c|c|}
\hline \multirow[t]{10}{*}{$\begin{array}{l}0.187 \\
(1.06)\end{array}$} & & & & & & & & & & $\begin{array}{l}5.920 \\
(1.07)\end{array}$ \\
\hline & & & & & & & & & $\begin{array}{l}5.932 \\
(0.47)\end{array}$ & $\begin{array}{l}5.983 \\
(0.45)\end{array}$ \\
\hline & & & & & & & & $\begin{array}{l}5.779 \\
(0.48)\end{array}$ & $\begin{array}{l}5.826 \\
(0.33)\end{array}$ & $\begin{array}{l}5.906 \\
(0.48)\end{array}$ \\
\hline & & & & & & & $\begin{array}{l}5.446 \\
(0.46)\end{array}$ & $\begin{array}{l}5.598 \\
(0.33)\end{array}$ & $\begin{array}{l}5.704 \\
(0.33)\end{array}$ & $\begin{array}{l}5.705 \\
(0.45)\end{array}$ \\
\hline & & & & & & $\begin{array}{l}5.019 \\
(0.56)\end{array}$ & $\begin{array}{l}5.257 \\
(0.36)\end{array}$ & $\begin{array}{l}5.406 \\
(0.36)\end{array}$ & $\begin{array}{l}5.478 \\
(0.36)\end{array}$ & $\begin{array}{l}5.496 \\
(0.47)\end{array}$ \\
\hline & & & & & $\begin{array}{l}4.482 \\
(0.54)\end{array}$ & $\begin{array}{l}4.743 \\
(0.40)\end{array}$ & $\begin{array}{l}4.946 \\
(0.34)\end{array}$ & $\begin{array}{l}5.088 \\
(0.35)\end{array}$ & $\begin{array}{l}5.205 \\
(0.34)\end{array}$ & $\begin{array}{l}5.195 \\
(0.51)\end{array}$ \\
\hline & & & & $\begin{array}{c}3.988 \\
(0.58)\end{array}$ & $\begin{array}{l}4.209 \\
(0.38)\end{array}$ & $\begin{array}{l}4.447 \\
(0.36)\end{array}$ & $\begin{array}{l}4.582 \\
(0.37)\end{array}$ & $\begin{array}{l}4.738 \\
(0.36)\end{array}$ & $\begin{array}{l}4.822 \\
(0.36)\end{array}$ & $\begin{array}{l}4.866 \\
(0.54)\end{array}$ \\
\hline & & & $\begin{array}{l}3.317 \\
(0.60)\end{array}$ & $\begin{array}{l}3.625 \\
(0.44)\end{array}$ & $\begin{array}{l}3.856 \\
(0.39)\end{array}$ & $\begin{array}{l}4.081 \\
(0.38)\end{array}$ & $\begin{array}{l}4.245 \\
(0.37)\end{array}$ & $\begin{array}{l}4.400 \\
(0.36)\end{array}$ & $\begin{array}{l}4.439 \\
(0.34)\end{array}$ & $\begin{array}{l}4.515 \\
(0.51)\end{array}$ \\
\hline & & $\begin{array}{c}2.787 \\
(0.67)\end{array}$ & $\begin{array}{l}3.058 \\
(0.48)\end{array}$ & $\begin{array}{l}3.340 \\
(0.44)\end{array}$ & $\begin{array}{l}3.557 \\
(0.45)\end{array}$ & $\begin{array}{c}3.734 \\
(0.42)\end{array}$ & $\begin{array}{l}3.912 \\
(0.43)\end{array}$ & $\begin{array}{l}4.022 \\
(0.39)\end{array}$ & $\begin{array}{l}4.049 \\
(0.41)\end{array}$ & $\begin{array}{l}4.134 \\
(0.59)\end{array}$ \\
\hline & $\begin{array}{l}2.676 \\
(0.74)\end{array}$ & $\begin{array}{l}2.740 \\
(0.51)\end{array}$ & $\begin{array}{l}3.007 \\
(0.46)\end{array}$ & $\begin{array}{c}3.254 \\
(0.49)\end{array}$ & $\begin{array}{l}3.491 \\
(0.42)\end{array}$ & $\begin{array}{c}3.663 \\
(0.47)\end{array}$ & $\begin{array}{l}3.821 \\
(0.42)\end{array}$ & $\begin{array}{l}3.954 \\
(0.42)\end{array}$ & $\begin{array}{l}4.004 \\
(0.43)\end{array}$ & $\begin{array}{l}4.050 \\
(0.61)\end{array}$ \\
\hline $\begin{array}{l}4.515 \\
(0.53)\end{array}$ & $\begin{array}{l}3.758 \\
(0.45)\end{array}$ & $\begin{array}{c}3.949 \\
(0.41)\end{array}$ & $\begin{array}{l}4.335 \\
(0.40)\end{array}$ & $\begin{array}{l}4.695 \\
(0.39)\end{array}$ & $\begin{array}{l}5.019 \\
(0.36)\end{array}$ & $\begin{array}{l}5.313 \\
(0.36)\end{array}$ & $\begin{array}{l}5.493 \\
(0.38)\end{array}$ & $\begin{array}{l}5.685 \\
(0.38)\end{array}$ & $\begin{array}{l}5.746 \\
(0.34)\end{array}$ & $\begin{array}{l}5.823 \\
(0.46)\end{array}$ \\
\hline
\end{tabular}

Fig. 3.9b. Pin-power distribution for NITAWL benchmark SAXTON-3. Value in parentheses is percent standard deviation. 
Table 3.25. SAXTON-3 CENTRM reaction rates and fluxes

\begin{tabular}{ccccccc}
\hline \multirow{2}{*}{ Pin } & Region & $\begin{array}{c}\mathrm{G}_{\mathrm{a}} \mathrm{M} \\
\left(\mathrm{cm}^{-3}-\mathrm{s}^{-1}\right)\end{array}$ & $\begin{array}{c}\mathrm{G}_{\mathrm{f}} \mathrm{M} \\
\left(\mathrm{cm}^{-3}-\mathrm{s}^{-1}\right)\end{array}$ & $\begin{array}{c}\mathrm{G}_{\mathrm{f}} \mathrm{M} \\
\left(\mathrm{cm}^{-3}-\mathrm{s}^{-1}\right)\end{array}$ & $\begin{array}{c}\mathrm{M} \\
\left(\mathrm{cm}^{-1}\right)\end{array}$ & $\mathrm{MM}_{\mathrm{t}}$ \\
\hline \multirow{2}{*}{1} & Fuel & $2.543 \mathrm{E}-05$ & $4.469 \mathrm{E}-05$ & $1.562 \mathrm{E}-05$ & $6.051 \mathrm{E}-03$ & 0.295 \\
& Clad & $2.340 \mathrm{E}-07$ & 0.0 & 0.0 & $1.781 \mathrm{E}-03$ & 0.087 \\
& Mod. & $1.588 \mathrm{E}-06$ & 0.0 & 0.0 & $1.266 \mathrm{E}-02$ & 0.618 \\
\hline \multirow{2}{*}{66} & Fuel & $3.944 \mathrm{E}-05$ & $5.901 \mathrm{E}-05$ & $2.061 \mathrm{E}-05$ & $1.985 \mathrm{E}-02$ & 0.292 \\
& Clad & $5.323 \mathrm{E}-07$ & 0.0 & 0.0 & $5.950 \mathrm{E}-03$ & 0.087 \\
& Mod. & $1.782 \mathrm{E}-06$ & 0.0 & 0.0 & $4.227 \mathrm{E}-02$ & 0.621 \\
\hline
\end{tabular}

Table 3.26. SAXTON-3 NITAWL reaction rates and fluxes

\begin{tabular}{|c|c|c|c|c|c|c|}
\hline Pin & Region & $\begin{array}{c}\mathrm{G}_{\mathrm{a}} \mathrm{M} \\
\left(\mathrm{cm}^{-3}-\mathrm{s}^{-1}\right)\end{array}$ & $\begin{array}{c}\mathcal{G}_{\mathrm{f}} \mathrm{M} \\
\left(\mathrm{cm}^{-3}-\mathrm{s}^{-1}\right)\end{array}$ & $\begin{array}{c}\mathrm{G}_{\mathrm{f}} \mathrm{M} \\
\left(\mathrm{cm}^{-3}-\mathrm{s}^{-1}\right)\end{array}$ & $\begin{array}{c}\mathrm{M} \\
\left(\mathrm{cm}-\mathrm{s}^{-1}\right)\end{array}$ & $M M_{t}$ \\
\hline \multirow{3}{*}{1} & Fuel & $2.574 \mathrm{E}-05$ & $4.529 \mathrm{E}-05$ & $1.589 \mathrm{E}-05$ & $6.060 \mathrm{E}-03$ & 0.295 \\
\hline & Clad & $2.313 \mathrm{E}-07$ & 0.0 & 0.0 & $1.785 \mathrm{E}-03$ & 0.087 \\
\hline & Mod. & 7.923E-07 & 0.0 & 0.0 & $1.270 \mathrm{E}-02$ & 0.618 \\
\hline \multirow{3}{*}{66} & Fuel & $3.956 \mathrm{E}-05$ & $5.952 \mathrm{E}-05$ & $2.079 \mathrm{E}-05$ & $1.999 \mathrm{E}-02$ & 0.293 \\
\hline & Clad & $5.281 \mathrm{E}-07$ & 0.0 & 0.0 & $5.968 \mathrm{E}-03$ & 0.087 \\
\hline & Mod. & $8.857 \mathrm{E}-07$ & 0.0 & 0.0 & 4.235E-02 & 0.620 \\
\hline
\end{tabular}


Table 3.27. SAXTON-3, Pin-1 four-group fluxes

\begin{tabular}{ccc}
\hline Group & $\begin{array}{c}\text { CENTRM M } \\
\left(\mathrm{cm}^{-2}-\mathrm{s}^{-1}\right)\end{array}$ & $\begin{array}{c}\text { NITAWL M } \\
\left(\mathrm{cm}^{-2}-\mathrm{s}^{-1}\right)\end{array}$ \\
\hline 1 & $7.796 \mathrm{E}-05$ & $7.800 \mathrm{E}-05$ \\
2 & $2.181 \mathrm{E}-05$ & $2.192 \mathrm{E}-05$ \\
3 & $4.829 \mathrm{E}-06$ & $4.689 \mathrm{E}-06$ \\
4 & $1.453 \mathrm{E}-05$ & $1.470 \mathrm{E}-05$ \\
\hline
\end{tabular}

Table 3.28. SAXTON-3, Pin-1 four-group U-235 cross sections

\begin{tabular}{cccccccc}
\hline Group & \multicolumn{3}{c}{ CENTRM cross sections $\left(\mathrm{cm}^{-1}\right)$} & \multicolumn{3}{c}{ NITAWL cross sections $\left(\mathrm{cm}^{-1}\right)$} \\
\hline & $\frac{\mathrm{G}_{\mathrm{a}}}{4.32521 \mathrm{E}-04}$ & $\frac{\mathrm{G}_{\mathrm{f}}}{5.28612 \mathrm{E}-04}$ & $\frac{\mathrm{G}_{\mathrm{f}}}{2.03658 \mathrm{E}-04}$ & $\frac{\mathrm{G}_{\mathrm{a}}}{2.32862 \mathrm{E}-04}$ & $\frac{\mathrm{G}_{\mathrm{f}}}{5.29454 \mathrm{E}-04}$ & $\frac{\mathrm{G}_{\mathrm{f}}}{2.03931 \mathrm{E}-04}$ \\
1 & $5.50005 \mathrm{E}-03$ & $8.43228 \mathrm{E}-03$ & $3.46053 \mathrm{E}-03$ & $5.39193 \mathrm{E}-03$ & $8.28878 \mathrm{E}-03$ & $3.40165 \mathrm{E}-03$ \\
2 & $7.92452 \mathrm{E}-03$ & $1.60789 \mathrm{E}-02$ & $6.59865 \mathrm{E}-03$ & $7.90441 \mathrm{E}-03$ & $1.60146 \mathrm{E}-02$ & $6.57223 \mathrm{E}-03$ \\
3 & $7.31686 \mathrm{E}-02$ & $1.52279 \mathrm{E}-01$ & $6.24939 \mathrm{E}-02$ & $7.33599 \mathrm{E}-02$ & $1.52663 \mathrm{E}-01$ & $6.26517 \mathrm{E}-02$ \\
\hline
\end{tabular}

Table 3.29. SAXTON-3, Pin-1 four-group U-238 cross sections

\begin{tabular}{cccccccc}
\hline Group & \multicolumn{3}{c}{ CENTRM cross sections $\left(\mathrm{cm}^{-1}\right)$} & \multicolumn{3}{c}{ NITAWL cross sections $\left(\mathrm{cm}^{-1}\right)$} \\
\hline & $\frac{\mathrm{G}_{\mathrm{a}}}{4.20136 \mathrm{E}-03}$ & $\frac{\mathrm{G}_{\mathrm{f}}}{1.33762 \mathrm{E}-02}$ & $\frac{\mathrm{G}_{\mathrm{f}}}{4.75174 \mathrm{E}-03}$ & $\frac{\mathrm{G}_{\mathrm{a}}}{7.23290 \mathrm{E}-03}$ & $\frac{\mathrm{G}_{\mathrm{f}}}{1.34483 \mathrm{E}-02}$ & $\frac{\mathrm{G}_{\mathrm{f}}}{4.77628 \mathrm{E}-03}$ \\
1 & $5.41784 \mathrm{E}-02$ & $5.69922 \mathrm{E}-06$ & $2.36116 \mathrm{E}-06$ & $5.29886 \mathrm{E}-02$ & $5.86670 \mathrm{E}-06$ & $2.43054 \mathrm{E}-06$ \\
2 & $1.12448 \mathrm{E}-02$ & $3.86080 \mathrm{E}-08$ & $1.59959 \mathrm{E}-08$ & $1.12161 \mathrm{E}-02$ & $3.84319 \mathrm{E}-08$ & $1.59231 \mathrm{E}-08$ \\
3 & $4.20736 \mathrm{E}-02$ & $1.97550 \mathrm{E}-07$ & $8.18486 \mathrm{E}-08$ & $4.21529 \mathrm{E}-02$ & $1.97922 \mathrm{E}-07$ & $8.20034 \mathrm{E}-08$ \\
\hline
\end{tabular}

Table 3.30. SAXTON-3, Pin-1 four-group Pu-239 cross sections

\begin{tabular}{cccccccc}
\hline Group & \multicolumn{3}{c}{ CENTRM cross sections $\left(\mathrm{cm}^{-1}\right)$} & \multicolumn{3}{c}{ NITAWL cross sections $\left(\mathrm{cm}^{-1}\right)$} \\
\hline & $\frac{\mathrm{G}_{\mathrm{a}}}{4.51444 \mathrm{E}-03}$ & $\frac{\mathrm{G}_{\mathrm{f}}}{7.33436 \mathrm{E}-03}$ & $\frac{\mathrm{G}_{\mathrm{f}}}{2.36219 \mathrm{E}-03}$ & $\frac{\mathrm{G}_{\mathrm{a}}}{2.51691 \mathrm{E}-03}$ & $\frac{\mathrm{G}_{\mathrm{f}}}{7.34278 \mathrm{E}-03}$ & $\frac{\mathrm{G}_{\mathrm{f}}}{2.36421 \mathrm{E}-03}$ \\
1 & $4.43213 \mathrm{E}-02$ & $7.47680 \mathrm{E}-02$ & $2.59628 \mathrm{E}-02$ & $4.17771 \mathrm{E}-02$ & $7.10830 \mathrm{E}-02$ & $2.46832 \mathrm{E}-02$ \\
2 & $1.44573 \mathrm{E}-01$ & $2.86496 \mathrm{E}-01$ & $9.94842 \mathrm{E}-02$ & $1.40383 \mathrm{E}-01$ & $2.78776 \mathrm{E}-01$ & $9.68036 \mathrm{E}-02$ \\
3 & $1.27177 \mathrm{E}+00$ & $2.55010 \mathrm{E}+00$ & $8.82257 \mathrm{E}-01$ & $1.28214 \mathrm{E}+00$ & $2.56855 \mathrm{E}+00$ & $8.88646 \mathrm{E}-01$ \\
\hline
\end{tabular}


Table 3.31. SAXTON-3, Pin-66 four-group fluxes

\begin{tabular}{ccc}
\hline Group & $\begin{array}{c}\text { CENTRM M } \\
\left(\mathrm{cm}^{-2}-\mathrm{s}^{-1}\right)\end{array}$ & $\begin{array}{c}\text { NITAWL M } \\
\left(\mathrm{cm}^{-2}-\mathrm{s}^{-1}\right)\end{array}$ \\
\hline 1 & $2.666 \mathrm{E}-04$ & $2.684 \mathrm{E}-04$ \\
2 & $9.313 \mathrm{E}-05$ & $9.364 \mathrm{E}-05$ \\
3 & $1.707 \mathrm{E}-05$ & $1.685 \mathrm{E}-05$ \\
4 & $1.407 \mathrm{E}-05$ & $1.456 \mathrm{E}-05$ \\
\hline
\end{tabular}

Table 3.32. SAXTON-3, Pin-66 four-group U-235 cross sections

\begin{tabular}{cccccccc}
\hline Group & \multicolumn{3}{c}{ CENTRM cross sections $\left(\mathrm{cm}^{-1}\right)$} & \multicolumn{3}{c}{ NITAWL cross sections $\left(\mathrm{cm}^{-1}\right)$} \\
\hline & $\frac{\mathrm{G}_{\mathrm{a}}}{2.42733 \mathrm{E}-04}$ & $\frac{\mathrm{G}_{\mathrm{f}}}{5.37472 \mathrm{E}-04}$ & $\frac{\mathrm{G}_{\mathrm{f}}}{2.08870 \mathrm{E}-04}$ & $\frac{\mathrm{G}_{\mathrm{a}}}{2.43387 \mathrm{E}-04}$ & $\frac{\mathrm{G}_{\mathrm{f}}}{5.37876 \mathrm{E}-04}$ & $\frac{\mathrm{G}_{\mathrm{f}}}{2.09178 \mathrm{E}-04}$ \\
1 & $5.30011 \mathrm{E}-03$ & $8.16540 \mathrm{E}-03$ & $3.35100 \mathrm{E}-03$ & $5.27422 \mathrm{E}-03$ & $8.12879 \mathrm{E}-03$ & $3.33598 \mathrm{E}-03$ \\
2 & $7.70943 \mathrm{E}-03$ & $1.55532 \mathrm{E}-02$ & $6.38289 \mathrm{E}-03$ & $7.67703 \mathrm{E}-03$ & $1.54383 \mathrm{E}-02$ & $6.33579 \mathrm{E}-03$ \\
3 & $6.52259 \mathrm{E}-02$ & $1.35439 \mathrm{E}-01$ & $5.55832 \mathrm{E}-02$ & $6.49576 \mathrm{E}-02$ & $1.34906 \mathrm{E}-01$ & $5.53641 \mathrm{E}-02$ \\
\hline
\end{tabular}

Table 3.33. SAXTON-3, Pin-66 four-group U-238 cross sections

\begin{tabular}{cccccccc}
\hline Group & \multicolumn{3}{c}{ CENTRM cross sections $\left(\mathrm{cm}^{-1}\right)$} & \multicolumn{3}{c}{ NITAWL cross sections $\left(\mathrm{cm}^{-1}\right)$} \\
\hline & $\frac{\mathrm{G}_{\mathrm{a}}}{6.92119 \mathrm{E}-03}$ & $\frac{\mathrm{G}_{\mathrm{f}}}{1.15793 \mathrm{E}-02}$ & $\frac{\mathrm{G}_{\mathrm{f}}}{4.11808 \mathrm{E}-03}$ & $\frac{\mathrm{G}_{\mathrm{a}}}{6.86967 \mathrm{E}-03}$ & $\frac{\mathrm{G}_{\mathrm{f}}}{1.13727 \mathrm{E}-02}$ & $\frac{\mathrm{G}_{\mathrm{f}}}{4.04314 \mathrm{E}-03}$ \\
1 & $5.09692 \mathrm{E}-02$ & $6.02439 \mathrm{E}-06$ & $2.49588 \mathrm{E}-06$ & $4.92231 \mathrm{E}-02$ & $6.08021 \mathrm{E}-06$ & $2.51901 \mathrm{E}-06$ \\
2 & $1.11677 \mathrm{E}-02$ & $3.78609 \mathrm{E}-08$ & $1.56865 \mathrm{E}-08$ & $1.11602 \mathrm{E}-02$ & $3.77671 \mathrm{E}-08$ & $1.56476 \mathrm{E}-08$ \\
3 & $3.79241 \mathrm{E}-02$ & $1.77598 \mathrm{E}-07$ & $7.35821 \mathrm{E}-08$ & $3.78323 \mathrm{E}-02$ & $1.77181 \mathrm{E}-07$ & $7.34093 \mathrm{E}-08$ \\
\hline
\end{tabular}

Table 3.34. SAXTON-3, Pin-66 four-group Pu-239 cross sections

\begin{tabular}{cccccccc}
\hline Group & \multicolumn{3}{c}{ CENTRM cross sections $\left(\mathrm{cm}^{-1}\right)$} & \multicolumn{3}{c}{ NITAWL cross sections $\left(\mathrm{cm}^{-1}\right)$} \\
\hline & $\frac{\mathrm{G}_{\mathrm{a}}}{2.52720 \mathrm{E}-03}$ & $\frac{\mathrm{G}_{\mathrm{f}}}{7.21032 \mathrm{E}-03}$ & $\frac{\mathrm{G}_{\mathrm{f}}}{2.34053 \mathrm{E}-03}$ & $\frac{\mathrm{G}_{\mathrm{a}}}{2.52750 \mathrm{E}-03}$ & $\frac{\mathrm{G}_{\mathrm{f}}}{7.19788 \mathrm{E}-03}$ & $\frac{\mathrm{G}_{\mathrm{f}}}{2.33835 \mathrm{E}-03}$ \\
1 & $4.12518 \mathrm{E}-02$ & $6.95573 \mathrm{E}-02$ & $2.41534 \mathrm{E}-02$ & $4.03271 \mathrm{E}-02$ & $6.74581 \mathrm{E}-02$ & $2.34245 \mathrm{E}-02$ \\
2 & $1.37500 \mathrm{E}-01$ & $2.73101 \mathrm{E}-01$ & $9.48330 \mathrm{E}-02$ & $1.37757 \mathrm{E}-01$ & $2.73564 \mathrm{E}-01$ & $9.49941 \mathrm{E}-02$ \\
3 & $1.43800 \mathrm{E}+00$ & $2.77757 \mathrm{E}+00$ & $9.61635 \mathrm{E}-01$ & $1.41120 \mathrm{E}+00$ & $2.73126 \mathrm{E}+00$ & $9.45577 \mathrm{E}-01$ \\
\hline
\end{tabular}




\begin{tabular}{|c|c|c|c|c|c|c|}
\hline \multirow[t]{6}{*}{$\begin{array}{l}0.218 \\
(0.73)\end{array}$} & & & & & & $\begin{array}{c}18.89 \\
(0.69)\end{array}$ \\
\hline & & & & & $\begin{array}{l}18.60 \\
(0.33)\end{array}$ & $\begin{array}{c}18.82 \\
(0.36)\end{array}$ \\
\hline & & & & $\begin{array}{l}17.23 \\
(0.35)\end{array}$ & $\begin{array}{l}17.81 \\
(0.24)\end{array}$ & $\begin{array}{l}18.18 \\
(0.33)\end{array}$ \\
\hline & & & $\begin{array}{l}14.90 \\
(0.35)\end{array}$ & $\begin{array}{l}16.09 \\
(0.26)\end{array}$ & $\begin{array}{c}1.6 .9 \\
(0.26)\end{array}$ & $\begin{array}{l}16.95 \\
(0.35)\end{array}$ \\
\hline & & $\begin{array}{r}12.39 \\
(0.38)\end{array}$ & $\begin{array}{l}13.62 \\
(0.29)\end{array}$ & $\begin{array}{l}14.58 \\
(0.24)\end{array}$ & $\begin{array}{l}15.18 \\
(0.25)\end{array}$ & $\begin{array}{l}15.37 \\
(0.38)\end{array}$ \\
\hline & $\begin{array}{c}10.54 \\
(0.30)\end{array}$ & $\begin{array}{c}11.44 \\
(0.28)\end{array}$ & $\begin{array}{c}12.55 \\
(0.28)\end{array}$ & $\begin{array}{c}13.40 \\
(0.26)\end{array}$ & $\begin{array}{l}13.91 \\
(0.27)\end{array}$ & $\begin{array}{c}14.11 \\
(0.40)\end{array}$ \\
\hline $\begin{array}{l}13.71 \\
(0.41)\end{array}$ & $\begin{array}{l}12.57 \\
(0.29)\end{array}$ & $\begin{array}{l}13.86 \\
(0.29)\end{array}$ & $\begin{array}{c}15.06 \\
(0.26)\end{array}$ & $\begin{array}{l}16.18 \\
(0.25)\end{array}$ & $\begin{array}{l}16.83 \\
(0.25)\end{array}$ & $\begin{array}{c}17.04 \\
(0.30)\end{array}$ \\
\hline
\end{tabular}

Fig. 3.10a. Pin-power distribution for CENTRM benchmark SAXTON-4. Value in parentheses is percent standard deviation.

\begin{tabular}{|c|c|c|c|c|c|c|}
\hline \multirow[t]{6}{*}{$\begin{array}{l}0.216 \\
(0.71)\end{array}$} & & & & & & $\begin{array}{l}19.22 \\
(0.63)\end{array}$ \\
\hline & & & & & $\begin{array}{l}18.69 \\
(0.34)\end{array}$ & $\begin{array}{l}18.74 \\
(0.33)\end{array}$ \\
\hline & & & & $\begin{array}{l}17.22 \\
(0.35)\end{array}$ & $\begin{array}{c}17.97 \\
(0.23)\end{array}$ & $\begin{array}{l}18.11 \\
(0.32)\end{array}$ \\
\hline & & & $\begin{array}{l}15.01 \\
(0.37)\end{array}$ & $\begin{array}{l}16.06 \\
(0.26)\end{array}$ & $\begin{array}{l}16.70 \\
(0.24)\end{array}$ & $\begin{array}{l}16.94 \\
(0.35)\end{array}$ \\
\hline & & $\begin{array}{l}12.38 \\
(0.42)\end{array}$ & $\begin{array}{c}13.63 \\
(0.29)\end{array}$ & $\begin{array}{c}14.56 \\
(0.25)\end{array}$ & $\begin{array}{c}15.19 \\
(0.26)\end{array}$ & $\begin{array}{l}15.46 \\
(0.37)\end{array}$ \\
\hline & $\begin{array}{l}10.58 \\
(0.45)\end{array}$ & $\begin{array}{c}11.45 \\
(0.28)\end{array}$ & $\begin{array}{c}12.55 \\
(0.30)\end{array}$ & $\begin{array}{c}13.47 \\
(0.29)\end{array}$ & $\begin{array}{c}14.02 \\
(0.27)\end{array}$ & $\begin{array}{c}14.19 \\
(0.39)\end{array}$ \\
\hline $\begin{array}{l}13.72 \\
(0.38)\end{array}$ & $\begin{array}{c}12.61 \\
(0.27)\end{array}$ & $\begin{array}{r}13.89 \\
(0.27)\end{array}$ & $\begin{array}{l}15.18 \\
(0.25)\end{array}$ & $\begin{array}{l}16.16 \\
(0.28)\end{array}$ & $\begin{array}{l}16.86 \\
(0.24)\end{array}$ & $\begin{array}{l}17.12 \\
(0.33)\end{array}$ \\
\hline
\end{tabular}

Fig. 3.10b. Pin-power distribution for NITAWL benchmark SAXTON-4. Value in parentheses is percent standard deviation. 
Table 3.35. SAXTON-4 CENTRM reaction rates and fluxes

\begin{tabular}{ccccccc}
\hline \multirow{2}{*}{ Pin } & Region & $\begin{array}{c}\mathrm{G}_{\mathrm{a}} \mathrm{M} \\
\left(\mathrm{cm}^{-3}-\mathrm{s}^{-1}\right)\end{array}$ & $\begin{array}{c}\mathrm{G}_{\mathrm{f}} \mathrm{M} \\
\left(\mathrm{cm}^{-3}-\mathrm{s}^{-1}\right)\end{array}$ & $\begin{array}{c}\mathrm{G}_{\mathrm{f}} \mathrm{M} \\
\left(\mathrm{cm}^{-3}-\mathrm{s}^{-1}\right)\end{array}$ & $\begin{array}{c}\mathrm{M} \\
\left(\mathrm{cm}^{-1}\right)\end{array}$ & $\mathrm{MM}_{\mathrm{t}}$ \\
\hline \multirow{2}{*}{1} & Fuel & $7.531 \mathrm{E}-05$ & $1.363 \mathrm{E}-04$ & $4.765 \mathrm{E}-05$ & $1.085 \mathrm{E}-02$ & 0.172 \\
& Clad & $6.208 \mathrm{E}-07$ & 0.0 & 0.0 & $3.159 \mathrm{E}-03$ & 0.050 \\
& Mod. & $3.325 \mathrm{E}-06$ & 0.0 & 0.0 & $4.911 \mathrm{E}-02$ & 0.778 \\
\hline \multirow{2}{*}{28} & Fuel & $1.133 \mathrm{E}-04$ & $1.888 \mathrm{E}-04$ & $6.597 \mathrm{E}-05$ & $2.644 \mathrm{E}-02$ & 0.170 \\
& Clad & $1.093 \mathrm{E}-06$ & 0.0 & 0.0 & $7.857 \mathrm{E}-03$ & 0.051 \\
& Mod. & $4.152 \mathrm{E}-06$ & 0.0 & 0.0 & $1.212 \mathrm{E}-01$ & 0.779 \\
\hline
\end{tabular}

Table 3.36. SAXTON-4 NITAWL reaction rates and fluxes

\begin{tabular}{ccccccc}
\hline Pin & Region & $\begin{array}{c}\mathrm{G}_{\mathrm{a}} \mathrm{M} \\
\left(\mathrm{cm}^{-3}-\mathrm{s}^{-1}\right)\end{array}$ & $\begin{array}{c}\mathrm{G}_{\mathrm{f}} \mathrm{M} \\
\left(\mathrm{cm}^{-3}-\mathrm{s}^{-1}\right)\end{array}$ & $\begin{array}{c}\mathrm{G}_{\mathrm{f}} \mathrm{M} \\
\left(\mathrm{cm}^{-3}-\mathrm{s}^{-1}\right)\end{array}$ & $\begin{array}{c}\mathrm{M} \\
\left(\mathrm{cm} \mathrm{-} \mathrm{s}^{-1}\right)\end{array}$ & $\mathrm{MM}_{\mathrm{t}}$ \\
\hline \multirow{2}{*}{1} & Fuel & $7.555 \mathrm{E}-05$ & $1.372 \mathrm{E}-04$ & $4.796 \mathrm{E}-05$ & $1.091 \mathrm{E}-02$ & 0.172 \\
& Clad & $6.298 \mathrm{E}-07$ & 0.0 & 0.0 & $3.186 \mathrm{E}-03$ & 0.050 \\
& Mod. & $1.677 \mathrm{E}-06$ & 0.0 & 0.0 & $4.926 \mathrm{E}-02$ & 0.778 \\
\hline \multirow{2}{*}{28} & Fuel & $1.153 \mathrm{E}-04$ & $1.923 \mathrm{E}-04$ & $6.721 \mathrm{E}-05$ & $2.661 \mathrm{E}-02$ & 0.171 \\
& Clad & $1.090 \mathrm{E}-06$ & 0.0 & 0.0 & $7.874 \mathrm{E}-03$ & 0.050 \\
& Mod. & $2.076 \mathrm{E}-06$ & 0.0 & 0.0 & $1.214 \mathrm{E}-01$ & 0.779 \\
\hline
\end{tabular}


Table 3.37. SAXTON-4, Pin-1 four-group fluxes

\begin{tabular}{ccc}
\hline Group & $\begin{array}{c}\text { CENTRM M } \\
\left(\mathrm{cm}^{-2}-\mathrm{s}^{-1}\right)\end{array}$ & $\begin{array}{c}\text { NITAWL M } \\
\left(\mathrm{cm}^{-2}-\mathrm{s}^{-1}\right)\end{array}$ \\
\hline 1 & $1.767 \mathrm{E}-04$ & $1.772 \mathrm{E}-04$ \\
2 & $4.238 \mathrm{E}-05$ & $4.314 \mathrm{E}-05$ \\
3 & $9.788 \mathrm{E}-06$ & $9.606 \mathrm{E}-06$ \\
4 & $4.606 \mathrm{E}-05$ & $4.644 \mathrm{E}-05$ \\
\hline
\end{tabular}

Table 3.38. SAXTON-4, Pin-1 four-group U-235 cross sections

\begin{tabular}{cccccccc}
\hline Group & \multicolumn{3}{c}{ CENTRM cross sections $\left(\mathrm{cm}^{-1}\right)$} & \multicolumn{3}{c}{ NITAWL cross sections $\left(\mathrm{cm}^{-1}\right)$} \\
\hline & $\frac{\mathrm{G}_{\mathrm{a}}}{2.28768 \mathrm{E}-04}$ & $\frac{\mathrm{G}_{\mathrm{f}}}{5.25936 \mathrm{E}-04}$ & $\frac{\mathrm{G}_{\mathrm{f}}}{2.01824 \mathrm{E}-04}$ & $\frac{\mathrm{G}_{\mathrm{a}}}{2.28888 \mathrm{E}-04}$ & $\frac{\mathrm{G}_{\mathrm{f}}}{5.26218 \mathrm{E}-04}$ & $\frac{\mathrm{G}_{\mathrm{f}}}{2.01905 \mathrm{E}-04}$ \\
1 & $5.58388 \mathrm{E}-03$ & $8.56052 \mathrm{E}-03$ & $3.51317 \mathrm{E}-03$ & $5.50211 \mathrm{E}-03$ & $8.45190 \mathrm{E}-03$ & $3.46859 \mathrm{E}-03$ \\
2 & $8.12596 \mathrm{E}-03$ & $1.65337 \mathrm{E}-02$ & $6.78529 \mathrm{E}-03$ & $8.00644 \mathrm{E}-03$ & $1.62619 \mathrm{E}-02$ & $6.67374 \mathrm{E}-03$ \\
3 & $7.52875 \mathrm{E}-02$ & $1.56735 \mathrm{E}-01$ & $6.43226 \mathrm{E}-02$ & $7.54139 \mathrm{E}-02$ & $1.57003 \mathrm{E}-01$ & $6.44324 \mathrm{E}-02$ \\
\hline
\end{tabular}

Table 3.39. SAXTON-4, Pin-1 four-group U-238 cross sections

\begin{tabular}{cccccccc}
\hline Group & \multicolumn{3}{c}{ CENTRM cross sections $\left(\mathrm{cm}^{-1}\right)$} & \multicolumn{3}{c}{ NITAWL cross sections $\left(\mathrm{cm}^{-1}\right)$} \\
\hline & $\frac{\mathrm{G}_{\mathrm{a}}}{4.34533 \mathrm{E}-03}$ & $\frac{\mathrm{G}_{\mathrm{f}}}{1.41658 \mathrm{E}-02}$ & $\frac{\mathrm{G}_{\mathrm{f}}}{5.02602 \mathrm{E}-03}$ & $\frac{7.35536 \mathrm{E}-03}{1.41852 \mathrm{E}-02}$ & $\frac{\mathrm{G}_{\mathrm{f}}}{5.03150 \mathrm{E}-03}$ \\
1 & $5.77836 \mathrm{E}-02$ & $6.06902 \mathrm{E}-06$ & $2.51437 \mathrm{E}-06$ & $5.53505 \mathrm{E}-02$ & $5.94471 \mathrm{E}-06$ & $2.46287 \mathrm{E}-06$ \\
2 & $1.12800 \mathrm{E}-02$ & $3.90072 \mathrm{E}-08$ & $1.61613 \mathrm{E}-08$ & $1.12638 \mathrm{E}-02$ & $3.87636 \mathrm{E}-08$ & $1.60605 \mathrm{E}-08$ \\
3 & $4.31348 \mathrm{E}-02$ & $2.02633 \mathrm{E}-07$ & $8.39546 \mathrm{E}-08$ & $4.31960 \mathrm{E}-02$ & $2.02927 \mathrm{E}-07$ & $8.40764 \mathrm{E}-08$ \\
\hline
\end{tabular}

Table 3.40. SAXTON-4, Pin-1 four-group Pu-239 cross sections

\begin{tabular}{cccccccc}
\hline Group & \multicolumn{3}{c}{ CENTRM cross sections $\left(\mathrm{cm}^{-1}\right)$} & \multicolumn{3}{c}{ NITAWL cross sections $\left(\mathrm{cm}^{-1}\right)$} \\
\hline & $\frac{\mathrm{G}_{\mathrm{a}}}{2.51088 \mathrm{E}-03}$ & $\frac{\mathrm{G}_{\mathrm{f}}}{7.39032 \mathrm{E}-03}$ & $\frac{\mathrm{G}_{\mathrm{f}}}{2.37191 \mathrm{E}-03}$ & $\frac{\mathrm{G}_{\mathrm{a}}}{2.51148 \mathrm{E}-03}$ & $\frac{\mathrm{G}_{\mathrm{f}}}{7.39283 \mathrm{E}-03}$ & $\frac{\mathrm{G}_{\mathrm{f}}}{2.37221 \mathrm{E}-03}$ \\
1 & $4.48570 \mathrm{E}-02$ & $7.50776 \mathrm{E}-02$ & $2.60703 \mathrm{E}-02$ & $4.28458 \mathrm{E}-02$ & $7.19822 \mathrm{E}-02$ & $2.49954 \mathrm{E}-02$ \\
2 & $1.48740 \mathrm{E}-01$ & $2.94317 \mathrm{E}-01$ & $1.02200 \mathrm{E}-01$ & $1.46544 \mathrm{E}-01$ & $2.90227 \mathrm{E}-01$ & $1.00780 \mathrm{E}-01$ \\
3 & $1.25958 \mathrm{E}+00$ & $2.54316 \mathrm{E}+00$ & $8.79726 \mathrm{E}-01$ & $1.25886 \mathrm{E}+00$ & $2.54285 \mathrm{E}+00$ & $8.79615 \mathrm{E}-01$ \\
\hline
\end{tabular}


Table 3.41. SAXTON-4, Pin-28 four-group fluxes

\begin{tabular}{ccc}
\hline Group & $\begin{array}{c}\text { CENTRM M } \\
\left(\mathrm{cm}^{-2}-\mathrm{s}^{-1}\right)\end{array}$ & $\begin{array}{c}\text { NITAWL M } \\
\left(\mathrm{cm}^{-2}-\mathrm{s}^{-1}\right)\end{array}$ \\
\hline 1 & $4.435 \mathrm{E}-04$ & $4.456 \mathrm{E}-04$ \\
2 & $1.401 \mathrm{E}-04$ & $1.409 \mathrm{E}-04$ \\
3 & $2.995 \mathrm{E}-05$ & $2.997 \mathrm{E}-05$ \\
4 & $5.644 \mathrm{E}-05$ & $5.789 \mathrm{E}-05$ \\
\hline
\end{tabular}

Table 3.42. SAXTON-4, Pin-28 four-group U-235 cross sections

\begin{tabular}{cccccccc}
\hline Group & \multicolumn{3}{c}{ CENTRM cross sections $\left(\mathrm{cm}^{-1}\right)$} & \multicolumn{3}{c}{ NITAWL cross sections $\left(\mathrm{cm}^{-1}\right)$} \\
\hline & $\frac{\mathrm{G}_{\mathrm{a}}}{4.37576 \mathrm{E}-04}$ & $\frac{\mathrm{G}_{\mathrm{f}}}{5.33610 \mathrm{E}-04}$ & $\frac{\mathrm{G}_{\mathrm{f}}}{2.06345 \mathrm{E}-04}$ & $\frac{\mathrm{G}_{\mathrm{a}}}{2.36654 \mathrm{E}-04}$ & $\frac{\mathrm{G}_{\mathrm{f}}}{5.32933 \mathrm{E}-04}$ & $\frac{\mathrm{G}_{\mathrm{f}}}{2.05880 \mathrm{E}-04}$ \\
1 & $5.42624 \mathrm{E}-03$ & $8.34946 \mathrm{E}-03$ & $3.42656 \mathrm{E}-03$ & $5.49204 \mathrm{E}-03$ & $8.44613 \mathrm{E}-03$ & $3.46621 \mathrm{E}-03$ \\
2 & $8.00180 \mathrm{E}-03$ & $1.62249 \mathrm{E}-02$ & $6.65856 \mathrm{E}-03$ & $7.91521 \mathrm{E}-03$ & $1.60134 \mathrm{E}-02$ & $6.57174 \mathrm{E}-03$ \\
3 & $7.09472 \mathrm{E}-02$ & $1.47553 \mathrm{E}-01$ & $6.05542 \mathrm{E}-02$ & $7.02795 \mathrm{E}-02$ & $1.46150 \mathrm{E}-01$ & $5.99789 \mathrm{E}-02$ \\
\hline
\end{tabular}

Table 3.43. SAXTON-4, Pin-28 four-group U-238 cross sections

\begin{tabular}{cccccccc}
\hline Group & \multicolumn{3}{c}{ CENTRM cross sections $\left(\mathrm{cm}^{-1}\right)$} & \multicolumn{3}{c}{ NITAWL cross sections $\left(\mathrm{cm}^{-1}\right)$} \\
\hline & $\frac{\mathrm{G}_{\mathrm{a}}}{7.11109 \mathrm{E}-03}$ & $\frac{\mathrm{G}_{\mathrm{f}}}{1.26244 \mathrm{E}-02}$ & $\frac{\mathrm{G}_{\mathrm{f}}}{4.48453 \mathrm{E}-03}$ & $\frac{7.14856 \mathrm{E}-03}{\mathrm{G}}$ & $\frac{\mathrm{G}_{\mathrm{f}}}{1.28317 \mathrm{E}-02}$ & $\frac{\mathrm{G}_{\mathrm{f}}}{4.55624 \mathrm{E}-03}$ \\
1 & $5.52425 \mathrm{E}-02$ & $5.99818 \mathrm{E}-06$ & $2.48502 \mathrm{E}-06$ & $5.40680 \mathrm{E}-02$ & $5.89807 \mathrm{E}-06$ & $2.44355 \mathrm{E}-06$ \\
2 & $1.12522 \mathrm{E}-02$ & $3.86845 \mathrm{E}-08$ & $1.60276 \mathrm{E}-08$ & $1.12054 \mathrm{E}-02$ & $3.82593 \mathrm{E}-08$ & $1.58516 \mathrm{E}-08$ \\
3 & $4.09068 \mathrm{E}-02$ & $1.91933 \mathrm{E}-07$ & $7.95213 \mathrm{E}-08$ & $4.05719 \mathrm{E}-02$ & $1.90337 \mathrm{E}-07$ & $7.88606 \mathrm{E}-08$ \\
\hline
\end{tabular}

Table 3.44. SAXTON-4, Pin-28 four-group Pu-239 cross sections

\begin{tabular}{|c|c|c|c|c|c|c|}
\hline \multirow[t]{2}{*}{ Group } & \multicolumn{3}{|c|}{ CENTRM cross sections $\left(\mathrm{cm}^{-1}\right)$} & \multicolumn{3}{|c|}{ NITAWL cross sections $\left(\mathrm{cm}^{-1}\right)$} \\
\hline & $\mathrm{G}_{\mathrm{a}}$ & $<\mathrm{G}_{\mathrm{f}}$ & $\mathrm{G}_{\mathrm{f}}$ & $\mathrm{G}_{\mathrm{a}-}$ & $<\mathrm{G}_{\mathrm{f}}$ & $\mathrm{G}_{\mathrm{f}}$ \\
\hline 1 & $2.52286 \mathrm{E}-03$ & $\overline{7.28582 \mathrm{E}-03}$ & $\overline{2.354} \overline{16 \mathrm{E}-03}$ & $2.52139 \mathrm{E}-03$ & $\overline{7.29800 \mathrm{E}-03}$ & $\overline{2.35595 \mathrm{E}-03}$ \\
\hline 2 & $4.55287 \mathrm{E}-02$ & 7.65767E-02 & 2.65909E-02 & 4.36759E-02 & 7.33527E-02 & 2.54713E-02 \\
\hline 3 & $1.45672 \mathrm{E}-01$ & $2.88541 \mathrm{E}-01$ & $1.00195 \mathrm{E}-01$ & $1.41609 \mathrm{E}-01$ & $2.80817 \mathrm{E}-01$ & $9.75128 \mathrm{E}-02$ \\
\hline 4 & $1.32655 \mathrm{E}+00$ & $2.62741 \mathrm{E}+00$ & 9.09199E-01 & $1.32947 \mathrm{E}+00$ & $2.62807 \mathrm{E}+00$ & $9.09471 \mathrm{E}-01$ \\
\hline
\end{tabular}




\begin{tabular}{|c|c|c|c|c|c|}
\hline \multirow[t]{5}{*}{$\begin{array}{l}0.274 \\
(0.94) \\
\end{array}$} & & & & & $\begin{array}{l}20.09 \\
(0.35)\end{array}$ \\
\hline & & & & $\begin{array}{l}18.84 \\
(0.33)\end{array}$ & $\begin{array}{c}19.55 \\
(0.23)\end{array}$ \\
\hline & & & $\begin{array}{l}16.65 \\
(0.35)\end{array}$ & $\begin{array}{l}17.71 \\
(0.21)\end{array}$ & $\begin{array}{l}18.36 \\
(0.23)\end{array}$ \\
\hline & & $\begin{array}{c}13.79 \\
(0.34)\end{array}$ & $\begin{array}{l}15.16 \\
(0.22)\end{array}$ & $\begin{array}{l}16.11 \\
(0.24)\end{array}$ & $\begin{array}{l}16.66 \\
(0.24)\end{array}$ \\
\hline & $\begin{array}{l}11.35 \\
(0.40)\end{array}$ & $\begin{array}{l}12.45 \\
(0.27)\end{array}$ & $\begin{array}{l}13.69 \\
(0.26)\end{array}$ & $\begin{array}{l}14.59 \\
(0.26)\end{array}$ & $\begin{array}{l}15.00 \\
(0.23)\end{array}$ \\
\hline $\begin{array}{c}13.36 \\
(0.35)\end{array}$ & $\begin{array}{l}12.75 \\
(0.28)\end{array}$ & $\begin{array}{c}14.14 \\
(0.23)\end{array}$ & $\begin{array}{l}15.59 \\
(0.23)\end{array}$ & $\begin{array}{l}16.47 \\
(0.23)\end{array}$ & $\begin{array}{c}17.04 \\
(0.24)\end{array}$ \\
\hline
\end{tabular}

Fig. 3.11a. Pin-power distribution for CENTRM benchmark SAXTON-5. Value in parentheses is percent standard deviation.

\begin{tabular}{|c|c|c|c|c|c|}
\hline \multirow[t]{5}{*}{$\begin{array}{l}0.273 \\
(0.90)\end{array}$} & & & & & $\begin{array}{l}20.26 \\
(0.30)\end{array}$ \\
\hline & & & & $\begin{array}{l}19.04 \\
(0.33)\end{array}$ & $\begin{array}{l}19.51 \\
(0.21)\end{array}$ \\
\hline & & & $\begin{array}{c}16.69 \\
(0.35)\end{array}$ & $\begin{array}{l}17.88 \\
(0.23)\end{array}$ & $\begin{array}{l}18.32 \\
(0.22)\end{array}$ \\
\hline & & $\begin{array}{l}13.84 \\
(0.38)\end{array}$ & $\begin{array}{l}15.25 \\
(0.24)\end{array}$ & $\begin{array}{l}16.23 \\
(0.23)\end{array}$ & $\begin{array}{l}16.66 \\
(0.23)\end{array}$ \\
\hline & $\begin{array}{l}11.26 \\
(0.44)\end{array}$ & $\begin{array}{c}12.43 \\
(0.29)\end{array}$ & $\begin{array}{c}13.78 \\
(0.25)\end{array}$ & $\begin{array}{l}14.67 \\
(0.26)\end{array}$ & $\begin{array}{l}15.07 \\
(0.25)\end{array}$ \\
\hline $\begin{array}{c}13.29 \\
(0.34)\end{array}$ & $\begin{array}{l}12.74 \\
(0.26)\end{array}$ & $\begin{array}{l}14.20 \\
(0.24)\end{array}$ & $\begin{array}{l}15.58 \\
(0.22)\end{array}$ & $\begin{array}{l}16.46 \\
(0.22)\end{array}$ & $\begin{array}{l}17.07 \\
(0.24)\end{array}$ \\
\hline
\end{tabular}

Fig. 3.11b. Pin-power distribution for NITAWL Benchmark SAXTON-5. Value in parentheses is percent standard deviation. 
Table 3.45. SAXTON-5 CENTRM reaction rates and fluxes

\begin{tabular}{|c|c|c|c|c|c|c|}
\hline Pin & Region & $\begin{array}{c}\mathrm{G}_{\mathrm{a}} \mathrm{M} \\
\left(\mathrm{cm}^{-3}-\mathrm{s}^{-1}\right)\end{array}$ & $\begin{array}{c}\mathcal{G}_{\mathrm{f}} \mathrm{M} \\
\left(\mathrm{cm}^{-3}-\mathrm{s}^{-1}\right)\end{array}$ & $\begin{array}{c}\mathrm{G}_{\mathrm{f}} \mathrm{M} \\
\left(\mathrm{cm}^{-3}-\mathrm{s}^{-1}\right)\end{array}$ & $\begin{array}{c}\mathrm{M} \\
\left(\mathrm{cm}-\mathrm{s}^{-1}\right)\end{array}$ & $\mathrm{MM}_{\mathrm{t}}$ \\
\hline \multirow{3}{*}{1} & Fuel & $7.340 \mathrm{E}-05$ & $1.334 \mathrm{E}-04$ & $4.665 \mathrm{E}-05$ & $1.142 \mathrm{E}-02$ & 0.148 \\
\hline & Clad & $6.001 \mathrm{E}-07$ & 0.0 & 0.0 & $3.327 \mathrm{E}-03$ & 0.043 \\
\hline & Mod. & 3.369E-06 & 0.0 & 0.0 & $6.246 \mathrm{E}-02$ & 0.809 \\
\hline \multirow{3}{*}{21} & Fuel & $1.175 \mathrm{E}-04$ & $2.005 \mathrm{E}-04$ & $7.008 \mathrm{E}-05$ & $2.753 \mathrm{E}-02$ & 0.147 \\
\hline & Clad & $1.081 \mathrm{E}-06$ & 0.0 & 0.0 & $8.169 \mathrm{E}-03$ & 0.043 \\
\hline & Mod. & $4.571 \mathrm{E}-06$ & 0.0 & 0.0 & $1.519 \mathrm{E}-01$ & 0.810 \\
\hline
\end{tabular}

Table 3.46. SAXTON-5 NITAWL reaction rates and fluxes

\begin{tabular}{ccccccc}
\hline Pin & Region & $\begin{array}{c}\mathrm{G}_{\mathrm{a}} \mathrm{M} \\
\left(\mathrm{cm}^{-3}-\mathrm{s}^{-1}\right)\end{array}$ & $\begin{array}{c}\mathrm{G}_{\mathrm{f}} \mathrm{M} \\
\left(\mathrm{cm}^{-3}-\mathrm{s}^{-1}\right)\end{array}$ & $\begin{array}{c}\mathrm{G}_{\mathrm{f}} \mathrm{M} \\
\left(\mathrm{cm}^{-3}-\mathrm{s}^{-1}\right)\end{array}$ & $\begin{array}{c}\mathrm{M} \\
\left({\left.\mathrm{cm}-\mathrm{s}^{-1}\right)}^{2}\right.\end{array}$ & $\mathrm{MM}_{\mathrm{t}}$ \\
\hline \multirow{2}{*}{1} & Fuel & $7.276 \mathrm{E}-05$ & $1.326 \mathrm{E}-04$ & $4.636 \mathrm{E}-05$ & $1.134 \mathrm{E}-02$ & 0.147 \\
& Clad & $5.939 \mathrm{E}-07$ & 0.0 & 0.0 & $3.314 \mathrm{E}-03$ & 0.043 \\
& Mod. & $1.683 \mathrm{E}-06$ & 0.0 & 0.0 & $6.233 \mathrm{E}-02$ & 0.810 \\
\hline \multirow{2}{*}{21} & Fuel & $1.185 \mathrm{E}-04$ & $2.024 \mathrm{E}-04$ & $7.072 \mathrm{E}-05$ & $2.776 \mathrm{E}-02$ & 0.147 \\
& Clad & $1.081 \mathrm{E}-06$ & 0.0 & 0.0 & $8.220 \mathrm{E}-03$ & 0.044 \\
& Mod. & $2.306 \mathrm{E}-06$ & 0.0 & 0.0 & $1.528 \mathrm{E}-01$ & 0.809 \\
\hline
\end{tabular}


Table 3.47. SAXTON-5, Pin-1 four-group fluxes

\begin{tabular}{ccc}
\hline Group & $\begin{array}{c}\text { CENTRM M } \\
\left(\mathrm{cm}^{-2}-\mathrm{s}^{-1}\right)\end{array}$ & $\begin{array}{c}\text { NITAWL M } \\
\left(\mathrm{cm}^{-2}-\mathrm{s}^{-1}\right)\end{array}$ \\
\hline 1 & $1.642 \mathrm{E}-04$ & $1.632 \mathrm{E}-04$ \\
2 & $3.938 \mathrm{E}-05$ & $3.897 \mathrm{E}-05$ \\
3 & $9.086 \mathrm{E}-06$ & $8.830 \mathrm{E}-06$ \\
4 & $4.517 \mathrm{E}-05$ & $4.505 \mathrm{E}-05$ \\
\hline
\end{tabular}

Table 3.48. SAXTON-5, Pin-1 four-group U-235 cross sections

\begin{tabular}{cccccccc}
\hline Group & \multicolumn{3}{c}{ CENTRM cross sections $\left(\mathrm{cm}^{-1}\right)$} & \multicolumn{3}{c}{ NITAWL cross sections $\left(\mathrm{cm}^{-1}\right)$} \\
\hline & $\frac{\mathrm{G}_{\mathrm{a}}}{4.28516 \mathrm{E}-04}$ & $\frac{\mathrm{G}_{\mathrm{f}}}{5.26334 \mathrm{E}-04}$ & $\frac{\mathrm{G}_{\mathrm{f}}}{2.01776 \mathrm{E}-04}$ & $\frac{\mathrm{G}_{\mathrm{a}}}{2.28104 \mathrm{E}-04}$ & $\frac{\mathrm{G}_{\mathrm{f}}}{5.25678 \mathrm{E}-04}$ & $\frac{\mathrm{G}_{\mathrm{f}}}{2.01507 \mathrm{E}-04}$ \\
1 & $5.57692 \mathrm{E}-03$ & $8.53438 \mathrm{E}-03$ & $3.50244 \mathrm{E}-03$ & $5.57593 \mathrm{E}-03$ & $8.52166 \mathrm{E}-03$ & $3.49720 \mathrm{E}-03$ \\
2 & $8.00912 \mathrm{E}-03$ & $1.62733 \mathrm{E}-02$ & $6.67842 \mathrm{E}-03$ & $7.98496 \mathrm{E}-03$ & $1.61621 \mathrm{E}-02$ & $6.63276 \mathrm{E}-03$ \\
3 & $7.58687 \mathrm{E}-02$ & $1.57957 \mathrm{E}-01$ & $6.48244 \mathrm{E}-02$ & $7.56584 \mathrm{E}-02$ & $1.57516 \mathrm{E}-01$ & $6.46433 \mathrm{E}-02$ \\
\hline
\end{tabular}

Table 3.49. SAXTON-5, Pin-1 four-group U-238 cross sections

\begin{tabular}{cccccccc}
\hline Group & \multicolumn{3}{c}{ CENTRM cross sections $\left(\mathrm{cm}^{-1}\right)$} & \multicolumn{3}{c}{ NITAWL cross sections $\left(\mathrm{cm}^{-1}\right)$} \\
\hline & $\frac{\mathrm{G}_{\mathrm{a}}}{7.40499 \mathrm{E}-03}$ & $\frac{\mathrm{G}_{\mathrm{f}}}{1.43721 \mathrm{E}-02}$ & $\frac{\mathrm{G}_{\mathrm{f}}}{5.09562 \mathrm{E}-03}$ & $\frac{7.36961 \mathrm{E}-03}{\mathrm{G}}$ & $\frac{\mathrm{G}_{\mathrm{f}}}{1.43170 \mathrm{E}-02}$ & $\frac{\mathrm{G}_{\mathrm{f}}}{5.07374 \mathrm{E}-03}$ \\
1 & $6.01938 \mathrm{E}-02$ & $6.08276 \mathrm{E}-06$ & $2.52006 \mathrm{E}-06$ & $5.60690 \mathrm{E}-02$ & $5.89946 \mathrm{E}-06$ & $2.44412 \mathrm{E}-06$ \\
2 & $1.12744 \mathrm{E}-02$ & $3.88600 \mathrm{E}-08$ & $1.61004 \mathrm{E}-08$ & $1.12557 \mathrm{E}-02$ & $3.87165 \mathrm{E}-08$ & $1.60410 \mathrm{E}-08$ \\
3 & $4.34215 \mathrm{E}-02$ & $2.04004 \mathrm{E}-07$ & $8.45229 \mathrm{E}-08$ & $4.33185 \mathrm{E}-02$ & $2.03512 \mathrm{E}-07$ & $8.43190 \mathrm{E}-08$ \\
\hline
\end{tabular}

Table 3.50. SAXTON-5, Pin-1 four-group Pu-239 cross sections

\begin{tabular}{|c|c|c|c|c|c|c|}
\hline \multirow[t]{2}{*}{ Group } & \multicolumn{3}{|c|}{ CENTRM cross sections $\left(\mathrm{cm}^{-1}\right)$} & \multicolumn{3}{|c|}{ NITAWL cross sections $\left(\mathrm{cm}^{-1}\right)$} \\
\hline & $\mathrm{G}_{\mathrm{a}}$ & $<\mathrm{G}_{\mathrm{f}}$ & $\mathrm{G}_{\mathrm{f}}$ & $\mathrm{G}_{\mathrm{a}-}$ & $<\mathrm{G}_{\mathrm{f}}$ & $\mathrm{G}_{\mathrm{f}}$ \\
\hline 1 & $2 . \overline{51193 \mathrm{E}-03}$ & $\overline{7.40536 \mathrm{E}-03}$ & $\overline{2.37431 \mathrm{E}-03}$ & $2.51011 \mathrm{E}-03$ & $\overline{7.40294 \mathrm{E}-03}$ & $\overline{2.37356 \mathrm{E}-03}$ \\
\hline 2 & 4.59985E-02 & 7.75008E-02 & 2.69116E-02 & 4.40523E-02 & 7.41950E-02 & 2.57639E-02 \\
\hline 3 & $1.50511 \mathrm{E}-01$ & $2.97520 \mathrm{E}-01$ & $1.03313 \mathrm{E}-01$ & $1.47223 \mathrm{E}-01$ & $2.91456 \mathrm{E}-01$ & $1.01207 \mathrm{E}-01$ \\
\hline 4 & $1.25860 \mathrm{E}+00$ & $2.54527 \mathrm{E}+00$ & 8.80429E-01 & $1.25753 \mathrm{E}+00$ & $2.54209 \mathrm{E}+00$ & 8.79338E-01 \\
\hline
\end{tabular}


Table 3.51. SAXTON-5, Pin-21 four-group fluxes

\begin{tabular}{ccc}
\hline Group & $\begin{array}{c}\text { CENTRM M } \\
\left(\mathrm{cm}^{-2}-\mathrm{s}^{-1}\right)\end{array}$ & $\begin{array}{c}\text { NITAWL M } \\
\left(\mathrm{cm}^{-2}-\mathrm{s}^{-1}\right)\end{array}$ \\
\hline 1 & $4.092 \mathrm{E}-04$ & $4.112 \mathrm{E}-04$ \\
2 & $1.239 \mathrm{E}-04$ & $1.261 \mathrm{E}-04$ \\
3 & $2.677 \mathrm{E}-05$ & $2.696 \mathrm{E}-05$ \\
4 & $6.193 \mathrm{E}-05$ & $6.260 \mathrm{E}-05$ \\
\hline
\end{tabular}

Table 3.52. SAXTON-5, Pin-21 four-group U-235 cross sections

\begin{tabular}{cccccccc}
\hline Group & \multicolumn{3}{c}{ CENTRM cross sections $\left(\mathrm{cm}^{-1}\right)$} & \multicolumn{2}{c}{ NITAWL cross sections $\left(\mathrm{cm}^{-1}\right)$} \\
\hline & $\frac{\mathrm{G}_{\mathrm{a}}}{2.36162 \mathrm{E}-04}$ & $\frac{\mathrm{G}_{\mathrm{f}}}{5.32867 \mathrm{E}-04}$ & $\frac{\mathrm{G}_{\mathrm{f}}}{2.05677 \mathrm{E}-04}$ & $\frac{\mathrm{G}_{\mathrm{a}}}{2.35623 \mathrm{E}-04}$ & $\frac{\mathrm{G}_{\mathrm{f}}}{5.32179 \mathrm{E}-04}$ & $\frac{\mathrm{G}_{\mathrm{f}}}{2.05354 \mathrm{E}-04}$ \\
1 & $5.48935 \mathrm{E}-03$ & $8.40097 \mathrm{E}-03$ & $3.44768 \mathrm{E}-03$ & $5.44674 \mathrm{E}-03$ & $8.35559 \mathrm{E}-03$ & $3.42905 \mathrm{E}-03$ \\
2 & $7.96989 \mathrm{E}-03$ & $1.61823 \mathrm{E}-02$ & $6.64109 \mathrm{E}-03$ & $7.98750 \mathrm{E}-03$ & $1.62231 \mathrm{E}-02$ & $6.65779 \mathrm{E}-03$ \\
3 & $7.21016 \mathrm{E}-02$ & $1.49992 \mathrm{E}-01$ & $6.15555 \mathrm{E}-02$ & $7.19091 \mathrm{E}-02$ & $1.49587 \mathrm{E}-01$ & $6.13891 \mathrm{E}-02$ \\
\hline
\end{tabular}

Table 3.53. SAXTON-5, Pin-21 four-group U-238 cross sections

\begin{tabular}{cccccccc}
\hline Group & \multicolumn{3}{c}{ CENTRM cross sections $\left(\mathrm{cm}^{-1}\right)$} & \multicolumn{3}{c}{ NITAWL cross sections $\left(\mathrm{cm}^{-1}\right)$} \\
\hline & $\frac{\mathrm{G}_{\mathrm{a}}}{4.19130 \mathrm{E}-03}$ & $\frac{\varangle \mathrm{G}_{\mathrm{f}}}{1.30115 \mathrm{E}-02}$ & $\frac{\mathrm{G}_{\mathrm{f}}}{4.61767 \mathrm{E}-03}$ & $\frac{\mathrm{G}_{\mathrm{a}}}{7.19283 \mathrm{E}-03}$ & $\frac{\mathrm{G}_{\mathrm{f}}}{1.30642 \mathrm{E}-02}$ & $\frac{\mathrm{G}_{\mathrm{f}}}{4.63670 \mathrm{E}-03}$ \\
1 & $5.75995 \mathrm{E}-02$ & $6.14358 \mathrm{E}-06$ & $2.54525 \mathrm{E}-06$ & $5.55646 \mathrm{E}-02$ & $6.04202 \mathrm{E}-06$ & $2.50319 \mathrm{E}-06$ \\
2 & $1.12575 \mathrm{E}-02$ & $3.87725 \mathrm{E}-08$ & $1.60642 \mathrm{E}-08$ & $1.12617 \mathrm{E}-02$ & $3.87641 \mathrm{E}-08$ & $1.60607 \mathrm{E}-08$ \\
3 & $4.14883 \mathrm{E}-02$ & $1.94726 \mathrm{E}-07$ & $8.06790 \mathrm{E}-08$ & $4.13946 \mathrm{E}-02$ & $1.94278 \mathrm{E}-07$ & $8.04931 \mathrm{E}-08$ \\
\hline
\end{tabular}

Table 3.54. SAXTON-5, Pin-21 four-group Pu-239 cross sections

\begin{tabular}{cccccccc}
\hline Group & \multicolumn{3}{c}{ CENTRM cross sections $\left(\mathrm{cm}^{-1}\right)$} & \multicolumn{3}{c}{ NITAWL cross sections $\left(\mathrm{cm}^{-1}\right)$} \\
\hline & $\frac{\mathrm{G}_{\mathrm{a}}}{2.52182 \mathrm{E}-03}$ & $\frac{\mathrm{G}_{\mathrm{f}}}{7.31114 \mathrm{E}-03}$ & $\frac{\mathrm{G}_{\mathrm{f}}}{2.35818 \mathrm{E}-03}$ & $\frac{\mathrm{G}_{\mathrm{a}}}{2.52033 \mathrm{E}-03}$ & $\frac{\mathrm{G}_{\mathrm{f}}}{7.31286 \mathrm{E}-03}$ & $\frac{\mathrm{G}_{\mathrm{f}}}{2.35814 \mathrm{E}-03}$ \\
1 & $4.59107 \mathrm{E}-02$ & $7.65671 \mathrm{E}-02$ & $2.65875 \mathrm{E}-02$ & $4.38622 \mathrm{E}-02$ & $7.38489 \mathrm{E}-02$ & $2.56436 \mathrm{E}-02$ \\
2 & $1.46728 \mathrm{E}-01$ & $2.90546 \mathrm{E}-01$ & $1.00891 \mathrm{E}-01$ & $1.47469 \mathrm{E}-01$ & $2.91907 \mathrm{E}-01$ & $1.01364 \mathrm{E}-01$ \\
3 & $1.31306 \mathrm{E}+00$ & $2.61230 \mathrm{E}+00$ & $9.03898 \mathrm{E}-01$ & $1.31380 \mathrm{E}+00$ & $2.61230 \mathrm{E}+00$ & $9.03907 \mathrm{E}-01$ \\
\hline
\end{tabular}




\begin{tabular}{|c|c|c|c|c|c|}
\hline \multirow[t]{5}{*}{$\begin{array}{l}0.349 \\
(1.00) \\
\end{array}$} & & & & & $\begin{array}{l}26.71 \\
(0.54)\end{array}$ \\
\hline & & & & $\begin{array}{l}25.24 \\
(0.26)\end{array}$ & $\begin{array}{r}26.06 \\
(0.27)\end{array}$ \\
\hline & & & $\begin{array}{l}22.18 \\
(0.30)\end{array}$ & $\begin{array}{l}23.68 \\
(0.19)\end{array}$ & $\begin{array}{l}24.15 \\
(0.28)\end{array}$ \\
\hline & & $\begin{array}{l}17.62 \\
(0.35)\end{array}$ & $\begin{array}{l}19.75 \\
(0.21)\end{array}$ & $\begin{array}{l}21.10 \\
(0.19)\end{array}$ & $\begin{array}{l}21.46 \\
(0.29)\end{array}$ \\
\hline & $\begin{array}{l}12.89 \\
(0.35)\end{array}$ & $\begin{array}{l}15.03 \\
(0.22)\end{array}$ & $\begin{array}{l}16.85 \\
(0.22)\end{array}$ & $\begin{array}{l}17.95 \\
(0.20)\end{array}$ & $\begin{array}{r}18.30 \\
(0.31)\end{array}$ \\
\hline $\begin{array}{c}10.69 \\
(0.39)\end{array}$ & $\begin{array}{c}11.87 \\
(0.28)\end{array}$ & $\begin{array}{c}13.89 \\
(0.26)\end{array}$ & $\begin{array}{l}15.51 \\
(0.25)\end{array}$ & $\begin{array}{c}16.58 \\
(0.23)\end{array}$ & $\begin{array}{c}16.92 \\
(0.35)\end{array}$ \\
\hline
\end{tabular}

Fig. 3.12a. Pin-power distribution for CENTRM benchmark SAXTON-6. Value in parentheses is percent standard deviation.

\begin{tabular}{|c|c|c|c|c|c|}
\hline \multirow[t]{5}{*}{$\begin{array}{l}0.341 \\
(1.01) \\
\end{array}$} & & & & & $\begin{array}{l}26.34 \\
(0.53)\end{array}$ \\
\hline & & & & $\begin{array}{l}25.27 \\
(0.28)\end{array}$ & $\begin{array}{l}25.86 \\
(0.27)\end{array}$ \\
\hline & & & $\begin{array}{l}22.14 \\
(0.31)\end{array}$ & $\begin{array}{l}23.68 \\
(0.20)\end{array}$ & $\begin{array}{r}24.16 \\
(0.27)\end{array}$ \\
\hline & & $\begin{array}{l}17.65 \\
(0.32)\end{array}$ & $\begin{array}{c}19.77 \\
(0.21)\end{array}$ & $\begin{array}{l}21.15 \\
(0.19)\end{array}$ & $\begin{array}{l}21.58 \\
(0.30)\end{array}$ \\
\hline & $\begin{array}{c}12.84 \\
(0.40)\end{array}$ & $\begin{array}{l}15.03 \\
(0.24)\end{array}$ & $\begin{array}{l}16.86 \\
(0.23)\end{array}$ & $\begin{array}{l}17.98 \\
(0.20)\end{array}$ & $\begin{array}{r}18.46 \\
(0.31)\end{array}$ \\
\hline $\begin{array}{l}10.75 \\
(0.42)\end{array}$ & $\begin{array}{c}11.84 \\
(0.27)\end{array}$ & $\begin{array}{c}13.94 \\
(0.26)\end{array}$ & $\begin{array}{c}15.58 \\
(0.24)\end{array}$ & $\begin{array}{c}16.62 \\
(0.23)\end{array}$ & $\begin{array}{c}16.94 \\
(0.30)\end{array}$ \\
\hline
\end{tabular}

Fig. 3.12b. Pin-power distribution for NITAWL benchmark SAXTON-6. Value in parentheses is percent standard deviation. 
Table 3.55. SAXTON-6 CENTRM reaction rates and fluxes

\begin{tabular}{|c|c|c|c|c|c|c|}
\hline Pin & Region & $\begin{array}{c}\mathrm{G}_{\mathrm{a}} \mathrm{M} \\
\left(\mathrm{cm}^{-3}-\mathrm{s}^{-1}\right)\end{array}$ & $\begin{array}{c}\mathcal{G}_{\mathrm{f}} \mathrm{M} \\
\left(\mathrm{cm}^{-3}-\mathrm{s}^{-1}\right)\end{array}$ & $\begin{array}{c}\mathrm{G}_{\mathrm{f}} \mathrm{M} \\
\left(\mathrm{cm}^{-3}-\mathrm{s}^{-1}\right)\end{array}$ & $\begin{array}{c}\mathrm{M} \\
\left(\mathrm{cm}-\mathrm{s}^{-1}\right)\end{array}$ & $\mathrm{MM}_{\mathrm{t}}$ \\
\hline \multirow{3}{*}{1} & Fuel & $5.801 \mathrm{E}-05$ & $1.068 \mathrm{E}-04$ & $3.734 \mathrm{E}-05$ & $8.227 \mathrm{E}-03$ & 0.085 \\
\hline & Clad & $4.571 \mathrm{E}-07$ & 0.0 & 0.0 & $2.381 \mathrm{E}-03$ & 0.024 \\
\hline & Mod. & $2.974 \mathrm{E}-06$ & 0.0 & 0.0 & $8.646 \mathrm{E}-02$ & 0.891 \\
\hline \multirow{3}{*}{21} & Fuel & $1.483 \mathrm{E}-04$ & $2.649 \mathrm{E}-04$ & $9.259 \mathrm{E}-05$ & $2.605 \mathrm{E}-02$ & 0.085 \\
\hline & Clad & $1.224 \mathrm{E}-06$ & 0.0 & 0.0 & $7.665 \mathrm{E}-03$ & 0.025 \\
\hline & Mod. & $6.914 \mathrm{E}-06$ & 0.0 & 0.0 & $2.718 \mathrm{E}-01$ & 0.890 \\
\hline
\end{tabular}

Table 3.56. SAXTON-6 NITAWL reaction rates and fluxes

\begin{tabular}{|c|c|c|c|c|c|c|}
\hline Pin & Region & $\begin{array}{c}\mathrm{G}_{\mathrm{a}} \mathrm{M} \\
\left(\mathrm{cm}^{-3}-\mathrm{s}^{-1}\right)\end{array}$ & $\begin{array}{c}\mathcal{G}_{\mathrm{f}} \mathrm{M} \\
\left(\mathrm{cm}^{-3}-\mathrm{s}^{-1}\right)\end{array}$ & $\begin{array}{c}\mathrm{G}_{\mathrm{f}} \mathrm{M} \\
\left(\mathrm{cm}^{-3}-\mathrm{s}^{-1}\right)\end{array}$ & $\begin{array}{c}\mathrm{M} \\
\left(\mathrm{cm}-\mathrm{s}^{-1}\right)\end{array}$ & $M / M_{t}$ \\
\hline \multirow{3}{*}{1} & Fuel & $5.849 \mathrm{E}-05$ & $1.078 \mathrm{E}-04$ & $3.768 \mathrm{E}-05$ & $8.318 \mathrm{E}-03$ & 0.085 \\
\hline & Clad & $4.597 \mathrm{E}-07$ & 0.0 & 0.0 & $2.405 \mathrm{E}-03$ & 0.025 \\
\hline & Mod. & $1.489 \mathrm{E}-06$ & 0.0 & 0.0 & 8.696E-02 & 0.890 \\
\hline \multirow{3}{*}{21} & Fuel & $1.484 \mathrm{E}-04$ & $2.656 \mathrm{E}-04$ & $9.284 \mathrm{E}-05$ & $2.592 \mathrm{E}-02$ & 0.085 \\
\hline & Clad & $1.212 \mathrm{E}-06$ & 0.0 & 0.0 & $7.589 \mathrm{E}-03$ & 0.025 \\
\hline & Mod. & $3.439 \mathrm{E}-06$ & 0.0 & 0.0 & $2.708 \mathrm{E}-01$ & 0.890 \\
\hline
\end{tabular}


Table 3.57. SAXTON-6, Pin-1 four-group fluxes

\begin{tabular}{ccc}
\hline Group & $\begin{array}{c}\text { CENTRM M } \\
\left(\mathrm{cm}^{-2}-\mathrm{s}^{-1}\right)\end{array}$ & $\begin{array}{c}\text { NITAWL M } \\
\left(\mathrm{cm}^{-2}-\mathrm{s}^{-1}\right)\end{array}$ \\
\hline 1 & $1.127 \mathrm{E}-04$ & $1.136 \mathrm{E}-04$ \\
2 & $2.431 \mathrm{E}-05$ & $2.487 \mathrm{E}-05$ \\
3 & $5.596 \mathrm{E}-06$ & $5.708 \mathrm{E}-06$ \\
4 & $3.680 \mathrm{E}-05$ & $3.720 \mathrm{E}-05$ \\
\hline
\end{tabular}

Table 3.58. SAXTON-6, Pin-1 four-group U-235 cross sections

\begin{tabular}{cccccccc}
\hline Group & \multicolumn{2}{c}{ CENTRM cross sections $\left(\mathrm{cm}^{-1}\right)$} & \multicolumn{3}{c}{ NITAWL cross sections $\left(\mathrm{cm}^{-1}\right)$} \\
\hline & $\frac{\mathrm{G}_{\mathrm{a}}}{4.25419 \mathrm{E}-04}$ & $\frac{\leftarrow_{\mathrm{f}}}{5.23524 \mathrm{E}-04}$ & $\frac{\mathrm{G}_{\mathrm{f}}}{2.00192 \mathrm{E}-04}$ & $\frac{\mathrm{G}_{\mathrm{a}}}{2.25627 \mathrm{E}-04}$ & $\frac{\mathrm{G}_{\mathrm{f}}}{5.23460 \mathrm{E}-04}$ & $\frac{\mathrm{G}_{\mathrm{f}}}{2.00250 \mathrm{E}-04}$ \\
1 & $5.64554 \mathrm{E}-03$ & $8.59223 \mathrm{E}-03$ & $3.52618 \mathrm{E}-03$ & $5.66043 \mathrm{E}-03$ & $8.68922 \mathrm{E}-03$ & $3.56598 \mathrm{E}-03$ \\
2 & $8.10920 \mathrm{E}-03$ & $1.64809 \mathrm{E}-02$ & $6.76363 \mathrm{E}-03$ & $8.14676 \mathrm{E}-03$ & $1.65761 \mathrm{E}-02$ & $6.80268 \mathrm{E}-03$ \\
3 & $7.66356 \mathrm{E}-02$ & $1.59575 \mathrm{E}-01$ & $6.54883 \mathrm{E}-02$ & $7.61884 \mathrm{E}-02$ & $1.58640 \mathrm{E}-01$ & $6.51043 \mathrm{E}-02$ \\
\hline
\end{tabular}

Table 3.59. SAXTON-6, Pin-1 four-group U-238 cross sections

\begin{tabular}{cccccccc}
\hline Group & \multicolumn{3}{c}{ CENTRM cross sections $\left(\mathrm{cm}^{-1}\right)$} & \multicolumn{3}{c}{ NITAWL cross sections $\left(\mathrm{cm}^{-1}\right)$} \\
\hline & $\frac{\mathrm{G}_{\mathrm{a}}}{7.46961 \mathrm{E}-03}$ & $\frac{\mathrm{G}_{\mathrm{f}}}{1.48484 \mathrm{E}-02}$ & $\frac{\mathrm{G}_{\mathrm{f}}}{5.26453 \mathrm{E}-03}$ & $\frac{\mathrm{G}_{\mathrm{a}}}{7.45908 \mathrm{E}-03}$ & $\frac{\mathrm{G}_{\mathrm{f}}}{1.47830 \mathrm{E}-02}$ & $\frac{\mathrm{G}_{\mathrm{f}}}{5.24259 \mathrm{E}-03}$ \\
1 & $6.30267 \mathrm{E}-02$ & $6.27162 \mathrm{E}-06$ & $2.59830 \mathrm{E}-06$ & $5.76920 \mathrm{E}-02$ & $5.74105 \mathrm{E}-06$ & $2.37850 \mathrm{E}-06$ \\
3 & $1.12849 \mathrm{E}-02$ & $3.89762 \mathrm{E}-08$ & $1.61485 \mathrm{E}-08$ & $1.13126 \mathrm{E}-02$ & $3.92234 \mathrm{E}-08$ & $1.62510 \mathrm{E}-08$ \\
4 & $4.38120 \mathrm{E}-02$ & $2.05877 \mathrm{E}-07$ & $8.52986 \mathrm{E}-08$ & $4.35935 \mathrm{E}-02$ & $2.04834 \mathrm{E}-07$ & $8.48667 \mathrm{E}-08$ \\
\hline
\end{tabular}

Table 3.60. SAXTON-6, Pin-1 four-group Pu-239 cross sections

\begin{tabular}{cccccccc}
\hline Group & \multicolumn{3}{c}{ CENTRM cross sections $\left(\mathrm{cm}^{-1}\right)$} & \multicolumn{3}{c}{ NITAWL cross sections $\left(\mathrm{cm}^{-1}\right)$} \\
\hline & $\frac{\mathrm{G}_{\mathrm{a}}}{2.50829 \mathrm{E}-03}$ & $\frac{\mathrm{G}_{\mathrm{f}}}{7.44201 \mathrm{E}-03}$ & $\frac{\mathrm{G}_{\mathrm{f}}}{2.38103 \mathrm{E}-03}$ & $\frac{\mathrm{G}_{\mathrm{a}}}{2.50741 \mathrm{E}-03}$ & $\frac{\mathrm{G}_{\mathrm{f}}}{7.43347 \mathrm{E}-03}$ & $\frac{\mathrm{G}_{\mathrm{f}}}{2.37922 \mathrm{E}-03}$ \\
1 & $4.78379 \mathrm{E}-02$ & $8.01259 \mathrm{E}-02$ & $2.78233 \mathrm{E}-02$ & $4.63764 \mathrm{E}-02$ & $7.75412 \mathrm{E}-02$ & $2.69258 \mathrm{E}-02$ \\
2 & $1.52214 \mathrm{E}-01$ & $3.00611 \mathrm{E}-01$ & $1.04386 \mathrm{E}-01$ & $1.54150 \mathrm{E}-01$ & $3.04373 \mathrm{E}-01$ & $1.05692 \mathrm{E}-01$ \\
3 & $1.24949 \mathrm{E}+00$ & $2.53488 \mathrm{E}+00$ & $8.76780 \mathrm{E}-01$ & $1.24891 \mathrm{E}+00$ & $2.53120 \mathrm{E}+00$ & $8.75527 \mathrm{E}-01$ \\
\hline
\end{tabular}


Table 3.61. SAXTON-6, Pin-21 four-group fluxes

\begin{tabular}{ccc}
\hline Group & $\begin{array}{c}\text { CENTRM M } \\
\left(\mathrm{cm}^{-2}-\mathrm{s}^{-1}\right)\end{array}$ & $\begin{array}{c}\text { NITAWL M } \\
\left(\mathrm{cm}^{-2}-\mathrm{s}^{-1}\right)\end{array}$ \\
\hline 1 & $3.626 \mathrm{E}-04$ & $3.592 \mathrm{E}-04$ \\
2 & $9.528 \mathrm{E}-05$ & $9.575 \mathrm{E}-05$ \\
3 & $2.230 \mathrm{E}-05$ & $2.206 \mathrm{E}-05$ \\
4 & $8.798 \mathrm{E}-05$ & $8.816 \mathrm{E}-05$ \\
\hline
\end{tabular}

Table 3.62. SAXTON-6, Pin-21 four-group U-235 cross sections

\begin{tabular}{cccccccc}
\hline Group & \multicolumn{3}{c}{ CENTRM cross sections $\left(\mathrm{cm}^{-1}\right)$} & \multicolumn{3}{c}{ NITAWL cross sections $\left(\mathrm{cm}^{-1}\right)$} \\
\hline & $\frac{\mathrm{G}_{\mathrm{a}}}{4.30565 \mathrm{E}-04}$ & $\frac{\mathrm{G}_{\mathrm{f}}}{5.27350 \mathrm{E}-04}$ & $\frac{\mathrm{G}_{\mathrm{f}}}{2.02688 \mathrm{E}-04}$ & $\frac{\mathrm{G}_{\mathrm{a}}}{2.30477 \mathrm{E}-04}$ & $\frac{\mathrm{G}_{\mathrm{f}}}{5.28068 \mathrm{E}-04}$ & $\frac{\mathrm{G}_{\mathrm{f}}}{2.02763 \mathrm{E}-04}$ \\
1 & $5.63375 \mathrm{E}-03$ & $8.59935 \mathrm{E}-03$ & $3.52909 \mathrm{E}-03$ & $5.56879 \mathrm{E}-03$ & $8.53534 \mathrm{E}-03$ & $3.50283 \mathrm{E}-03$ \\
2 & $8.06789 \mathrm{E}-03$ & $1.64302 \mathrm{E}-02$ & $6.74278 \mathrm{E}-03$ & $8.03178 \mathrm{E}-03$ & $1.64159 \mathrm{E}-02$ & $6.73694 \mathrm{E}-03$ \\
3 & $7.49118 \mathrm{E}-02$ & $1.55931 \mathrm{E}-01$ & $6.39925 \mathrm{E}-02$ & $7.47374 \mathrm{E}-02$ & $1.55564 \mathrm{E}-01$ & $6.38420 \mathrm{E}-02$ \\
\hline
\end{tabular}

Table 3.63. SAXTON-6, Pin-21 four-group U-238 cross sections

\begin{tabular}{cccccccc}
\hline Group & \multicolumn{2}{c}{ CENTRM cross sections $\left(\mathrm{cm}^{-1}\right)$} & \multicolumn{2}{c}{ NITAWL cross sections $\left(\mathrm{cm}^{-1}\right)$} \\
& $\frac{\mathrm{G}_{\mathrm{a}}}{7.31139 \mathrm{E}-03}$ & $\frac{\mathrm{G}_{\mathrm{f}}}{1.38701 \mathrm{E}-02}$ & $\frac{\mathrm{G}_{\mathrm{f}}}{4.92308 \mathrm{E}-03}$ & $\overline{7.34112 \mathrm{E}-03}$ & $\frac{\mathrm{G}_{\mathrm{f}}}{1.40133 \mathrm{E}-02}$ & $\frac{\mathrm{G}_{\mathrm{f}}}{4.96478 \mathrm{E}-03}$ \\
1 & $6.04901 \mathrm{E}-02$ & $6.16474 \mathrm{E}-06$ & $2.55402 \mathrm{E}-06$ & $5.89630 \mathrm{E}-02$ & $6.22799 \mathrm{E}-06$ & $2.58023 \mathrm{E}-06$ \\
2 & $1.12898 \mathrm{E}-02$ & $3.90791 \mathrm{E}-08$ & $1.61912 \mathrm{E}-08$ & $1.12885 \mathrm{E}-02$ & $3.90972 \mathrm{E}-08$ & $1.61987 \mathrm{E}-08$ \\
3 & $4.29311 \mathrm{E}-02$ & $2.01652 \mathrm{E}-07$ & $8.35480 \mathrm{E}-08$ & $4.28370 \mathrm{E}-02$ & $2.01200 \mathrm{E}-07$ & $8.33613 \mathrm{E}-08$ \\
\hline
\end{tabular}

Table 3.64. SAXTON-6, Pin-21 four-group Pu-239 cross sections

\begin{tabular}{cccccccc}
\hline Group & \multicolumn{3}{c}{ CENTRM cross sections $\left(\mathrm{cm}^{-1}\right)$} & \multicolumn{3}{c}{ NITAWL cross sections $\left(\mathrm{cm}^{-1}\right)$} \\
\hline & $\frac{\mathrm{G}_{\mathrm{a}}}{2.51210 \mathrm{E}-03}$ & $\frac{\mathrm{G}_{\mathrm{f}}}{7.36437 \mathrm{E}-03}$ & $\frac{\mathrm{G}_{\mathrm{f}}}{2.36645 \mathrm{E}-03}$ & $\frac{\mathrm{G}_{\mathrm{a}}}{2.51355 \mathrm{E}-03}$ & $\frac{\mathrm{G}_{\mathrm{f}}}{7.37904 \mathrm{E}-03}$ & $\frac{\mathrm{G}_{\mathrm{f}}}{2.36928 \mathrm{E}-03}$ \\
1 & $4.63848 \mathrm{E}-02$ & $7.74581 \mathrm{E}-02$ & $2.68969 \mathrm{E}-02$ & $4.49666 \mathrm{E}-02$ & $7.52761 \mathrm{E}-02$ & $2.61392 \mathrm{E}-02$ \\
2 & $1.47063 \mathrm{E}-01$ & $2.91411 \mathrm{E}-01$ & $1.01191 \mathrm{E}-01$ & $1.47665 \mathrm{E}-01$ & $2.92500 \mathrm{E}-01$ & $1.01570 \mathrm{E}-01$ \\
3 & $1.27191 \mathrm{E}+00$ & $2.56133 \mathrm{E}+00$ & $8.86051 \mathrm{E}-01$ & $1.27598 \mathrm{E}+00$ & $2.56709 \mathrm{E}+00$ & $8.88067 \mathrm{E}-01$ \\
\hline
\end{tabular}




\section{MIX-COMP-THERM-4 (TCA-1 TO TCA-11)}

\subsection{DESCRIPTION}

This section describes a set of 11 critical experiments, each consisting of a square-pitched array of mixed Plutonium-Uranium fuel rods partially submerged in water surrounded by a water reflector. The water-to-fuel ratios for the arrays range from 2.4 to 5.6. This set of experiments is contained in the International Handbook of Evaluated Criticality Safety Benchmark Experiment. 3

This set of experiments was performed between 1972 and 1975 at the Tokai Research Establishment of JAERI. The Tank-Type Critical Assembly (TCA) benchmark cases are light-water moderated critical assemblies, consisting of a core array supported by upper, middle, and lower grid plates. The grid plates do not pass through the fuel region. The fuel is sufficiently long such that the water level is below the top of the fuel region in all cases. The reactor is brought to critical by raising the water level in the tank, thus avoiding the use of control rods. The fuel rods sit on a support plate above the bottom of the tank. The tank is wide enough to assume an infinite moderator on the sides ( $30 \mathrm{~cm}$ of water).

All fuel rods have the same physical dimensions. A schematic diagram of the fuel rods and bottom reflector is given in Fig. 4.1. Each fuel rod has an active fuel length of $70.6 \mathrm{~cm}$ and a $16.83-$ $\mathrm{cm}$-long bottom aluminum end plug that sits on a $1.27-\mathrm{cm}$-thick aluminum support plate. The fuel has a radius of $0.5325 \mathrm{~cm}$. The cladding has an outside radius of $0.6115 \mathrm{~cm}$. For the calculation, the cladding is extended $9.97 \mathrm{~cm}$ above the active fuel to the bottom of the middle grid plate. The middle grid plate and everything above are assumed to be insignificant and thus excluded from the model. The fuel lattice is surrounded by $30 \mathrm{~cm}$ of water on the four sides from the bottom of the tank to the top of the critical water level.

From the top of the aluminum support plate is a $4.445-\mathrm{cm}$ water gap and $0.601-\mathrm{cm}$ lower aluminum grid plate. Below the aluminum support plate is a 2.2-cm-thick stainless steel (SS) support plate, a 13.8-cm water gap, 0.5-cm-thick SS tank liner and $37.0 \mathrm{~cm}$ of concrete.

The primary differences between the 11 benchmarks are lattice pitch, number of rods in the lattice, water level, and Pu-241 and Am-241 number densities. All other benchmark characteristics are constant. The 11 benchmarks are divided into four different lattice pitches: $1.825 \mathrm{~cm}, 1.956 \mathrm{~cm}$, $2.225 \mathrm{~cm}$, and $2.474 \mathrm{~cm}$. For a given pitch, the number of pins in the lattice is given. The fuel is arranged in a square-pitched square lattice. The characteristics of each of the four lattices are given in Table 4.1. The critical fuel height variations are due to the changes in $\mathrm{Pu}-241$ and $\mathrm{Am}-241$ atom densities. Table 4.2 contains the atom densities for all the materials in the problem except $\mathrm{Pu}-241$ and Am-241. The atom densities in Table 4.2 are constant for all benchmarks. Table 4.3 contains the atom densities for Pu-241 and Am-241 for each benchmark. All material temperatures are assumed to be $20^{\circ} \mathrm{C}$. 

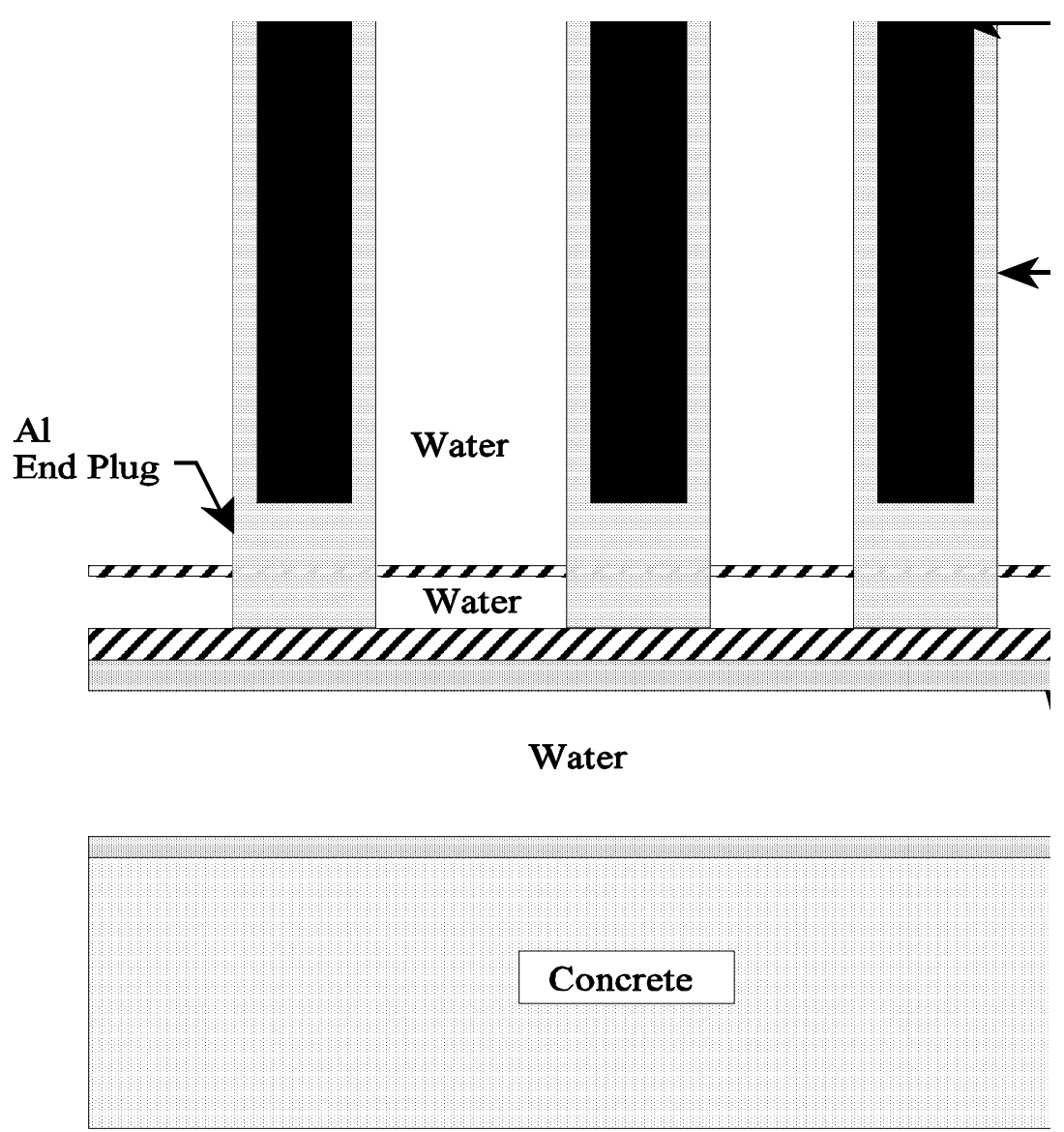

Fig. 4.1. Schematic of the TCA fuel rods and bottom reflector. 
Table 4.1. Lattice description for benchmarks

\begin{tabular}{cccccc}
\hline $\begin{array}{c}\text { Benchmark } \\
\text { No. }\end{array}$ & $\begin{array}{c}\text { Water/Fuel } \\
\text { volume ratio } \\
(\text { H/Pu ratio })\end{array}$ & $\begin{array}{c}\text { Lattice } \\
\text { pitch } \\
(\mathrm{cm})\end{array}$ & $\begin{array}{c}\text { Number } \\
\text { of rods } \\
\text { on a Side }\end{array}$ & $\begin{array}{c}\text { Lattice side } \\
\text { dimension } \\
(\mathrm{cm})\end{array}$ & $\begin{array}{c}\text { Critical } \\
\text { water level } \\
(\mathrm{cm})\end{array}$ \\
\hline 1 & & & & & 59.5 \\
2 & $\begin{array}{c}2.42 \\
(402)\end{array}$ & 1.825 & 23 & 41.975 & 91.90 \\
3 & & & & & 64.06 \\
\hline 4 & 2.98 & 1.956 & 21 & 41.076 & 61.50 \\
5 & $(494)$ & & & & 64.40 \\
6 & & & & & 69.40 \\
\hline 7 & 4.24 & $\mathrm{p} 2.225$ & 20 & 44.500 & 60.32 \\
8 & $(703)$ & & & & 62.99 \\
9 & & & & & 65.63 \\
\hline 10 & 5.55 & 2.474 & 21 & 51.954 & 62.05 \\
11 & $(921)$ & & & & 64.53 \\
\hline
\end{tabular}

The first three benchmarks; TCA-1, TCA-2, and TCA-3 have a pitch of $1.825 \mathrm{~cm}$ and $23 \times 23$ pin lattice. This pitch produces a water-to-fuel volume ratio of $2.42 \mathrm{~cm}$ and a $\mathrm{H} / \mathrm{Pu}$ ratio of 402 . To simplify the production of the lattice pin-power distribution and improve the statistics, the lattice advantage is taken of the lattice symmetry. Only $1 / 8$ th of the lattice needs to be modeled precisely to produce the pin-power distribution. The lattice is then filled from the $1 / 8$ th section by inserting additional pins in a mirror image. Figure 4.2 is a lattice map of the pin locations.

The same approach is used for the next three benchmarks: TCA-4, TCA-5, and TCA-6. These benchmarks have a pitch of $1.956 \mathrm{~cm}$ and $21 \times 21$ pin lattice. This pitch produces a water-to-fuel volume ratio of $2.98 \mathrm{~cm}$ and a $\mathrm{H} / \mathrm{Pu}$ ratio of 494 . The symmetry of the lattice is used in the same way it was used in the first three benchmarks. One-eighth of the lattice is filled, and the remaining lattice is filled assuming a mirror image. Figure 4.3 is a lattice map of the pin locations.

The next three benchmark problemsCTCA-7, TCA-8, and TCA-9Cagain use the same approach. These benchmarks have a pitch of $2.225 \mathrm{~cm}$ and a $20 \times 20$ pin lattice. This pitch produces a water-to-fuel volume ratio of $4.24 \mathrm{~cm}$ and a $\mathrm{H} / \mathrm{Pu}$ ratio of 703 . Figure 4.4 is a lattice map of the pin locations.

The last benchmark problemsCTCA-10 and TCA-11Chave the same lattice arrangement as the second set of benchmarks (see Fig. 4.3). However, these benchmarks have of pitch of $2.474 \mathrm{~cm}$ which produces a water-to-fuel volume ratio of $5.55 \mathrm{~cm}$ and a $\mathrm{H} / \mathrm{Pu}$ ratio of 921 . The input files for each of the benchmark problems can be found in Appendix C. 


\subsection{ANALYSIS}

All 11 computational benchmarks in this section were processed twice using SCALE 5.0. The set labeled NITAWL uses the NITAWL resonance processor to self-shield the resolved resonance region for all nuclides in the fuel and clad. An identical set, labeled CENTRM, replaces NITAWL with the CENTRM/PMC code sequence. The NITAWL and CENTRM results are then compared.

Table 4.4 contains the $k_{\text {eff }}$ and Energy of the Average Lethargy Causing Fission (EALCF). The $k_{\text {eff }}$ values for all the benchmark cases are very close to 1.0: the worst NITAWL benchmark is $0.26 \%$ high, and the worst CENTRM case is $0.37 \%$ low. There does appear to be a small negative bias between NITAWL and CENTRM, the CENTRM cases being about $0.2 \%$ lower on average than the NITAWL cases. The EALCF values listed are from the CENTRM cases. For all cases the difference between this value for CENTRM and NITAWL was less than $0.1 \%$. Both CENTRM and NITAWL produce excellent results for the $k_{\text {eff }}$ and fission energy for this set of problems.

Also, calculated for each problem is the: pin-power distributions; absorption, $\mathbb{G}_{\mathrm{f}}$, and fission reaction rates and fluxes in the pin fuel, clad, and moderator; and four group cross sections and fluxes for a corner outside pin and the innermost pin. Each of these sets of data are calculated using both CENTRM and NITAWL. The data for each case are contained in Figs. 4.5a through 4.15b and Tables 4.114 through 4.5 .

The pin-power distributions for this problem assumed 1/8th core symmetry with a surrounding reflector. The pin-power data consist of a value and a standard deviation for each pin. The values for the pin-powers are actually in units of fissions per $\mathrm{cm}^{3}$-s-source particle $\times 10^{-5}$. The value in parentheses is the percent standard deviation of the pin-power value. In most cases, the CENTRM and NITAWL results for each benchmark case agree within 2 standard deviations. The pin-power distributions represent that portion of the pin covered by water. The value off to the side in the pinpower distributions represents all the fuel not covered by water. The peak-to-low power changed with respect to pitch. The peak-to-low-power ratio changed from a low of 2.7 for the smallest pitch to a high of 4.9 at the largest pitch.

The reaction rates, total fluxes, and flux ratios for the CENTRM and NITAWL cases of each benchmark case are also in good agreement, seldom varying by more than $1 \%$. These values are included for a corner pin and the center pin for each case. The flux ratios over the fuel, clad, and moderator stay relatively constant for a given pitch. However, as the pitch increases the proportion of the total pin flux in the moderator increases.

Finally, four-group fluxes and cross sections were calculated for selected nuclides in the fuel region of the same corner and center pins. The macroscopic cross sections include the radiative capture, fission, and nu*fission cross sections for U-235, U-238, and $\mathrm{Pu}-239$. The four groups are collapsed from the 238-group multigroup cross-section set using the flux profile calculated in KENOV.a as follows: group 1 is from $20 \mathrm{Mev}$ to $9.5 \mathrm{keV}$, group 2 is from $9.5 \mathrm{keV}$ to $3.0 \mathrm{eV}$, Group 3 is from $3 \mathrm{eV}$ to $0.4 \mathrm{eV}$, and group 4 is from $0.4 \mathrm{eV}$ to $10^{-5} \mathrm{eV}$. The $0.4 \mathrm{eV}$ was chosen as a boundary because it is the cadmium cutoff energy. Groups 2 and 3 contain the resolved resonance regions for most of the nuclides used in these cases. Most of the cross-section data for the CENTRM and NITAWL cases of a given benchmark case agree within $1 \%$. 


\subsection{CONCLUSIONS}

For this set of benchmark cases, using either NITAWL and CENTRM as the resonance region processor produces acceptable results of the $k_{\text {eff }}$ values. All other values produced using CENTRM and NITAWL are also consistent with each. The $k_{\text {eff }}$ values are slightly lower, $\sim 0.2 \%$, for all CENTRM cases. The pin-power distributions, fluxes, reaction rates, and macroscopic cross sections generally agree within approximately $1 \%$ between CENTRM and NITAWL. No significant differences were identified between the results produced by NITAWL and CENTRM for these benchmark cases. 
Table 4.2. Benchmark atom densities

\begin{tabular}{|c|c|c|c|c|c|}
\hline Material & Isotope & $\begin{array}{c}\text { Atom density } \\
\text { (atoms/barn-cm) }\end{array}$ & Material & Isotope & $\begin{array}{c}\text { Atom density } \\
\text { (atoms/barn-cm) }\end{array}$ \\
\hline Fuel & $\begin{array}{c}\mathrm{U}-234 \\
\mathrm{U}-235 \\
\mathrm{U}-238 \\
\mathrm{Pu}-238 \\
\mathrm{Pu}-239 \\
\mathrm{Pu}-240 \\
\mathrm{Pu}-241 \\
\mathrm{Pu}-242 \\
\mathrm{Am}-241 \\
\mathrm{O}-16 \\
\mathrm{~B}-10 \\
\mathrm{~B}-11\end{array}$ & $\begin{array}{c}7.1749 \times 10^{-7} \\
9.3926 \times 10^{-5} \\
1.2951 \times 10^{-2} \\
2.0003 \times 10^{-6} \\
2.7491 \times 10^{-4} \\
8.8417 \times 10^{-5} \\
(\text { See Table 3.3) } \\
8.1234 \times 10^{-6} \\
(\text { See Table 3.3) } \\
2.7837 \times 10^{-2} \\
6.0418 \times 10^{-8} \\
2.4319 \times 10^{-7}\end{array}$ & $\begin{array}{l}\text { Ordinary } \\
\text { concrete }\end{array}$ & $\begin{array}{c}\mathrm{H} \\
\mathrm{O} \\
\mathrm{C} \\
\mathrm{Na} \\
\mathrm{Mg} \\
\mathrm{Al} \\
\mathrm{Si} \\
\mathrm{K} \\
\mathrm{Ca} \\
\mathrm{Fe}\end{array}$ & $\begin{array}{l}1.3742 \times 10^{-2} \\
4.5919 \times 10^{-2} \\
1.1532 \times 10^{-4} \\
9.6395 \times 10^{-4} \\
1.2388 \times 10^{-4} \\
1.7409 \times 10^{-3} \\
1.6617 \times 10^{-2} \\
4.6052 \times 10^{-4} \\
1.5025 \times 10^{-3} \\
3.4492 \times 10^{-4}\end{array}$ \\
\hline $\begin{array}{l}\text { Cladding } \\
+ \text { Air gap }\end{array}$ & $\begin{array}{l}\mathrm{Zr} \\
\mathrm{Sn} \\
\mathrm{Fe} \\
\mathrm{Cr} \\
\mathrm{Ni}\end{array}$ & $\begin{array}{l}3.7772 \times 10^{-2} \\
4.3737 \times 10^{-4} \\
8.8570 \times 10^{-5} \\
6.6119 \times 10^{-5} \\
3.5864 \times 10^{-5}\end{array}$ & $\begin{array}{c}\text { Stainless } \\
\text { steel } \\
(\mathrm{SS}-304 \mathrm{~L})\end{array}$ & $\begin{array}{c}\mathrm{C} \\
\mathrm{Si} \\
\mathrm{Mn} \\
\mathrm{P} \\
\mathrm{S} \\
\mathrm{Ni} \\
\mathrm{Cr} \\
\mathrm{Fe}\end{array}$ & $\begin{array}{l}1.1928 \times 10^{-4} \\
1.7003 \times 10^{-3} \\
1.7385 \times 10^{-3} \\
6.9381 \times 10^{-5} \\
4.4673 \times 10^{-5} \\
8.9506 \times 10^{-3} \\
1.7450 \times 10^{-2} \\
5.7202 \times 10^{-2}\end{array}$ \\
\hline $\begin{array}{l}\text { Water }\left(20^{\circ} \mathrm{C}\right) \\
0.9982 \mathrm{~g} / \mathrm{cc}\end{array}$ & $\begin{array}{l}\mathrm{H} \\
\mathrm{O}\end{array}$ & $\begin{array}{l}6.6735 \times 10^{-2} \\
3.3368 \times 10^{-2}\end{array}$ & Aluminum & $\mathrm{Al}$ & $6.0224 \times 10^{-2}$ \\
\hline
\end{tabular}


Table 4.3. Pu-241 and Am-241 atom densities

\begin{tabular}{ccc}
\hline & \multicolumn{2}{c}{ Atom densities (atoms/barn-cm $)$} \\
\cline { 2 - 3 } $\begin{array}{c}\text { Benchmark } \\
\text { No. }\end{array}$ & Pu-241 & Am-241 \\
\hline 1 & $2.7923 \times 10^{-5}$ & $1.3531 \times 10^{-6}$ \\
2 & $2.6701 \times 10^{-5}$ & $2.5812 \times 10^{-6}$ \\
3 & $2.5447 \times 10^{-5}$ & $3.8361 \times 10^{-6}$ \\
4 & $2.8003 \times 10^{-5}$ & $1.2793 \times 10^{-6}$ \\
5 & $2.6670 \times 10^{-5}$ & $2.6129 \times 10^{-6}$ \\
6 & $2.4228 \times 10^{-5}$ & $5.0543 \times 10^{-6}$ \\
7 & $2.8133 \times 10^{-5}$ & $1.1498 \times 10^{-6}$ \\
8 & $2.6649 \times 10^{-5}$ & $2.6340 \times 10^{-6}$ \\
9 & $2.5373 \times 10^{-5}$ & $3.9098 \times 10^{-6}$ \\
10 & $2.8077 \times 10^{-5}$ & $1.2053 \times 10^{-6}$ \\
11 & $2.6617 \times 10^{-5}$ & $2.6656 \times 10^{-6}$ \\
\hline
\end{tabular}

Table 4.4. Comparison of $k_{\text {eff }}$ and EALCF from CENTRM and NITAWL ${ }^{a}$

\begin{tabular}{ccccc}
\hline $\begin{array}{c}\text { Benchmark } \\
\text { identification }\end{array}$ & $\begin{array}{c}\text { CENTRM } \\
k_{\text {eff }}( \pm \mathrm{F})\end{array}$ & $\begin{array}{c}\text { NITAWL } \\
k_{\text {eff }}( \pm \mathrm{F})\end{array}$ & $\begin{array}{c}\text { EALF } \\
(\mathrm{eV})\end{array}$ & $\begin{array}{c}k_{\text {eff }} \\
\% \text { DIFF }\end{array}$ \\
\hline TCA-1 & $0.9963(0.0005)$ & $0.9993(0.0004)$ & 0.145 & -0.3 \\
TCA-2 & $0.9969(0.0004)$ & $1.0001(0.0004)$ & 0.144 & -0.32 \\
TCA-3 & $0.9985(0.0005)$ & $1.0002(0.0004)$ & 0.143 & -0.17 \\
TCA-4 & $0.9973(0.0004)$ & $0.9995(0.0004)$ & 0.119 & -0.22 \\
TCA-5 & $0.9982(0.0004)$ & $1.0008(0.0004)$ & 0.118 & -0.26 \\
TCA-6 & $0.9988(0.0004)$ & $1.0003(0.0003)$ & 0.117 & -0.15 \\
TCA-7 & $0.9994(0.0004)$ & $1.0016(0.0004)$ & 0.0927 & -0.22 \\
TCA-8 & $1.0002(0.0004)$ & $1.0017(0.0003)$ & 0.0923 & -0.15 \\
TCA-9 & $1.0009(0.0004)$ & $1.0024(0.0004)$ & 0.0916 & -0.15 \\
TCA-10 & $1.0003(0.0003)$ & $1.0020(0.0004)$ & 0.0797 & -0.17 \\
TCA-11 & $1.0010(0.0004)$ & $1.0026(0.0004)$ & 0.0792 & -0.16 \\
\hline
\end{tabular}

${ }^{a}$ Value in parentheses is percent standard deviation. 


\begin{tabular}{|c|c|c|c|c|c|c|c|c|c|c|c|}
\hline & 78 \\
\hline & & & & & & & & & & 76 & 77 \\
\hline & & & & & & & & & 73 & 74 & 75 \\
\hline & & & & & & & & 69 & 70 & 71 & 72 \\
\hline & & & & & & & 64 & 65 & 66 & 67 & 68 \\
\hline & & & & & & 58 & 59 & 60 & 61 & 62 & 63 \\
\hline & & & & & 51 & 52 & 53 & 54 & 55 & 56 & 57 \\
\hline & & & & 43 & 44 & 45 & 46 & 47 & 48 & 49 & 50 \\
\hline & & & 34 & 35 & 36 & 37 & 38 & 39 & 40 & 41 & 42 \\
\hline & & 24 & 25 & 26 & 27 & 28 & 29 & 30 & 31 & 32 & 33 \\
\hline & 13 & 14 & 15 & 16 & 17 & 18 & 19 & 20 & 21 & 22 & 23 \\
\hline 1 & 2 & 3 & 4 & 5 & 6 & 7 & 8 & 9 & 10 & 11 & 12 \\
\hline
\end{tabular}

Fig. 4.2. Pin layout of benchmark cases TCA-1, TCA-2, and TCA-3.

\begin{tabular}{|c|c|c|c|c|c|c|c|c|c|c|}
\hline & & & & & & & & & & 66 \\
\hline & & & & & & & & & 64 & 65 \\
\hline & & & & & & & & 61 & 62 & 63 \\
\hline & & & & & & & 57 & 58 & 59 & 60 \\
\hline & & & & & & 52 & 53 & 54 & 55 & 56 \\
\hline & & & & & 46 & 47 & 48 & 49 & 50 & 51 \\
\hline & & & & 39 & 40 & 41 & 42 & 43 & 44 & 45 \\
\hline & & & 31 & 32 & 33 & 34 & 35 & 36 & 37 & 38 \\
\hline & & 22 & 23 & 24 & 25 & 26 & 27 & 28 & 29 & 30 \\
\hline & 12 & 13 & 14 & 15 & 16 & 17 & 18 & 19 & 20 & 21 \\
\hline 1 & 2 & 3 & 4 & 5 & 6 & 7 & 8 & 9 & 10 & 11 \\
\hline
\end{tabular}

Fig. 4.3. Pin layout of benchmark cases TCA-4, TCA-5 ,TCA-6, TCA-10, and TCA-11. 


\begin{tabular}{|c|c|c|c|c|c|c|c|c|c|}
\hline & & & & & & & & & 55 \\
\hline & & & & & & & & 53 & 54 \\
\hline & & & & & & & 50 & 51 & 52 \\
\hline & & & & & & 46 & 47 & 48 & 49 \\
\hline & & & & & 41 & 42 & 43 & 44 & 45 \\
\hline & & & & 35 & 36 & 37 & 38 & 39 & 40 \\
\hline & & & 28 & 29 & 30 & 31 & 32 & 33 & 34 \\
\hline & & 20 & 21 & 22 & 23 & 24 & 25 & 26 & 27 \\
\hline & 11 & 12 & 13 & 14 & 15 & 16 & 17 & 18 & 19 \\
\hline 1 & 2 & 3 & 4 & 5 & 6 & 7 & 8 & 9 & 10 \\
\hline
\end{tabular}

Fig. 4.4. Pin layout of benchmark cases TCA-7, TCA-8, and TCA-9. 


\begin{tabular}{|c|c|c|c|c|c|c|c|c|c|c|c|}
\hline & & & & & & & & & & & $\begin{array}{l}5.299 \\
(0.91)\end{array}$ \\
\hline & $\begin{array}{c}0.131 \\
(0.59)\end{array}$ & & & & & & & & & $\begin{array}{c}5.191 \\
(0.46)\end{array}$ & $\begin{array}{l}5.175 \\
(0.48)\end{array}$ \\
\hline & & & & & & & & & $\begin{array}{l}4.954 \\
(0.48)\end{array}$ & $\begin{array}{c}5104 \\
(0.36)\end{array}$ & $\begin{array}{l}5.242 \\
(0.48)\end{array}$ \\
\hline & & & & & & & & $\begin{array}{l}4.722 \\
(0.51)\end{array}$ & $\begin{array}{l}4.871 \\
(0.33)\end{array}$ & $\begin{array}{l}4.971 \\
(0.34)\end{array}$ & $\begin{array}{l}4.937 \\
(0.48)\end{array}$ \\
\hline & & & & & & & $\begin{array}{l}4.426 \\
(0.51)\end{array}$ & $\begin{array}{l}4.601 \\
(0.33)\end{array}$ & $\begin{array}{l}4.697 \\
(0.36)\end{array}$ & $\begin{array}{l}4.759 \\
(0.36)\end{array}$ & $\begin{array}{l}4.815 \\
(0.54)\end{array}$ \\
\hline & & & & & & $\begin{array}{l}3.982 \\
(0.50)\end{array}$ & $\begin{array}{l}4.212 \\
(0.39)\end{array}$ & $\begin{array}{l}4.340 \\
(0.37)\end{array}$ & $\begin{array}{l}4.479 \\
0.36)\end{array}$ & $\begin{array}{l}4.554 \\
(0.33)\end{array}$ & $\begin{array}{l}4.551 \\
(0.44)\end{array}$ \\
\hline & & & & & $\begin{array}{l}3.535 \\
(0.54)\end{array}$ & $\begin{array}{l}3.766 \\
(0.44)\end{array}$ & $\begin{array}{l}3.934 \\
(0.40)\end{array}$ & $\begin{array}{l}4.068 \\
(0.40)\end{array}$ & $\begin{array}{l}4.184 \\
(0.38)\end{array}$ & $\begin{array}{l}4.252 \\
(0.38)\end{array}$ & $\begin{array}{l}4.286 \\
(0.53)\end{array}$ \\
\hline & & & & $\begin{array}{l}3.058 \\
(060)\end{array}$ & $\begin{array}{l}3.295 \\
(0.43)\end{array}$ & $\begin{array}{l}3.456 \\
(0.45)\end{array}$ & $\begin{array}{l}3.650 \\
(0.35)\end{array}$ & $\begin{array}{l}3.781 \\
(0.36)\end{array}$ & $\begin{array}{l}3.895 \\
(0.37)\end{array}$ & $\begin{array}{l}3.942 \\
(0.39)\end{array}$ & $\begin{array}{l}3.989 \\
(0.53)\end{array}$ \\
\hline & & & $\begin{array}{l}2.518 \\
(0.75)\end{array}$ & $\begin{array}{l}2.769 \\
(0.48)\end{array}$ & $\begin{array}{l}2.963 \\
(0.45)\end{array}$ & $\begin{array}{l}3.180 \\
(0.44)\end{array}$ & $\begin{array}{l}3.354 \\
(0.46)\end{array}$ & $\begin{array}{l}3.457 \\
(0.41)\end{array}$ & $\begin{array}{c}3.555 \\
(0.38)\end{array}$ & $\begin{array}{l}3.617 \\
(0.40)\end{array}$ & $\begin{array}{c}3.640 \\
(0.56)\end{array}$ \\
\hline & & $\begin{array}{l}2.104 \\
(0.83)\end{array}$ & $\begin{array}{l}2.321 \\
(0.51)\end{array}$ & $\begin{array}{c}2.533 \\
(0.48)\end{array}$ & $\begin{array}{l}2.736 \\
(0.45)\end{array}$ & $\begin{array}{c}2.893 \\
(0.47)\end{array}$ & $\begin{array}{l}3.058 \\
(0.39)\end{array}$ & $\begin{array}{r}3.159 \\
(0.41)\end{array}$ & $\begin{array}{c}3.257 \\
(0.42)\end{array}$ & $\begin{array}{c}3.271 \\
(0.41)\end{array}$ & $\begin{array}{l}3.331 \\
(0.58)\end{array}$ \\
\hline & $\begin{array}{c}1.889 \\
(0.78)\end{array}$ & $\begin{array}{c}1.980 \\
(0.57)\end{array}$ & $\begin{array}{l}2.223 \\
(0.55)\end{array}$ & $\begin{array}{l}2.402 \\
(0.51)\end{array}$ & $\begin{array}{l}2.624 \\
(0.50)\end{array}$ & $\begin{array}{l}2.789 \\
(0.41)\end{array}$ & $\begin{array}{l}2.917 \\
(0.41)\end{array}$ & $\begin{array}{l}3.037 \\
(0.47)\end{array}$ & $\begin{array}{l}3.092 \\
(0.45)\end{array}$ & $\begin{array}{l}3.150 \\
(0.42)\end{array}$ & $\begin{array}{l}3.184 \\
(0.65)\end{array}$ \\
\hline $\begin{array}{l}2.329 \\
(0.66)\end{array}$ & $\begin{array}{c}2.178 \\
(0.54)\end{array}$ & $\begin{array}{l}2.356 \\
(0.50)\end{array}$ & $\begin{array}{l}2.589 \\
(0.47)\end{array}$ & $\begin{array}{l}2.844 \\
(0.47)\end{array}$ & $\begin{array}{l}3.066 \\
(0.41)\end{array}$ & $\begin{array}{l}3.258 \\
(0.42)\end{array}$ & $\begin{array}{l}3.398 \\
(0.43)\end{array}$ & $\begin{array}{l}3.576 \\
(0.39)\end{array}$ & $\begin{array}{c}3.644 \\
(0.44)\end{array}$ & $\begin{array}{c}3.710 \\
(0.40)\end{array}$ & $\begin{array}{l}3.729 \\
(0.57)\end{array}$ \\
\hline
\end{tabular}

Fig. 4.5a. Pin-power distribution for CENTRM benchmark TCA-1. Value in parentheses is percent standard deviation. 


\begin{tabular}{|c|c|c|c|c|c|c|c|c|c|c|c|}
\hline & & & & & & & & & & & $\begin{array}{l}5.219 \\
(0.86)\end{array}$ \\
\hline & $\begin{array}{l}0.131 \\
(0.53)\end{array}$ & & & & & & & & & $\begin{array}{l}5.153 \\
(0.50)\end{array}$ & $\begin{array}{l}5.235 \\
(0.47)\end{array}$ \\
\hline & & & & & & & & & $\begin{array}{l}5.022 \\
(0.49)\end{array}$ & $\begin{array}{l}5.083 \\
(0.36)\end{array}$ & $\begin{array}{l}5.140 \\
(0.46)\end{array}$ \\
\hline & & & & & & & & $\begin{array}{l}4.775 \\
(0.48)\end{array}$ & $\begin{array}{l}4.893 \\
(0.35)\end{array}$ & $\begin{array}{l}4.968 \\
(0.38)\end{array}$ & $\begin{array}{l}5.010 \\
(0.49)\end{array}$ \\
\hline & & & & & & & $\begin{array}{l}4.453 \\
(0.54\end{array}$ & $\begin{array}{l}4.607 \\
(0.35)\end{array}$ & $\begin{array}{l}4.712 \\
(0.36)\end{array}$ & $\begin{array}{l}4.779 \\
(0.33)\end{array}$ & $\begin{array}{l}4.840 \\
(0.50)\end{array}$ \\
\hline & & & & & & $\begin{array}{l}4.006 \\
(0.55)\end{array}$ & $\begin{array}{l}4.225 \\
(0.41)\end{array}$ & $\begin{array}{l}4.384 \\
(0.36)\end{array}$ & $\begin{array}{l}4.474 \\
(0.36)\end{array}$ & $\begin{array}{l}4.594 \\
(0.35)\end{array}$ & $\begin{array}{l}4.547 \\
(0.53)\end{array}$ \\
\hline & & & & & $\begin{array}{l}3.541 \\
(0.55)\end{array}$ & $\begin{array}{l}3.807 \\
(0.39)\end{array}$ & $\begin{array}{l}3.978 \\
(0.41)\end{array}$ & $\begin{array}{l}4.086 \\
(0.36)\end{array}$ & $\begin{array}{l}4.200 \\
(0.39)\end{array}$ & $\begin{array}{l}4.270 \\
(0.37)\end{array}$ & $\begin{array}{l}4.341 \\
(0.47)\end{array}$ \\
\hline & & & & $\begin{array}{l}3.013 \\
(0.60)\end{array}$ & $\begin{array}{l}3.298 \\
(0.40)\end{array}$ & $\begin{array}{l}3.496 \\
(0.39)\end{array}$ & $\begin{array}{l}3.645 \\
(0.42)\end{array}$ & $\begin{array}{l}3.821 \\
(0.38)\end{array}$ & $\begin{array}{l}3.921 \\
(0.43)\end{array}$ & $\begin{array}{l}3.981 \\
(0.37)\end{array}$ & $\begin{array}{c}3.948 \\
(0.55)\end{array}$ \\
\hline & & & $\begin{array}{l}2.552 \\
(0.65)\end{array}$ & $\begin{array}{l}2.767 \\
(0.46)\end{array}$ & $\begin{array}{l}2.999 \\
(0.44)\end{array}$ & $\begin{array}{l}3.200 \\
(0.40)\end{array}$ & $\begin{array}{l}3.362 \\
(0.36)\end{array}$ & $\begin{array}{l}3.471 \\
(0.38)\end{array}$ & $\begin{array}{l}3.581 \\
(0.41)\end{array}$ & $\begin{array}{l}3.631 \\
(0.41)\end{array}$ & $\begin{array}{l}3.624 \\
(0.59)\end{array}$ \\
\hline & & $\begin{array}{l}2.113 \\
(0.73)\end{array}$ & $\begin{array}{l}2.284 \\
(0.48)\end{array}$ & $\begin{array}{l}2.509 \\
0.50)\end{array}$ & $\begin{array}{l}2.749 \\
(0.46)\end{array}$ & $\begin{array}{l}2.906 \\
(0.47)\end{array}$ & $\begin{array}{l}3.036 \\
(0.42)\end{array}$ & $\begin{array}{l}3.153 \\
(0.42)\end{array}$ & $\begin{array}{l}3.238 \\
(0.41)\end{array}$ & $\begin{array}{c}3.276 \\
(0.44)\end{array}$ & $\begin{array}{l}3.329 \\
(0.53)\end{array}$ \\
\hline & $\begin{array}{c}1.911 \\
(0.77)\end{array}$ & $\begin{array}{l}1.997 \\
(0.47)\end{array}$ & $\begin{array}{l}2.202 \\
(0.57)\end{array}$ & $\begin{array}{l}2.417 \\
(0.51)\end{array}$ & $\begin{array}{l}2.623 \\
(0.44)\end{array}$ & $\begin{array}{l}2.768 \\
(0.46)\end{array}$ & $\begin{array}{l}2.927 \\
(0.46)\end{array}$ & $\begin{array}{l}3.029 \\
(0.40)\end{array}$ & $\begin{array}{l}3.071 \\
(0.44)\end{array}$ & $\begin{array}{c}3.176 \\
(0.41)\end{array}$ & $\begin{array}{l}3.180 \\
(0.65)\end{array}$ \\
\hline $\begin{array}{l}2.279 \\
(0.69)\end{array}$ & $\begin{array}{l}2.171 \\
(0.53)\end{array}$ & $\begin{array}{l}2.347 \\
(0.50)\end{array}$ & $\begin{array}{l}2.571 \\
(0.48)\end{array}$ & $\begin{array}{l}2.845 \\
(0.43)\end{array}$ & $\begin{array}{c}3.066 \\
(0.47)\end{array}$ & $\begin{array}{c}3.274 \\
(0.39)\end{array}$ & $\begin{array}{l}3.446 \\
(0.41)\end{array}$ & $\begin{array}{l}3.601 \\
(0.38)\end{array}$ & $\begin{array}{c}3.673 \\
(0.38)\end{array}$ & $\begin{array}{c}3.729 \\
(0.41)\end{array}$ & $\begin{array}{c}3.776 \\
(0.55)\end{array}$ \\
\hline
\end{tabular}

Fig. 4.5b. Pin-power distribution for NITAWL benchmark TCA-1. Value in parentheses is percent standard deviation. 
Table 4.5. Selected reaction rates for CENTRM case TCA-1

\begin{tabular}{ccccccc}
\hline Pin & Region & $\begin{array}{c}\mathrm{G}_{\mathrm{a}} \mathrm{M} \\
\left(\mathrm{cm}^{-3}-\mathrm{s}^{-1}\right)\end{array}$ & $\begin{array}{c}\mathrm{G}_{\mathrm{f}} \mathrm{M} \\
\left(\mathrm{cm}^{-3}-\mathrm{s}^{-1}\right)\end{array}$ & $\begin{array}{c}\mathrm{G}_{\mathrm{f}} \mathrm{M} \\
\left(\mathrm{cm}^{-3}-\mathrm{s}^{-1}\right)\end{array}$ & $\begin{array}{c}\mathrm{M} \\
\left(\mathrm{cm}-\mathrm{s}^{-1}\right)\end{array}$ & $\mathrm{M}_{\mathrm{t}}$ \\
\hline \multirow{2}{*}{1} & Fuel & $1.400 \mathrm{E}-05$ & $2.320 \mathrm{E}-05$ & $8.219 \mathrm{E}-06$ & $5.406 \mathrm{E}-03$ & 0.271 \\
& Clad & $2.686 \mathrm{E}-07$ & 0.0 & 0.0 & $1.687 \mathrm{E}-03$ & 0.085 \\
& Mod. & $7.396 \mathrm{E}-07$ & 0.0 & 0.0 & $1.285 \mathrm{E}-02$ & 0.644 \\
\hline \multirow{2}{*}{78} & Fuel & $3.547 \mathrm{E}-05$ & $5.287 \mathrm{E}-05$ & $1.869 \mathrm{E}-05$ & $2.162 \mathrm{E}-02$ & 0.270 \\
& Clad & $7.404 \mathrm{E}-07$ & 0.0 & 0.0 & $6.840 \mathrm{E}-03$ & 0.085 \\
& Mod. & $1.542 \mathrm{E}-06$ & 0.0 & 0.0 & $5.169 \mathrm{E}-02$ & 0.645 \\
\hline
\end{tabular}

Table 4.6. Selected reaction rates for NITAWL case TCA-1

\begin{tabular}{ccccccc}
\hline \multirow{2}{*}{ Pin } & Region & $\begin{array}{c}\mathrm{G}_{\mathrm{a}} \mathrm{M} \\
\left(\mathrm{cm}^{-3}-\mathrm{s}^{-1}\right)\end{array}$ & $\begin{array}{c}\mathrm{G}_{\mathrm{f}} \mathrm{M} \\
\left(\mathrm{cm}^{-3}-\mathrm{s}^{-1}\right)\end{array}$ & $\begin{array}{c}\mathrm{G}_{\mathrm{f}} \mathrm{M} \\
\left(\mathrm{cm}^{-3}-\mathrm{s}^{-1}\right)\end{array}$ & $\begin{array}{c}\mathrm{M} \\
\left(\mathrm{cm}-\mathrm{s}^{-1}\right)\end{array}$ & $\mathrm{M}_{\mathrm{t}}$ \\
\hline \multirow{2}{*}{1} & Fuel & $1.386 \mathrm{E}-05$ & $2.292 \mathrm{E}-05$ & $8.121 \mathrm{E}-06$ & $5.354 \mathrm{E}-03$ & 0.271 \\
& Clad & $2.641 \mathrm{E}-07$ & 0.0 & 0.0 & $1.665 \mathrm{E}-03$ & 0.084 \\
& Mod. & $7.348 \mathrm{E}-07$ & 0.0 & 0.0 & $1.277 \mathrm{E}-02$ & 0.645 \\
\hline \multirow{2}{*}{78} & Fuel & $3.457 \mathrm{E}-05$ & $5.164 \mathrm{E}-05$ & $1.827 \mathrm{E}-05$ & $2.134 \mathrm{E}-02$ & 0.268 \\
& Clad & $7.151 \mathrm{E}-07$ & 0.0 & 0.0 & $6.771 \mathrm{E}-03$ & 0.085 \\
& Mod. & $1.529 \mathrm{E}-06$ & 0.0 & 0.0 & $5.146 \mathrm{E}-02$ & 0.647 \\
\hline
\end{tabular}


Table 4.7. TCA-1, Pin-1 four-group fluxes

\begin{tabular}{ccc}
\hline Group & $\begin{array}{c}\text { CENTRM M } \\
\left(\mathrm{cm}^{-2}-\mathrm{s}^{-1}\right)\end{array}$ & $\begin{array}{c}\text { NITAWL M } \\
\left(\mathrm{cm}^{-2}-\mathrm{s}^{-1}\right)\end{array}$ \\
\hline 1 & $5.205 \mathrm{E}-05$ & $5.150 \mathrm{E}-05$ \\
2 & $1.550 \mathrm{E}-05$ & $1.533 \mathrm{E}-05$ \\
3 & $3.677 \mathrm{E}-06$ & $3.705 \mathrm{E}-06$ \\
4 & $3.068 \mathrm{E}-05$ & $3.040 \mathrm{E}-05$ \\
\hline
\end{tabular}

Table 4.8. TCA-1, Pin-1 four-group U-235 cross sections

\begin{tabular}{ccccccc}
\hline Group & \multicolumn{2}{c}{ CENTRM cross sections $\left(\mathrm{cm}^{-1}\right)$} & \multicolumn{3}{c}{ NITAWL cross sections $\left(\mathrm{cm}^{-1}\right)$} \\
\hline & $\frac{\mathrm{G}_{\mathrm{a}}}{1.43276 \mathrm{E}-04}$ & $\frac{\mathrm{G}_{\mathrm{f}}}{3.25948 \mathrm{E}-04}$ & $\frac{\mathrm{G}_{\mathrm{f}}}{1.25443 \mathrm{E}-04}$ & $\frac{\mathrm{G}_{\mathrm{a}}}{1.43133 \mathrm{E}-04}$ & $\frac{\mathrm{G}_{\mathrm{f}}}{3.25491 \mathrm{E}-04}$ & $\frac{\mathrm{G}_{\mathrm{f}}}{1.25308 \mathrm{E}-04}$ \\
1 & $3.47383 \mathrm{E}-03$ & $5.32334 \mathrm{E}-03$ & $2.18465 \mathrm{E}-03$ & $3.41501 \mathrm{E}-03$ & $5.21704 \mathrm{E}-03$ & $2.14103 \mathrm{E}-03$ \\
2 & $5.07797 \mathrm{E}-03$ & $1.03603 \mathrm{E}-02$ & $4.25178 \mathrm{E}-03$ & $5.13482 \mathrm{E}-03$ & $1.05164 \mathrm{E}-02$ & $4.31584 \mathrm{E}-03$ \\
3 & $4.86542 \mathrm{E}-02$ & $1.01291 \mathrm{E}-01$ & $4.15691 \mathrm{E}-02$ & $4.83612 \mathrm{E}-02$ & $1.00680 \mathrm{E}-01$ & $4.13181 \mathrm{E}-02$ \\
\hline
\end{tabular}

Table 4.9. TCA-1, Pin-1 four-group U-238 cross sections

\begin{tabular}{ccccccc}
\hline Group & \multicolumn{3}{c}{ CENTRM cross sections $\left(\mathrm{cm}^{-1}\right)$} & \multicolumn{2}{c}{ NITAWL cross sections $\left(\mathrm{cm}^{-1}\right)$} \\
\hline & $\frac{\mathrm{G}_{\mathrm{a}}}{4.48868 \mathrm{E}-03}$ & $\frac{\mathrm{G}_{\mathrm{f}}}{8.37908 \mathrm{E}-03}$ & $\frac{\mathrm{G}_{\mathrm{f}}}{2.97182 \mathrm{E}-03}$ & $\frac{\mathrm{G}_{\mathrm{a}}}{4.45235 \mathrm{E}-03}$ & $\frac{\mathrm{G}_{\mathrm{f}}}{8.27583 \mathrm{E}-03}$ & $\frac{\mathrm{G}_{\mathrm{f}}}{2.93388 \mathrm{E}-03}$ \\
1 & $3.79279 \mathrm{E}-02$ & $3.70803 \mathrm{E}-06$ & $1.53622 \mathrm{E}-06$ & $3.78200 \mathrm{E}-02$ & $3.60508 \mathrm{E}-06$ & $1.49357 \mathrm{E}-06$ \\
2 & $6.96274 \mathrm{E}-03$ & $2.42790 \mathrm{E}-08$ & $1.00592 \mathrm{E}-08$ & $6.99725 \mathrm{E}-03$ & $2.45979 \mathrm{E}-08$ & $1.01914 \mathrm{E}-08$ \\
3 & $2.76422 \mathrm{E}-02$ & $1.29929 \mathrm{E}-07$ & $5.38321 \mathrm{E}-08$ & $2.75005 \mathrm{E}-02$ & $1.29251 \mathrm{E}-07$ & $5.35513 \mathrm{E}-08$ \\
\hline
\end{tabular}

Table 4.10. TCA-1, Pin-1 four-group Pu-239 cross sections

\begin{tabular}{cccccccc}
\hline Group & \multicolumn{3}{c}{ CENTRM cross sections $\left(\mathrm{cm}^{-1}\right)$} & \multicolumn{3}{c}{ NITAWL cross sections $\left(\mathrm{cm}^{-1}\right)$} \\
\hline & $\frac{\mathrm{G}_{\mathrm{a}}}{}$ & $\frac{\mathrm{G}_{\mathrm{f}}}{12119 \mathrm{E}-04}$ & $\frac{\mathrm{G}_{\mathrm{f}}}{1.49477 \mathrm{E}-03}$ & $\frac{4.80859 \mathrm{E}-04}{5.11680 \mathrm{E}-04}$ & $\frac{\mathrm{G}_{\mathrm{f}}}{1.49305 \mathrm{E}-03}$ & $\frac{\mathrm{G}_{\mathrm{f}}}{4.80499 \mathrm{E}-04}$ \\
2 & $1.13759 \mathrm{E}-02$ & $1.87090 \mathrm{E}-02$ & $6.49658 \mathrm{E}-03$ & $1.06255 \mathrm{E}-02$ & $1.76083 \mathrm{E}-02$ & $6.11439 \mathrm{E}-03$ \\
3 & $3.27337 \mathrm{E}-02$ & $6.44523 \mathrm{E}-02$ & $2.23808 \mathrm{E}-02$ & $3.35760 \mathrm{E}-02$ & $6.60534 \mathrm{E}-02$ & $2.29368 \mathrm{E}-02$ \\
4 & $2.66846 \mathrm{E}-01$ & $5.40163 \mathrm{E}-01$ & $1.86831 \mathrm{E}-01$ & $2.66855 \mathrm{E}-01$ & $5.39595 \mathrm{E}-01$ & $1.86638 \mathrm{E}-01$ \\
\hline
\end{tabular}


Table 4.11. TCA-1, Pin-78 four-group fluxes

\begin{tabular}{ccc}
\hline Group & $\begin{array}{c}\text { CENTRM M } \\
\left(\mathrm{cm}^{-2}-\mathrm{s}^{-1}\right)\end{array}$ & $\begin{array}{c}\text { NITAWL M } \\
\left(\mathrm{cm}^{-2}-\mathrm{s}^{-1}\right)\end{array}$ \\
\hline 1 & $2.399 \mathrm{E}-04$ & $2.360 \mathrm{E}-04$ \\
2 & $8.524 \mathrm{E}-05$ & $8.507 \mathrm{E}-05$ \\
3 & $1.867 \mathrm{E}-05$ & $1.851 \mathrm{E}-05$ \\
4 & $6.380 \mathrm{E}-05$ & $6.276 \mathrm{E}-05$ \\
\hline
\end{tabular}

Table 4.12. TCA-1, Pin-78 four-group U-235 cross sections

\begin{tabular}{cccccccc}
\hline Group & \multicolumn{3}{c}{ CENTRM cross sections $\left(\mathrm{cm}^{-1}\right)$} & \multicolumn{3}{c}{ NITAWL cross sections $\left(\mathrm{cm}^{-1}\right)$} \\
\hline & $\frac{\mathrm{G}_{\mathrm{a}}}{1.48131 \mathrm{E}-04}$ & $\frac{\mathrm{G}_{\mathrm{f}}}{3.30102 \mathrm{E}-04}$ & $\frac{\mathrm{G}_{\mathrm{f}}}{1.27921 \mathrm{E}-04}$ & $\frac{\mathrm{G}_{\mathrm{a}}}{1.47652 \mathrm{E}-04}$ & $\frac{\mathrm{G}_{\mathrm{f}}}{3.28787 \mathrm{E}-04}$ & $\frac{\mathrm{G}_{\mathrm{f}}}{1.27540 \mathrm{E}-04}$ \\
1 & $3.33968 \mathrm{E}-03$ & $5.12318 \mathrm{E}-03$ & $2.10251 \mathrm{E}-03$ & $3.40930 \mathrm{E}-03$ & $5.23865 \mathrm{E}-03$ & $2.14990 \mathrm{E}-03$ \\
3 & $5.10391 \mathrm{E}-03$ & $1.04015 \mathrm{E}-02$ & $4.26869 \mathrm{E}-03$ & $5.13030 \mathrm{E}-03$ & $1.04663 \mathrm{E}-02$ & $4.29526 \mathrm{E}-03$ \\
4 & $4.54602 \mathrm{E}-02$ & $9.45345 \mathrm{E}-02$ & $3.87961 \mathrm{E}-02$ & $4.53139 \mathrm{E}-02$ & $9.42388 \mathrm{E}-02$ & $3.86748 \mathrm{E}-02$ \\
\hline
\end{tabular}

Table 4.13. TCA-1, Pin-78 four-group U-238 cross sections

\begin{tabular}{cccccccc}
\hline Group & \multicolumn{3}{c}{ CENTRM cross sections $\left(\mathrm{cm}^{-1}\right)$} & \multicolumn{3}{c}{ NITAWL cross sections $\left(\mathrm{cm}^{-1}\right)$} \\
\hline & $\frac{\mathrm{G}_{\mathrm{a}}}{4.34327 \mathrm{E}-03}$ & $\frac{\mathrm{G}_{\mathrm{f}}}{7.48462 \mathrm{E}-03}$ & $\frac{\mathrm{G}_{\mathrm{f}}}{2.65627 \mathrm{E}-03}$ & $\frac{\mathrm{G}_{\mathrm{a}}}{4.30530 \mathrm{E}-03}$ & $\frac{\mathrm{G}_{\mathrm{f}}}{7.37008 \mathrm{E}-03}$ & $\frac{\mathrm{G}_{\mathrm{f}}}{2.62250 \mathrm{E}-03}$ \\
1 & $3.98175 \mathrm{E}-02$ & $3.68536 \mathrm{E}-06$ & $1.52683 \mathrm{E}-06$ & $3.74190 \mathrm{E}-02$ & $3.60909 \mathrm{E}-06$ & $1.49523 \mathrm{E}-06$ \\
2 & $6.98275 \mathrm{E}-03$ & $2.43235 \mathrm{E}-08$ & $1.00777 \mathrm{E}-08$ & $6.98957 \mathrm{E}-03$ & $2.44139 \mathrm{E}-08$ & $1.01151 \mathrm{E}-08$ \\
3 & $2.59889 \mathrm{E}-02$ & $1.21997 \mathrm{E}-07$ & $5.05458 \mathrm{E}-08$ & $2.59301 \mathrm{E}-02$ & $1.21719 \mathrm{E}-07$ & $5.04304 \mathrm{E}-08$ \\
\hline
\end{tabular}

Table 4.14. TCA-1, Pin-78 four-group Pu-239 cross sections

\begin{tabular}{|c|c|c|c|c|c|c|}
\hline \multirow[t]{2}{*}{ Group } & \multicolumn{3}{|c|}{ CENTRM cross sections $\left(\mathrm{cm}^{-1}\right)$} & \multicolumn{3}{|c|}{ NITAWL cross sections $\left(\mathrm{cm}^{-1}\right)$} \\
\hline & $\mathrm{G}_{\mathrm{a}}$ & $\mathrm{G}_{\mathrm{f}}$ & $\mathrm{G}_{\mathrm{f}}$ & $\mathrm{G}_{\mathrm{a}}$ & $\mathrm{G}_{\mathrm{f}}$ & $\mathrm{G}_{\mathrm{f}}$ \\
\hline 1 & $5.14181 \mathrm{E}-04$ & $\overline{1.47485 \mathrm{E}-03}$ & $\overline{4.77461 \mathrm{E}-04}$ & $5.13292 \mathrm{E}-04$ & $\overline{1.47159 \mathrm{E}-03}$ & $\overline{4.76847 \mathrm{E}-04}$ \\
\hline 2 & 1.06372E-02 & $1.75772 \mathrm{E}-02$ & $6.10359 \mathrm{E}-03$ & $1.08006 \mathrm{E}-02$ & 1.79107E-02 & $6.21938 \mathrm{E}-03$ \\
\hline 3 & 3.42975E-02 & $6.73203 \mathrm{E}-02$ & $2.33768 \mathrm{E}-02$ & 3.44553E-02 & 6.76072E-02 & $2.34764 \mathrm{E}-02$ \\
\hline 4 & $2.85886 \mathrm{E}-01$ & 5.65284E-01 & $1.95610 \mathrm{E}-01$ & $2.82398 \mathrm{E}-01$ & $5.59148 \mathrm{E}-01$ & $1.93483 \mathrm{E}-01$ \\
\hline
\end{tabular}




\begin{tabular}{|c|c|c|c|c|c|c|c|c|c|c|c|}
\hline & & & & & & & & & & & $\begin{array}{l}4.965 \\
(0.99)\end{array}$ \\
\hline & $\begin{array}{l}0.139 \\
(0.64)\end{array}$ & & & & & & & & & $\begin{array}{l}4.937 \\
(0.46)\end{array}$ & $\begin{array}{l}4.994 \\
(0.49)\end{array}$ \\
\hline & & & & & & & & & $\begin{array}{l}4.776 \\
(0.50)\end{array}$ & $\begin{array}{l}4.866 \\
(0.34)\end{array}$ & $\begin{array}{l}4.932 \\
(0.47)\end{array}$ \\
\hline & & & & & & & & $\begin{array}{l}4.569 \\
(0.53)\end{array}$ & $\begin{array}{l}4.688 \\
(0.32)\end{array}$ & $\begin{array}{l}4.750 \\
(0.35)\end{array}$ & $\begin{array}{l}4.781 \\
(0.47)\end{array}$ \\
\hline & & & & & & & $\begin{array}{l}4.283 \\
(0.57)\end{array}$ & $\begin{array}{l}4.402 \\
(0.38)\end{array}$ & $\begin{array}{l}4.527 \\
(0.36)\end{array}$ & $\begin{array}{l}4.600 \\
(0.35)\end{array}$ & $\begin{array}{l}4.677 \\
(0.48)\end{array}$ \\
\hline & & & & & & $\begin{array}{l}3.846 \\
(0.55)\end{array}$ & $\begin{array}{l}4.048 \\
(0.38)\end{array}$ & $\begin{array}{l}4.205 \\
(0.37)\end{array}$ & $\begin{array}{l}4.302 \\
(0.36)\end{array}$ & $\begin{array}{l}4.359 \\
(0.34)\end{array}$ & $\begin{array}{l}4.376 \\
(0.50)\end{array}$ \\
\hline & & & & & $\begin{array}{l}3.405 \\
(0.57)\end{array}$ & $\begin{array}{l}3.636 \\
(0.43)\end{array}$ & $\begin{array}{l}3.795 \\
(0.37)\end{array}$ & $\begin{array}{l}3.946 \\
(0.36)\end{array}$ & $\begin{array}{l}4.058 \\
(0.34)\end{array}$ & $\begin{array}{l}4.111 \\
(0.39)\end{array}$ & $\begin{array}{l}4.125 \\
(0.49)\end{array}$ \\
\hline & & & & $\begin{array}{l}2.910 \\
(0.64)\end{array}$ & $\begin{array}{l}3.171 \\
(0.47)\end{array}$ & $\begin{array}{l}3.388 \\
(0.42)\end{array}$ & $\begin{array}{l}3.532 \\
(0.36)\end{array}$ & $\begin{array}{c}3.649 \\
(0.41)\end{array}$ & $\begin{array}{l}3.739 \\
(0.41)\end{array}$ & $\begin{array}{l}3.822 \\
(0.40)\end{array}$ & $\begin{array}{c}3.849 \\
(0.55)\end{array}$ \\
\hline & & & $\begin{array}{l}2.455 \\
(0.64)\end{array}$ & $\begin{array}{l}2.666 \\
(0.48)\end{array}$ & $\begin{array}{l}2.882 \\
(0.43)\end{array}$ & $\begin{array}{l}3.063 \\
(0.42)\end{array}$ & $\begin{array}{l}3.207 \\
(0.41)\end{array}$ & $\begin{array}{l}3.324 \\
(0.42)\end{array}$ & $\begin{array}{l}3.449 \\
(0.41)\end{array}$ & $\begin{array}{l}3.489 \\
(0.42)\end{array}$ & $\begin{array}{c}3.486 \\
(0.55)\end{array}$ \\
\hline & & $\begin{array}{l}2.021 \\
(0.74)\end{array}$ & $\begin{array}{l}2.222 \\
(0.54)\end{array}$ & $\begin{array}{l}2.433 \\
0.46)\end{array}$ & $\begin{array}{l}2.634 \\
(0.46)\end{array}$ & $\begin{array}{l}2.808 \\
(0.47)\end{array}$ & $\begin{array}{l}2.906 \\
(0.42)\end{array}$ & $\begin{array}{l}3.043 \\
(0.45)\end{array}$ & $\begin{array}{l}3.141 \\
(0.44)\end{array}$ & $\begin{array}{c}3.166 \\
(0.45)\end{array}$ & $\begin{array}{l}3.189 \\
(0.61)\end{array}$ \\
\hline & $\begin{array}{l}1.841 \\
(0.80)\end{array}$ & $\begin{array}{l}1.935 \\
(0.54)\end{array}$ & $\begin{array}{c}2.120 \\
(0.48)\end{array}$ & $\begin{array}{l}2.324 \\
(0.52)\end{array}$ & $\begin{array}{l}2.503 \\
(0.44)\end{array}$ & $\begin{array}{l}2.645 \\
(0.46)\end{array}$ & $\begin{array}{l}2.825 \\
(0.44)\end{array}$ & $\begin{array}{l}2.902 \\
(0.43)\end{array}$ & $\begin{array}{l}2.982 \\
(0.43)\end{array}$ & $\begin{array}{c}3.040 \\
(0.46)\end{array}$ & $\begin{array}{c}3.036 \\
(0.61)\end{array}$ \\
\hline $\begin{array}{l}2.226 \\
(0.69)\end{array}$ & $\begin{array}{l}2.099 \\
(0.51)\end{array}$ & $\begin{array}{l}2.276 \\
(0.50)\end{array}$ & $\begin{array}{l}2.506 \\
(0.50)\end{array}$ & $\begin{array}{l}2.739 \\
(0.44)\end{array}$ & $\begin{array}{l}2.952 \\
(0.41)\end{array}$ & $\begin{array}{l}3.138 \\
(0.43)\end{array}$ & $\begin{array}{l}3.341 \\
(0.35)\end{array}$ & $\begin{array}{l}3.425 \\
(0.39)\end{array}$ & $\begin{array}{c}3.533 \\
(0.37)\end{array}$ & $\begin{array}{l}3.606 \\
(0.40)\end{array}$ & $\begin{array}{l}3.590 \\
(0.59)\end{array}$ \\
\hline
\end{tabular}

Fig. 4.6a. Pin-power distribution for CENTRM benchmark TCA-2. Value in parentheses is percent standard deviation. 


\begin{tabular}{|c|c|c|c|c|c|c|c|c|c|c|c|}
\hline & & & & & & & & & & & $\begin{array}{l}5.023 \\
(0.99)\end{array}$ \\
\hline & $\begin{array}{l}0.139 \\
(0.63)\end{array}$ & & & & & & & & & $\begin{array}{l}4.960 \\
(0.46)\end{array}$ & $\begin{array}{l}5.042 \\
(0.47)\end{array}$ \\
\hline & & & & & & & & & $\begin{array}{l}4.835 \\
(0.48)\end{array}$ & $\begin{array}{l}4.922 \\
(0.33)\end{array}$ & $\begin{array}{l}4.929 \\
(0.47)\end{array}$ \\
\hline & & & & & & & & $\begin{array}{l}4.569 \\
(0.52)\end{array}$ & $\begin{array}{l}4.721 \\
(0.32)\end{array}$ & $\begin{array}{l}4.780 \\
(0.36)\end{array}$ & $\begin{array}{l}4.776 \\
(0.48)\end{array}$ \\
\hline & & & & & & & $\begin{array}{l}4.250 \\
(0.50)\end{array}$ & $\begin{array}{l}4.420 \\
(0.35)\end{array}$ & $\begin{array}{l}4.521 \\
(0.36)\end{array}$ & $\begin{array}{l}4.608 \\
(0.35)\end{array}$ & $\begin{array}{l}4.612 \\
(0.50)\end{array}$ \\
\hline & & & & & & $\begin{array}{l}3.803 \\
(0.58)\end{array}$ & $\begin{array}{l}4.057 \\
(0.34)\end{array}$ & $\begin{array}{l}4.179 \\
(0.39)\end{array}$ & $\begin{array}{l}4.303 \\
(0.35)\end{array}$ & $\begin{array}{l}4.406 \\
(0.35)\end{array}$ & $\begin{array}{l}4.409 \\
(0.51)\end{array}$ \\
\hline & & & & & $\begin{array}{l}3.430 \\
(0.58)\end{array}$ & $\begin{array}{l}3.641 \\
(0.41)\end{array}$ & $\begin{array}{c}3.836 \\
(0.39)\end{array}$ & $\begin{array}{c}3.972 \\
(0.37)\end{array}$ & $\begin{array}{l}4.049 \\
(0.39)\end{array}$ & $\begin{array}{l}4.121 \\
(0.37)\end{array}$ & $\begin{array}{l}4.127 \\
(0.54)\end{array}$ \\
\hline & & & & $\begin{array}{l}2.949 \\
(0.66)\end{array}$ & $\begin{array}{l}3.164 \\
(0.42)\end{array}$ & $\begin{array}{l}3.374 \\
(0.41)\end{array}$ & $\begin{array}{l}3.549 \\
(0.43)\end{array}$ & $\begin{array}{l}3.649 \\
(0.40)\end{array}$ & $\begin{array}{l}3.775 \\
(0.38)\end{array}$ & $\begin{array}{l}3.806 \\
(0.42)\end{array}$ & $\begin{array}{l}3.858 \\
(0.53)\end{array}$ \\
\hline & & & $\begin{array}{l}2.447 \\
(0.66)\end{array}$ & $\begin{array}{l}2.659 \\
(0.43)\end{array}$ & $\begin{array}{l}2.876 \\
(0.44)\end{array}$ & $\begin{array}{l}3.101 \\
(0.45)\end{array}$ & $\begin{array}{l}3.229 \\
(0.44)\end{array}$ & $\begin{array}{l}3.359 \\
(0.40)\end{array}$ & $\begin{array}{l}3.459 \\
(0.38)\end{array}$ & $\begin{array}{l}3.487 \\
(0.43)\end{array}$ & $\begin{array}{l}3.504 \\
(0.59)\end{array}$ \\
\hline & & $\begin{array}{l}2.029 \\
(0.71)\end{array}$ & $\begin{array}{l}2.232 \\
(0.51)\end{array}$ & $\begin{array}{l}2.431 \\
0.48)\end{array}$ & $\begin{array}{l}2.644 \\
(0.45)\end{array}$ & $\begin{array}{l}2.793 \\
(0.49)\end{array}$ & $\begin{array}{l}2.940 \\
(0.44)\end{array}$ & $\begin{array}{l}3.042 \\
(0.45)\end{array}$ & $\begin{array}{l}3.132 \\
(0.39)\end{array}$ & $\begin{array}{l}3.194 \\
(0.43)\end{array}$ & $\begin{array}{l}3.205 \\
(0.58)\end{array}$ \\
\hline & $\begin{array}{l}1.837 \\
(0.75)\end{array}$ & $\begin{array}{l}1.930 \\
(0.56)\end{array}$ & $\begin{array}{l}2.140 \\
(0.51)\end{array}$ & $\begin{array}{l}2.325 \\
(0.53)\end{array}$ & $\begin{array}{l}2.522 \\
(0.47)\end{array}$ & $\begin{array}{l}2.677 \\
(0.44)\end{array}$ & $\begin{array}{l}2.816 \\
(0.44)\end{array}$ & $\begin{array}{l}2.936 \\
(0.40)\end{array}$ & $\begin{array}{l}3.008 \\
(0.44)\end{array}$ & $\begin{array}{l}3.035 \\
(0.40)\end{array}$ & $\begin{array}{l}3.064 \\
(0.61)\end{array}$ \\
\hline $\begin{array}{l}2.222 \\
(0.68)\end{array}$ & $\begin{array}{l}2.106 \\
(0.51)\end{array}$ & $\begin{array}{l}2.274 \\
(0.52)\end{array}$ & $\begin{array}{l}2.527 \\
(0.47)\end{array}$ & $\begin{array}{l}2.756 \\
(0.43)\end{array}$ & $\begin{array}{l}2.976 \\
(0.41)\end{array}$ & $\begin{array}{l}3.149 \\
(0.44)\end{array}$ & $\begin{array}{c}3.323 \\
(0.39)\end{array}$ & $\begin{array}{l}3.477 \\
(0.38)\end{array}$ & $\begin{array}{c}3.562 \\
(0.38)\end{array}$ & $\begin{array}{c}3.583 \\
(0.41)\end{array}$ & $\begin{array}{l}3.598 \\
(0.59)\end{array}$ \\
\hline
\end{tabular}

Fig. 4.6b. Pin-power distribution for NITAWL benchmark TCA-2. Value in parentheses is percent standard deviation. 
Table 4.15. Selected reaction rates for CENTRM case TCA-2

\begin{tabular}{ccccccc}
\hline Pin & Region & $\begin{array}{c}\mathrm{G}_{\mathrm{a}} \mathrm{M} \\
\left(\mathrm{cm}^{-3}-\mathrm{s}^{-1}\right)\end{array}$ & $\begin{array}{c}\mathrm{G}_{\mathrm{f}} \mathrm{M} \\
\left(\mathrm{cm}^{-3}-\mathrm{s}^{-1}\right)\end{array}$ & $\begin{array}{c}\mathrm{G}_{\mathrm{f}} \mathrm{M} \\
\left(\mathrm{cm}^{-3}-\mathrm{s}^{-1}\right)\end{array}$ & $\begin{array}{c}\mathrm{M} \\
\left(\mathrm{cm}^{-1}\right)\end{array}$ & $\mathrm{M}_{\mathrm{t}}$ \\
\hline \multirow{2}{*}{1} & Fuel & $1.356 \mathrm{E}-05$ & $2.236 \mathrm{E}-05$ & $7.923 \mathrm{E}-06$ & $5.453 \mathrm{E}-03$ & 0.270 \\
& Clad & $2.613 \mathrm{E}-07$ & 0.0 & 0.0 & $1.705 \mathrm{E}-03$ & 0.085 \\
& Mod. & $7.206 \mathrm{E}-07$ & 0.0 & 0.0 & $1.301 \mathrm{E}-02$ & 0.645 \\
\hline \multirow{2}{*}{78} & Fuel & $3.394 \mathrm{E}-05$ & $5.051 \mathrm{E}-05$ & $1.786 \mathrm{E}-05$ & $2.155 \mathrm{E}-02$ & 0.269 \\
& Clad & $7.023 \mathrm{E}-07$ & 0.0 & 0.0 & $6.812 \mathrm{E}-03$ & 0.085 \\
& Mod. & $1.495 \mathrm{E}-06$ & 0.0 & 0.0 & $5.179 \mathrm{E}-02$ & 0.646 \\
\hline
\end{tabular}

Table 4.16. Selected reaction rates for NITAWL case TCA-2

\begin{tabular}{|c|c|c|c|c|c|c|}
\hline Pin & Region & $\begin{array}{c}\mathrm{G}_{\mathrm{a}} \mathrm{M} \\
\left(\mathrm{cm}^{-3}-\mathrm{s}^{-1}\right)\end{array}$ & $\begin{array}{c}\mathcal{G}_{\mathrm{f}} \mathrm{M} \\
\left(\mathrm{cm}^{-3}-\mathrm{s}^{-1}\right)\end{array}$ & $\begin{array}{c}\mathrm{G}_{\mathrm{f}} \mathrm{M} \\
\left(\mathrm{cm}^{-3}-\mathrm{s}^{-1}\right)\end{array}$ & $\begin{array}{c}\mathrm{M} \\
\left(\mathrm{cm}-\mathrm{s}^{-1}\right)\end{array}$ & $\mathrm{M} \mathrm{M}_{\mathrm{t}}$ \\
\hline \multirow{3}{*}{1} & Fuel & $1.356 \mathrm{E}-05$ & $2.241 \mathrm{E}-05$ & $7.942 \mathrm{E}-06$ & $5.429 \mathrm{E}-03$ & 0.271 \\
\hline & Clad & $2.591 \mathrm{E}-07$ & 0.0 & 0.0 & $1.693 \mathrm{E}-03$ & 0.084 \\
\hline & Mod. & $7.211 \mathrm{E}-07$ & 0.0 & 0.0 & $1.293 \mathrm{E}-02$ & 0.645 \\
\hline \multirow{3}{*}{78} & Fuel & $3.354 \mathrm{E}-05$ & $5.009 \mathrm{E}-05$ & $1.771 \mathrm{E}-05$ & $2.137 \mathrm{E}-02$ & 0.268 \\
\hline & Clad & $6.772 \mathrm{E}-07$ & 0.0 & 0.0 & $6.756 \mathrm{E}-03$ & 0.084 \\
\hline & Mod. & $1.478 \mathrm{E}-06$ & 0.0 & 0.0 & $5.172 \mathrm{E}-02$ & 0.648 \\
\hline
\end{tabular}


Table 4.17. TCA-2, Pin-1 four-group fluxes

\begin{tabular}{ccc}
\hline Group & $\begin{array}{c}\text { CENTRM M } \\
\left(\mathrm{cm}^{-2}-\mathrm{s}^{-1}\right)\end{array}$ & $\begin{array}{c}\text { NITAWL M } \\
\left(\mathrm{cm}^{-2}-\mathrm{s}^{-1}\right)\end{array}$ \\
\hline 1 & $5.046 \mathrm{E}-05$ & $5.017 \mathrm{E}-05$ \\
2 & $1.503 \mathrm{E}-05$ & $1.485 \mathrm{E}-05$ \\
3 & $3.705 \mathrm{E}-06$ & $3.620 \mathrm{E}-06$ \\
4 & $2.970 \mathrm{E}-05$ & $2.981 \mathrm{E}-05$ \\
\hline
\end{tabular}

Table 4.18. TCA-2, Pin-1 four-group U-235 cross sections

\begin{tabular}{cccccccc}
\hline Group & \multicolumn{2}{c}{ CENTRM cross sections $\left(\mathrm{cm}^{-1}\right)$} & \multicolumn{3}{c}{ NITAWL cross sections $\left(\mathrm{cm}^{-1}\right)$} \\
\hline & $\frac{\mathrm{G}_{\mathrm{a}}}{1.43119 \mathrm{E}-04}$ & $\frac{\mathrm{G}_{\mathrm{f}}}{3.25379 \mathrm{E}-04}$ & $\frac{\mathrm{G}_{\mathrm{f}}}{1.25277 \mathrm{E}-04}$ & $\frac{\mathrm{G}_{\mathrm{a}}}{1.42796 \mathrm{E}-04}$ & $\frac{\mathrm{G}_{\mathrm{f}}}{3.25595 \mathrm{E}-04}$ & $\frac{\mathrm{G}_{\mathrm{f}}}{1.25157 \mathrm{E}-04}$ \\
1 & $3.53013 \mathrm{E}-03$ & $5.42307 \mathrm{E}-03$ & $2.22558 \mathrm{E}-03$ & $3.53101 \mathrm{E}-03$ & $5.37976 \mathrm{E}-03$ & $2.20780 \mathrm{E}-03$ \\
2 & $5.06839 \mathrm{E}-03$ & $1.03472 \mathrm{E}-02$ & $4.24642 \mathrm{E}-03$ & $5.21793 \mathrm{E}-03$ & $1.06593 \mathrm{E}-02$ & $4.37445 \mathrm{E}-03$ \\
3 & $4.85643 \mathrm{E}-02$ & $1.01103 \mathrm{E}-01$ & $4.14919 \mathrm{E}-02$ & $4.86105 \mathrm{E}-02$ & $1.01201 \mathrm{E}-01$ & $4.15317 \mathrm{E}-02$ \\
\hline
\end{tabular}

Table 4.19. TCA-2, Pin-1 four-group U-238 cross sections

\begin{tabular}{cccccccc}
\hline Group & \multicolumn{3}{c}{ CENTRM cross sections $\left(\mathrm{cm}^{-1}\right)$} & \multicolumn{3}{c}{ NITAWL cross sections $\left(\mathrm{cm}^{-1}\right)$} \\
\hline & $\frac{\mathrm{G}_{\mathrm{a}}}{4.44405 \mathrm{E}-03}$ & $\frac{\mathrm{G}_{\mathrm{f}}}{8.24665 \mathrm{E}-03}$ & $\frac{\mathrm{G}_{\mathrm{f}}}{2.92378 \mathrm{E}-03}$ & $\frac{\mathrm{G}_{\mathrm{a}}}{4.49711 \mathrm{E}-03}$ & $\frac{\mathrm{G}_{\mathrm{f}}}{8.46895 \mathrm{E}-03}$ & $\frac{\mathrm{G}_{\mathrm{f}}}{2.99705 \mathrm{E}-03}$ \\
1 & $3.85408 \mathrm{E}-02$ & $3.81629 \mathrm{E}-06$ & $1.58108 \mathrm{E}-06$ & $3.63117 \mathrm{E}-02$ & $3.48554 \mathrm{E}-06$ & $1.44405 \mathrm{E}-06$ \\
2 & $6.97263 \mathrm{E}-03$ & $2.42518 \mathrm{E}-08$ & $1.00480 \mathrm{E}-08$ & $6.99494 \mathrm{E}-03$ & $2.45706 \mathrm{E}-08$ & $1.01801 \mathrm{E}-08$ \\
3 & $2.75966 \mathrm{E}-02$ & $1.29711 \mathrm{E}-07$ & $5.37418 \mathrm{E}-08$ & $2.76225 \mathrm{E}-02$ & $1.29835 \mathrm{E}-07$ & $5.37930 \mathrm{E}-08$ \\
\hline
\end{tabular}

Table 4.20. TCA-2, Pin-1 four-group $\mathrm{Pu}-239$ cross sections

\begin{tabular}{|c|c|c|c|c|c|c|}
\hline \multirow[t]{2}{*}{ Group } & \multicolumn{3}{|c|}{ CENTRM cross sections $\left(\mathrm{cm}^{-1}\right)$} & \multicolumn{3}{|c|}{ NITAWL cross sections $\left(\mathrm{cm}^{-1}\right)$} \\
\hline & $\mathrm{G}_{\mathrm{a}}$ & $<\mathrm{G}_{\mathrm{f}}$ & $\mathrm{G}_{\mathrm{f}}$ & $\mathrm{G}_{\mathrm{a}-}$ & $<\mathrm{G}_{\mathrm{f}}$ & $\mathrm{G}_{\mathrm{f}}$ \\
\hline 1 & $5.11583 \mathrm{E}-04$ & $\overline{1.49239 \mathrm{E}-03}$ & $\overline{4.80303 \mathrm{E}-04}$ & $\overline{5.11437 \mathrm{E}-04}$ & $1.49567 \mathrm{E}-03$ & $4.80666 \mathrm{E}-04$ \\
\hline 2 & $1.10651 \mathrm{E}-02$ & $1.80525 \mathrm{E}-02$ & $6.26864 \mathrm{E}-03$ & 1.07872E-02 & $1.76378 \mathrm{E}-02$ & $6.12465 \mathrm{E}-03$ \\
\hline 3 & 3.33360E-02 & 6.55390E-02 & $2.27581 \mathrm{E}-02$ & 3.37318E-02 & $6.63232 \mathrm{E}-02$ & 2.30304E-02 \\
\hline 4 & 2.67093E-01 & 5.40384E-01 & $1.86909 \mathrm{E}-01$ & $2.66605 \mathrm{E}-01$ & 5.39661E-01 & $1.86656 \mathrm{E}-01$ \\
\hline
\end{tabular}


Table 4.21. TCA-2, Pin-78 four-group fluxes

\begin{tabular}{ccc}
\hline Group & $\begin{array}{c}\text { CENTRM M } \\
\left(\mathrm{cm}^{-2}-\mathrm{s}^{-1}\right)\end{array}$ & $\begin{array}{c}\text { NITAWL M } \\
\left(\mathrm{cm}^{-2}-\mathrm{s}^{-1}\right)\end{array}$ \\
\hline 1 & $2.302 \mathrm{E}-04$ & $2.272 \mathrm{E}-04$ \\
2 & $8.173 \mathrm{E}-05$ & $8.176 \mathrm{E}-05$ \\
3 & $1.759 \mathrm{E}-05$ & $1.791 \mathrm{E}-05$ \\
4 & $6.122 \mathrm{E}-05$ & $6.071 \mathrm{E}-05$ \\
\hline
\end{tabular}

Table 4.22. TCA-2, Pin-78 four-group U-235 cross sections

\begin{tabular}{cccccccc}
\hline Group & \multicolumn{3}{c}{ CENTRM cross sections $\left(\mathrm{cm}^{-1}\right)$} & \multicolumn{3}{c}{ NITAWL cross sections $\left(\mathrm{cm}^{-1}\right)$} \\
\hline & $\frac{\mathrm{G}_{\mathrm{a}}}{1.47775 \mathrm{E}-04}$ & $\frac{\mathrm{G}_{\mathrm{f}}}{3.29361 \mathrm{E}-04}$ & $\frac{\mathrm{G}_{\mathrm{f}}}{1.27654 \mathrm{E}-04}$ & $\frac{\mathrm{G}_{\mathrm{a}}}{1.47727 \mathrm{E}-04}$ & $\frac{\mathrm{G}_{\mathrm{f}}}{3.29384 \mathrm{E}-04}$ & $\frac{\mathrm{G}_{\mathrm{f}}}{1.27666 \mathrm{E}-04}$ \\
1 & $3.40270 \mathrm{E}-03$ & $5.23267 \mathrm{E}-03$ & $2.14744 \mathrm{E}-03$ & $3.44284 \mathrm{E}-03$ & $5.28022 \mathrm{E}-03$ & $2.16695 \mathrm{E}-03$ \\
2 & $5.19904 \mathrm{E}-03$ & $1.06173 \mathrm{E}-02$ & $4.35723 \mathrm{E}-03$ & $5.12518 \mathrm{E}-03$ & $1.04631 \mathrm{E}-02$ & $4.29398 \mathrm{E}-03$ \\
3 & $4.56001 \mathrm{E}-02$ & $9.48282 \mathrm{E}-02$ & $3.89167 \mathrm{E}-02$ & $4.54184 \mathrm{E}-02$ & $9.44484 \mathrm{E}-02$ & $3.87607 \mathrm{E}-02$ \\
\hline
\end{tabular}

Table 4.23. TCA-2, Pin-78 four-group U-238 cross sections

\begin{tabular}{|c|c|c|c|c|c|c|}
\hline \multirow[t]{2}{*}{ Group } & \multicolumn{3}{|c|}{ CENTRM cross sections $\left(\mathrm{cm}^{-1}\right)$} & \multicolumn{3}{|c|}{ NITAWL cross sections $\left(\mathrm{cm}^{-1}\right)$} \\
\hline & $\mathrm{G}_{3}$ & $<\mathrm{G}_{\mathrm{f}}$ & $\mathrm{G}_{\mathrm{f}}$ & $\mathrm{G}_{\mathrm{a}}$ & $\mathrm{G}_{\mathrm{f}}$ & $\mathrm{G}_{\mathrm{f}}$ \\
\hline 1 & $4.32970 \mathrm{E}-03$ & $7.46321 \mathrm{E}-03$ & $2.65044 \mathrm{E}-03$ & $4.32945 \mathrm{E}-03$ & $7.46893 \mathrm{E}-03$ & $2.65359 \mathrm{E}-03$ \\
\hline 2 & 3.93173E-02 & $3.71651 \mathrm{E}-06$ & $1.53973 \mathrm{E}-06$ & $3.66084 \mathrm{E}-02$ & 3.70903E-06 & $1.53663 \mathrm{E}-06$ \\
\hline 3 & 7.00819E-03 & $2.46566 \mathrm{E}-08$ & $1.02156 \mathrm{E}-08$ & 6.99481E-03 & $2.44808 \mathrm{E}-08$ & 1.01429E-08 \\
\hline 4 & $2.60606 \mathrm{E}-02$ & $1.22341 \mathrm{E}-07$ & $5.06882 \mathrm{E}-08$ & 2.59687E-02 & $1.21901 \mathrm{E}-07$ & $5.05058 \mathrm{E}-08$ \\
\hline
\end{tabular}

Table 4.24. TCA-2, Pin-78 four-group Pu-239 cross sections

\begin{tabular}{|c|c|c|c|c|c|c|}
\hline \multirow[t]{2}{*}{ Group } & \multicolumn{3}{|c|}{ CENTRM cross sections $\left(\mathrm{cm}^{-1}\right)$} & \multicolumn{3}{|c|}{ NITAWL cross sections $\left(\mathrm{cm}^{-1}\right)$} \\
\hline & $\mathrm{G}_{\mathrm{a}}$ & $<\mathrm{G}_{\mathrm{f}}$ & $\mathrm{G}_{\mathrm{f}}$ & $\mathrm{G}_{\mathrm{a}}$ & $\mathrm{G}_{\mathrm{f}}$ & $\mathrm{G}_{\mathrm{f}}$ \\
\hline 1 & $5.13614 \mathrm{E}-04$ & $\overline{1.47354 \mathrm{E}-03}$ & $\overline{4.77076 \mathrm{E}-04}$ & $5.13794 \mathrm{E}-04$ & $\overline{1.47461 \mathrm{E}-03}$ & $\overline{4.77474 \mathrm{E}-04}$ \\
\hline 2 & 1.10415E-02 & $1.82425 \mathrm{E}-02$ & $6.33460 \mathrm{E}-03$ & $1.06940 \mathrm{E}-02$ & 1.76497E-02 & $6.12879 \mathrm{E}-03$ \\
\hline 3 & 3.48073E-02 & 6.83161E-02 & 2.37224E-02 & 3.39004E-02 & $6.66510 \mathrm{E}-02$ & 2.31443E-02 \\
\hline 4 & $2.84748 \mathrm{E}-01$ & $5.63652 \mathrm{E}-01$ & $1.95041 \mathrm{E}-01$ & $2.85625 \mathrm{E}-01$ & 5.64792E-01 & $1.95441 \mathrm{E}-01$ \\
\hline
\end{tabular}




\begin{tabular}{|c|c|c|c|c|c|c|c|c|c|c|c|}
\hline & & & & & & & & & & & $\begin{array}{l}4.889 \\
(1.00)\end{array}$ \\
\hline & $\begin{array}{l}0.143 \\
(0.72)\end{array}$ & & & & & & & & & $\begin{array}{l}4.823 \\
(0.45)\end{array}$ & $\begin{array}{l}4.841 \\
(0.48)\end{array}$ \\
\hline & & & & & & & & & $\begin{array}{r}4.680 \\
(0.49)\end{array}$ & $\begin{array}{l}4.775 \\
(0.35)\end{array}$ & $\begin{array}{l}4.801 \\
(0.51)\end{array}$ \\
\hline & & & & & & & & $\begin{array}{l}4.418 \\
(0.49)\end{array}$ & $\begin{array}{l}4.556 \\
(0.35)\end{array}$ & $\begin{array}{l}4.662 \\
(0.34)\end{array}$ & $\begin{array}{l}4.671 \\
(0.44)\end{array}$ \\
\hline & & & & & & & $\begin{array}{l}4.131 \\
(0.53)\end{array}$ & $\begin{array}{l}4.290 \\
(0.37)\end{array}$ & $\begin{array}{l}4.381 \\
(0.40)\end{array}$ & $\begin{array}{l}4.473 \\
(0.37)\end{array}$ & $\begin{array}{l}4.486 \\
(0.50)\end{array}$ \\
\hline & & & & & & $\begin{array}{l}3.736 \\
(0.54)\end{array}$ & $\begin{array}{l}3.929 \\
(0.37)\end{array}$ & $\begin{array}{l}4.067 \\
(0.37)\end{array}$ & $\begin{array}{l}4.181 \\
(0.37)\end{array}$ & $\begin{array}{l}4.232 \\
(0.35)\end{array}$ & $\begin{array}{l}4.253 \\
(0.47)\end{array}$ \\
\hline & & & & & $\begin{array}{l}3.282 \\
(0.51)\end{array}$ & $\begin{array}{c}3.485 \\
(0.38)\end{array}$ & $\begin{array}{l}3.672 \\
(0.38)\end{array}$ & $\begin{array}{l}3.812 \\
(0.35)\end{array}$ & $\begin{array}{l}3.911 \\
(0.36)\end{array}$ & $\begin{array}{l}3.997 \\
(0.36)\end{array}$ & $\begin{array}{l}4.014 \\
(0.57)\end{array}$ \\
\hline & & & & $\begin{array}{c}2.803 \\
(0.63)\end{array}$ & $\begin{array}{l}3.040 \\
(0.42)\end{array}$ & $\begin{array}{l}3.236 \\
(0.40)\end{array}$ & $\begin{array}{l}3.429 \\
(0.41)\end{array}$ & $\begin{array}{l}3.556 \\
(0.41)\end{array}$ & $\begin{array}{c}3.634 \\
(0.40)\end{array}$ & $\begin{array}{c}3.682 \\
(0.39)\end{array}$ & $\begin{array}{l}3.716 \\
(0.53)\end{array}$ \\
\hline & & & $\begin{array}{l}2.356 \\
(0.60)\end{array}$ & $\begin{array}{l}2.576 \\
(0.50)\end{array}$ & $\begin{array}{l}2.763 \\
(0.42)\end{array}$ & $\begin{array}{l}2.948 \\
(0.40)\end{array}$ & $\begin{array}{l}3.121 \\
(0.39)\end{array}$ & $\begin{array}{l}3.244 \\
(0.43)\end{array}$ & $\begin{array}{l}3.319 \\
(0.40)\end{array}$ & $\begin{array}{l}3.364 \\
(0.43)\end{array}$ & $\begin{array}{l}3.384 \\
(0.59)\end{array}$ \\
\hline & & $\begin{array}{c}1.962 \\
(0.81)\end{array}$ & $\begin{array}{l}2.133 \\
(0.49)\end{array}$ & $\begin{array}{l}2.338 \\
0.47)\end{array}$ & $\begin{array}{l}2.571 \\
(0.43)\end{array}$ & $\begin{array}{l}2.695 \\
(0.42)\end{array}$ & $\begin{array}{l}2.838 \\
(0.44)\end{array}$ & $\begin{array}{l}2.947 \\
(0.47)\end{array}$ & $\begin{array}{l}3.038 \\
(0.46)\end{array}$ & $\begin{array}{l}3.085 \\
(0.43)\end{array}$ & $\begin{array}{l}3.097 \\
(0.55)\end{array}$ \\
\hline & $\begin{array}{c}1.782 \\
(0.78)\end{array}$ & $\begin{array}{c}1.874 \\
(0.52)\end{array}$ & $\begin{array}{l}2.046 \\
(0.52)\end{array}$ & $\begin{array}{c}2.263 \\
(0.47)\end{array}$ & $\begin{array}{l}2.423 \\
(0.44)\end{array}$ & $\begin{array}{l}2.573 \\
(0.48)\end{array}$ & $\begin{array}{l}2.708 \\
(0.46)\end{array}$ & $\begin{array}{l}2.819 \\
(0.40)\end{array}$ & $\begin{array}{l}2.891 \\
(0.44)\end{array}$ & $\begin{array}{l}2.907 \\
(0.42)\end{array}$ & $\begin{array}{r}2.936 \\
(0.54)\end{array}$ \\
\hline $\begin{array}{l}2.156 \\
(0.74)\end{array}$ & $\begin{array}{l}2.021 \\
(0.52)\end{array}$ & $\begin{array}{l}2.204 \\
(0.50)\end{array}$ & $\begin{array}{l}2.429 \\
(0.49)\end{array}$ & $\begin{array}{c}2.663 \\
(0.44)\end{array}$ & $\begin{array}{l}2.876 \\
(0.42)\end{array}$ & $\begin{array}{l}3.083 \\
(0.41)\end{array}$ & $\begin{array}{l}3.215 \\
(0.40)\end{array}$ & $\begin{array}{l}3.338 \\
(0.42)\end{array}$ & $\begin{array}{l}3.414 \\
(0.40)\end{array}$ & $\begin{array}{l}3.474 \\
(0.33)\end{array}$ & $\begin{array}{l}3.480 \\
(0.59)\end{array}$ \\
\hline
\end{tabular}

Fig. 4.7a. Pin-power distribution for CENTRM benchmark TCA-3. Value in parentheses is percent standard deviation. 


\begin{tabular}{|c|c|c|c|c|c|c|c|c|c|c|c|}
\hline & & & & & & & & & & & $\begin{array}{l}4.857 \\
(1.00)\end{array}$ \\
\hline & $\begin{array}{l}0.143 \\
(0.73)\end{array}$ & & & & & & & & & $\begin{array}{l}4.845 \\
(0.46)\end{array}$ & $\begin{array}{l}4.826 \\
(0.50)\end{array}$ \\
\hline & & & & & & & & & $\begin{array}{l}4.692 \\
(0.49)\end{array}$ & $\begin{array}{l}4.713 \\
(0.37)\end{array}$ & $\begin{array}{l}4.814 \\
(0.46)\end{array}$ \\
\hline & & & & & & & & $\begin{array}{l}4.430 \\
(0.53)\end{array}$ & $\begin{array}{l}4.565 \\
(0.34)\end{array}$ & $\begin{array}{l}4.635 \\
(0.34)\end{array}$ & $\begin{array}{l}4.646 \\
(0.48)\end{array}$ \\
\hline & & & & & & & $\begin{array}{l}4.145 \\
(0.54)\end{array}$ & $\begin{array}{l}4.284 \\
(0.39)\end{array}$ & $\begin{array}{l}4.400 \\
(0.36)\end{array}$ & $\begin{array}{l}4.464 \\
(0.36)\end{array}$ & $\begin{array}{l}4.512 \\
(0.50)\end{array}$ \\
\hline & & & & & & $\begin{array}{l}3.718 \\
(0.51)\end{array}$ & $\begin{array}{l}3.929 \\
(0.35)\end{array}$ & $\begin{array}{l}4.066 \\
(0.38)\end{array}$ & $\begin{array}{l}4.195 \\
(0.37)\end{array}$ & $\begin{array}{l}4.262 \\
(0.33)\end{array}$ & $\begin{array}{l}4.242 \\
(0.50)\end{array}$ \\
\hline & & & & & $\begin{array}{l}3.318 \\
(0.57)\end{array}$ & $\begin{array}{l}3.525 \\
(0.36)\end{array}$ & $\begin{array}{c}3.701 \\
(0.40)\end{array}$ & $\begin{array}{l}3.836 \\
(0.37)\end{array}$ & $\begin{array}{l}3.934 \\
(0.37)\end{array}$ & $\begin{array}{l}3.997 \\
(0.35)\end{array}$ & $\begin{array}{r}4.006 \\
(0.51)\end{array}$ \\
\hline & & & & $\begin{array}{l}2.807 \\
(0.61)\end{array}$ & $\begin{array}{l}3.095 \\
(0.41)\end{array}$ & $\begin{array}{l}3.287 \\
(0.39)\end{array}$ & $\begin{array}{l}3.406 \\
(0.41)\end{array}$ & $\begin{array}{l}3.538 \\
(0.40)\end{array}$ & $\begin{array}{l}3.646 \\
(0.36)\end{array}$ & $\begin{array}{l}3.714 \\
(0.36)\end{array}$ & $\begin{array}{l}3.704 \\
(0.49)\end{array}$ \\
\hline & & & $\begin{array}{l}2.365 \\
(0.67)\end{array}$ & $\begin{array}{l}2.589 \\
(0.48)\end{array}$ & $\begin{array}{l}2.799 \\
(0.44)\end{array}$ & $\begin{array}{l}2.975 \\
(0.41)\end{array}$ & $\begin{array}{c}3.132 \\
(0.41)\end{array}$ & $\begin{array}{l}3.230 \\
(0.40)\end{array}$ & $\begin{array}{l}3.314 \\
(0.41)\end{array}$ & $\begin{array}{l}3.391 \\
(0.39)\end{array}$ & $\begin{array}{l}3.396 \\
(0.59)\end{array}$ \\
\hline & & $\begin{array}{l}1.949 \\
(0.76)\end{array}$ & $\begin{array}{l}2.150 \\
(0.51)\end{array}$ & $\begin{array}{l}2.363 \\
0.47)\end{array}$ & $\begin{array}{l}2.541 \\
(0.48)\end{array}$ & $\begin{array}{l}2.706 \\
(0.45)\end{array}$ & $\begin{array}{l}2.838 \\
(0.44)\end{array}$ & $\begin{array}{l}2.977 \\
(0.42)\end{array}$ & $\begin{array}{l}3.035 \\
(0.43)\end{array}$ & $\begin{array}{l}3.082 \\
(0.43)\end{array}$ & $\begin{array}{l}3.092 \\
(0.55)\end{array}$ \\
\hline & $\begin{array}{l}1.762 \\
(0.77)\end{array}$ & $\begin{array}{l}1.861 \\
(0.58)\end{array}$ & $\begin{array}{l}2.063 \\
(0.49)\end{array}$ & $\begin{array}{l}2.238 \\
(0.53)\end{array}$ & $\begin{array}{l}2.433 \\
(0.47)\end{array}$ & $\begin{array}{l}2.572 \\
(0.46)\end{array}$ & $\begin{array}{l}2.729 \\
(0.48)\end{array}$ & $\begin{array}{l}2.840 \\
(0.40)\end{array}$ & $\begin{array}{l}2.894 \\
(0.44)\end{array}$ & $\begin{array}{l}2.944 \\
(0.42)\end{array}$ & $\begin{array}{l}2.962 \\
(0.59)\end{array}$ \\
\hline $\begin{array}{l}2.155 \\
(0.76)\end{array}$ & $\begin{array}{l}2.037 \\
(0.53)\end{array}$ & $\begin{array}{l}2.194 \\
(0.47\end{array}$ & $\begin{array}{l}2.420 \\
(0.50)\end{array}$ & $\begin{array}{l}2.654 \\
(0.46)\end{array}$ & $\begin{array}{l}2.866 \\
(0.42)\end{array}$ & $\begin{array}{l}3.058 \\
(0.41)\end{array}$ & $\begin{array}{l}3.225 \\
(0.40)\end{array}$ & $\begin{array}{l}3.315 \\
(0.41)\end{array}$ & $\begin{array}{l}3.430 \\
(0.39)\end{array}$ & $\begin{array}{l}3.480 \\
(0.37)\end{array}$ & $\begin{array}{c}3.483 \\
(0.58)\end{array}$ \\
\hline
\end{tabular}

Fig. 4.7b. Pin-power distribution for NITAWL benchmark TCA-3. Value in parentheses is percent standard deviation. 
Table 4.25. Selected reaction rates for CENTRM case TCA-3

\begin{tabular}{|c|c|c|c|c|c|c|}
\hline Pin & Region & $\begin{array}{c}\mathrm{G}_{\mathrm{a}} \mathrm{M} \\
\left(\mathrm{cm}^{-3}-\mathrm{s}^{-1}\right)\end{array}$ & $\begin{array}{c}\mathcal{G}_{\mathrm{f}} \mathrm{M} \\
\left(\mathrm{cm}^{-3}-\mathrm{s}^{-1}\right)\end{array}$ & $\begin{array}{c}\mathrm{G}_{\mathrm{f}} \mathrm{M} \\
\left(\mathrm{cm}^{-3}-\mathrm{s}^{-1}\right)\end{array}$ & $\begin{array}{c}\mathrm{M} \\
\left(\mathrm{cm}-\mathrm{s}^{-1}\right)\end{array}$ & $\mathrm{M}_{\mathrm{t}}$ \\
\hline \multirow{3}{*}{1} & Fuel & $1.307 \mathrm{E}-05$ & $2.156 \mathrm{E}-05$ & 7.643E-06 & $5.476 \mathrm{E}-03$ & 0.271 \\
\hline & Clad & $2.496 \mathrm{E}-07$ & 0.0 & 0.0 & $1.701 \mathrm{E}-03$ & 0.085 \\
\hline & Mod. & $6.938 \mathrm{E}-07$ & 0.0 & 0.0 & $1.300 \mathrm{E}-02$ & 0.644 \\
\hline \multirow{3}{*}{78} & Fuel & $3.318 \mathrm{E}-05$ & $4.938 \mathrm{E}-05$ & $1.747 \mathrm{E}-05$ & $2.182 \mathrm{E}-02$ & 0.269 \\
\hline & Clad & $6.794 \mathrm{E}-07$ & 0.0 & 0.0 & $6.875 \mathrm{E}-03$ & 0.085 \\
\hline & Mod. & $1.442 \mathrm{E}-06$ & 0.0 & 0.0 & $5.233 \mathrm{E}-02$ & 0.646 \\
\hline
\end{tabular}

Table 4.26. Selected reaction rates for NITAWL case TCA-3

\begin{tabular}{|c|c|c|c|c|c|c|}
\hline Pin & Region & $\begin{array}{c}\mathrm{G}_{\mathrm{a}} \mathrm{M} \\
\left(\mathrm{cm}^{-3}-\mathrm{s}^{-1}\right)\end{array}$ & $\begin{array}{c}\mathcal{G}_{\mathrm{f}} \mathrm{M} \\
\left(\mathrm{cm}^{-3}-\mathrm{s}^{-1}\right)\end{array}$ & $\begin{array}{c}\mathrm{G}_{\mathrm{f}} \mathrm{M} \\
\left(\mathrm{cm}^{-3}-\mathrm{s}^{-1}\right)\end{array}$ & $\begin{array}{c}\mathrm{M} \\
\left(\mathrm{cm}-\mathrm{s}^{-1}\right)\end{array}$ & $\mathrm{M} / \mathrm{M}_{\mathrm{t}}$ \\
\hline \multirow{3}{*}{1} & Fuel & $1.296 \mathrm{E}-05$ & $2.142 \mathrm{E}-05$ & $7.591 \mathrm{E}-06$ & $5.418 \mathrm{E}-03$ & 0.272 \\
\hline & Clad & $2.463 \mathrm{E}-07$ & 0.0 & 0.0 & $1.676 \mathrm{E}-03$ & 0.084 \\
\hline & Mod. & $6.886 \mathrm{E}-07$ & 0.0 & 0.0 & $1.285 \mathrm{E}-02$ & 0.644 \\
\hline \multirow{3}{*}{78} & Fuel & $3.290 \mathrm{E}-05$ & $4.896 \mathrm{E}-05$ & $1.732 \mathrm{E}-05$ & $2.172 \mathrm{E}-02$ & 0.269 \\
\hline & Clad & $6.746 \mathrm{E}-07$ & 0.0 & 0.0 & $6.861 \mathrm{E}-03$ & 0.085 \\
\hline & Mod. & $1.449 \mathrm{E}-06$ & 0.0 & 0.0 & $5.222 \mathrm{E}-02$ & 0.646 \\
\hline
\end{tabular}


Table 4.27. TCA-3, Pin-1 four-group fluxes

\begin{tabular}{ccc}
\hline Group & $\begin{array}{c}\text { CENTRM M } \\
\left(\mathrm{cm}^{-2}-\mathrm{s}^{-1}\right)\end{array}$ & $\begin{array}{c}\text { NITAWL M } \\
\left(\mathrm{cm}^{-2}-\mathrm{s}^{-1}\right)\end{array}$ \\
\hline 1 & $4.915 \mathrm{E}-05$ & $4.860 \mathrm{E}-05$ \\
2 & $1.453 \mathrm{E}-05$ & $1.440 \mathrm{E}-05$ \\
3 & $3.519 \mathrm{E}-06$ & $3.300 \mathrm{E}-06$ \\
4 & $2.876 \mathrm{E}-05$ & $2.864 \mathrm{E}-05$ \\
\hline
\end{tabular}

Table 4.28. TCA-3, Pin-1 four-group U-235 cross sections

\begin{tabular}{cccccccc}
\hline Group & \multicolumn{2}{c}{ CENTRM cross sections $\left(\mathrm{cm}^{-1}\right)$} & \multicolumn{3}{c}{ NITAWL cross sections $\left(\mathrm{cm}^{-1}\right)$} \\
\hline & $\frac{\mathrm{G}_{\mathrm{a}}}{1.42818 \mathrm{E}-04}$ & $\frac{\mathrm{G}_{\mathrm{f}}}{3.25054 \mathrm{E}-04}$ & $\frac{\mathrm{G}_{\mathrm{f}}}{1.25124 \mathrm{E}-04}$ & $\frac{\mathrm{G}_{\mathrm{a}}}{1.43107 \mathrm{E}-04}$ & $\frac{\mathrm{G}_{\mathrm{f}}}{3.25739 \mathrm{E}-04}$ & $\frac{\mathrm{G}_{\mathrm{f}}}{1.25325 \mathrm{E}-04}$ \\
1 & $3.36929 \mathrm{E}-03$ & $5.19745 \mathrm{E}-03$ & $2.13299 \mathrm{E}-03$ & $3.46178 \mathrm{E}-03$ & $5.31513 \mathrm{E}-03$ & $2.18128 \mathrm{E}-03$ \\
2 & $5.17928 \mathrm{E}-03$ & $1.05930 \mathrm{E}-02$ & $4.34726 \mathrm{E}-03$ & $5.19818 \mathrm{E}-03$ & $1.06224 \mathrm{E}-02$ & $4.35933 \mathrm{E}-03$ \\
3 & $4.86050 \mathrm{E}-02$ & $1.01190 \mathrm{E}-01$ & $4.15275 \mathrm{E}-02$ & $4.84951 \mathrm{E}-02$ & $1.00958 \mathrm{E}-01$ & $4.14323 \mathrm{E}-02$ \\
\hline
\end{tabular}

Table 4.29. TCA-3, Pin-1 four-group U-238 cross sections

\begin{tabular}{cccccccc}
\hline Group & \multicolumn{3}{c}{ CENTRM cross sections $\left(\mathrm{cm}^{-1}\right)$} & \multicolumn{2}{c}{ NITAWL cross sections $\left(\mathrm{cm}^{-1}\right)$} \\
\hline & $\frac{\mathrm{G}_{\mathrm{a}}}{4.44993 \mathrm{E}-03}$ & $\frac{\mathrm{G}_{\mathrm{f}}}{8.27601 \mathrm{E}-03}$ & $\frac{\mathrm{G}_{\mathrm{f}}}{2.93516 \mathrm{E}-03}$ & $\frac{\mathrm{G}_{\mathrm{a}}}{4.47772 \mathrm{E}-03}$ & $\frac{\mathrm{G}_{\mathrm{f}}}{8.36366 \mathrm{E}-03}$ & $\frac{\mathrm{G}_{\mathrm{f}}}{2.96352 \mathrm{E}-03}$ \\
1 & $3.65872 \mathrm{E}-02$ & $3.54715 \mathrm{E}-06$ & $1.46957 \mathrm{E}-06$ & $3.65101 \mathrm{E}-02$ & $3.54449 \mathrm{E}-06$ & $1.46847 \mathrm{E}-06$ \\
2 & $7.01523 \mathrm{E}-03$ & $2.46279 \mathrm{E}-08$ & $1.02038 \mathrm{E}-08$ & $7.00700 \mathrm{E}-03$ & $2.46391 \mathrm{E}-08$ & $1.02084 \mathrm{E}-08$ \\
3 & $2.76173 \mathrm{E}-02$ & $1.29810 \mathrm{E}-07$ & $5.37830 \mathrm{E}-08$ & $2.75642 \mathrm{E}-02$ & $1.29555 \mathrm{E}-07$ & $5.36770 \mathrm{E}-08$ \\
\hline
\end{tabular}

Table 4.30. TCA-3, Pin-1 four-group Pu-239 cross sections

\begin{tabular}{ccccccc}
\hline Group & \multicolumn{3}{c}{ CENTRM cross sections $\left(\mathrm{cm}^{-1}\right)$} & \multicolumn{3}{c}{ NITAWL cross sections $\left(\mathrm{cm}^{-1}\right)$} \\
\hline & $\frac{\mathrm{G}_{\mathrm{a}}}{1}$ & $\frac{\mathrm{G}_{\mathrm{f}}}{1.11491 \mathrm{E}-04}$ & $\frac{\mathrm{G}_{\mathrm{f}}}{1.49361 \mathrm{E}-03}$ & $\frac{4.80637 \mathrm{E}-04}{4.11798 \mathrm{E}-04}$ & $\frac{\mathrm{G}_{\mathrm{f}}}{1.49459 \mathrm{E}-03}$ & $\frac{\mathrm{G}_{\mathrm{f}}}{4.80689 \mathrm{E}-04}$ \\
2 & $1.09264 \mathrm{E}-02$ & $1.82048 \mathrm{E}-02$ & $6.32153 \mathrm{E}-03$ & $1.06379 \mathrm{E}-02$ & $1.74194 \mathrm{E}-02$ & $6.04882 \mathrm{E}-03$ \\
3 & $3.56962 \mathrm{E}-02$ & $6.99270 \mathrm{E}-02$ & $2.42818 \mathrm{E}-02$ & $3.44527 \mathrm{E}-02$ & $6.76673 \mathrm{E}-02$ & $2.34972 \mathrm{E}-02$ \\
4 & $2.66921 \mathrm{E}-01$ & $5.40191 \mathrm{E}-01$ & $1.86841 \mathrm{E}-01$ & $2.66795 \mathrm{E}-01$ & $5.39735 \mathrm{E}-01$ & $1.86684 \mathrm{E}-01$ \\
\hline
\end{tabular}


Table 4.31. TCA-3, Pin-78 four-group fluxes

\begin{tabular}{ccc}
\hline Group & $\begin{array}{c}\text { CENTRM M } \\
\left(\mathrm{cm}^{-2}-\mathrm{s}^{-1}\right)\end{array}$ & $\begin{array}{c}\text { NITAWL M } \\
\left(\mathrm{cm}^{-2}-\mathrm{s}^{-1}\right)\end{array}$ \\
\hline 1 & $2.243 \mathrm{E}-04$ & $2.215 \mathrm{E}-04$ \\
2 & $8.038 \mathrm{E}-05$ & $8.190 \mathrm{E}-05$ \\
3 & $1.810 \mathrm{E}-05$ & $1.765 \mathrm{E}-05$ \\
4 & $5.962 \mathrm{E}-05$ & $5.956 \mathrm{E}-05$ \\
\hline
\end{tabular}

Table 4.32. TCA-3, Pin-78 four-group U-235 cross sections

\begin{tabular}{cccccccc}
\hline Group & \multicolumn{3}{c}{ CENTRM cross sections $\left(\mathrm{cm}^{-1}\right)$} & \multicolumn{3}{c}{ NITAWL cross sections $\left(\mathrm{cm}^{-1}\right)$} \\
\hline & $\frac{\mathrm{G}_{\mathrm{a}}}{1.47728 \mathrm{E}-04}$ & $\frac{\mathrm{G}_{\mathrm{f}}}{3.29228 \mathrm{E}-04}$ & $\frac{\mathrm{G}_{\mathrm{f}}}{1.27610 \mathrm{E}-04}$ & $\frac{\mathrm{G}_{\mathrm{a}}}{1.47738 \mathrm{E}-04}$ & $\frac{\mathrm{G}_{\mathrm{f}}}{3.29429 \mathrm{E}-04}$ & $\frac{\mathrm{G}_{\mathrm{f}}}{1.27667 \mathrm{E}-04}$ \\
1 & $3.36825 \mathrm{E}-03$ & $5.14716 \mathrm{E}-03$ & $2.11235 \mathrm{E}-03$ & $3.40828 \mathrm{E}-03$ & $5.21512 \mathrm{E}-03$ & $2.14023 \mathrm{E}-03$ \\
2 & $5.12983 \mathrm{E}-03$ & $1.04660 \mathrm{E}-02$ & $4.29517 \mathrm{E}-03$ & $5.13172 \mathrm{E}-03$ & $1.04717 \mathrm{E}-02$ & $4.29747 \mathrm{E}-03$ \\
3 & $4.59777 \mathrm{E}-02$ & $9.56112 \mathrm{E}-02$ & $3.92380 \mathrm{E}-02$ & $4.54550 \mathrm{E}-02$ & $9.45238 \mathrm{E}-02$ & $3.87918 \mathrm{E}-02$ \\
\hline
\end{tabular}

Table 4.33. TCA-3, Pin-78 four-group U-238 cross sections

\begin{tabular}{|c|c|c|c|c|c|c|}
\hline \multirow[t]{2}{*}{ Group } & \multicolumn{3}{|c|}{ CENTRM cross sections $\left(\mathrm{cm}^{-1}\right)$} & \multicolumn{3}{|c|}{ NITAWL cross sections $\left(\mathrm{cm}^{-1}\right)$} \\
\hline & $\mathrm{G}_{3}$ & $<\mathrm{G}_{\mathrm{f}}$ & $\mathrm{G}_{\mathrm{f}}$ & $\mathrm{G}_{\mathrm{a}}$ & $\mathrm{G}_{\mathrm{f}}$ & $\mathrm{G}_{\mathrm{f}}$ \\
\hline 1 & $4.31758 \mathrm{E}-03$ & $7.42746 \mathrm{E}-03$ & $2.63695 \mathrm{E}-03$ & $4.34475 \mathrm{E}-03$ & $7.50781 \mathrm{E}-03$ & $2.66748 \mathrm{E}-03$ \\
\hline 2 & 3.69603E-02 & 3.91179E-06 & $1.62064 \mathrm{E}-06$ & 3.60504E-02 & 3.75949E-06 & $1.55755 \mathrm{E}-06$ \\
\hline 3 & 6.98884E-03 & $2.44361 \mathrm{E}-08$ & 1.01243E-08 & $6.98652 \mathrm{E}-03$ & 2.44809E-08 & 1.01429E-08 \\
\hline 4 & $2.62422 \mathrm{E}-02$ & $1.23201 \mathrm{E}-07$ & $5.10444 \mathrm{E}-08$ & $2.59876 \mathrm{E}-02$ & $1.21988 \mathrm{E}-07$ & $5.05418 \mathrm{E}-08$ \\
\hline
\end{tabular}

Table 4.34. TCA-3, Pin-78 four-group Pu-239 cross sections

\begin{tabular}{ccccccc}
\hline Group & \multicolumn{3}{c}{ CENTRM cross sections $\left(\mathrm{cm}^{-1}\right)$} & \multicolumn{3}{c}{ NITAWL cross sections $\left(\mathrm{cm}^{-1}\right)$} \\
\hline & $\frac{\mathrm{G}_{\mathrm{a}}}{4}$ & $\frac{\mathrm{G}_{\mathrm{f}}}{1.43304 \mathrm{E}-04}$ & $\frac{\mathrm{G}_{\mathrm{f}}}{1.47258 \mathrm{E}-03}$ & $\frac{4.76835 \mathrm{E}-04}{5.13774 \mathrm{E}-04}$ & $\frac{\mathrm{G}_{\mathrm{f}}}{1.47465 \mathrm{E}-03}$ & $\frac{\mathrm{G}_{\mathrm{f}}}{4.77404 \mathrm{E}-04}$ \\
2 & $1.08802 \mathrm{E}-02$ & $1.78741 \mathrm{E}-02$ & $6.20669 \mathrm{E}-03$ & $1.05497 \mathrm{E}-02$ & $1.73471 \mathrm{E}-02$ & $6.02371 \mathrm{E}-03$ \\
3 & $3.36764 \mathrm{E}-02$ & $6.62210 \mathrm{E}-02$ & $2.29950 \mathrm{E}-02$ & $3.26723 \mathrm{E}-02$ & $6.44142 \mathrm{E}-02$ & $2.23675 \mathrm{E}-02$ \\
4 & $2.87561 \mathrm{E}-01$ & $5.69182 \mathrm{E}-01$ & $1.96949 \mathrm{E}-01$ & $2.86127 \mathrm{E}-01$ & $5.65714 \mathrm{E}-01$ & $1.95759 \mathrm{E}-01$ \\
\hline
\end{tabular}




\begin{tabular}{|c|c|c|c|c|c|c|c|c|c|c|}
\hline & & & & & & & & & & $\begin{array}{l}6.107 \\
(0.90)\end{array}$ \\
\hline & $\begin{array}{l}0.155 \\
(0.61)\end{array}$ & & & & & & & & $\begin{array}{l}6.067 \\
(0.43)\end{array}$ & $\begin{array}{l}6.093 \\
(0.43)\end{array}$ \\
\hline & & & & & & & & $\begin{array}{l}5.865 \\
(0.43)\end{array}$ & $\begin{array}{l}5.933 \\
(0.35)\end{array}$ & $\begin{array}{l}5.977 \\
(0.46)\end{array}$ \\
\hline & & & & & & & $\begin{array}{l}5.480 \\
(0.46)\end{array}$ & $\begin{array}{l}5.673 \\
(0.35)\end{array}$ & $\begin{array}{l}5.759 \\
(0.31)\end{array}$ & $\begin{array}{l}5.797 \\
(0.43)\end{array}$ \\
\hline & & & & & & $\begin{array}{l}4.989 \\
(0.43)\end{array}$ & $\begin{array}{l}5.244 \\
(0.34)\end{array}$ & $\begin{array}{l}5.447 \\
(0.34)\end{array}$ & $\begin{array}{l}5.513 \\
(0.35)\end{array}$ & $\begin{array}{l}5.571 \\
(0.47)\end{array}$ \\
\hline & & & & & $\begin{array}{l}4.464 \\
(0.56)\end{array}$ & $\begin{array}{l}4.722 \\
(0.35)\end{array}$ & $\begin{array}{l}4.957 \\
(0.33)\end{array}$ & $\begin{array}{l}5.109 \\
(0.36)\end{array}$ & $\begin{array}{l}5.207 \\
(0.35)\end{array}$ & $\begin{array}{l}5.226 \\
(0.47)\end{array}$ \\
\hline & & & & $\begin{array}{c}3.839 \\
(0.55)\end{array}$ & $\begin{array}{l}4.125 \\
(0.38)\end{array}$ & $\begin{array}{l}4.412 \\
(0.33)\end{array}$ & $\begin{array}{l}4.611 \\
(0.35)\end{array}$ & $\begin{array}{l}4.762 \\
(0.32)\end{array}$ & $\begin{array}{l}4.805 \\
(0.34)\end{array}$ & $\begin{array}{l}4.824 \\
(0.46)\end{array}$ \\
\hline & & & $\begin{array}{c}3.152 \\
(0.60)\end{array}$ & $\begin{array}{l}3.489 \\
(0.40)\end{array}$ & $\begin{array}{l}3.765 \\
(0.38)\end{array}$ & $\begin{array}{l}3.994 \\
(0.35)\end{array}$ & $\begin{array}{l}4.201 \\
(0.34)\end{array}$ & $\begin{array}{l}4.308 \\
(0.38)\end{array}$ & $\begin{array}{l}4.384 \\
(0.35)\end{array}$ & $\begin{array}{l}4.402 \\
(0.52)\end{array}$ \\
\hline & & $\begin{array}{l}2.601 \\
(0.64)\end{array}$ & $\begin{array}{l}2.827 \\
(0.45)\end{array}$ & $\begin{array}{l}3.120 \\
(0.42)\end{array}$ & $\begin{array}{l}3.374 \\
(0.40)\end{array}$ & $\begin{array}{l}3.577 \\
(0.41)\end{array}$ & $\begin{array}{c}3.735 \\
(0.37)\end{array}$ & $\begin{array}{l}3.861 \\
(0.39)\end{array}$ & $\begin{array}{l}3.933 \\
(0.37)\end{array}$ & $\begin{array}{l}3.954 \\
(0.56)\end{array}$ \\
\hline & $\begin{array}{l}2.180 \\
(0.76)\end{array}$ & $\begin{array}{l}2.373 \\
(0.47)\end{array}$ & $\begin{array}{l}2.634 \\
(0.45)\end{array}$ & $\begin{array}{l}2.897 \\
(0.44)\end{array}$ & $\begin{array}{c}3.116 \\
(0.42)\end{array}$ & $\begin{array}{l}3.317 \\
(0.44)\end{array}$ & $\begin{array}{c}3.488 \\
(0.39)\end{array}$ & $\begin{array}{l}3.597 \\
(0.37)\end{array}$ & $\begin{array}{l}3.641 \\
(0.41)\end{array}$ & $\begin{array}{c}3.642 \\
(0.57)\end{array}$ \\
\hline $\begin{array}{l}2.423 \\
(0.70)\end{array}$ & $\begin{array}{l}2.365 \\
(0.50)\end{array}$ & $\begin{array}{c}2.614 \\
(0.49)\end{array}$ & $\begin{array}{l}2.928 \\
(0.42)\end{array}$ & $\begin{array}{c}3.239 \\
(0.43)\end{array}$ & $\begin{array}{l}3.492 \\
(0.39)\end{array}$ & $\begin{array}{l}3.697 \\
(0.36)\end{array}$ & $\begin{array}{c}3.883 \\
(0.36)\end{array}$ & $\begin{array}{l}3.998 \\
(0.39)\end{array}$ & $\begin{array}{l}4.081 \\
(0.36)\end{array}$ & $\begin{array}{l}4.134 \\
(0.53)\end{array}$ \\
\hline
\end{tabular}

Fig. 4.8a. Pin-power distribution for CENTRM benchmark TCA-4. Value in parentheses is percent standard deviation. 


\begin{tabular}{|c|c|c|c|c|c|c|c|c|c|c|}
\hline & & & & & & & & & & $\begin{array}{l}6.212 \\
(0.86)\end{array}$ \\
\hline & $\begin{array}{c}1.54 \\
(0.66)\end{array}$ & & & & & & & & $\begin{array}{l}6.114 \\
(0.44)\end{array}$ & $\begin{array}{l}6.159 \\
(0.45)\end{array}$ \\
\hline & & & & & & & & $\begin{array}{l}5.831 \\
(0.44)\end{array}$ & $\begin{array}{l}5.974 \\
(0.31)\end{array}$ & $\begin{array}{l}5.984 \\
(0.46)\end{array}$ \\
\hline & & & & & & & $\begin{array}{l}5.529 \\
(0.46)\end{array}$ & $\begin{array}{l}5.708 \\
(0.33)\end{array}$ & $\begin{array}{l}5.770 \\
(0.31)\end{array}$ & $\begin{array}{l}5.819 \\
(0.41)\end{array}$ \\
\hline & & & & & & $\begin{array}{l}5.102 \\
(0.47)\end{array}$ & $\begin{array}{l}5.229 \\
(0.35)\end{array}$ & $\begin{array}{l}5.445 \\
(0.32)\end{array}$ & $\begin{array}{l}5.522 \\
(0.33)\end{array}$ & $\begin{array}{l}5.533 \\
(0.42)\end{array}$ \\
\hline & & & & & $\begin{array}{l}4.470 \\
(0.51)\end{array}$ & $\begin{array}{l}4.777 \\
(0.34)\end{array}$ & $\begin{array}{l}4.968 \\
(0.33)\end{array}$ & $\begin{array}{l}5.140 \\
(0.35)\end{array}$ & $\begin{array}{l}5.202 \\
(0.33)\end{array}$ & $\begin{array}{l}5.243 \\
(0.45)\end{array}$ \\
\hline & & & & $\begin{array}{c}3.832 \\
(0.54)\end{array}$ & $\begin{array}{l}4.116 \\
(0.40)\end{array}$ & $\begin{array}{l}4.387 \\
(0.37)\end{array}$ & $\begin{array}{l}4.630 \\
(0.35)\end{array}$ & $\begin{array}{l}4.727 \\
(0.32)\end{array}$ & $\begin{array}{l}4.837 \\
(0.33)\end{array}$ & $\begin{array}{l}4.850 \\
(0.46)\end{array}$ \\
\hline & & & $\begin{array}{l}3.129 \\
(0.62)\end{array}$ & $\begin{array}{l}3.495 \\
(0.38)\end{array}$ & $\begin{array}{l}3.735 \\
(0.41)\end{array}$ & $\begin{array}{l}4.030 \\
(0.39)\end{array}$ & $\begin{array}{l}4.197 \\
(0.38)\end{array}$ & $\begin{array}{l}4.309 \\
(0.34)\end{array}$ & $\begin{array}{l}4.374 \\
(0.35)\end{array}$ & $\begin{array}{l}4.458 \\
(0.50)\end{array}$ \\
\hline & & $\begin{array}{l}2.582 \\
(0.69)\end{array}$ & $\begin{array}{l}2.840 \\
(0.45)\end{array}$ & $\begin{array}{c}3.132 \\
(0.46)\end{array}$ & $\begin{array}{l}3.388 \\
(0.42)\end{array}$ & $\begin{array}{l}3.581 \\
(0.39)\end{array}$ & $\begin{array}{c}3.754 \\
(0.40)\end{array}$ & $\begin{array}{l}3.870 \\
(0.38)\end{array}$ & $\begin{array}{l}3.961 \\
(0.38)\end{array}$ & $\begin{array}{l}3.971 \\
(0.51)\end{array}$ \\
\hline & $\begin{array}{c}2.185 \\
(0.71)\end{array}$ & $\begin{array}{l}2.350 \\
(0.49)\end{array}$ & $\begin{array}{l}2.643 \\
(0.44)\end{array}$ & $\begin{array}{c}2.904 \\
(0.44)\end{array}$ & $\begin{array}{c}3.128 \\
(0.43)\end{array}$ & $\begin{array}{l}3.330 \\
(0.42)\end{array}$ & $\begin{array}{c}3.500 \\
(0.40)\end{array}$ & $\begin{array}{l}3.601 \\
(0.40)\end{array}$ & $\begin{array}{c}3.672 \\
(0.39)\end{array}$ & $\begin{array}{l}3.710 \\
(0.48)\end{array}$ \\
\hline $\begin{array}{l}2.436 \\
(0.71)\end{array}$ & $\begin{array}{l}2.386 \\
(0.49)\end{array}$ & $\begin{array}{l}2.626 \\
(0.48)\end{array}$ & $\begin{array}{l}2.916 \\
(0.44)\end{array}$ & $\begin{array}{c}3.228 \\
(0.44)\end{array}$ & $\begin{array}{l}3.505 \\
(0.41)\end{array}$ & $\begin{array}{l}3.706 \\
(0.43)\end{array}$ & $\begin{array}{c}3.883 \\
(0.39)\end{array}$ & $\begin{array}{l}3.981 \\
(0.41)\end{array}$ & $\begin{array}{l}4.062 \\
(0.38)\end{array}$ & $\begin{array}{l}4.100 \\
(0.55)\end{array}$ \\
\hline
\end{tabular}

Fig. 4.8b. Pin-power distribution for NITAWL benchmark TCA-4. Value in parentheses is percent standard deviation. 
Table 4.35. Selected reaction rates for CENTRM case TCA-4

\begin{tabular}{|c|c|c|c|c|c|c|}
\hline Pin & Region & $\begin{array}{c}\mathrm{G}_{\mathrm{a}} \mathrm{M} \\
\left(\mathrm{cm}^{-3}-\mathrm{s}^{-1}\right)\end{array}$ & $\begin{array}{c}\mathcal{G}_{\mathrm{f}} \mathrm{M} \\
\left(\mathrm{cm}^{-3}-\mathrm{s}^{-1}\right)\end{array}$ & $\begin{array}{c}\mathrm{G}_{\mathrm{f}} \mathrm{M} \\
\left(\mathrm{cm}^{-3}-\mathrm{s}^{-1}\right)\end{array}$ & $\begin{array}{c}\mathrm{M} \\
\left(\mathrm{cm}-\mathrm{s}^{-1}\right)\end{array}$ & $M / M_{t}$ \\
\hline \multirow{3}{*}{1} & Fuel & $1.440 \mathrm{E}-05$ & $2.393 \mathrm{E}-05$ & $8.480 \mathrm{E}-06$ & $5.581 \mathrm{E}-03$ & 0.235 \\
\hline & Clad & $2.746 \mathrm{E}-07$ & 0.0 & 0.0 & $1.739 \mathrm{E}-03$ & 0.073 \\
\hline & Mod. & 7.889E-07 & 0.0 & 0.0 & $1.642 \mathrm{E}-02$ & 0.692 \\
\hline \multirow{3}{*}{66} & Fuel & $4.055 \mathrm{E}-05$ & $6.181 \mathrm{E}-05$ & $2.186 \mathrm{E}-05$ & $2.281 \mathrm{E}-02$ & 0.235 \\
\hline & Clad & $8.066 \mathrm{E}-07$ & 0.0 & 0.0 & $7.166 \mathrm{E}-03$ & 0.074 \\
\hline & Mod. & $1.867 \mathrm{E}-06$ & 0.0 & 0.0 & $6.712 \mathrm{E}-02$ & 0.691 \\
\hline
\end{tabular}

Table 4.36. Selected reaction rates for NITAWL case TCA-4

\begin{tabular}{|c|c|c|c|c|c|c|}
\hline Pin & Region & $\begin{array}{c}\mathrm{G}_{\mathrm{a}} \mathrm{M} \\
\left(\mathrm{cm}^{-3}-\mathrm{s}^{-1}\right)\end{array}$ & $\begin{array}{c}\mathcal{G}_{\mathrm{f}} \mathrm{M} \\
\left(\mathrm{cm}^{-3}-\mathrm{s}^{-1}\right)\end{array}$ & $\begin{array}{c}\mathrm{G}_{\mathrm{f}} \mathrm{M} \\
\left(\mathrm{cm}^{-3}-\mathrm{s}^{-1}\right) \\
\end{array}$ & $\begin{array}{c}\mathrm{M} \\
\left(\mathrm{cm}-\mathrm{s}^{-1}\right)\end{array}$ & $M / M_{t}$ \\
\hline \multirow{3}{*}{1} & Fuel & $1.464 \mathrm{E}-05$ & $2.446 \mathrm{E}-05$ & $8.665 \mathrm{E}-06$ & $5.642 \mathrm{E}-03$ & 0.236 \\
\hline & Clad & $2.772 \mathrm{E}-07$ & 0.0 & 0.0 & $1.754 \mathrm{E}-03$ & 0.073 \\
\hline & Mod. & $7.942 \mathrm{E}-07$ & 0.0 & 0.0 & $1.652 \mathrm{E}-02$ & 0.691 \\
\hline \multirow{3}{*}{66} & Fuel & $4.061 \mathrm{E}-05$ & $6.263 \mathrm{E}-05$ & $2.216 \mathrm{E}-05$ & $2.295 \mathrm{E}-02$ & 0.234 \\
\hline & Clad & $8.106 \mathrm{E}-07$ & 0.0 & 0.0 & $7.227 \mathrm{E}-03$ & 0.074 \\
\hline & Mod. & $1.885 \mathrm{E}-06$ & 0.0 & 0.0 & $6.790 \mathrm{E}-02$ & 0.692 \\
\hline
\end{tabular}


Table 4.37. TCA-4, Pin-1 four-group fluxes

\begin{tabular}{ccc}
\hline Group & $\begin{array}{c}\text { CENTRM M } \\
\left(\mathrm{cm}^{-2}-\mathrm{s}^{-1}\right)\end{array}$ & $\begin{array}{c}\text { NITAWL M } \\
\left(\mathrm{cm}^{-2}-\mathrm{s}^{-1}\right)\end{array}$ \\
\hline 1 & $5.137 \mathrm{E}-05$ & $5.198 \mathrm{E}-05$ \\
2 & $1.508 \mathrm{E}-05$ & $1.488 \mathrm{E}-05$ \\
3 & $3.637 \mathrm{E}-06$ & $3.684 \mathrm{E}-06$ \\
4 & $3.178 \mathrm{E}-05$ & $3.244 \mathrm{E}-05$ \\
\hline
\end{tabular}

Table 4.38. TCA-4, Pin-1 four-group U-235 cross sections

\begin{tabular}{cccccccc}
\hline Group & \multicolumn{3}{c}{ CENTRM cross sections $\left(\mathrm{cm}^{-1}\right)$} & \multicolumn{3}{c}{ NITAWL cross sections $\left(\mathrm{cm}^{-1}\right)$} \\
\hline & $\frac{\mathrm{G}_{\mathrm{a}}}{1.42972 \mathrm{E}-04}$ & $\frac{\mathrm{G}_{\mathrm{f}}}{3.25729 \mathrm{E}-04}$ & $\frac{\mathrm{G}_{\mathrm{f}}}{1.25284 \mathrm{E}-04}$ & $\frac{\mathrm{G}_{\mathrm{a}}}{1.42717 \mathrm{E}-04}$ & $\frac{\mathrm{G}_{\mathrm{f}}}{3.25108 \mathrm{E}-04}$ & $\frac{\mathrm{G}_{\mathrm{f}}}{1.25072 \mathrm{E}-04}$ \\
1 & $3.54777 \mathrm{E}-03$ & $5.40060 \mathrm{E}-03$ & $2.21636 \mathrm{E}-03$ & $3.49262 \mathrm{E}-03$ & $5.32638 \mathrm{E}-03$ & $2.18590 \mathrm{E}-03$ \\
2 & $5.19612 \mathrm{E}-03$ & $1.06384 \mathrm{E}-02$ & $4.36591 \mathrm{E}-03$ & $5.19563 \mathrm{E}-03$ & $1.06220 \mathrm{E}-02$ & $4.35917 \mathrm{E}-03$ \\
3 & $4.87823 \mathrm{E}-02$ & $1.01563 \mathrm{E}-01$ & $4.16806 \mathrm{E}-02$ & $4.89171 \mathrm{E}-02$ & $1.01845 \mathrm{E}-01$ & $4.17962 \mathrm{E}-02$ \\
\hline
\end{tabular}

Table 4.39. TCA-4, Pin-1 four-group U-238 cross sections

\begin{tabular}{cccccccc}
\hline Group & \multicolumn{3}{c}{ CENTRM cross sections $\left(\mathrm{cm}^{-1}\right)$} & \multicolumn{2}{c}{ NITAWL cross sections $\left(\mathrm{cm}^{-1}\right)$} \\
\hline & $\frac{\mathrm{G}_{\mathrm{a}}}{4.51308 \mathrm{E}-03}$ & $\frac{\mathrm{G}_{\mathrm{f}}}{8.46385 \mathrm{E}-03}$ & $\frac{\mathrm{G}_{\mathrm{f}}}{3.00241 \mathrm{E}-03}$ & $\overline{4.47482 \mathrm{E}-03}$ & $\overline{8.38222 \mathrm{E}-03}$ & $\overline{2.97205 \mathrm{E}-03}$ \\
1 & $3.86113 \mathrm{E}-02$ & $3.72395 \mathrm{E}-06$ & $1.54282 \mathrm{E}-06$ & $3.54513 \mathrm{E}-02$ & $3.52350 \mathrm{E}-06$ & $1.45977 \mathrm{E}-06$ \\
3 & $7.00737 \mathrm{E}-03$ & $2.46436 \mathrm{E}-08$ & $1.02103 \mathrm{E}-08$ & $7.01355 \mathrm{E}-03$ & $2.47048 \mathrm{E}-08$ & $1.02357 \mathrm{E}-08$ \\
4 & $2.77151 \mathrm{E}-02$ & $1.30278 \mathrm{E}-07$ & $5.39767 \mathrm{E}-08$ & $2.77735 \mathrm{E}-02$ & $1.30557 \mathrm{E}-07$ & $5.40922 \mathrm{E}-08$ \\
\hline
\end{tabular}

Table 4.40. TCA-4, Pin-1 four-group Pu-239 cross sections

\begin{tabular}{|c|c|c|c|c|c|c|}
\hline \multirow[t]{2}{*}{ Group } & \multicolumn{3}{|c|}{ CENTRM cross sections $\left(\mathrm{cm}^{-1}\right)$} & \multicolumn{3}{|c|}{ NITAWL cross sections $\left(\mathrm{cm}^{-1}\right)$} \\
\hline & $\mathrm{G}_{\mathrm{a}}$ & $<\mathrm{G}_{\mathrm{f}}$ & $\mathrm{G}_{\mathrm{f}}$ & $\mathrm{G}_{\mathrm{a}}$ & $\mathcal{G}_{\mathrm{f}}$ & $\mathrm{G}_{\mathrm{f}}$ \\
\hline 1 & $5.12178 \mathrm{E}-04$ & $1.49692 \mathrm{E}-03$ & $4.81232 \mathrm{E}-04$ & $5.11274 \mathrm{E}-04$ & $1.49406 \mathrm{E}-03$ & $4.80446 \mathrm{E}-04$ \\
\hline 2 & $1.11540 \mathrm{E}-02$ & $1.83841 \mathrm{E}-02$ & $6.38377 \mathrm{E}-03$ & $1.08239 \mathrm{E}-02$ & $1.78689 \mathrm{E}-02$ & 6.20489E-03 \\
\hline 3 & $3.46250 \mathrm{E}-02$ & 6.79736E-02 & 2.36035E-02 & $3.48586 \mathrm{E}-02$ & 6.84167E-02 & 2.37574E-02 \\
\hline 4 & $2.65578 \mathrm{E}-01$ & 5.38310E-01 & $1.86183 \mathrm{E}-01$ & 2.66229E-01 & $5.39667 \mathrm{E}-01$ & $1.86654 \mathrm{E}-01$ \\
\hline
\end{tabular}


Table 4.41. TCA-4, Pin-66 four-group fluxes

\begin{tabular}{ccc}
\hline Group & $\begin{array}{c}\text { CENTRM M } \\
\left(\mathrm{cm}^{-2}-\mathrm{s}^{-1}\right)\end{array}$ & $\begin{array}{c}\text { NITAWL M } \\
\left(\mathrm{cm}^{-2}-\mathrm{s}^{-1}\right)\end{array}$ \\
\hline 1 & $2.378 \mathrm{E}-04$ & $2.383 \mathrm{E}-04$ \\
2 & $8.358 \mathrm{E}-05$ & $8.422 \mathrm{E}-05$ \\
3 & $1.882 \mathrm{E}-05$ & $1.898 \mathrm{E}-05$ \\
4 & $7.623 \mathrm{E}-05$ & $7.746 \mathrm{E}-05$ \\
\hline
\end{tabular}

Table 4.42. TCA-4, Pin-66 four-group U-235 cross sections

\begin{tabular}{ccccccc}
\hline Group & \multicolumn{2}{c}{ CENTRM cross sections $\left(\mathrm{cm}^{-1}\right)$} & \multicolumn{3}{c}{ NITAWL cross sections $\left(\mathrm{cm}^{-1}\right)$} \\
\hline & $\frac{\mathrm{G}_{\mathrm{a}}}{}$ & $\frac{\mathrm{G}_{\mathrm{f}}}{1.47181 \mathrm{E}-04}$ & $\frac{3.29323 \mathrm{E}-04}{1.27404 \mathrm{E}-04}$ & $\frac{\mathrm{G}_{\mathrm{f}}}{1.47030 \mathrm{E}-04}$ & $\frac{\mathrm{G}_{\mathrm{f}}}{3.28757 \mathrm{E}-04}$ & $\frac{\mathrm{G}_{\mathrm{f}}}{1.27281 \mathrm{E}-04}$ \\
1 & $3.46397 \mathrm{E}-03$ & $5.28525 \mathrm{E}-03$ & $2.16902 \mathrm{E}-03$ & $3.45056 \mathrm{E}-03$ & $5.27803 \mathrm{E}-03$ & $2.16605 \mathrm{E}-03$ \\
3 & $5.05350 \mathrm{E}-03$ & $1.03193 \mathrm{E}-02$ & $4.23494 \mathrm{E}-03$ & $5.07799 \mathrm{E}-03$ & $1.03695 \mathrm{E}-02$ & $4.25556 \mathrm{E}-03$ \\
4 & $4.64067 \mathrm{E}-02$ & $9.65304 \mathrm{E}-02$ & $3.96152 \mathrm{E}-02$ & $4.62458 \mathrm{E}-02$ & $9.61957 \mathrm{E}-02$ & $3.94779 \mathrm{E}-02$ \\
\hline
\end{tabular}

Table 4.43. TCA-4, Pin-66 four-group U-238 cross sections

\begin{tabular}{cccccccc}
\hline Group & \multicolumn{3}{c}{ CENTRM cross sections $\left(\mathrm{cm}^{-1}\right)$} & \multicolumn{3}{c}{ NITAWL cross sections $\left(\mathrm{cm}^{-1}\right)$} \\
\hline & $\frac{\mathrm{G}_{\mathrm{a}}}{1}$ & $4.36716 \mathrm{E}-03$ & $\frac{\mathrm{G}_{\mathrm{f}}}{7.64672 \mathrm{E}-03}$ & $\frac{\mathrm{G}_{\mathrm{f}}}{2.70927 \mathrm{E}-03}$ & $\frac{\mathrm{G}_{\mathrm{a}}}{4.35812 \mathrm{E}-03}$ & $\frac{\mathrm{G}_{\mathrm{f}}}{7.61288 \mathrm{E}-03}$ & $\frac{\mathrm{G}_{\mathrm{f}}}{2.70342 \mathrm{E}-03}$ \\
2 & $4.02182 \mathrm{E}-02$ & $3.82265 \mathrm{E}-06$ & $1.58372 \mathrm{E}-06$ & $3.67393 \mathrm{E}-02$ & $3.69650 \mathrm{E}-06$ & $1.53144 \mathrm{E}-06$ \\
3 & $6.96068 \mathrm{E}-03$ & $2.41878 \mathrm{E}-08$ & $1.00214 \mathrm{E}-08$ & $6.97476 \mathrm{E}-03$ & $2.43271 \mathrm{E}-08$ & $1.00792 \mathrm{E}-08$ \\
4 & $2.64749 \mathrm{E}-02$ & $1.24324 \mathrm{E}-07$ & $5.15096 \mathrm{E}-08$ & $2.63939 \mathrm{E}-02$ & $1.23940 \mathrm{E}-07$ & $5.13506 \mathrm{E}-08$ \\
\hline
\end{tabular}

Table 4.44. TCA-4, Pin-66 four-group Pu-239 cross sections

\begin{tabular}{|c|c|c|c|c|c|c|}
\hline \multirow[t]{2}{*}{ Group } & \multicolumn{3}{|c|}{ CENTRM cross sections $\left(\mathrm{cm}^{-1}\right)$} & \multicolumn{3}{|c|}{ NITAWL cross sections $\left(\mathrm{cm}^{-1}\right)$} \\
\hline & $\mathrm{G}_{\mathrm{a}}$ & $\leftarrow_{\mathrm{f}}$ & $\mathrm{G}_{\mathrm{f}}$ & $\mathrm{G}_{\mathrm{a}}$ & $<\mathrm{G}_{f}$ & $\mathrm{G}_{\mathrm{f}}$ \\
\hline 1 & $5.13616 \mathrm{E}-04$ & $\overline{1.47840 \mathrm{E}-03}$ & $\overline{4.77839 \mathrm{E}-04}$ & $5 . \overline{13256 \mathrm{E}-04}$ & $\overline{1.47677 \mathrm{E}-03}$ & $\overline{4.77633 \mathrm{E}-04}$ \\
\hline 2 & $1.11268 \mathrm{E}-02$ & 1.82952E-02 & 6.35292E-03 & 1.07160E-02 & $1.75431 \mathrm{E}-02$ & $6.09175 \mathrm{E}-03$ \\
\hline 3 & $3.23976 \mathrm{E}-02$ & $6.38268 \mathrm{E}-02$ & $2.21636 \mathrm{E}-02$ & $3.30910 \mathrm{E}-02$ & $6.51217 \mathrm{E}-02$ & $2.26133 \mathrm{E}-02$ \\
\hline 4 & $2.81830 \mathrm{E}-01$ & $5.60508 \mathrm{E}-01$ & $1.93933 \mathrm{E}-01$ & 2.81951E-01 & $5.60363 \mathrm{E}-01$ & $1.93887 \mathrm{E}-01$ \\
\hline
\end{tabular}




\begin{tabular}{|c|c|c|c|c|c|c|c|c|c|c|}
\hline & & & & & & & & & & $\begin{array}{l}5.973 \\
(0.85)\end{array}$ \\
\hline & \multirow[t]{8}{*}{$\begin{array}{l}0.164 \\
(0.72) \\
\end{array}$} & & & & & & & & $\begin{array}{l}5.841 \\
(0.44)\end{array}$ & $\begin{array}{l}5.875 \\
(0.49)\end{array}$ \\
\hline & & & & & & & & $\begin{array}{l}5.617 \\
(0.45)\end{array}$ & $\begin{array}{l}5.723 \\
(0.34)\end{array}$ & $\begin{array}{l}5.821 \\
(0.42)\end{array}$ \\
\hline & & & & & & & $\begin{array}{l}5.245 \\
(0.44)\end{array}$ & $\begin{array}{l}5.464 \\
(0.33)\end{array}$ & $\begin{array}{l}5.537 \\
(0.33)\end{array}$ & $\begin{array}{l}5.623 \\
(0.44)\end{array}$ \\
\hline & & & & & & $\begin{array}{l}4.824 \\
(0.47) \\
\end{array}$ & $\begin{array}{l}5.071 \\
(0.31) \\
\end{array}$ & $\begin{array}{l}5.205 \\
(0.30) \\
\end{array}$ & $\begin{array}{l}5.288 \\
(0.29) \\
\end{array}$ & $\begin{array}{l}5.352 \\
(0.47) \\
\end{array}$ \\
\hline & & & & & $\begin{array}{l}4.271 \\
(0.53)\end{array}$ & $\begin{array}{l}4.523 \\
(0.33)\end{array}$ & $\begin{array}{l}4.753 \\
(0.30)\end{array}$ & $\begin{array}{l}4.916 \\
(0.31)\end{array}$ & $\begin{array}{l}4.981 \\
(0.34)\end{array}$ & $\begin{array}{l}5.050 \\
(0.44)\end{array}$ \\
\hline & & & & $\begin{array}{l}3.643 \\
(0.55)\end{array}$ & $\begin{array}{l}3.966 \\
(0.37)\end{array}$ & $\begin{array}{l}4.187 \\
(0.38)\end{array}$ & $\begin{array}{l}4.389 \\
(0.35)\end{array}$ & $\begin{array}{l}4.507 \\
(0.34)\end{array}$ & $\begin{array}{l}4.630 \\
(0.34)\end{array}$ & $\begin{array}{l}4.648 \\
(0.50)\end{array}$ \\
\hline & & & $\begin{array}{l}3.029 \\
(0.59)\end{array}$ & $\begin{array}{l}3.330 \\
(0.42)\end{array}$ & $\begin{array}{l}3.604 \\
(0.41)\end{array}$ & $\begin{array}{l}3.798 \\
(0.40)\end{array}$ & $\begin{array}{l}3.987 \\
(0.38)\end{array}$ & $\begin{array}{l}4.110 \\
(0.36)\end{array}$ & $\begin{array}{l}4.165 \\
(0.36)\end{array}$ & $\begin{array}{l}4.228 \\
(0.48)\end{array}$ \\
\hline & & $\begin{array}{l}2.450 \\
(0.65)\end{array}$ & $\begin{array}{l}2.713 \\
(0.44)\end{array}$ & $\begin{array}{l}2.973 \\
(0.41)\end{array}$ & $\begin{array}{l}3.230 \\
(0.39)\end{array}$ & $\begin{array}{l}3.385 \\
(0.41)\end{array}$ & $\begin{array}{l}3.574 \\
(0.39)\end{array}$ & $\begin{array}{l}3.709 \\
(0.42)\end{array}$ & $\begin{array}{l}3.777 \\
(0.37)\end{array}$ & $\begin{array}{l}3.781 \\
(0.56)\end{array}$ \\
\hline & $\begin{array}{l}2.050 \\
(0.75)\end{array}$ & $\begin{array}{l}2.254 \\
(0.48)\end{array}$ & $\begin{array}{l}2.522 \\
(0.47)\end{array}$ & $\begin{array}{l}2.750 \\
(0.45)\end{array}$ & $\begin{array}{l}2.981 \\
(0.46)\end{array}$ & $\begin{array}{c}3.176 \\
(0.38)\end{array}$ & $\begin{array}{l}3.331 \\
(0.38)\end{array}$ & $\begin{array}{l}3.429 \\
(0.35)\end{array}$ & $\begin{array}{c}3.508 \\
(0.41)\end{array}$ & $\begin{array}{l}3.534 \\
(0.55)\end{array}$ \\
\hline $\begin{array}{l}2.283 \\
(0.66)\end{array}$ & $\begin{array}{l}2.260 \\
(0.50)\end{array}$ & $\begin{array}{l}2.500 \\
(0.47)\end{array}$ & $\begin{array}{l}2.828 \\
(0.43)\end{array}$ & $\begin{array}{l}3.060 \\
(0.41)\end{array}$ & $\begin{array}{l}3.331 \\
(0.40)\end{array}$ & $\begin{array}{l}3.528 \\
(0.37)\end{array}$ & $\begin{array}{l}3.725 \\
(0.37)\end{array}$ & $\begin{array}{l}3.815 \\
(0.39)\end{array}$ & $\begin{array}{c}3.867 \\
(0.37)\end{array}$ & $\begin{array}{l}3.921 \\
(0.50)\end{array}$ \\
\hline
\end{tabular}

Fig. 4.9a. Pin-power distribution for CENTRM benchmark TCA-5. Value in parentheses is percent standard deviation. 


\begin{tabular}{|c|c|c|c|c|c|c|c|c|c|c|}
\hline & & & & & & & & & & $\begin{array}{l}5.872 \\
(0.90)\end{array}$ \\
\hline & \multirow[t]{8}{*}{$\begin{array}{l}0.161 \\
(0.77) \\
\end{array}$} & & & & & & & & $\begin{array}{l}5.824 \\
(0.44)\end{array}$ & $\begin{array}{l}5.930 \\
(0.43)\end{array}$ \\
\hline & & & & & & & & $\begin{array}{l}5.663 \\
(0.47)\end{array}$ & $\begin{array}{l}5.775 \\
(0.33)\end{array}$ & $\begin{array}{l}5.815 \\
(0.46)\end{array}$ \\
\hline & & & & & & & $\begin{array}{l}5.278 \\
(0.44)\end{array}$ & $\begin{array}{l}5.523 \\
(0.31)\end{array}$ & $\begin{array}{l}5.575 \\
(0.32)\end{array}$ & $\begin{array}{l}5.572 \\
(0.46)\end{array}$ \\
\hline & & & & & & $\begin{array}{r}4.844 \\
(0.50) \\
\end{array}$ & $\begin{array}{l}5.069 \\
(0.31) \\
\end{array}$ & $\begin{array}{l}5.219 \\
(0.32) \\
\end{array}$ & $\begin{array}{l}5.311 \\
(0.31) \\
\end{array}$ & $\begin{array}{l}5.323 \\
(0.47) \\
\end{array}$ \\
\hline & & & & & $\begin{array}{l}4.326 \\
(0.49)\end{array}$ & $\begin{array}{l}4.538 \\
(0.35)\end{array}$ & $\begin{array}{l}4.742 \\
(0.33)\end{array}$ & $\begin{array}{l}4.945 \\
(0.33)\end{array}$ & $\begin{array}{l}5.011 \\
(0.32)\end{array}$ & $\begin{array}{l}5.054 \\
(0.46)\end{array}$ \\
\hline & & & & $\begin{array}{l}3.679 \\
(0.54)\end{array}$ & $\begin{array}{c}3.942 \\
(0.34)\end{array}$ & $\begin{array}{l}4.189 \\
(0.36)\end{array}$ & $\begin{array}{l}4.400 \\
(0.36)\end{array}$ & $\begin{array}{l}4.534 \\
(0.35)\end{array}$ & $\begin{array}{l}4.638 \\
(0.33)\end{array}$ & $\begin{array}{l}4.691 \\
(0.53)\end{array}$ \\
\hline & & & $\begin{array}{l}3.002 \\
(0.59)\end{array}$ & $\begin{array}{l}3.342 \\
(0.40)\end{array}$ & $\begin{array}{l}3.606 \\
(0.38)\end{array}$ & $\begin{array}{l}3.840 \\
(0.35)\end{array}$ & $\begin{array}{l}4.021 \\
(0.35)\end{array}$ & $\begin{array}{l}4.138 \\
(0.38)\end{array}$ & $\begin{array}{l}4.209 \\
(0.38)\end{array}$ & $\begin{array}{l}4.213 \\
(0.54)\end{array}$ \\
\hline & & $\begin{array}{l}2.468 \\
(0.64)\end{array}$ & $\begin{array}{l}2.720 \\
(0.49)\end{array}$ & $\begin{array}{l}2.999 \\
(0.42)\end{array}$ & $\begin{array}{l}3.225 \\
(0.45)\end{array}$ & $\begin{array}{l}3.440 \\
(0.39)\end{array}$ & $\begin{array}{c}3.582 \\
(0.41)\end{array}$ & $\begin{array}{l}3.702 \\
(0.40)\end{array}$ & $\begin{array}{c}3.771 \\
(0.38)\end{array}$ & $\begin{array}{l}3.783 \\
(0.53)\end{array}$ \\
\hline & $\begin{array}{l}2.075 \\
(0.66)\end{array}$ & $\begin{array}{l}2.259 \\
(0.54)\end{array}$ & $\begin{array}{l}2.518 \\
(0.45)\end{array}$ & $\begin{array}{l}2.768 \\
(0.45)\end{array}$ & $\begin{array}{l}3.015 \\
(0.41)\end{array}$ & $\begin{array}{c}3.168 \\
(0.43)\end{array}$ & $\begin{array}{l}3.345 \\
(0.39)\end{array}$ & $\begin{array}{c}3.455 \\
(0.40)\end{array}$ & $\begin{array}{c}3.474 \\
(0.39)\end{array}$ & $\begin{array}{l}3.504 \\
(0.54)\end{array}$ \\
\hline $\begin{array}{l}2.297 \\
(0.69)\end{array}$ & $\begin{array}{l}2.263 \\
(0.45)\end{array}$ & $\begin{array}{l}2.480 \\
(0.49)\end{array}$ & $\begin{array}{l}2.792 \\
(0.42)\end{array}$ & $\begin{array}{l}3.046 \\
(0.38)\end{array}$ & $\begin{array}{l}3.316 \\
(0.41)\end{array}$ & $\begin{array}{l}3.535 \\
(0.39)\end{array}$ & $\begin{array}{l}3.708 \\
(0.38)\end{array}$ & $\begin{array}{l}3.801 \\
(0.38)\end{array}$ & $\begin{array}{c}3.876 \\
(0.36)\end{array}$ & $\begin{array}{l}3.904 \\
(0.55)\end{array}$ \\
\hline
\end{tabular}

Fig. 4.9b. Pin-power distribution for NITAWL benchmark TCA-5. Value in parentheses is percent standard deviation. 
Table 4.45. Selected reaction rates for CENTRM case TCA-5

\begin{tabular}{|c|c|c|c|c|c|c|}
\hline Pin & Region & $\begin{array}{c}\mathrm{G}_{\mathrm{a}} \mathrm{M} \\
\left(\mathrm{cm}^{-3}-\mathrm{s}^{-1}\right)\end{array}$ & $\begin{array}{c}\mathcal{G}_{\mathrm{f}} \mathrm{M} \\
\left(\mathrm{cm}^{-3}-\mathrm{s}^{-1}\right)\end{array}$ & $\begin{array}{c}\mathrm{G}_{\mathrm{f}} \mathrm{M} \\
\left(\mathrm{cm}^{-3}-\mathrm{s}^{-1}\right)\end{array}$ & $\begin{array}{c}\mathrm{M} \\
\left(\mathrm{cm}-\mathrm{s}^{-1}\right)\end{array}$ & M/M \\
\hline \multirow{3}{*}{1} & Fuel & $1.374 \mathrm{E}-05$ & $2.280 \mathrm{E}-05$ & $8.078 \mathrm{E}-06$ & $5.603 \mathrm{E}-03$ & 0.235 \\
\hline & Clad & $2.618 \mathrm{E}-07$ & 0.0 & 0.0 & $1.747 \mathrm{E}-03$ & 0.074 \\
\hline & Mod. & $7.503 \mathrm{E}-07$ & 0.0 & 0.0 & $1.646 \mathrm{E}-02$ & 0.691 \\
\hline \multirow{3}{*}{66} & Fuel & $3.907 \mathrm{E}-05$ & $5.970 \mathrm{E}-05$ & $2.112 \mathrm{E}-05$ & $2.311 \mathrm{E}-02$ & 0.235 \\
\hline & Clad & $7.783 \mathrm{E}-07$ & 0.0 & 0.0 & $7.263 \mathrm{E}-03$ & 0.074 \\
\hline & Mod. & $1.793 \mathrm{E}-06$ & 0.0 & 0.0 & $6.806 \mathrm{E}-02$ & 0.691 \\
\hline
\end{tabular}

Table 4.46. Selected reaction rates for NITAWL case TCA-5

\begin{tabular}{ccccccc}
\hline Pin & Region & $\begin{array}{c}\mathrm{G}_{\mathrm{a}} \mathrm{M} \\
\left(\mathrm{cm}^{-3}-\mathrm{s}^{-1}\right)\end{array}$ & $\begin{array}{c}\mathrm{G}_{\mathrm{f}} \mathrm{M} \\
\left(\mathrm{cm}^{-3}-\mathrm{s}^{-1}\right)\end{array}$ & $\begin{array}{c}\mathrm{G}_{\mathrm{f}} \mathrm{M} \\
\left(\mathrm{cm}^{-3}-\mathrm{s}^{-1}\right)\end{array}$ & $\begin{array}{c}\mathrm{M} \\
\left(\mathrm{cm}^{-1}\right)\end{array}$ & $\mathrm{sM}_{\mathrm{t}}$ \\
\hline \multirow{2}{*}{1} & Fuel & $1.378 \mathrm{E}-05$ & $2.293 \mathrm{E}-05$ & $8.127 \mathrm{E}-06$ & $5.603 \mathrm{E}-03$ & 0.235 \\
& Clad & $2.619 \mathrm{E}-07$ & 0.0 & 0.0 & $1.745 \mathrm{E}-03$ & 0.074 \\
& Mod. & $7.543 \mathrm{E}-07$ & 0.0 & 0.0 & $1.646 \mathrm{E}-02$ & 0.691 \\
\hline \multirow{2}{*}{66} & Fuel & $3.868 \mathrm{E}-05$ & $5.889 \mathrm{E}-05$ & $2.084 \mathrm{E}-05$ & $2.304 \mathrm{E}-02$ & 0.234 \\
& Clad & $7.727 \mathrm{E}-07$ & 0.0 & 0.0 & $7.281 \mathrm{E}-03$ & 0.074 \\
& Mod. & $1.803 \mathrm{E}-06$ & 0.0 & 0.0 & $6.824 \mathrm{E}-02$ & 0.692 \\
\hline
\end{tabular}


Table 4.47. TCA-5, Pin-1 four-group fluxes

\begin{tabular}{ccc}
\hline Group & $\begin{array}{c}\text { CENTRM M } \\
\left(\mathrm{cm}^{-2}-\mathrm{s}^{-1}\right)\end{array}$ & $\begin{array}{c}\text { NITAWL M } \\
\left(\mathrm{cm}^{-2}-\mathrm{s}^{-1}\right)\end{array}$ \\
\hline 1 & $4.939 \mathrm{E}-05$ & $4.938 \mathrm{E}-05$ \\
2 & $1.446 \mathrm{E}-05$ & $1.412 \mathrm{E}-05$ \\
3 & $3.466 \mathrm{E}-06$ & $3.579 \mathrm{E}-06$ \\
4 & $3.035 \mathrm{E}-05$ & $3.059 \mathrm{E}-05$ \\
\hline
\end{tabular}

Table 4.48. TCA-5, Pin-1 four-group U-235 cross sections

\begin{tabular}{cccccccc}
\hline Group & \multicolumn{2}{c}{ CENTRM cross sections $\left(\mathrm{cm}^{-1}\right)$} & \multicolumn{3}{c}{ NITAWL cross sections $\left(\mathrm{cm}^{-1}\right)$} \\
\hline & $\frac{\mathrm{G}_{\mathrm{a}}}{1.42994 \mathrm{E}-04}$ & $\frac{\mathrm{G}_{\mathrm{f}}}{3.26070 \mathrm{E}-04}$ & $\frac{\mathrm{G}_{\mathrm{f}}}{1.25328 \mathrm{E}-04}$ & $\frac{\mathrm{G}_{\mathrm{a}}}{1.43122 \mathrm{E}-04}$ & $\frac{4 \mathrm{G}_{\mathrm{f}}}{3.25555 \mathrm{E}-04}$ & $\frac{\mathrm{G}_{\mathrm{f}}}{1.25325 \mathrm{E}-04}$ \\
1 & $3.42309 \mathrm{E}-03$ & $5.26214 \mathrm{E}-03$ & $2.15954 \mathrm{E}-03$ & $3.47849 \mathrm{E}-03$ & $5.29943 \mathrm{E}-03$ & $2.17484 \mathrm{E}-03$ \\
2 & $5.14146 \mathrm{E}-03$ & $1.04884 \mathrm{E}-02$ & $4.30436 \mathrm{E}-03$ & $5.22434 \mathrm{E}-03$ & $1.06706 \mathrm{E}-02$ & $4.37913 \mathrm{E}-03$ \\
3 & $4.87407 \mathrm{E}-02$ & $1.01475 \mathrm{E}-01$ & $4.16445 \mathrm{E}-02$ & $4.86829 \mathrm{E}-02$ & $1.01355 \mathrm{E}-01$ & $4.15953 \mathrm{E}-02$ \\
\hline
\end{tabular}

Table 4.49. TCA-5, Pin-1 four-group U-238 cross sections

\begin{tabular}{cccccccc}
\hline Group & \multicolumn{3}{c}{ CENTRM cross sections $\left(\mathrm{cm}^{-1}\right)$} & \multicolumn{3}{c}{ NITAWL cross sections $\left(\mathrm{cm}^{-1}\right)$} \\
\hline & $\frac{\mathrm{G}_{\mathrm{a}}}{4.50583 \mathrm{E}-03}$ & $\frac{\mathrm{G}_{\mathrm{f}}}{8.47688 \mathrm{E}-03}$ & $\frac{\mathrm{G}_{\mathrm{f}}}{2.99982 \mathrm{E}-03}$ & $\frac{\mathrm{G}_{\mathrm{a}}}{4.48281 \mathrm{E}-03}$ & $\frac{\mathrm{G}_{\mathrm{f}}}{8.35527 \mathrm{E}-03}$ & $\frac{\mathrm{G}_{\mathrm{f}}}{2.96632 \mathrm{E}-03}$ \\
1 & $3.83667 \mathrm{E}-02$ & $3.84273 \mathrm{E}-06$ & $1.59203 \mathrm{E}-06$ & $3.62671 \mathrm{E}-02$ & $3.53982 \mathrm{E}-06$ & $1.46653 \mathrm{E}-06$ \\
3 & $7.00150 \mathrm{E}-03$ & $2.45134 \mathrm{E}-08$ & $1.01563 \mathrm{E}-08$ & $7.02110 \mathrm{E}-03$ & $2.47448 \mathrm{E}-08$ & $1.02522 \mathrm{E}-08$ \\
4 & $2.76871 \mathrm{E}-02$ & $1.30144 \mathrm{E}-07$ & $5.39213 \mathrm{E}-08$ & $2.76564 \mathrm{E}-02$ & $1.30000 \mathrm{E}-07$ & $5.38614 \mathrm{E}-08$ \\
\hline
\end{tabular}

Table 4.50. TCA-5, Pin-1 four-group Pu-239 cross sections

\begin{tabular}{|c|c|c|c|c|c|c|}
\hline \multirow[t]{2}{*}{ Group } & \multicolumn{3}{|c|}{ CENTRM cross sections $\left(\mathrm{cm}^{-1}\right)$} & \multicolumn{3}{|c|}{ NITAWL cross sections $\left(\mathrm{cm}^{-1}\right)$} \\
\hline & $\mathrm{G}_{\mathrm{a}}$ & $<\mathrm{G}_{\mathrm{f}}$ & $\mathrm{G}_{\mathrm{f}}$ & $\mathrm{G}_{\mathrm{a}}$ & $<\mathrm{G}_{\mathrm{f}}$ & $\mathrm{G}_{\mathrm{f}}$ \\
\hline 1 & $5.12069 \mathrm{E}-04$ & $1.49754 \mathrm{E}-03$ & $4.81215 \mathrm{E}-04$ & $5.11841 \mathrm{E}-04$ & $1.49393 \mathrm{E}-03$ & 4.80664E-04 \\
\hline 2 & $1.09615 \mathrm{E}-02$ & $1.82420 \mathrm{E}-02$ & $6.33444 \mathrm{E}-03$ & $1.10261 \mathrm{E}-02$ & $1.81861 \mathrm{E}-02$ & $6.31503 \mathrm{E}-03$ \\
\hline 3 & $3.50990 \mathrm{E}-02$ & 6.88148E-02 & 2.38956E-02 & $3.48620 \mathrm{E}-02$ & 6.84733E-02 & 2.37771E-02 \\
\hline 4 & $2.66585 \mathrm{E}-01$ & 5.39901E-01 & $1.86737 \mathrm{E}-01$ & $2.66263 \mathrm{E}-01$ & 5.39232E-01 & $1.86508 \mathrm{E}-01$ \\
\hline
\end{tabular}


Table 4.51. TCA-5, Pin-66 four-group fluxes

\begin{tabular}{ccc}
\hline Group & $\begin{array}{c}\text { CENTRM M } \\
\left(\mathrm{cm}^{-2}-\mathrm{s}^{-1}\right)\end{array}$ & $\begin{array}{c}\text { NITAWL M } \\
\left(\mathrm{cm}^{-2}-\mathrm{s}^{-1}\right)\end{array}$ \\
\hline 1 & $2.303 \mathrm{E}-04$ & $2.296 \mathrm{E}-04$ \\
2 & $8.041 \mathrm{E}-05$ & $8.059 \mathrm{E}-05$ \\
3 & $1.804 \mathrm{E}-05$ & $1.799 \mathrm{E}-05$ \\
4 & $7.411 \mathrm{E}-05$ & $7.336 \mathrm{E}-05$ \\
\hline
\end{tabular}

Table 4.52. TCA-5, Pin-66 four-group U-235 cross sections

\begin{tabular}{cccccccc}
\hline Group & \multicolumn{2}{c}{ CENTRM cross sections $\left(\mathrm{cm}^{-1}\right)$} & \multicolumn{3}{c}{ NITAWL cross sections $\left(\mathrm{cm}^{-1}\right)$} \\
\hline & $\frac{\mathrm{G}_{\mathrm{a}}}{}$ & $\frac{\mathrm{G}_{\mathrm{f}}}{1.47516 \mathrm{E}-04}$ & $\frac{\mathrm{G}_{\mathrm{f}}}{3.29683 \mathrm{E}-04}$ & $\frac{1.27617 \mathrm{E}-04}{1.46906 \mathrm{E}-04}$ & $\frac{\mathrm{G}_{\mathrm{f}}}{3.28595 \mathrm{E}-04}$ & $\frac{\mathrm{G}_{\mathrm{f}}}{1.27226 \mathrm{E}-04}$ \\
1 & $3.45804 \mathrm{E}-03$ & $5.28540 \mathrm{E}-03$ & $2.16908 \mathrm{E}-03$ & $3.42799 \mathrm{E}-03$ & $5.19278 \mathrm{E}-03$ & $2.13107 \mathrm{E}-03$ \\
3 & $5.21778 \mathrm{E}-03$ & $1.06625 \mathrm{E}-02$ & $4.37578 \mathrm{E}-03$ & $5.17950 \mathrm{E}-03$ & $1.05856 \mathrm{E}-02$ & $4.34425 \mathrm{E}-03$ \\
4 & $4.63091 \mathrm{E}-02$ & $9.63284 \mathrm{E}-02$ & $3.95323 \mathrm{E}-02$ & $4.61333 \mathrm{E}-02$ & $9.59635 \mathrm{E}-02$ & $3.93825 \mathrm{E}-02$ \\
\hline
\end{tabular}

Table 4.53. TCA-5, Pin-66 four-group U-238 cross sections

\begin{tabular}{cccccccc}
\hline Group & \multicolumn{3}{c}{ CENTRM cross sections $\left(\mathrm{cm}^{-1}\right)$} & \multicolumn{3}{c}{ NITAWL cross sections $\left(\mathrm{cm}^{-1}\right)$} \\
\hline & $\frac{\mathrm{G}_{\mathrm{a}}}{1}$ & $4.38259 \mathrm{E}-03$ & $\frac{\mathrm{G}_{\mathrm{f}}}{7.65041 \mathrm{E}-03}$ & $\frac{\mathrm{G}_{\mathrm{f}}}{2.71517 \mathrm{E}-03}$ & $\frac{\mathrm{G}_{\mathrm{a}}}{4.35318 \mathrm{E}-03}$ & $\frac{\mathrm{G}_{\mathrm{f}}}{7.60571 \mathrm{E}-03}$ & $\frac{\mathrm{G}_{\mathrm{f}}}{2.70175 \mathrm{E}-03}$ \\
2 & $3.76966 \mathrm{E}-02$ & $3.76282 \mathrm{E}-06$ & $1.55892 \mathrm{E}-06$ & $3.76657 \mathrm{E}-02$ & $3.47384 \mathrm{E}-06$ & $1.43920 \mathrm{E}-06$ \\
3 & $7.01397 \mathrm{E}-03$ & $2.46319 \mathrm{E}-08$ & $1.02054 \mathrm{E}-08$ & $6.99839 \mathrm{E}-03$ & $2.45177 \mathrm{E}-08$ & $1.01581 \mathrm{E}-08$ \\
4 & $2.64310 \mathrm{E}-02$ & $1.24116 \mathrm{E}-07$ & $5.14236 \mathrm{E}-08$ & $2.63412 \mathrm{E}-02$ & $1.23688 \mathrm{E}-07$ & $5.12462 \mathrm{E}-08$ \\
\hline
\end{tabular}

Table 4.54. TCA-5, Pin-66 four-group Pu-239 cross sections

\begin{tabular}{|c|c|c|c|c|c|c|}
\hline \multirow[t]{2}{*}{ Group } & \multicolumn{3}{|c|}{ CENTRM cross sections $\left(\mathrm{cm}^{-1}\right)$} & \multicolumn{3}{|c|}{ NITAWL cross sections $\left(\mathrm{cm}^{-1}\right)$} \\
\hline & $\mathrm{G}_{\mathrm{a}}$ & $\mathcal{G}_{f}$ & $\mathrm{G}_{\mathrm{f}}$ & $\mathrm{G}_{3}$ & $<\mathrm{G}_{\mathrm{f}}$ & $\mathrm{G}_{\mathrm{f}}$ \\
\hline 1 & $5.14155 \mathrm{E}-04$ & $1.47850 \mathrm{E}-03$ & $4.78094 \mathrm{E}-04$ & $5.13349 \mathrm{E}-04$ & $1.47737 \mathrm{E}-03$ & $4.77888 \mathrm{E}-04$ \\
\hline 2 & $1.09920 \mathrm{E}-02$ & $1.80785 \mathrm{E}-02$ & $6.27768 \mathrm{E}-03$ & $1.06624 \mathrm{E}-02$ & 1.75079E-02 & $6.07953 \mathrm{E}-03$ \\
\hline 3 & $3.48251 \mathrm{E}-02$ & 6.83614E-02 & 2.37382E-02 & $3.45711 \mathrm{E}-02$ & 6.78516E-02 & 2.35613E-02 \\
\hline 4 & 2.81194E-01 & $5.59228 \mathrm{E}-01$ & $1.93491 \mathrm{E}-01$ & 2.80259E-01 & 5.57291E-01 & $1.92823 \mathrm{E}-01$ \\
\hline
\end{tabular}




\begin{tabular}{|c|c|c|c|c|c|c|c|c|c|c|}
\hline & & & & & & & & & & $\begin{array}{l}5.446 \\
(0.87)\end{array}$ \\
\hline & \multirow[t]{8}{*}{$\begin{array}{l}0.191 \\
(1.58) \\
\end{array}$} & & & & & & & & $\begin{array}{l}5.462 \\
(0.45)\end{array}$ & $\begin{array}{l}5.433 \\
(0.42)\end{array}$ \\
\hline & & & & & & & & $\begin{array}{l}5.225 \\
(0.40)\end{array}$ & $\begin{array}{l}5.327 \\
(0.30)\end{array}$ & $\begin{array}{l}5.357 \\
(0.42)\end{array}$ \\
\hline & & & & & & & $\begin{array}{l}4.915 \\
(0.44)\end{array}$ & $\begin{array}{l}5.049 \\
(0.30)\end{array}$ & $\begin{array}{l}5.160 \\
(0.34)\end{array}$ & $\begin{array}{l}5.185 \\
(0.43)\end{array}$ \\
\hline & & & & & & $\begin{array}{r}4.477 \\
(0.48) \\
\end{array}$ & $\begin{array}{l}4.691 \\
(0.37) \\
\end{array}$ & $\begin{array}{r}4.860 \\
(0.33) \\
\end{array}$ & $\begin{array}{l}4.954 \\
(0.36) \\
\end{array}$ & $\begin{array}{l}4.957 \\
(0.47) \\
\end{array}$ \\
\hline & & & & & $\begin{array}{l}3.976 \\
(0.52)\end{array}$ & $\begin{array}{l}4.231 \\
(0.37)\end{array}$ & $\begin{array}{l}4.430 \\
(0.37)\end{array}$ & $\begin{array}{l}4.569 \\
(0.33)\end{array}$ & $\begin{array}{l}4.651 \\
(0.31)\end{array}$ & $\begin{array}{l}4.688 \\
(0.46)\end{array}$ \\
\hline & & & & $\begin{array}{l}3.440 \\
(0.56)\end{array}$ & $\begin{array}{l}3.685 \\
(0.42)\end{array}$ & $\begin{array}{l}3.916 \\
(0.36)\end{array}$ & $\begin{array}{l}4.084 \\
(0.36)\end{array}$ & $\begin{array}{l}4.239 \\
(0.34)\end{array}$ & $\begin{array}{l}4.317 \\
(0.35)\end{array}$ & $\begin{array}{l}4.338 \\
(0.50)\end{array}$ \\
\hline & & & $\begin{array}{l}2.811 \\
(0.62)\end{array}$ & $\begin{array}{l}3.097 \\
(0.39)\end{array}$ & $\begin{array}{l}3.347 \\
(0.37)\end{array}$ & $\begin{array}{l}3.552 \\
(0.34)\end{array}$ & $\begin{array}{l}3.704 \\
(0.36)\end{array}$ & $\begin{array}{l}3.855 \\
(0.39)\end{array}$ & $\begin{array}{l}3.900 \\
(0.33)\end{array}$ & $\begin{array}{l}3.925 \\
(0.48)\end{array}$ \\
\hline & & $\begin{array}{l}2.272 \\
(0.65)\end{array}$ & $\begin{array}{l}2.533 \\
(0.42)\end{array}$ & $\begin{array}{c}2797 \\
(0.37)\end{array}$ & $\begin{array}{l}3.010 \\
(0.43)\end{array}$ & $\begin{array}{l}3.216 \\
(0.43)\end{array}$ & $\begin{array}{l}3.339 \\
(0.40)\end{array}$ & $\begin{array}{l}3.445 \\
(0.33)\end{array}$ & $\begin{array}{l}3.528 \\
(0.38)\end{array}$ & $\begin{array}{l}3.528 \\
(0.59)\end{array}$ \\
\hline & $\begin{array}{l}1.928 \\
(0.76)\end{array}$ & $\begin{array}{l}2.090 \\
(0.51)\end{array}$ & $\begin{array}{l}2.327 \\
(0.47)\end{array}$ & $\begin{array}{l}2.570 \\
(0.45)\end{array}$ & $\begin{array}{l}2.789 \\
(0.40)\end{array}$ & $\begin{array}{l}2.962 \\
(0.40)\end{array}$ & $\begin{array}{c}3.081 \\
(0.42)\end{array}$ & $\begin{array}{l}3.182 \\
(0.38)\end{array}$ & $\begin{array}{c}3.274 \\
(0.42)\end{array}$ & $\begin{array}{l}3.260 \\
(0.56)\end{array}$ \\
\hline $\begin{array}{l}2.123 \\
(0.72)\end{array}$ & $\begin{array}{l}2.094 \\
(0.47)\end{array}$ & $\begin{array}{l}2.324 \\
(0.47)\end{array}$ & $\begin{array}{c}2.602 \\
(0.41)\end{array}$ & $\begin{array}{l}2.847 \\
(0.39)\end{array}$ & $\begin{array}{c}3.072 \\
(0.40)\end{array}$ & $\begin{array}{l}3.294 \\
(0.40)\end{array}$ & $\begin{array}{l}3.461 \\
(0.41)\end{array}$ & $\begin{array}{l}3.543 \\
(0.36)\end{array}$ & $\begin{array}{c}3.638 \\
(0.36)\end{array}$ & $\begin{array}{l}3.680 \\
(0.54)\end{array}$ \\
\hline
\end{tabular}

Fig. 4.10a. Pin-power distribution for CENTRM benchmark TCA-6. Value in parentheses is percent standard deviation. 


\begin{tabular}{|c|c|c|c|c|c|c|c|c|c|c|}
\hline & & & & & & & & & & $\begin{array}{l}5.535 \\
(0.82)\end{array}$ \\
\hline & $\begin{array}{c}0.192 \\
(1.50)\end{array}$ & & & & & & & & $\begin{array}{l}5.433 \\
(0.45)\end{array}$ & $\begin{array}{l}5.485 \\
(0.41)\end{array}$ \\
\hline & & & & & & & & $\begin{array}{l}5.208 \\
(0.43)\end{array}$ & $\begin{array}{l}5.336 \\
(0.30)\end{array}$ & $\begin{array}{l}5.380 \\
(0.44)\end{array}$ \\
\hline & & & & & & & $\begin{array}{l}4.903 \\
(0.45)\end{array}$ & $\begin{array}{l}5.056 \\
(0.32)\end{array}$ & $\begin{array}{l}5.155 \\
(0.31)\end{array}$ & $\begin{array}{l}5.224 \\
(0.42)\end{array}$ \\
\hline & & & & & & $\begin{array}{l}4.478 \\
(0.50)\end{array}$ & $\begin{array}{l}4.745 \\
(0.32)\end{array}$ & $\begin{array}{l}4.840 \\
(0.32)\end{array}$ & $\begin{array}{l}4.949 \\
(0.31)\end{array}$ & $\begin{array}{l}5.009 \\
(0.47)\end{array}$ \\
\hline & & & & & $\begin{array}{c}3.945 \\
(0.51)\end{array}$ & $\begin{array}{l}4.219 \\
(0.35)\end{array}$ & $\begin{array}{l}4.437 \\
(0.34)\end{array}$ & $\begin{array}{l}4.592 \\
(0.34)\end{array}$ & $\begin{array}{l}4.656 \\
(0.35)\end{array}$ & $\begin{array}{l}4.662 \\
(0.46)\end{array}$ \\
\hline & & & & $\begin{array}{l}3.402 \\
(0.55)\end{array}$ & $\begin{array}{l}3.677 \\
(0.37)\end{array}$ & $\begin{array}{l}3.935 \\
(0.36)\end{array}$ & $\begin{array}{l}4.103 \\
(0.36)\end{array}$ & $\begin{array}{l}4.259 \\
(0.35)\end{array}$ & $\begin{array}{l}4.279 \\
(0.33)\end{array}$ & $\begin{array}{l}4.347 \\
(0.51)\end{array}$ \\
\hline & & & $\begin{array}{l}2.808 \\
(0.56)\end{array}$ & $\begin{array}{l}3.096 \\
(0.43)\end{array}$ & $\begin{array}{l}3.349 \\
(0.38)\end{array}$ & $\begin{array}{l}3.556 \\
(0.37)\end{array}$ & $\begin{array}{l}3.711 \\
(0.36)\end{array}$ & $\begin{array}{l}3.858 \\
(0.35)\end{array}$ & $\begin{array}{c}3.908 \\
(0.35)\end{array}$ & $\begin{array}{l}3.932 \\
(0.47)\end{array}$ \\
\hline & & $\begin{array}{l}2.286 \\
(0.62)\end{array}$ & $\begin{array}{l}2.545 \\
(0.44)\end{array}$ & $\begin{array}{c}2795 \\
(0.38)\end{array}$ & $\begin{array}{l}3.014 \\
(0.42)\end{array}$ & $\begin{array}{l}3.211 \\
(0.41)\end{array}$ & $\begin{array}{l}3.340 \\
(0.38)\end{array}$ & $\begin{array}{l}3.434 \\
(0.35)\end{array}$ & $\begin{array}{l}3.501 \\
(0.39)\end{array}$ & $\begin{array}{l}3.547 \\
(0.56)\end{array}$ \\
\hline & $\begin{array}{l}1.930 \\
(0.79)\end{array}$ & $\begin{array}{l}2.114 \\
(0.47)\end{array}$ & $\begin{array}{c}2.328 \\
(0.48)\end{array}$ & $\begin{array}{c}2.571 \\
(0.47)\end{array}$ & $\begin{array}{l}2.784 \\
(0.43)\end{array}$ & $\begin{array}{l}2.977 \\
(0.38)\end{array}$ & $\begin{array}{l}3.106 \\
(0.36)\end{array}$ & $\begin{array}{l}3.198 \\
(0.41)\end{array}$ & $\begin{array}{c}3.269 \\
(0.38)\end{array}$ & $\begin{array}{l}3.308 \\
(0.52)\end{array}$ \\
\hline $\begin{array}{l}2.129 \\
(0.65)\end{array}$ & $\begin{array}{l}2.105 \\
(0.45)\end{array}$ & $\begin{array}{l}2.328 \\
(0.49)\end{array}$ & $\begin{array}{c}2.622 \\
(0.41)\end{array}$ & $\begin{array}{l}2.861 \\
(0.43)\end{array}$ & $\begin{array}{c}3.096 \\
(0.38)\end{array}$ & $\begin{array}{l}3.292 \\
(0.40)\end{array}$ & $\begin{array}{l}3.450 \\
(0.38)\end{array}$ & $\begin{array}{l}3.563 \\
(0.40)\end{array}$ & $\begin{array}{c}3.618 \\
(0.35)\end{array}$ & $\begin{array}{l}3.656 \\
(0.57)\end{array}$ \\
\hline
\end{tabular}

Fig. 4.10b. Pin-power distribution for NITAWL benchmark TCA-6. Value in parentheses is percent standard deviation. 
Table 4.55. Selected reaction rates for CENTRM case TCA-6

\begin{tabular}{|c|c|c|c|c|c|c|}
\hline Pin & Region & $\begin{array}{c}\mathrm{G}_{\mathrm{a}} \mathrm{M} \\
\left(\mathrm{cm}^{-3}-\mathrm{s}^{-1}\right)\end{array}$ & $\begin{array}{c}\mathcal{G}_{\mathrm{f}} \mathrm{M} \\
\left(\mathrm{cm}^{-3}-\mathrm{s}^{-1}\right)\end{array}$ & $\begin{array}{c}\mathrm{G}_{\mathrm{f}} \mathrm{M} \\
\left(\mathrm{cm}^{-3}-\mathrm{s}^{-1}\right)\end{array}$ & $\begin{array}{c}\mathrm{M} \\
\left(\mathrm{cm}-\mathrm{s}^{-1}\right)\end{array}$ & M/M \\
\hline \multirow{3}{*}{1} & Fuel & $1.298 \mathrm{E}-05$ & $2.136 \mathrm{E}-05$ & 7.574E-06 & $5.637 \mathrm{E}-03$ & 0.235 \\
\hline & Clad & $2.474 \mathrm{E}-07$ & 0.0 & 0.0 & $1.759 \mathrm{E}-03$ & 0.073 \\
\hline & Mod. & 7.049E-07 & 0.0 & 0.0 & $1.659 \mathrm{E}-02$ & 0.692 \\
\hline \multirow{3}{*}{66} & Fuel & $3.599 \mathrm{E}-05$ & $5.470 \mathrm{E}-05$ & $1.936 \mathrm{E}-05$ & $2.295 \mathrm{E}-02$ & 0.234 \\
\hline & Clad & $7.226 \mathrm{E}-07$ & 0.0 & 0.0 & $7.268 \mathrm{E}-03$ & 0.074 \\
\hline & Mod. & $1.669 \mathrm{E}-06$ & 0.0 & 0.0 & $6.776 \mathrm{E}-02$ & 0.692 \\
\hline
\end{tabular}

Table 4.56. Selected reaction rates for NITAWL case TCA-6

\begin{tabular}{ccccccc}
\hline Pin & Region & $\begin{array}{c}\mathrm{G}_{\mathrm{a}} \mathrm{M} \\
\left(\mathrm{cm}^{-3}-\mathrm{s}^{-1}\right)\end{array}$ & $\begin{array}{c}\mathrm{G}_{\mathrm{f}} \mathrm{M} \\
\left(\mathrm{cm}^{-3}-\mathrm{s}^{-1}\right)\end{array}$ & $\begin{array}{c}\mathrm{G}_{\mathrm{f}} \mathrm{M} \\
\left(\mathrm{cm}^{-3}-\mathrm{s}^{-1}\right)\end{array}$ & $\begin{array}{c}\mathrm{M} \\
\left(\mathrm{cm}^{-1}\right)\end{array}$ & $\mathrm{sM}_{\mathrm{t}}$ \\
\hline \multirow{2}{*}{1} & Fuel & $1.301 \mathrm{E}-05$ & $2.152 \mathrm{E}-05$ & $7.630 \mathrm{E}-06$ & $5.677 \mathrm{E}-03$ & 0.236 \\
& Clad & $2.482 \mathrm{E}-07$ & 0.0 & 0.0 & $1.771 \mathrm{E}-03$ & 0.073 \\
& Mod. & $7.104 \mathrm{E}-07$ & 0.0 & 0.0 & $1.663 \mathrm{E}-02$ & 0.691 \\
\hline \multirow{2}{*}{66} & Fuel & $3.641 \mathrm{E}-05$ & $5.549 \mathrm{E}-05$ & $1.964 \mathrm{E}-05$ & $2.328 \mathrm{E}-02$ & 0.235 \\
& Clad & $7.250 \mathrm{E}-07$ & 0.0 & 0.0 & $7.322 \mathrm{E}-03$ & 0.074 \\
& Mod. & $1.692 \mathrm{E}-06$ & 0.0 & 0.0 & $6.857 \mathrm{E}-02$ & 0.691 \\
\hline
\end{tabular}


Table 4.57. TCA-6, Pin-1 four-group fluxes

\begin{tabular}{ccc}
\hline Group & $\begin{array}{c}\text { CENTRM M } \\
\left(\mathrm{cm}^{-2}-\mathrm{s}^{-1}\right)\end{array}$ & $\begin{array}{c}\text { NITAWL M } \\
\left(\mathrm{cm}^{-2}-\mathrm{s}^{-1}\right)\end{array}$ \\
\hline 1 & $4.562 \mathrm{E}-05$ & $4.614 \mathrm{E}-05$ \\
2 & $1.353 \mathrm{E}-05$ & $1.361 \mathrm{E}-05$ \\
3 & $3.260 \mathrm{E}-06$ & $3.167 \mathrm{E}-06$ \\
4 & $2.877 \mathrm{E}-05$ & $2.891 \mathrm{E}-05$ \\
\hline
\end{tabular}

Table 4.58. TCA-6, Pin-1 four-group U-235 cross sections

\begin{tabular}{cccccccc}
\hline Group & \multicolumn{3}{c}{ CENTRM cross sections $\left(\mathrm{cm}^{-1}\right)$} & \multicolumn{3}{c}{ NITAWL cross sections $\left(\mathrm{cm}^{-1}\right)$} \\
\hline & $\frac{\mathrm{G}_{\mathrm{a}}}{1.43180 \mathrm{E}-04}$ & $\frac{\mathrm{G}_{\mathrm{f}}}{3.25454 \mathrm{E}-04}$ & $\frac{\mathrm{G}_{\mathrm{f}}}{1.25334 \mathrm{E}-04}$ & $\frac{\mathrm{G}_{\mathrm{a}}}{1.42890 \mathrm{E}-04}$ & $\frac{\mathrm{G}_{\mathrm{f}}}{3.25301 \mathrm{E}-04}$ & $\frac{\mathrm{G}_{\mathrm{f}}}{1.25170 \mathrm{E}-04}$ \\
1 & $3.45574 \mathrm{E}-03$ & $5.26068 \mathrm{E}-03$ & $2.15894 \mathrm{E}-03$ & $3.46874 \mathrm{E}-03$ & $5.30446 \mathrm{E}-03$ & $2.17691 \mathrm{E}-03$ \\
2 & $5.12187 \mathrm{E}-03$ & $1.04432 \mathrm{E}-02$ & $4.28580 \mathrm{E}-03$ & $5.18636 \mathrm{E}-03$ & $1.05942 \mathrm{E}-02$ & $4.34774 \mathrm{E}-03$ \\
3 & $4.87091 \mathrm{E}-02$ & $1.01408 \mathrm{E}-01$ & $4.16173 \mathrm{E}-02$ & $4.87392 \mathrm{E}-02$ & $1.01468 \mathrm{E}-01$ & $4.16413 \mathrm{E}-02$ \\
\hline
\end{tabular}

Table 4.59. TCA-6, Pin-1 four-group U-238 cross sections

\begin{tabular}{ccccccc}
\hline Group & \multicolumn{2}{c}{ CENTRM cross sections $\left(\mathrm{cm}^{-1}\right)$} & \multicolumn{3}{c}{ NITAWL cross sections $\left(\mathrm{cm}^{-1}\right)$} \\
\hline & $\frac{\mathrm{G}_{\mathrm{a}}}{4.44759 \mathrm{E}-03}$ & $\frac{\mathrm{G}_{\mathrm{f}}}{8.24022 \mathrm{E}-03}$ & $\frac{\mathrm{G}_{\mathrm{f}}}{2.92337 \mathrm{E}-03}$ & $\frac{\mathrm{G}_{\mathrm{a}}}{4.46693 \mathrm{E}-03}$ & $\overline{8.35598 \mathrm{E}-03}$ & $\overline{2.96179 \mathrm{E}-03}$ \\
1 & $3.90256 \mathrm{E}-02$ & $3.50820 \mathrm{E}-06$ & $1.45343 \mathrm{E}-06$ & $3.57765 \mathrm{E}-02$ & $3.56985 \mathrm{E}-06$ & $1.47897 \mathrm{E}-06$ \\
2 & $6.98181 \mathrm{E}-03$ & $2.44734 \mathrm{E}-08$ & $1.01398 \mathrm{E}-08$ & $7.00395 \mathrm{E}-03$ & $2.46168 \mathrm{E}-08$ & $1.01992 \mathrm{E}-08$ \\
3 & $2.76742 \mathrm{E}-02$ & $1.30082 \mathrm{E}-07$ & $5.38954 \mathrm{E}-08$ & $2.76818 \mathrm{E}-02$ & $1.30118 \mathrm{E}-07$ & $5.39104 \mathrm{E}-08$ \\
\hline
\end{tabular}

Table 4.60. TCA-6, Pin-1 four-group Pu-239 cross sections

\begin{tabular}{cccccccc}
\hline Group & \multicolumn{3}{c}{ CENTRM cross sections $\left(\mathrm{cm}^{-1}\right)$} & \multicolumn{3}{c}{ NITAWL cross sections $\left(\mathrm{cm}^{-1}\right)$} \\
\hline & $\frac{\mathrm{G}_{\mathrm{a}}}{1}$ & $5.11662 \mathrm{E}-04$ & $\frac{\leftarrow_{\mathrm{f}}}{1.49257 \mathrm{E}-03}$ & $\frac{\mathrm{G}_{\mathrm{f}}}{4.80489 \mathrm{E}-04}$ & $\frac{\mathrm{G}_{\mathrm{a}}}{5.11324 \mathrm{E}-04}$ & $\frac{\mathrm{G}_{\mathrm{f}}}{1.49329 \mathrm{E}-03}$ & $\frac{\mathrm{G}_{\mathrm{f}}}{4.80338 \mathrm{E}-04}$ \\
2 & $1.13135 \mathrm{E}-02$ & $1.86601 \mathrm{E}-02$ & $6.47963 \mathrm{E}-03$ & $1.07085 \mathrm{E}-02$ & $1.75213 \mathrm{E}-02$ & $6.08419 \mathrm{E}-03$ \\
3 & $3.39736 \mathrm{E}-02$ & $6.67666 \mathrm{E}-02$ & $2.31844 \mathrm{E}-02$ & $3.36473 \mathrm{E}-02$ & $6.62052 \mathrm{E}-02$ & $2.29895 \mathrm{E}-02$ \\
4 & $2.66162 \mathrm{E}-01$ & $5.39117 \mathrm{E}-01$ & $1.86465 \mathrm{E}-01$ & $2.67553 \mathrm{E}-01$ & $5.41501 \mathrm{E}-01$ & $1.87294 \mathrm{E}-01$ \\
\hline
\end{tabular}


Table 4.61. TCA-6, Pin-66 four-group fluxes

\begin{tabular}{ccc}
\hline Group & $\begin{array}{c}\text { CENTRM M } \\
\left(\mathrm{cm}^{-2}-\mathrm{s}^{-1}\right)\end{array}$ & $\begin{array}{c}\text { NITAWL M } \\
\left(\mathrm{cm}^{-2}-\mathrm{s}^{-1}\right)\end{array}$ \\
\hline 1 & $2.129 \mathrm{E}-04$ & $2.141 \mathrm{E}-04$ \\
2 & $7.322 \mathrm{E}-05$ & $7.593 \mathrm{E}-05$ \\
3 & $1.668 \mathrm{E}-05$ & $1.698 \mathrm{E}-05$ \\
4 & $6.844 \mathrm{E}-05$ & $6.955 \mathrm{E}-05$ \\
\hline
\end{tabular}

Table 4.62. TCA-6, Pin-66 four-group U-235 cross sections

\begin{tabular}{cccccccc}
\hline Group & \multicolumn{3}{c}{ CENTRM cross sections $\left(\mathrm{cm}^{-1}\right)$} & \multicolumn{3}{c}{ NITAWL cross sections $\left(\mathrm{cm}^{-1}\right)$} \\
\hline & $\frac{\mathrm{G}_{\mathrm{a}}}{1.46734 \mathrm{E}-04}$ & $\frac{\mathrm{G}_{\mathrm{f}}}{3.28401 \mathrm{E}-04}$ & $\frac{\mathrm{G}_{\mathrm{f}}}{1.27150 \mathrm{E}-04}$ & $\frac{\mathrm{G}_{\mathrm{a}}}{1.47522 \mathrm{E}-04}$ & $\frac{\mathrm{G}_{\mathrm{f}}}{3.29450 \mathrm{E}-04}$ & $\frac{\mathrm{G}_{\mathrm{f}}}{1.27598 \mathrm{E}-04}$ \\
1 & $3.38204 \mathrm{E}-03$ & $5.17330 \mathrm{E}-03$ & $2.12307 \mathrm{E}-03$ & $3.41355 \mathrm{E}-03$ & $5.23610 \mathrm{E}-03$ & $2.14885 \mathrm{E}-03$ \\
2 & $5.13990 \mathrm{E}-03$ & $1.04899 \mathrm{E}-02$ & $4.30497 \mathrm{E}-03$ & $5.04984 \mathrm{E}-03$ & $1.02714 \mathrm{E}-02$ & $4.21529 \mathrm{E}-03$ \\
3 & $4.64337 \mathrm{E}-02$ & $9.65915 \mathrm{E}-02$ & $3.96403 \mathrm{E}-02$ & $4.61970 \mathrm{E}-02$ & $9.60939 \mathrm{E}-02$ & $3.94361 \mathrm{E}-02$ \\
\hline
\end{tabular}

Table 4.63. TCA-6, Pin-66 four-group U-238 cross sections

\begin{tabular}{cccccccc}
\hline Group & \multicolumn{3}{c}{ CENTRM cross sections $\left(\mathrm{cm}^{-1}\right)$} & \multicolumn{3}{c}{ NITAWL cross sections $\left(\mathrm{cm}^{-1}\right)$} \\
\hline & $\frac{\mathrm{G}_{\mathrm{a}}}{4.34898 \mathrm{E}-03}$ & $\frac{\mathrm{G}_{\mathrm{f}}}{7.60907 \mathrm{E}-03}$ & $\frac{\mathrm{G}_{\mathrm{f}}}{2.70457 \mathrm{E}-03}$ & $\frac{\mathrm{G}_{\mathrm{a}}}{4.36295 \mathrm{E}-03}$ & $\frac{\mathrm{G}_{\mathrm{f}}}{7.58351 \mathrm{E}-03}$ & $\frac{\mathrm{G}_{\mathrm{f}}}{2.69381 \mathrm{E}-03}$ \\
1 & $3.85262 \mathrm{E}-02$ & $3.68752 \mathrm{E}-06$ & $1.52772 \mathrm{E}-06$ & $3.74174 \mathrm{E}-02$ & $3.59244 \mathrm{E}-06$ & $1.48833 \mathrm{E}-06$ \\
3 & $6.99508 \mathrm{E}-03$ & $2.44889 \mathrm{E}-08$ & $1.01462 \mathrm{E}-08$ & $6.96908 \mathrm{E}-03$ & $2.42193 \mathrm{E}-08$ & $1.00345 \mathrm{E}-08$ \\
4 & $2.64864 \mathrm{E}-02$ & $1.24380 \mathrm{E}-07$ & $5.15332 \mathrm{E}-08$ & $2.63675 \mathrm{E}-02$ & $1.23812 \mathrm{E}-07$ & $5.12979 \mathrm{E}-08$ \\
\hline
\end{tabular}

Table 4.64. TCA-6, Pin-66 four-group Pu-239 cross sections

\begin{tabular}{cccccccc}
\hline Group & \multicolumn{2}{c}{ CENTRM cross sections $\left(\mathrm{cm}^{-1}\right)$} & \multicolumn{3}{c}{ NITAWL cross sections $\left(\mathrm{cm}^{-1}\right)$} \\
\hline & $\frac{\mathrm{G}_{\mathrm{a}}}{5.13250 \mathrm{E}-04}$ & $\frac{\mathrm{G}_{\mathrm{f}}}{1.47784 \mathrm{E}-03}$ & $\frac{\mathrm{G}_{\mathrm{f}}}{4.78051 \mathrm{E}-04}$ & $\frac{\mathrm{G}_{\mathrm{a}}}{5.13989 \mathrm{E}-04}$ & $\frac{\mathrm{G}_{\mathrm{f}}}{1.47716 \mathrm{E}-03}$ & $\frac{\mathrm{G}_{\mathrm{f}}}{4.77940 \mathrm{E}-04}$ \\
1 & $1.13093 \mathrm{E}-02$ & $1.84779 \mathrm{E}-02$ & $6.41636 \mathrm{E}-03$ & $1.07147 \mathrm{E}-02$ & $1.76559 \mathrm{E}-02$ & $6.13092 \mathrm{E}-03$ \\
3 & $3.39521 \mathrm{E}-02$ & $6.67206 \mathrm{E}-02$ & $2.31683 \mathrm{E}-02$ & $3.32187 \mathrm{E}-02$ & $6.53557 \mathrm{E}-02$ & $2.26946 \mathrm{E}-02$ \\
4 & $2.81522 \mathrm{E}-01$ & $5.60045 \mathrm{E}-01$ & $1.93774 \mathrm{E}-01$ & $2.81843 \mathrm{E}-01$ & $5.60105 \mathrm{E}-01$ & $1.93799 \mathrm{E}-01$ \\
\hline
\end{tabular}




\begin{tabular}{|c|c|c|c|c|c|c|c|c|c|}
\hline & & & & & & & & & $\begin{array}{c}7.308 \\
(0.44)\end{array}$ \\
\hline & $\begin{array}{l}0.155 \\
(0.61)\end{array}$ & & & & & & & $\begin{array}{c}7.112 \\
(0.39)\end{array}$ & $\begin{array}{l}7.241 \\
(0.35)\end{array}$ \\
\hline & & & & & & & $\begin{array}{l}6.694 \\
(0.43)\end{array}$ & $\begin{array}{l}6.907 \\
(0.26)\end{array}$ & $\begin{array}{r}7.038 \\
(0.31)\end{array}$ \\
\hline & & & & & & $\begin{array}{l}6.068 \\
(0.46)\end{array}$ & $\begin{array}{l}6.408 \\
(0.30)\end{array}$ & $\begin{array}{l}6.595 \\
(0.30)\end{array}$ & $\begin{array}{l}6.694 \\
(0.30)\end{array}$ \\
\hline & & & & & $\begin{array}{l}5.368 \\
(0.55)\end{array}$ & $\begin{array}{l}5.743 \\
(0.35)\end{array}$ & $\begin{array}{l}6.028 \\
(0.33)\end{array}$ & $\begin{array}{c}6.153 \\
(0.34)\end{array}$ & $\begin{array}{l}6.308 \\
(0.29)\end{array}$ \\
\hline & & & & $\begin{array}{l}4.563 \\
(0.47)\end{array}$ & $\begin{array}{l}4.998 \\
(0.34)\end{array}$ & $\begin{array}{l}5.297 \\
(0.35)\end{array}$ & $\begin{array}{l}5.515 \\
(0.33)\end{array}$ & $\begin{array}{l}5.710 \\
(0.30)\end{array}$ & $\begin{array}{l}5.792 \\
(0.32)\end{array}$ \\
\hline & & & $\begin{array}{c}3.708 \\
(0.54)\end{array}$ & $\begin{array}{l}4.107 \\
(0.38)\end{array}$ & $\begin{array}{l}4.467 \\
(0.38)\end{array}$ & $\begin{array}{l}4.726 \\
(0.34)\end{array}$ & $\begin{array}{l}4.996 \\
(0.34)\end{array}$ & $\begin{array}{l}5.135 \\
(0.34)\end{array}$ & $\begin{array}{l}5.182 \\
(0.36)\end{array}$ \\
\hline & & $\begin{array}{l}2.884 \\
(0.72)\end{array}$ & $\begin{array}{l}3.250 \\
(0.41)\end{array}$ & $\begin{array}{l}3.615 \\
(0.38)\end{array}$ & $\begin{array}{l}3.950 \\
(0.39)\end{array}$ & $\begin{array}{l}4.203 \\
(0.34)\end{array}$ & $\begin{array}{l}4.373 \\
(0.37)\end{array}$ & $\begin{array}{l}4.511 \\
(0.36)\end{array}$ & $\begin{array}{l}4.579 \\
(0.39)\end{array}$ \\
\hline & $\begin{array}{l}2.218 \\
(0.76)\end{array}$ & $\begin{array}{l}2.524 \\
(0.48)\end{array}$ & $\begin{array}{l}2.866 \\
(0.44)\end{array}$ & $\begin{array}{l}3.179 \\
(0.45)\end{array}$ & $\begin{array}{l}3.463 \\
(0.41)\end{array}$ & $\begin{array}{l}3.694 \\
(0.41)\end{array}$ & $\begin{array}{l}3.852 \\
(0.37)\end{array}$ & $\begin{array}{l}3.966 \\
(0.40)\end{array}$ & $\begin{array}{l}4.027 \\
(0.40)\end{array}$ \\
\hline $\begin{array}{l}2.112 \\
(0.73)\end{array}$ & $\begin{array}{l}2.218 \\
(0.54)\end{array}$ & $\begin{array}{l}2.550 \\
(0.48)\end{array}$ & $\begin{array}{l}2.879 \\
(0.44)\end{array}$ & $\begin{array}{l}3.211 \\
(0.40)\end{array}$ & $\begin{array}{l}3.481 \\
(0.45)\end{array}$ & $\begin{array}{l}3.706 \\
(0.38)\end{array}$ & $\begin{array}{l}3.893 \\
(0.38)\end{array}$ & $\begin{array}{l}4.026 \\
(0.39)\end{array}$ & $\begin{array}{l}4.085 \\
(0.37)\end{array}$ \\
\hline
\end{tabular}

Fig. 4.11a. Pin-power distribution for CENTRM benchmark TCA-7. Value in parentheses is percent standard deviation. 


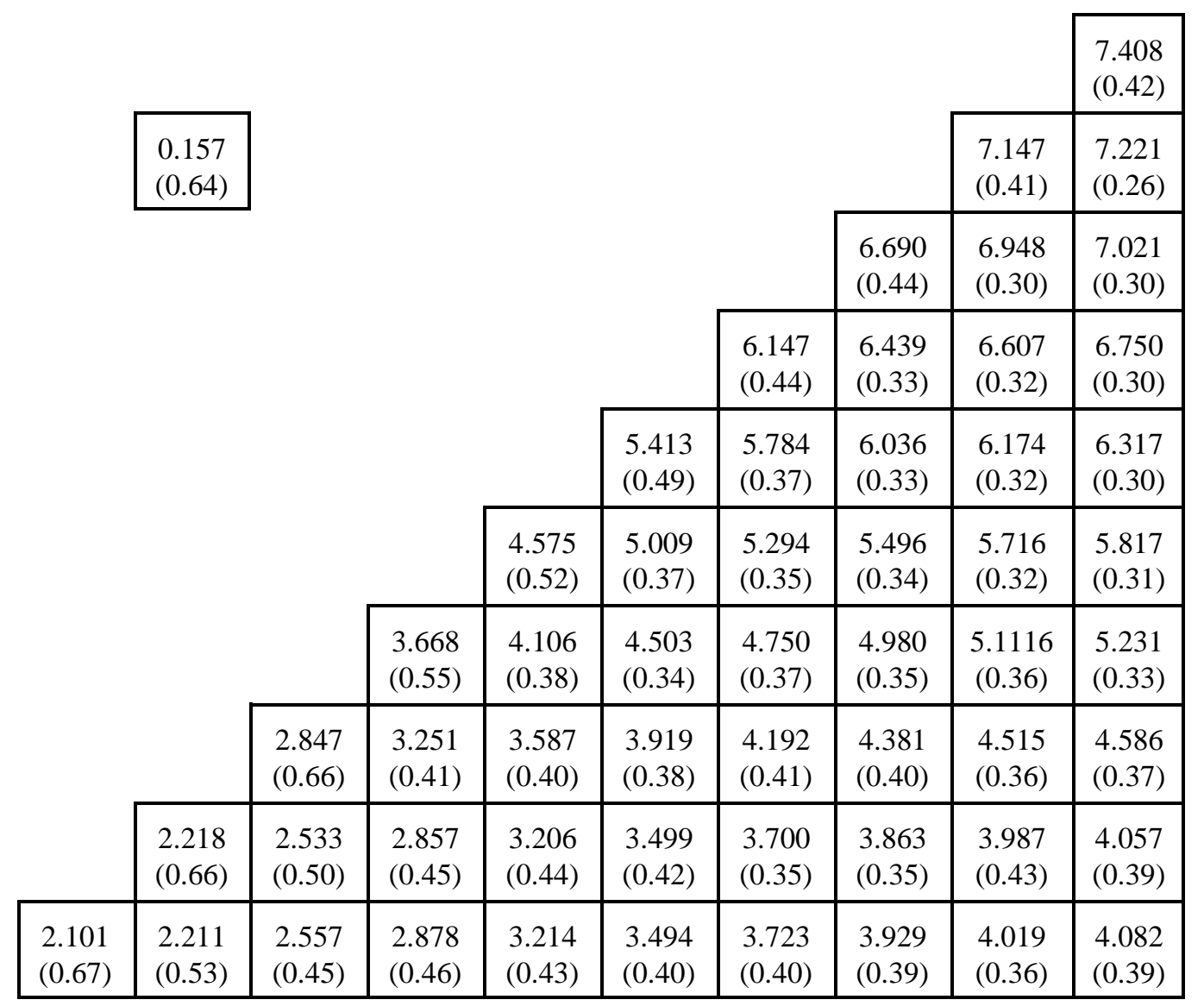

Fig. 4.11b. Pin-power distribution for NITAWL benchmark TCA-7. Value in parentheses is percent standard deviation. 
Table 4.65. Selected reaction rates for CENTRM case TCA-7

\begin{tabular}{|c|c|c|c|c|c|c|}
\hline Pin & Region & $\begin{array}{c}\mathrm{G}_{\mathrm{a}} \mathrm{M} \\
\left(\mathrm{cm}^{-3}-\mathrm{s}^{-1}\right)\end{array}$ & $\begin{array}{c}\mathcal{G}_{\mathrm{f}} \mathrm{M} \\
\left(\mathrm{cm}^{-3}-\mathrm{s}^{-1}\right)\end{array}$ & $\begin{array}{c}\mathrm{G}_{\mathrm{f}} \mathrm{M} \\
\left(\mathrm{cm}^{-3}-\mathrm{s}^{-1}\right)\end{array}$ & $\begin{array}{c}\mathrm{M} \\
\left(\mathrm{cm}-\mathrm{s}^{-1}\right)\end{array}$ & M/M \\
\hline \multirow{3}{*}{1} & Fuel & $1.250 \mathrm{E}-05$ & $2.098 \mathrm{E}-05$ & 7.436E-06 & 4.475E-03 & 0.181 \\
\hline & Clad & $2.338 \mathrm{E}-07$ & 0.0 & 0.0 & $1.390 \mathrm{E}-03$ & 0.056 \\
\hline & Mod. & $7.159 \mathrm{E}-07$ & 0.0 & 0.0 & $1.884 \mathrm{E}-02$ & 0.763 \\
\hline \multirow{3}{*}{55} & Fuel & $4.638 \mathrm{E}-05$ & 7.357E-05 & $2.604 \mathrm{E}-05$ & $2.167 \mathrm{E}-02$ & 0.182 \\
\hline & Clad & $8.926 \mathrm{E}-07$ & 0.0 & 0.0 & $6.796 \mathrm{E}-03$ & 0.057 \\
\hline & Mod. & $2.331 \mathrm{E}-06$ & 0.0 & 0.0 & $9.081 \mathrm{E}-02$ & 0.761 \\
\hline
\end{tabular}

Table 4.66. Selected reaction rates for NITAWL case TCA-7

\begin{tabular}{ccccccc}
\hline Pin & Region & $\begin{array}{c}\mathrm{G}_{\mathrm{a}} \mathrm{M} \\
\left(\mathrm{cm}^{-3}-\mathrm{s}^{-1}\right)\end{array}$ & $\begin{array}{c}\mathrm{G}_{\mathrm{f}} \mathrm{M} \\
\left(\mathrm{cm}^{-3}-\mathrm{s}^{-1}\right)\end{array}$ & $\begin{array}{c}\mathrm{G}_{\mathrm{f}} \mathrm{M} \\
\left(\mathrm{cm}^{-3}-\mathrm{s}^{-1}\right)\end{array}$ & $\begin{array}{c}\mathrm{M} \\
\left(\mathrm{cm}^{-1}\right)\end{array}$ & $\mathrm{MM}_{\mathrm{t}}$ \\
\hline \multirow{2}{*}{1} & Fuel & $1.239 \mathrm{E}-05$ & $2.081 \mathrm{E}-05$ & $7.375 \mathrm{E}-06$ & $4.405 \mathrm{E}-03$ & 0.181 \\
& Clad & $2.328 \mathrm{E}-07$ & 0.0 & 0.0 & $1.370 \mathrm{E}-03$ & 0.056 \\
& Mod. & $7.088 \mathrm{E}-07$ & 0.0 & 0.0 & $1.861 \mathrm{E}-02$ & 0.763 \\
\hline \multirow{2}{*}{55} & Fuel & $4.646 \mathrm{E}-05$ & $7.384 \mathrm{E}-05$ & $2.619 \mathrm{E}-05$ & $2.180 \mathrm{E}-02$ & 0.182 \\
& Clad & $8.849 \mathrm{E}-07$ & 0.0 & 0.0 & $6.807 \mathrm{E}-03$ & 0.057 \\
& Mod. & $2.324 \mathrm{E}-06$ & 0.0 & 0.0 & $9.112 \mathrm{E}-02$ & 0.761 \\
\hline
\end{tabular}


Table 4.67. TCA-7, Pin-1 four-group fluxes

\begin{tabular}{ccc}
\hline Group & $\begin{array}{c}\text { CENTRM M } \\
\left(\mathrm{cm}^{-2}-\mathrm{s}^{-1}\right)\end{array}$ & $\begin{array}{c}\text { NITAWL M } \\
\left(\mathrm{cm}^{-2}-\mathrm{s}^{-1}\right)\end{array}$ \\
\hline 1 & $4.076 \mathrm{E}-05$ & $4.002 \mathrm{E}-05$ \\
2 & $1.156 \mathrm{E}-05$ & $1.122 \mathrm{E}-05$ \\
3 & $2.916 \mathrm{E}-06$ & $2.849 \mathrm{E}-06$ \\
4 & $2.805 \mathrm{E}-05$ & $2.789 \mathrm{E}-05$ \\
\hline
\end{tabular}

Table 4.68. TCA-7, Pin-1 four-group U-235 cross sections

\begin{tabular}{cccccccc}
\hline Group & \multicolumn{3}{c}{ CENTRM cross sections $\left(\mathrm{cm}^{-1}\right)$} & \multicolumn{3}{c}{ NITAWL cross sections $\left(\mathrm{cm}^{-1}\right)$} \\
\hline & $\frac{\mathrm{G}_{\mathrm{a}}}{}$ & $\frac{\varangle_{\mathrm{f}}}{1.42491 \mathrm{E}-04}$ & $\frac{\mathrm{G}_{\mathrm{f}}}{3.25106 \mathrm{E}-04}$ & $\frac{\mathrm{G}_{\mathrm{f}}}{1.25005 \mathrm{E}-04}$ & $\frac{\mathrm{G}_{\mathrm{a}}}{1.41299 \mathrm{E}-04}$ & $\frac{\varangle_{\mathrm{f}}}{3.24300 \mathrm{E}-04}$ & $\frac{\mathrm{G}_{\mathrm{f}}}{1.24414 \mathrm{E}-04}$ \\
2 & $3.44975 \mathrm{E}-03$ & $5.22773 \mathrm{E}-03$ & $2.14542 \mathrm{E}-03$ & $3.47152 \mathrm{E}-03$ & $5.26026 \mathrm{E}-03$ & $2.15876 \mathrm{E}-03$ \\
3 & $5.21159 \mathrm{E}-03$ & $1.06607 \mathrm{E}-02$ & $4.37503 \mathrm{E}-03$ & $5.27529 \mathrm{E}-03$ & $1.08126 \mathrm{E}-02$ & $4.43738 \mathrm{E}-03$ \\
4 & $4.89248 \mathrm{E}-02$ & $1.01867 \mathrm{E}-01$ & $4.18053 \mathrm{E}-02$ & $4.88655 \mathrm{E}-02$ & $1.01743 \mathrm{E}-01$ & $4.17544 \mathrm{E}-02$ \\
\hline
\end{tabular}

Table 4.69. TCA-7, Pin-1 four-group U-238 cross sections

\begin{tabular}{cccccccc}
\hline Group & \multicolumn{3}{c}{ CENTRM cross sections $\left(\mathrm{cm}^{-1}\right)$} & \multicolumn{3}{c}{ NITAWL cross sections $\left(\mathrm{cm}^{-1}\right)$} \\
\hline & $\frac{\mathrm{G}_{\mathrm{a}}}{4.5114 \mathrm{E}-03}$ & $\frac{\mathrm{G}_{\mathrm{f}}}{8.48982 \mathrm{E}-03}$ & $\frac{\mathrm{G}_{\mathrm{f}}}{3.01170 \mathrm{E}-03}$ & $\frac{4.53551 \mathrm{E}-03}{\mathrm{G}_{\mathrm{a}}}$ & $\frac{{ }_{8}}{8.70929 \mathrm{E}-03}$ & $\frac{\mathrm{G}_{\mathrm{f}}}{3.08361 \mathrm{E}-03}$ \\
1 & $3.80608 \mathrm{E}-02$ & $3.86401 \mathrm{E}-06$ & $1.60085 \mathrm{E}-06$ & $3.81145 \mathrm{E}-02$ & $3.57483 \mathrm{E}-06$ & $1.48104 \mathrm{E}-06$ \\
3 & $7.01523 \mathrm{E}-03$ & $2.46902 \mathrm{E}-08$ & $1.02296 \mathrm{E}-08$ & $7.02506 \mathrm{E}-03$ & $2.48652 \mathrm{E}-08$ & $1.03021 \mathrm{E}-08$ \\
4 & $2.77876 \mathrm{E}-02$ & $1.30626 \mathrm{E}-07$ & $5.41212 \mathrm{E}-08$ & $2.77567 \mathrm{E}-02$ & $1.30480 \mathrm{E}-07$ & $5.40602 \mathrm{E}-08$ \\
\hline
\end{tabular}

Table 4.70. TCA-7, Pin-1 four-group Pu-239 cross sections

\begin{tabular}{cccccccc}
\hline Group & \multicolumn{3}{c}{ CENTRM cross sections $\left(\mathrm{cm}^{-1}\right)$} & \multicolumn{3}{c}{ NITAWL cross sections $\left(\mathrm{cm}^{-1}\right)$} \\
\hline & $\frac{\mathrm{G}_{\mathrm{a}}}{}$ & $\frac{\mathrm{G}_{\mathrm{f}}}{1.11889 \mathrm{E}-04}$ & $\frac{\mathrm{G}_{\mathrm{f}}}{4.49757 \mathrm{E}-03}$ & $4.81327 \mathrm{E}-04$ & $\frac{\mathrm{G}_{\mathrm{a}}}{5.11022 \mathrm{E}-04}$ & $\frac{\mathrm{G}_{\mathrm{f}}}{1.50238 \mathrm{E}-03}$ & $\frac{\mathrm{G}_{\mathrm{f}}}{4.81994 \mathrm{E}-04}$ \\
1 & $1.09768 \mathrm{E}-02$ & $1.79177 \mathrm{E}-02$ & $6.22185 \mathrm{E}-03$ & $1.09889 \mathrm{E}-02$ & $1.81187 \mathrm{E}-02$ & $6.29163 \mathrm{E}-03$ \\
3 & $3.46245 \mathrm{E}-02$ & $6.80175 \mathrm{E}-02$ & $2.36188 \mathrm{E}-02$ & $3.47959 \mathrm{E}-02$ & $6.83577 \mathrm{E}-02$ & $2.37369 \mathrm{E}-02$ \\
4 & $2.64817 \mathrm{E}-01$ & $5.37348 \mathrm{E}-01$ & $1.85848 \mathrm{E}-01$ & $2.63994 \mathrm{E}-01$ & $5.35812 \mathrm{E}-01$ & $1.85317 \mathrm{E}-01$ \\
\hline
\end{tabular}


Table 4.71. TCA-7, Pin-55 four-group fluxes

\begin{tabular}{ccc}
\hline Group & $\begin{array}{c}\text { CENTRM M } \\
\left(\mathrm{cm}^{-2}-\mathrm{s}^{-1}\right)\end{array}$ & $\begin{array}{c}\text { NITAWL M } \\
\left(\mathrm{cm}^{-2}-\mathrm{s}^{-1}\right)\end{array}$ \\
\hline 1 & $2.186 \mathrm{E}-04$ & $2.205 \mathrm{E}-04$ \\
2 & $7.342 \mathrm{E}-05$ & $7.405 \mathrm{E}-05$ \\
3 & $1.743 \mathrm{E}-05$ & $1.715 \mathrm{E}-05$ \\
4 & $9.388 \mathrm{E}-05$ & $9.404 \mathrm{E}-05$ \\
\hline
\end{tabular}

Table 4.72. TCA-7, Pin-55 four-group U-235 cross sections

\begin{tabular}{cccccccc}
\hline Group & \multicolumn{2}{c}{ CENTRM cross sections $\left(\mathrm{cm}^{-1}\right)$} & \multicolumn{3}{c}{ NITAWL cross sections $\left(\mathrm{cm}^{-1}\right)$} \\
\hline & $\frac{\mathrm{G}_{\mathrm{a}}}{}$ & $\frac{\mathrm{G}_{\mathrm{f}}}{1.46008 \mathrm{E}-04}$ & $\frac{3.28325 \mathrm{E}-04}{1.26835 \mathrm{E}-04}$ & $\frac{\mathrm{G}_{\mathrm{f}}}{1.45867 \mathrm{E}-04}$ & $\frac{\mathrm{G}_{\mathrm{f}}}{3.28061 \mathrm{E}-04}$ & $\frac{\mathrm{G}_{\mathrm{f}}}{1.26745 \mathrm{E}-04}$ \\
1 & $3.45407 \mathrm{E}-03$ & $5.28814 \mathrm{E}-03$ & $2.17020 \mathrm{E}-03$ & $3.44685 \mathrm{E}-03$ & $5.26736 \mathrm{E}-03$ & $2.16167 \mathrm{E}-03$ \\
3 & $5.22363 \mathrm{E}-03$ & $1.06689 \mathrm{E}-02$ & $4.37843 \mathrm{E}-03$ & $5.15115 \mathrm{E}-03$ & $1.05143 \mathrm{E}-02$ & $4.31500 \mathrm{E}-03$ \\
4 & $4.73673 \mathrm{E}-02$ & $9.85703 \mathrm{E}-02$ & $4.04524 \mathrm{E}-02$ & $4.74047 \mathrm{E}-02$ & $9.86425 \mathrm{E}-02$ & $4.04820 \mathrm{E}-02$ \\
\hline
\end{tabular}

Table 4.73. TCA-7, Pin-55 four-group U-238 cross sections

\begin{tabular}{cccccccc}
\hline Group & \multicolumn{3}{c}{ CENTRM cross sections $\left(\mathrm{cm}^{-1}\right)$} & \multicolumn{3}{c}{ NITAWL cross sections $\left(\mathrm{cm}^{-1}\right)$} \\
\hline & $\frac{\mathrm{G}_{\mathrm{a}}}{1}$ & $\frac{\mathrm{G}_{\mathrm{f}}}{7.42752 \mathrm{E}-03}$ & $\frac{\mathrm{G}_{\mathrm{f}}}{2.91693 \mathrm{E}-03}$ & $\frac{2.81011 \mathrm{E}-03}{4.41279 \mathrm{E}-03}$ & $\frac{\mathrm{G}_{\mathrm{f}}}{7.88612 \mathrm{E}-03}$ & $\frac{\mathrm{G}_{\mathrm{f}}}{2.79909 \mathrm{E}-03}$ \\
2 & $3.99843 \mathrm{E}-02$ & $3.82517 \mathrm{E}-06$ & $1.58476 \mathrm{E}-06$ & $3.88724 \mathrm{E}-02$ & $3.70637 \mathrm{E}-06$ & $1.53553 \mathrm{E}-06$ \\
3 & $7.01291 \mathrm{E}-03$ & $2.46832 \mathrm{E}-08$ & $1.02267 \mathrm{E}-08$ & $6.99207 \mathrm{E}-03$ & $2.45220 \mathrm{E}-08$ & $1.01599 \mathrm{E}-08$ \\
4 & $2.69759 \mathrm{E}-02$ & $1.26733 \mathrm{E}-07$ & $5.25080 \mathrm{E}-08$ & $2.69906 \mathrm{E}-02$ & $1.26799 \mathrm{E}-07$ & $5.25354 \mathrm{E}-08$ \\
\hline
\end{tabular}

Table 4.74. TCA-7, Pin-55 four-group Pu-239 cross sections

\begin{tabular}{cccccccc}
\hline Group & \multicolumn{3}{c}{ CENTRM cross sections $\left(\mathrm{cm}^{-1}\right)$} & \multicolumn{3}{c}{ NITAWL cross sections $\left(\mathrm{cm}^{-1}\right)$} \\
\hline & $\frac{\mathrm{G}_{\mathrm{a}}}{4}$ & $5.13362 \mathrm{E}-04$ & $\frac{\mathrm{G}_{\mathrm{f}}}{1.48414 \mathrm{E}-03}$ & $\frac{\mathrm{G}_{\mathrm{f}}}{4.78998 \mathrm{E}-04}$ & $\overline{5.13238 \mathrm{E}-04}$ & $\frac{\mathrm{G}_{\mathrm{f}}}{1.48384 \mathrm{E}-03}$ & $\frac{\mathrm{G}_{\mathrm{f}}}{4.78975 \mathrm{E}-04}$ \\
2 & $1.13505 \mathrm{E}-02$ & $1.86607 \mathrm{E}-02$ & $6.47983 \mathrm{E}-03$ & $1.09736 \mathrm{E}-02$ & $1.80388 \mathrm{E}-02$ & $6.26385 \mathrm{E}-03$ \\
3 & $3.48495 \mathrm{E}-02$ & $6.84159 \mathrm{E}-02$ & $2.37571 \mathrm{E}-02$ & $3.38470 \mathrm{E}-02$ & $6.65440 \mathrm{E}-02$ & $2.31071 \mathrm{E}-02$ \\
4 & $2.74694 \mathrm{E}-01$ & $5.50591 \mathrm{E}-01$ & $1.90475 \mathrm{E}-01$ & $2.75966 \mathrm{E}-01$ & $5.52786 \mathrm{E}-01$ & $1.91235 \mathrm{E}-01$ \\
\hline
\end{tabular}




\begin{tabular}{|c|c|c|c|c|c|c|c|c|c|}
\hline & & & & & & & & & $\begin{array}{l}7.058 \\
(0.42)\end{array}$ \\
\hline & \multirow[t]{7}{*}{$\begin{array}{l}0.163 \\
(0.75) \\
\end{array}$} & & & & & & & $\begin{array}{l}6.778 \\
(0.45)\end{array}$ & $\begin{array}{l}6.930 \\
(0.30)\end{array}$ \\
\hline & & & & & & & $\begin{array}{l}6.434 \\
(0.40)\end{array}$ & $\begin{array}{l}6.596 \\
(0.28)\end{array}$ & $\begin{array}{l}6.745 \\
(0.31) \\
\end{array}$ \\
\hline & & & & & & $\begin{array}{l}5.871 \\
(0.47)\end{array}$ & $\begin{array}{l}6.133 \\
(0.33)\end{array}$ & $\begin{array}{l}6.327 \\
(0.30)\end{array}$ & $\begin{array}{l}6.451 \\
(0.29)\end{array}$ \\
\hline & & & & & $\begin{array}{l}5.168 \\
(0.48)\end{array}$ & $\begin{array}{l}5.489 \\
(0.32)\end{array}$ & $\begin{array}{l}5.742 \\
(0.35)\end{array}$ & $\begin{array}{l}5.939 \\
(0.31)\end{array}$ & $\begin{array}{l}6.053 \\
(0.31)\end{array}$ \\
\hline & & & & $\begin{array}{l}4.377 \\
(0.49)\end{array}$ & $\begin{array}{l}4.767 \\
(0.33)\end{array}$ & $\begin{array}{l}5.039 \\
(0.34)\end{array}$ & $\begin{array}{l}5.336 \\
(0.33)\end{array}$ & $\begin{array}{l}5.465 \\
(0.33)\end{array}$ & $\begin{array}{l}5.519 \\
(0.32)\end{array}$ \\
\hline & & & $\begin{array}{l}3.520 \\
(0.56)\end{array}$ & $\begin{array}{l}3.936 \\
(0.40)\end{array}$ & $\begin{array}{l}4.294 \\
(0.38)\end{array}$ & $\begin{array}{l}4.552 \\
(0.35)\end{array}$ & $\begin{array}{l}4.754 \\
(0.36)\end{array}$ & $\begin{array}{l}4.920 \\
(0.32)\end{array}$ & $\begin{array}{l}4.979 \\
(0.34)\end{array}$ \\
\hline & & $\begin{array}{l}2.777 \\
(0.66) \\
\end{array}$ & $\begin{array}{l}3.119 \\
(0.45) \\
\end{array}$ & $\begin{array}{l}3.500 \\
(0.39) \\
\end{array}$ & $\begin{array}{l}3.787 \\
(0.45) \\
\end{array}$ & $\begin{array}{l}4.040 \\
(0.37) \\
\end{array}$ & $\begin{array}{l}4.186 \\
(0.35) \\
\end{array}$ & $\begin{array}{l}4.325 \\
(0.33) \\
\end{array}$ & $\begin{array}{r}4.397 \\
(0.36) \\
\end{array}$ \\
\hline & $\begin{array}{l}2.148 \\
(0.70)\end{array}$ & $\begin{array}{l}2.439 \\
(0.51)\end{array}$ & $\begin{array}{l}2.772 \\
(0.46)\end{array}$ & $\begin{array}{l}3.062 \\
(0.39)\end{array}$ & $\begin{array}{l}3.330 \\
(0.39)\end{array}$ & $\begin{array}{l}3.540 \\
(0.40)\end{array}$ & $\begin{array}{l}3.714 \\
(0.42)\end{array}$ & $\begin{array}{l}3.803 \\
(0.37)\end{array}$ & $\begin{array}{l}3.857 \\
(0.39)\end{array}$ \\
\hline $\begin{array}{l}2.037 \\
(0.75)\end{array}$ & $\begin{array}{l}2.140 \\
(0.50)\end{array}$ & $\begin{array}{c}2.445 \\
(0.49)\end{array}$ & $\begin{array}{l}2.777 \\
(0.46)\end{array}$ & $\begin{array}{c}3.109 \\
(0.42)\end{array}$ & $\begin{array}{l}3.342 \\
(0.42)\end{array}$ & $\begin{array}{c}3.553 \\
(0.38)\end{array}$ & $\begin{array}{c}3.728 \\
(0.41)\end{array}$ & $\begin{array}{c}3.851 \\
(0.40)\end{array}$ & $\begin{array}{l}3.923 \\
(0.39)\end{array}$ \\
\hline
\end{tabular}

Fig. 4.12a. Pin-power distribution for CENTRM benchmark TCA-8. Value in parentheses is percent standard deviation. 


\begin{tabular}{|c|c|c|c|c|c|c|c|c|c|}
\hline & & & & & & & & & $\begin{array}{l}7.087 \\
(0.46)\end{array}$ \\
\hline & \multirow[t]{7}{*}{$\begin{array}{l}0.162 \\
(0.72) \\
\end{array}$} & & & & & & & $\begin{array}{l}6.790 \\
(0.41)\end{array}$ & $\begin{array}{l}6.919 \\
(0.28)\end{array}$ \\
\hline & & & & & & & $\begin{array}{l}6.419 \\
(0.42)\end{array}$ & $\begin{array}{l}6.669 \\
(0.27)\end{array}$ & $\begin{array}{l}6.737 \\
(0.32)\end{array}$ \\
\hline & & & & & & $\begin{array}{l}5.900 \\
(0.43)\end{array}$ & $\begin{array}{l}6.151 \\
(0.30)\end{array}$ & $\begin{array}{l}6.328 \\
(0.27)\end{array}$ & $\begin{array}{l}6.378 \\
(0.33)\end{array}$ \\
\hline & & & & & $\begin{array}{l}5.179 \\
(0.50)\end{array}$ & $\begin{array}{l}5.512 \\
(0.31)\end{array}$ & $\begin{array}{l}5.799 \\
(0.30)\end{array}$ & $\begin{array}{l}5.942 \\
(0.31)\end{array}$ & $\begin{array}{l}6.021 \\
(0.31)\end{array}$ \\
\hline & & & & $\begin{array}{l}4.388 \\
(0.53)\end{array}$ & $\begin{array}{l}4.751 \\
(0.35)\end{array}$ & $\begin{array}{l}5.082 \\
(0.35)\end{array}$ & $\begin{array}{l}5.308 \\
(0.31)\end{array}$ & $\begin{array}{l}5.501 \\
(0.31)\end{array}$ & $\begin{array}{l}5.566 \\
(0.31)\end{array}$ \\
\hline & & & $\begin{array}{l}3.547 \\
(0.55)\end{array}$ & $\begin{array}{l}3.954 \\
(0.39)\end{array}$ & $\begin{array}{l}4.305 \\
(0.40)\end{array}$ & $\begin{array}{l}4.595 \\
(0.33)\end{array}$ & $\begin{array}{l}4.764 \\
(0.36)\end{array}$ & $\begin{array}{l}4.941 \\
(0.34)\end{array}$ & $\begin{array}{l}4.987 \\
(0.34)\end{array}$ \\
\hline & & $\begin{array}{r}2.717 \\
(0.67) \\
\end{array}$ & $\begin{array}{l}3.141 \\
(0.42) \\
\end{array}$ & $\begin{array}{l}3.469 \\
(0.43) \\
\end{array}$ & $\begin{array}{l}3.797 \\
(0.38) \\
\end{array}$ & $\begin{array}{l}4.026 \\
(0.36) \\
\end{array}$ & $\begin{array}{l}4.223 \\
(0.36) \\
\end{array}$ & $\begin{array}{l}4.326 \\
(0.36) \\
\end{array}$ & $\begin{array}{r}4.387 \\
(0.37) \\
\end{array}$ \\
\hline & $\begin{array}{l}2.140 \\
(0.71)\end{array}$ & $\begin{array}{l}2.434 \\
(0.47)\end{array}$ & $\begin{array}{l}2.753 \\
(0.43)\end{array}$ & $\begin{array}{l}3.061 \\
(0.44)\end{array}$ & $\begin{array}{l}3.313 \\
(0.40)\end{array}$ & $\begin{array}{l}3.540 \\
(0.40)\end{array}$ & $\begin{array}{l}3.695 \\
(0.40)\end{array}$ & $\begin{array}{l}3.832 \\
(0.42)\end{array}$ & $\begin{array}{l}3.886 \\
(0.38)\end{array}$ \\
\hline $\begin{array}{l}2.055 \\
(0.75)\end{array}$ & $\begin{array}{c}2.128 \\
(0.49)\end{array}$ & $\begin{array}{c}2.452 \\
(0.45)\end{array}$ & $\begin{array}{l}2.780 \\
(0.44)\end{array}$ & $\begin{array}{c}3.082 \\
(0.44)\end{array}$ & $\begin{array}{l}3.377 \\
(0.39)\end{array}$ & $\begin{array}{l}3.615 \\
(0.40)\end{array}$ & $\begin{array}{c}3.740 \\
(0.39)\end{array}$ & $\begin{array}{l}3.859 \\
(0.40)\end{array}$ & $\begin{array}{l}3.928 \\
(0.38)\end{array}$ \\
\hline
\end{tabular}

Fig. 4.12b. Pin-power distribution for NITAWL benchmark TCA-8. Value in parentheses is percent standard deviation. 
Table 4.75. Selected reaction rates for CENTRM case TCA-8

\begin{tabular}{|c|c|c|c|c|c|c|}
\hline Pin & Region & $\begin{array}{c}\mathrm{G}_{\mathrm{a}} \mathrm{M} \\
\left(\mathrm{cm}^{-3}-\mathrm{s}^{-1}\right)\end{array}$ & $\begin{array}{c}\mathcal{G}_{\mathrm{f}} \mathrm{M} \\
\left(\mathrm{cm}^{-3}-\mathrm{s}^{-1}\right)\end{array}$ & $\begin{array}{c}\mathrm{G}_{\mathrm{f}} \mathrm{M} \\
\left(\mathrm{cm}^{-3}-\mathrm{s}^{-1}\right)\end{array}$ & $\begin{array}{c}\mathrm{M} \\
\left(\mathrm{cm}-\mathrm{s}^{-1}\right)\end{array}$ & M/M \\
\hline \multirow{3}{*}{1} & Fuel & $1.219 \mathrm{E}-05$ & $2.033 \mathrm{E}-05$ & 7.204E-06 & $4.521 \mathrm{E}-03$ & 0.182 \\
\hline & Clad & $2.267 \mathrm{E}-07$ & 0.0 & 0.0 & $1.397 \mathrm{E}-03$ & 0.056 \\
\hline & Mod. & $6.904 \mathrm{E}-07$ & 0.0 & 0.0 & $1.890 \mathrm{E}-02$ & 0.762 \\
\hline \multirow{3}{*}{55} & Fuel & 4.454E-05 & 7.082E-05 & $2.507 \mathrm{E}-05$ & $2.182 \mathrm{E}-02$ & 0.182 \\
\hline & Clad & $8.569 \mathrm{E}-07$ & 0.0 & 0.0 & $6.833 \mathrm{E}-03$ & 0.057 \\
\hline & Mod. & $2.247 \mathrm{E}-06$ & 0.0 & 0.0 & $9.140 \mathrm{E}-02$ & 0.761 \\
\hline
\end{tabular}

Table 4.76. Selected reaction rates for NITAWL case TCA-8

\begin{tabular}{ccccccc}
\hline \multirow{2}{*}{ Pin } & Region & $\begin{array}{c}\mathrm{G}_{\mathrm{a}} \mathrm{M} \\
\left(\mathrm{cm}^{-3}-\mathrm{s}^{-1}\right)\end{array}$ & $\begin{array}{c}\mathrm{G}_{\mathrm{f}} \mathrm{M} \\
\left(\mathrm{cm}^{-3}-\mathrm{s}^{-1}\right)\end{array}$ & $\begin{array}{c}\mathrm{G}_{\mathrm{f}} \mathrm{M} \\
\left(\mathrm{cm}^{-3}-\mathrm{s}^{-1}\right)\end{array}$ & $\begin{array}{c}\mathrm{M} \\
\left({\left.\mathrm{cm}-\mathrm{s}^{-1}\right)}^{2}\right.\end{array}$ & $\mathrm{MM}_{\mathrm{t}}$ \\
\hline \multirow{2}{*}{1} & Fuel & $1.228 \mathrm{E}-05$ & $2.056 \mathrm{E}-05$ & $7.288 \mathrm{E}-06$ & $4.508 \mathrm{E}-03$ & 0.182 \\
& Clad & $2.273 \mathrm{E}-07$ & 0.0 & 0.0 & $1.396 \mathrm{E}-03$ & 0.056 \\
& Mod. & $6.917 \mathrm{E}-07$ & 0.0 & 0.0 & $1.890 \mathrm{E}-02$ & 0.762 \\
\hline \multirow{2}{*}{55} & Fuel & $4.442 \mathrm{E}-05$ & $7.058 \mathrm{E}-05$ & $2.499 \mathrm{E}-05$ & $2.172 \mathrm{E}-02$ & 0.182 \\
& Clad & $8.512 \mathrm{E}-07$ & 0.0 & 0.0 & $6.810 \mathrm{E}-03$ & 0.057 \\
& Mod. & $2.244 \mathrm{E}-06$ & 0.0 & 0.0 & $9.104 \mathrm{E}-02$ & 0.761 \\
\hline
\end{tabular}


Table 4.77. TCA-8, Pin-1 four-group fluxes

\begin{tabular}{ccc}
\hline Group & $\begin{array}{c}\text { CENTRM M } \\
\left(\mathrm{cm}^{-2}-\mathrm{s}^{-1}\right)\end{array}$ & $\begin{array}{c}\text { NITAWL M } \\
\left(\mathrm{cm}^{-2}-\mathrm{s}^{-1}\right)\end{array}$ \\
\hline 1 & $3.944 \mathrm{E}-05$ & $3.890 \mathrm{E}-05$ \\
2 & $1.103 \mathrm{E}-05$ & $1.116 \mathrm{E}-05$ \\
3 & $2.823 \mathrm{E}-06$ & $2.632 \mathrm{E}-06$ \\
4 & $2.727 \mathrm{E}-05$ & $2.765 \mathrm{E}-05$ \\
\hline
\end{tabular}

Table 4.78. TCA-8, Pin-1 four-group U-235 cross sections

\begin{tabular}{cccccccc}
\hline Group & \multicolumn{2}{c}{ CENTRM cross sections $\left(\mathrm{cm}^{-1}\right)$} & \multicolumn{3}{c}{ NITAWL cross sections $\left(\mathrm{cm}^{-1}\right)$} \\
\hline & $\frac{\mathrm{G}_{\mathrm{a}}}{1.42334 \mathrm{E}-04}$ & $\frac{\mathrm{G}_{\mathrm{f}}}{3.25312 \mathrm{E}-04}$ & $\frac{\mathrm{G}_{\mathrm{f}}}{1.24980 \mathrm{E}-04}$ & $\frac{\mathrm{G}_{\mathrm{a}}}{1.42088 \mathrm{E}-04}$ & $\frac{\mathrm{G}_{\mathrm{f}}}{3.25219 \mathrm{E}-04}$ & $\frac{\mathrm{G}_{\mathrm{f}}}{1.24828 \mathrm{E}-04}$ \\
1 & $3.53127 \mathrm{E}-03$ & $5.35172 \mathrm{E}-03$ & $2.19630 \mathrm{E}-03$ & $3.46204 \mathrm{E}-03$ & $5.26653 \mathrm{E}-03$ & $2.16133 \mathrm{E}-03$ \\
2 & $5.12848 \mathrm{E}-03$ & $1.04583 \mathrm{E}-02$ & $4.29199 \mathrm{E}-03$ & $5.21250 \mathrm{E}-03$ & $1.06698 \mathrm{E}-02$ & $4.37876 \mathrm{E}-03$ \\
3 & $4.88629 \mathrm{E}-02$ & $1.01736 \mathrm{E}-01$ & $4.17514 \mathrm{E}-02$ & $4.88872 \mathrm{E}-02$ & $1.01784 \mathrm{E}-01$ & $4.17714 \mathrm{E}-02$ \\
\hline
\end{tabular}

Table 4.79. TCA-8, Pin-1 four-group U-238 cross sections

\begin{tabular}{cccccccc}
\hline Group & \multicolumn{3}{c}{ CENTRM cross sections $\left(\mathrm{cm}^{-1}\right)$} & \multicolumn{3}{c}{ NITAWL cross sections $\left(\mathrm{cm}^{-1}\right)$} \\
\hline & $\frac{\mathrm{G}_{\mathrm{a}}}{4.53489 \mathrm{E}-03}$ & $\frac{\mathrm{G}_{\mathrm{f}}}{8.60396 \mathrm{E}-03}$ & $\frac{\mathrm{G}_{\mathrm{f}}}{3.05003 \mathrm{E}-03}$ & $\frac{\mathrm{G}_{\mathrm{a}}}{4.52758 \mathrm{E}-03}$ & $\frac{\mathrm{G}_{\mathrm{f}}}{8.62663 \mathrm{E}-03}$ & $\frac{\mathrm{G}_{\mathrm{f}}}{3.05064 \mathrm{E}-03}$ \\
1 & $4.11256 \mathrm{E}-02$ & $3.90524 \mathrm{E}-06$ & $1.61793 \mathrm{E}-06$ & $3.90452 \mathrm{E}-02$ & $3.64034 \mathrm{E}-06$ & $1.50818 \mathrm{E}-06$ \\
3 & $6.97793 \mathrm{E}-03$ & $2.43563 \mathrm{E}-08$ & $1.00913 \mathrm{E}-08$ & $7.01079 \mathrm{E}-03$ & $2.46993 \mathrm{E}-08$ & $1.02334 \mathrm{E}-08$ \\
4 & $2.77529 \mathrm{E}-02$ & $1.30460 \mathrm{E}-07$ & $5.40521 \mathrm{E}-08$ & $2.77661 \mathrm{E}-02$ & $1.30523 \mathrm{E}-07$ & $5.40785 \mathrm{E}-08$ \\
\hline
\end{tabular}

Table 4.80. TCA-8, Pin-1 four-group Pu-239 cross sections

\begin{tabular}{|c|c|c|c|c|c|c|}
\hline \multirow[t]{2}{*}{ Group } & \multicolumn{3}{|c|}{ CENTRM cross sections $\left(\mathrm{cm}^{-1}\right)$} & \multicolumn{3}{|c|}{ NITAWL cross sections $\left(\mathrm{cm}^{-1}\right)$} \\
\hline & $\mathrm{G}_{\mathrm{a}}$ & $<\mathrm{G}_{\mathrm{f}}$ & $\mathrm{G}_{\mathrm{f}}$ & $\mathrm{G}_{\mathrm{a}}$ & $<\mathrm{G}_{\mathrm{f}}$ & $\mathrm{G}_{\mathrm{f}}$ \\
\hline 1 & $5.11790 \mathrm{E}-04$ & $1.49952 \mathrm{E}-03$ & $4.81643 \mathrm{E}-04$ & $5.11429 \mathrm{E}-04$ & $1.50018 \mathrm{E}-03$ & 4.81447E-04 \\
\hline 2 & $1.15051 \mathrm{E}-02$ & $1.87178 \mathrm{E}-02$ & $6.49966 \mathrm{E}-03$ & $1.06573 \mathrm{E}-02$ & $1.75647 \mathrm{E}-02$ & 6.09924E-03 \\
\hline 3 & 3.42439E-02 & $6.71877 \mathrm{E}-02$ & $2.33306 \mathrm{E}-02$ & $3.42088 \mathrm{E}-02$ & $6.72576 \mathrm{E}-02$ & $2.33550 \mathrm{E}-02$ \\
\hline 4 & $2.65649 \mathrm{E}-01$ & $5.38621 \mathrm{E}-01$ & $1.86291 \mathrm{E}-01$ & 2.65522E-01 & $5.38441 \mathrm{E}-01$ & $1.86228 \mathrm{E}-01$ \\
\hline
\end{tabular}


Table 4.81. TCA-8, Pin-55 four-group fluxes

\begin{tabular}{ccc}
\hline Group & $\begin{array}{c}\text { CENTRM M } \\
\left(\mathrm{cm}^{-2}-\mathrm{s}^{-1}\right)\end{array}$ & $\begin{array}{c}\text { NITAWL M } \\
\left(\mathrm{cm}^{-2}-\mathrm{s}^{-1}\right)\end{array}$ \\
\hline 1 & $2.110 \mathrm{E}-04$ & $2.107 \mathrm{E}-04$ \\
2 & $7.037 \mathrm{E}-05$ & $6.962 \mathrm{E}-05$ \\
3 & $1.673 \mathrm{E}-05$ & $1.667 \mathrm{E}-05$ \\
4 & $9.070 \mathrm{E}-05$ & $9.010 \mathrm{E}-05$ \\
\hline
\end{tabular}

Table 4.82. TCA-8, Pin-55 four-group U-235 cross sections

\begin{tabular}{cccccccc}
\hline Group & \multicolumn{2}{c}{ CENTRM cross sections $\left(\mathrm{cm}^{-1}\right)$} & \multicolumn{3}{c}{ NITAWL cross sections $\left(\mathrm{cm}^{-1}\right)$} \\
\hline & $\frac{\mathrm{G}_{\mathrm{a}}}{1.45480 \mathrm{E}-04}$ & $\frac{\mathrm{G}_{\mathrm{f}}}{3.27674 \mathrm{E}-04}$ & $\frac{\mathrm{G}_{\mathrm{f}}}{1.26526 \mathrm{E}-04}$ & $\frac{\mathrm{G}_{\mathrm{a}}}{1.45435 \mathrm{E}-04}$ & $\frac{\mathrm{G}_{\mathrm{f}}}{3.27709 \mathrm{E}-04}$ & $\frac{\mathrm{G}_{\mathrm{f}}}{1.26510 \mathrm{E}-04}$ \\
1 & $3.39048 \mathrm{E}-03$ & $5.20276 \mathrm{E}-03$ & $2.13517 \mathrm{E}-03$ & $3.47111 \mathrm{E}-03$ & $5.30325 \mathrm{E}-03$ & $2.17640 \mathrm{E}-03$ \\
2 & $5.13229 \mathrm{E}-03$ & $1.04562 \mathrm{E}-02$ & $4.29112 \mathrm{E}-03$ & $5.19965 \mathrm{E}-03$ & $1.06013 \mathrm{E}-02$ & $4.35069 \mathrm{E}-03$ \\
3 & $4.74630 \mathrm{E}-02$ & $9.87671 \mathrm{E}-02$ & $4.05332 \mathrm{E}-02$ & $4.77188 \mathrm{E}-02$ & $9.92999 \mathrm{E}-02$ & $4.07518 \mathrm{E}-02$ \\
\hline
\end{tabular}

Table 4.83. TCA-8, Pin-55 four-group U-238 cross sections

\begin{tabular}{cccccccc}
\hline Group & \multicolumn{3}{c}{ CENTRM cross sections $\left(\mathrm{cm}^{-1}\right)$} & \multicolumn{3}{c}{ NITAWL cross sections $\left(\mathrm{cm}^{-1}\right)$} \\
\hline & $\frac{\mathrm{G}_{\mathrm{a}}}{1}$ & $\frac{\mathrm{G}_{\mathrm{f}}}{7.41327 \mathrm{E}-03}$ & $\frac{\mathrm{G}_{\mathrm{f}}}{7.93014 \mathrm{E}-03}$ & $\frac{2.81274 \mathrm{E}-03}{4.42132 \mathrm{E}-03}$ & $\frac{\mathrm{G}_{\mathrm{f}}}{7.95819 \mathrm{E}-03}$ & $\frac{\mathrm{G}_{\mathrm{f}}}{2.82199 \mathrm{E}-03}$ \\
2 & $3.87344 \mathrm{E}-02$ & $3.80772 \mathrm{E}-06$ & $1.57752 \mathrm{E}-06$ & $3.76439 \mathrm{E}-02$ & $3.63710 \mathrm{E}-06$ & $1.50684 \mathrm{E}-06$ \\
3 & $6.98380 \mathrm{E}-03$ & $2.43899 \mathrm{E}-08$ & $1.01051 \mathrm{E}-08$ & $7.00174 \mathrm{E}-03$ & $2.45717 \mathrm{E}-08$ & $1.01805 \mathrm{E}-08$ \\
4 & $2.70204 \mathrm{E}-02$ & $1.26942 \mathrm{E}-07$ & $5.25946 \mathrm{E}-08$ & $2.71446 \mathrm{E}-02$ & $1.27535 \mathrm{E}-07$ & $5.28402 \mathrm{E}-08$ \\
\hline
\end{tabular}

Table 4.84. TCA-8, Pin-55 four-group Pu-239 cross sections

\begin{tabular}{|c|c|c|c|c|c|c|}
\hline \multirow[t]{2}{*}{ Group } & \multicolumn{3}{|c|}{ CENTRM cross sections $\left(\mathrm{cm}^{-1}\right)$} & \multicolumn{3}{|c|}{ NITAWL cross sections $\left(\mathrm{cm}^{-1}\right)$} \\
\hline & $\mathrm{G}_{\mathrm{a}}$ & $\mathrm{G}_{\mathrm{f}}$ & $\mathrm{G}_{\mathrm{f}}$ & $\mathrm{G}_{\mathrm{a}-}$ & $<\mathrm{G}_{\mathrm{f}}$ & $\mathrm{G}_{\mathrm{f}}$ \\
\hline 1 & $5 . \overline{12787 \mathrm{E}-04}$ & $\overline{1.48453 \mathrm{E}-03}$ & $\overline{4.78981 \mathrm{E}-04}$ & $5.12848 \mathrm{E}-04$ & $\overline{1.48514 \mathrm{E}-03}$ & $\overline{4.79084 \mathrm{E}-04}$ \\
\hline 2 & 1.11398E-02 & $1.83762 \mathrm{E}-02$ & 6.38104E-03 & $1.10696 \mathrm{E}-02$ & $1.81030 \mathrm{E}-02$ & $6.28615 \mathrm{E}-03$ \\
\hline 3 & $3.39692 \mathrm{E}-02$ & $6.67376 \mathrm{E}-02$ & 2.31743E-02 & $3.43528 \mathrm{E}-02$ & $6.74775 \mathrm{E}-02$ & 2.34313E-02 \\
\hline 4 & $2.75637 \mathrm{E}-01$ & $5.52375 \mathrm{E}-01$ & $1.91090 \mathrm{E}-01$ & $2.76264 \mathrm{E}-01$ & $5.53948 \mathrm{E}-01$ & 1.91632E-01 \\
\hline
\end{tabular}




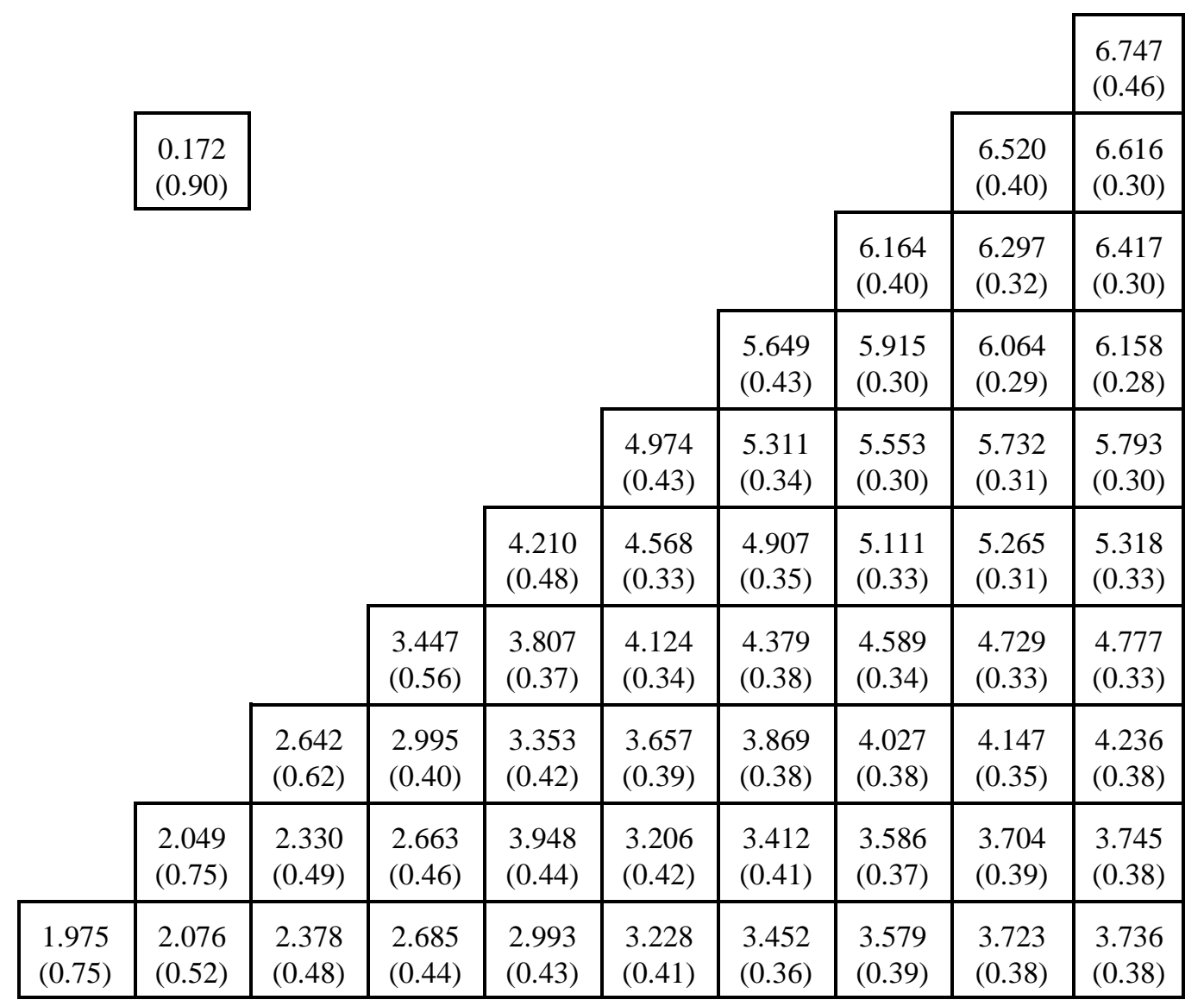

Fig. 4.13a. Pin-power distribution for CENTRM benchmark TCA-9. Value in parentheses is percent standard deviation. 


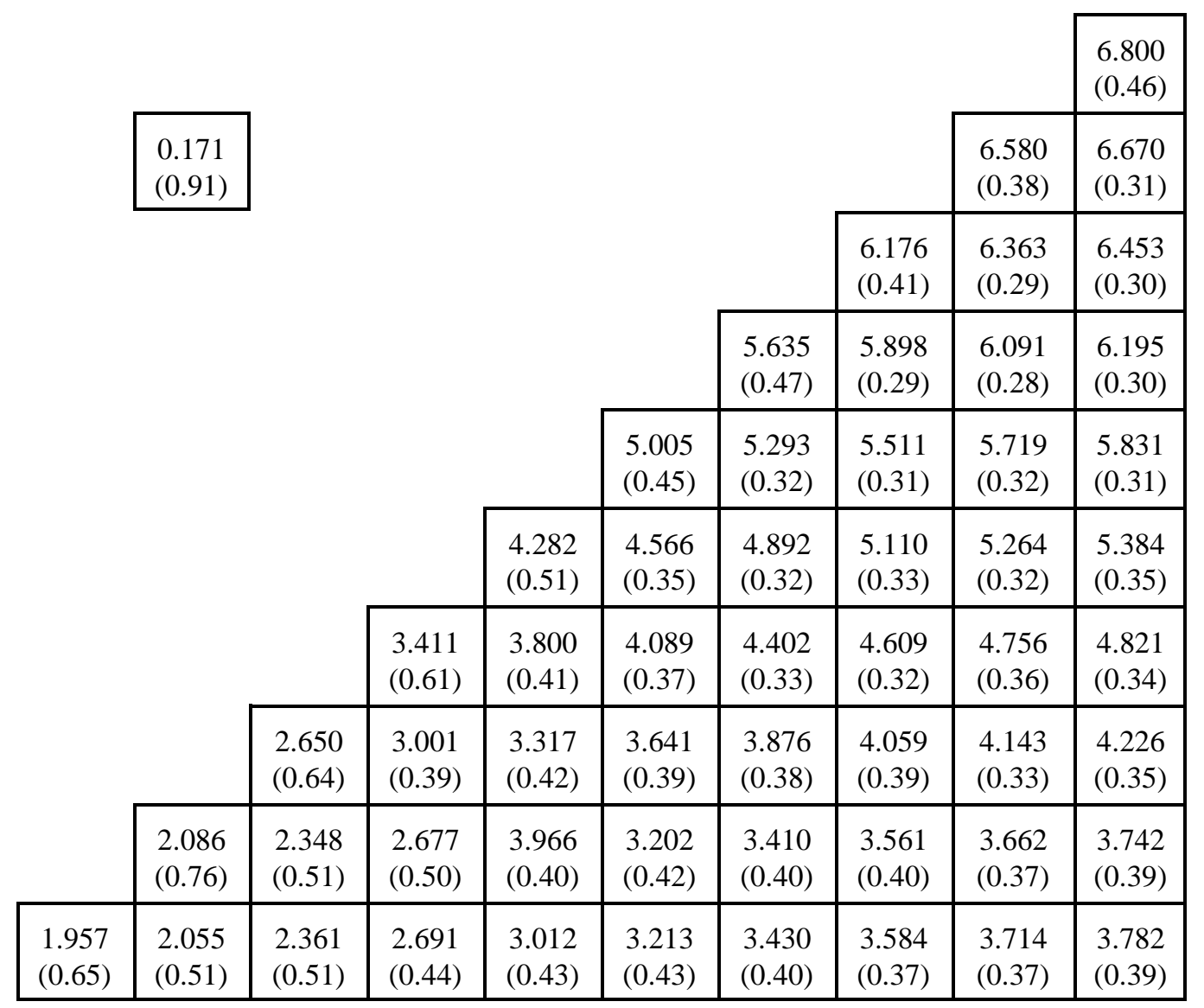

Fig. 4.13b. Pin-power distribution for NITAWL benchmark TCA-9. Value in parentheses is percent standard deviation. 
Table 4.85. Selected reaction rates for CENTRM case TCA-9

\begin{tabular}{|c|c|c|c|c|c|c|}
\hline Pin & Region & $\begin{array}{c}\mathrm{G}_{\mathrm{a}} \mathrm{M} \\
\left(\mathrm{cm}^{-3}-\mathrm{s}^{-1}\right)\end{array}$ & $\begin{array}{c}\mathcal{G}_{\mathrm{f}} \mathrm{M} \\
\left(\mathrm{cm}^{-3}-\mathrm{s}^{-1}\right)\end{array}$ & $\begin{array}{c}\mathrm{G}_{\mathrm{f}} \mathrm{M} \\
\left(\mathrm{cm}^{-3}-\mathrm{s}^{-1}\right)\end{array}$ & $\begin{array}{c}\mathrm{M} \\
\left(\mathrm{cm}-\mathrm{s}^{-1}\right)\end{array}$ & M/M \\
\hline \multirow{3}{*}{1} & Fuel & $1.197 \mathrm{E}-05$ & $1.995 \mathrm{E}-05$ & 7.072E-06 & 4.613E-03 & 0.183 \\
\hline & Clad & $2.247 \mathrm{E}-07$ & 0.0 & 0.0 & $1.425 \mathrm{E}-03$ & 0.056 \\
\hline & Mod. & $6.729 \mathrm{E}-07$ & 0.0 & 0.0 & $1.919 \mathrm{E}-02$ & 0.761 \\
\hline \multirow{3}{*}{55} & Fuel & $4.255 \mathrm{E}-05$ & $6.730 \mathrm{E}-05$ & $2.383 \mathrm{E}-05$ & $2.168 \mathrm{E}-02$ & 0.181 \\
\hline & Clad & $8.192 \mathrm{E}-07$ & 0.0 & 0.0 & $6.794 \mathrm{E}-03$ & 0.057 \\
\hline & Mod. & $2.155 \mathrm{E}-06$ & 0.0 & 0.0 & $9.111 \mathrm{E}-02$ & 0.762 \\
\hline
\end{tabular}

Table 4.86. Selected reaction rates for NITAWL case TCA-9

\begin{tabular}{ccccccc}
\hline Pin & Region & $\begin{array}{c}\mathrm{G}_{\mathrm{a}} \mathrm{M} \\
\left(\mathrm{cm}^{-3}-\mathrm{s}^{-1}\right)\end{array}$ & $\begin{array}{c}\mathrm{G}_{\mathrm{f}} \mathrm{M} \\
\left(\mathrm{cm}^{-3}-\mathrm{s}^{-1}\right)\end{array}$ & $\begin{array}{c}\mathrm{G}_{\mathrm{f}} \mathrm{M} \\
\left(\mathrm{cm}^{-3}-\mathrm{s}^{-1}\right)\end{array}$ & $\begin{array}{c}\mathrm{M} \\
\left(\mathrm{cm} \mathrm{-} \mathrm{s}^{-1}\right)\end{array}$ & $\mathrm{MM}_{\mathrm{t}}$ \\
\hline \multirow{2}{*}{1} & Fuel & $1.173 \mathrm{E}-05$ & $1.955 \mathrm{E}-05$ & $6.931 \mathrm{E}-06$ & $4.554 \mathrm{E}-03$ & 0.182 \\
& Clad & $2.206 \mathrm{E}-07$ & 0.0 & 0.0 & $1.420 \mathrm{E}-03$ & 0.056 \\
& Mod. & $6.680 \mathrm{E}-07$ & 0.0 & 0.0 & $1.910 \mathrm{E}-02$ & 0.762 \\
\hline \multirow{2}{*}{55} & Fuel & $4.279 \mathrm{E}-05$ & $6.771 \mathrm{E}-05$ & $2.398 \mathrm{E}-05$ & $2.188 \mathrm{E}-02$ & 0.182 \\
& Clad & $8.213 \mathrm{E}-07$ & 0.0 & 0.0 & $6.844 \mathrm{E}-03$ & 0.057 \\
& Mod. & $2.172 \mathrm{E}-06$ & 0.0 & 0.0 & $9.168 \mathrm{E}-02$ & 0.761 \\
\hline
\end{tabular}


Table 4.87. TCA-9, Pin-1 four-group fluxes

\begin{tabular}{ccc}
\hline Group & $\begin{array}{c}\text { CENTRM M } \\
\left(\mathrm{cm}^{-2}-\mathrm{s}^{-1}\right)\end{array}$ & $\begin{array}{c}\text { NITAWL M } \\
\left(\mathrm{cm}^{-2}-\mathrm{s}^{-1}\right)\end{array}$ \\
\hline 1 & $3.851 \mathrm{E}-05$ & $3.808 \mathrm{E}-05$ \\
2 & $1.088 \mathrm{E}-05$ & $1.068 \mathrm{E}-05$ \\
3 & $2.665 \mathrm{E}-06$ & $2.690 \mathrm{E}-06$ \\
4 & $2.684 \mathrm{E}-05$ & $2.644 \mathrm{E}-05$ \\
\hline
\end{tabular}

Table 4.88. TCA-9, Pin-1 four-group U-235 cross sections

\begin{tabular}{cccccccc}
\hline Group & \multicolumn{2}{c}{ CENTRM cross sections $\left(\mathrm{cm}^{-1}\right)$} & \multicolumn{3}{c}{ NITAWL cross sections $\left(\mathrm{cm}^{-1}\right)$} \\
\hline & $\frac{\mathrm{G}_{\mathrm{a}}}{1.42657 \mathrm{E}-04}$ & $\frac{\mathrm{G}_{\mathrm{f}}}{3.25414 \mathrm{E}-04}$ & $\frac{\mathrm{G}_{\mathrm{f}}}{1.25101 \mathrm{E}-04}$ & $\frac{\mathrm{G}_{\mathrm{a}}}{1.41875 \mathrm{E}-04}$ & $\frac{\mathrm{G}_{\mathrm{f}}}{3.24320 \mathrm{E}-04}$ & $\frac{\mathrm{G}_{\mathrm{f}}}{1.24670 \mathrm{E}-04}$ \\
1 & $3.42835 \mathrm{E}-03$ & $5.22619 \mathrm{E}-03$ & $2.14479 \mathrm{E}-03$ & $3.51588 \mathrm{E}-03$ & $5.33745 \mathrm{E}-03$ & $2.19045 \mathrm{E}-03$ \\
2 & $5.02206 \mathrm{E}-03$ & $1.01901 \mathrm{E}-02$ & $4.18195 \mathrm{E}-03$ & $5.22963 \mathrm{E}-03$ & $1.07319 \mathrm{E}-02$ & $4.40428 \mathrm{E}-03$ \\
4 & $4.91554 \mathrm{E}-02$ & $1.02344 \mathrm{E}-01$ & $4.20007 \mathrm{E}-02$ & $4.86914 \mathrm{E}-02$ & $1.01377 \mathrm{E}-01$ & $4.16040 \mathrm{E}-02$ \\
\hline
\end{tabular}

Table 4.89. TCA-9, Pin-1 four-group U-238 cross sections

\begin{tabular}{cccccccc}
\hline Group & \multicolumn{2}{c}{ CENTRM cross sections $\left(\mathrm{cm}^{-1}\right)$} & \multicolumn{3}{c}{ NITAWL cross sections $\left(\mathrm{cm}^{-1}\right)$} \\
\hline & $\frac{\mathrm{G}_{\mathrm{a}}}{4.50192 \mathrm{E}-03}$ & $\frac{\mathrm{G}_{\mathrm{f}}}{8.46604 \mathrm{E}-03}$ & $\frac{\mathrm{G}_{\mathrm{f}}}{2.99920 \mathrm{E}-03}$ & $\frac{\mathrm{G}_{\mathrm{a}}}{4.50218 \mathrm{E}-03}$ & $\frac{\mathrm{G}_{\mathrm{f}}}{8.53582 \mathrm{E}-03}$ & $\frac{\mathrm{G}_{\mathrm{f}}}{3.03046 \mathrm{E}-03}$ \\
1 & $4.14411 \mathrm{E}-02$ & $3.76574 \mathrm{E}-06$ & $1.56013 \mathrm{E}-06$ & $3.83075 \mathrm{E}-02$ & $3.58055 \mathrm{E}-06$ & $1.48341 \mathrm{E}-06$ \\
2 & $6.94867 \mathrm{E}-03$ & $2.40724 \mathrm{E}-08$ & $9.97362 \mathrm{E}-09$ & $7.02602 \mathrm{E}-03$ & $2.48603 \mathrm{E}-08$ & $1.03001 \mathrm{E}-08$ \\
3 & $2.78966 \mathrm{E}-02$ & $1.31143 \mathrm{E}-07$ & $5.43353 \mathrm{E}-08$ & $2.76690 \mathrm{E}-02$ & $1.30061 \mathrm{E}-07$ & $5.38869 \mathrm{E}-08$ \\
\hline
\end{tabular}

Table 4.90 TCA-9, Pin-1 four-group Pu-239 cross sections

\begin{tabular}{|c|c|c|c|c|c|c|}
\hline \multirow[t]{2}{*}{ Group } & \multicolumn{3}{|c|}{ CENTRM cross sections $\left(\mathrm{cm}^{-1}\right)$} & \multicolumn{3}{|c|}{ NITAWL cross sections $\left(\mathrm{cm}^{-1}\right)$} \\
\hline & $\mathrm{G}_{\mathrm{a}}$ & $<\mathrm{G}_{\mathrm{f}}$ & $\mathrm{G}_{\mathrm{f}}$ & $\mathrm{G}_{\mathrm{a}}$ & $<\mathrm{G}_{f}$ & $\mathrm{G}_{\mathrm{f}}$ \\
\hline 1 & $5 . \overline{11763 \mathrm{E}-04}$ & $1.49708 \mathrm{E}-03$ & $\overline{4.81127 \mathrm{E}-04}$ & $\overline{5.10995 \mathrm{E}-04}$ & $1.49750 \mathrm{E}-03$ & $4.81258 \mathrm{E}-04$ \\
\hline 2 & 1.10924E-02 & $1.84326 \mathrm{E}-02$ & $6.40062 \mathrm{E}-03$ & 1.10329E-02 & 1.80993E-02 & $6.28488 \mathrm{E}-03$ \\
\hline 3 & $3.20870 \mathrm{E}-02$ & $6.32248 \mathrm{E}-02$ & $2.19544 \mathrm{E}-02$ & $3.47840 \mathrm{E}-02$ & $6.83283 \mathrm{E}-02$ & 2.37267E-02 \\
\hline 4 & $2.66228 \mathrm{E}-01$ & $5.40183 \mathrm{E}-01$ & $1.86826 \mathrm{E}-01$ & $2.64971 \mathrm{E}-01$ & 5.37118E-01 & $1.85773 \mathrm{E}-01$ \\
\hline
\end{tabular}


Table 4.91. TCA-9, Pin-55 four-group fluxes

\begin{tabular}{ccc}
\hline Group & $\begin{array}{c}\text { CENTRM M } \\
\left(\mathrm{cm}^{-2}-\mathrm{s}^{-1}\right)\end{array}$ & $\begin{array}{c}\text { NITAWL M } \\
\left(\mathrm{cm}^{-2}-\mathrm{s}^{-1}\right)\end{array}$ \\
\hline 1 & $2.010 \mathrm{E}-04$ & $2.034 \mathrm{E}-04$ \\
2 & $6.739 \mathrm{E}-05$ & $6.769 \mathrm{E}-05$ \\
3 & $1.576 \mathrm{E}-05$ & $1.591 \mathrm{E}-05$ \\
4 & $8.667 \mathrm{E}-05$ & $8.728 \mathrm{E}-05$ \\
\hline
\end{tabular}

Table 4.92. TCA-9, Pin-55 four-group U-235 cross sections

\begin{tabular}{cccccccc}
\hline Group & \multicolumn{3}{c}{ CENTRM cross sections $\left(\mathrm{cm}^{-1}\right)$} & \multicolumn{3}{c}{ NITAWL cross sections $\left(\mathrm{cm}^{-1}\right)$} \\
\hline & $\frac{\mathrm{G}_{\mathrm{a}}}{1.45454 \mathrm{E}-04}$ & $\frac{\mathrm{G}_{\mathrm{f}}}{3.27423 \mathrm{E}-04}$ & $\frac{\mathrm{G}_{\mathrm{f}}}{1.26486 \mathrm{E}-04}$ & $\frac{\mathrm{G}_{\mathrm{a}}}{1.45607 \mathrm{E}-04}$ & $\frac{\mathrm{G}_{\mathrm{f}}}{3.27771 \mathrm{E}-04}$ & $\frac{\mathrm{G}_{\mathrm{f}}}{1.26597 \mathrm{E}-04}$ \\
1 & $3.41193 \mathrm{E}-03$ & $5.23067 \mathrm{E}-03$ & $2.14662 \mathrm{E}-03$ & $3.48574 \mathrm{E}-03$ & $5.30232 \mathrm{E}-03$ & $2.17602 \mathrm{E}-03$ \\
2 & $5.17154 \mathrm{E}-03$ & $1.05678 \mathrm{E}-02$ & $4.33692 \mathrm{E}-03$ & $5.13163 \mathrm{E}-03$ & $1.04658 \mathrm{E}-02$ & $4.29508 \mathrm{E}-03$ \\
3 & $4.74074 \mathrm{E}-02$ & $9.86494 \mathrm{E}-02$ & $4.04848 \mathrm{E}-02$ & $4.74735 \mathrm{E}-02$ & $9.87892 \mathrm{E}-02$ & $4.05423 \mathrm{E}-02$ \\
\hline
\end{tabular}

Table 4.93. TCA-9, Pin-55 four-group U-238 cross sections

\begin{tabular}{|c|c|c|c|c|c|c|}
\hline \multirow[t]{2}{*}{ Group } & \multicolumn{3}{|c|}{ CENTRM cross sections $\left(\mathrm{cm}^{-1}\right)$} & \multicolumn{3}{|c|}{ NITAWL cross sections $\left(\mathrm{cm}^{-1}\right)$} \\
\hline & $\mathrm{G}_{\mathrm{a}}$ & $\mathrm{G}_{\mathrm{f}}$ & $\mathrm{G}_{\mathrm{f}}$ & $\mathrm{G}_{\mathrm{a}}$ & $\mathrm{G}_{\mathrm{f}}$ & $\mathrm{G}_{\mathrm{f}}$ \\
\hline 1 & $4.40327 \mathrm{E}-03$ & $7.89249 \mathrm{E}-03$ & $2.80210 \mathrm{E}-03$ & $4.40840 \mathrm{E}-03$ & $7.90103 \mathrm{E}-03$ & $2.80352 \mathrm{E}-03$ \\
\hline 2 & 3.82984E-02 & $3.87376 \mathrm{E}-06$ & $1.60488 \mathrm{E}-06$ & 3.80297E-02 & 3.68119E-06 & $1.52510 \mathrm{E}-06$ \\
\hline 3 & 6.99689E-03 & $2.45553 \mathrm{E}-08$ & $1.01737 \mathrm{E}-08$ & $6.98259 \mathrm{E}-03$ & 2.44124E-08 & $1.01145 \mathrm{E}-08$ \\
\hline 4 & 2.69919E-02 & $1.26807 \mathrm{E}-07$ & $5.25386 \mathrm{E}-08$ & 2.70262E-02 & $1.26972 \mathrm{E}-07$ & 5.26067E-08 \\
\hline
\end{tabular}

Table 4.94. TCA-9, Pin-55 four-group Pu-239 cross sections

\begin{tabular}{cccccccc}
\hline Group & \multicolumn{2}{c}{ CENTRM cross sections $\left(\mathrm{cm}^{-1}\right)$} & \multicolumn{3}{c}{ NITAWL cross sections $\left(\mathrm{cm}^{-1}\right)$} \\
\hline & $\frac{\mathrm{G}_{\mathrm{a}}}{5.12642 \mathrm{E}-04}$ & $\frac{\mathrm{G}_{\mathrm{f}}}{1.48335 \mathrm{E}-03}$ & $\frac{\mathrm{G}_{\mathrm{f}}}{4.78804 \mathrm{E}-04}$ & $\frac{\mathrm{G}_{\mathrm{a}}}{5.12945 \mathrm{E}-04}$ & $\frac{\mathrm{G}_{\mathrm{f}}}{1.48419 \mathrm{E}-03}$ & $\frac{\mathrm{G}_{\mathrm{f}}}{4.78995 \mathrm{E}-04}$ \\
1 & $1.10458 \mathrm{E}-02$ & $1.82405 \mathrm{E}-02$ & $6.33394 \mathrm{E}-03$ & $1.08837 \mathrm{E}-02$ & $1.78922 \mathrm{E}-02$ & $6.21299 \mathrm{E}-03$ \\
3 & $3.38276 \mathrm{E}-02$ & $6.65209 \mathrm{E}-02$ & $2.30991 \mathrm{E}-02$ & $3.35685 \mathrm{E}-02$ & $6.60145 \mathrm{E}-02$ & $2.29233 \mathrm{E}-02$ \\
4 & $2.75620 \mathrm{E}-01$ & $5.52218 \mathrm{E}-01$ & $1.91037 \mathrm{E}-01$ & $2.75080 \mathrm{E}-01$ & $5.51449 \mathrm{E}-01$ & $1.90770 \mathrm{E}-01$ \\
\hline
\end{tabular}




\begin{tabular}{|c|c|c|c|c|c|c|c|c|c|c|}
\hline & & & & & & & & & & $\begin{array}{l}6.856 \\
(0.85)\end{array}$ \\
\hline & \multirow[t]{8}{*}{$\begin{array}{l}0.141 \\
(0.76)\end{array}$} & & & & & & & & $\begin{array}{l}6.768 \\
(0.41)\end{array}$ & $\begin{array}{l}6.823 \\
(0.42)\end{array}$ \\
\hline & & & & & & & & $\begin{array}{l}6.535 \\
(0.43)\end{array}$ & $\begin{array}{l}6.644 \\
(0.30)\end{array}$ & $\begin{array}{l}6.692 \\
(0.44)\end{array}$ \\
\hline & & & & & & & $\begin{array}{l}6.073 \\
(0.44)\end{array}$ & $\begin{array}{l}6.278 \\
(0.31)\end{array}$ & $\begin{array}{l}6.452 \\
(0.32)\end{array}$ & $\begin{array}{l}6.462 \\
(0.48)\end{array}$ \\
\hline & & & & & & $\begin{array}{l}5.443 \\
(0.46)\end{array}$ & $\begin{array}{l}5.760 \\
(0.35)\end{array}$ & $\begin{array}{l}5.984 \\
(0.30) \\
\end{array}$ & $\begin{array}{l}6.074 \\
(0.35)\end{array}$ & $\begin{array}{l}6.161 \\
(0.44)\end{array}$ \\
\hline & & & & & $\begin{array}{l}4.729 \\
(0.48)\end{array}$ & $\begin{array}{l}5.080 \\
(0.34)\end{array}$ & $\begin{array}{l}5.339 \\
(0.33)\end{array}$ & $\begin{array}{l}5.561 \\
(0.32)\end{array}$ & $\begin{array}{l}5.648 \\
(0.33)\end{array}$ & $\begin{array}{l}5.659 \\
(0.48)\end{array}$ \\
\hline & & & & $\begin{array}{c}3.888 \\
(0.57)\end{array}$ & $\begin{array}{l}4.322 \\
(0.37)\end{array}$ & $\begin{array}{l}4.641 \\
(0.36)\end{array}$ & $\begin{array}{l}4.858 \\
(0.32)\end{array}$ & $\begin{array}{l}5.062 \\
(0.33)\end{array}$ & $\begin{array}{l}5.192 \\
(0.34)\end{array}$ & $\begin{array}{l}5.200 \\
(0.49)\end{array}$ \\
\hline & & & $\begin{array}{l}3.115 \\
(0.64)\end{array}$ & $\begin{array}{l}3.500 \\
(0.43)\end{array}$ & $\begin{array}{l}3.840 \\
(0.39)\end{array}$ & $\begin{array}{l}4.093 \\
(0.37)\end{array}$ & $\begin{array}{l}4.339 \\
(0.38)\end{array}$ & $\begin{array}{l}4.483 \\
(0.39)\end{array}$ & $\begin{array}{l}4.605 \\
(0.39)\end{array}$ & $\begin{array}{l}4.604 \\
(0.53)\end{array}$ \\
\hline & & $\begin{array}{l}2.358 \\
(0.72)\end{array}$ & $\begin{array}{l}2.681 \\
(0.47)\end{array}$ & $\begin{array}{l}3.033 \\
(0.43)\end{array}$ & $\begin{array}{l}3.323 \\
(0.38)\end{array}$ & $\begin{array}{l}3.532 \\
(0.43)\end{array}$ & $\begin{array}{l}3.760 \\
(0.40)\end{array}$ & $\begin{array}{l}3.875 \\
(0.42)\end{array}$ & $\begin{array}{l}3.955 \\
(0.37)\end{array}$ & $\begin{array}{l}3.936 \\
(0.55)\end{array}$ \\
\hline & $\begin{array}{l}1.684 \\
(0.85)\end{array}$ & $\begin{array}{l}1.983 \\
(0.56)\end{array}$ & $\begin{array}{l}2.291 \\
(0.51)\end{array}$ & $\begin{array}{l}2.553 \\
(0.46)\end{array}$ & $\begin{array}{l}2.823 \\
(0.46)\end{array}$ & $\begin{array}{l}3.029 \\
(0.41)\end{array}$ & $\begin{array}{l}3.208 \\
(0.45)\end{array}$ & $\begin{array}{c}3.286 \\
(0.41)\end{array}$ & $\begin{array}{c}3.384 \\
(0.39)\end{array}$ & $\begin{array}{c}3.418 \\
(0.59)\end{array}$ \\
\hline $\begin{array}{l}1.428 \\
(0.77)\end{array}$ & $\begin{array}{c}1.569 \\
(0.61)\end{array}$ & $\begin{array}{l}1.859 \\
(0.57)\end{array}$ & $\begin{array}{l}2.158 \\
(0.49)\end{array}$ & $\begin{array}{l}2.398 \\
(0.51)\end{array}$ & $\begin{array}{l}2.643 \\
(0.49)\end{array}$ & $\begin{array}{l}2.831 \\
(0.45)\end{array}$ & $\begin{array}{l}2.995 \\
(0.47)\end{array}$ & $\begin{array}{l}3.099 \\
(0.42)\end{array}$ & $\begin{array}{l}3.141 \\
(0.43)\end{array}$ & $\begin{array}{l}3.185 \\
(0.58)\end{array}$ \\
\hline
\end{tabular}

Fig. 4.14a. Pin-power distribution for CENTRM benchmark TCA-10. Value in parentheses is percent standard deviation. 


\begin{tabular}{|c|c|c|c|c|c|c|c|c|c|c|}
\hline & & & & & & & & & & $\begin{array}{l}6.908 \\
(0.85)\end{array}$ \\
\hline & \multirow[t]{8}{*}{$\begin{array}{l}0.144 \\
(0.76) \\
\end{array}$} & & & & & & & & $\begin{array}{l}6.810 \\
(0.41)\end{array}$ & $\begin{array}{l}6.858 \\
(0.43)\end{array}$ \\
\hline & & & & & & & & $\begin{array}{l}6.535 \\
(0.42)\end{array}$ & $\begin{array}{l}6.640 \\
(0.31)\end{array}$ & $\begin{array}{l}6.685 \\
(0.43)\end{array}$ \\
\hline & & & & & & & $\begin{array}{l}6.057 \\
(0.47)\end{array}$ & $\begin{array}{l}6.279 \\
(0.33)\end{array}$ & $\begin{array}{l}6.387 \\
(0.31)\end{array}$ & $\begin{array}{r}6.470 \\
(0.43)\end{array}$ \\
\hline & & & & & & $\begin{array}{l}5.486 \\
(0.45)\end{array}$ & $\begin{array}{l}5.776 \\
(0.31)\end{array}$ & $\begin{array}{c}6.008 \\
(0.31)\end{array}$ & $\begin{array}{c}6.085 \\
(0.33)\end{array}$ & $\begin{array}{l}6.152 \\
(0.45)\end{array}$ \\
\hline & & & & & $\begin{array}{l}4.750 \\
(0.51)\end{array}$ & $\begin{array}{l}5.094 \\
(0.33)\end{array}$ & $\begin{array}{l}5.371 \\
(0.31)\end{array}$ & $\begin{array}{l}5.545 \\
(0.30)\end{array}$ & $\begin{array}{l}5.669 \\
(0.34)\end{array}$ & $\begin{array}{l}5.702 \\
(0.44)\end{array}$ \\
\hline & & & & $\begin{array}{l}3.961 \\
(0.55)\end{array}$ & $\begin{array}{l}4.306 \\
(0.33)\end{array}$ & $\begin{array}{l}4.671 \\
(0.38)\end{array}$ & $\begin{array}{l}4.906 \\
(0.32)\end{array}$ & $\begin{array}{l}5.079 \\
(0.34)\end{array}$ & $\begin{array}{l}5.159 \\
(0.36)\end{array}$ & $\begin{array}{l}5.240 \\
(0.48)\end{array}$ \\
\hline & & & $\begin{array}{l}3.100 \\
(0.59)\end{array}$ & $\begin{array}{l}3.517 \\
(0.38)\end{array}$ & $\begin{array}{l}3.850 \\
(0.40)\end{array}$ & $\begin{array}{l}4.088 \\
(0.40)\end{array}$ & $\begin{array}{l}4.342 \\
(0.36)\end{array}$ & $\begin{array}{l}4.512 \\
(0.35)\end{array}$ & $\begin{array}{l}4.599 \\
(0.34)\end{array}$ & $\begin{array}{l}4.621 \\
(0.50)\end{array}$ \\
\hline & & $\begin{array}{l}2.323 \\
(0.70)\end{array}$ & $\begin{array}{l}2.712 \\
(0.44)\end{array}$ & $\begin{array}{l}3.010 \\
(0.46)\end{array}$ & $\begin{array}{l}3.318 \\
(0.45)\end{array}$ & $\begin{array}{l}3.576 \\
(0.42)\end{array}$ & $\begin{array}{l}3.745 \\
(0.40)\end{array}$ & $\begin{array}{c}3.898 \\
(0.41)\end{array}$ & $\begin{array}{c}3.962 \\
(0.39)\end{array}$ & $\begin{array}{l}4.025 \\
(0.59)\end{array}$ \\
\hline & $\begin{array}{l}1.667 \\
(0.84)\end{array}$ & $\begin{array}{l}2.010 \\
(0.56)\end{array}$ & $\begin{array}{l}2.295 \\
(0.51)\end{array}$ & $\begin{array}{l}2.584 \\
(0.45)\end{array}$ & $\begin{array}{l}2.808 \\
(0.48)\end{array}$ & $\begin{array}{c}3.022 \\
(0.46)\end{array}$ & $\begin{array}{c}3.184 \\
(0.45)\end{array}$ & $\begin{array}{c}3.312 \\
(0.43)\end{array}$ & $\begin{array}{c}3.374 \\
(0.41)\end{array}$ & $\begin{array}{c}3.412 \\
(0.57)\end{array}$ \\
\hline $\begin{array}{l}1.422 \\
(0.85)\end{array}$ & $\begin{array}{l}1.576 \\
(0.65)\end{array}$ & $\begin{array}{c}1.865 \\
(0.58)\end{array}$ & $\begin{array}{c}2.122 \\
(0.51)\end{array}$ & $\begin{array}{l}2.401 \\
(0.50)\end{array}$ & $\begin{array}{l}2.644 \\
(0.46)\end{array}$ & $\begin{array}{l}2.824 \\
(0.47)\end{array}$ & $\begin{array}{l}2.996 \\
(0.45)\end{array}$ & $\begin{array}{c}3.102 \\
(0.39)\end{array}$ & $\begin{array}{c}3.155 \\
(0.44)\end{array}$ & $\begin{array}{l}3.217 \\
(0.56)\end{array}$ \\
\hline
\end{tabular}

Fig. 4.14b. Pin-power distribution for NITAWL benchmark TCA-10. Value in parentheses is percent standard deviation. 
Table 4.95. Selected reaction rates for CENTRM case TCA-10

\begin{tabular}{|c|c|c|c|c|c|c|}
\hline Pin & Region & $\begin{array}{c}\mathrm{G}_{\mathrm{a}} \mathrm{M} \\
\left(\mathrm{cm}^{-3}-\mathrm{s}^{-1}\right)\end{array}$ & $\begin{array}{c}\mathcal{G}_{\mathrm{f}} \mathrm{M} \\
\left(\mathrm{cm}^{-3}-\mathrm{s}^{-1}\right)\end{array}$ & $\begin{array}{c}\mathrm{G}_{\mathrm{f}} \mathrm{M} \\
\left(\mathrm{cm}^{-3}-\mathrm{s}^{-1}\right)\end{array}$ & $\begin{array}{c}\mathrm{M} \\
\left(\mathrm{cm}-\mathrm{s}^{-1}\right)\end{array}$ & $\mathrm{MM}_{\mathrm{t}}$ \\
\hline \multirow{3}{*}{1} & Fuel & $8.487 \mathrm{E}-06$ & $1.430 \mathrm{E}-05$ & $5.068 \mathrm{E}-06$ & $2.952 \mathrm{E}-03$ & 0.146 \\
\hline & Clad & $1.587 \mathrm{E}-07$ & 0.0 & 0.0 & $9.168 \mathrm{E}-04$ & 0.046 \\
\hline & Mod. & 4.949E-07 & 0.0 & 0.0 & $1.630 \mathrm{E}-02$ & 0.808 \\
\hline \multirow{3}{*}{66} & Fuel & $4.238 \mathrm{E}-05$ & $6.861 \mathrm{E}-05$ & $2.429 \mathrm{E}-05$ & $1.833 \mathrm{E}-02$ & 0.147 \\
\hline & Clad & $8.072 \mathrm{E}-07$ & 0.0 & 0.0 & $5.748 \mathrm{E}-03$ & 0.046 \\
\hline & Mod. & $2.263 \mathrm{E}-06$ & 0.0 & 0.0 & $1.007 \mathrm{E}-01$ & 0.807 \\
\hline
\end{tabular}

Table 4.96. Selected reaction rates for NITAWL case TCA-10

\begin{tabular}{ccccccc}
\hline Pin & Region & $\begin{array}{c}\mathrm{G}_{\mathrm{a}} \mathrm{M} \\
\left(\mathrm{cm}^{-3}-\mathrm{s}^{-1}\right)\end{array}$ & $\begin{array}{c}\mathrm{G}_{\mathrm{f}} \mathrm{M} \\
\left(\mathrm{cm}^{-3}-\mathrm{s}^{-1}\right)\end{array}$ & $\begin{array}{c}\mathrm{G}_{\mathrm{f}} \mathrm{M} \\
\left(\mathrm{cm}^{-3}-\mathrm{s}^{-1}\right)\end{array}$ & $\begin{array}{c}\mathrm{M} \\
\left(\mathrm{cm} \mathrm{-} \mathrm{s}^{-1}\right)\end{array}$ & $\mathrm{MM}_{\mathrm{t}}$ \\
\hline \multirow{2}{*}{1} & Fuel & $8.415 \mathrm{E}-06$ & $1.413 \mathrm{E}-05$ & $5.006 \mathrm{E}-06$ & $2.931 \mathrm{E}-03$ & 0.146 \\
& Clad & $1.562 \mathrm{E}-07$ & 0.0 & 0.0 & $9.141 \mathrm{E}-04$ & 0.046 \\
& Mod. & $4.895 \mathrm{E}-07$ & 0.0 & 0.0 & $1.618 \mathrm{E}-02$ & 0.808 \\
\hline \multirow{2}{*}{66} & Fuel & $4.266 \mathrm{E}-05$ & $6.930 \mathrm{E}-05$ & $2.454 \mathrm{E}-05$ & $1.832 \mathrm{E}-02$ & 0.147 \\
& Clad & $8.014 \mathrm{E}-07$ & 0.0 & 0.0 & $5.729 \mathrm{E}-03$ & 0.045 \\
& Mod. & $2.297 \mathrm{E}-06$ & 0.0 & 0.0 & $1.009 \mathrm{E}-01$ & 0.808 \\
\hline
\end{tabular}


Table 4.97. TCA-10, Pin-1 four-group fluxes

\begin{tabular}{ccc}
\hline Group & $\begin{array}{c}\text { CENTRM M } \\
\left(\mathrm{cm}^{-2}-\mathrm{s}^{-1}\right)\end{array}$ & $\begin{array}{c}\text { NITAWL M } \\
\left(\mathrm{cm}^{-2}-\mathrm{s}^{-1}\right)\end{array}$ \\
\hline 1 & $2.547 \mathrm{E}-05$ & $2.512 \mathrm{E}-05$ \\
2 & $7.003 \mathrm{E}-06$ & $7.231 \mathrm{E}-06$ \\
3 & $1.709 \mathrm{E}-06$ & $1.737 \mathrm{E}-06$ \\
4 & $1.922 \mathrm{E}-05$ & $1.894 \mathrm{E}-05$ \\
\hline
\end{tabular}

Table 4.98. TCA-10, Pin-1 four-group U-235 cross sections

\begin{tabular}{cccccccc}
\hline Group & \multicolumn{2}{c}{ CENTRM cross sections $\left(\mathrm{cm}^{-1}\right)$} & \multicolumn{3}{c}{ NITAWL cross sections $\left(\mathrm{cm}^{-1}\right)$} \\
\hline & $\frac{\mathrm{G}_{\mathrm{a}}}{1.40788 \mathrm{E}-04}$ & $\frac{\mathrm{G}_{\mathrm{f}}}{3.23363 \mathrm{E}-04}$ & $\frac{\mathrm{G}_{\mathrm{f}}}{1.24110 \mathrm{E}-04}$ & $\frac{\mathrm{G}_{\mathrm{a}}}{1.41106 \mathrm{E}-04}$ & $\frac{4 \mathrm{G}_{\mathrm{f}}}{3.24338 \mathrm{E}-04}$ & $\frac{\mathrm{G}_{\mathrm{f}}}{1.24311 \mathrm{E}-04}$ \\
1 & $3.64170 \mathrm{E}-03$ & $5.55391 \mathrm{E}-03$ & $2.27928 \mathrm{E}-03$ & $3.51527 \mathrm{E}-03$ & $5.32391 \mathrm{E}-03$ & $2.18488 \mathrm{E}-03$ \\
2 & $5.30350 \mathrm{E}-03$ & $1.08803 \mathrm{E}-02$ & $4.46517 \mathrm{E}-03$ & $5.18458 \mathrm{E}-03$ & $1.06225 \mathrm{E}-02$ & $4.35935 \mathrm{E}-03$ \\
4 & $4.91298 \mathrm{E}-02$ & $1.02298 \mathrm{E}-01$ & $4.19822 \mathrm{E}-02$ & $4.92076 \mathrm{E}-02$ & $1.02458 \mathrm{E}-01$ & $4.20476 \mathrm{E}-02$ \\
\hline
\end{tabular}

Table 4.99. TCA-10, Pin-1 four-group U-238 cross sections

\begin{tabular}{cccccccc}
\hline Group & \multicolumn{3}{c}{ CENTRM cross sections $\left(\mathrm{cm}^{-1}\right)$} & \multicolumn{3}{c}{ NITAWL cross sections $\left(\mathrm{cm}^{-1}\right)$} \\
\hline & $\frac{\mathrm{G}_{\mathrm{a}}}{4.53168 \mathrm{E}-03}$ & $\frac{\mathrm{G}_{\mathrm{f}}}{8.72356 \mathrm{E}-03}$ & $\frac{\mathrm{G}_{\mathrm{f}}}{3.09660 \mathrm{E}-03}$ & $\frac{\mathrm{G}_{\mathrm{a}}}{4.53467 \mathrm{E}-03}$ & $\frac{\mathrm{G}_{\mathrm{f}}}{8.75482 \mathrm{E}-03}$ & $\frac{\mathrm{G}_{\mathrm{f}}}{3.09219 \mathrm{E}-03}$ \\
1 & $4.29217 \mathrm{E}-02$ & $3.81724 \mathrm{E}-06$ & $1.58147 \mathrm{E}-06$ & $4.01630 \mathrm{E}-02$ & $3.94182 \mathrm{E}-06$ & $1.63309 \mathrm{E}-06$ \\
3 & $7.03335 \mathrm{E}-03$ & $2.49018 \mathrm{E}-08$ & $1.03173 \mathrm{E}-08$ & $7.00443 \mathrm{E}-03$ & $2.45869 \mathrm{E}-08$ & $1.01868 \mathrm{E}-08$ \\
4 & $2.78924 \mathrm{E}-02$ & $1.31130 \mathrm{E}-07$ & $5.43293 \mathrm{E}-08$ & $2.79251 \mathrm{E}-02$ & $1.31284 \mathrm{E}-07$ & $5.43933 \mathrm{E}-08$ \\
\hline
\end{tabular}

Table 4.100. TCA-10, Pin-1 four-group Pu-239 cross sections

\begin{tabular}{|c|c|c|c|c|c|c|}
\hline \multirow[t]{2}{*}{ Group } & \multicolumn{3}{|c|}{ CENTRM cross sections $\left(\mathrm{cm}^{-1}\right)$} & \multicolumn{3}{|c|}{ NITAWL cross sections $\left(\mathrm{cm}^{-1}\right)$} \\
\hline & $\mathrm{G}_{\mathrm{a}}$ & $\mathbb{G}_{\mathrm{f}}$ & $\mathrm{G}_{\mathrm{f}}$ & $\mathrm{G}_{\mathrm{a}}$ & $<\mathrm{G}_{\mathrm{f}}$ & $\mathrm{G}_{\mathrm{f}}$ \\
\hline 1 & $5 . \overline{10742 \mathrm{E}-04}$ & $\overline{1.50232 \mathrm{E}-03}$ & $\overline{4.82148 \mathrm{E}-04}$ & $\overline{5.10768 \mathrm{E}-04}$ & $\overline{1.50322 \mathrm{E}-03}$ & $\overline{4.81907 \mathrm{E}-04}$ \\
\hline 2 & $1.13157 \mathrm{E}-02$ & $1.86831 \mathrm{E}-02$ & $6.48763 \mathrm{E}-03$ & $1.14534 \mathrm{E}-02$ & 1.87359E-02 & $6.50593 \mathrm{E}-03$ \\
\hline 3 & $3.56099 \mathrm{E}-02$ & $6.98250 \mathrm{E}-02$ & $2.42465 \mathrm{E}-02$ & $3.42803 \mathrm{E}-02$ & $6.73431 \mathrm{E}-02$ & 2.33847E-02 \\
\hline 4 & 2.63607E-01 & 5.35739E-01 & $1.85285 \mathrm{E}-01$ & $2.64216 \mathrm{E}-01$ & $5.36885 \mathrm{E}-01$ & $1.85682 \mathrm{E}-01$ \\
\hline
\end{tabular}


Table 4.101. TCA-10, Pin-66 four-group fluxes

\begin{tabular}{ccc}
\hline Group & $\begin{array}{c}\text { CENTRM M } \\
\left(\mathrm{cm}^{-2}-\mathrm{s}^{-1}\right)\end{array}$ & $\begin{array}{c}\text { NITAWL M } \\
\left(\mathrm{cm}^{-2}-\mathrm{s}^{-1}\right)\end{array}$ \\
\hline 1 & $1.721 \mathrm{E}-04$ & $1.716 \mathrm{E}-04$ \\
2 & $5.659 \mathrm{E}-05$ & $5.611 \mathrm{E}-05$ \\
3 & $1.360 \mathrm{E}-05$ & $1.336 \mathrm{E}-05$ \\
4 & $8.928 \mathrm{E}-05$ & $9.041 \mathrm{E}-05$ \\
\hline
\end{tabular}

Table 4.102. TCA-10, Pin-66 four-group U-235 cross sections

\begin{tabular}{cccccccc}
\hline Group & \multicolumn{2}{c}{ CENTRM cross sections $\left(\mathrm{cm}^{-1}\right)$} & \multicolumn{3}{c}{ NITAWL cross sections $\left(\mathrm{cm}^{-1}\right)$} \\
\hline & $\frac{\mathrm{G}_{\mathrm{a}}}{1.44765 \mathrm{E}-04}$ & $\frac{\mathrm{G}_{\mathrm{f}}}{3.27146 \mathrm{E}-04}$ & $\frac{\mathrm{G}_{\mathrm{f}}}{1.26179 \mathrm{E}-04}$ & $\frac{\mathrm{G}_{\mathrm{a}}}{1.44207 \mathrm{E}-04}$ & $\frac{\mathrm{G}_{\mathrm{f}}}{3.26568 \mathrm{E}-04}$ & $\frac{\mathrm{G}_{\mathrm{f}}}{1.25881 \mathrm{E}-04}$ \\
1 & $3.42458 \mathrm{E}-03$ & $5.21627 \mathrm{E}-03$ & $2.14071 \mathrm{E}-03$ & $3.45678 \mathrm{E}-03$ & $5.26794 \mathrm{E}-03$ & $2.16191 \mathrm{E}-03$ \\
2 & $5.10952 \mathrm{E}-03$ & $1.04097 \mathrm{E}-02$ & $4.27204 \mathrm{E}-03$ & $5.28578 \mathrm{E}-03$ & $1.08415 \mathrm{E}-02$ & $4.44925 \mathrm{E}-03$ \\
3 & $4.80156 \mathrm{E}-02$ & $9.99418 \mathrm{E}-02$ & $4.10152 \mathrm{E}-02$ & $4.77411 \mathrm{E}-02$ & $9.93647 \mathrm{E}-02$ & $4.07783 \mathrm{E}-02$ \\
\hline
\end{tabular}

Table 4.103. TCA-10, Pin-66 four-group U-238 cross sections

\begin{tabular}{cccccccc}
\hline Group & \multicolumn{2}{c}{ CENTRM cross sections $\left(\mathrm{cm}^{-1}\right)$} & \multicolumn{3}{c}{ NITAWL cross sections $\left(\mathrm{cm}^{-1}\right)$} \\
\hline & $\frac{\mathrm{G}_{\mathrm{a}}}{4.43964 \mathrm{E}-03}$ & $\frac{\mathrm{G}_{\mathrm{f}}}{8.07600 \mathrm{E}-03}$ & $\frac{\mathrm{G}_{\mathrm{f}}}{2.86439 \mathrm{E}-03}$ & $\frac{\mathrm{G}_{\mathrm{a}}}{4.43787 \mathrm{E}-03}$ & $\frac{\mathrm{G}_{\mathrm{f}}}{8.12821 \mathrm{E}-03}$ & $\frac{\mathrm{G}_{\mathrm{f}}}{2.88138 \mathrm{E}-03}$ \\
1 & $3.89892 \mathrm{E}-02$ & $3.80924 \mathrm{E}-06$ & $1.57815 \mathrm{E}-06$ & $3.85192 \mathrm{E}-02$ & $3.58239 \mathrm{E}-06$ & $1.48417 \mathrm{E}-06$ \\
2 & $6.97667 \mathrm{E}-03$ & $2.43379 \mathrm{E}-08$ & $1.00837 \mathrm{E}-08$ & $7.03071 \mathrm{E}-03$ & $2.48865 \mathrm{E}-08$ & $1.03109 \mathrm{E}-08$ \\
3 & $2.73091 \mathrm{E}-02$ & $1.28331 \mathrm{E}-07$ & $5.31703 \mathrm{E}-08$ & $2.71792 \mathrm{E}-02$ & $1.27709 \mathrm{E}-07$ & $5.29124 \mathrm{E}-08$ \\
\hline
\end{tabular}

Table 4.104. TCA-10, Pin-66 four-group Pu-239 cross sections

\begin{tabular}{|c|c|c|c|c|c|c|}
\hline \multirow[t]{2}{*}{ Group } & \multicolumn{3}{|c|}{ CENTRM cross sections $\left(\mathrm{cm}^{-1}\right)$} & \multicolumn{3}{|c|}{ NITAWL cross sections $\left(\mathrm{cm}^{-1}\right)$} \\
\hline & $\mathrm{G}_{\mathrm{a}-}$ & $\mathrm{G}_{\mathrm{f}}$ & $\mathrm{G}_{\mathrm{f}}$ & $\mathrm{G}_{\mathrm{a}}$ & $<\mathrm{G}_{\mathrm{f}}$ & $\mathrm{G}_{\mathrm{f}}$ \\
\hline 1 & $5.12740 \mathrm{E}-04$ & $1.48833 \mathrm{E}-03$ & $\overline{4.79719 \mathrm{E}-04}$ & $5.12294 \mathrm{E}-04$ & $1.48952 \mathrm{E}-03$ & $4.79891 \mathrm{E}-04$ \\
\hline 2 & 1.11449E-02 & 1.83349E-02 & $6.36671 \mathrm{E}-03$ & $1.12214 \mathrm{E}-02$ & $1.84762 \mathrm{E}-02$ & $6.41579 \mathrm{E}-03$ \\
\hline 3 & 3.30546E-02 & $6.50683 \mathrm{E}-02$ & $2.25947 \mathrm{E}-02$ & $3.57615 \mathrm{E}-02$ & 7.00931E-02 & $2.43396 \mathrm{E}-02$ \\
\hline 4 & 2.70972E-01 & $5.45716 \mathrm{E}-01$ & $1.88771 \mathrm{E}-01$ & 2.70533E-01 & $5.44404 \mathrm{E}-01$ & 1.88319E-01 \\
\hline
\end{tabular}




\begin{tabular}{|c|c|c|c|c|c|c|c|c|c|c|}
\hline & & & & & & & & & & $\begin{array}{l}6.654 \\
(0.79)\end{array}$ \\
\hline & $\begin{array}{c}0.147 \\
(0.81)\end{array}$ & & & & & & & & $\begin{array}{c}6.562 \\
(0.44)\end{array}$ & $\begin{array}{l}6.611 \\
(0.46)\end{array}$ \\
\hline & & & & & & & & $\begin{array}{r}6.263 \\
(0.44)\end{array}$ & $\begin{array}{c}6.409 \\
(0.32)\end{array}$ & $\begin{array}{l}6.449 \\
(0.43)\end{array}$ \\
\hline & & & & & & & $\begin{array}{l}5.818 \\
(0.46)\end{array}$ & $\begin{array}{r}6.026 \\
(0.31)\end{array}$ & $\begin{array}{l}6.170 \\
(0.31)\end{array}$ & $\begin{array}{l}6.207 \\
(0.44)\end{array}$ \\
\hline & & & & & & $\begin{array}{l}5.216 \\
(0.46)\end{array}$ & $\begin{array}{l}5.552 \\
(0.35)\end{array}$ & $\begin{array}{l}5.746 \\
(0.30)\end{array}$ & $\begin{array}{l}5.878 \\
(0.30)\end{array}$ & $\begin{array}{l}5.943 \\
(0.39)\end{array}$ \\
\hline & & & & & $\begin{array}{l}4.527 \\
(0.58)\end{array}$ & $\begin{array}{l}4.898 \\
(0.35)\end{array}$ & $\begin{array}{l}5.157 \\
(0.31)\end{array}$ & $\begin{array}{l}5.351 \\
(0.31)\end{array}$ & $\begin{array}{l}5.483 \\
(0.32)\end{array}$ & $\begin{array}{l}5.471 \\
(0.45)\end{array}$ \\
\hline & & & & $\begin{array}{c}3.745 \\
(0.51)\end{array}$ & $\begin{array}{l}4.139 \\
(0.38)\end{array}$ & $\begin{array}{l}4.467 \\
(0.35)\end{array}$ & $\begin{array}{l}4.724 \\
(0.35)\end{array}$ & $\begin{array}{l}4.888 \\
(0.33)\end{array}$ & $\begin{array}{l}5.001 \\
(0.33)\end{array}$ & $\begin{array}{l}4.991 \\
(0.49)\end{array}$ \\
\hline & & & $\begin{array}{c}3.030 \\
(0.65)\end{array}$ & $\begin{array}{l}3.377 \\
(0.41)\end{array}$ & $\begin{array}{c}3.691 \\
(0.40)\end{array}$ & $\begin{array}{l}3.991 \\
(0.38)\end{array}$ & $\begin{array}{l}4.178 \\
(0.39)\end{array}$ & $\begin{array}{l}4.331 \\
(0.34)\end{array}$ & $\begin{array}{l}4.457 \\
(0.36)\end{array}$ & $\begin{array}{l}4.431 \\
(0.50)\end{array}$ \\
\hline & & $\begin{array}{l}2.212 \\
(0.70)\end{array}$ & $\begin{array}{l}2.576 \\
(0.52)\end{array}$ & $\begin{array}{l}2.912 \\
(0.43)\end{array}$ & $\begin{array}{l}3.174 \\
(0.42)\end{array}$ & $\begin{array}{c}3.439 \\
(0.38)\end{array}$ & $\begin{array}{l}3.611 \\
(0.39)\end{array}$ & $\begin{array}{l}3.748 \\
(0.36)\end{array}$ & $\begin{array}{l}3.825 \\
(0.40)\end{array}$ & $\begin{array}{l}3.884 \\
(0.50)\end{array}$ \\
\hline & $\begin{array}{c}1.604 \\
(0.82)\end{array}$ & $\begin{array}{l}1.905 \\
(0.50)\end{array}$ & $\begin{array}{l}2.200 \\
(0.49)\end{array}$ & $\begin{array}{c}2.472 \\
(0.45)\end{array}$ & $\begin{array}{l}2.710 \\
(0.48)\end{array}$ & $\begin{array}{l}2.905 \\
(0.41)\end{array}$ & $\begin{array}{c}3.084 \\
(0.47)\end{array}$ & $\begin{array}{l}3.215 \\
(0.41)\end{array}$ & $\begin{array}{l}3.268 \\
(0.43)\end{array}$ & $\begin{array}{l}3.263 \\
(0.55)\end{array}$ \\
\hline $\begin{array}{l}1.350 \\
(0.84)\end{array}$ & $\begin{array}{c}1.492 \\
(0.56)\end{array}$ & $\begin{array}{l}1.764 \\
(0.57)\end{array}$ & $\begin{array}{l}2.050 \\
(0.50)\end{array}$ & $\begin{array}{l}2.302 \\
(0.49)\end{array}$ & $\begin{array}{l}2.553 \\
(0.45)\end{array}$ & $\begin{array}{l}2.722 \\
(0.44)\end{array}$ & $\begin{array}{l}2.886 \\
(0.45)\end{array}$ & $\begin{array}{r}2.980 \\
(0.46)\end{array}$ & $\begin{array}{l}3.036 \\
(0.44)\end{array}$ & $\begin{array}{l}3.092 \\
(0.56)\end{array}$ \\
\hline
\end{tabular}

Fig. 4.15a. Pin-power distribution for CENTRM benchmark TCA-11. Value in parentheses is percent standard deviation. 


\begin{tabular}{|c|c|c|c|c|c|c|c|c|c|c|}
\hline & & & & & & & & & & $\begin{array}{l}6.756 \\
(0.82)\end{array}$ \\
\hline & \multirow[t]{8}{*}{$\begin{array}{l}0.148 \\
(0.84)\end{array}$} & & & & & & & & $\begin{array}{l}6.609 \\
(0.41)\end{array}$ & $\begin{array}{l}6.637 \\
(0.39)\end{array}$ \\
\hline & & & & & & & & $\begin{array}{l}6.358 \\
(0.42)\end{array}$ & $\begin{array}{l}6.453 \\
(0.31)\end{array}$ & $\begin{array}{l}6.510 \\
(0.44)\end{array}$ \\
\hline & & & & & & & $\begin{array}{l}5.866 \\
(0.43)\end{array}$ & $\begin{array}{c}6.087 \\
(0.31)\end{array}$ & $\begin{array}{l}6.184 \\
(0.31)\end{array}$ & $\begin{array}{l}6.208 \\
(0.44)\end{array}$ \\
\hline & & & & & & $\begin{array}{l}5.248 \\
(0.45) \\
\end{array}$ & $\begin{array}{l}5.518 \\
(0.31) \\
\end{array}$ & $\begin{array}{l}5.748 \\
(0.29) \\
\end{array}$ & $\begin{array}{l}5.874 \\
(0.31) \\
\end{array}$ & $\begin{array}{l}5.922 \\
(0.43)\end{array}$ \\
\hline & & & & & $\begin{array}{l}4.565 \\
(0.51)\end{array}$ & $\begin{array}{l}4.901 \\
(0.35)\end{array}$ & $\begin{array}{l}5.149 \\
(0.31)\end{array}$ & $\begin{array}{l}5.364 \\
(0.31)\end{array}$ & $\begin{array}{l}5.480 \\
(0.32)\end{array}$ & $\begin{array}{l}5.535 \\
(0.47)\end{array}$ \\
\hline & & & & $\begin{array}{c}3.782 \\
(0.57)\end{array}$ & $\begin{array}{l}4.171 \\
(0.39)\end{array}$ & $\begin{array}{l}4.444 \\
(0.38)\end{array}$ & $\begin{array}{l}4.672 \\
(0.37)\end{array}$ & $\begin{array}{l}4.911 \\
(0.33)\end{array}$ & $\begin{array}{l}4.984 \\
(0.30)\end{array}$ & $\begin{array}{l}5.039 \\
(0.47)\end{array}$ \\
\hline & & & $\begin{array}{l}2.969 \\
(0.61)\end{array}$ & $\begin{array}{l}3.380 \\
(0.38)\end{array}$ & $\begin{array}{l}3.683 \\
(0.38)\end{array}$ & $\begin{array}{l}3.961 \\
(0.37)\end{array}$ & $\begin{array}{l}4.175 \\
(0.40)\end{array}$ & $\begin{array}{l}4.337 \\
(0.35)\end{array}$ & $\begin{array}{l}4.431 \\
(0.36)\end{array}$ & $\begin{array}{l}4.489 \\
(0.53)\end{array}$ \\
\hline & & $\begin{array}{l}2.266 \\
(0.73)\end{array}$ & $\begin{array}{l}2.599 \\
(0.49)\end{array}$ & $\begin{array}{l}2.912 \\
(0.47)\end{array}$ & $\begin{array}{l}3.209 \\
(0.40)\end{array}$ & $\begin{array}{l}3.419 \\
(0.44)\end{array}$ & $\begin{array}{l}3.625 \\
(0.39)\end{array}$ & $\begin{array}{l}3.746 \\
(0.38)\end{array}$ & $\begin{array}{c}3.823 \\
(0.39)\end{array}$ & $\begin{array}{l}3.855 \\
(0.60)\end{array}$ \\
\hline & $\begin{array}{l}1.628 \\
(0.86)\end{array}$ & $\begin{array}{c}1.918 \\
(0.54)\end{array}$ & $\begin{array}{l}2.205 \\
(0.51)\end{array}$ & $\begin{array}{l}2.470 \\
(0.48)\end{array}$ & $\begin{array}{l}2.727 \\
(0.45)\end{array}$ & $\begin{array}{l}2.946 \\
(0.52)\end{array}$ & $\begin{array}{c}3.062 \\
(0.42)\end{array}$ & $\begin{array}{c}3.184 \\
(0.42)\end{array}$ & $\begin{array}{c}3.282 \\
(0.42)\end{array}$ & $\begin{array}{l}3.293 \\
(0.59)\end{array}$ \\
\hline $\begin{array}{l}1.376 \\
(0.81)\end{array}$ & $\begin{array}{l}1.498 \\
(0.61)\end{array}$ & $\begin{array}{c}1.770 \\
(0.54)\end{array}$ & $\begin{array}{c}2.049 \\
(0.51)\end{array}$ & $\begin{array}{l}2.320 \\
(0.50)\end{array}$ & $\begin{array}{l}2.536 \\
(0.45)\end{array}$ & $\begin{array}{l}2.723 \\
(0.45)\end{array}$ & $\begin{array}{l}2.861 \\
(0.49)\end{array}$ & $\begin{array}{c}2.983 \\
(0.44)\end{array}$ & $\begin{array}{l}3.037 \\
(0.42)\end{array}$ & $\begin{array}{l}3.032 \\
(0.61)\end{array}$ \\
\hline
\end{tabular}

Fig. 4.15b. Pin-power distribution for NITAWL benchmark TCA-11. Value in parentheses is percent standard deviation. 
Table 4.105. Selected reaction rates for CENTRM case TCA-11

\begin{tabular}{ccccccc}
\hline Pin & Region & $\begin{array}{c}\mathrm{G}_{\mathrm{a}} \mathrm{M} \\
\left(\mathrm{cm}^{-3}-\mathrm{s}^{-1}\right)\end{array}$ & $\begin{array}{c}\mathrm{G}_{\mathrm{f}} \mathrm{M} \\
\left(\mathrm{cm}^{-3}-\mathrm{s}^{-1}\right)\end{array}$ & $\begin{array}{c}\mathrm{G}_{\mathrm{f}} \mathrm{M} \\
\left(\mathrm{cm}^{-3}-\mathrm{s}^{-1}\right)\end{array}$ & $\begin{array}{c}\mathrm{M} \\
\left(\mathrm{cm}^{-1}\right)\end{array}$ & $\mathrm{MM}_{\mathrm{t}}$ \\
\hline \multirow{2}{*}{1} & Fuel & $7.995 \mathrm{E}-06$ & $1.342 \mathrm{E}-05$ & $4.759 \mathrm{E}-06$ & $2.922 \mathrm{E}-03$ & 0.146 \\
& Clad & $1.494 \mathrm{E}-07$ & 0.0 & 0.0 & $8.994 \mathrm{E}-04$ & 0.046 \\
& Mod. & $4.727 \mathrm{E}-07$ & 0.0 & 0.0 & $1.613 \mathrm{E}-02$ & 0.808 \\
\hline \multirow{2}{*}{66} & Fuel & $4.112 \mathrm{E}-05$ & $6.652 \mathrm{E}-05$ & $2.356 \mathrm{E}-05$ & $1.831 \mathrm{E}-02$ & 0.147 \\
& Clad & $7.801 \mathrm{E}-07$ & 0.0 & 0.0 & $5.719 \mathrm{E}-03$ & 0.045 \\
& Mod. & $2.193 \mathrm{E}-06$ & 0.0 & 0.0 & $1.009 \mathrm{E}-01$ & 0.808 \\
\hline
\end{tabular}

Table 4.106. Selected reaction rates for NITAWL case TCA-11

\begin{tabular}{ccccccc}
\hline Pin & Region & $\begin{array}{c}\mathrm{G}_{\mathrm{a}} \mathrm{M} \\
\left(\mathrm{cm}^{-3}-\mathrm{s}^{-1}\right)\end{array}$ & $\begin{array}{c}\mathrm{G}_{\mathrm{f}} \mathrm{M} \\
\left(\mathrm{cm}^{-3}-\mathrm{s}^{-1}\right)\end{array}$ & $\begin{array}{c}\mathrm{G}_{\mathrm{f}} \mathrm{M} \\
\left(\mathrm{cm}^{-3}-\mathrm{s}^{-1}\right)\end{array}$ & $\begin{array}{c}\mathrm{M} \\
\left(\mathrm{cm}^{-1}\right)\end{array}$ & $\mathrm{M}_{\mathrm{t}}$ \\
\hline \multirow{2}{*}{1} & Fuel & $8.237 \mathrm{E}-06$ & $1.382 \mathrm{E}-05$ & $4.898 \mathrm{E}-06$ & $2.946 \mathrm{E}-03$ & 0.146 \\
& Clad & $1.536 \mathrm{E}-07$ & 0.0 & 0.0 & $9.201 \mathrm{E}-04$ & 0.046 \\
& Mod. & $4.780 \mathrm{E}-07$ & 0.0 & 0.0 & $1.632 \mathrm{E}-02$ & 0.808 \\
\hline \multirow{2}{*}{66} & Fuel & $4.210 \mathrm{E}-05$ & $6.785 \mathrm{E}-05$ & $2.403 \mathrm{E}-05$ & $1.881 \mathrm{E}-02$ & 0.149 \\
& Clad & $7.944 \mathrm{E}-07$ & 0.0 & 0.0 & $5.881 \mathrm{E}-03$ & 0.045 \\
& Mod. & $2.226 \mathrm{E}-06$ & 0.0 & 0.0 & $1.019 \mathrm{E}-01$ & 0.805 \\
\hline
\end{tabular}


Table 4.107. TCA-11, Pin-1 four-group fluxes

\begin{tabular}{ccc}
\hline Group & $\begin{array}{c}\text { CENTRM M } \\
\left(\mathrm{cm}^{-2}-\mathrm{s}^{-1}\right)\end{array}$ & $\begin{array}{c}\text { NITAWL M } \\
\left(\mathrm{cm}^{-2}-\mathrm{s}^{-1}\right)\end{array}$ \\
\hline 1 & $2.428 \mathrm{E}-05$ & $2.418 \mathrm{E}-05$ \\
2 & $6.754 \mathrm{E}-06$ & $6.771 \mathrm{E}-06$ \\
3 & $1.616 \mathrm{E}-06$ & $1.716 \mathrm{E}-06$ \\
4 & $1.818 \mathrm{E}-05$ & $1.859 \mathrm{E}-05$ \\
\hline
\end{tabular}

Table 4.108. TCA-11, Pin-1 four-group U-235 cross sections

\begin{tabular}{cccccccc}
\hline Group & \multicolumn{2}{c}{ CENTRM cross sections $\left(\mathrm{cm}^{-1}\right)$} & \multicolumn{3}{c}{ NITAWL cross sections $\left(\mathrm{cm}^{-1}\right)$} \\
\hline & $\frac{\mathrm{G}_{\mathrm{a}}}{1.41872 \mathrm{E}-04}$ & $\frac{\mathrm{G}_{\mathrm{f}}}{3.24906 \mathrm{E}-04}$ & $\frac{\mathrm{G}_{\mathrm{f}}}{1.24701 \mathrm{E}-04}$ & $\frac{\mathrm{G}_{\mathrm{a}}}{1.41020 \mathrm{E}-04}$ & $\frac{4 \mathrm{G}_{\mathrm{f}}}{3.23700 \mathrm{E}-04}$ & $\frac{\mathrm{G}_{\mathrm{f}}}{1.24222 \mathrm{E}-04}$ \\
1 & $3.59017 \mathrm{E}-03$ & $5.46494 \mathrm{E}-03$ & $2.24276 \mathrm{E}-03$ & $3.58779 \mathrm{E}-03$ & $5.48985 \mathrm{E}-03$ & $2.25299 \mathrm{E}-03$ \\
2 & $5.18994 \mathrm{E}-03$ & $1.06233 \mathrm{E}-02$ & $4.35970 \mathrm{E}-03$ & $5.10914 \mathrm{E}-03$ & $1.04368 \mathrm{E}-02$ & $4.28316 \mathrm{E}-03$ \\
4 & $4.91039 \mathrm{E}-02$ & $1.02248 \mathrm{E}-01$ & $4.19618 \mathrm{E}-02$ & $4.92082 \mathrm{E}-02$ & $1.02458 \mathrm{E}-01$ & $4.20477 \mathrm{E}-02$ \\
\hline
\end{tabular}

Table 4.109. TCA-11, Pin-1 four-group U-238 cross sections

\begin{tabular}{cccccccc}
\hline Group & \multicolumn{3}{c}{ CENTRM cross sections $\left(\mathrm{cm}^{-1}\right)$} & \multicolumn{3}{c}{ NITAWL cross sections $\left(\mathrm{cm}^{-1}\right)$} \\
\hline & $\frac{\mathrm{G}_{\mathrm{a}}}{4.53707 \mathrm{E}-03}$ & $\frac{\leftarrow_{\mathrm{f}}}{8.65445 \mathrm{E}-03}$ & $\frac{\mathrm{G}_{\mathrm{f}}}{3.06352 \mathrm{E}-03}$ & $\frac{\mathrm{G}_{\mathrm{a}}}{4.50757 \mathrm{E}-03}$ & $\frac{\mathrm{G}_{\mathrm{f}}}{8.64413 \mathrm{E}-03}$ & $\frac{\mathrm{G}_{\mathrm{f}}}{3.06142 \mathrm{E}-03}$ \\
1 & $3.77442 \mathrm{E}-02$ & $3.89227 \mathrm{E}-06$ & $1.61256 \mathrm{E}-06$ & $4.14119 \mathrm{E}-02$ & $3.44468 \mathrm{E}-06$ & $1.42711 \mathrm{E}-06$ \\
3 & $6.97847 \mathrm{E}-03$ & $2.44523 \mathrm{E}-08$ & $1.01310 \mathrm{E}-08$ & $6.97541 \mathrm{E}-03$ & $2.44034 \mathrm{E}-08$ & $1.01108 \mathrm{E}-08$ \\
4 & $2.78818 \mathrm{E}-02$ & $1.31080 \mathrm{E}-07$ & $5.43088 \mathrm{E}-08$ & $2.79222 \mathrm{E}-02$ & $1.31269 \mathrm{E}-07$ & $5.43873 \mathrm{E}-08$ \\
\hline
\end{tabular}

Table 4.110. TCA-11, Pin-1 four-group Pu-239 cross sections

\begin{tabular}{|c|c|c|c|c|c|c|}
\hline \multirow[t]{2}{*}{ Group } & \multicolumn{3}{|c|}{ CENTRM cross sections $\left(\mathrm{cm}^{-1}\right)$} & \multicolumn{3}{|c|}{ NITAWL cross sections $\left(\mathrm{cm}^{-1}\right)$} \\
\hline & $\mathrm{G}_{\mathrm{a}}$ & $\leftarrow_{\mathrm{f}}$ & $\mathrm{G}_{\mathrm{f}}$ & $\mathrm{G}_{\mathrm{a}}$ & $\mathbb{G}_{f}$ & $\mathrm{G}_{\mathrm{f}}$ \\
\hline 1 & $5 . \overline{11446 \mathrm{E}-04}$ & $\overline{1.50099 \mathrm{E}-03}$ & $\overline{4.81647 \mathrm{E}-04}$ & $\overline{5.10571 \mathrm{E}-04}$ & $\overline{1.50108 \mathrm{E}-03}$ & $\overline{4.81762 \mathrm{E}-04}$ \\
\hline 2 & $1.15859 \mathrm{E}-02$ & $1.88543 \mathrm{E}-02$ & $6.54708 \mathrm{E}-03$ & $1.15115 \mathrm{E}-02$ & 1.90902E-02 & 6.62899E-03 \\
\hline 3 & $3.43188 \mathrm{E}-02$ & $6.73416 \mathrm{E}-02$ & $2.33841 \mathrm{E}-02$ & $3.42125 \mathrm{E}-02$ & $6.71562 \mathrm{E}-02$ & $2.33196 \mathrm{E}-02$ \\
\hline 4 & $2.62911 \mathrm{E}-01$ & $5.34534 \mathrm{E}-01$ & $1.84867 \mathrm{E}-01$ & 2.65492E-01 & 5.39053E-01 & $1.86434 \mathrm{E}-01$ \\
\hline
\end{tabular}


Table 4.111. TCA-11, Pin-66 four-group fluxes

\begin{tabular}{ccc}
\hline Group & $\begin{array}{c}\text { CENTRM M } \\
\left(\mathrm{cm}^{-2}-\mathrm{s}^{-1}\right)\end{array}$ & $\begin{array}{c}\text { NITAWL M } \\
\left(\mathrm{cm}^{-2}-\mathrm{s}^{-1}\right)\end{array}$ \\
\hline 1 & $1.657 \mathrm{E}-04$ & $1.701 \mathrm{E}-04$ \\
2 & $5.293 \mathrm{E}-05$ & $5.558 \mathrm{E}-05$ \\
3 & $1.316 \mathrm{E}-05$ & $1.301 \mathrm{E}-05$ \\
4 & $8.674 \mathrm{E}-05$ & $8.849 \mathrm{E}-05$ \\
\hline
\end{tabular}

Table 4.112. TCA-11, Pin-66 four-group U-235 cross sections

\begin{tabular}{cccccccc}
\hline Group & \multicolumn{2}{c}{ CENTRM cross sections $\left(\mathrm{cm}^{-1}\right)$} & \multicolumn{3}{c}{ NITAWL cross sections $\left(\mathrm{cm}^{-1}\right)$} \\
\hline & $\frac{\mathrm{G}_{\mathrm{a}}}{1.44711 \mathrm{E}-04}$ & $\frac{\mathrm{G}_{\mathrm{f}}}{3.27220 \mathrm{E}-04}$ & $\frac{\mathrm{G}_{\mathrm{f}}}{1.26172 \mathrm{E}-04}$ & $\frac{\mathrm{G}_{\mathrm{a}}}{1.44755 \mathrm{E}-04}$ & $\frac{\mathrm{G}_{\mathrm{f}}}{3.27436 \mathrm{E}-04}$ & $\frac{\mathrm{G}_{\mathrm{f}}}{1.26225 \mathrm{E}-04}$ \\
1 & $3.46220 \mathrm{E}-03$ & $5.28876 \mathrm{E}-03$ & $2.17045 \mathrm{E}-03$ & $3.45516 \mathrm{E}-03$ & $5.25847 \mathrm{E}-03$ & $2.15802 \mathrm{E}-03$ \\
2 & $5.16589 \mathrm{E}-03$ & $1.05595 \mathrm{E}-02$ & $4.33352 \mathrm{E}-03$ & $5.18064 \mathrm{E}-03$ & $1.05839 \mathrm{E}-02$ & $4.34357 \mathrm{E}-03$ \\
4 & $4.81825 \mathrm{E}-02$ & $1.00288 \mathrm{E}-01$ & $4.11571 \mathrm{E}-02$ & $4.80322 \mathrm{E}-02$ & $9.99713 \mathrm{E}-02$ & $4.10273 \mathrm{E}-02$ \\
\hline
\end{tabular}

Table 4.113. TCA-11, Pin-66 four-group U-238 cross sections

\begin{tabular}{cccccccc}
\hline Group & \multicolumn{3}{c}{ CENTRM cross sections $\left(\mathrm{cm}^{-1}\right)$} & \multicolumn{3}{c}{ NITAWL cross sections $\left(\mathrm{cm}^{-1}\right)$} \\
\hline & $\frac{\mathrm{G}_{\mathrm{a}}}{4.47467 \mathrm{E}-03}$ & $\frac{\mathrm{G}_{\mathrm{f}}}{8.17996 \mathrm{E}-03}$ & $\frac{\mathrm{G}_{\mathrm{f}}}{2.90346 \mathrm{E}-03}$ & $\frac{\mathrm{G}_{\mathrm{a}}}{4.47739 \mathrm{E}-03}$ & $\frac{\mathrm{G}_{\mathrm{f}}}{8.19447 \mathrm{E}-03}$ & $\frac{\mathrm{G}_{\mathrm{f}}}{2.90690 \mathrm{E}-03}$ \\
1 & $3.91615 \mathrm{E}-02$ & $3.93752 \mathrm{E}-06$ & $1.63130 \mathrm{E}-06$ & $4.02064 \mathrm{E}-02$ & $3.76053 \mathrm{E}-06$ & $1.55797 \mathrm{E}-06$ \\
3 & $6.99303 \mathrm{E}-03$ & $2.44910 \mathrm{E}-08$ & $1.01470 \mathrm{E}-08$ & $6.99198 \mathrm{E}-03$ & $2.45410 \mathrm{E}-08$ & $1.01678 \mathrm{E}-08$ \\
4 & $2.73917 \mathrm{E}-02$ & $1.28724 \mathrm{E}-07$ & $5.33326 \mathrm{E}-08$ & $2.73197 \mathrm{E}-02$ & $1.28377 \mathrm{E}-07$ & $5.31893 \mathrm{E}-08$ \\
\hline
\end{tabular}

Table 4.114. TCA-11, Pin-66 four-group Pu-239 cross sections

\begin{tabular}{cccccccc}
\hline Group & \multicolumn{3}{c}{ CENTRM cross sections $\left(\mathrm{cm}^{-1}\right)$} & \multicolumn{3}{c}{ NITAWL cross sections $\left(\mathrm{cm}^{-1}\right)$} \\
\hline & $\frac{\mathrm{G}_{\mathrm{a}}}{4.12696 \mathrm{E}-04}$ & $\frac{\mathrm{G}_{\mathrm{f}}}{1.48906 \mathrm{E}-03}$ & $\frac{\mathrm{G}_{\mathrm{f}}}{4.79759 \mathrm{E}-04}$ & $\frac{5.13031 \mathrm{E}-04}{1.49057 \mathrm{E}-03}$ & $\frac{\mathrm{G}_{\mathrm{a}}}{4.80148 \mathrm{E}-04}$ \\
1 & $1.14152 \mathrm{E}-02$ & $1.88409 \mathrm{E}-02$ & $6.54241 \mathrm{E}-03$ & $1.11940 \mathrm{E}-02$ & $1.81913 \mathrm{E}-02$ & $6.31686 \mathrm{E}-03$ \\
2 & $3.48957 \mathrm{E}-02$ & $6.84264 \mathrm{E}-02$ & $2.37608 \mathrm{E}-02$ & $3.45817 \mathrm{E}-02$ & $6.78782 \mathrm{E}-02$ & $2.35705 \mathrm{E}-02$ \\
3 & $2.71706 \mathrm{E}-01$ & $5.47283 \mathrm{E}-01$ & $1.89309 \mathrm{E}-01$ & $2.72174 \mathrm{E}-01$ & $5.47792 \mathrm{E}-01$ & $1.89488 \mathrm{E}-01$ \\
\hline
\end{tabular}




\section{CALCULATIONAL BENCHMARKS}

\subsection{DESCRIPTION}

The calculational benchmarks examined in this report are divided into two distinct geometric sets of cases. The first set of cases consists of an infinitely long pin in an infinite triangular-pitched lattice. The second set of cases consists of a buckled pin in an infinite triangular-pitched lattice. The radii of the fuel, clad, and moderator are all the same for all variants and states. Also, the buckling height is the same in all buckled problems. All variants and states are calculated in both geometric configurations. The state parameters are given in Table 5.1. ${ }^{4}$

The calculational benchmarks in each geometric set are divided into variants, with five states in each of the variants. The variants differ only by the isotopic composition of the fuel. Within each variant, the fuel composition is the same for each state, with the exception of the presence or absence of ${ }^{135} \mathrm{Xe}$ and ${ }^{149} \mathrm{Sm}$. The differences in the states are due to the presence or absence of ${ }^{135} \mathrm{Xe}$ and ${ }^{149} \mathrm{Sm}$ in the fuel, the fuel temperature, and the type of moderator.

There are four basic types of fuel: fresh uranium fuel, fresh MOX fuel, spent uranium fuel and fresh MOX fuel containing Pu recycled from a reactor. The spent uranium fuel is used in two variants: variant V3 uses spent uranium fuel with only the actinides, whereas variant V4 uses spent uranium fuel with actinides and fission products. There are also four variations of the fresh MOX fuel: fresh MOX fuel, MOX fuel with only ${ }^{239} \mathrm{Pu}$, MOX fuel with only ${ }^{240} \mathrm{Pu}$, and MOX fuel with only ${ }^{241} \mathrm{Pu}$. The basic fuel material isotopic composition for each variant is given in Table 5.2.

There are four nonfuel material compositions in the set of calculational benchmarks: 1 clad composition, which is common to all problems, and 3 moderator material compositions. The variations in the moderator compositions are due to the presence or absence of ${ }^{10} \mathrm{~B}$ and ${ }^{11} \mathrm{~B}$, the clad and moderator temperature, and the moderator density. The material isotopic compositions for the clad and each moderator are given in Table 5.3.

Table 5.1. State parameters for the calculational benchmark variants

\begin{tabular}{cccccc}
\hline State & $\begin{array}{c}\text { Fuel zone } \\
\text { temp }(\mathrm{K})\end{array}$ & $\begin{array}{c}\text { Nonfuel zone } \\
\text { temp }(\mathrm{K})\end{array}$ & $\begin{array}{c}{ }^{135} \mathrm{Xe},{ }^{149} \mathrm{Sm} \\
\text { in fuel }\end{array}$ & $\begin{array}{c}{ }^{10} \mathrm{~B},{ }^{11} \mathrm{~B} \text { in } \\
\text { moderator }\end{array}$ & $\begin{array}{c}\text { Moderator } \\
\text { material }\end{array}$ \\
\hline S1 & 1027 & 579 & Yes & Yes & MOD1 \\
S3 & 1027 & 579 & Yes & No & MOD2 \\
S4 & 1027 & 579 & No & Yes & MOD1 \\
S5 & 579 & 579 & No & Yes & MOD1 \\
S6 & 300 & 300 & No & Yes & MOD3 \\
\hline
\end{tabular}


Table 5.2. Basic fuel isotopic composition for each computational benchmark variant

\begin{tabular}{|c|c|c|c|c|c|}
\hline Variant & Comment & Isotope & $\begin{array}{l}\text { Atom density } \\
\left(10^{-24} \mathrm{~cm}^{-3}\right)\end{array}$ & $\begin{array}{c}\text { Isotop } \\
\mathrm{e}\end{array}$ & $\begin{array}{l}\text { Atom density } \\
\left(10^{-24} \mathrm{~cm}^{-3}\right)\end{array}$ \\
\hline V1 & Fresh uranium fuel & ${ }^{235} \mathrm{U}$ & $\begin{array}{l}8.7370 \times 10^{-4} \\
1.8744 \times 10^{-2}\end{array}$ & ${ }^{16} \mathrm{O}$ & $3.9235 \times 10^{-2}$ \\
\hline V2 & Fresh MOX fuel & $\begin{array}{l}{ }^{235} \mathrm{U} \\
{ }^{238} \mathrm{U} \\
{ }^{16} \mathrm{O}\end{array}$ & $\begin{array}{l}3.8393 \times 10^{-5} \\
1.8917 \times 10^{-2} \\
4.1707 \times 10^{-2}\end{array}$ & $\begin{array}{l}{ }^{239} \mathrm{Pu} \\
{ }^{240} \mathrm{Pu} \\
{ }^{41} \mathrm{Pu}\end{array}$ & $\begin{array}{l}6.5875 \times 10^{-4} \\
4.2323 \times 10^{-5} \\
7.0246 \times 10^{-4}\end{array}$ \\
\hline V3 & $\begin{array}{l}\text { Spent uranium fuel with } \\
\text { no fission products } \\
\text { ( actinides only ) }\end{array}$ & $\begin{array}{l}{ }^{235} \mathrm{U} \\
{ }^{236} \mathrm{U} \\
{ }^{238} \mathrm{U} \\
{ }^{237} \mathrm{~Np} \\
{ }^{238} \mathrm{Pu} \\
{ }^{239} \mathrm{~Np} \\
{ }^{239} \mathrm{Pu} \\
{ }^{240} \mathrm{Pu} \\
{ }^{241} \mathrm{Pu}\end{array}$ & $\begin{array}{l}3.7843 \times 10^{-4} \\
8.6365 \times 10^{-5} \\
1.8327 \times 10^{-2} \\
2.4823 \times 10^{-5} \\
6.7254 \times 10^{-6} \\
1.8332 \times 10^{-6} \\
1.3111 \times 10^{-4} \\
3.6233 \times 10^{-5} \\
2.1701 \times 10^{-5}\end{array}$ & $\begin{array}{l}{ }^{242} \mathrm{Pu} \\
{ }^{241} \mathrm{Am} \\
{ }^{242 \mathrm{~m}} \mathrm{Am} \\
{ }^{243} \mathrm{Am} \\
{ }^{242} \mathrm{Cm} \\
{ }^{243} \mathrm{Cm} \\
{ }^{244} \mathrm{Cm} \\
{ }^{16} \mathrm{O}\end{array}$ & $\begin{array}{l}4.7576 \times 10^{-6} \\
4.9491 \times 10^{-7} \\
7.9194 \times 10^{-9} \\
6.6925 \times 10^{-7} \\
1.2582 \times 10^{-7} \\
2.0629 \times 10^{-9} \\
1.2387 \times 10^{-7} \\
3.9235 \times 10^{-2}\end{array}$ \\
\hline V4 & $\begin{array}{l}\text { Spent uranium fuel } \\
\text { ( actinides }+ \\
\text { fission products ) } \\
\text { ( V3 + listed isotopes ) }\end{array}$ & 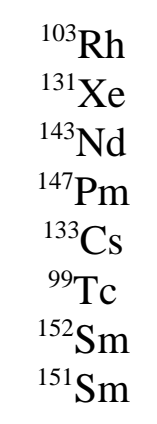 & $\begin{array}{l}1.8890 \times 10^{-4} \\
1.4255 \times 10^{-5} \\
2.6692 \times 10^{-2} \\
6.1574 \times 10^{-5} \\
3.5974 \times 10^{-6} \\
3.3320 \times 10^{-6} \\
2.6842 \times 10^{-4} \\
3.0757 \times 10^{-5}\end{array}$ & $\begin{array}{l}{ }^{{ }^{145} \mathrm{Nd}} \\
{ }^{153} \mathrm{Eu} \\
{ }^{109} \mathrm{Ag} \\
{ }^{155} \mathrm{Eu} \\
{ }^{95} \mathrm{Mo} \\
{ }^{154} \mathrm{Eu} \\
{ }^{101} \mathrm{Ru}\end{array}$ & $\begin{array}{l}1.9975 \times 10^{-6} \\
2.4801 \times 10^{-7} \\
2.2037 \times 10^{-9} \\
9.6857 \times 10^{-7} \\
3.3720 \times 10^{-7} \\
5.1189 \times 10^{-9} \\
3.1134 \times 10^{-7}\end{array}$ \\
\hline V7 & $\begin{array}{l}\text { Fresh MOX fuel with } \\
{ }^{239} \mathrm{Pu} \text { as only } \mathrm{Pu}\end{array}$ & ${ }^{235} \mathrm{U}$ & $\begin{array}{l}3.8393 \times 10^{-5} \\
1.8917 \times 10^{-2}\end{array}$ & ${ }^{{ }^{16} \mathrm{O}} \mathrm{O}$ & $\begin{array}{l}6.5875 \times 10^{-4} \\
4.1707 \times 10^{-2}\end{array}$ \\
\hline V8 & $\begin{array}{l}\text { Fresh MOX fuel with } \\
{ }^{240} \mathrm{Pu} \text { as only } \mathrm{Pu}\end{array}$ & ${ }^{235} \mathrm{U}$ & $\begin{array}{l}6.9714 \times 10^{-4} \\
1.8917 \times 10^{-2}\end{array}$ & ${ }^{240} \mathrm{Pu}$ & $\begin{array}{l}4.2323 \times 10^{-5} \\
4.1707 \times 10^{-2}\end{array}$ \\
\hline V9 & $\begin{array}{l}\text { Fresh MOX fuel with } \\
{ }^{241} \mathrm{Pu} \text { as only } \mathrm{Pu}\end{array}$ & ${ }^{235} \mathrm{U}$ & $\begin{array}{l}3.8393 \times 10^{-5} \\
1.8917 \times 10^{-2}\end{array}$ & ${ }^{41} \mathrm{Pu}$ & $\begin{array}{l}6.6577 \times 10^{-4} \\
4.1707 \times 10^{-2}\end{array}$ \\
\hline V10 & $\begin{array}{l}\text { Fresh MOX fuel with } \\
\text { recycled reactor } \mathrm{Pu}\end{array}$ & $\begin{array}{c}{ }^{235} \mathrm{U} \\
{ }^{238} \mathrm{U} \\
{ }^{16} \mathrm{O} \\
{ }^{238} \mathrm{Pu} \\
{ }^{239} \mathrm{Pu}\end{array}$ & $\begin{array}{l}5.0000 \times 10^{-5} \\
2.2100 \times 10^{-2} \\
4.6300 \times 10^{-2} \\
3.0000 \times 10^{-5} \\
1.1600 \times 10^{-3}\end{array}$ & $\begin{array}{l}{ }^{240} \mathrm{Pu} \\
{ }^{241} \mathrm{Pu} \\
{ }^{242} \mathrm{Pu} \\
{ }^{241} \mathrm{Am}\end{array}$ & $\begin{array}{l}4.9000 \times 10^{-4} \\
1.9000 \times 10^{-4} \\
1.0500 \times 10^{-4} \\
2.5000 \times 10^{-5}\end{array}$ \\
\hline
\end{tabular}


Table 5.3. Nonfuel material isotopic composition

\begin{tabular}{|c|c|c|c|c|c|}
\hline Material & Comment & Isotope & $\begin{array}{l}\text { Atom density } \\
\left(10^{-24} \mathrm{~cm}^{-3}\right)\end{array}$ & Isotope & $\begin{array}{l}\text { Atom density } \\
\left(10^{-24} \mathrm{~cm}^{-3}\right)\end{array}$ \\
\hline Clad & Cladding & $\mathrm{Zr}$ & $4.2300 \times 10^{-2}$ & & \\
\hline MOD1 & $\begin{array}{l}\text { Hot moderator } \\
\text { with boric acid }\end{array}$ & $\begin{array}{c}\mathrm{H} \\
{ }^{16} \mathrm{O}\end{array}$ & $\begin{array}{l}4.7830 \times 10^{-2} \\
2.3910 \times 10^{-2}\end{array}$ & $\begin{array}{l}{ }^{10} \mathrm{~B} \\
{ }^{11} \mathrm{~B}\end{array}$ & $\begin{array}{l}4.7344 \times 10^{-6} \\
1.9177 \times 10^{-5}\end{array}$ \\
\hline MOD2 & $\begin{array}{l}\text { Hot moderator } \\
\text { without boric acid }\end{array}$ & $\begin{array}{c}\mathrm{H} \\
{ }^{16} \mathrm{O}\end{array}$ & $\begin{array}{l}4.7830 \times 10^{-2} \\
2.3910 \times 10^{-2}\end{array}$ & & \\
\hline MOD3 & $\begin{array}{l}\text { Cold moderator } \\
\text { with boric acid }\end{array}$ & $\begin{array}{c}\mathrm{H} \\
{ }^{16} \mathrm{O}\end{array}$ & $\begin{array}{l}6.6940 \times 10^{-2} \\
3.3470 \times 10^{-2}\end{array}$ & $\begin{array}{l}{ }^{10} \mathrm{~B} \\
{ }^{11} \mathrm{~B}\end{array}$ & $\begin{array}{l}6.6262 \times 10^{-6} \\
2.6839 \times 10^{-5}\end{array}$ \\
\hline
\end{tabular}

\subsection{ANALYSIS}

All computational benchmarks were processed using SCALE 5.0. The cases labeled NITAWL utilize the following sequence: CSAS, BONAMI, NITAWL, XSDRNPM. The cases labeled CENTRM utilize the following sequence: CSAS, BONAMI, NITAWL, CENTRM, PMC, WORKER, XSDRNPM. In the NITAWL cases the resolved resonance region is self-shielded using NITAWL. In the CENTRM cases the resolved resonance region is self-shielded using CENTRM and PMC, which removes the limitations of the Nordheim Integral Treatment. Table 5.4 contains the results for the infinite arrays, and Table 5.5 contains the results for the buckled arrays.

For each variant and state, the differences between the NITAWL and CENTRM multiplication factors $\left(k_{4}\right.$ and buckled $\left.k_{4}\right)$ and the energy of the average lethargy causing fission (EALCF) are almost identical for the infinite and buckled geometries as shown in Tables 5.4 and 5.5. The differences in the multiplication between the CENTRM and NITAWL cases range from $-0.09 \%$ to $-0.55 \%$. Trends are apparent based on both fuel material and temperature. The smallest difference occurs for variant V4, state S6, which is spent uranium fuel with actinides and fission products at room temperature. The largest difference occurs for variant V9, state S1, which is fresh MOX fuel with all the $\mathrm{Pu}$ as ${ }^{241} \mathrm{Pu}$ at reactor temperatures.

The selection and number of nuclides in the fuel has little effect on the difference between NITAWL and CENTRM, at most $\sim 0.25 \%$, which occurs when the amount of ${ }^{241} \mathrm{Pu}$ is at its maximum. The effects of resonance overlap do not seem to be significant. The lowest difference at any given temperature occurs for spent uranium fuel with actinides and fission products. The amount of absorbers in the fuel $\left({ }^{135} \mathrm{Xe}\right.$ and $\left.{ }^{149} \mathrm{Sm}\right)$ and in the moderator $\left({ }^{10} \mathrm{~B}\right)$ also does not affect the differences.

Fuel and moderator temperature have the most obvious, albeit still small, effect on the difference between NITAWL and CENTRM results. For a given variant, the largest difference always occurs with the fuel at $1027 \mathrm{~K}$ and the moderator at $579 \mathrm{~K}$. Decreasing the fuel temperature to $579 \mathrm{~K}$ always decreases the difference, as much as $0.1 \%$. Further decreasing the fuel and moderator temperature to $300 \mathrm{~K}$ always further decreases the difference, as much as another $0.1 \%$. Both NITAWL and 
CENTRM interpolate data between temperatures.

The EALCF is also calculated for each variant and state. The differences in the average-energy between the NITAWL and CENTRM cases range from a low of $-0.41 \%$ to a high of $1.66 \%$. Trends are apparent based only on temperature. The smallest differences with each variant occur in state S6, and the largest differences occurs for states 1,2, and 3. As the pin temperature drops, the differences between the CSAS and CENTRM results lessen.

\subsection{CONCLUSIONS}

For this set of cases using either NITAWL or CENTRM as the resonance region processor produces consistent results. The $k_{\text {eff }}$ values are slightly lower, $\sim 0.3 \%$, for all CENTRM cases. The nuclides selected do not appear to have an effect on the difference, but the temperature of the system does have a slight effect: the point library has temperature data well above that available in the multigroup library. The approximations used to process the resolved resonance data in NITAWL do not significantly affect the results, as shown by the good agreement with the CENTRM results. 
Table 5.4a. $k_{4}$ and EALCF values and differences for the variant cases

\begin{tabular}{|c|c|c|c|c|c|c|}
\hline $\begin{array}{c}\text { VARIANTS } \\
\text { V1 - V4 }\end{array}$ & $\begin{array}{c}\text { NITAWL } \\
k_{4}\end{array}$ & $\begin{array}{c}\text { CENTRM } \\
k_{4}\end{array}$ & $\begin{array}{ll} & k_{4} \\
\% & \text { DIFF }\end{array}$ & $\begin{array}{c}\text { NITAWL } \\
\text { EALCF } \\
(\mathrm{eV})\end{array}$ & $\begin{array}{c}\text { CENTRM } \\
\text { EALCF } \\
(\mathrm{eV})\end{array}$ & $\begin{array}{l}\text { EALCF } \\
\% \text { DIFF }\end{array}$ \\
\hline V1S1 & 1.2593 & 1.2541 & -0.41 & 0.7766 & 0.7806 & 0.52 \\
\hline V1S3 & 1.3144 & 1.3090 & -0.41 & 0.6984 & 0.7019 & 0.5 \\
\hline V1S4 & 1.3069 & 1.3015 & -0.41 & 0.7052 & 0.7087 & 0.5 \\
\hline V1S5 & 1.3249 & 1.3205 & -0.33 & 0.6846 & 0.6877 & 0.45 \\
\hline V1S6 & 1.3669 & 1.3634 & -0.26 & 0.2808 & 0.2819 & 0.39 \\
\hline V2S1 & 1.2074 & 1.2017 & -0.47 & 1.5678 & 1.5873 & 1.24 \\
\hline V2S3 & 1.2360 & 1.2300 & -0.49 & 1.4690 & 1.4871 & 1.23 \\
\hline V2S4 & 1.2270 & 1.2212 & -0.47 & 1.4907 & 1.5090 & 1.23 \\
\hline V2S5 & 1.2474 & 1.2428 & -0.37 & 1.4305 & 1.4460 & 1.08 \\
\hline V2S6 & 1.3256 & 1.3210 & -0.35 & 0.5514 & 0.5568 & 0.98 \\
\hline V3S1 & 1.0821 & 1.0767 & -0.5 & 0.9976 & 1.0064 & 0.88 \\
\hline V3S3 & 1.1267 & 1.1210 & -0.51 & 0.8982 & 0.9058 & 0.85 \\
\hline V3S4 & 1.1218 & 1.1162 & -0.5 & 0.9027 & 0.9103 & 0.84 \\
\hline V3S5 & 1.1412 & 1.1357 & -0.48 & 0.8694 & 0.877 & 0.87 \\
\hline V3S6 & 1.1902 & 1.1862 & -0.34 & 0.3271 & 0.3289 & 0.55 \\
\hline V4S1 & 1.0158 & 1.0140 & -0.18 & 1.1496 & 1.1532 & 0.31 \\
\hline V4S3 & 1.0558 & 1.0536 & -0.21 & 1.0346 & 1.0385 & 0.38 \\
\hline V4S4 & 1.0510 & 1.0490 & -0.19 & 1.0411 & 1.0442 & 0.3 \\
\hline V4S5 & 1.0688 & 1.0669 & -0.18 & 1.0081 & 1.0055 & -0.26 \\
\hline V4S6 & 1.1197 & 1.1187 & -0.09 & 0.3678 & 0.368 & 0.05 \\
\hline
\end{tabular}


Table 5.4b. $k_{4}$ and EALCF values and differences for the variant cases (cont.)

\begin{tabular}{|c|c|c|c|c|c|c|}
\hline $\begin{array}{c}\text { VARIANTS } \\
\text { V7 - V10 } \\
\end{array}$ & $\begin{array}{c}\text { CSAS } \\
k_{4} \\
\end{array}$ & $\begin{array}{c}\text { CENTRM } \\
k_{4} \\
\end{array}$ & $\begin{array}{c}k_{4} \\
\% \text { DIFF } \\
\end{array}$ & $\begin{array}{c}\text { CSAS } \\
\text { EALCF } \\
(\mathrm{eV}) \\
\end{array}$ & $\begin{array}{c}\text { CENTRM } \\
\text { EALCF } \\
(\mathrm{eV}) \\
\end{array}$ & $\begin{array}{l}\text { EALCF } \\
\% \text { DIFF }\end{array}$ \\
\hline V7S1 & 1.3047 & 1.2984 & -0.48 & 1.2704 & 1.2851 & 1.16 \\
\hline V7S3 & 1.3372 & 1.3307 & -0.49 & 1.1922 & 1.2058 & 1.14 \\
\hline V7S4 & 1.3270 & 1.3206 & -0.48 & 1.2090 & 1.2228 & 1.14 \\
\hline V7S5 & 1.3459 & 1.3404 & -0.41 & 1.1687 & 1.1816 & 1.1 \\
\hline V7S6 & 1.4112 & 1.4067 & -0.32 & 0.4793 & 0.4835 & 0.88 \\
\hline V8S1 & 1.0904 & 1.0859 & -0.41 & 0.8038 & 0.8083 & 0.56 \\
\hline V8S3 & 1.1442 & 1.1395 & -0.41 & 0.7108 & 0.7146 & 0.53 \\
\hline V8S4 & 1.1399 & 1.1351 & -0.42 & 0.7147 & 0.7186 & 0.55 \\
\hline V8S5 & 1.1596 & 1.1557 & -0.34 & 0.6881 & 0.6912 & 0.45 \\
\hline V8S6 & 1.2115 & 1.2084 & -0.26 & 0.2694 & 0.2704 & 0.37 \\
\hline V9S1 & 1.5290 & 1.5207 & -0.54 & 1.4193 & 1.4140 & -0.37 \\
\hline V9S3 & 1.5644 & 1.5561 & -0.53 & 1.3346 & 1.3293 & -0.4 \\
\hline V9S4 & 1.5515 & 1.5432 & -0.53 & 1.3585 & 1.3529 & -0.41 \\
\hline V9S5 & 1.5736 & 1.5668 & -0.43 & 1.3191 & 1.3181 & -0.08 \\
\hline V9S6 & 1.6195 & 1.6140 & -0.34 & 0.5601 & 0.5602 & 0.02 \\
\hline V10S1 & 1.0952 & 1.0902 & -0.46 & 9.5809 & 9.7345 & 1.6 \\
\hline V10S3 & 1.1078 & 1.1027 & -0.46 & 9.118 & 9.2636 & 1.6 \\
\hline V10S4 & 1.1010 & 1.0958 & -0.47 & 9.3374 & 9.4863 & 1.59 \\
\hline V10S5 & 1.1196 & 1.1160 & -0.32 & 8.7717 & 8.8831 & 1.27 \\
\hline V10S6 & 1.1937 & 1.1917 & -0.17 & 2.614 & 2.6373 & 0.89 \\
\hline
\end{tabular}


Table 5.5a. Buckled $k_{4}$ and EALCF values and differences for the variant cases

\begin{tabular}{|c|c|c|c|c|c|c|}
\hline $\begin{array}{c}\text { VARIANTS } \\
\text { V1 - V4 }\end{array}$ & $\begin{array}{c}\text { CSAS } \\
\text { buckled } \\
k_{4}\end{array}$ & $\begin{array}{c}\text { CENTRM } \\
\text { buckled } \\
k_{4} \\
\end{array}$ & $\begin{array}{c}k_{4} \\
\% \text { DIFF }\end{array}$ & $\begin{array}{c}\text { CSAS } \\
\text { EALCF } \\
(\mathrm{eV})\end{array}$ & $\begin{array}{c}\text { CENTRM } \\
\text { EALCF } \\
(\mathrm{eV})\end{array}$ & $\begin{array}{l}\text { EALCF } \\
\% \text { DIFF }\end{array}$ \\
\hline V1S1 & 1.0802 & 1.0757 & -0.42 & 0.8408 & 0.8455 & 0.56 \\
\hline V1S3 & 1.1266 & 1.1218 & -0.43 & 0.755 & 0.7592 & 0.56 \\
\hline V1S4 & 1.1201 & 1.1154 & -0.42 & 0.7628 & 0.7670 & 0.55 \\
\hline V1S5 & 1.1353 & 1.1314 & -0.34 & 0.7402 & 0.7438 & 0.49 \\
\hline V1S6 & 1.2286 & 1.2253 & -0.27 & 0.2932 & 0.2944 & 0.41 \\
\hline V2S1 & 1.0396 & 1.0346 & -0.48 & 1.6927 & 1.7146 & 1.29 \\
\hline V2S3 & 1.0638 & 1.0586 & -0.49 & 1.5847 & 1.605 & 1.28 \\
\hline V2S4 & 1.0562 & 1.0511 & -0.48 & 1.6086 & 1.6293 & 1.29 \\
\hline V2S5 & 1.1050 & 1.1007 & -0.39 & 1.5477 & 1.5659 & 1.18 \\
\hline V2S6 & 1.2167 & 1.2125 & -0.35 & 0.5755 & 0.5814 & 1.03 \\
\hline V3S1 & 0.9547 & 0.9499 & -0.5 & 1.0872 & 1.0973 & 0.93 \\
\hline V3S3 & 0.9932 & 0.9882 & -0.5 & 0.9775 & 0.9863 & 0.9 \\
\hline V3S4 & 0.9889 & 0.9839 & -0.51 & 0.9827 & 0.9917 & 0.92 \\
\hline V3S5 & 1.0059 & 1.0011 & -0.48 & 0.9457 & 0.9542 & 0.9 \\
\hline V3S6 & 1.0887 & 1.0850 & -0.34 & 0.3425 & 0.3445 & 0.58 \\
\hline V4S1 & 0.8975 & 0.8958 & -0.19 & 1.2555 & 1.2600 & 0.36 \\
\hline V4S3 & 0.9322 & 0.9301 & -0.23 & 1.1285 & 1.1334 & 0.43 \\
\hline V4S4 & 0.9279 & 0.9261 & -0.19 & 1.1361 & 1.1400 & 0.34 \\
\hline V4S5 & 0.9435 & 0.9418 & -0.18 & 1.0923 & 1.0963 & 0.37 \\
\hline V4S6 & 1.0253 & 1.0242 & -0.11 & 0.3847 & 0.3858 & 0.29 \\
\hline
\end{tabular}


Table 5.5b. Buckled $k_{4}$ and EALCF values and differences for the variant cases (cont.)

\begin{tabular}{|c|c|c|c|c|c|c|}
\hline $\begin{array}{c}\text { VARIANTS } \\
\text { V7 - V10 }\end{array}$ & $\begin{array}{c}\text { CSAS } \\
\text { buckled } \\
k_{4} \\
\end{array}$ & $\begin{array}{c}\text { CENTRM } \\
\text { buckled } \\
k_{4} \\
\end{array}$ & $\begin{aligned} & k_{4} \\
& \% \text { DIFF } \\
&\end{aligned}$ & $\begin{array}{c}\text { CSAS } \\
\text { EALCF } \\
(\mathrm{eV})\end{array}$ & $\begin{array}{c}\text { CENTRM } \\
\text { EALCF } \\
(\mathrm{eV}) \\
\end{array}$ & $\begin{array}{l}\text { EALCF } \\
\% \text { DIFF }\end{array}$ \\
\hline V7S 1 & 1.1560 & 1.1504 & -0.48 & 1.3698 & 1.3865 & 1.22 \\
\hline V7S3 & 1.1844 & 1.1786 & -0.49 & 1.2846 & 1.300 & 1.2 \\
\hline V7S4 & 1.1755 & 1.1697 & -0.49 & 1.3031 & 1.3187 & 1.2 \\
\hline V7S5 & 1.1920 & 1.1870 & -0.42 & 1.2589 & 1.2734 & 1.15 \\
\hline V7S6 & 1.2955 & 1.2913 & -0.32 & 0.4991 & 0.5037 & 0.92 \\
\hline V8S1 & 0.9628 & 0.9588 & -0.42 & 0.8777 & 0.8831 & 0.62 \\
\hline V8S3 & 1.0093 & 1.0050 & -0.43 & 0.7748 & 0.7794 & 0.59 \\
\hline V8S4 & 1.0055 & 1.0012 & -0.43 & 0.7794 & 0.7841 & 0.6 \\
\hline V8S5 & 1.0227 & 1.0192 & -0.34 & 0.7496 & 0.7534 & 0.51 \\
\hline V8S6 & 1.1095 & 1.1066 & -0.26 & 0.2819 & 0.2829 & 0.35 \\
\hline V9S1 & 1.3591 & 1.3515 & -0.56 & 1.5135 & 1.5087 & -0.32 \\
\hline V9S3 & 1.3901 & 1.3825 & -0.55 & 1.4225 & 1.4177 & -0.34 \\
\hline V9S4 & 1.3788 & 1.3712 & -0.55 & 1.4481 & 1.4431 & -0.35 \\
\hline V9S5 & 1.3982 & 1.3920 & -0.44 & 1.4055 & 1.4052 & -0.02 \\
\hline V9S6 & 1.4906 & 1.4855 & -0.34 & 0.5802 & 0.5803 & 0.02 \\
\hline V10S1 & 0.9876 & 0.9831 & -0.46 & 10.483 & 10.657 & 1.66 \\
\hline V10S3 & 0.9988 & 0.9941 & -0.47 & 9.9769 & 10.142 & 1.65 \\
\hline V10S4 & 0.9927 & 0.9881 & -0.46 & 10.216 & 10.385 & 1.65 \\
\hline V10S5 & 1.0094 & 1.0061 & -0.33 & 9.5901 & 9.7165 & 1.32 \\
\hline V10S6 & 1.1087 & 1.1069 & -0.16 & 2.7455 & 2.7705 & 0.91 \\
\hline
\end{tabular}




\section{REFERENCES}

1. SCALE: A Modular Code System for Performing Standardized Analyses for Licensing Evaluations, NUREG/CR-0200, Rev. 4 (ORNL/NUREG/CSD-2/R4), Vols. I, II, and III, April 1995. Available from Radiation Safety Information Computational Center at Oak Ridge National Laboratory as CCC-545.

2. M. Williams and M. Asgari, "Computation of Continuous-Energy Neutron Spectra with Discrete Ordinates Transport Theory," Nucl. Sci. Eng. 121, 173B201 (1995).

3. International Handbook of Evaluated Criticality Safety Benchmark Experiments, HEU-METFAST-035 Benchmark, The Uranium/Iron Benchmark Assembly: A 235U(93\%)/Iron Cylinder Reflected by Stainless Steel, NEA/NSC/DOC(95)03 (September 1998).

4. Neutronics Benchmarks for the Utilization of Mixed-oxide Fuel: Joint U.S./Russian Progress Report for Fiscal Year 1997, Volume 3 - Calculations Performed in the Russian Federation, ORNL/TM-13603/V3, June 1998 



\section{INTERNAL DISTRIBUTION}

1. B. B. Bevard

2. S. M. Bowman

3. B. L. Broadhead

4-8. W. C. Carter (5)

9. E. D. Collins

10. B. S. Cowell

11. M. D. DeHart

12. M. E. Dunn

13. K. R. Elam

14. R. J Ellis

15. M. B. Emmett

16. I. C. Gauld

17. J. C. Gehin

18. N. M. Greene

19. S. R. Greene

20. O. W. Hermann

21-25. D. F. Hollenbach (5)

26. C. M. Hopper

27. O. W. Hermann

28. D. T. Ingersoll
29. H. T. Kerr

30. M. A. Kuliasha

31. L. C. Leal

32. S. B. Ludwig

33. G. E. Michaels

34. D. L. Moses

35. B. D. Murphy

36. L. M. Petrie

37-41. C. V. Parks (5)

42. L. M. Petrie

43-47. R. T. Primm (5)

48. B. T. Rearden

49. C. H. Shappert

50. T. E. Valentine

51. J. C. Wagner

52. R. M. Westfall

53. R. Q. Wright

54. Central Research Library Document Reference Section

55-56. ORNL Laboratory Records (2) for submission to OSTI

57. ORNL Laboratory Records - RC

\section{EXTERNAL DISTRIBUTION}

58. M. L. Adams, Department of Nuclear Engineering, Texas A\&M University, Zachry 129, College Station, TX 77843

59. R. E. Anderson, Los Alamos National Laboratory, P.O. Box 1663, NIS-6, MS J562, Los Alamos, NM 87545

60. W. L. Andrews, Defense Nuclear Facilities Safety Board, 625 Indiana Ave., Washington, DC 20004

61. M. G. Bailey, U.S. Nuclear Regulatory Commission, MS O6-G22, Washington, DC 20555

62. J. B. Briggs, INEEL, P.O. Box 1625, MS-3855, Idaho Falls, ID 83402

63. T. Burns, Defense Nuclear Facilities Safety Board, 625 Indiana Ave., Washington, DC 20004

64. D. E. Carlson, U.S. Nuclear Regulatory Commission, MS O6-F18, Washington, DC 20555 
65. I. Carron, Department of Nuclear Engineering, Texas A\&M University, 129 Zachry, College Station, TX 77843-3133

66. D. R. Damon, U.S. Nuclear Regulatory Commission, MS T8-H7, Washington, DC 20555

67. J. R. Davis, U.S. Nuclear Regulatory Commission, MS T8-A33, Washington, DC 20555

68. R. L. Dintaman, U.S. Department of Energy, DP-13, 19901 Germantown Rd., Germantown, MD 20874-1290

69. H. L. Dodds, University of Tennessee, Nuclear Engineering Dept., 214 Pasqua Engineering Bldg., Knoxville, TN 37922

70. T. W. Doering, Framatome Cogema Fuels, 1261 Town Center Drive, Las Vegas, NV 89124

71. J. D. Evans, U.S. Department of Energy, S-3.1/VISTA, 1000 Independence Ave., S.W., Washington, DC 22085

72. H. D. Felsher, U.S. Nuclear Regulatory Commission, MS T8-H7, Washington, DC 20555

73. P. Felsher, Rocky Flats Environment Technology Site, Bldg. T886B, P.O. Box 464, Golden, CO 80402-0464

74. J. R. Felty, Science Applications Int'l Corp., 2418 N. Dickerson St., Arlington, VA 22207

75. I. E. Fergus, U.S. Department of Energy, EH-22, 20300 Century Blvd., Germantown, MD 20874

76. E. K. Fujita, Argonne National Laboratory, 9700 South Cass Avenue, RA/208, Argonne, IL 60439-4842

77. A. S. Garcia, U.S. Department of Energy, Idaho Operations Office, 850 Energy Dr., MS 1154, Idaho Falls, ID 83401-1563

78. S. C. Keeton, Lawrence Livermore National Laboratory, P.O. Box 808 (L-128), Livermore, CA 94550

79. R. C. Little, Los Alamos National Laboratory, MS F663, P.O. Box 1663, Los Alamos, NM 87545

80. J. McKamy, U.S. Department of Energy, EH-34, 19901 Germantown Road, Germantown, MD 20874-1290

81. R. D. McKnight, Argonne National Laboratory, 9700 S. Cass Ave., Argonne, IL 604394842

82. T. P. McLaughlin, Los Alamos National Laboratory, P.O. Box 1663, Los Alamos, NM 87545

83. D. E.I. Mennerdal, E M Systems, Starvãgen 12, Täby, SWEDEN S-18357

84. D. C. Morey, U.S. Nuclear Regulatory Commission, MS T8-A33, Washington, DC 20555

85. J. A. Morman, Argonne National Laboratory, 9700 S. Cass Ave., Bldg. 208, C237B, Argonne, IL 60439-4842

86. R. D. Mosteller, Los Alamos National Laboratory, MS J562, Los Alamos, NM 87545

87. K. A. Niemer, Duke Engineering \& Services, 400 S. Tryon St., WC26B, P.O. Box 1004, Charlotte, NC 28201-1004

88. H. Okuno, Japan Atomic Energy Research Institute, 2-4 Shirakara-shirane, Tokai-mura, Ibaraki-ken, JAPAN 319-1195 
89. R. E. Pevey, University of Tennessee, Nuclear Engineering Dept., 214 Pasqua Engineering Bldg., Knoxville, TN 37922

90. M. Rahimi, U.S. Nuclear Regulatory Commission, M/S T7F3, Washington, DC 20555

91. P. T. Rhoads, Office of Fissile Materials Disposition, U.S. Department of Energy, MD4, 1000 Independence Avenue SW, Washington, DC 20585

92. B. Rothleder, U.S. Department of Energy, EH-31, 19901 Germantown Rd., Germantown, MD 20874-1290

93. M. K. Sheaffer, Lawrence Livermore National Laboratory, P.O. Box 808, L-634, Livermore, CA 94550

94. T. Taylor, Lockheed Martin Idaho Tech. Co., P.O. Box 1625, MS 3458, Idaho Falls, ID 83415-3458

95. C. Tripp, U.S. Nuclear Regulatory Commission, MS T8-A33, Washington, DC 20555

96. J. A. Thornton, Duke Engineering \& Services, 400 S. Tryon St., WC26B, P.O. Box 1004, Charlotte, NC 28201-1004

97. H. Toffer, Fluor Daniel Northwest, Inc., P.O. Box 1050, MSIN B4-44, 1100 Jadwin Ave., Richland, WA 99352-1050

98. E. F. Trumble, Westinghouse Safety Management Solutions, P.O. Box 5388, 1993 South Centennial Dr., Aiken, SC 29803

99. M. Wangler, Office of Site Operations, EM-70/CLV-1059, U.S. Department of Energy, 19901 Germantown Road, Germantown, MD 20874-1290

100. L. Wetzel, BWX Technologies, Inc., Naval Nuclear Fuel, P.O. Box 785, Lynchburg, VA 24505

101. B. H. White IV, U.S. Nuclear Regulatory Commission, MS O6-F18, Washington, DC 20555

102. Mark Williams, Louisiana State University, Baton Rouge, LA 70803-5820

103. R. E. Wilson, Safe Sites of Colorado, P.O. Box 464, Golden, CO 80402-0464

104 C. J. Withee, U.S. Nuclear Regulatory Commission, MS O6-G22, Washington, DC 20555 